\title{
Unusual Sesquiterpenes: Gorgonenes and Further Bioactive Secondary Metabolites Derived from Marine and Terrestrial Bacteria
}

\author{
Dissertation \\ zur Erlangung des Doktorgrades \\ der Mathematisch-Naturwissenschaftlichen Fakultäten \\ der Georg-August-Universität zu Göttingen
}

vorgelegt von

Md. Hafizur Rahman

aus Satkhira, Bangladesh

Göttingen 2008 
Referent: Prof. Dr. Hartmut Laatsch

Korreferent: Prof. Dr. A. Zeeck

Tag der mündlichen Prüfung: 29. Oktober 2008 
Die vorliegende Dissertation wurde im Institut für Organische und Biomolekulare Chemie der Universität Göttingen in der Zeit vom August 2004 bis Oktober 2008 unter der Leitung von Herrn Prof. Dr. Hartmut Laatsch durchgeführt. 


\section{Dedicated to my beloved parents Md Fazlur Rahman and Mrs. Fazilatunnessa}




\section{Contents}

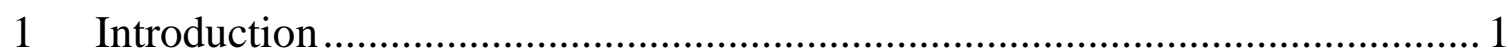

1.1 History of the use of natural products for medical applications ........................2

1.2 Approaches for the search of new natural products ......................................

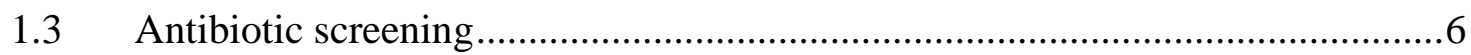

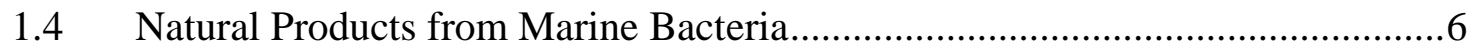

1.5 Therapeutic areas influenced by natural products ........................................

1.6 Natural products against infectious diseases; bacterial pathogens ....................9

1.7 Antifungal compounds from natural sources................................................. 10

1.8 Antibacterial compounds from natural sources ..........................................11

1.9 Potential antiparasitic drugs originated from natural products........................13

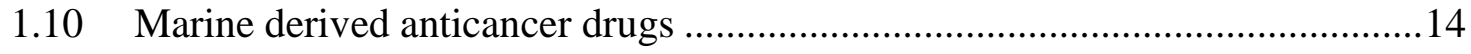

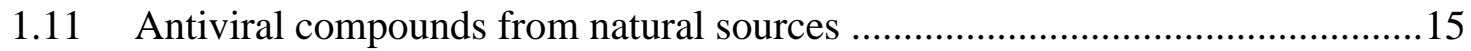

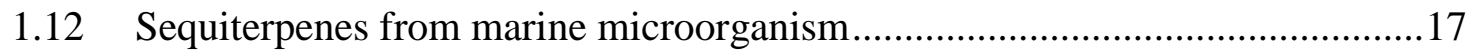

2 Objectives of the present investigation ..................................................... 18

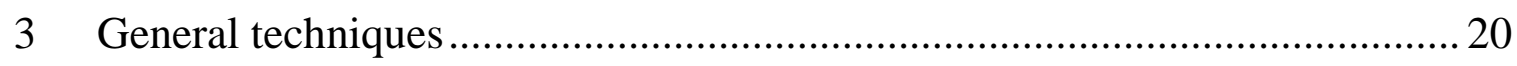

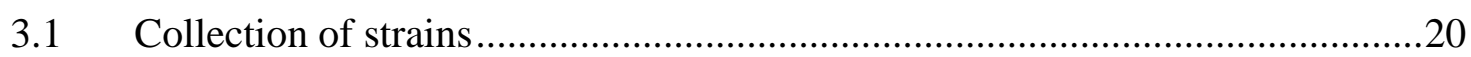

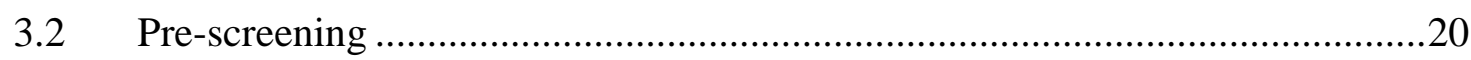

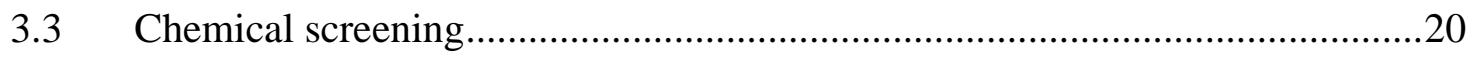

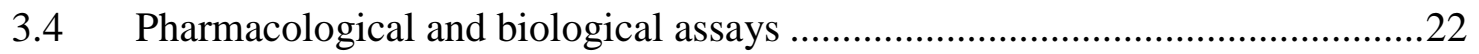

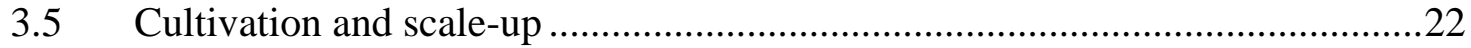

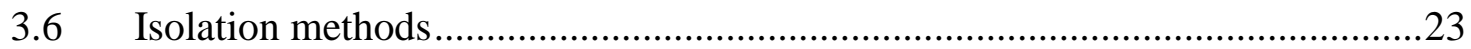

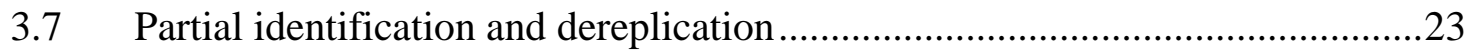

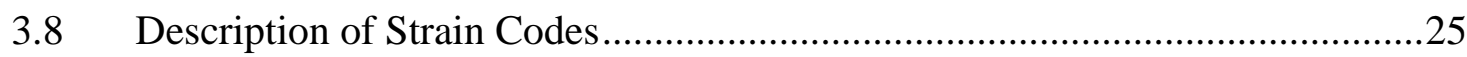

4 Investigation of selected marine and terrestrial Streptomycetes ................... 25

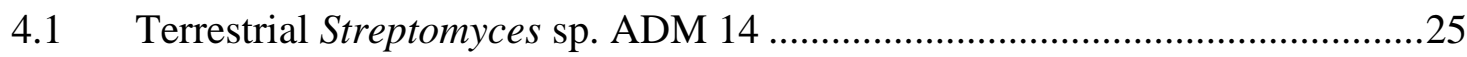

4.1.1 1-Hydroxy-4-methoxynaphthalene-2-caboxylic acid ....................................27

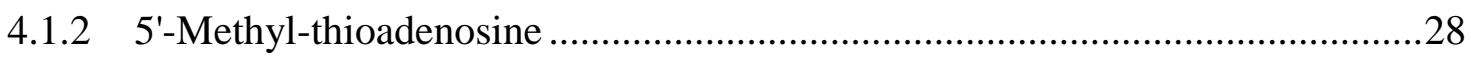

4.1.3 5,7,9,11-Tetrahydroxy-4,6,8,10-tetramethyl-tridec-2-enoic acid.....................30

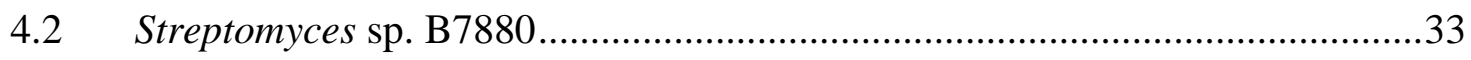

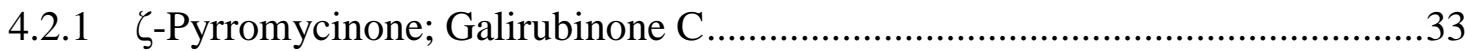

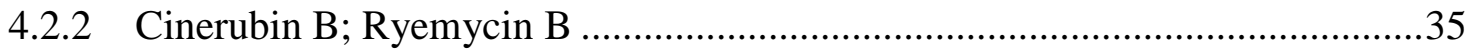




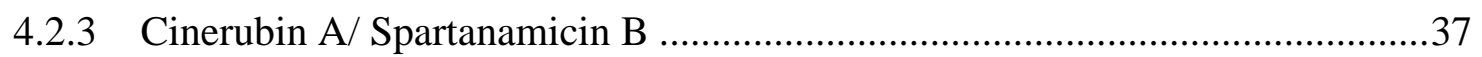

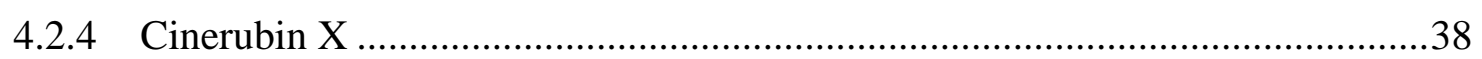

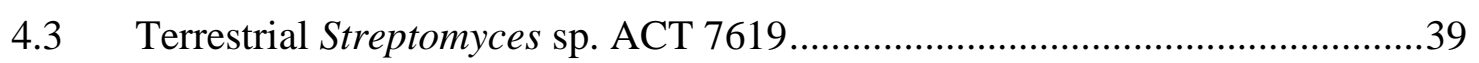

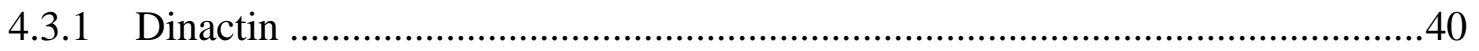

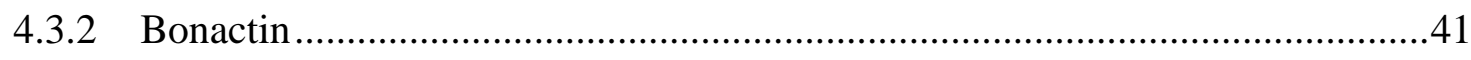

4.3.3 4,10-Dihydroxy-10-methyl-dodec-2-en-1,4-olide ........................................42

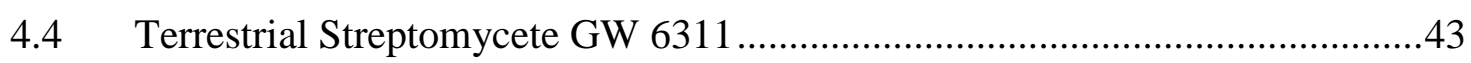

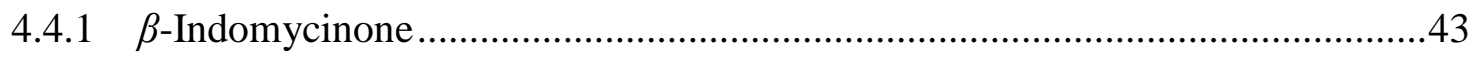

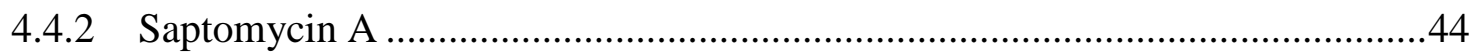

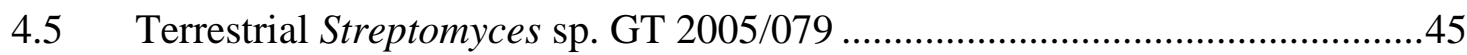

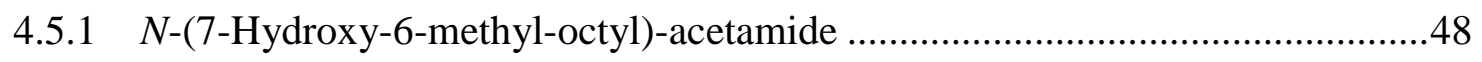

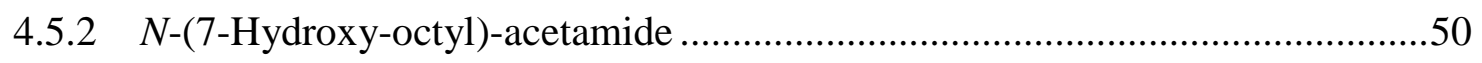

4.5.3 $N$-(6-Hydroxy-6-methyl-heptyl)-acetamide ….............................................53

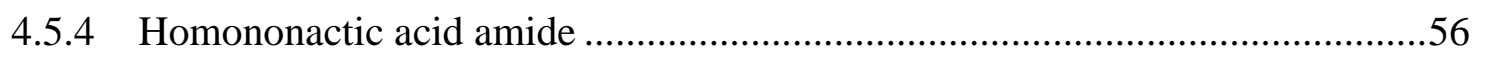

4.5.5 2-Phenylbenzoxazole-4-carboxylic acid......................................................59

4.5.6 2-(3-Chloro-2-hydroxy phenyl) benzoxazole-4-carbxylic acid........................61

4.6 Terrestrial Actinomycete $\mathrm{N} 435$.......................................................................63

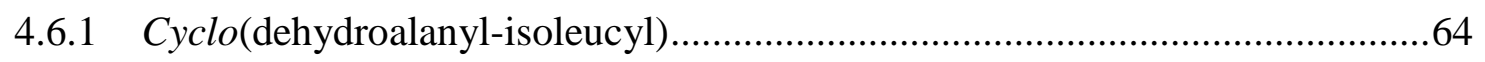

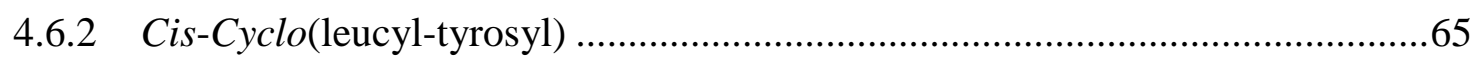

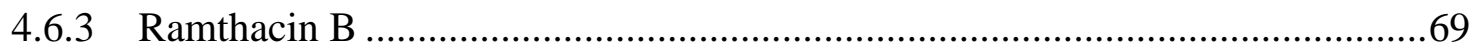

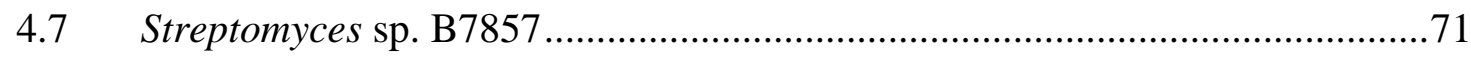

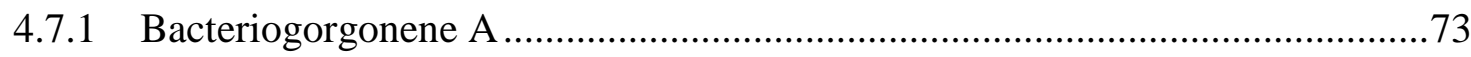

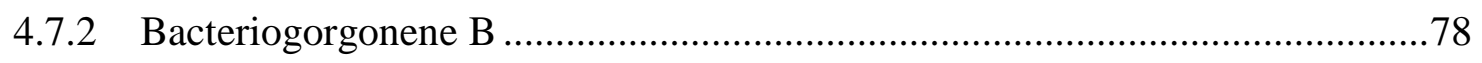

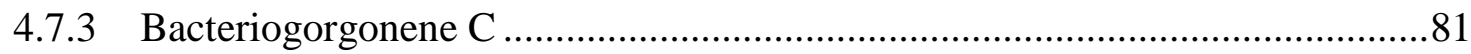

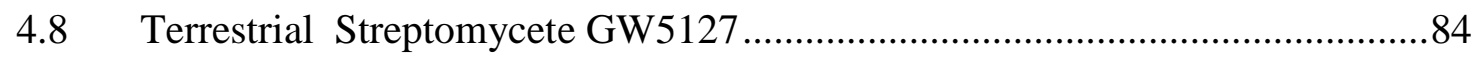

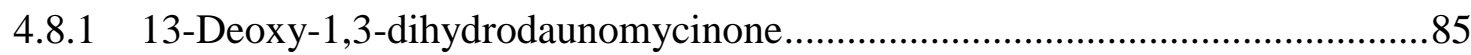

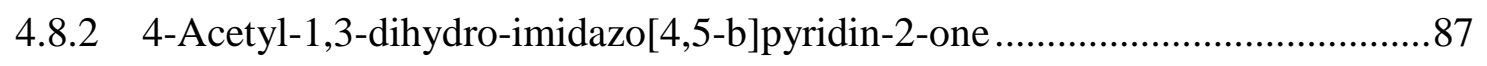

4.8.3 $N$-[2-(3-Hydroxy-4-methoxy-phenyl)-ethyl]-acetamide .................................90

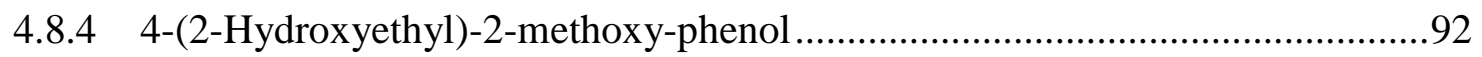

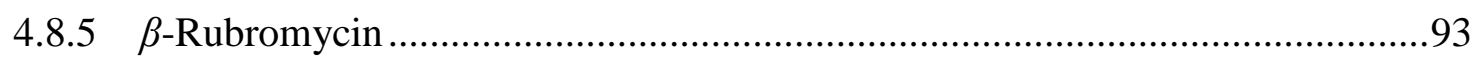




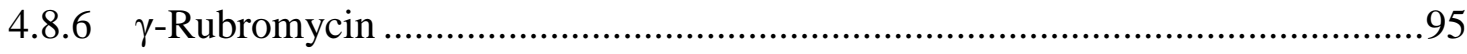

$4.9 \quad$ Terrestrial Streptomyces sp. GW 18/1811 ….................................................96

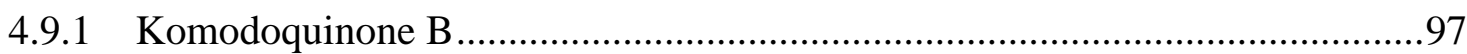

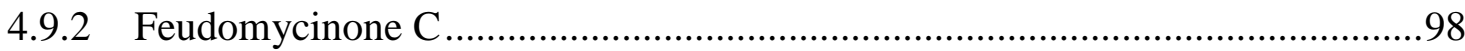

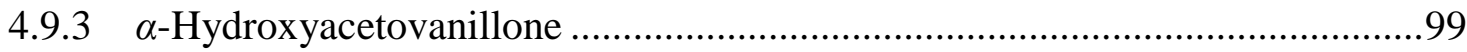

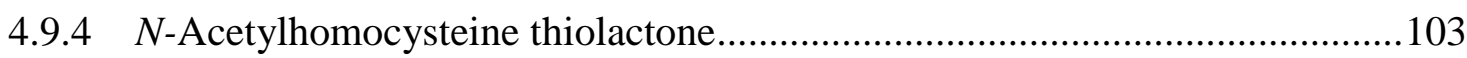

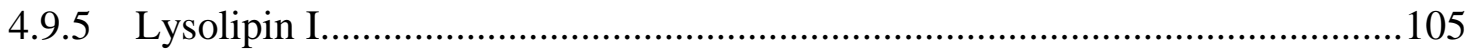

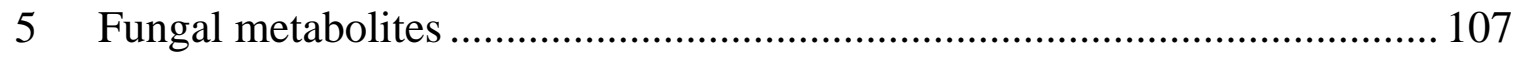

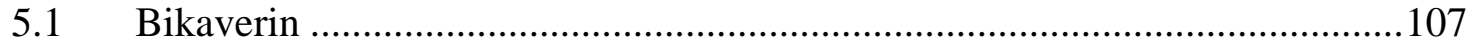

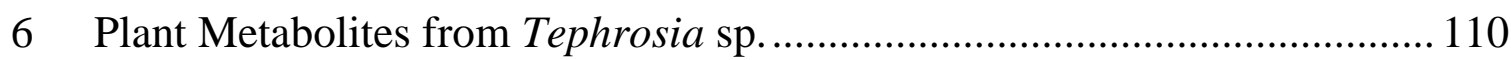

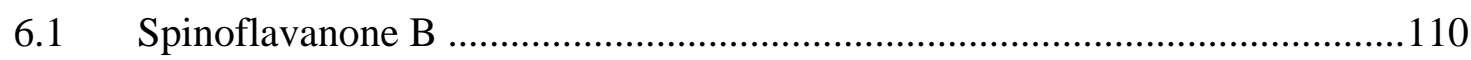

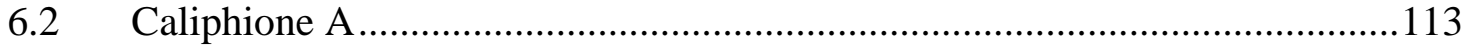

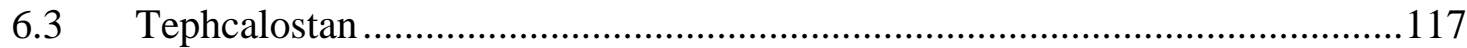

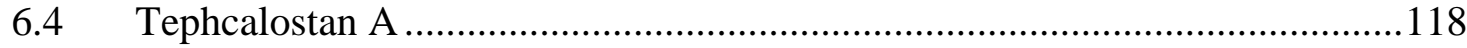

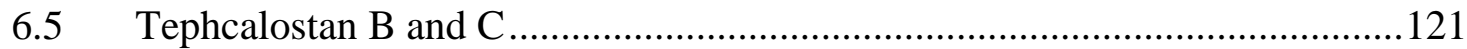

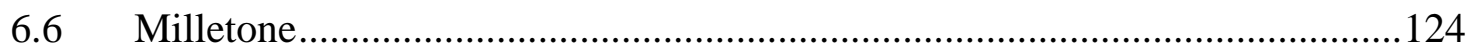

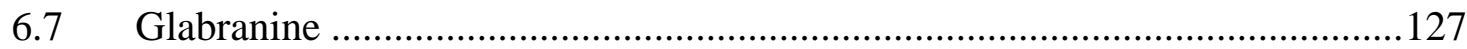

6.8 Betulinic acid and Betulinic acid methyl ester ..........................................129

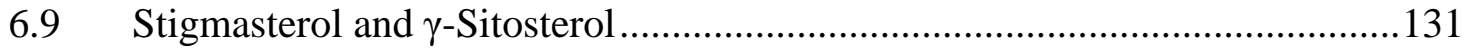

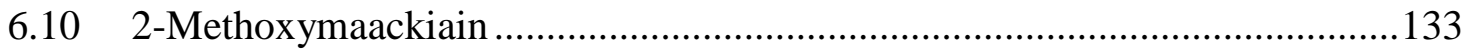

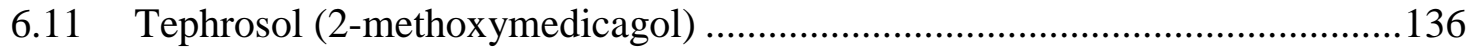

6.12 Didehydrovillosin (mixture of two stereoisomers at C-6) .............................138

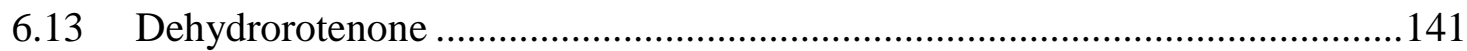

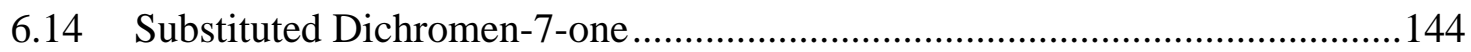

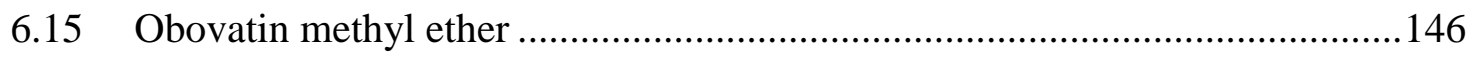

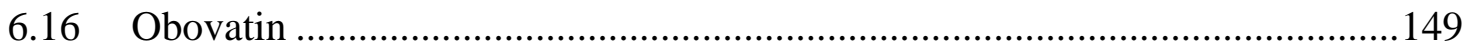

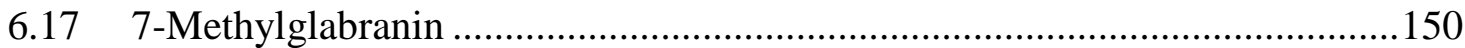

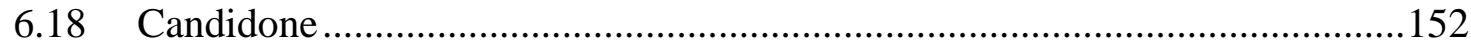

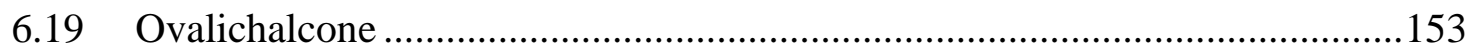

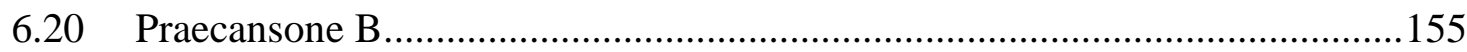

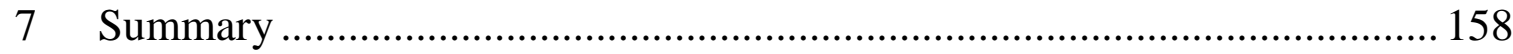

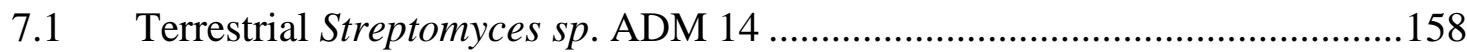




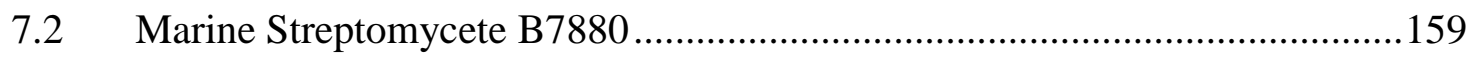

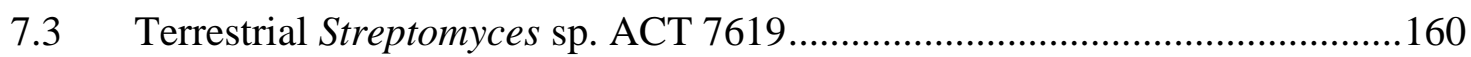

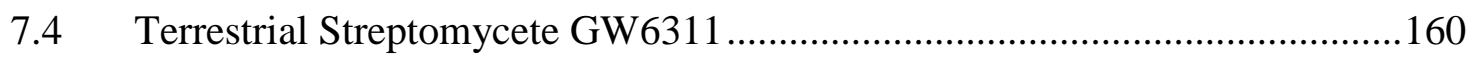

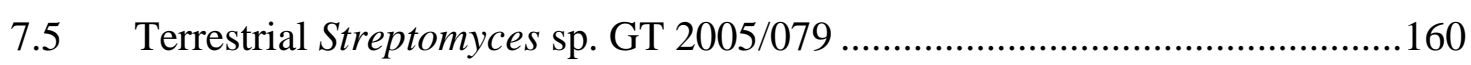

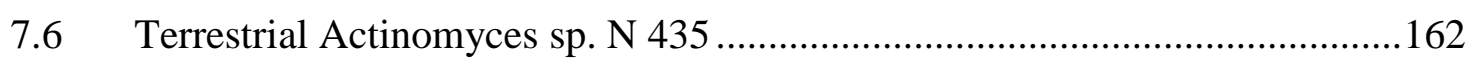

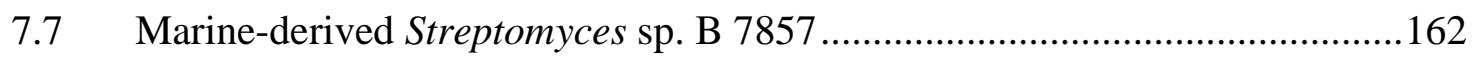

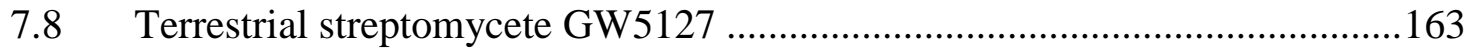

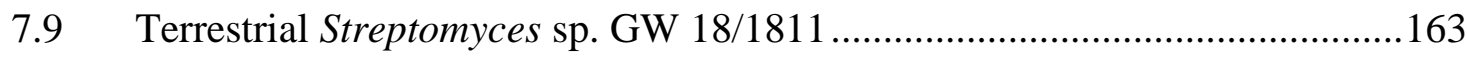

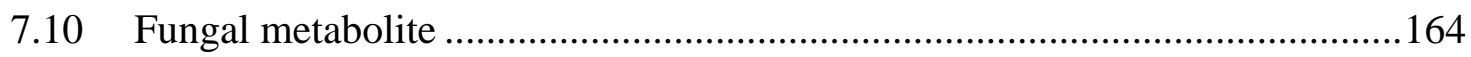

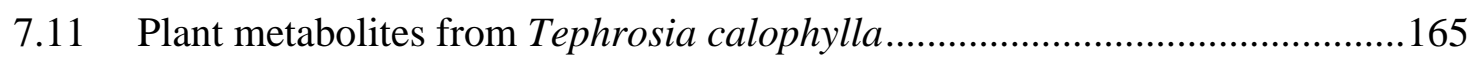

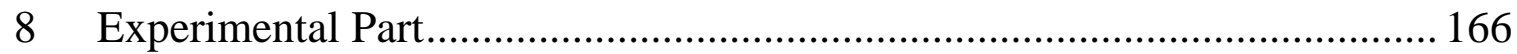

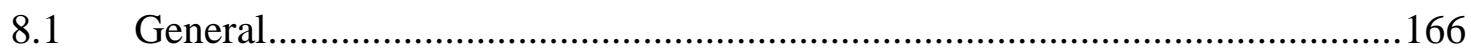

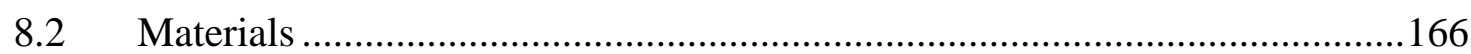

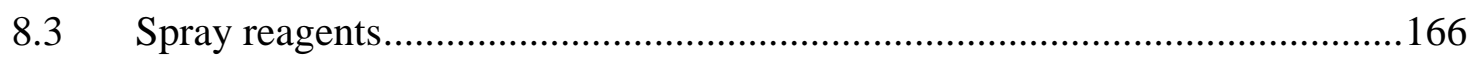

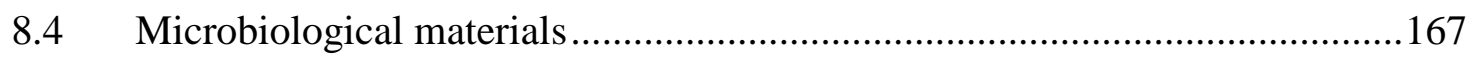

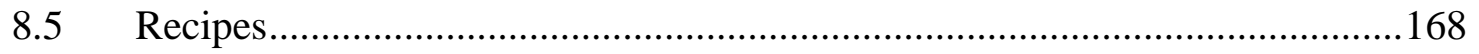

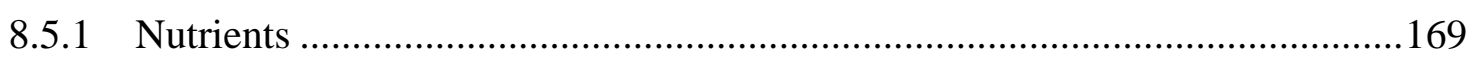

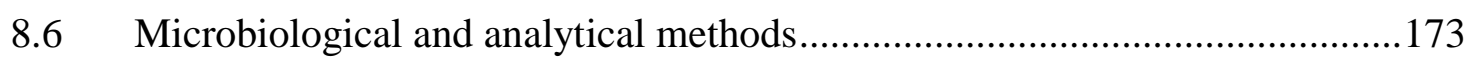

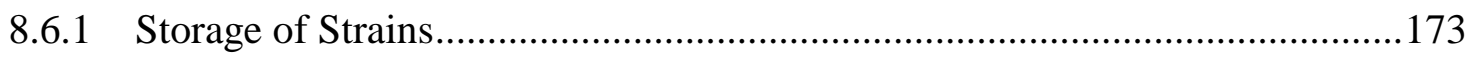

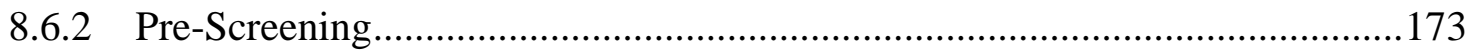

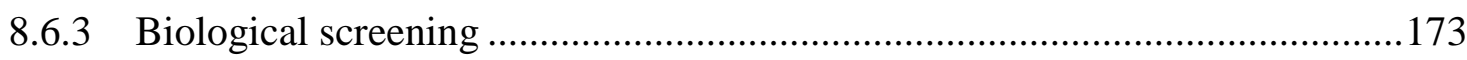

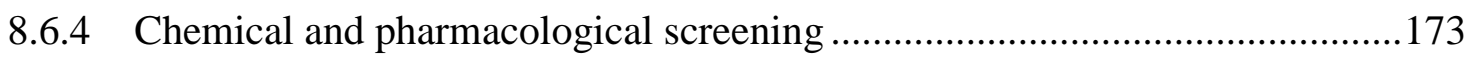

8.6.5 Brine shrimp microwell cytotoxicity assay .................................................. 174

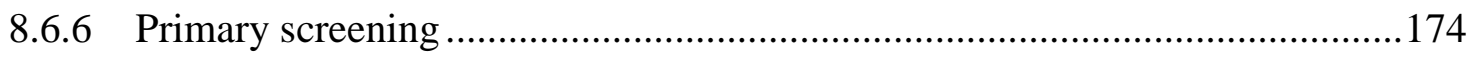

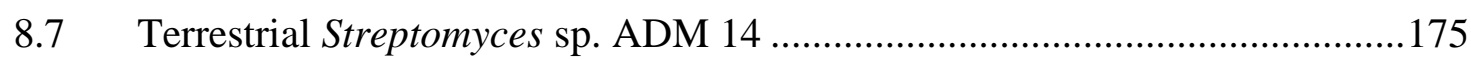

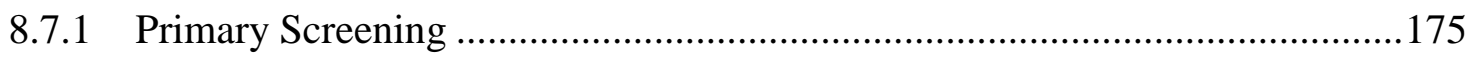

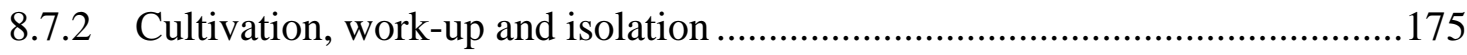

8.7.3 Synthesis of 1-hydroxy-4-methoxynaphthoic acid (43) ................................176

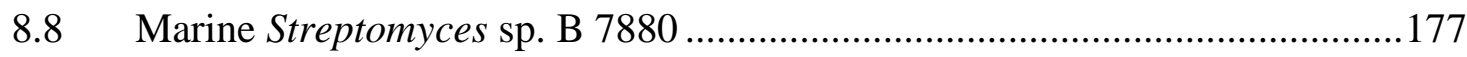

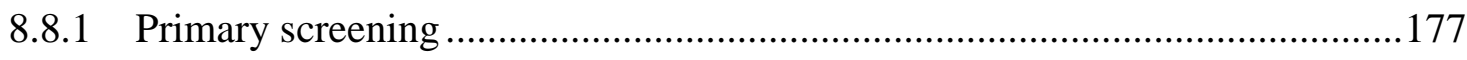

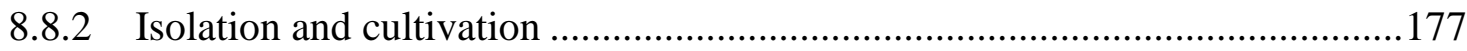




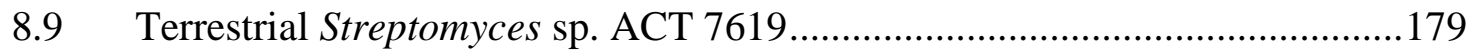

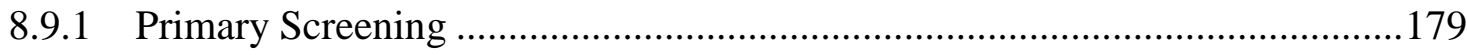

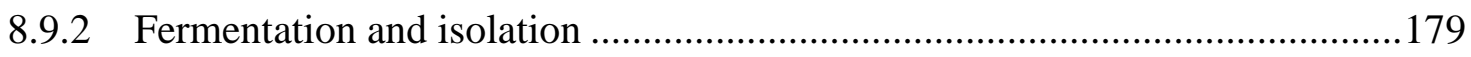

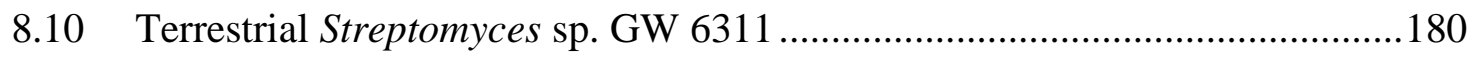

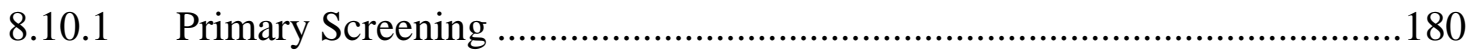

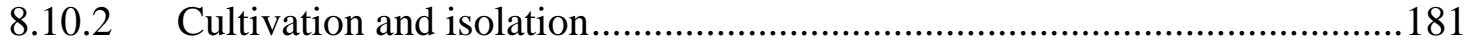

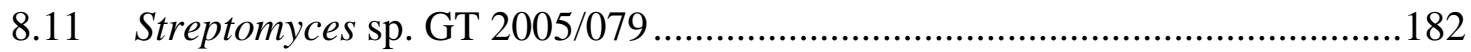

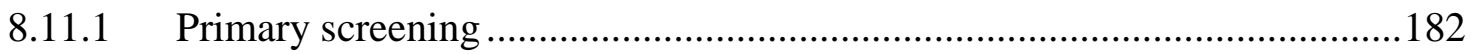

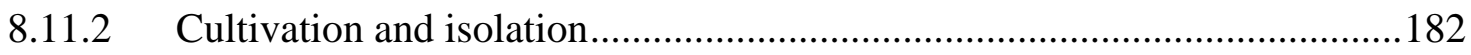

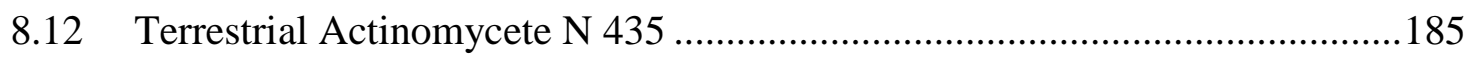

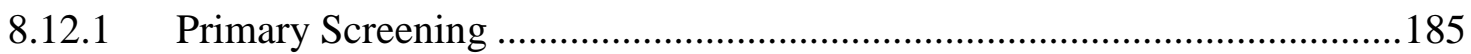

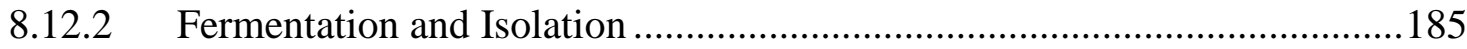

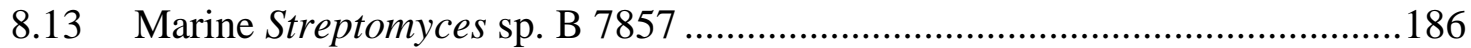

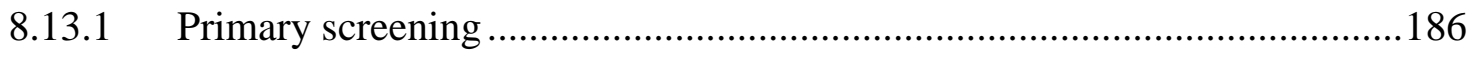

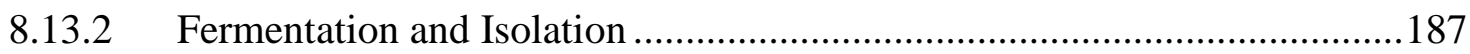

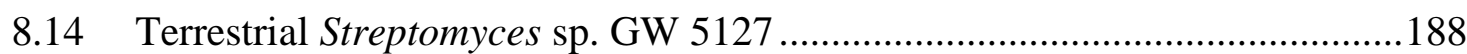

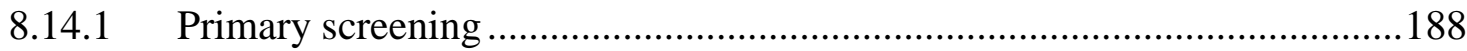

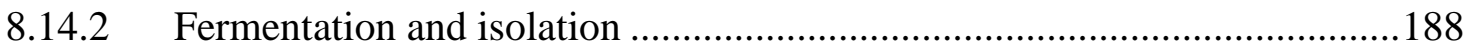

8.15 Terrestrial Streptomyces sp. GW 18/1811 .................................................191

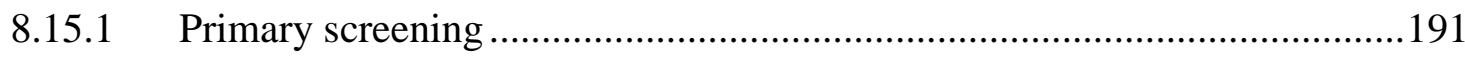

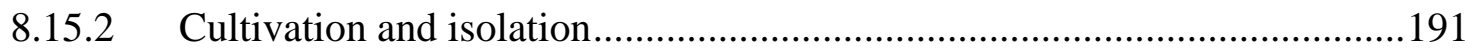

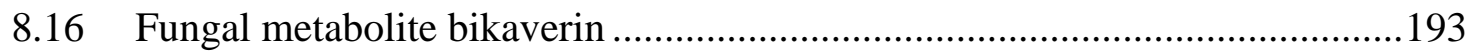

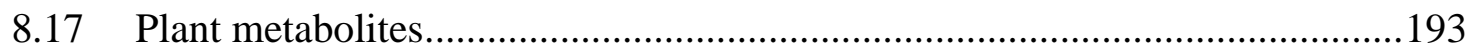

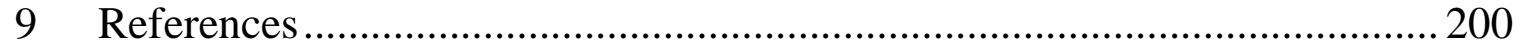





\section{Introduction}

The seas and oceans occupy almost three quarters of the earth's surface. They have numerous resources, which partly comprise of vegetation, algae, bacteria, fish shellfish and fungi. Recently these natural resources have drawn attention as a potential reservoir of many biologically active compounds. As the habitats for the marine organism are substantially different from that of terrestrial organisms, it can be supposed that their secondary metabolites will also differ considerably from terrestrial organisms. $^{1-3}$

Research on the chemistry of natural products derived from marine microorganism has increased tremendously in recent years due to the demand of compounds having potential pharmaceutical applications or economical value such as for cosmetics, drugs, fine chemicals, and functional personal-care products. ${ }^{4}$ Until now, quiet a number of species have been assayed for their activity and a huge number of bioactive molecules with unique structural features has been isolated. Those bioactive compounds often showed toxic properties. ${ }^{5}$ To this end, more than 15 compounds from marine source are in human trials. ${ }^{1}$

Natural products were the basis of most early traditional medicines and have played an important role in drug discovery. Natural products have contributed significant value to the pharmaceutical industries over the last decades. The term "natural product" implies the compounds derived from organism, plants or animals. Human societies have been using natural products for millennia. Historically therapeutic formulations from relatively crude plant extracts were common in pharmaceutical companies but with the advancement of technology, drug formulations of fairly purified compounds have become more popular, cost effective and convenient.

Numerous drug classes derived from natural product sources have benefited the therapeutic areas of oncology, immunosuppression, metabolic and infectious diseases. ${ }^{14}$ Modern techniques like X-ray crystallography, NMR spectroscopy and alternative drug discovery methods e.g. rational drug design, combinatorial chemistry have provided significant advances in natural product drug discovery research over the last 15 to 20 years. The development of resistance in infectious microorganisms to the available drugs and the increase of new diseases such as AIDS, Ebola and SARS require the discovery and development of new drugs. ${ }^{6}$ Microorganisms continue to be a productive and successful focus for marine natural products research and in particular the number of marine derived drug candidates remains high. ${ }^{7}$ 


\subsection{History of the use of natural products for medical applications}

Research of natural product has enormous unexploited potential, and the significant advantages and disadvantages of natural product derived molecules as drug candidates for development has been reported in many articles. ${ }^{8}$ The blossoming of natural product discovery efforts came into focus after the large-scale production of penicillin (1) during World War II. The pharmaceutical companies engaged their efforts during the wartime to build stocks of penicillin but after the war ended, they refocused on the search for new antibiotics. Natural product discovery programs were developed by all of the major pharmaceutical companies. Their screening programs included anti-bacterial and anti-fungal targets, as well as targets for other diseases. In the 1970s for example, cholesterol biosynthesis inhibitors, compactin ${ }^{9}$ (2) and mevinolin ${ }^{10}(3)$ were discovered. The discovery of compactin and mevinolin made it possible to develop the hugely successful $\operatorname{statin}^{11}$ (4) therapeutics, which even today are considered as successful drugs in both medical treatment and in pharmaceutical business fortunes. The discovery of streptomycin, gentamicin, omegamycin (5) and other antibiotics pushed the industry to implement large research and developing programs around natural product discovery, particularly microbial fermentation based technologies.<smiles>CC1(C)S[C@H]2[C@H](NC(=O)Cc3ccccc3)C(=O)N2C1C(=O)O</smiles>

1<smiles>CC[C@H](C)C(=O)O[C@H]1CCC=C2C=C[C@H](C)[C@H](CCC3C[C@@H](O)CC(=O)O3)[C@H]21</smiles>

2<smiles>CC[C@H](C)C(=O)O[C@H]1C[C@H](C)C=C2C=C[C@H](C)C(CCC3C[C@@H](O)CC(=O)O3)[C@H]21</smiles> 
<smiles>CCC(C)(C)C(=O)O[C@H]1C[C@@H](C)C=C2C=C[C@H](C)[C@H](CCC3C[C@@H](O)CC(=O)O3)[C@H]21</smiles>

4<smiles>CN(C)[C@H]1C(O)=C(C(N)=O)C(=O)[C@@]2(O)C=C3C(=O)c4c(O)cccc4[C@@](C)(O)[C@@H]3C[C@H]12</smiles>

5

Shu's review ${ }^{12}$ revealed that at least twenty-one natural products and natural product-derived drugs have been introduced onto the market in the USA, Europe or Japan in the last 8 years. In addition, a semi-synthetic camptothecin derivative, belotecan $^{13}$ (6) was launched in Korea in 2004. Table 1 represents twenty-one drugs which can be classified as 3 natural products, 10 semi-synthetic natural products and 8 natural product-derived drugs and include the "first in kind" drug caspofungin which functions as echinocandin antifungal, galantamine used as novel antiAlzheimer's drug, nitisinone (new treatment for the hereditary disease tyrosinemia type I), miglustat (new treatment of Type 1 Gaucher Disease).

Table 1: NP-derived drugs launched in the United States, Europe or Japan since 1998 by year with reference to their lead compound, classification and therapeutic area Year Generic name (trade name) Lead compound Classification Disease. ${ }^{14}$

\begin{tabular}{|c|c|c|c|c|}
\hline Year & Generic name & Lead compound & Classification & Disease area \\
\hline 1998 & $\begin{array}{l}\text { Orlistat } \\
\left(\text { Xenical }^{\mathrm{R}}\right)\end{array}$ & Lipstatin & semi-synthetic-NP & antiobesity \\
\hline 1998 & $\begin{array}{l}\text { Cefoselis } \\
\left(\text { Wincel }^{\mathrm{R}}\right)\end{array}$ & cephalosporin & semi-synthetic-NP & antibacterial \\
\hline 1999 & $\begin{array}{l}\text { Dalfopristin and } \\
\text { quinupristin }\end{array}$ & $\begin{array}{l}\text { Streptogramin } \\
\text { and B Strepto- } \\
\text { gramin A }\end{array}$ & semi-synthetic-NP & antibacterial \\
\hline 1999 & $\begin{array}{l}\text { Valrubicin } \\
\left(\text { Valstar }^{\mathrm{R}}\right)\end{array}$ & doxorubicin & NP-derived & oncology \\
\hline 1999 & $\begin{array}{l}\text { colforsin daropate } \\
\left(\text { Adele, } \text { Adehl }^{\mathrm{R}}\right)\end{array}$ & forskolin & semi-synthetic-NP & cardiotonic \\
\hline 2000 & $\begin{array}{l}\text { arteether } \\
\left(\text { Artemotil }^{\mathrm{R}}\right)\end{array}$ & artemisinin & semi-synthetic NP & antimalarial \\
\hline 2001 & $\begin{array}{l}\text { ertapenem } \\
\left(\text { Invanz }^{\mathrm{TM}}\right)\end{array}$ & thienamycin & NP-derived & antibacterial \\
\hline 2001 & $\begin{array}{l}\text { caspofungin (Can- } \\
\text { cidas }{ }^{R} \text { ) }\end{array}$ & pneumocandin B & semi-synthetic NP & antifunga \\
\hline
\end{tabular}




\begin{tabular}{|c|c|c|c|c|}
\hline 2001 & $\begin{array}{l}\text { telithromycin } \\
\left(\text { Ketek }^{\mathrm{R}}\right)\end{array}$ & erythromycin & semi-synthetic NP & antibacterial \\
\hline 2001 & $\begin{array}{l}\text { pimecrolimus } \\
\left(\text { Elidel }^{\mathrm{R}}\right)\end{array}$ & Ascomycin & semi-synthetic NP & $\begin{array}{l}\text { atopic derma- } \\
\text { titis }\end{array}$ \\
\hline 2002 & $\begin{array}{l}\text { galantamine } \\
\left(\text { Reminyl }^{\mathrm{R}}\right)\end{array}$ & galantamine & NP & $\begin{array}{l}\text { Alzheimer's } \\
\text { disease }\end{array}$ \\
\hline 2002 & $\begin{array}{l}\text { micafungin (Fun- } \\
\text { guard }^{\mathrm{R}} \text { ) }\end{array}$ & FR901379 & semi-synthetic NP & antifungal \\
\hline 2002 & $\begin{array}{l}\text { amrubicin hydro- } \\
\text { chloride }\left(\text { Calsed }^{\mathrm{R}}\right)\end{array}$ & doxorubicin & NP-derived & oncology \\
\hline 2002 & $\begin{array}{l}\text { biapenem } \\
\left(\text { Omegacin }^{\mathrm{R}}\right)\end{array}$ & thienamycin & NP-derived & antibacterial \\
\hline 2002 & $\begin{array}{l}\text { Nitisinone } \\
\left(\text { Orfadin }^{\mathrm{R}}\right)\end{array}$ & leptospermone & NP-derived & $\begin{array}{l}\text { antity- } \\
\text { rosinaemia }\end{array}$ \\
\hline 2003 & $\begin{array}{l}\text { Miglustat } \\
\left.\text { (Zavesca }^{\mathrm{R}}\right)\end{array}$ & deoxynojirimycin & semi-synthetic NP & $\begin{array}{l}\text { Gaucher dis- } \\
\text { ease }\end{array}$ \\
\hline 2003 & $\begin{array}{l}\text { mycophenolate } \\
\text { sodium } \\
\left(\text { Myfortic }^{\mathrm{R}}\right)\end{array}$ & $\begin{array}{l}\text { mycophenolic } \\
\text { acid }\end{array}$ & NP & $\begin{array}{l}\text { immunosup- } \\
\text { pression }\end{array}$ \\
\hline 2003 & $\begin{array}{l}\text { rosuvastatin } \\
\left(\text { Crestor }^{\mathrm{R}}\right)\end{array}$ & mevastatin & NP derived & dyslipidemia \\
\hline 2003 & $\begin{array}{l}\text { pitavastatin } \\
\left(\text { Livalo }^{\mathrm{R}}\right)\end{array}$ & mevastatin & NP derived & dyslipidemia \\
\hline 2003 & $\begin{array}{l}\text { daptomycin } \\
\left(\text { Cubicin }^{\mathrm{TM}}\right)\end{array}$ & daptomycin & NP & antibacterial \\
\hline 2004 & $\begin{array}{l}\text { everolimus } \\
\left(\text { Certican }^{\mathrm{TM}}\right)\end{array}$ & sirolimus & semi-synthetic & $\begin{array}{l}\text { immunosup- } \\
\text { pression }\end{array}$ \\
\hline
\end{tabular}

(NP- Natural Product)<smiles>CC(C)NCCc1c2c(nc3ccccc13)C1CC3=C(COC(=O)[C@]3(C)O)C(=O)N1C2</smiles>

6

\subsection{Approaches for the search of new natural products}

To obtain new bioactive compounds in nature is not a single-step procedure. However, the first step is to select suitable sources. Physical, chemical or biological in- 
teractions of metabolites with test systems can then be quantitatively or qualitatively evaluated.

In recent times, the genes for example of polyketide synthesis are being located and isolated. In addition to that, genetic engineering technology has made it possible to produce genetically manipulated microorganisms, which could be used to generate new metabolites by realigning the synthetic capacities of different species. Depending on the biological activities, novel natural products could be optimised to yield effective chemotherapeutic as well as other bioactive agents. ${ }^{15}$ To investigate new natural products biotransformation techniques are also being used. Biotransformation enables derivatisation of known compounds by esterfication, reduction, oxidation, demethylation, or glycosylation utilizing the enzymes of living microorganisms. For example, antibiotics or antineoplastic agents, e.g. bleomycin $\mathrm{A} 1{ }^{16}$ and glycopeptide antitumor metabolites were isolated from Streptomyces verticillus. ${ }^{17} \mathrm{Cur}-$ rently bleomycins are being used for clinical treatment of Hodkin's lymphoma, carcinomas of the head, skin, neck, and tumors of testis. ${ }^{18}$ To achieve the same goal by chemical transformations would be time consuming as well as involving many steps and produces by-products.

In order to optimise their pharmacological properties, some antibiotics derived from natural resources need structural transformation. The antibacterial macrolide erythromycin (7) was isolated in $1952^{19}$ while its semi-synthetic derivative telithromycin is 10-40 times more active than the parent antibiotic, which contains a 3-keto substituent instead of cladinose. ${ }^{20}$ The ketolides are a new class of antibiotics having excellent inhibitory abilities against Gram-negative or Gram-positive cocci like Staphylococcus, and Enterococcus, which are resistant against macrolides and other antibiotics. $^{21}$

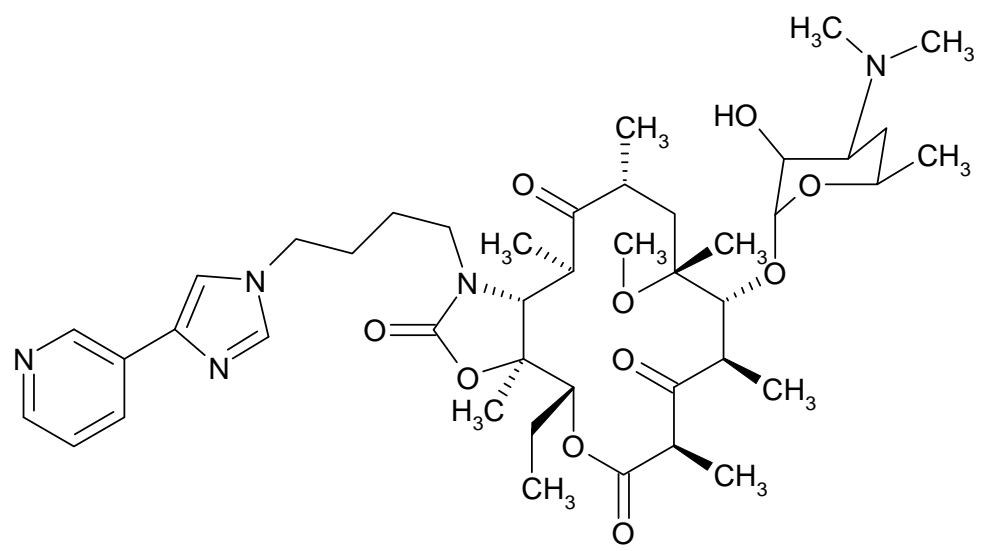




\subsection{Antibiotic screening}

A new research field "antibiotic screening" became important after the discovery of penicillin and the actinomycins. In the continuous search to discover new bioactive compounds, screening approaches are applied to crude extracts to evaluate chemical, biological and pharmaceutical characteristics. The pharmaceutical industries focused on bioactive substances and often contributed the advantages of greater sensitivity and sample throughput that is High-throughput-Screening (HTS) ${ }^{22}$ However, it is plausible that novel compounds could be neglected which may be active against other targets. To solve this problem, in the 1980s, Zähner and Zeeck introduced a systematic chemical screening of the crude extracts. ${ }^{23}$ The chromatographic features of metabolites on thin layer chromatography (TLC) plates followed by their detection with staining reagents under defined reaction conditions approved the visualization of an almost complete fingerprint of the secondary metabolite content of that particular crude extract. ${ }^{22}$ The application of this method made it possible to isolate nearly all metabolites of a given strain, and the variety of unidentified compounds could then be biologically tested in uncontaminated state. Due to the rapid developments in the sensitivity of mass spectroscopy (MS), nuclear magnetic resonance (NMR) instruments, and the recent growing chemical databases (AntiBase, DNP, CA), the dereplication of known chemical compounds has greatly improved. In current years, screening is accomplished in combination with ultraviolet (UV), high performance liquid chromatography (HPLC), HPLC-MS, HPLC-CD, HPLC-NMRMS or GC-MS methods.

At present, substantial concentration has been placed on marine microorganisms as the most permissible source for new bioactive natural products. However, under normal incubation conditions unlike the terrestrial bacteria the marine bacteria show a low production rate. To amplify the efficacy of screening for secondary metabolites of marine bacteria, polymerase chain reaction (PCR)-based screening is being used by microbiologists for assaying genes for example, non-ribosomal polypeptide synthases (NRPSs), dNDP-glucose dehydratases, polyketide synthases and halogenases. The dilemma of this method however, is, that all these genes occur very frequently, but may not be expressed in every case (silent genes).

\subsection{Natural Products from Marine Bacteria}

It is not so easy to define exactly what a marine bacterium is. But marine bacteria must even not have common criteria such as sodium chloride requirements, which should be unequivocal physiological needs for autochthonous marine bacteria of the 
free water column. Certain marine bacteria like Salinospora spp. use respiratory chain-linked sodium dependent NADH: quinone reductase instead of the common proton-coupled enzyme. Korormycin ${ }^{24}(\mathbf{8})$, which was isolated from a marine Vibrio alginolyticusis, is a specific inhibitor of this enzyme and may be a tool to identify such sodium-dependent bacteria. ${ }^{25}$

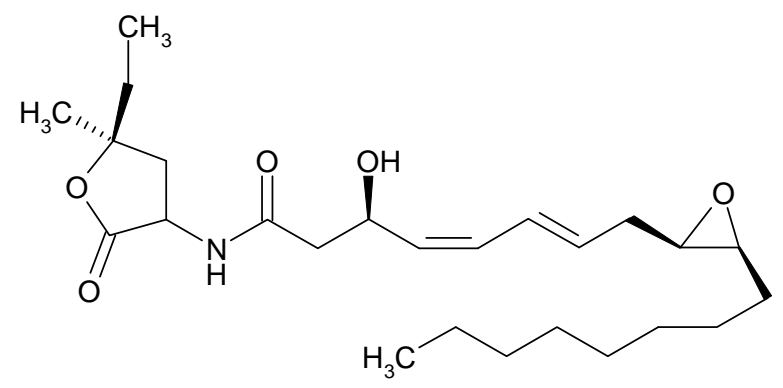

8

Highly biologically active quinones are commonly found in streptomycetes; these compounds are easily detected by naked eyes because for their pink, yellow or red colours. Many quinones also have been isolated from marine sources. The Cglycosides himalomycin $\mathrm{A}(\mathbf{9})$ and $\mathrm{B}^{26}$ (10)are two recent examples of anthraquinones with the rare fridamycin $\mathrm{E}$ chromophor which act as a precursor of the anthracycline antibiotics. These two compounds were reported from Streptomyces sp. B 6921 which was isolated from a littoral sample from Mauritius having strong antibacterial activity.

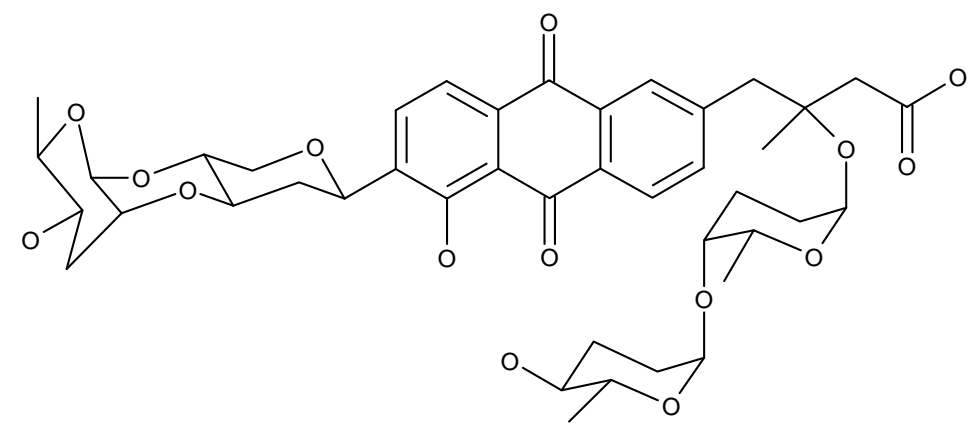

9

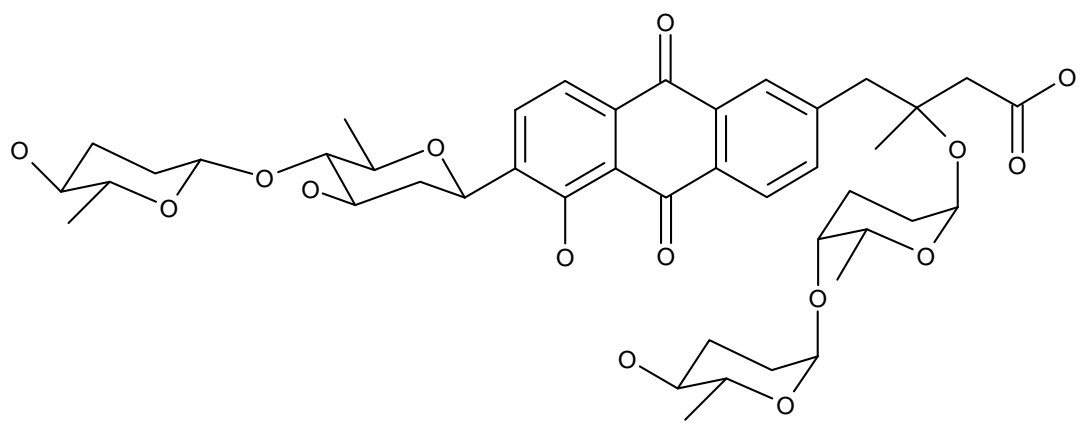


Among the first findings from the deep-sea microbiota were the macrolactins ${ }^{27}$ (11 $\mathbf{a}, \mathbf{b}, \mathbf{c})$, macrolactin E (12), macrolactin D (13), macrolactin F (14) and isomacrolactinic acid (15), which corresponded to a class of remarkable antiviral and cytotoxic macrocyclic lactones produced by an un-identified Gram-positive marine bacterium.

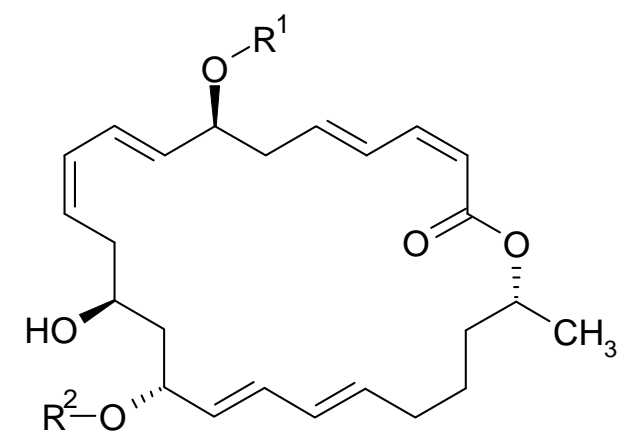

11

$11 \mathrm{a} \mathrm{R}_{1}=\mathrm{R}_{2}=\mathrm{H}$

11b $\mathrm{R}_{1}=\beta$-glucosyl, $\mathrm{R}_{2}=\mathrm{H}$

11c $\mathrm{R}_{1}=\mathrm{H}, \mathrm{R}_{2}=\beta$-glucosyl

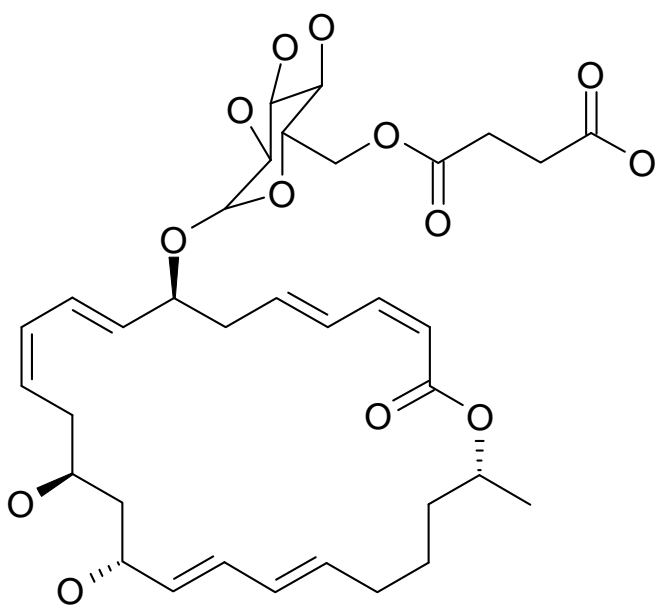

12<smiles></smiles>

14<smiles></smiles>

13<smiles>C[C@H](O)CCC/C=C/CC[C@@H](O)C[C@@H](O)C/C=C\C=C\[C@H](O)C/C=C/C=C\C(=O)O</smiles>

15

Utilization of marine derived natural products for medicinal purposes is not a new initiative as seahorses were being utilized in China, Japan and Taiwan for centuries 
in long-established treatments for sexual disorders, circulatory and respiratory problems as well as kidney and liver dysfunctions, amongst other aliments. Compounds derived from marine sources usually have small to medium molecular weight. Many of the compounds produced by marine plants, invertebrates or microbes have inspired interdisciplinary studies by chemists and biologists. Some bacteria are collected from tropical and cold temperate ocean habitats and continue to be the subject of extensive chemical investigation. The extracts found from this type of bacteria are being examined as a source of new cytotoxic secondary metabolites that are prospective to guide for the development of new drugs.

In 1966, from a culture of Pseudomonas bromoutilis Burkholder and coworkers reported the highly brominated pyrrole antibiotic pentabromopseudiline ${ }^{28}(\mathbf{1 6})$, which was the first marine bacterial natural product. It exhibited remarkable antibiotic activity against Gram-positive bacteria.<smiles>Oc1c(Br)cc(Br)cc1-c1[nH]c(Br)c(Br)c1Br</smiles>

16

\subsection{Therapeutic areas influenced by natural products}

It can be said that it is the evolutionary selection, which makes many of the secondary metabolites highly potent and selective. For example, some are inferred to be signal molecules or demonstrate protecting mechanisms against competitors, herbivores or pathogens. Reproductivity of the producing organism is being assisted by these compounds and they help in maintaining survival. These characteristics make them ideal as lead compounds for development as therapeutic agents for oncology and infectious diseases wherein death or injury to populations of cells is desired.

\subsection{Natural products against infectious diseases; bacterial pathogens}

During the last century natural products were considered as the "silver bullet" for treating bacterial diseases because natural product directed discovery provided a vast majority of the antibiotics being used today. Nevertheless, many famous pharmaceutical companies ended their HTS (high-throughput screening) efforts for discovery of antibiotics. As a result, they did not update their natural product libraries. A re- 
cent survey revealed that from pharmaceutical companies and academic laboratories conducting HTS, only a handful of anti-viral candidates were identified from activities originally identified but no anti-bacterial drug candidates were identified through HTS. A significant number of pathogens gradually arose and become resistant to antibiotics. In a recent report the US Center for Disease Control and Prevention indicated the emergence of Mycobacterium tuberculosis strains within the United States and the rest of the world, which are resistant to all available therapeutics. Due to the growing resistance to existing antibiotics and the indispensable medical needs the development of new classes of antibiotics which are in urgent need and new targets are therefore vital for drug discovery. In this context, the recently reported discovery of platensimycin ${ }^{29}(\mathbf{1 7})$, a new class of inhibitor, by a group at Merck can be considered as very encouraging. Plantensimycin (17) demonstrated strong, broad-spectrum Gram-positive anti-bacterial activity by inhibiting cellular lipid biosynthesis. Platensimycin (17) was first time isolated and elucidated in our group, ${ }^{30}$ however, had not been published. It has been shown that cerulenin and thiolactomycin inhibit condensing enzymes in the biosynthesis pathways of fatty acids (Fab F/B and Fab H), however till now no drugs targeting these condensing enzymes are used clinically. Selective and specific activity against the Fab/F enzyme was shown by platensimycin in the synthetic pathway of fatty acids.

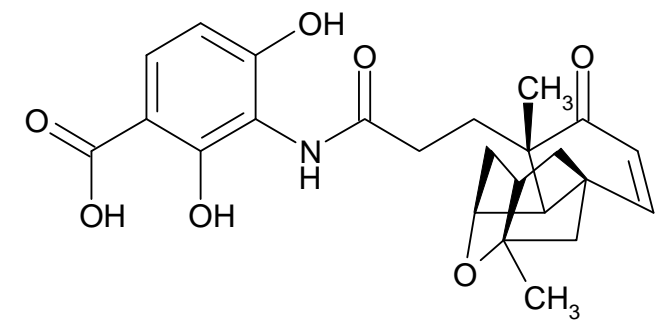

17

\subsection{Antifungal compounds from natural sources}

Existing antifungal drugs, which are in use to date have some connection to natural products. For example, some polyenes as well as griseofulvin are natural products, whereas the echinocandin derivatives are semi-synthetically originated from natural products. Another example is 5-fluorocytosine, which is a nucleoside and interferes with DNA and RNA synthesis, is chiefly used together with the polyene amphotericin B. Even though in general the azoles are considered to be synthetic in origin, their drug prototype pathway belongs to the Streptomyces metabolite azomycin. The antifungal activity of azoles is due to the inhibition P450 3A-dependant C14-amethylase, which is an enzyme that changes lanosterol to ergosterol that leads to ergosterol depletion and disruption of the fungal cell membrane integrity. ${ }^{31-33}$ 
Various fungi produce a group of naturally occurring lipopeptides called echinocandins which display potent antifungal activity by inhibition of $1,3-\beta$-D-glucan synthesis in the fungal cell wall. ${ }^{34,35}$ Until now, two echinocandin-based drugs have been approved for clinical use. A semi-synthetic drug "caspofungin" which is a derivative of pneumocandin $\mathrm{B}$, was first introduced by Merck in the US in 2001, ${ }^{36,37}$ whereas micafungin is a semisynthetic derivative of FR901379 that was first launched by Fujisawa in Japan in 2002. There are two echinocandins, anidulafungi and aminocandin (structure not available), currently undergoing clinical evaluation. $2 H$-Purin-2-one (18) and 7H-pyrrolo[2,3-d]pyrimidine-5-carboximidamide (19) are two further examples of antifungal compounds from natural sources.<smiles>Cn1c(=O)c2[nH]c(SCCN3CCCC3)nc2n(C)c1=S</smiles>

18<smiles>N=C(NO)c1cn([C@@H]2O[C@H](CO)[C@@H](O)[C@H]2O)c2ncnc(NO)c12</smiles>

19

\subsection{Antibacterial compounds from natural sources}

In the development of antibacterial drugs, natural products have played a central role, as most have been derived from natural product leads. For example, $\beta$-lactams were first introduced in 1941, aminoglycosides in 1944, cephalosporins in 1945, chloramphenicol in 1949, tetracyclines in 1950, macrolides in 1952, lincosamides in 1952, streptogramins in 1952, glycopeptides in 1956, rifamycins in 1957 and lipopeptides in 2003 which is quite recent. Only a few antibacterials are synthetically derived such as sulphonamides (20) (1935), nitroimidazole (21) (1959), quinolone (1962), trimethoprim (1968) and oxazolidinones (2000).

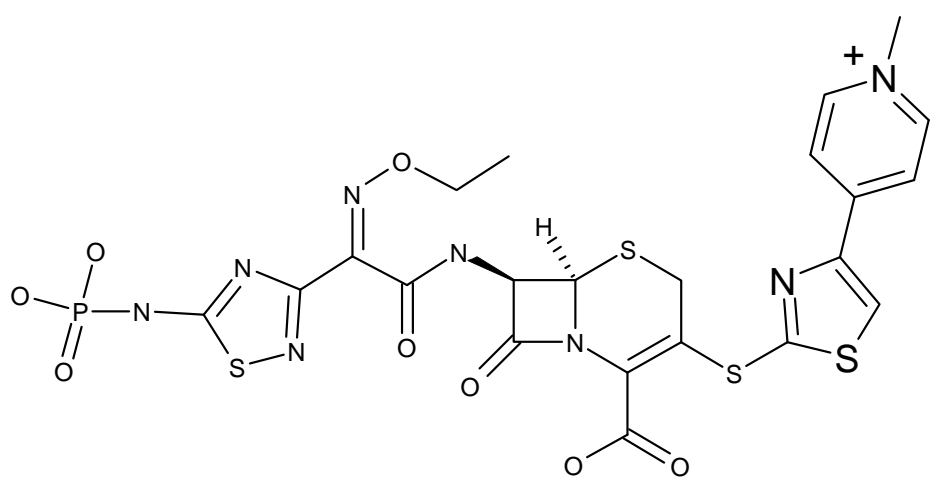


<smiles>Cc1oc(=O)oc1COC(=O)C1=C(C2CCCC2)SC2[C@H](C(C)O)C(=O)N12</smiles>

21<smiles>CC(O)[C@H]1C(=O)N2C(C(=O)OCOC(=O)C(C)(C)C)=C(SC3CN(C4=NCCS4)C3)[C@H](C)[C@H]12</smiles>

22

The immense pressure for developing new and improved antibacterial drugs has increased tremendously due to widespread antibacterial drug resistance. However, only a few major pharmaceutical companies are actively engaged in the field despite the introduction of only a few recent novel antibacterial classes since 1968. Due to the huge investments required to bring an antibacterial drug from clinical trials to the market, an acceptable financial return may not be forthcoming and this may be a concern for major pharmaceutical companies. Anxieties also have been elevated that nowadays the regulatory procedures make approval complicated for new antibacterials as they need to compete head to head with the established drugs in sensitive strains, while activity against resistant strains is not satisfactorily weighted. After all, most mechanism based antibacterial screening programmes accepted to date have not been successful in identifying new in vivo active antibacterial drugs. ${ }^{17,38}$

$\beta$-Lactam antibacterials derived from total synthesis, semi-synthesis or from a natural product drugs can be further classified into seven subclasses: penicillins, cephalosporins, cephamycins, cephems, carbapenems, penems and monobactams. At present, four cephalosporins are existing (ceftizoxime alapivoxil (23), ceftobiprole $^{39}$ (24), RWJ-442831 and PPI-0903), one penem (faropenem daloxate) and three carbapenems (CS-023, tebipenem and doripenem) in clinical development.<smiles>CO/N=C(\C(=O)N[C@@H]1C(=O)N2C=C(C(=O)OCOC(O)C(C)(C)C)CS[C@H]12)C1CSC(NC(=O)C(C)N)=N1</smiles> 
<smiles>CO/N=C(\C(=O)NC1C(=O)N2C=C(Sc3ccncc3CSCCNC(=O)[C@H](N)CC(=O)O)CS[C@H]12)c1nc(N)sc1Cl</smiles><smiles>CC(O)[C@H]1C(=O)N2C(C(=O)S)=C(S[C@@H]3CC(C(=O)N4CC[C@@H](NC(=O)CNC(=N)N)C4)N(C)C3)C[C@H]12</smiles>

\subsection{Potential antiparasitic drugs originated from natural products}

Since ancient times, medicinal plants are being used for the treatment of parasitic diseases, which is well known and documented. For example, Cinchona succiruba (Rubiaceae) has been used as antimalarial. A very promising compound licochalcone $\mathrm{A}^{40}$ (26), isolated from Glycyrrhiza glabra (Fabaceae) is now in intensive preclinical studies for the treatment of intestinal microsporidiosis, Phase III clinical trials of fumagillin had been done by Sanofi-Aventis which was granted EU orphan drug status for this use in March 2002. The spore-forming unicellular parasite Enterocytozoon bieneusi causes microsporidiosis, which is of major concern to immuno-compromised patients as it causes chronic diarrhoea. In year 1949, fumagillin was first isolated from Aspergillus fumigatus, which was used shortly after its discovery as an effective drug for intestinal amoebiasis. Amphotericin B (27) is used as a drug for the therapy of visceral and mucocutaneous leishmaniasis. Different analogues of amphotericin B (27) such as hamycins $A^{41}$ (28) also inhibit parasite growth. Hamycin is now extensive used in the treatment of candidiasis and otomycosis, which is found to be satisfactorily effective in killing Leishmania donovani promastigotes. 
<smiles>C=CC(C)(C)c1cc(/C=C/C(=O)c2ccc(O)cc2)c(OC)cc1O</smiles>

26

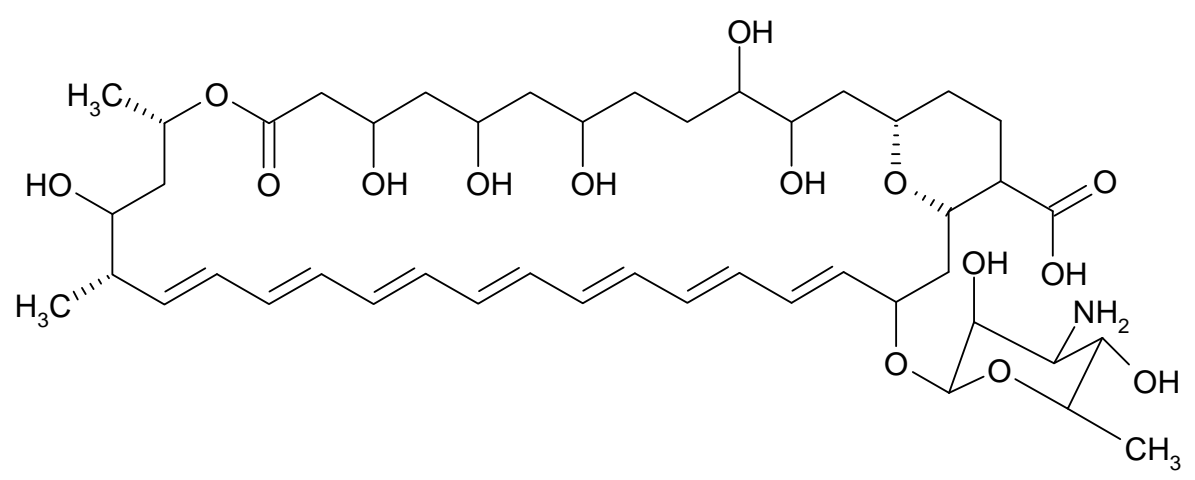

27

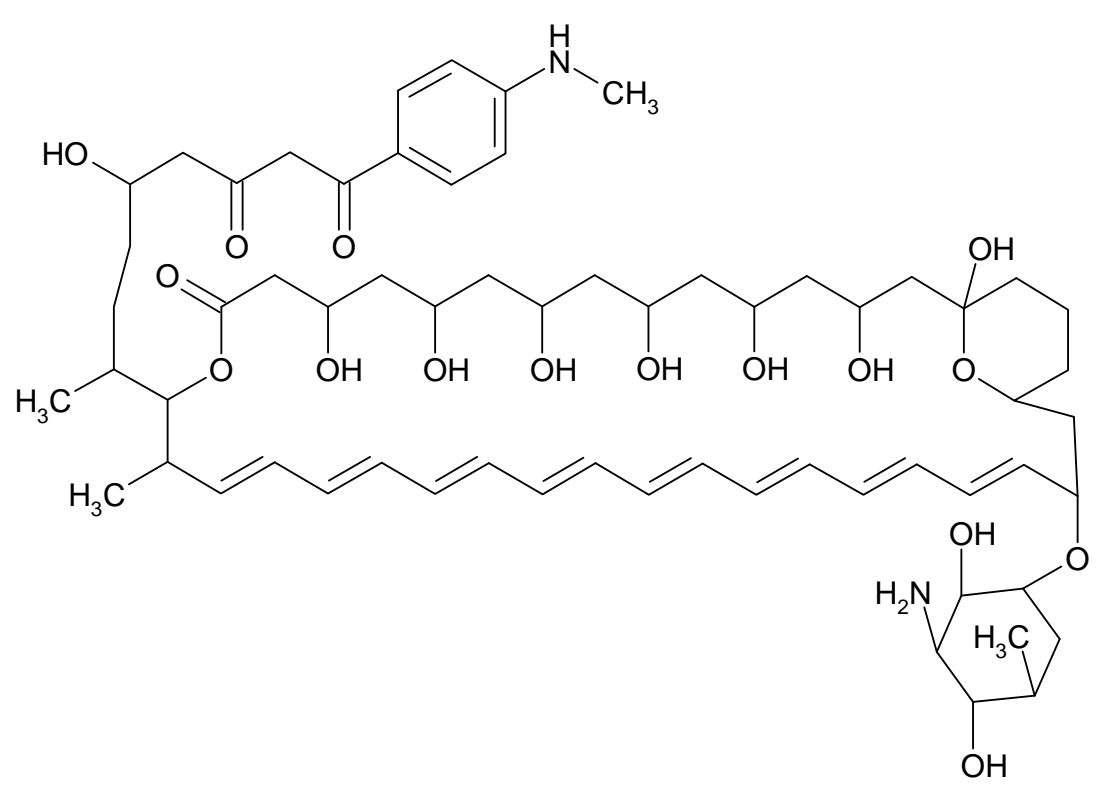

28

\subsection{Marine derived anticancer drugs}

The marine environment is infinitely diversified according to their chemical and biological characteristics. Consequently it is an astonishing resource for the discovery of new anticancer drugs. Modern technology and methodology in structure elucida- 
tion, organic synthesis, and biological assay have made it possible for the isolation and clinical evaluation of various novel anticancer agents.

Table 2. Some examples of marine natural products as anticancer agents in pre clinical or clinical trails

\begin{tabular}{llll}
\hline Compound & Chemical Class & Molecular Target & $\begin{array}{c}\text { Current } \\
\text { status }\end{array}$ \\
\hline Ecteinascidin 743 & $\begin{array}{l}\text { Tetrahydroisoquinolone } \\
\text { Alkaloid }\end{array}$ & Tubulin & Phase 2 \\
Dolastatin & Linear peptide & Tubulin & Phase 2 \\
Bryostatin & Macrocyclic lactone & PKC & Phase 2 \\
Synthadotin & Linear peptide & Tubulin & Phase 2 \\
Kahalalide F & Cyclic dipeptide & Lysosomes & Phase 2 \\
Squalamine & Aminosteroid & Phospholipid bilayer & Phase 2 \\
Dehydrodidemnin B & Cyclic dipeptide & Ornithine decarboxy- & Phase 2 \\
& & lase & Phase 2 \\
\hline Didemnin B & Cyclic dipeptide & FK-506 bp &
\end{tabular}

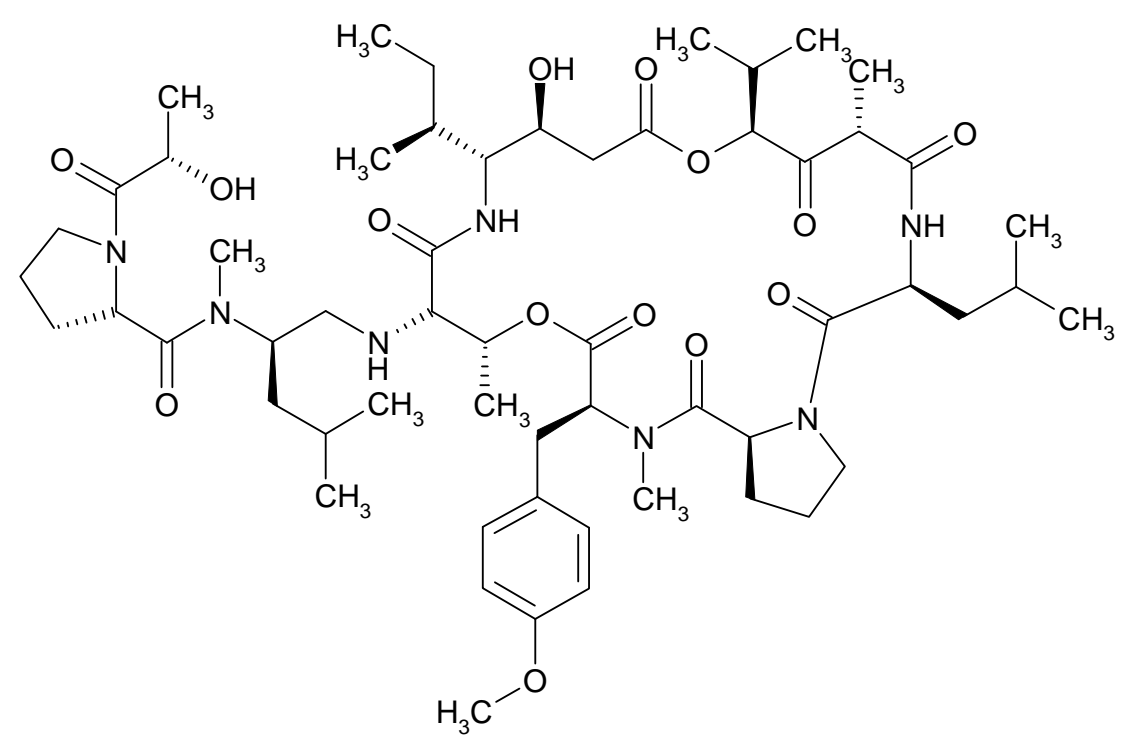

\subsection{Antiviral compounds from natural sources}

Viral diseases such as HIV, hepatitis B and C (HCV), Ebola, influenza, dengue fever and yellow fever cause some of the greatest health risks known to humans. The 2003 outbreak of the new Severe Acute Respiratory Syndrome (SARS) illustrates the potential danger and disruption, which can be caused by viral epidemics. Consequently, there has been considerable effort over the last 20 years invested into antivi- 
ral drug discovery, especially in the field of HIV. In addition to development of small molecule antiviral drugs, vaccines also are commonly used to try to prevent diseases like influenza, measles, mumps, polio and smallpox. One of the most promising compounds being evaluated to treat HIV is PA-457, a semi-synthetic derivative of the plant triterpenoid betulinic acid. Betulinic acid was found to be a weak inhibitor of HIV replication and a concerted medicinal chemistry programme by Lee and co-workers at the University of North Carolina identified a semi-synthetic derivative as the promising candidate for further evaluation. Panacos Pharmaceuticals licensed and started antiretroviral Phase I clinical trials in March 2004 and plans for Phase II clinical development were announced in December 2004. Workers at Panacos have reported that the antiretroviral activity of PA-457 was due to a novel mechanism of action targeting a late step in the Gag processing. This finding has prompted great interest as it represents a new class of HIV inhibitor. ${ }^{42}$

In 1992, workers at the National Cancer Institute (NCI), a branch of the US National Institutes for Health, reported the isolation of coumarins, which they named calanolides, with potent activity against HIV-1. The calanolides were obtained from the tree, Calophyllum lanigerum, collected in Sarawak, Malaysia. The right to develop these compounds was licensed to Sarawak Medichem Pharmaceuticals who have progressed with the most promising candidate, $(+)$-calanolide A (31) ${ }^{43}$ through to Phase II clinical trials in combination with other anti-HIV agents. Calanolide A for use in preclinical and clinical studies was produced by total synthesis as the original plant source was not readily accessible and produced only small quantities of calanolide A. The later was also found to have activity against all Mycobacterium tuberculosis and anti $\mathrm{HIV}^{44}$ strains tested, including some which are resistant to standard antitubercular drugs. This property is unique amongst and may allow more efficient treatment of patients infected with both HIV and tuberculosis. The related coumarins calanolide B (30) (costatolide), dihydrocalanolide B (31) and oxocalano$\operatorname{lide}^{45}$ (32) are also under preclinical development by Sarawak Medichem and the NCI.<smiles>CCCc1cc(=O)oc2c3c(c4c(c12)OC(C)(C)CC4)O[C@H](C)[C@H](C)[C@H]3O</smiles><smiles>CCCc1cc(=O)oc2c3c(c4c(c12)OC(C)(C)C=C4)O[C@H](C)[C@H](C)[C@H]3O</smiles> 


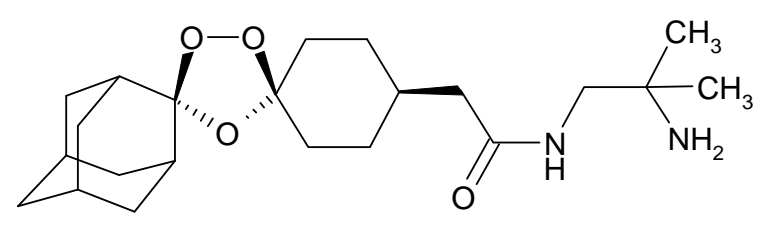

32

\subsection{Sequiterpenes from marine microorganism}

During the last two decades sesquiterpenoids and their biological activities from $A s-$ teraceae species have been the focus of numerous phytochemical, pharmacological and synthetic studies. Because the sesquiterpenoids exhibit a wide range of biological activities, and include compounds that are plant growth regulators, insect antifeedants, antifungals, anti-tumour compounds and antibacterials, there has been much interest in relating structure and oxygenation patterns to function. Eudistomins C (33) and eudistomins E46 (34) are two examples of antiviral compounds isolated from nature.<smiles>N[C@@H]1CSCON2CCC3c4cc(O)c(Br)cc4NC3C12</smiles>

33<smiles>N[C@H]1CSCOC2CCc3c([nH]c4ccc(O)c(Br)c34)[C@H]21</smiles>

34

To date, only a few sesquiterpenes have been reported from marine microorganism although they are common metabolites from fungi or plants. From the few reported sesquiterpenes, africantriol (35), 4S,7R-germacra-1E,5E-dien-11-ol (36), 1-epibicyclosesquiphellandrene (37), 2-methylisoborneol (39) and the antitumor pentalenolactones pentalenolactone $\mathrm{H} \mathrm{(40)}$ and arenamycin-E (41) are shown as examples. Marine-derived Streptomyces sp. M491 yielded new sesquiterpenes; leukotriene biosynthesis can be inhibited by some sesquiterpenes and some exhibit activity against human colon tumor cell line. 
<smiles>C[C@H]1[C@@H](O)C[C@]2(C)[C@@H]1CC(C)(C)C(O)[C@@H]1C[C@@]12C</smiles>

35<smiles>C=C1C=C2[C@@H](C(=C)C)CC[C@H](C)[C@H]2CC1</smiles>

37<smiles>CC1C(O)[C@@H]2CC[C@]1(C)C2(C)C</smiles>

39<smiles>C[C@H]1C=CC(CCC(C=C2C=CCC[C@H]2C)C(C)(C)O)CC1</smiles>

36<smiles>C[C@@H]1CCC[C@@]2(C)CCCC[C@]12O</smiles>

38

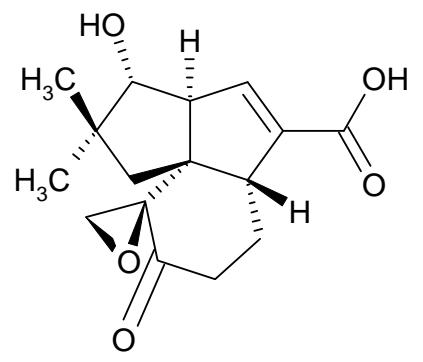

40<smiles>CC1=C[C@]23C(=O)OC[C@H]2C(C(=O)O)=C[C@H]3[C@@H]1C</smiles>

41

\section{Objectives of the present investigation}

The goal of this research is the isolation, purification and structure elucidation of new secondary metabolites with pharmacological activity from marine microorganisms. To attain this goal, a series of sequential chemical (TLC/LC-MS) and biological screening processes should be undertaken.

For the isolation and purification of the different constituents of crude extracts obtained from the fermentation, various chromatographic methods (silica gel, Sephadex LH-20, PTLC, HPLC, etc.) will be used. Various spectroscopic methods 
(NMR, MS, IR, UV, X-ray crystal analysis if possible) are utilized for the structure elucidation in addition to databases (AntiBase, Dictionary of Natural Products and Chemicals Abstracts), for rapid dereplication.

Fractions and pure compounds are submitted for different bioassays (i.e. antimicrobial test, antifungal, brine shrimp test) to explore new bioactivities against targets. 


\section{General techniques}

\subsection{Collection of strains}

Through the cooperation with different microbiological groups the strains to be investigated were supplied. All the streptomycetes reported in this dissertation were obtained from the strain collection of the former BioLeads Company in Heidelberg and the Alfred-Wegener Institute for Polar and Marine Research in Bremerhaven, Germany. Some plant extracts were obtained from Prof. S. Ganapaty, Andhra University, India. In most cases, the taxonomy on the species level was unknown in the beginning.

\subsection{Pre-screening}

From the strains received, only few were expected to produce metabolites with significant bioactivity or other interesting properties. Therefore, in order to select such strains, a pre-screening was performed. The pre-screening method includes a number of suitable qualitative or quantitative criteria, such as physical, biological, or chemical interactions of metabolites with different test systems.

To perform these tests, sub-culturing of the strains was done on agar plates for 3-7 days, which then were checked for contaminations under the microscope. In $1 \mathrm{~L} \mathrm{Er-}$ lenmeyer flasks with inflections containing $250 \mathrm{ml}$ of medium, small pieces of the agar culture were then used for inoculation, followed by incubation on a rotary shaker at $28{ }^{\circ} \mathrm{C}$. The culture broth was lyophilised and the dried residue was extracted with ethyl acetate. The crude extract obtained was used for chemical, biological, and pharmacological screenings. TLC with different spray reagents as well as HPLC played a vital role in the chemical screening.

\subsection{Chemical screening}

It is an expensive task and a multiple step procedure, to search for and isolate pure bioactive compounds from bacteria. Therefore, it is wise to reduce the unnecessary work, such as the re-isolation of known metabolites either from the crude extract or from a partially purified fraction. The most important step for this purpose is the chemical screening, which allows reaching this goal at the earliest stages of separation. One of the cheapest and simplest methods is TLC (thin layer chromatography), which is used for the detection of bacterial constituents in the crude extract. TLC is quick and easy to perform and requires simple equipment with results that are suffi- 
ciently reproducible, when compared with other methods like HPLC or LC MS. Based on the $R_{\mathrm{f}}$ value, a suitable organic solvent system is chosen for elution. After developing, the TLC plate is visualized under UV light. Further interesting zones are localized by exposure to different spray reagents. Numerous spray reagents are available for the detection of specific compounds while others are universal. Only the following spray reagents listed below were used in this study.

Many structural elements show different colour reactions with anisaldehyde/sulphuric acid.

Indoles turn pink, blue or violet, when Ehrlich's reagent is sprayed on TLC. It is a rather specific reagent for these compounds. Some other nitrogen containing compounds and furans become brown, anthranilic acid derivatives change to yellow.

Addition of concentrated sulphuric acid on TLC is especially used for polyenes. Short conjugated chains show a brown or black colour while carotenoids develop a blue or green colour.

Sodium hydroxide can be used for the detection of peri-hydroxyquinones, which turn red, blue or violet, while the deep red prodigiosins show a yellow colour.

For the detection of peptides, the chlorine/o-dianisidin reaction is used as a universal reagent for the amide bond.

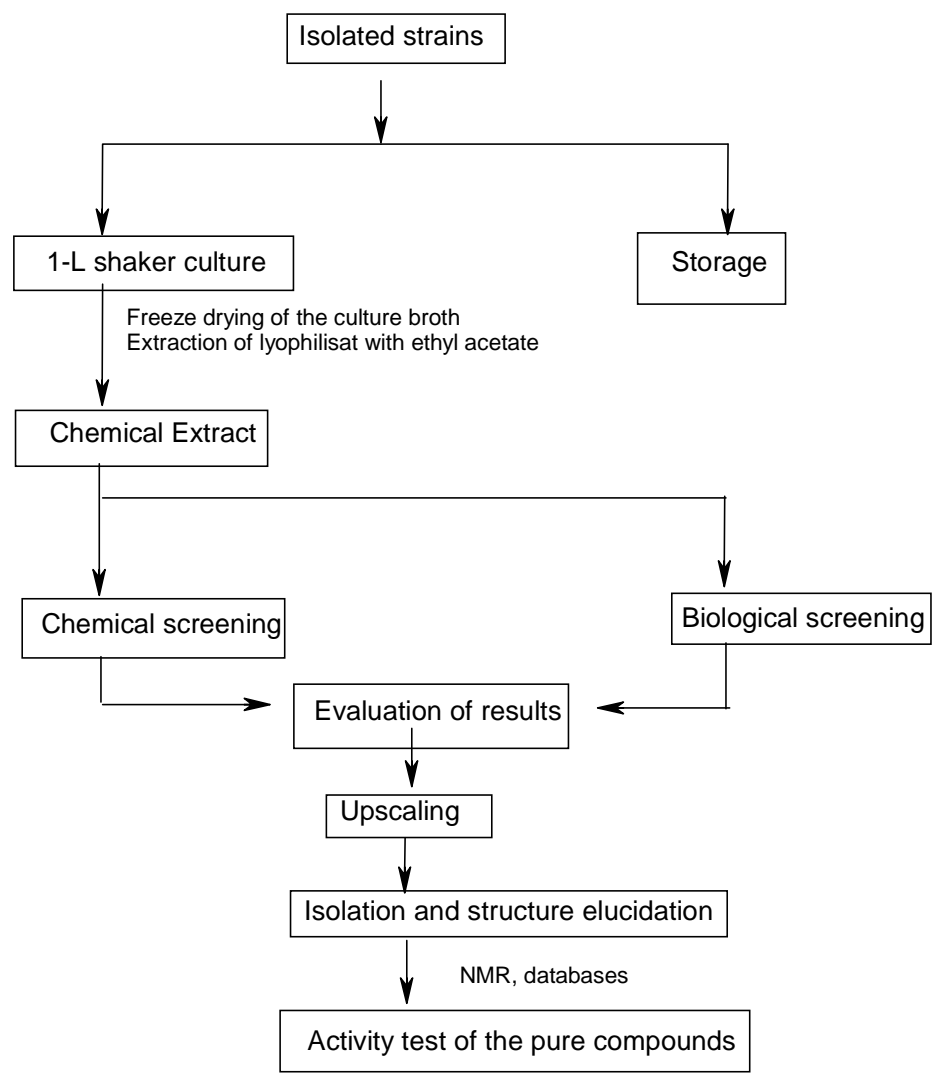

Figure 1 : General screening of the selected strains 


\subsection{Pharmacological and biological assays}

An appropriate test is needed to screen a crude extract for bioactive substances. The screening programs developed in natural product chemistry are usually divided into two groups: specialized screens and general screening bioassays. The screening methods are different for pharmaceutical industry and university research groups. In any bioassay method, high capacity, sensitive, low cost, and quiet solutions take preference. Generally, two types of screening are available: 1) the vertical screening mostly used in industry shows high selectivity and narrow results (1:10.000-1:20.000); it is used in combination with high throughput techniques. 2) The horizontal screening, which exhibits low selectivity, however, a higher responds rate (1:3-1:100) and gives therefore a quick overview. In our research group, the screening of the crude extract is performed by using the agar diffusion test with bacteria (Gram-positive, Gramnegative), fungi, plant pathogens and higher organisms for cytotoxicity: The crude extracts are tested against Escherichia coli, Bacillus subtilis, Mucor miehei, Candida albicans, Streptomyces viridochromogenes (Tü 57), and Staphylococcus aureus as well as the microalgae Chlorella sorokiniana, Chlorella vulgaris and Scenedesmus subspicatus.

Cellular cytotoxicity can be tested using the brine shrimp assay with larvae of Artemia salina. The brine shrimp test is a good indicator for potential anticancer activity and has a parallelism of about $95 \%$ with cellular systems.

Further pharmacological tests in our group are carried out by the collaborative industries, for example Oncotest (Freiburg) or BASF (Ludwigshafen). Both the results from chemical and biological screening complement each other and allow us to choose suitable strains for further scale-up.

\subsection{Cultivation and scale-up}

After a primary screening, the cultivation and scale-up steps are carried out. In order to choose the best medium for highest activity, an optimisation of the culture conditions may sometimes be done to improve the yield or the metabolite pattern. Either the fermentation in shaking flask or in a fermentor is used for the culture of bacteria. For the fermentor, a pre-culture of 2 or $5 \mathrm{~L}$ is used for the inoculation.

After harvesting of the selected strain, the culture broth is mixed with Celite and filtered under vacuum. After separation of the mycelium, the water phase can be extracted with a suitable organic solvent like ethyl acetate. However, it is highly recommend using a solid phase extraction with XAD resin as it is not harmful and reduces considerably the volume of solvents used and can adsorb more polar com- 
pounds effectively. The mycelium is extracted with ethyl acetate and acetone. The organic extracts are evaporated to dryness and the crude extract obtained is used for separations.

\subsection{Isolation methods}

Polarity or molecular weight of the compounds of interest play the most important role in the separation but the amount of the crude extract is always a factor in choosing an appropriate method. Fat is produced by every strain and makes the purification sometimes difficult. Therefore, the crude extract is first defatted using cyclohexane, followed by silica gel chromatography using a gradient of increasing polarity with various solvent systems $\left(\mathrm{CH}_{2} \mathrm{Cl}_{2} / \mathrm{MeOH}\right.$ or cyclohexane/ethyl acetate etc.). Another commonly used and effective method is size-exclusion chromatography (Sephadex LH-20), which offers the advantage of a high recovery rate and minimizes the destruction of compounds. It is used preferentially when the amount of the crude extract is relatively less and already partially purified by silica column chromatography. Some further methods like RP-18 chromatography, PTLC and HPLC are also used for final purification.

\subsection{Partial identification and dereplication}

Researchers are still trying to find more effective methods for the discovery of unexplored bioactive compounds and to minimize the re-isolation of known metabolites. This steadily increases the challenge for the natural product chemist. To meet this challenge a number of methods have already been developed to identify known metabolites at the earliest stages of the purification or from a partially purified mixture of metabolites. The complimentary processes for the rapid identification of already reported compounds or the elucidation of a partial structure of an unknown compound is called "dereplication". ${ }^{47}$ In the recent years, several databases became available to identify known or relevant compounds from NMR derived sub-structures or physical and chemical properties by using computers. ${ }^{48}$ To date, the most effective and comprehensive tools for the detection of microbial and marine natural products are the data collections AntiBase ${ }^{49}$ and AntiMarin. For the detection of natural compounds from any other source the "Dictionary of Natural Products (DNP)" choice. These databases are also important tools in the identification of new metabolites with respect to compounds classes and chromophors. Presently, dereplication is being done with liquid chromatography in combination with NMR spectroscopy (HPLC-NMR) as well as tandem mass spectroscopy (HPLC-MS/MS) using refer- 
ences. The advantage of using HPLC-NMR or HPLC-MS/MS is that both methods require minimum amounts of material to obtain information about known constituents from biological matrices e.g. extracts from marine microorganisms ${ }^{51}$ or plants ${ }^{52}$. Most of the isolated natural compounds of interest are thermally labile. Therefore, HPLCESIMS/MS method has made it possible to identify known metabolites from multicomponent mixtures with high selectivity and sensitivity. ${ }^{53}$ Due to the achiral character of mass techniques and NMR spectroscopy, the information concerning the full absolute three-dimensional structures can not be obtained by HPLC NMR or HPLCMS/MS techniques. Two further methods, CD (circular dichroism) and ORD (optical dichroism) spectroscopy are widely used for the attribution of the absolute configuration by comparing the experimental data with those of structurally closely related compounds or quantum-chemical calculations. 


\subsection{Description of Strain Codes}

Bacterial strains and other samples were obtained from various sources:

1) Strains with the prefix ADM were isolated by Prof. H. Anke (Kaiserslautern) from soil samples provided by Professor Armin de Meijere from our institute.

2) Strains with the prefix B are marine Streptomyces, collected and identified by Dr.

E. Helmke, Alfred-Wagner Institute (AWI) for Polar Marine Research, Bremen, Germany; Act strains are marine actinomycetes from the same origin.

3) Strains with the prefix GT are terrestrial Streptomyces sp., which were received from the Hans Knöll Institute, Germany, in June 2006.

4) The terrestrial actinomycete N 435 was obtained from Prof. Wolf, one of our research collaborators. The goal in selecting this strain was to find active compounds against plant pathogenic fungi.

5) Strains with the prefix GW are terrestrial Streptomyces sp., which were obtained from the strain collections of bioLeads, Heidelberg, Germany.

6) A fungal metabolite was sent by Prof. Dr. H. Anke, Institute for Biotechnology and Drug Research, Kaiserslautern, Germany.

7) The plant samples from Tephrosia calophylla were obtained from Professor Seru Ganapaty from The Pharmacognosy and Photochemistry Division, Andhra University, India.

\section{$4 \quad$ Investigation of selected marine and terrestrial Streptomycetes}

\subsection{Terrestrial Streptomyces sp. ADM 14}

The crude extract of the terrestrial Streptomyces sp. ADM 14 showed moderate activity in the biological screening against the test organisms Bacillus subtilis, Staphylococcus aureus and Escherichia coli, and a good activity against Streptomyces viridochromogenes. In the TLC screening, the extract showed nonpolar yellow zones, and medium to high polar grey-violet bands, after spraying with anisaldehyde/sulphuric acid. In addition, numerous UV absorbing and fluorescent zones were observed, which were coloured orange to violet when sprayed with anisaldehyde/sulphuric acid, or pink to blue with Ehrlich's reagent. All major compounds showed medium to high polarity on TLC. 
Well-grown agar plates of the strain ADM 14 were used to inoculate 20 litres of $\mathrm{M}_{2}$ medium and cultivated as shaker culture for 5 days. The obtained broth was filtered over Celite, the water phase was extracted with XAD-16, and the resin eluted with methanol; the biomass was extracted with ethyl acetate. The combined extracts were evaporated to dryness under vacuum to afford a brown crude extract. The extract was defatted with cyclohexane and the methanolic part was pre-separated by column chromatography into five fractions. Further purification on Sephadex LH-20 followed by PTLC and silica gel column chromatography afforded 14 compounds, mostly primary metabolites. These compounds were 1-hydroxy-4-methoxy-naphthalene-2caboxylic acid (43), 5'-methyl-thioadenosine (44), 5,7,9,11-tetrahydroxy-4,6,8,10tetramethyl-tridec-2-enoic acid (45), uridine, adenine, uracil, indole-3-acetic acid, indole-3-carboxylic acid, tryptophol, tyrosol, phenylacetic acid, anthranilic acid and a new compound. Description of trivial compounds like uracil, adenine, indole-3-acetic acid, indole-3-carboxylic acid, tryptophol, and tyrosol are not included here, as they have been isolated frequently in our group.

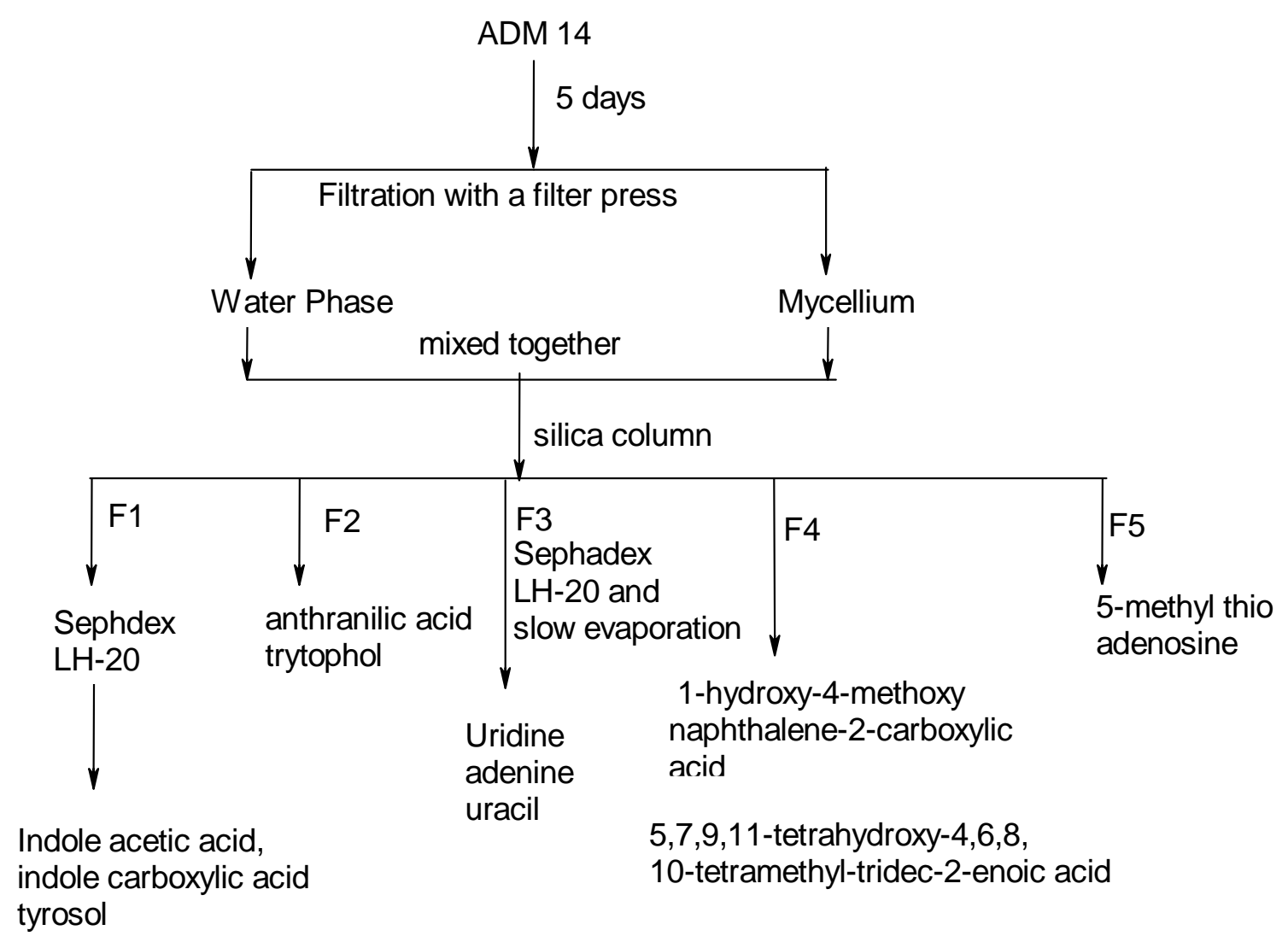

Figure 2: Working-up scheme for terrestrial Streptomyces sp. ADM 14. 


\subsubsection{1-Hydroxy-4-methoxynaphthalene-2-caboxylic acid}

From fraction 2, a grey coloured UV active solid was obtained, which gave a molecular weight of $m / z 218$ by (-)-ESIMS spectrometry. The ${ }^{1} \mathrm{H}$ NMR spectrum showed a methoxy signal at $\delta 3.96$, but no further signal in the aliphatic region. Five protons were observed in the aromatic region, an ABCD system and a singlet at $\delta 7.29$ of a proton, which was obviously present in another ring. With the help of ESI mass spectrometry and ${ }^{1} \mathrm{H}$ NMR measurements, the compound was tentatively identified as 1hydroxy-4-methoxy-naphthalene-2-carboxylic acid. The ${ }^{1} \mathrm{H}$ NMR data were, however, not completely identical with the published values. ${ }^{54}$ To confirm the structure of the natural product, a sample was synthesized for comparison.

1-Hydroxy-4-methoxynaphthol (42) and freshly heated $\mathrm{K}_{2} \mathrm{CO}_{3}$ were mixed homogeneously. It was then taken into a $250 \mathrm{ml}$ autoclave and dry ice was added. The reaction mixture was heated in the sealed autoclave to $150{ }^{\circ} \mathrm{C}$ for 6 hours under a pressure of 70-80 bar to produce 1-hydroxy-4-methoxynaphthoic acid (43).<smiles>COc1cc(C(=O)[18OH])ccc1-c1ccc2c(O)c(C(=O)O)cc(OC)c2c1</smiles>

42

43

Figure 3: Synthesis of 1-hydroxy-4-methoxy-naphthalene-2-caboxylic acid (43).

The synthetic compound also showed substantial deviations from the values of the natural acid. The isomeric 4-hydroxy-1-methoxy-2-naphthoic acid was definitely excluded due to the chelated 1-OH signal in natural and synthetic samples. As also no further alternative came into consideration, natural and synthetic product were finally mixed. The ${ }^{1} \mathrm{H}$ NMR spectrum of this mixture showed only a single data set, so that impurities or concentration effects must explain the previous differences. 


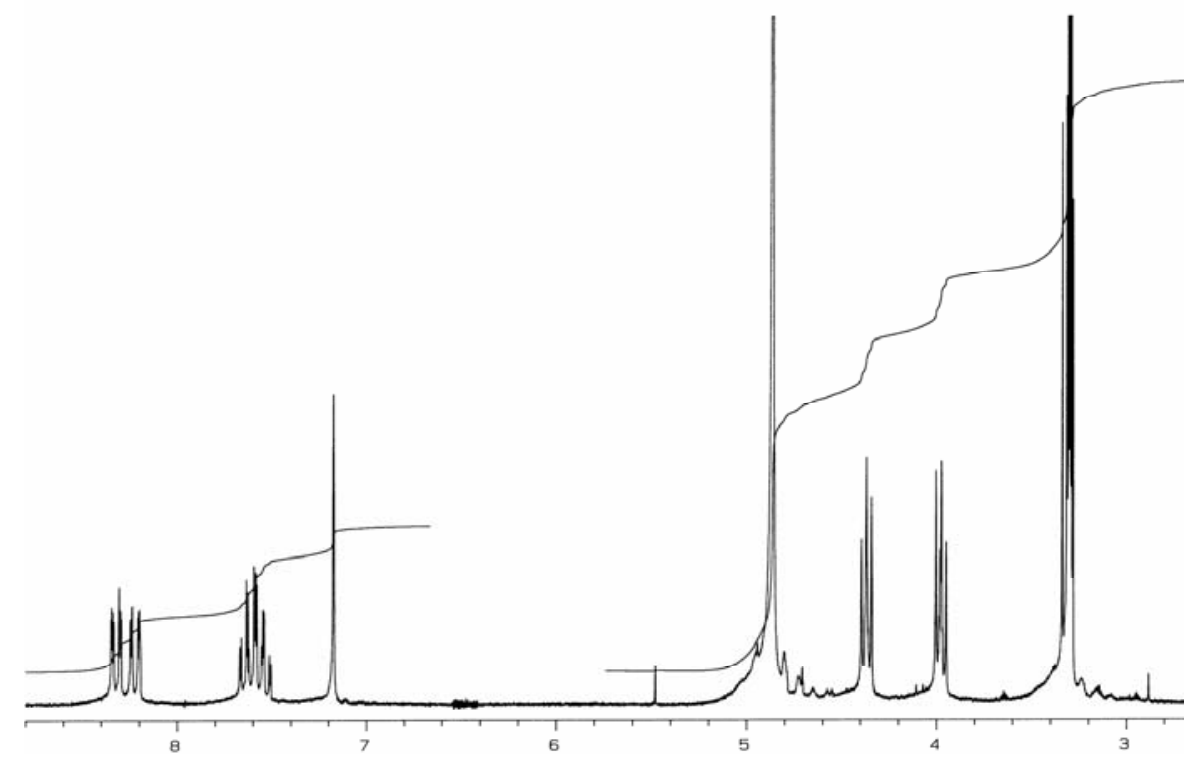

Figure 4: $\quad{ }^{1} \mathrm{H}$ NMR spectrum $\left(\mathrm{CD}_{3} \mathrm{OD}, 300 \mathrm{MHz}\right)$ of 1-hydroxy-4-methoxynaphthalene-2-carboxylic acid (43).<smiles>COc1cc(C(=O)O)c(O)c2ccccc12</smiles>

43

\subsubsection{5'-Methyl-thioadenosine}

Fraction 5 contained a colourless UV absorbing band of a polar component, which was coloured to brown after spraying with anisaldehyde/sulphuric acid and heating. The constituent was isolated as a colourless solid by silica gel column chromatography followed by slow evaporation of the sample.

The ${ }^{1} \mathrm{H}$ NMR spectrum showed two $1 \mathrm{H}$ singlets in the aromatic region at $\delta 8.35$ and 8.15 , and a broad $2 \mathrm{H}$ singlet at $\delta 7.23$ (H/D exchangeable), which is characteristic for an adenine moiety. In the aliphatic region, it displayed a doublet at $\delta 5.92\left({ }^{3} J=6.1\right.$ $\mathrm{Hz}$ ) of an anomeric proton, a broad singlet of $2 \mathrm{OH}$ groups at $\delta 5.65$, in addition to three oxygenated methine protons at $\delta 4.73\left(\mathrm{t},{ }^{3} J=6.3 \mathrm{~Hz}\right), 4.16(\mathrm{~m})$, and $4.07(\mathrm{~m})$. This resembled the spectrum of adenosine, however, the signal between $\delta 3.64-3.57$ of the oxygenated methylene group in adenosine had disappeared and was replaced up- 
field by an ABX 2H signal between $\delta 2.90-2.75\left(\mathrm{dd},{ }^{3} J=14.1 \mathrm{~Hz}, 6 \mathrm{~Hz}\right)$. In addition, a $3 \mathrm{H}$ singlet at $\delta 2.05$ was interpreted as a methyl group attached to an $s p^{2}$ carbon moiety or as a thiomethyl group.

In the aliphatic region, the ${ }^{1} \mathrm{H}$ NMR data were characteristic for a ribose moiety except that the oxygenated methylene group at 5'-postion (displayed normally between $\delta$ .3.5-3.7) was exchanged against an ABX signal of a methylene group at $\delta$ 2.90-2.75.

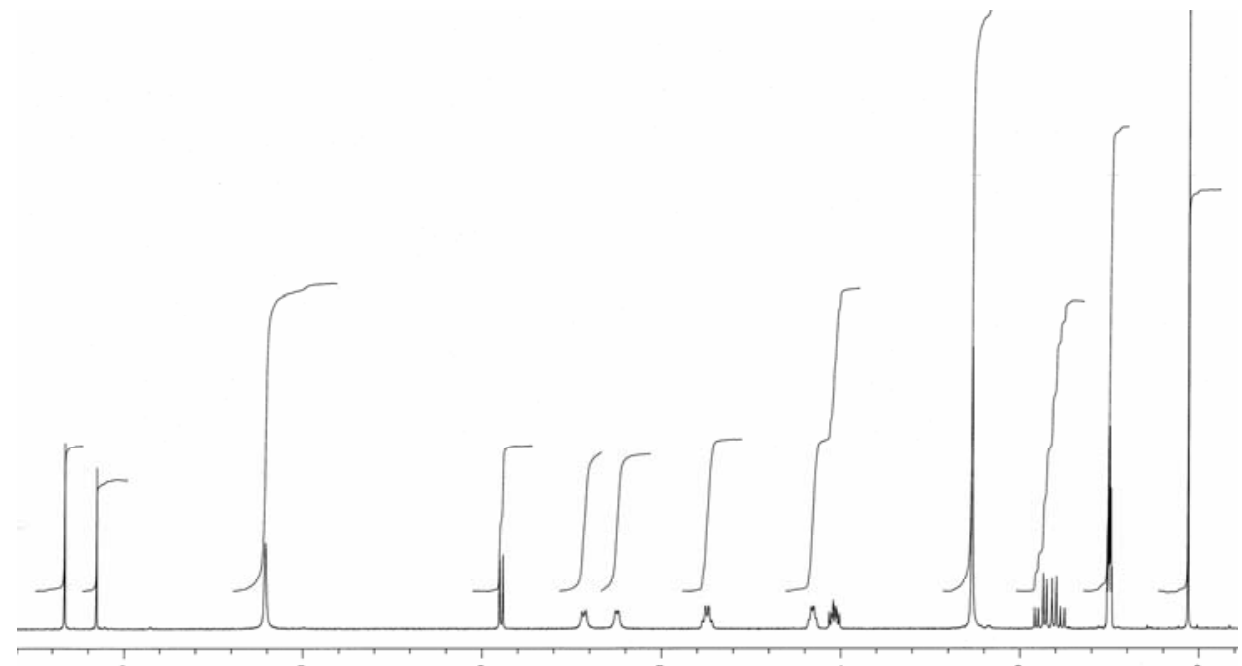

Figure 5: ${ }^{1} \mathrm{H}$ NMR spectrum ([D6]DMSO, $300 \mathrm{MHz}$ ) of 5'-methyl-thioadenosine (44).

The (+)-ESI mass spectrometry showed three quasi-molecular ions at $\mathrm{m} / z, 298([\mathrm{M}+$ $\left.\mathrm{H}]^{+}\right), 320\left([\mathrm{M}+\mathrm{Na}]^{+}\right)$, and $616\left([2 \mathrm{M}+\mathrm{Na}]^{+}\right)$. (-)-ESIMS showed two quasimolecular ion peaks at $296\left(\left[\mathrm{M}-\mathrm{H}^{-}\right)\right.$, and $593\left([2 \mathrm{M}-\mathrm{H}]^{-}\right)$pointing to 297 as the molecular mass of the compound.

A search in AntiBase using the above spectral data resulted in 5'-methyl-thioadenosine, which was further confirmed by direct comparison with an authentic spectrum as well as with literature data. ${ }^{55}{ }^{5}$-Methyl-thioadenosine is a common bacterial metabolite and was isolated previously in our group.<smiles>CSCC1O[C](n2cnc3c(N)ncnc32)C(O)C1O</smiles> 


\subsubsection{5,7,9,11-Tetrahydroxy-4,6,8,10-tetramethyl-tridec-2-enoic acid}

5,7,9,11-Tetrahydroxy-4,6,8,10-tetramethyl-tridec-2-enoic acid (45) was obtained as a colourless liquid, which showed a violet colour reaction with anisaldehyde/sulphuric acid. In the ${ }^{1} \mathrm{H}$ NMR spectrum, there were two protons observed, which were attached with $s p^{2}$ carbon. The ${ }^{1} \mathrm{H}$ NMR spectrum showed several methyl groups between $\delta$ 0.98-0.78. There were two $s p^{2}$ protons at $\delta 6.32$ and 5.86 and four oxygenated methine protons at $\delta 3.74-3.41$.

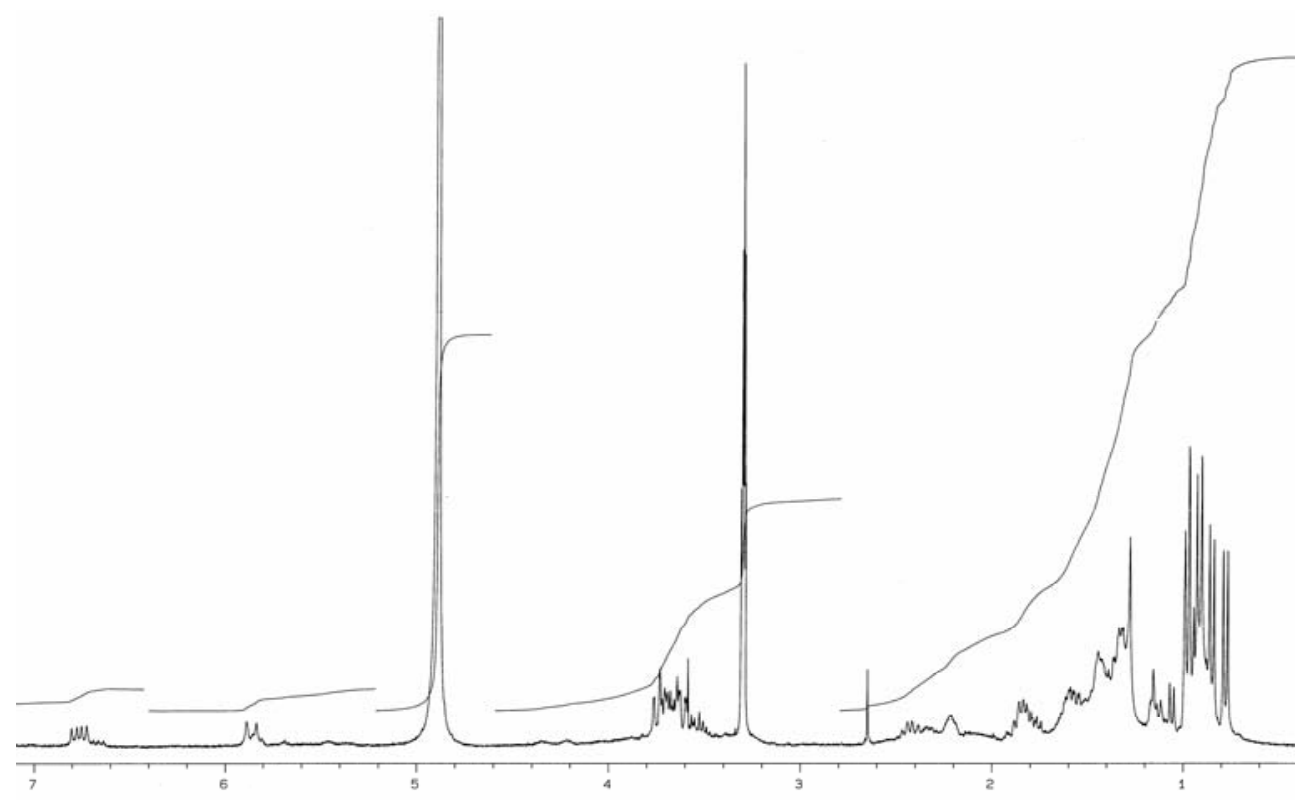

Figure 6: ${ }^{1} \mathrm{H} \mathrm{NMR}$ spectrum $\left(\mathrm{CDCl}_{3}, 300 \mathrm{MHz}\right)$ of 5,7,9,11-tetrahydroxy-4,6,8,10tetramethyl-tridec-2-enoic acid (45).

The ${ }^{13} \mathrm{C}$ NMR spectrum delivered 17 signals as expected for the molecular formula, a carbonyl at $\delta 171.7$ of an ester or acid, and two $s p^{2}$ carbon signals at $\delta 150.4$ and 126.4 which seemed to be adjacent to each other. There were four oxygenated methine carbons observed at $\delta 80.3,79.2,76.4$, and 74.6. Moreover, five methyl and four methine carbons were also present in the spectra. 


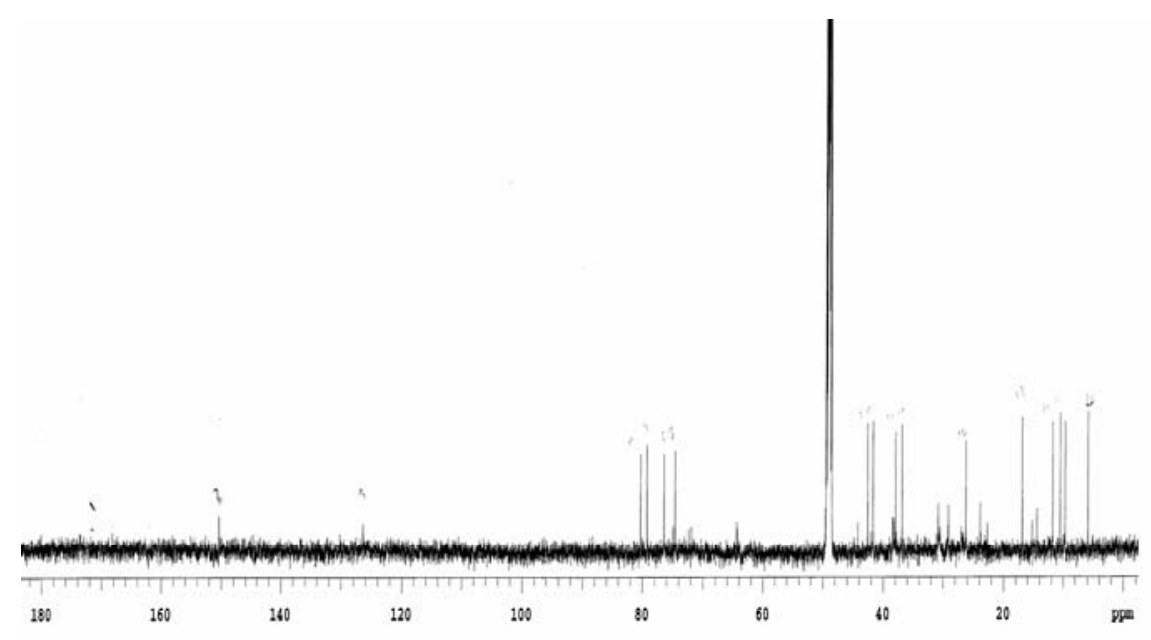

Figure 7: ${ }^{13} \mathrm{C}$ NMR spectrum $\left(\mathrm{CDCl}_{3}, 125 \mathrm{MHz}\right)$ of 5,7,9,11-tetrahydroxy-4,6,8,10tetramethyl-tridec-2-enoic acid (45).

The H-H COSY spectrum showed that the four methyl groups appeared as doublets because of adjacent methine protons. A methyl triplet indicated a methylene group as next neighbour. The spectra showed further a long aliphatic chain.

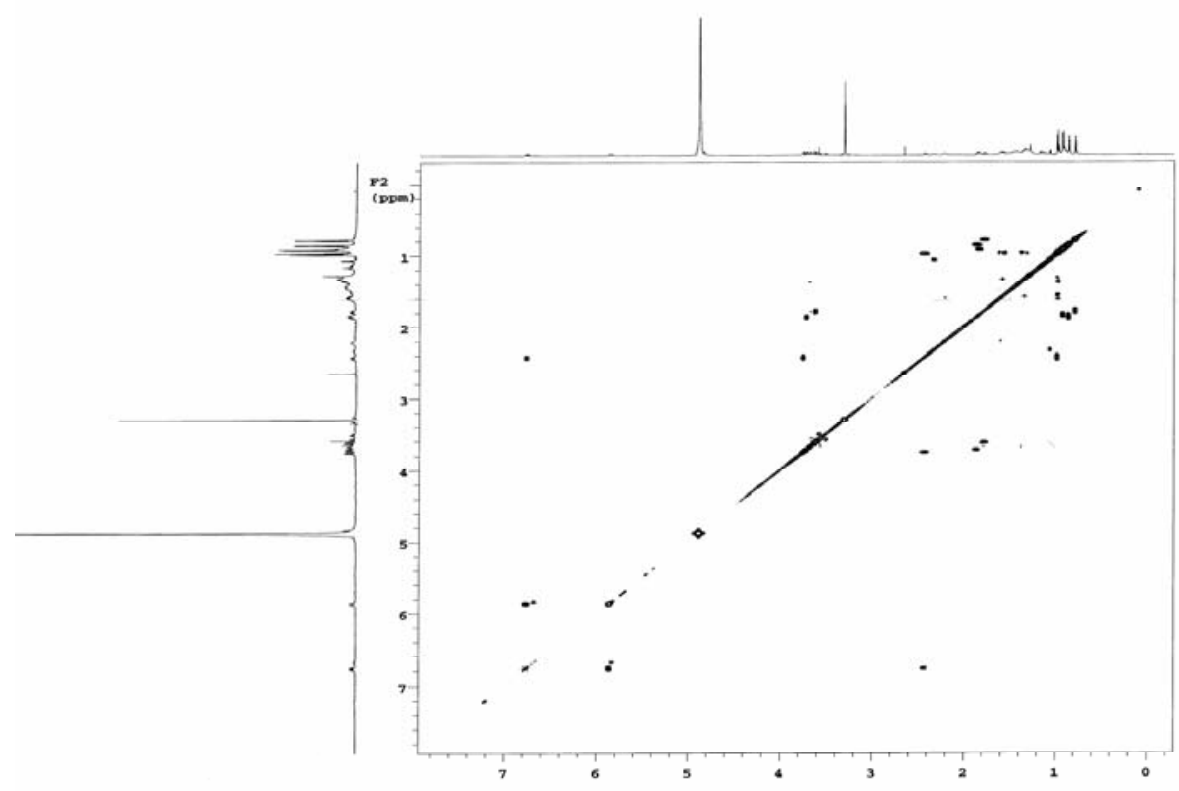

Figure 8: $\mathrm{H}-\mathrm{H}$ COSY spectrum $\left(\mathrm{CDCl}_{3}, 600 \mathrm{MHz}\right)$ of 5,7,9,11-tetrahydroxy4,6,8,10-tetramethyl-tridec-2-enoic acid (45).

In the HMBC spectrum, the $s p^{2}$ protons showed correlations with the carbonyl signal. The structure of 5,7,9,11-tetrahydroxy-4,6,8,10-tetramethyl-tridec-2-enoic acid resulted taking all further correlations into account. The structure was confirmed by 
comparing the spectroscopic data with those of the related 10-deoxymethynolide (46). ${ }^{56}$<smiles>CCC(O)C(C)/C=C/C(O)C(C)CC(C)C(O)C(C)C(=O)O</smiles>

45<smiles>CCC(OC(=O)C(C)C(O)C(C)CC(C)C(O)C=CC(C)C(C)C)C(C)C</smiles>

46

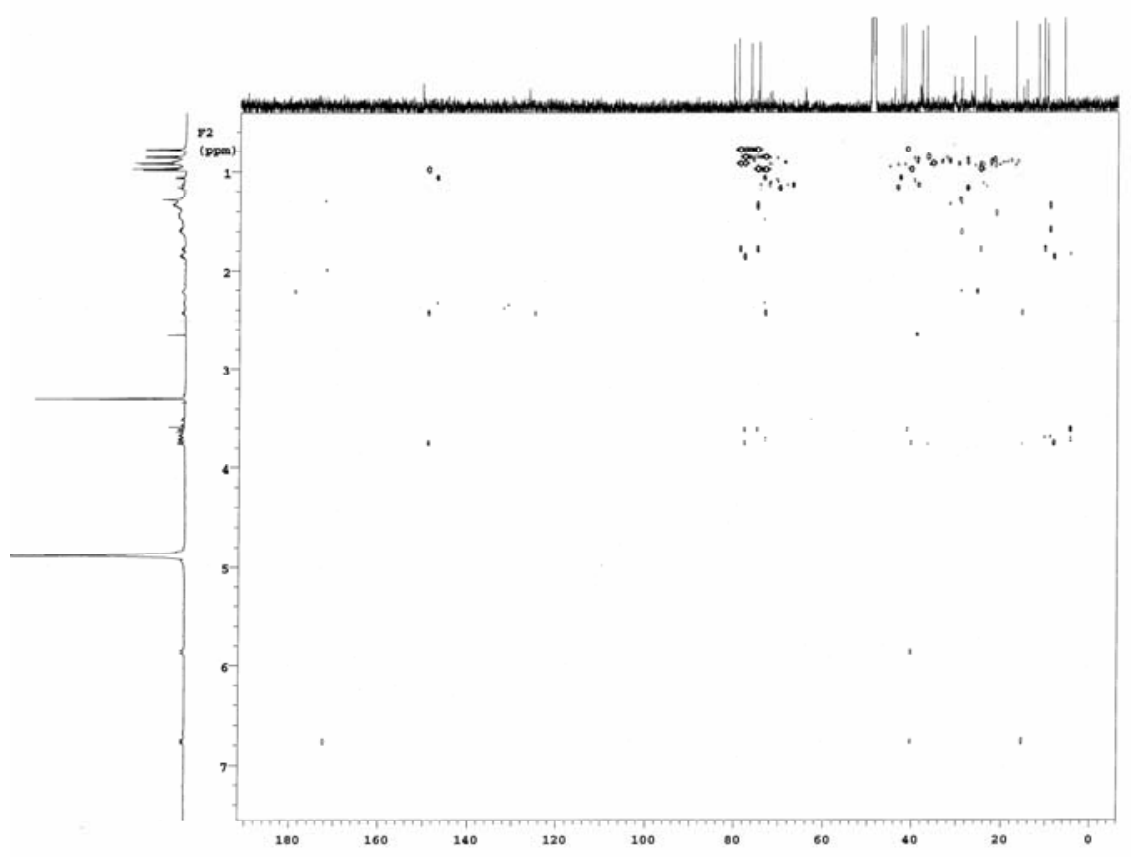

Figure 9: $\mathrm{HMBC}$ spectrum $\left(\mathrm{CDCl}_{3}, 600 \mathrm{MHz}\right)$ of 5,7,9,11-tetrahydroxy-4,6,8,10tetramethyl-tridec-2-enoic acid (45).

Compound 45 is part of macrolides like erythromycin and may be a biosynthetic intermediate of the macrolides. Compound $\mathbf{4 6}$ showed significant antibiotic activity. ${ }^{57}$

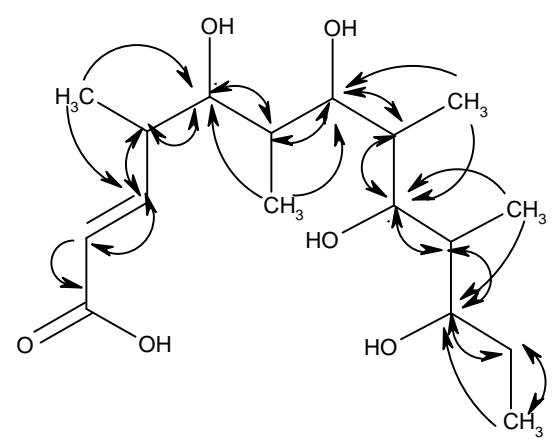

Figure 10: HMBC correlation of compound 45 


\subsection{Streptomyces sp. B7880}

The ethyl acetate extract of the marine-derived streptomycete isolate B7880 drew our attention due to the presence of several pink to red coloured, UV absorbing zones. The strain exhibited high biological activity against Mucor miehei (Tü284), Candida albicans, and the alga Chlorella sorokiniana.

The strain B7880 was cultivated on a 20 -liter scale for 7 days at $28^{\circ} \mathrm{C}$ as shaker culture $(110 \mathrm{rpm})$. After filtration, the dark red broth was filtered to separate the water phase and mycelium. The water phase was extracted by XAD-16 and the mycelium was extracted with ethyl acetate. The extracts from both phases were combined, as TLC did not show much difference. For isolation and purification of the metabolites, the resulting product was chromatographed on silica gel column using a chloroformmethanol gradient. Further separation of the fractions containing the reddish-orange peri-hydroxyquinones delivered four cinerubin derivatives: $\zeta$-pyrromycinone (47), cinerubin B (49), cinerubin A (51), and cinerubin X (52).

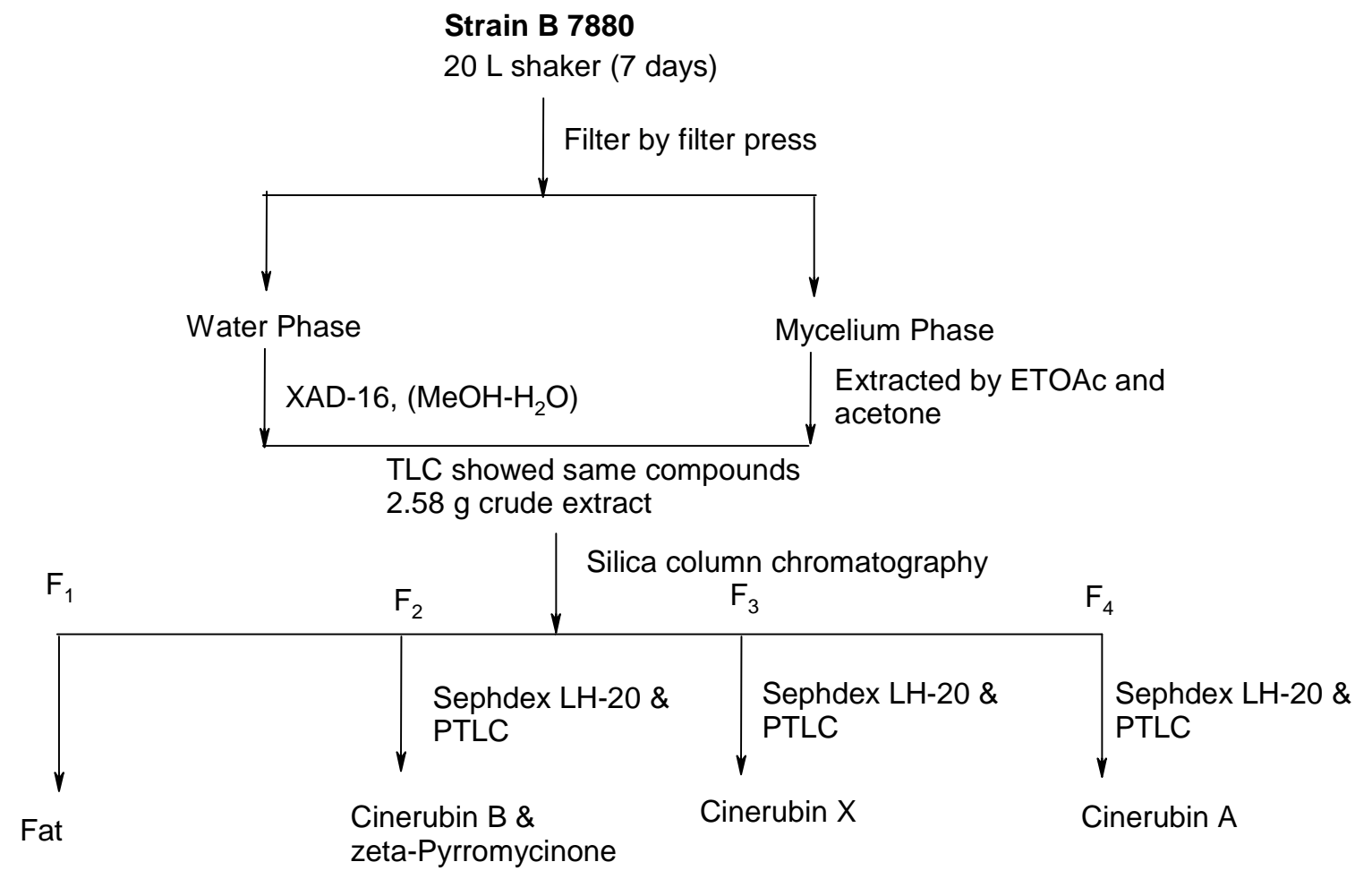

Figure 11: Working-up scheme for strain B 7880

\subsection{1 $\zeta$-Pyrromycinone; Galirubinone C}

Through the further isolation and purification of the fast moving fraction 2, compound was isolated as low polar main product from an orange fluorescent zone (366 nm), 
which turned to violet by treating with sodium hydroxide, thus indicating a perihydroxyquinone. It delivered a red-orange solid on further PTLC and chromatography on Sephadex LH-20. The ${ }^{1} \mathrm{H}$ NMR spectrum showed three $1 \mathrm{H}$ singlets at $\delta 13.02$, 12.61 , and 12.28 , which are characteristic for peri-hydroxy groups in a quinone moiety. In the aromatic region, one singlet of $1 \mathrm{H}$ at $\delta 7.69$, and an $\mathrm{AB}$ system $\delta 7.29$ and 7.28 was detected, as for ortho-coupled protons in a 1,2,3,4-tetrasubstituted benzene ring. In addition, a $1 \mathrm{H}$ singlet was observed at $\delta 3.96$, which could be assigned as oxymethine or as methine group flanked by two $s p^{2}$ carbon atoms.

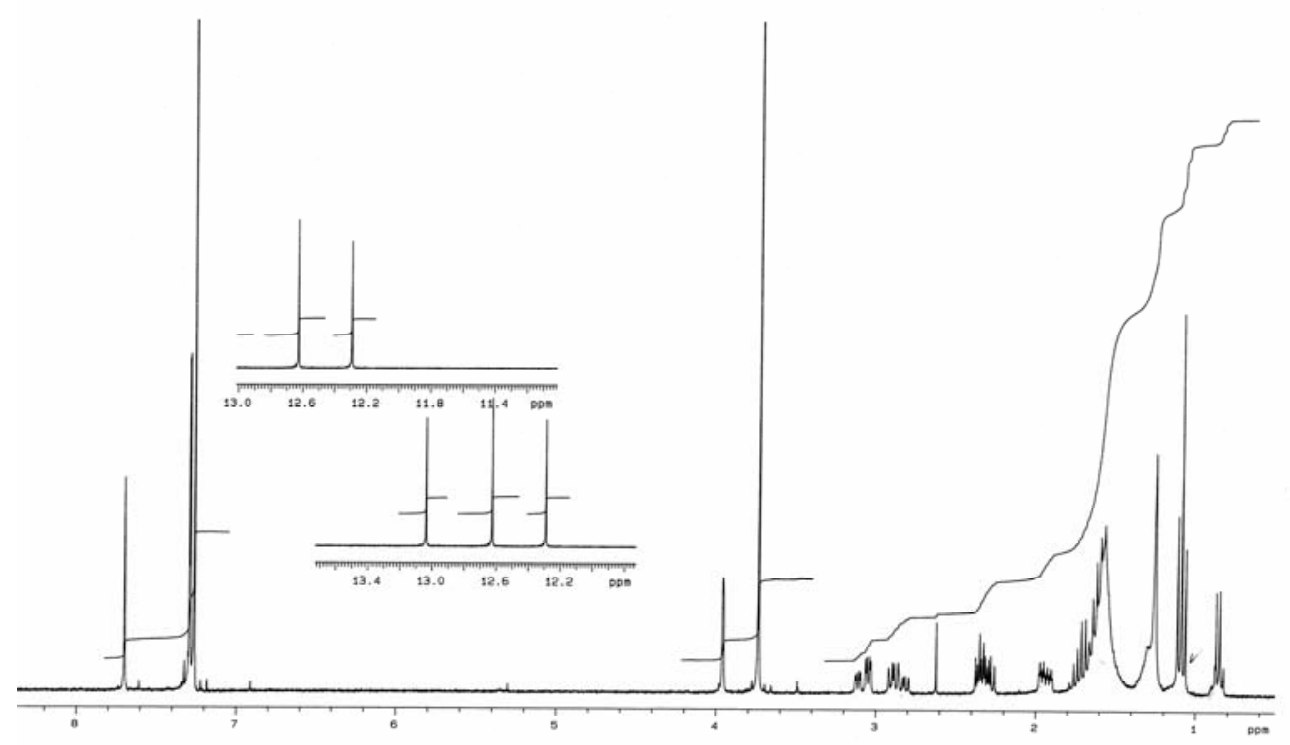

Figure 12: ${ }^{1} \mathrm{H} \mathrm{NMR}$ spectrum $\left(\mathrm{CDCl}_{3}, 300 \mathrm{MHz}\right)$ of galirubinone $\mathrm{C}$ (47).

One methoxy group was observed at $\delta 3.74$ (s). Four multiplets, each of $1 \mathrm{H}$ of two vicinal methylene groups were displayed at $\delta 3.07\left(7-\mathrm{H}_{\mathrm{A}}\right), 2.86\left(7-\mathrm{H}_{\mathrm{B}}\right), 2.30\left(8-\mathrm{H}_{\mathrm{A}}\right)$ and $1.95\left(8-\mathrm{H}_{\mathrm{B}}\right)$. The first methylene group $(\delta 3.07$ and 2.86$)$ should be next to an $s p^{2}$ carbon, while the other one ( $\delta 2.30$ and 1.95$)$ should be next to an oxygenated quaternary $s p^{3}$ carbon. Furthermore, a third methylene group was giving two multiplets between $\delta 1.70-1.58$, which could be adjacent to a methyl group, observed as triplet at $\delta$ 1.09 , to constitute an ethyl group. The (-)-ESI mass spectrometry exhibited two quasimolecular ions at $\mathrm{m} / \mathrm{z} 845\left(\left[2 \mathrm{M}+\mathrm{Na}-2 \mathrm{H}^{-}\right)\right.$and $411\left([\mathrm{M}-\mathrm{H}]^{-}\right)$, establishing the molecular weight as 412 Dalton. It was further confirmed by a CI mass spectrometry, which exhibited an ion peak at $\mathrm{m} / z 430\left[\mathrm{M}+\mathrm{NH}_{4}\right]^{+}$. Based on the above chromatographic and spectroscopic data and searching in AntiBase, one structure was suggested, $\zeta$-pyrromycinone ${ }^{58}$ (47). The compound was further confirmed by direct comparison of the 1D NMR and MS data with authentic values. 


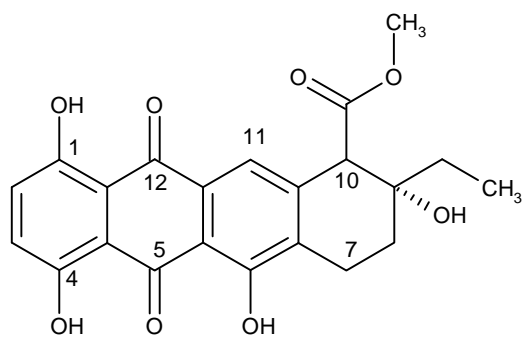

47

\subsubsection{Cinerubin B; Ryemycin B}

Cinerubin B was isolated as an UV fluorescent major product from fraction 3 as redorange solid. By using sodium hydroxide, it showed a colour change to violet, indicating a peri-hydroxyquinone. The ${ }^{1} \mathrm{H}$ NMR spectrum showed a close similarity with $\zeta$ pyrromycinone. Three peri-hydroxy groups of the pyrromycinone moiety were observed at $\delta 12.97,12.83$ and 12.25 . In addition, the $1 \mathrm{H}$ singlet of an aromatic proton at $\delta 7.73(11-\mathrm{H})$, and two $\mathrm{AB}$ protons were observed at $\delta 7.32$ and $7.28(2,3-\mathrm{H})$, as part of the pyrromycinone skeleton. Also, the ester methoxy group and the 10-H singlet were present at $\delta 3.70$ and 4.13, respectively. Moreover, an ethyl group was established due to a triplet at $\delta 1.09$ and a methylene group at $\delta 1.87$ and 1.52 . Besides, 8$\mathrm{H}_{2}$ protons were observed between $\delta 2.48-2.32$. This pointed to the same pyrromycinone skeleton, substituted at position 7 . In the sugar region, it displayed four oxymethine at $\delta 5.51(\mathrm{~s} \mathrm{br}), 5.28(\mathrm{~m}), 5.19(\mathrm{~d})$ and $5.13(\mathrm{~m})$. The proton displayed at $\delta 5.28$ is characteristic for 7- $\mathrm{H}$ in pyrromycinone. In addition, three methyl groups were observed as three doublets at $\delta 1.37,1.30$ and 1.25. This pointed to the presence of three sugar moieties in this compound. In addition, a $6 \mathrm{H}$ singlet of two equivalent $\mathrm{N}$ methyl groups was displayed at $\delta 2.16$. Furthermore, seven hydroxymethines were displayed between $\delta 4.81$ and 3.72, in addition to a methylene doublet at $\delta 2.60$ linked most likely to $s p^{2}$ carbon. A further multiplet of a methylene group was exhibited at $\delta 2.09$ $\left(2 "-\mathrm{H}_{2}\right)$. 


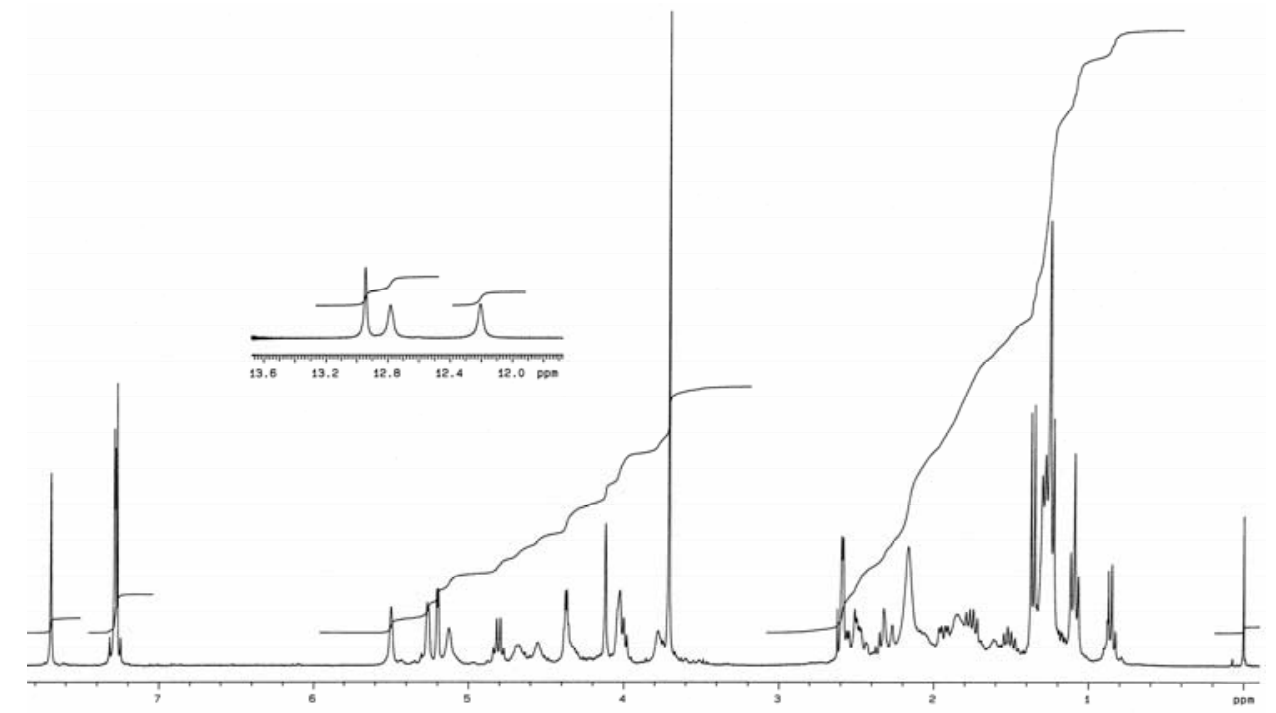

Figure 13: ${ }^{1} \mathrm{H}$ NMR spectrum $\left(\mathrm{CDCl}_{3}, 300 \mathrm{MHz}\right)$ of cinerubin $\mathrm{B}(\mathbf{4 9})$.

In accordance, this compound had a pyrromycinone chromophore with three sugar moieties attached at 7-position, which could be rhodosamine and 2-deoxyfucose. The ESI mass spectrometry fixed its molecular weight as 825 Dalton. A search in AntiBase with the help of MS and ${ }^{1} \mathrm{H}$ NMR data resulted in three structures: cinerubin $B$ (49), spartanamicin A (48) and pyrraculomycin (50). However, the ${ }^{1} \mathrm{H}$ NMR spectra exhibited no olefinic double bond, and hence pyrraculomycin (50) was excluded. Spartanamicin A (48) and cinerubin B (49) are configurational isomers. They differ only in the stereochemistry of the methyl group at 5 "'-position, which is equatorial $(\mathrm{H}$ 5 '"axial at $\delta 4.37)$ in cinerubin B (49) and axial in spartanamicin A (48) (H-5"' equatorial at $\delta$ 4.62). By comparing ${ }^{1} \mathrm{H}$ NMR and mass spectra of both compounds 49 and 48 with the present values, the compound was elucidated as cinerubin B (49). Cinerubin B (49) is characterised by its pronounced activity against Gram-positive bacteria, viruses and tumours. Cinerubin B (49) was isolated and identified previously from different Streptomyces sp. and described by Ettlinger et al. as red/orange antibiotic. The aglycone of the cinerubins named previously by Brockmann et al. as $\eta$ pyrromycinone. Cinerubin $\mathrm{B}^{59}(\mathbf{4 9})$ was isolated frequently from bacteria e.g. Streptomyces antibioticus, Streptomyces griseorubiginosus, and Streptomyces galilaeus. 


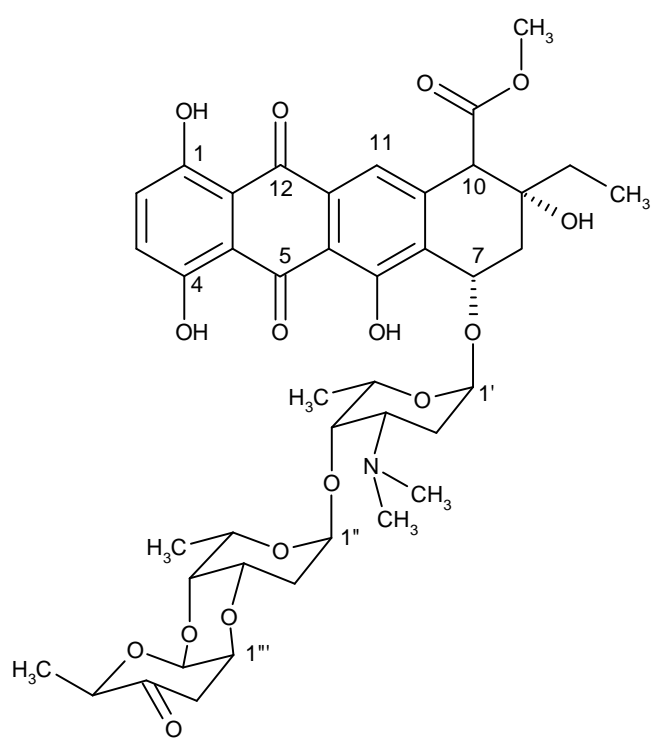

48

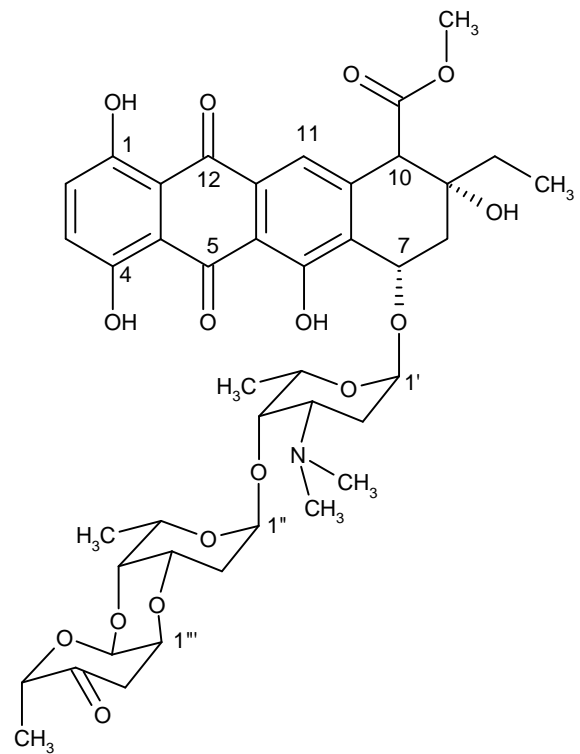

49

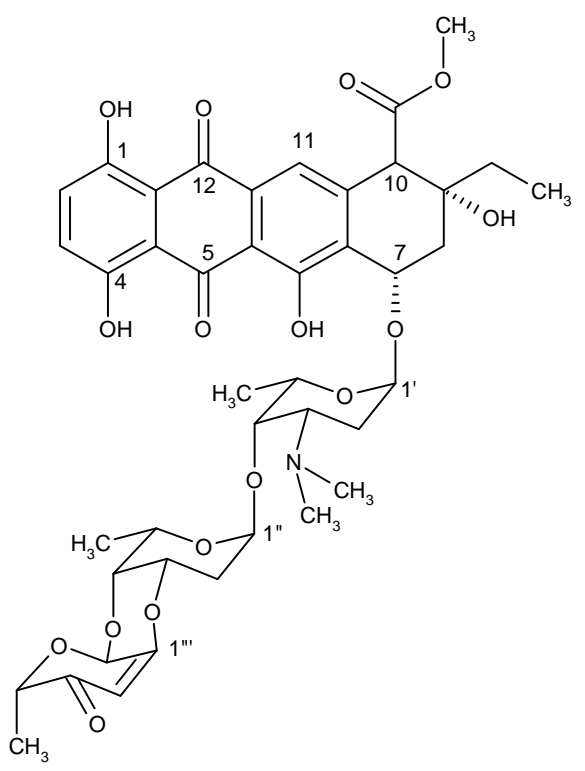

\subsubsection{Cinerubin A/ Spartanamicin B}

A further red compound was separated from fraction 4. The mass was 827 Dalton confirmed by ESIMS with $m / z, 828\left[\mathrm{M}+\mathrm{H}^{+}, 826(\mathrm{M}-\mathrm{H})^{-}\right.$, which was only 2 Dalton more than that of cinerubin B. The ${ }^{1} \mathrm{H}$ NMR spectrum showed similarity with that of the previously described compound 49. A difference in the ${ }^{1} \mathrm{H}$ NMR spectrum was observed in the sugar region. There was one proton signal less at $\delta 4-5$. By searching with mass and ${ }^{1} \mathrm{H}$ NMR data in AntiBase, the compound was identified as cinerubin A. The structure of cinerubin $A^{60}$ (51) was confirmed by comparing the ${ }^{1} \mathrm{H}$ NMR and MS data of an authentic sample. 


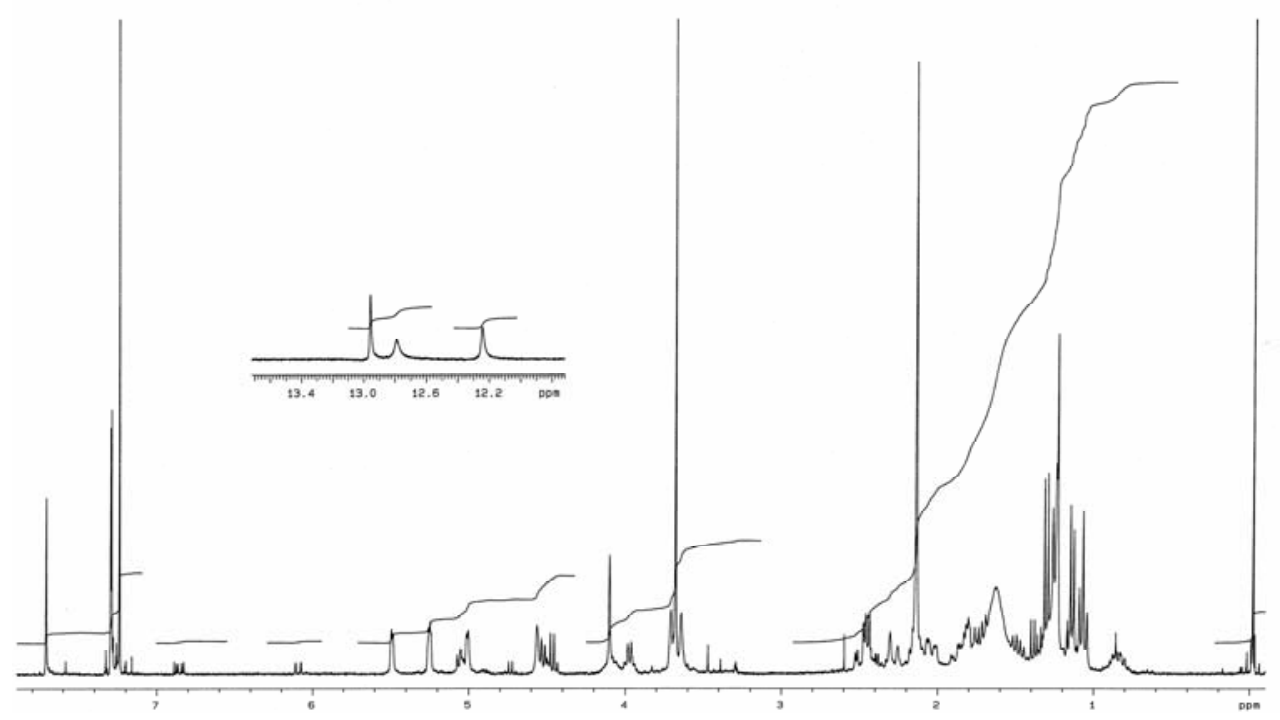

Figure 14: ${ }^{1} \mathrm{H}$ NMR spectrum $\left(\mathrm{CDCl}_{3}, 300 \mathrm{MHz}\right)$ of cinerubin $\mathrm{A}(\mathbf{5 1})$

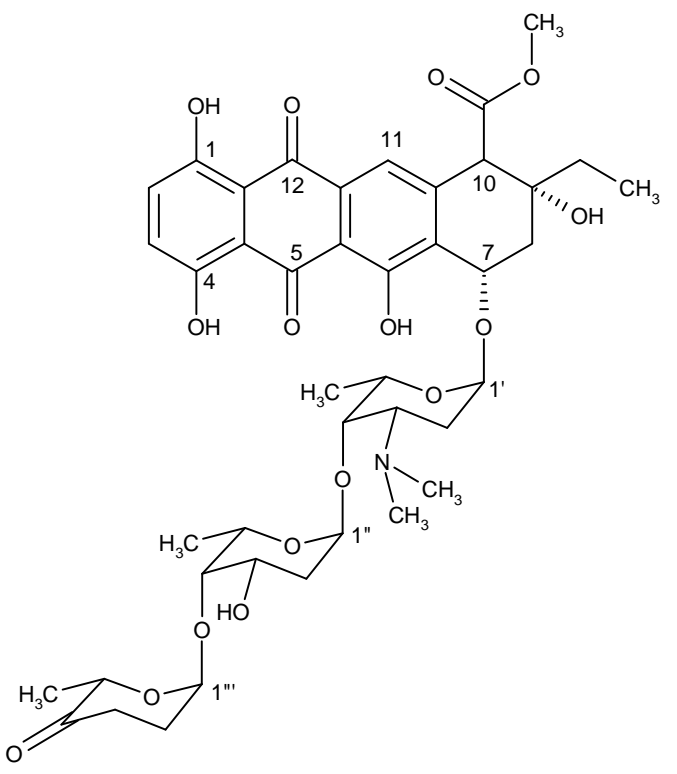

51

\subsubsection{Cinerubin $X$}

Cinerubin X (52) had the same colour and appearance as cinerubin A (51) but a different $R_{f}$ value, and the N,N-dimethyl signal at $\delta 2.20$ in cinerubin A was not observed in ${ }^{1} \mathrm{H}$ NMR spectrum for cinerubin X (52). ESI mass spectrometry showed $\mathrm{m} / \mathrm{z} 783$ $(\mathrm{M}-\mathrm{H})^{-}$, which was 43 less than for cinerubin A (51). This could be explained by the loss of the N,N-dimethyl group from the first sugar. A search in AntiBase resulted in cinerubin $X$ (52). The assumption was found to be correct and the ${ }^{1} \mathrm{H}$ NMR spectrum corresponded with that of the authentic sample. ${ }^{61}$ 


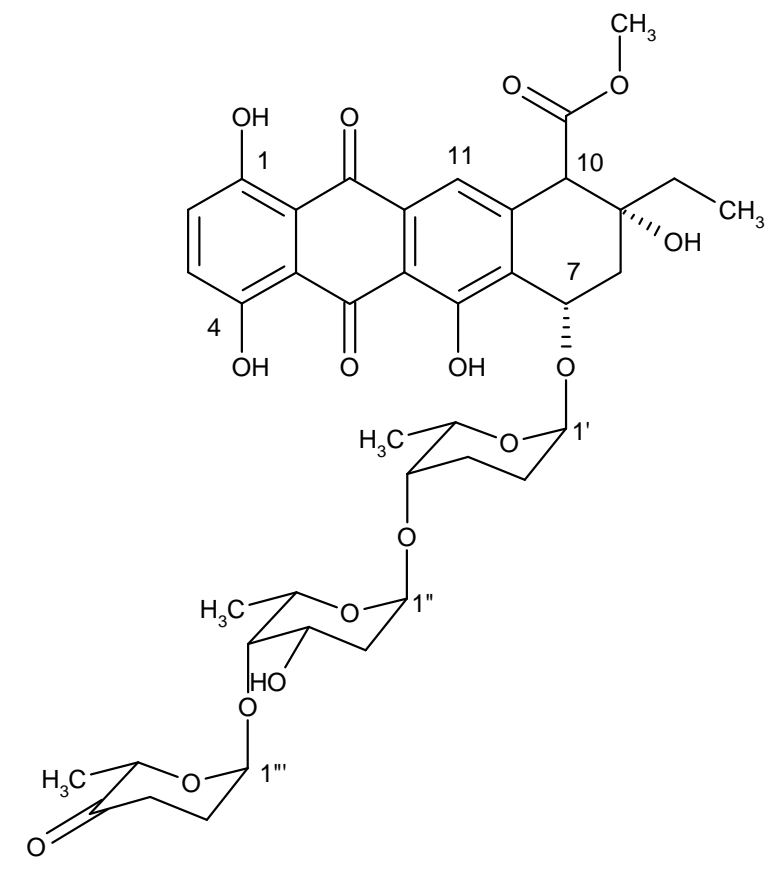

52

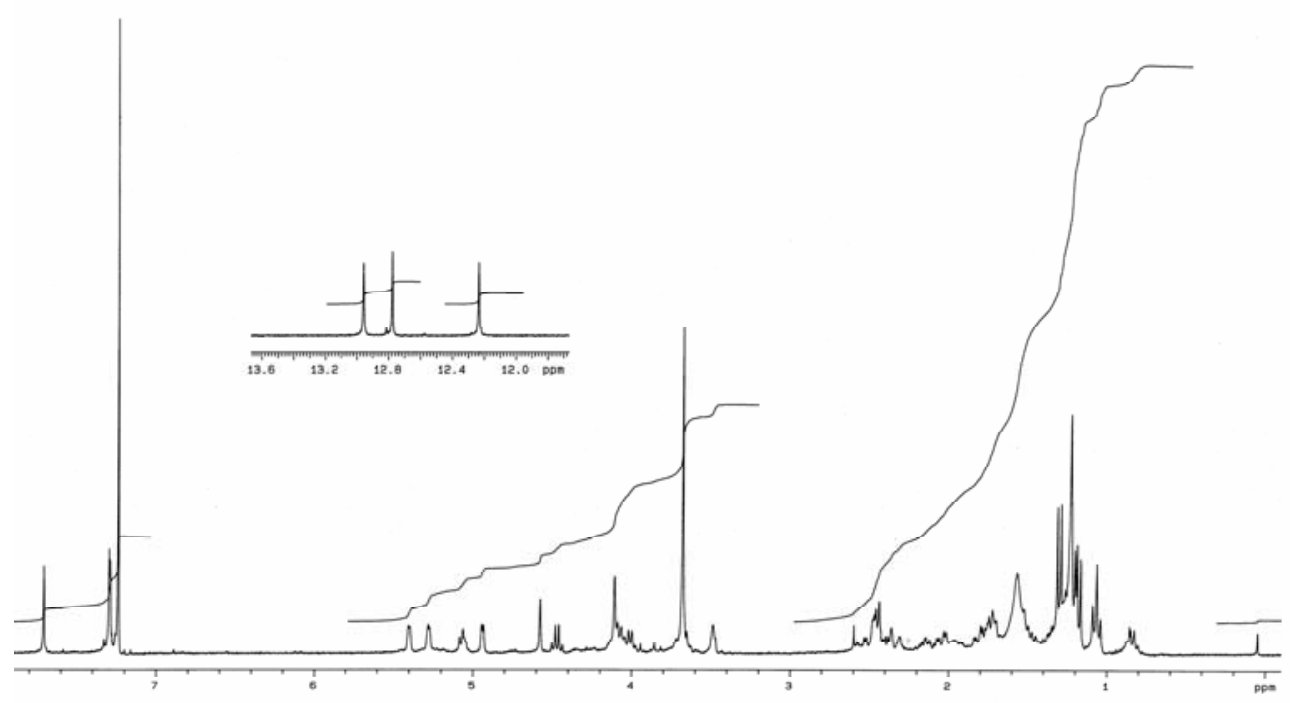

Figure 15: ${ }^{1} \mathrm{H}$ NMR spectrum $\left(\mathrm{CDCl}_{3}, 300 \mathrm{MHz}\right)$ of cinerubin $\mathrm{X}(\mathbf{5 2})$.

\subsection{Terrestrial Streptomyces sp. ACT 7619}

The ethyl acetate extract of the terrestrial Streptomyces sp. isolate ACT 7619 was found to be highly active against bacteria and fungi. Some colourless weakly UV absorbing bands gave violet colouration with anisaldehyde/sulphuric acid. The $25 \mathrm{~L}$ fermentation of the strain using $\mathrm{M}_{2}$ medium was performed as a shaker culture during five days, followed by extraction with ethyl acetate and XAD-16. This strain provided dinactin (53), bonactin (54), 4,10-dihydroxy-10-methyl-dodec-2-en-1,4-olide (56), $\mathrm{N}_{\beta}$-acetyltryptamine, tyrosol and maltol. 


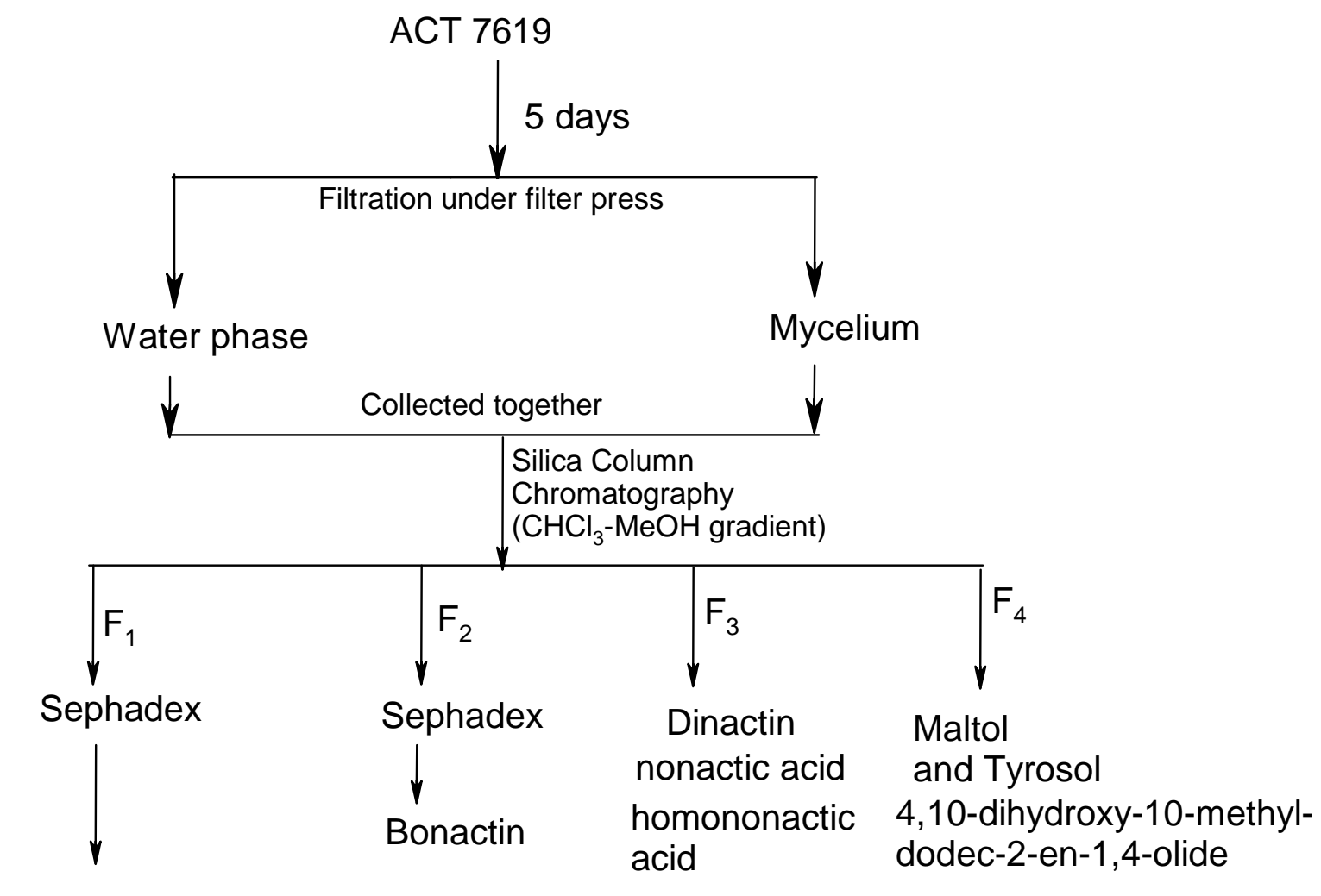

$N^{B}$ Acetyl-tryptamine

Figure 16: Working-up scheme of terrestrial strain ACT 7619.

\subsubsection{Dinactin}

A light yellow coloured liquid was obtained from fraction 3 after fractionation by column chromatography. The TLC chromatography showed a green-grey colour reaction with anisaldehyde/ sulphuric acid. ${ }^{1} \mathrm{H}$ NMR spectrum showed the absence of aromatic protons. There were two protons observed at $\delta 4.93$. At $\delta 2.52$, a $2 \mathrm{H}$ multiplet was present, whose shift was due to the connection with an $s p^{2}$ carbon. Seven methylene multiplets were also observed in the range of $\delta$ 2.01-1.40. Three $3 \mathrm{H}$ doublets and one $3 \mathrm{H}$ triplet were present at $\delta 1.23,1.10,1.08$, and 0.87 respectively. The ESIMS showed the mass as $m / z 788[\mathrm{M}+\mathrm{Na})^{+}$. With the help of ${ }^{1} \mathrm{H}$ NMR spectrum and mass a search in AntiBase detected a possible candidate. The structure was confirmed by comparing the mass and ${ }^{1} \mathrm{H}$ NMR spectrum with the published literature as dinactin (53). ${ }^{62}$

Dinactin (53) is a member of the macrotetrolide complex produced by a number of Streptomyces species. It is a monovalent cation ionophore with high selectivity for 
ammonium and potassium. It also inhibited T-cell proliferation induced by IL-2 and cytokine production at nanomolar levels for IL-2, IL-4, IL-5 and interferon- $\gamma{ }^{63}$

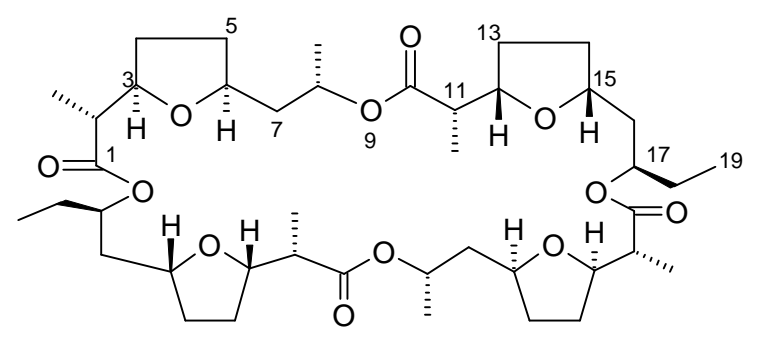

53

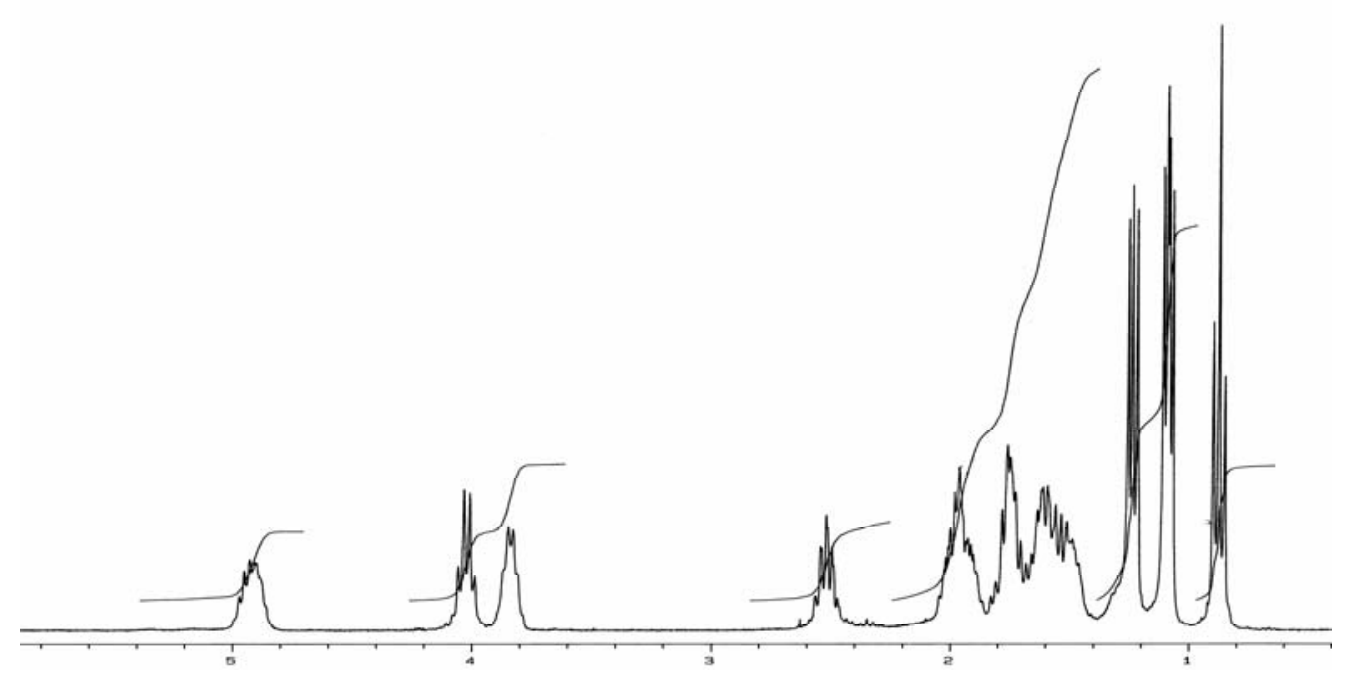

Figure 17: ${ }^{1} \mathrm{H}$ NMR spectrum $\left(\mathrm{CDCl}_{3}, 300 \mathrm{MHz}\right)$ of dinactin $(\mathbf{5 3})$.

\subsubsection{Bonactin}

The ${ }^{1} \mathrm{H}$ NMR spectrum displayed close similarity with those of dinactin (53). Dinactin is a dimer of two molecules of bonactin. The ESIMS showed a molecular ion peak at $\mathrm{m} / \mathrm{z} 423\left([\mathrm{M}+\mathrm{Na}]^{+}\right)$affording a molecular weight of 400 Dalton. Searching in AntiBase using the above ${ }^{1} \mathrm{H}$ NMR data and the molecular mass as well as the 'Rule of 13' afforded the molecular formula $\mathrm{C}_{21} \mathrm{H}_{36} \mathrm{O}_{7}$ resulting in bonactin (54), which was further confirmed by direct comparison with the literature. ${ }^{64}$ 


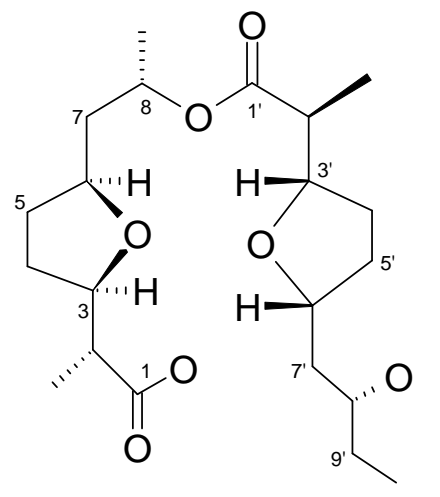

54

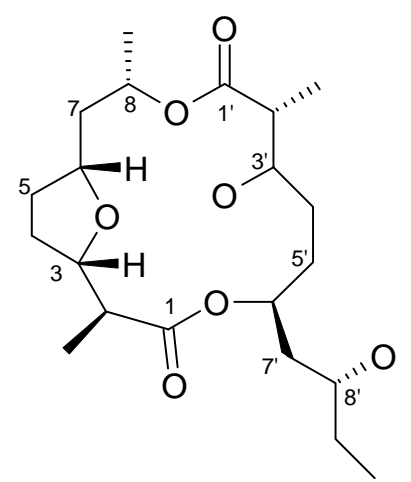

55

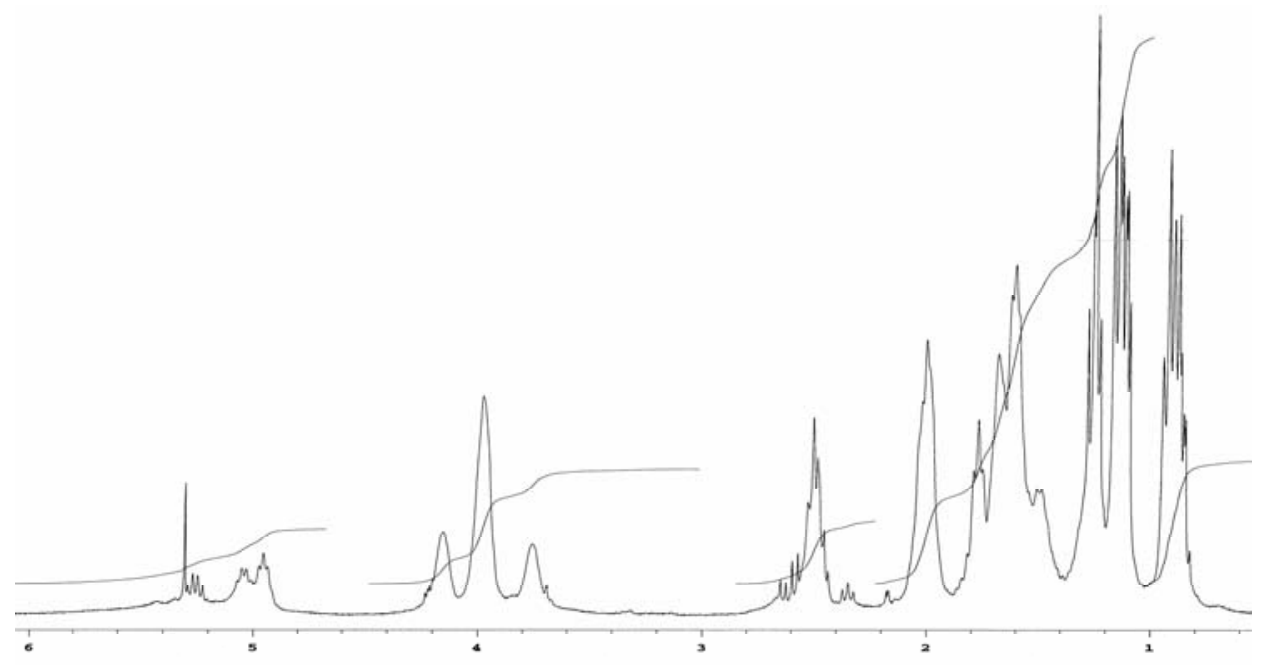

Figure 18: ${ }^{1} \mathrm{H}$ NMR spectrum $\left(\mathrm{CDCl}_{3}, 300 \mathrm{MHz}\right)$ of bonactin (54).

Lee and co-worker succeeded in synthesizing bonactin (54) and its C-3' epimer which was previously published with a wrong structure as feigrisolide $\mathrm{C}(\mathbf{5 5}) .{ }^{65}$ Bonactin (54) showed moderate inhibition of the $3 \alpha$-hydroxysteroid dehydrogenase, moderate cytotoxicity against L-929, K562 and HeLa cell lines, and 50\% inhibition of Coxackie virus B3 at $25 \mu \mathrm{g} / \mathrm{mL}^{6}{ }^{6}$

\subsubsection{4,10-Dihydroxy-10-methyl-dodec-2-en-1,4-olide}

Purification of fraction 1 delivered a colourless oil, which showed no UV absorbance on TLC and turned to violet-red colour with anisaldehyde/sulphuric acid. In the ${ }^{1} \mathrm{H}$ NMR spectrum, the three signals at $\delta 7.44(\mathrm{dd}), 6.12(\mathrm{dd})$ and $5.04(\mathrm{~m})$ are typical for monosubstituted butenolides. In the aliphatic region, one methyl triplet at $\delta 0.90$, one 
methyl singlet at 1.12 and multiplet of some methylene groups between 1.43 and 1.70 were observed.

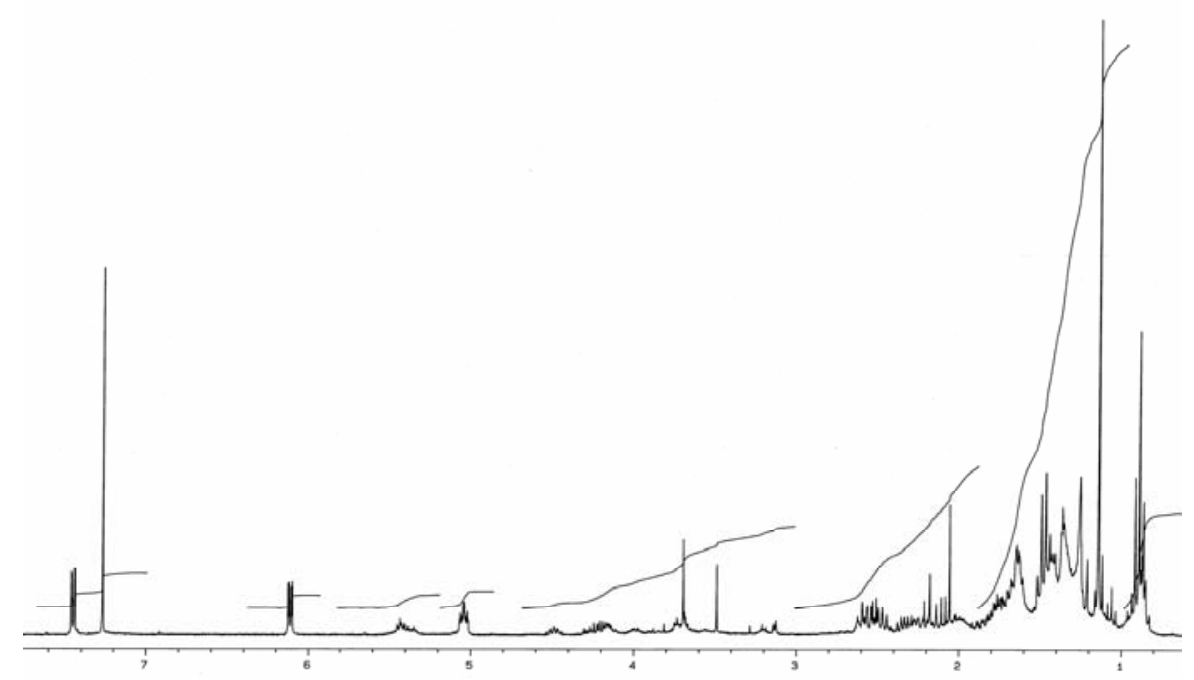

Figure 19: ${ }^{1} \mathrm{H}$ NMR spectrum $\left(\mathrm{CDCl}_{3}, 300 \mathrm{MHz}\right)$ of 4,10-dihydroxy-10-methyldodec-2-en-1,4-olide (56).

By searching in AntiBase using the above ${ }^{1} \mathrm{H}$ NMR data as well as comparison with authentic spectra, the isolated compound was assigned as 4,10-dihydroxy-10-methyldodec-2-en-1,4-olide (56). ${ }^{67,68}$<smiles>CCC(C)(O)CCCCCC1C=CC(=O)O1</smiles>

56

\subsection{Terrestrial Streptomycete GW 6311}

The culture filtrate of the terrestrial streptomycete GW6311 was antibiotically active against Staphylococcus aureus, Escherichia coli, Bacillus subtilis, Streptomyces viridochromogenes (Tü57), Mucor miehei (Tü284), and Candida albicans. In the chemical screening, it exhibited two low-polar red-orange bands, which turned to blueviolet by treatment with sodium hydroxide, pointing to peri-hydroxyquinones. Additionally, an UV absorbing band was present, which was stained to yellow-orange by Ehrlich's reagent.

\subsection{1 $\beta$-Indomycinone}

Compound (57) was isolated as a yellow amorphous compound from fraction 2. The ${ }^{1} \mathrm{H}$ NMR spectrum showed singlets at $\delta 12.85(\mathrm{OH})$ and $\delta 8.08(\mathrm{H}-6)$, three ortho- 
coupled aromatic protons at $\delta 7.83,7.70$ and 7.38, a $1 \mathrm{H}$ singlet at $\delta 6.56$, and the singlet of a peri-methyl group at $\delta 3.02$. In addition, two multiplets of an olefinic double bond were detected at $\delta 5.74$ and 5.38. In the aliphatic region, two methylene protons revealing an $\mathrm{ABX}$ system were detected at $\delta 2.91$ and 2.78. Finally, two methyl groups linked to $s p^{2}$ carbons were displayed at $\delta 1.72$ and 1.64, of which the first gave a singlet, and the second a doublet. The molecular weight of this compound was established as 404 Dalton by ESIMS. By using ${ }^{1} \mathrm{H}$ NMR and MS data the structure was found as $\beta$-indomycinone (57). The structure was further confirmed by comparing with an authentic sample. ${ }^{69,70}$<smiles>C/C=C/CC(C)(O)c1cc(=O)c2c(C)cc3c(c2o1)C(=O)c1c(O)cccc1C3=O</smiles>

57

\subsubsection{Saptomycin A}

Compound 58 was another peri-hydroxyquinone obtained as a yellow solid from the same fraction, from which $\beta$-indomycinone (57) was isolated. The ${ }^{1} \mathrm{H}$ NMR spectrum showed nearly the same signal pattern as in $\beta$-indomycinone (57). A singlet of a perihydroxy group at $\delta 12.64$ was exhibited. In the aromatic region, a $1 \mathrm{H}$ singlet at $\delta 8.09$, three aromatic protons at $\delta 7.83,7.69$ and 7.36 of a 1,2,3-trisubstituted aromatic ring, a singlet at $\delta 6.28(3-\mathrm{H})$, in addition to the singlet of a peri-methyl group at $\delta 3.02$ $\left(13-\mathrm{H}_{3}\right)$. In addition, two multiplets of an olefinic double bond were detected at $\delta 5.65$ and 5.50. Moreover, two methine protons were displayed at $\delta 5.01$ and 2.98, of which the first could be oxygenated, while the second was a methine flanked most likely by two $s p^{2}$ carbons. Finally, two methyl doublets were detected at $\delta 1.71$ and 1.45 , of which the first could be attached to a $s p^{2}$ carbon. Based on the revealed spectral data and the molecular formula, a search in AntiBase directed to saptomycin A. This compound was further confirmed by comparing the data with those in the literature. ${ }^{71}$ 
<smiles>C/C=C/C(O)C(C)c1cc(=O)c2c(C)cc3c(c2o1)C(=O)c1c(O)cccc1C3=O</smiles>

58

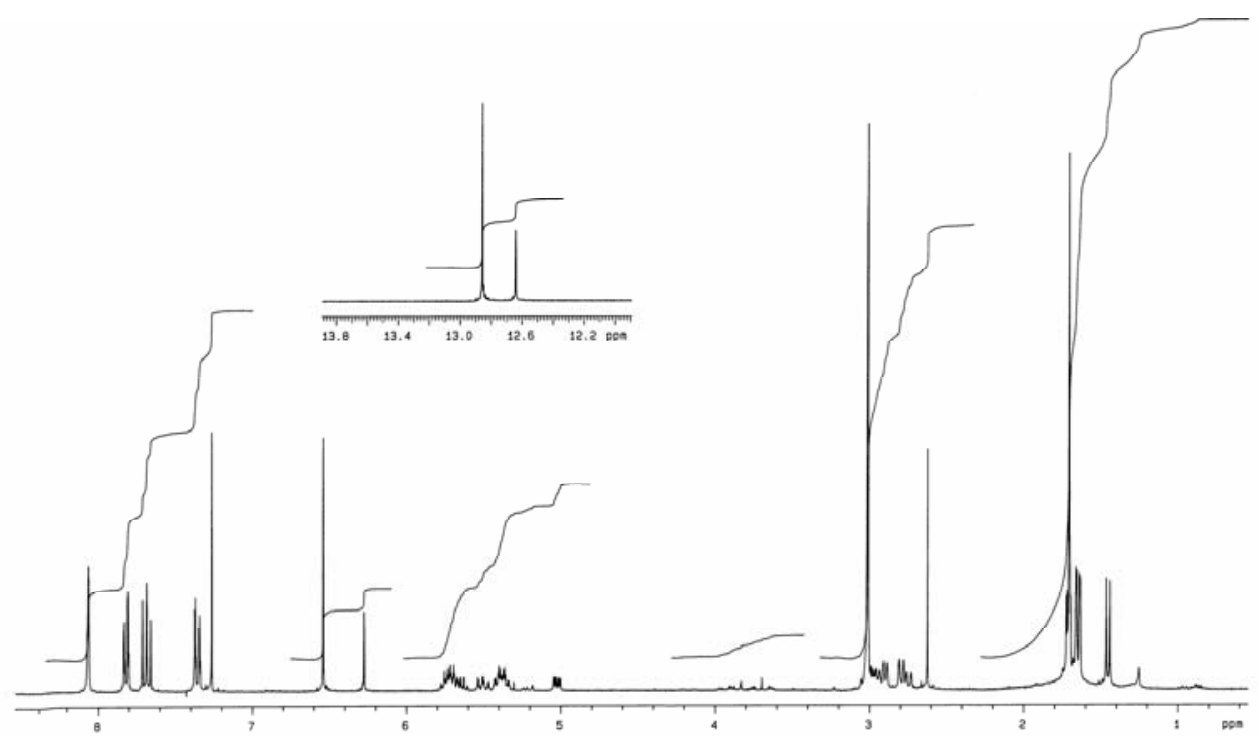

Figure 20: ${ }^{1} \mathrm{H}$ NMR spectrum $\left(\mathrm{CDCl}_{3}, 300 \mathrm{MHz}\right)$ of mixture of $\beta$-indomycinone (57) and saptomycin A (58).

$\beta$-indomycinone (57) and saptomycin A (58) are composed of an anthraquinone $\gamma$ pyrone nucleus, but bear a different side chain. Both of them belong to pluramycin group and showed antimicrobial and potent antitumor activities against human or murine tumor cell lines in vitro and Meth A fibrosarcoma in vivo. ${ }^{72}$

\subsection{Terrestrial Streptomyces sp. GT 2005/079}

The biological screening of the crude extract of the terrestrial Streptomyces sp. GT 2005/079 exhibited pronounced antibacterial activity against Staphylococcus aureus, Bacillus subtilis, and Escherichia coli. The TLC screening revealed several polar green fluorescent zones as well as some middle polar zones, which were not observable under UV at 254 or $366 \mathrm{~nm}$ but gave a dark green colour reaction on TLC with anisaldehyde/sulphuric acid. The strain was cultivated on $\mathrm{M}_{2}$ medium in a $20 \mathrm{~L}$ scale during seven days. The grey culture broth was mixed with Celite and filtered through a filter press. Both water and mycelium phases were extracted with ethyl acetate and 
the organic phase brought to dryness. The dark oily crude extract was defatted using cyclohexane and submitted to column chromatography on silica gel using a $\mathrm{CH}_{2} \mathrm{Cl}_{2} / \mathrm{MeOH}$ gradient to get 6 new compounds: four new antifungal amides 59-62 together with two new oxazole antibiotics. The structures of these compounds were deduced by interpretation of high resolution mass, 1D and 2D NMR spectra.

The crude extract was chromatographed on a silica gel column eluting with a gradient system of $\mathrm{CH}_{2} \mathrm{Cl}_{2}$ : $\mathrm{MeOH}$ (100:0 to 90:10) to give five main fractions. Fraction 1 contained mostly lipids, whereas fraction 2 showed three main spots on TLC. This fraction was further purified on a silica gel and on Sephadex LH-20 followed by a RP-8 column eluted with 50-60\% aqueous methanol to get compound 59 and compound 60. Fraction 3 was subjected to silica gel and Sephadex LH-20 followed by RP-8 column chromatography yielding compound 61. Fraction 4 was subjected to RP-18 column chromatography with $30 \%$ aqueous methanol to give two sub-fractions. Further purification with methanol on Sephadex LH-20 yielded 62. Fraction 5 contained a highly fluorescent polar compound. It was separated on Sephadex LH-20 into two sub-fractions. The solubility of these compounds was low in dichloromethane or methanol or even in a mixture of both solvents. Therefore, the solids were washed several times with a mixture of dichloromethane and methanol to obtain compound $\mathbf{6 3}$ and 64. 


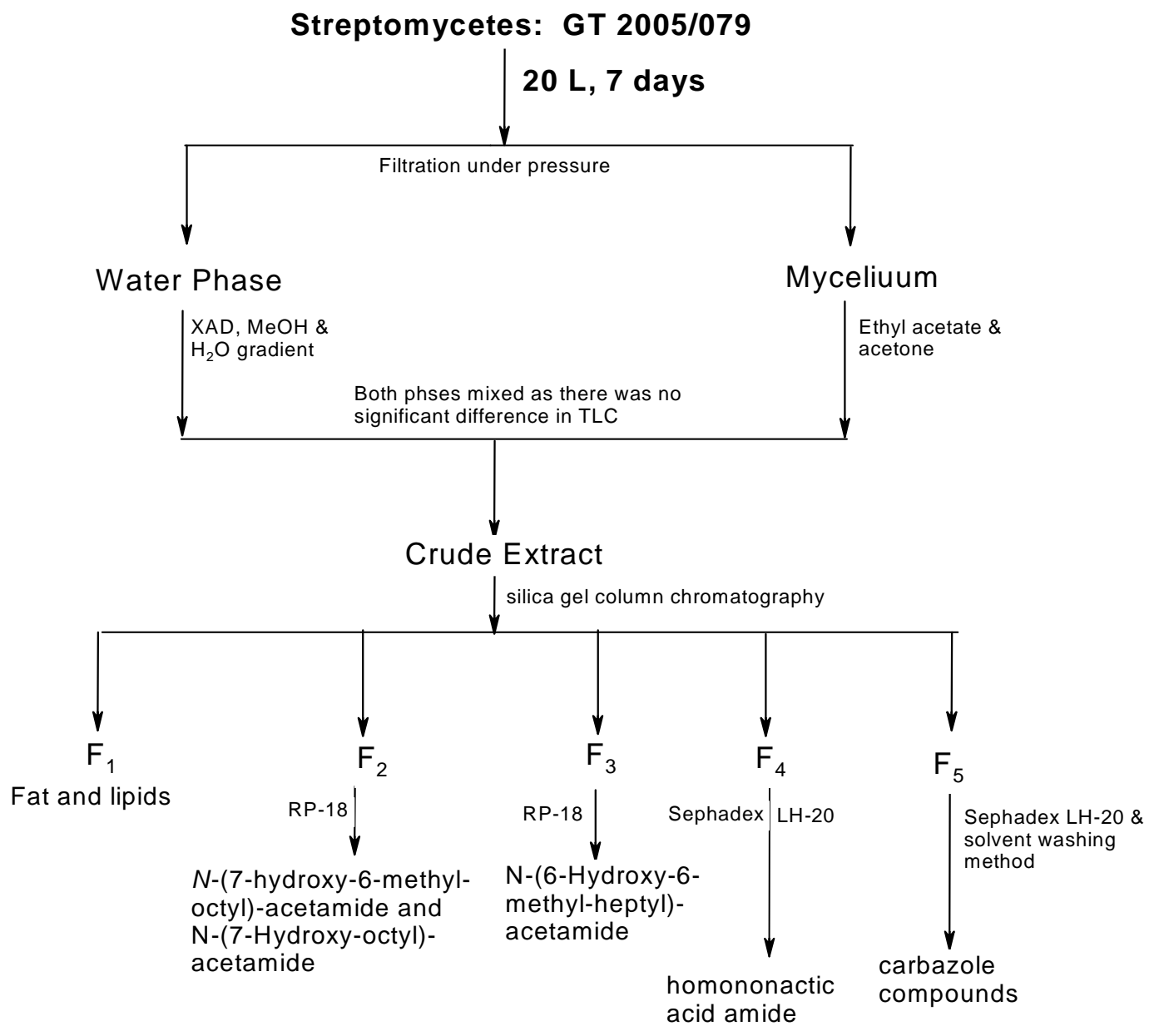

Figure 21: Work-up scheme of the strain Streptomyces sp. GT 2005/079.<smiles>CC(=O)NCCCCCCC(C)O</smiles>

59<smiles>CC(=O)NCCCCCC(C)(C)O</smiles>

61<smiles>O=C(O)c1cccc2oc(-c3ccccc3O)nc12</smiles>

63<smiles>CC(=O)NCCCCCCC(C)O</smiles>

60<smiles>CCC(O)CC1CCC(C(C)C(N)=O)O1</smiles>

62<smiles>O=C(O)c1cccc2oc(-c3cccc(Cl)c3O)nc12</smiles> 


\subsubsection{N-(7-Hydroxy-6-methyl-octyl)-acetamide}

On ESIMS, $N$-(7-hydroxy-6-methyl-octyl)-acetamide (59). showed a pseudomolecular ion at $m / z 224[\mathrm{M}+\mathrm{Na}]^{+}$. (+)-HRESIMS established the formula as $\mathrm{C}_{11} \mathrm{H}_{23} \mathrm{NO}_{2}$ with 1 double bond equivalent (DBE). The ${ }^{1} \mathrm{H}$ NMR spectrum displayed a broad signal at $\delta$ 5.70, which could be due to $\mathrm{NH} / \mathrm{OH}$, as in the HSQC spectrum there was no cross peak with a carbon signal visible. The quartet of a methylene group at $\delta 3.22$ showed an $\mathrm{H}-\mathrm{H}$ COSY correlation with this acidic proton at $\delta 5.70$ as well as with another methylene group at $\delta 3.22$. There were 3 methyl signals observed at $\delta 1.97$ (s), 1.15 (d) and 0.85 (d). From the value of the methyl singlet at $\delta 1.97$, it could be assumed that it was attached to an $s p^{2}$ carbon or near a carbonyl group or a nitrogen atom. The other two methyl groups are neighbours of two $\mathrm{CH}$ groups as they were doublets. There were overlapping signals of four methylene groups in the range of $\delta 1.2-1.4$. A CH quartet at $\delta 3.65$ depicted that it was attached to a methyl group and a hetero atom.

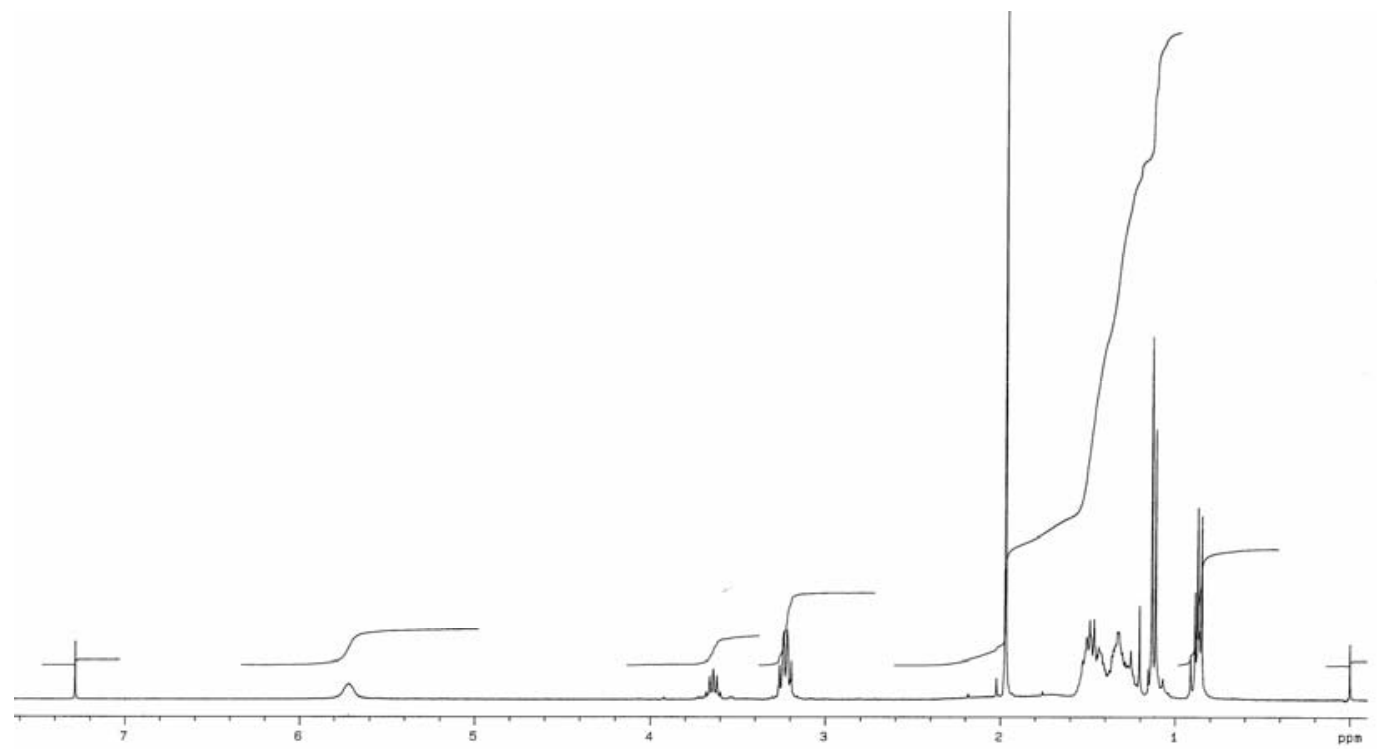

Figure 22: ${ }^{1} \mathrm{H}$ NMR spectrum $(\mathrm{CDCl} 3,300 \mathrm{MHz})$ of $N$-(7-hydroxy-6-methyl-octyl)acetamide (59).

The ${ }^{13} \mathrm{C}$ NMR spectrum showed a carbon signal at $\delta 170.1$, which suggested the presence of an ester, acid or amide group. The methine carbon at $\delta 71.6$ depicted an attachment of oxygen, while the methylene carbons at $\delta 43.3$ and 39.3 were either attached to a nitrogen atom or a $s p^{2}$ carbon. There were seven more methylene carbons observed between $\delta 30-14$. 


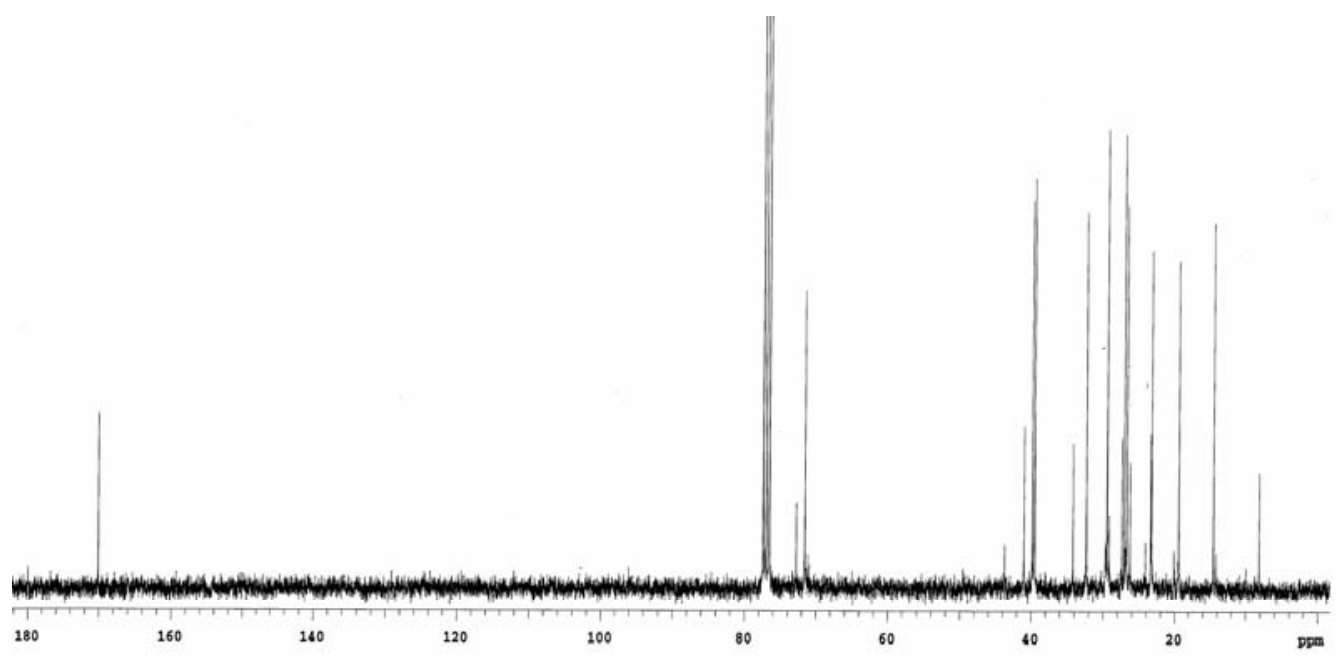

Figure 23: ${ }^{13} \mathrm{C}$ NMR spectrum $\left(\mathrm{CDCl}_{3}, 125 \mathrm{MHz}\right)$ of $\mathrm{N}$-(7-hydroxy-6-methyl-octyl)acetamide (59).

In the H-H COSY spectrum, the acidic proton at $\delta 5.70$ showed a correlation with the methylene at $\delta 3.15$.

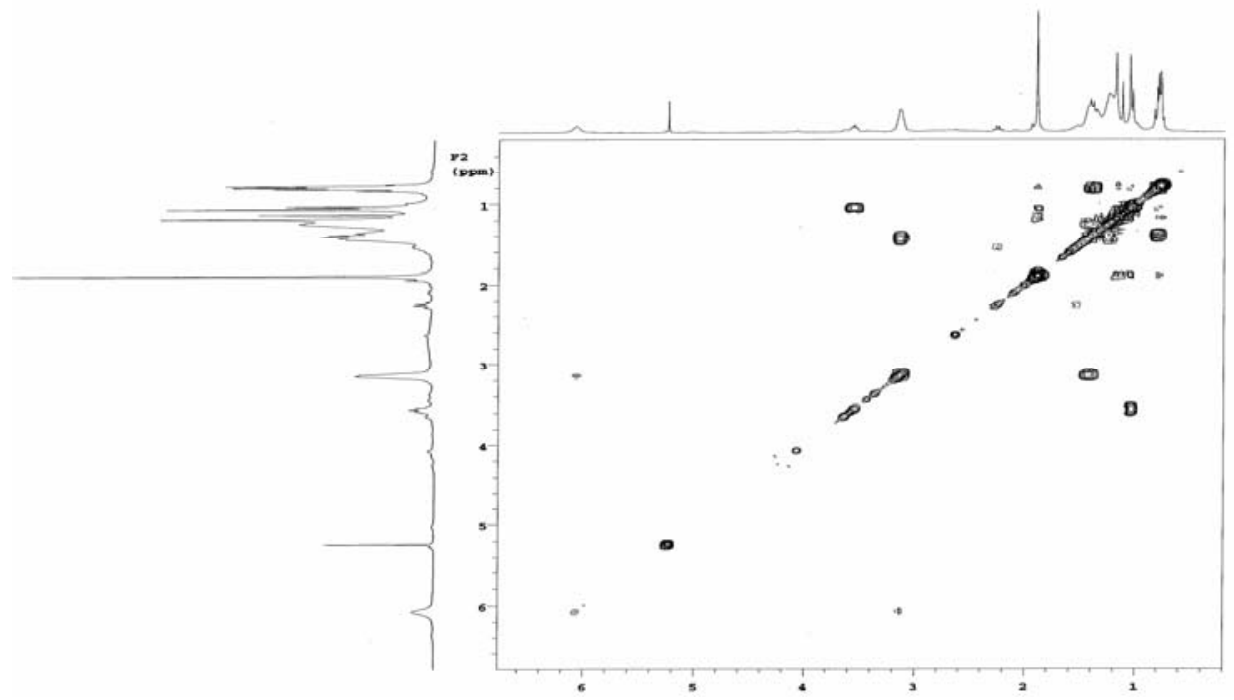

Figure 24: $\mathrm{H}-\mathrm{H}$ COSY spectrum $\left(\mathrm{CDCl}_{3}, 600 \mathrm{MHz}\right)$ of $N$-(7-hydroxy-6-methyloctyl)-acetamide (59).

From HMBC spectrum the methyl group at $\delta 1.97$ showed a ${ }^{2} J$ coupling with the carbonyl at $\delta$ 170.6. The carbonyl also displayed correlation with the acidic proton, which is probably an $\mathrm{NH}$, as only two oxygen atoms are present and one was already used for the carbonyl. The other one was attached to the $\mathrm{CH}$ at $\delta 71.5$, so the substructures A and B could be drawn. 


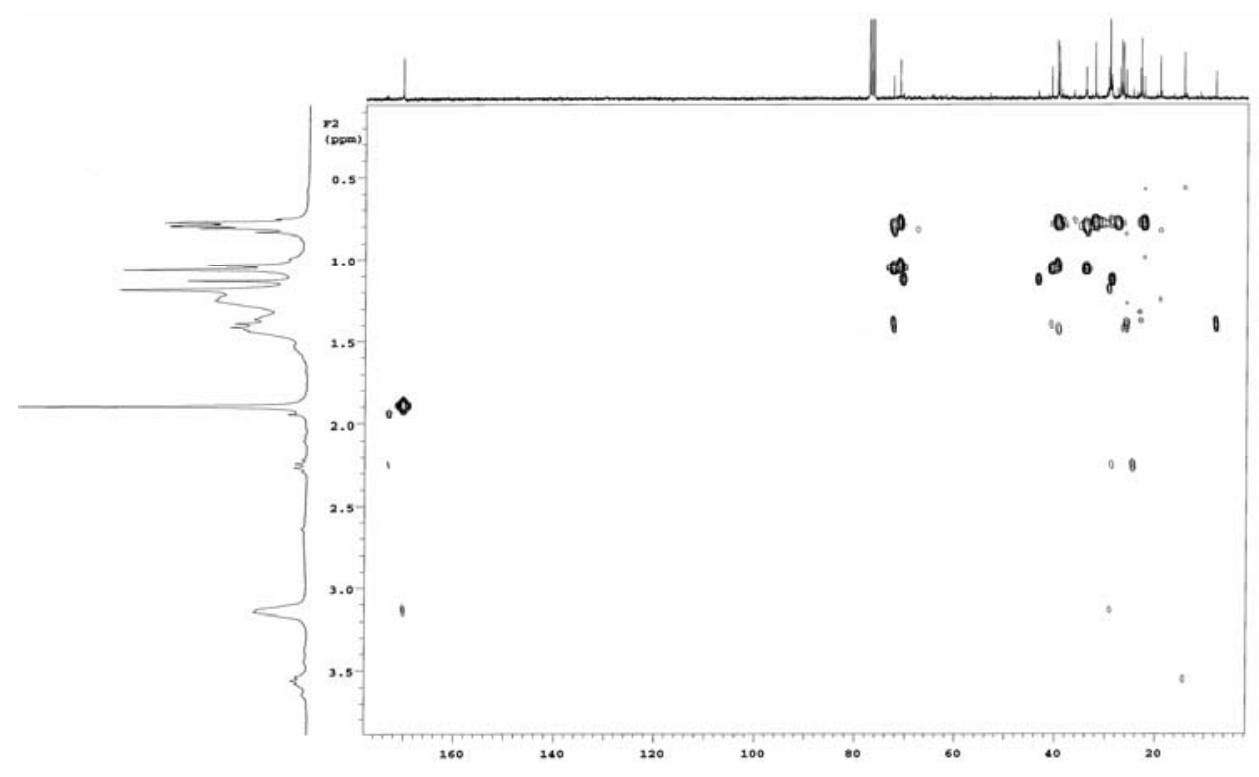

Figure 25: $\mathrm{HMBC}$ spectrum $\left(\mathrm{CDCl}_{3}, 600 \mathrm{MHz}\right)$ of $N$-(7-Hydroxy-6-methyl-octyl)acetamide (59).<smiles>CC(=O)NCCCC1CC1</smiles>

A<smiles>CC(C)C(C)C(C)O</smiles>

B

Figure 26: Substructure A and B of $N$-(7-Hydroxy-6-methyl-octyl)-acetamide (59).

Using all the 1D and 2D NMR correlations, the structure of compound 59 was established. $^{73}$

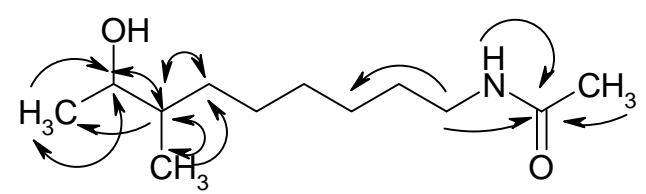

Figure 27: H-H COSY $(\leftrightarrow)$ and HMBC $(\rightarrow)$ correlation of (7-hydroxy-6-methyloctyl)-acetamide (59).

\subsubsection{N-(7-Hydroxy-octyl)-acetamide}

$N$-(7-Hydroxy-octyl)-acetamide (60) showed similarity with $N$-(7-hydroxy-6-methyloctyl)-acetamide (60) in the ${ }^{1} \mathrm{H}$ NMR spectrum and physical properties. From the HRESIMS the molecular formula was established as $\mathrm{C}_{10} \mathrm{H}_{21} \mathrm{NO}_{2}$, which was 14 Daltons less than that of compound 60. The ${ }^{1} \mathrm{H}$ NMR pattern depicted a difference of a methylene group. The methyl signal at $\delta 0.85$ was observed as doublet, while a 
number of methylene groups appeared between $\delta$ 1.40-1.15. The acetyl methyl group appeared at $\delta 1.97$ as in $\mathbf{5 9}$.

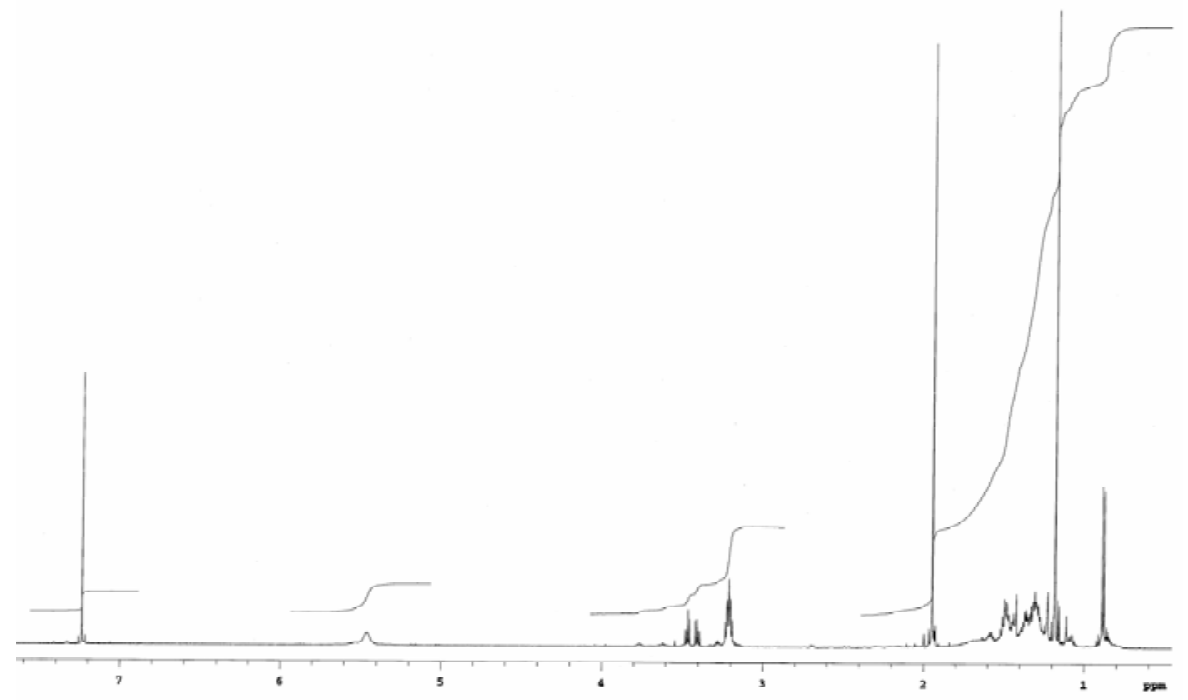

Figure 28: ${ }^{1} \mathrm{H}$ NMR spectrum $\left(\mathrm{CDCl}_{3}, 300 \mathrm{MHz}\right)$ spectra of $N$-(7-hydroxy-octyl)acetamide $(\mathbf{6 0})$.

In the ${ }^{13} \mathrm{C}$ NMR spectrum of $\mathbf{6 0}$, there was a carbonyl signal at $\delta 171.3$ observed which could be that of an amide as in 59. There was an oxymethylene group at $\delta 68.2$ along with the methylene near nitrogen at $\delta$ 39.6. The methyl signal of the acetyl group was at $\delta 23.4$, while another methyl was seen at $\delta 16.0$.

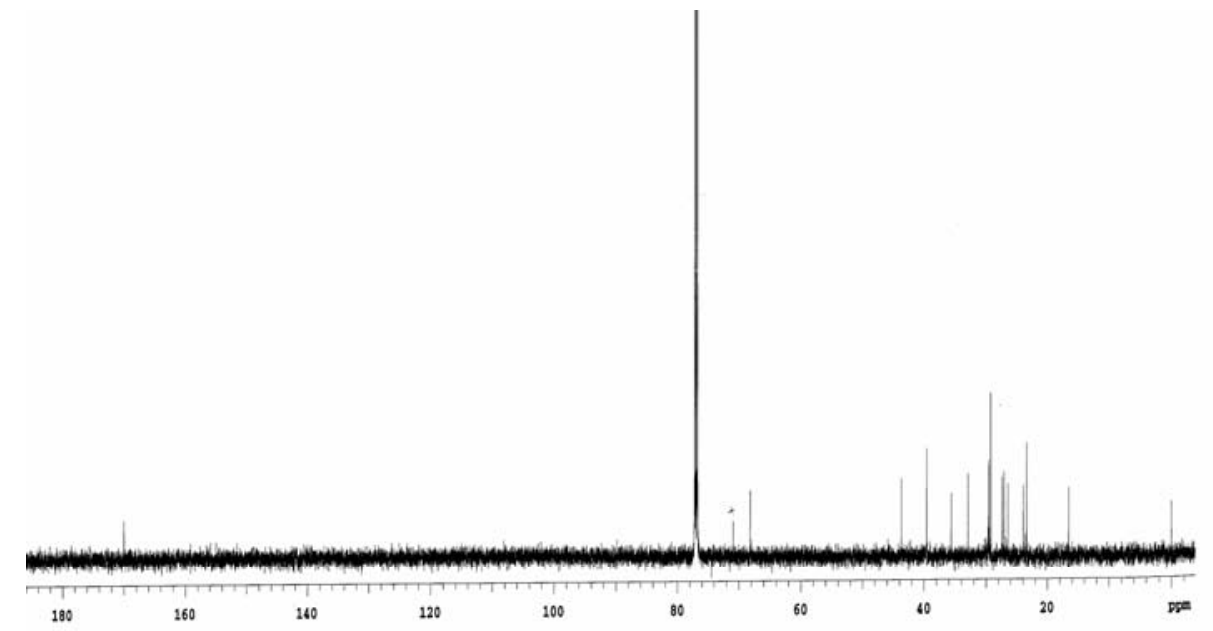

Figure 29: ${ }^{13} \mathrm{C}$ NMR spectrum $(\mathrm{CDCl} 3,150 \mathrm{MHz})$ of $N$-(7-hydroxy-octyl)-acetamide $(60)$.

In the $\mathrm{H}-\mathrm{H}$ COSY spectrum the methyl doublet showed a correlation with a methine proton, and the $\mathrm{NH}$ group showed a correlation with a methylene group. All other methylene signals appeared nearly in the same position as in 59. From the pattern it was clear that they were attached to each other. 


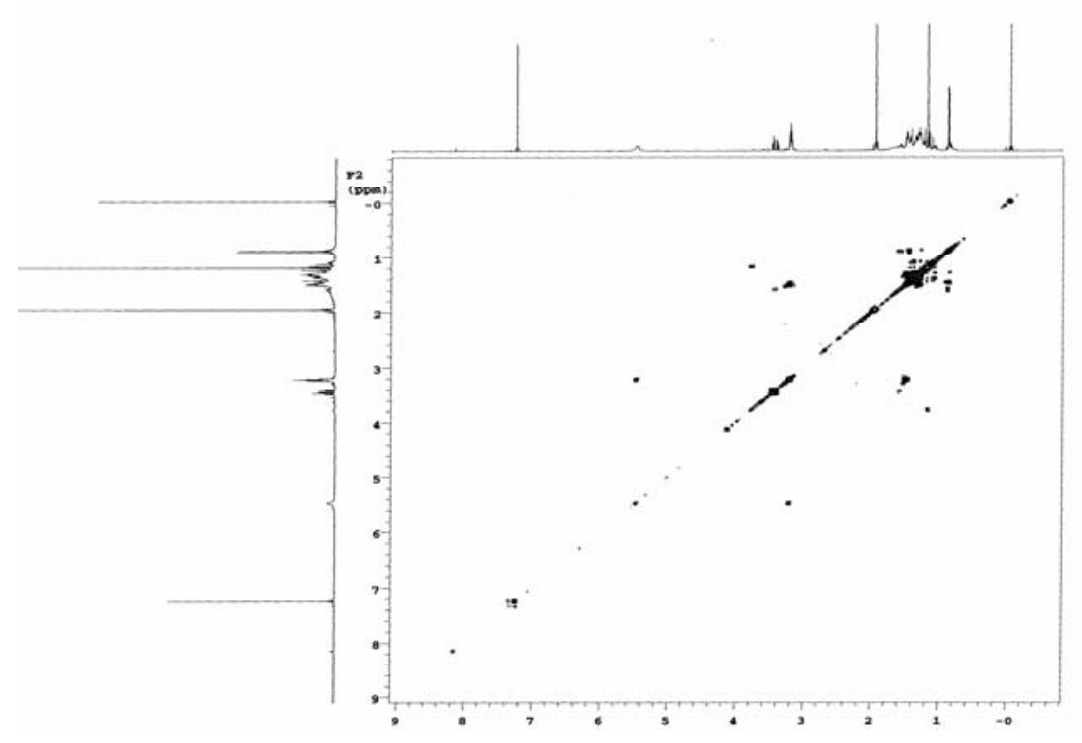

Figure 30: $\mathrm{H}-\mathrm{H}$ COSY spectrum $\left(\mathrm{CDCl}_{3}, 600 \mathrm{MHz}\right)$ spectra of $N$-(7-hydroxy-octyl)acetamide (60).

Based on the correlations as described above and by comparing with the data of published compounds structure $\mathbf{6 0}$ was derived. ${ }^{74,75}$

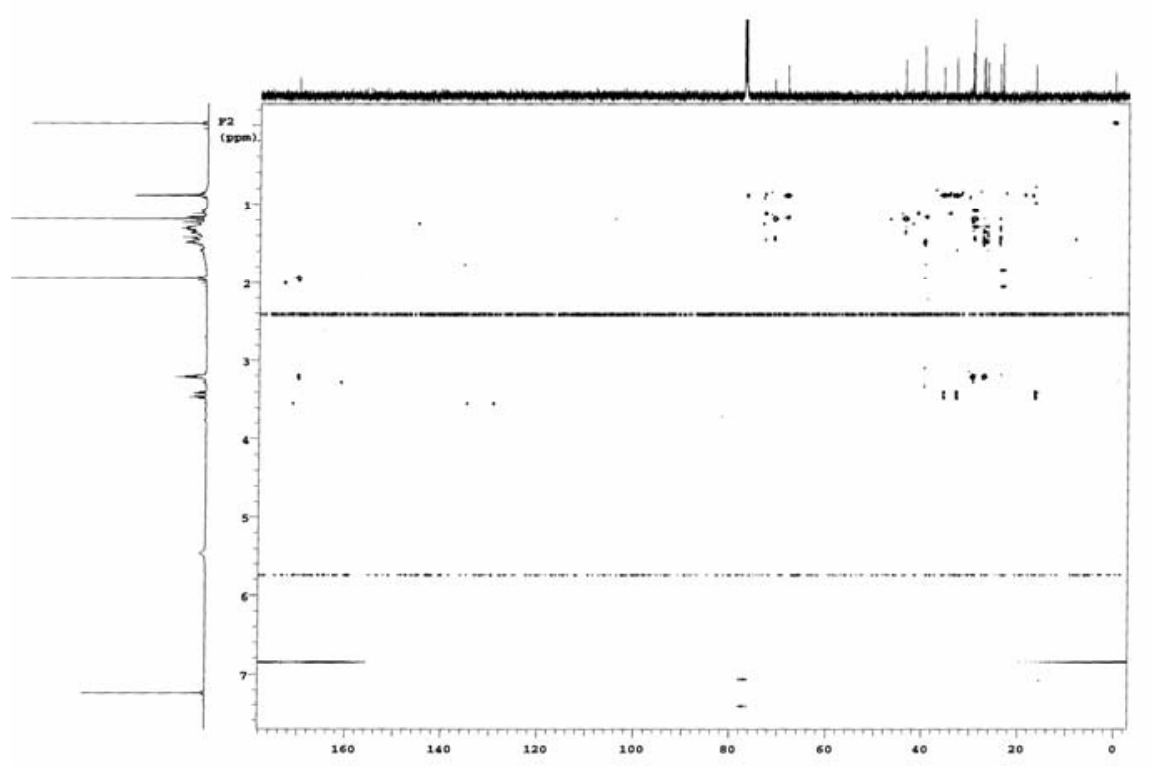

Figure 31: $\mathrm{HMBC}$ spectrum $\left(\mathrm{CDCl}_{3}, 600 \mathrm{MHz}\right)$ of $N$-(7-hydroxy-octyl)-acetamide (60).

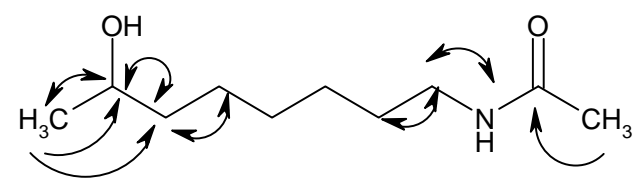

Figure 32:H-H COSY $(\leftrightarrow)$ correlations and HMBC $(\longrightarrow)$ correlations. 


\subsubsection{N-(6-Hydroxy-6-methyl-heptyl)-acetamide}

$N$-(6-Hydroxy-6-methyl-heptyl)-acetamide (61) was a further acetamide isolated from the same strain. The molecular formula $\mathrm{C}_{10} \mathrm{H}_{21} \mathrm{NO}_{2}$ was derived from the HRESI mass spectrum. The molecular formula was same as for compound $\mathbf{6 0}$. There were two methyl groups, which appeared as a singlet in this compound at $\delta 1.19$. So, it was one possibility that the methyls were present in an isopropyl group, while the methine proton of that isopropyl group was substituted with oxygen, as the shift of the respective carbon atom was observed at $\delta 70.5$. The $\mathrm{NH}$ proton and the adjacent methylene group at $\delta 3.20$ showed the same shifts as for $\mathbf{5 9}$ and $\mathbf{6 0}$.

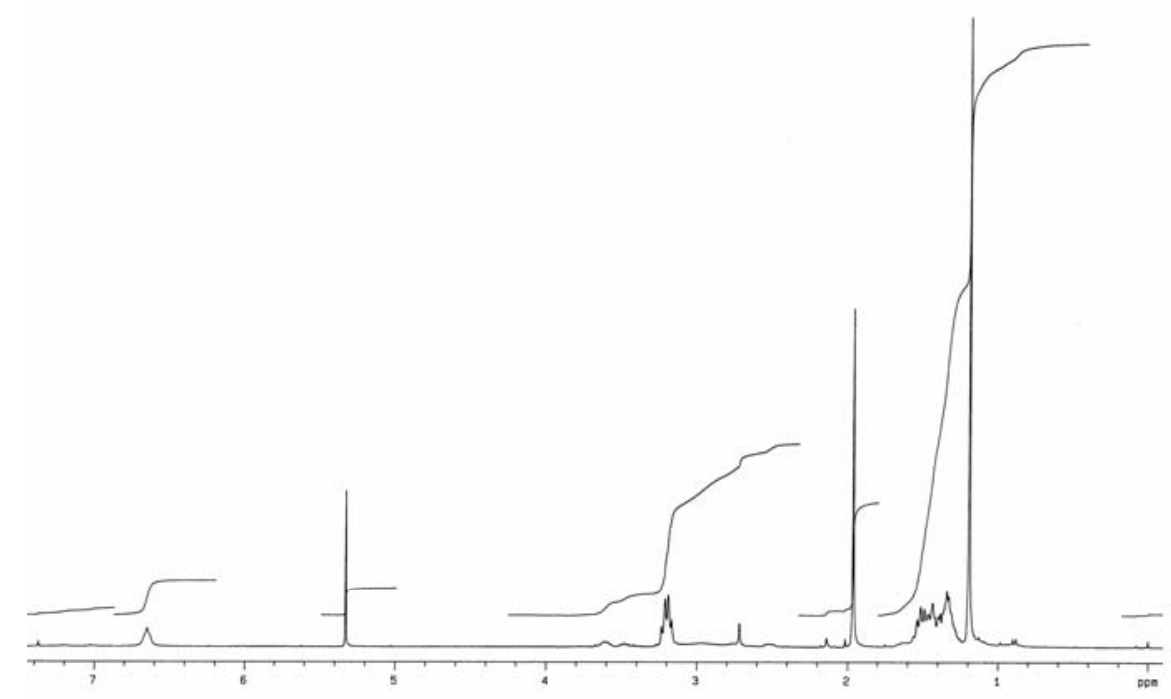

Figure 33: ${ }^{1} \mathrm{H}$ NMR spectrum $(\mathrm{CDCl} 3,300 \mathrm{MHz})$ spectrum of $\mathrm{N}-(6$-hydroxy-6methyl-heptyl)-acetamide (61).

In the ${ }^{13} \mathrm{C}$ NMR spectrum, 10 carbon signals appeared. The acetamide carbonyl signal appeared at $\delta 170.4$ and a quaternary oxygenated $s p^{3}$ carbon was observed at $\delta$ 70.5. The other carbons showed values in the same range as for the previously described amides. 


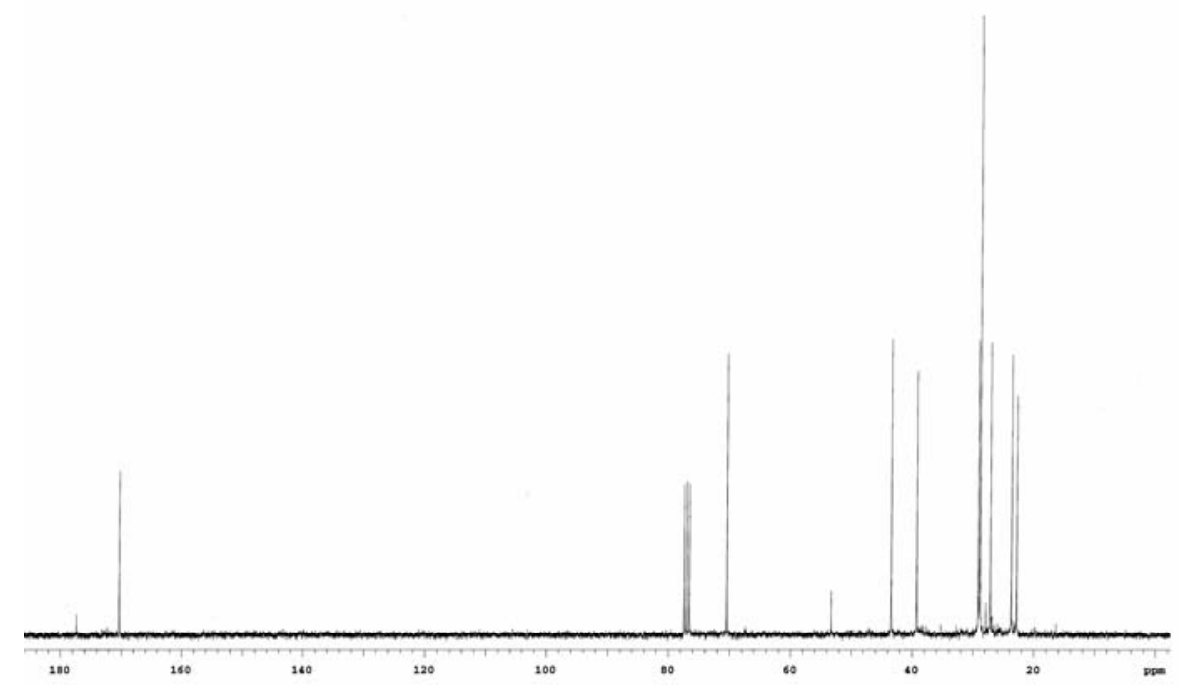

Figure 34: ${ }^{13} \mathrm{C}$ NMR spectrum $(\mathrm{CDCl} 3,125 \mathrm{MHz})$ spectra of $\mathrm{N}$-(6-hydroxy-6methyl-heptyl)-acetamide (61).

In the H-H COSY spectrum the methylene at $\delta 3.20$ showed correlations with the NH signal and another methylene. The other correlations were similar to those of $\mathbf{5 9}$ and 60, except for the methyl groups, which gave a singlet.

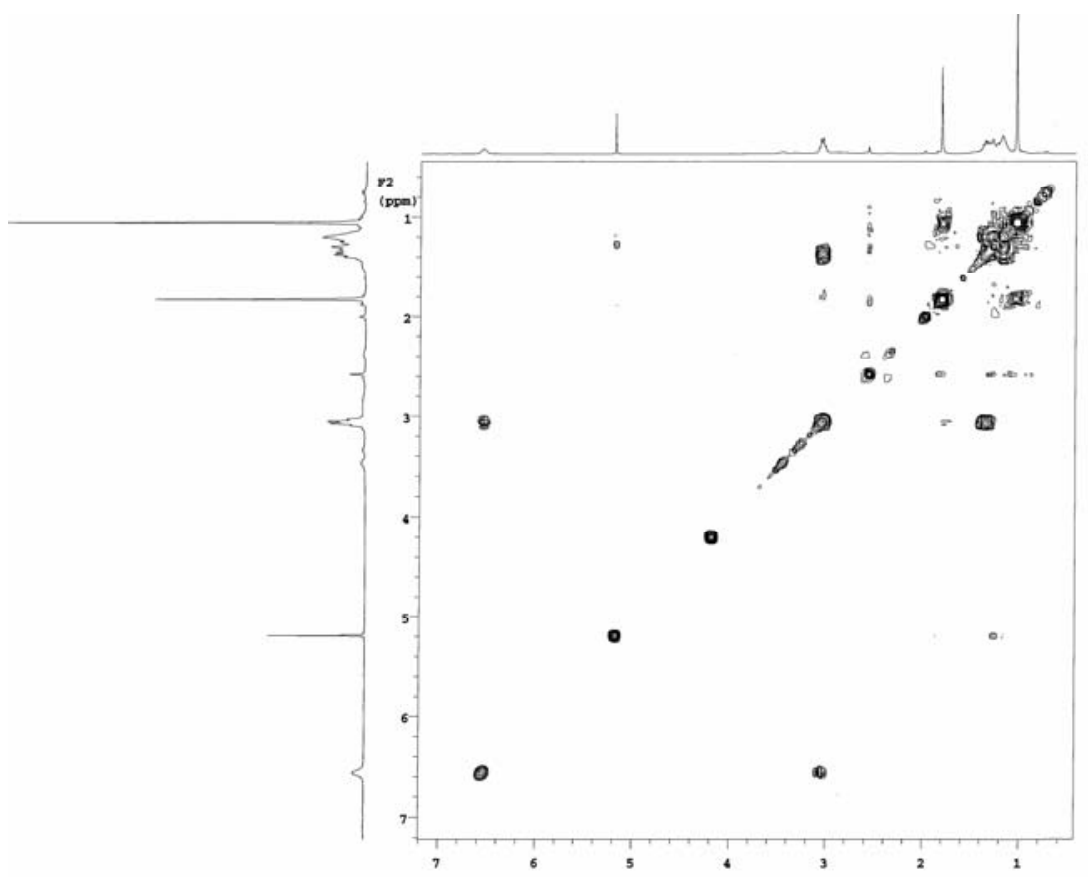

Figure 35: $\mathrm{H}-\mathrm{H}$ COSY spectrum $\left(\mathrm{CDCl}_{3}, 600 \mathrm{MHz}\right)$ of $\mathrm{N}$-(6-hydroxy-6-methylheptyl)-acetamide (61).

In the HMBC spectrum the methylene at $\delta 3.20$ and the nearby $\mathrm{NH}$ group showed correlation with the carbonyl at $\delta 170.4$. The isopropyl group showed correlation with the 
quaternary carbon at $\delta 70.4$. The other correlations were the same as in $N$-(7-hydroxyoctyl)-acetamide (59) and $N$-(7-hydroxy-6-methyl-octyl)-acetamide (60).

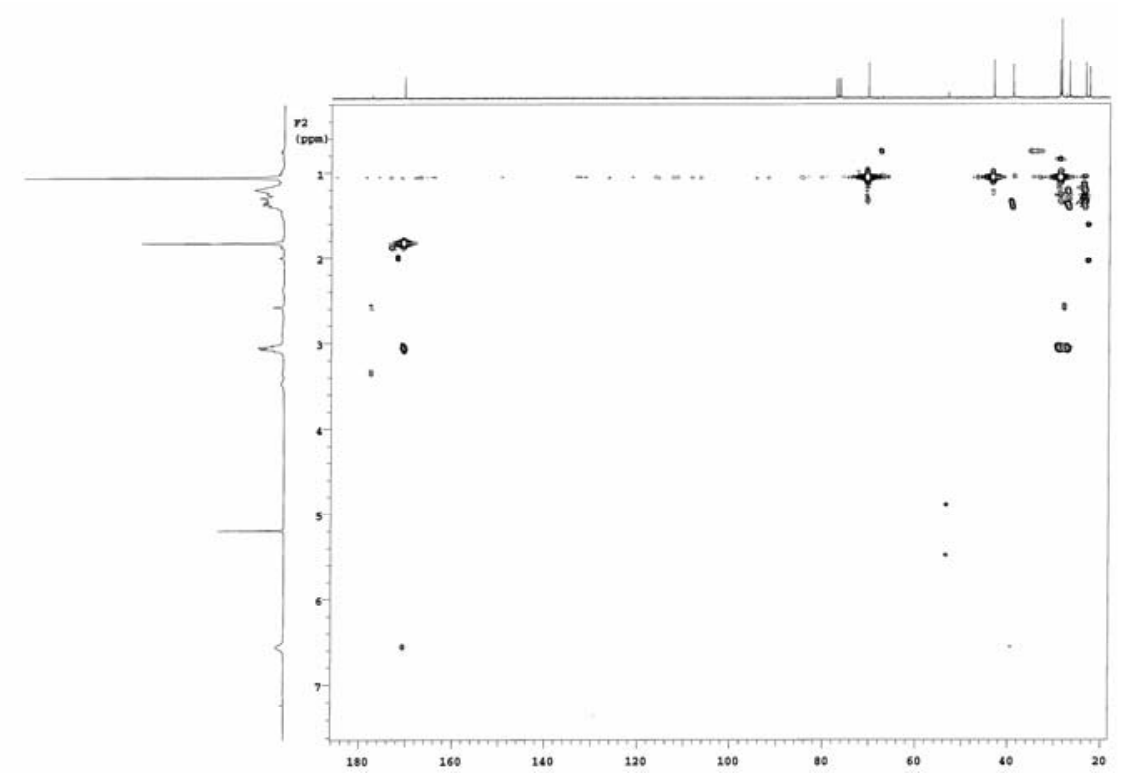

Figure 36: $\mathrm{HMBC}$ Spectrum $\left(\mathrm{CDCl}_{3}, 600 \mathrm{MHz}\right)$ of $N$-(6-hydroxy-6-methyl-heptyl)acetamide (61).

Based on the 1D and 2D NMR data and comparing with related compounds (e.g. 65), structure 61 was confirmed. ${ }^{76}$

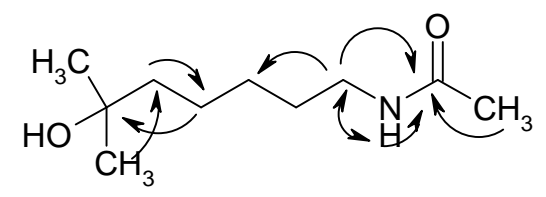

61<smiles>O=C([P])N[P]O</smiles>

65

Figure 37: $\mathrm{R}^{\prime}=\left(\mathrm{CH}_{2}\right)_{\mathrm{n}}$ with $\mathrm{n}=3,4,5,6$ and $\mathrm{R}^{\prime \prime}=\mathrm{Me}$ or Pr.

Amides are interesting compounds due to the natural occurrence of their derivatives, e.g., peptides. There is considerable interest of amides of carboxylic acid derivatives in the formation of peptides and lactams. ${ }^{77}$ Bellenamine (66) is an antibiotic which inhibits the growth of Gram-positive bacteria and has activity against the human immunodeficiency virus and enhances both delayed-type hypersensitivity to sheep red blood cells and antibody formation in the mouse spleen. ${ }^{78}$ 
<smiles>NCCCCCCC(=O)NCN</smiles>

66

\subsubsection{Homononactic acid amide}

The ${ }^{1} \mathrm{H}$ NMR spectrum of compound $\mathbf{6 2} \mathbf{b}$ showed similarity with that of homononactic acid (62a). ESIMS showed a quasimolecular ion at $m / z 216[\mathrm{M}+\mathrm{H}]^{+}$. The odd mass number was an indication of an odd number of nitrogen atoms in the molecule. HRMS established the molecular formula as $\mathrm{C}_{11} \mathrm{H}_{22} \mathrm{NO}_{3}$ and confirmed the presence of one nitrogen atom in this compound.

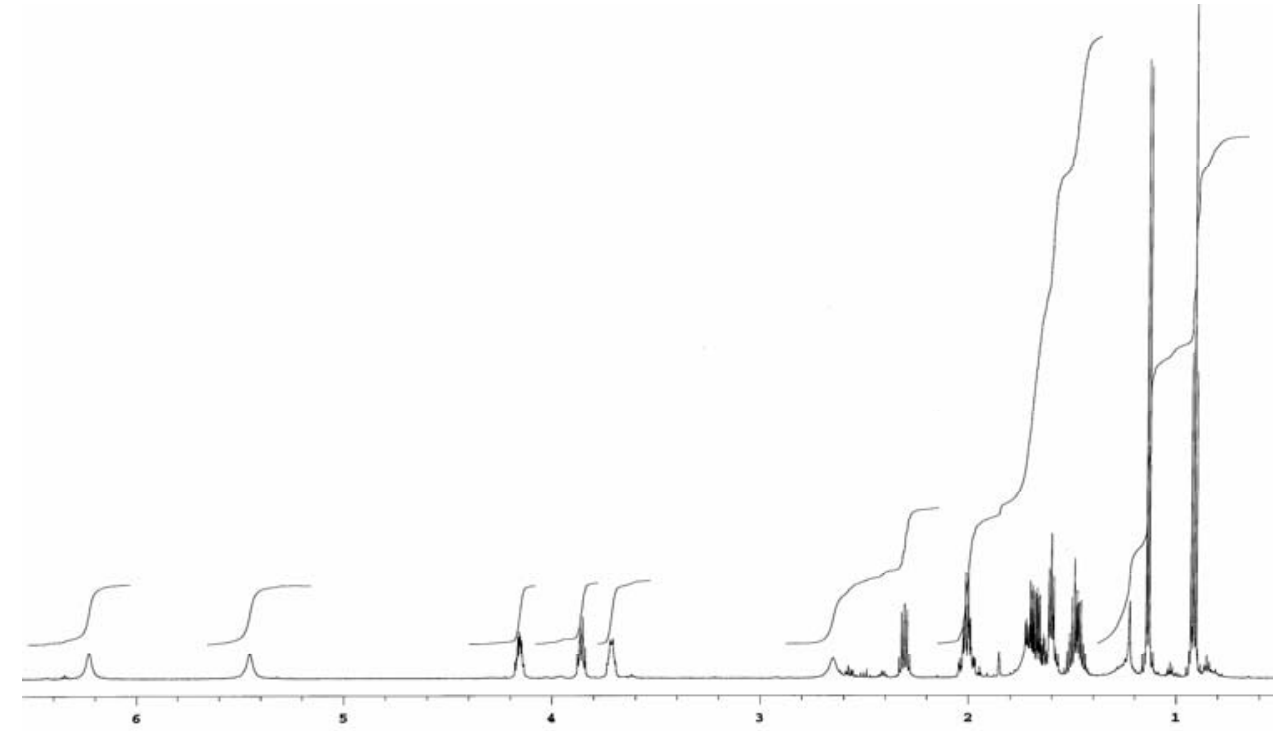

Figure 38: ${ }^{1} \mathrm{H}$ NMR spectrum $\left(\mathrm{CDCl}_{3}, 300 \mathrm{MHz}\right)$ of homononactic acid amide (62).

The ${ }^{1} \mathrm{H}$ NMR spectrum showed a methyl triplet and another $3 \mathrm{H}$ doublet at $\delta 0.94$ and 1.17, respectively. There were three protons present at $\delta 4.18(\mathrm{~m}), 3.90(\mathrm{q})$ and 3.80 (m) which were a hint for attached hetero atoms. There were two H/D exchangeable protons observed as broad singlets at $\delta 6.27$ and 5.50. 


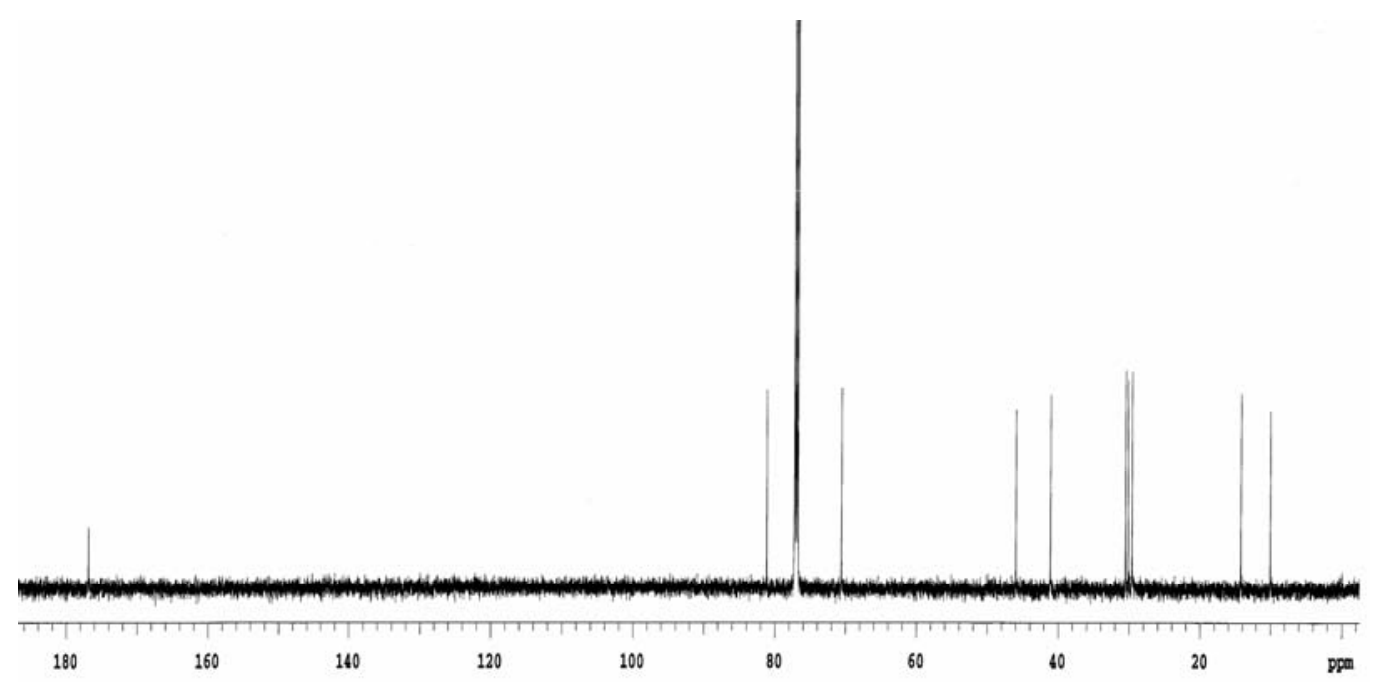

Figure 39: ${ }^{13} \mathrm{C} \mathrm{NMR}$ spectrum $\left(\mathrm{CDCl}_{3}, 150 \mathrm{MHz}\right)$ of homononactic acid amide (62).

In ${ }^{13} \mathrm{C}$ NMR spectrum there were ten carbon signals observed. The carbon signal at $\delta$ 176.9 can be attributed to the carbonyl of an ester, acid or amide. There were three methine carbons at $\delta 81.1,77.0$ and 70.6, which were attached to oxygen. Another methine appeared at $\delta$ 46.0, and four methylene signals at $\delta 41.1,30.6,30.2$ and 29.6. There were two methyl signals observed at $\delta 14.2,14.2$.

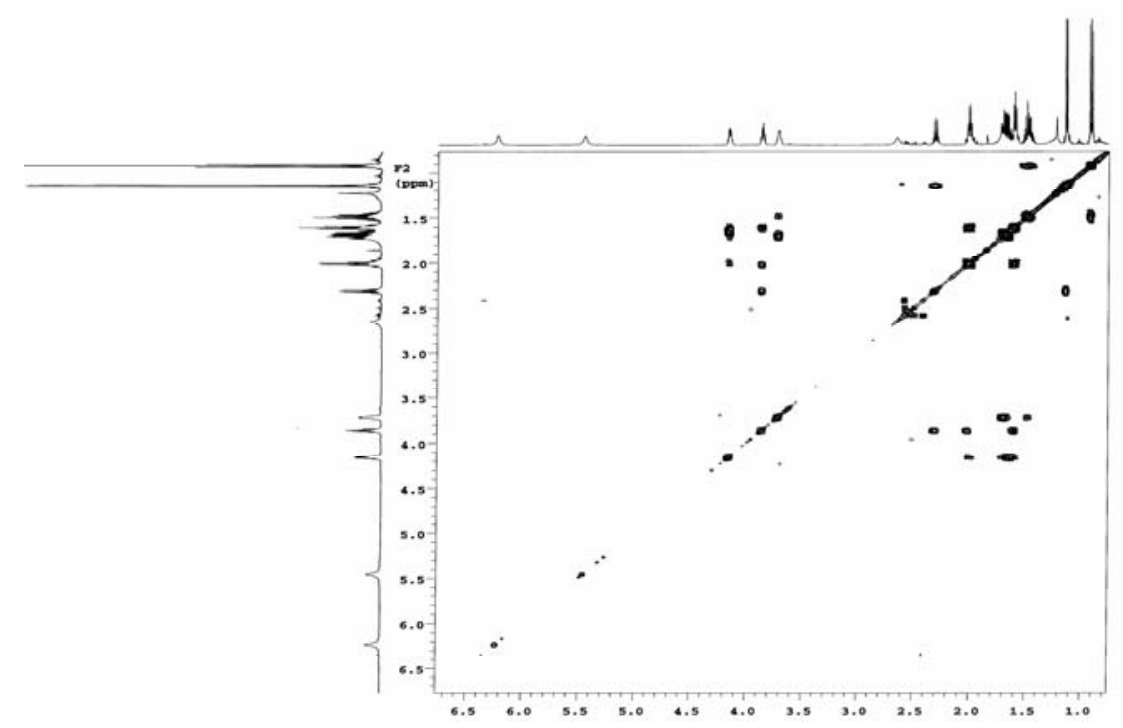

Figure 40: $\mathrm{H}-\mathrm{H}$ COSY spectrum $\left(\mathrm{CDCl}_{3}, 600 \mathrm{MHz}\right)$ of homononactic acid amide (62).

In the H-H COSY spectrum, one of the methyl signals showed correlations with a methylene and another one with a methine. The three oxygenated methines showed correlations with methylene groups. 


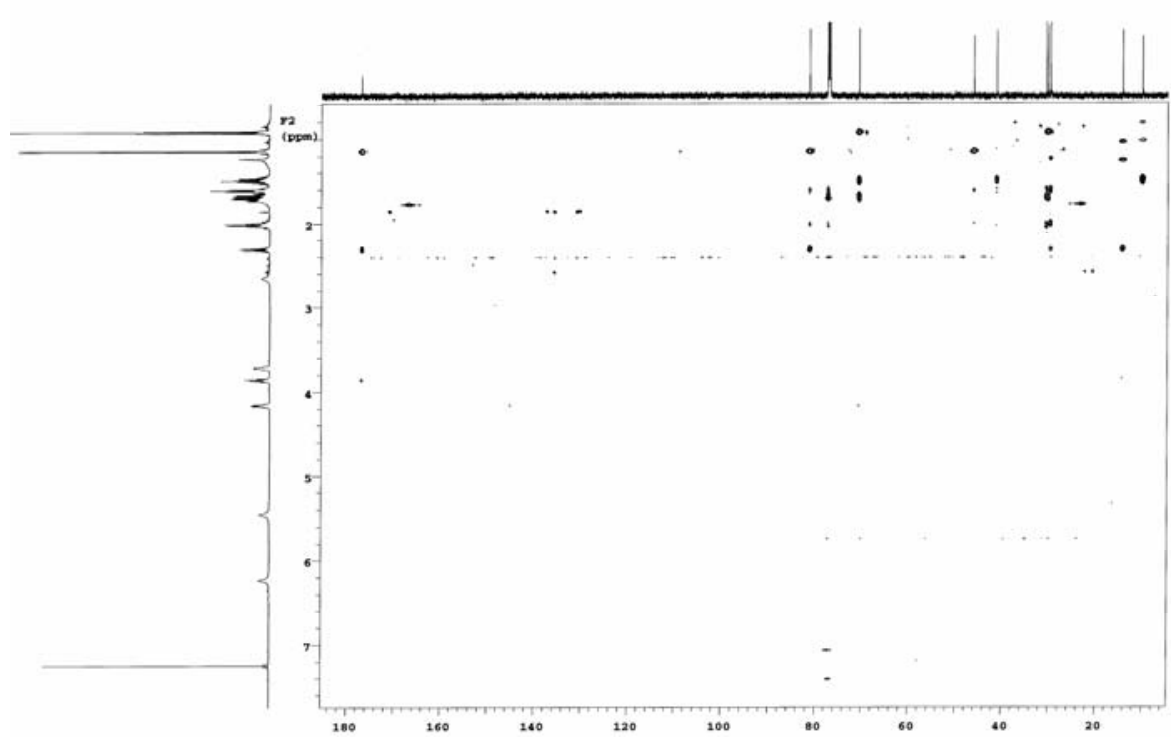

Figure 41: $\mathrm{HMBC}$ Spectrum $\left(\mathrm{CDCl}_{3}, 600 \mathrm{MHz}\right)$ of homononactic acid amide (62).

In the HMBC spectrum, the methyl group at $\delta 1.17$ showed correlation with the carbonyl at $\delta 176.9$ and with the methine $(\mathrm{CH})$ carbon at $\delta 81.1$, which was probably attached to oxygen. There was another methyl triplet at $\delta 0.94$, which showed a longrange correlation with one proton at position $\delta 3.80$, which was also at a methine carbon attached to oxygen. Protons at $\delta 5.44$ and 6.25 did not show any correlation in the HSQC spectrum and were obviously $\mathrm{H} / \mathrm{D}$ exchangeable. The HRESIMS $\left(\mathrm{C}_{11} \mathrm{H}_{21} \mathrm{NO}_{3}\right)$ showed two double bond equivalents in compound $\mathbf{6 2 b}$. The ${ }^{1} \mathrm{H}$ and ${ }^{13} \mathrm{C}$ NMR as well as H-H COSY spectra suggested the structure of homononactic acid (62a). But the HRESIMS as well as two H/D protons at $\delta 5.44$ and 6.25 were different from that of homononactic acid (62a). The carbonyl at $\delta 176.9$ presented the value as ester, amide or acid.<smiles>[R]C(=O)C(C)C1CCC(CC(O)CC)O1</smiles>

62a: Homononactic acid, $\mathrm{R}=\mathrm{OH}$.

62b: Homononactic acid amide, $\mathrm{R}=\mathrm{NH}_{2}$.

On the basis of 1D and 2D NMR data and in comparison with the data of homononactic acid (62a) ${ }^{79}$ the structure of homononactic acid amide (62) was confirmed. 


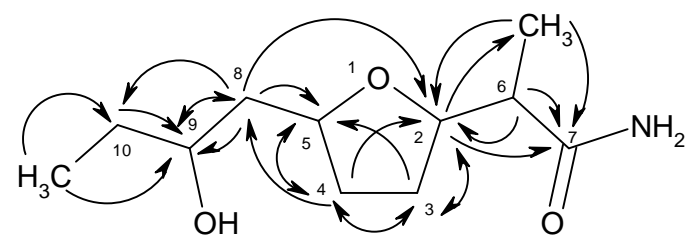

Figure 42: HMBC correlations of homononactic acid amide (62).

\subsubsection{2-Phenylbenzoxazole-4-carboxylic acid}

2-Phenylbenzoxazole-4-carboxylic (63) acid was isolated as a polar, green fluorescent pale yellow solid. In the ${ }^{1} \mathrm{H}$ NMR spectrum, 9 protons were found in the $s p^{2}$ region, but there were no aliphatic proton signals. Two of the protons were acidic and appeared as a broad signal at $\delta 11.5$.

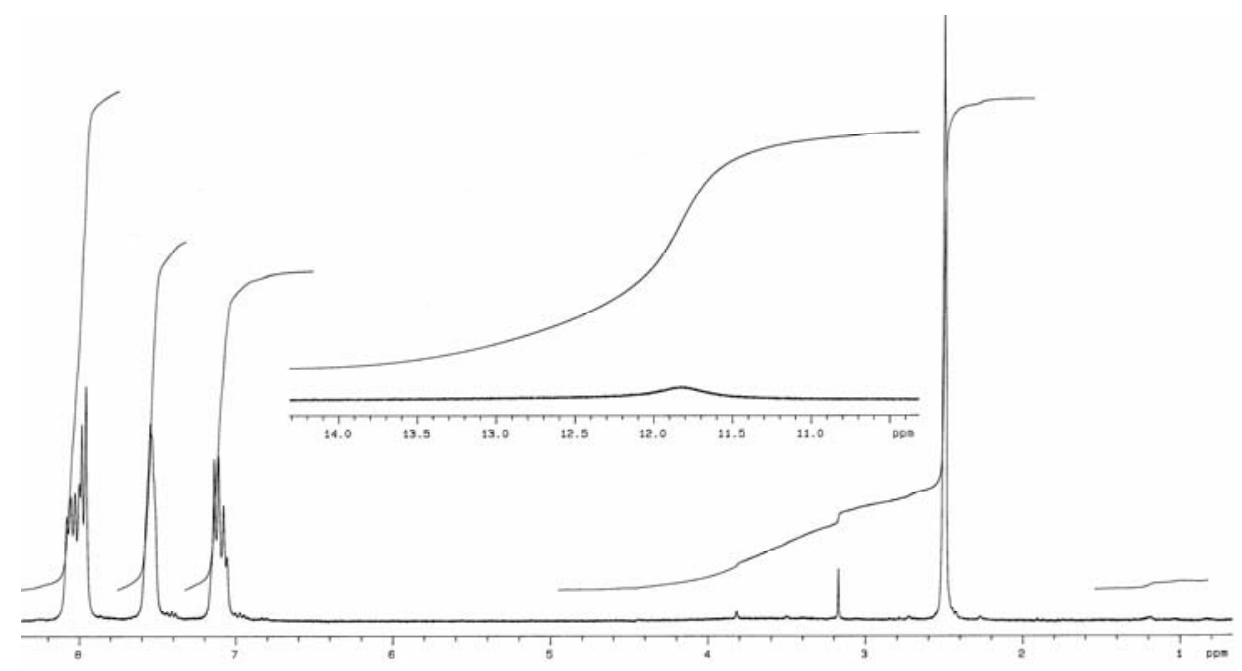

Figure 43: ${ }^{1} \mathrm{H}$ NMR spectrum $\left(\left[\mathrm{D}_{6}\right] \mathrm{DMSO}, 300 \mathrm{MHz}\right)$ of 2-phenylbenzoxazole-4carboxylic acid (63).

HRESIMS afforded the molecular formula $\mathrm{C}_{14} \mathrm{H}_{10} \mathrm{NO}_{4}$. From the $\mathrm{H}-\mathrm{H}$ COSY spectrum two aromatic rings were confirmed with two substructures $\mathrm{A}$ and $\mathrm{B}$.<smiles>CC(C)(C)c1ccccc1I</smiles>

A<smiles>CC(C)(C)c1ccccc1</smiles>

B

Figure 44: Substructures A and B of compound 63. 


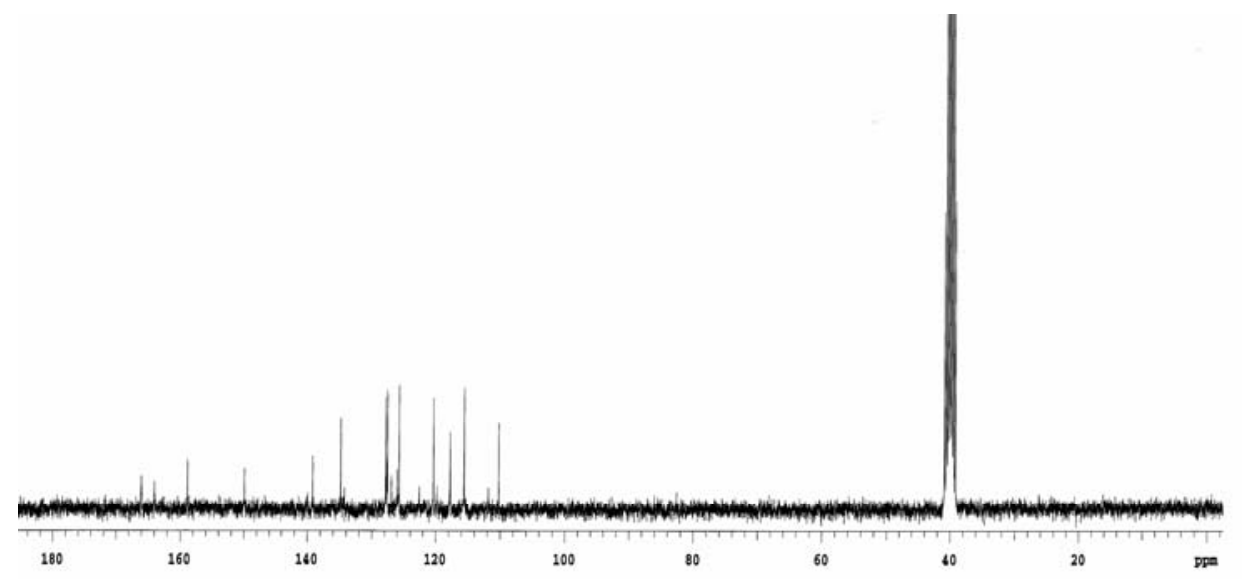

Figure 45: ${ }^{13} \mathrm{C}$ NMR spectrum ([D6]DMSO, $125 \mathrm{MHz}$ ) of 2-phenylbenzoxazole-4carboxylic acid (63).

From the ${ }^{13} \mathrm{C}$ NMR spectrum there was a quaternary carbon signal observed at $\delta$ 165.5, which could be a carbonyl of an ester, amide or an acid. As the presence of an acidic proton was already described, a carboxylic acid or an amide was more likely.

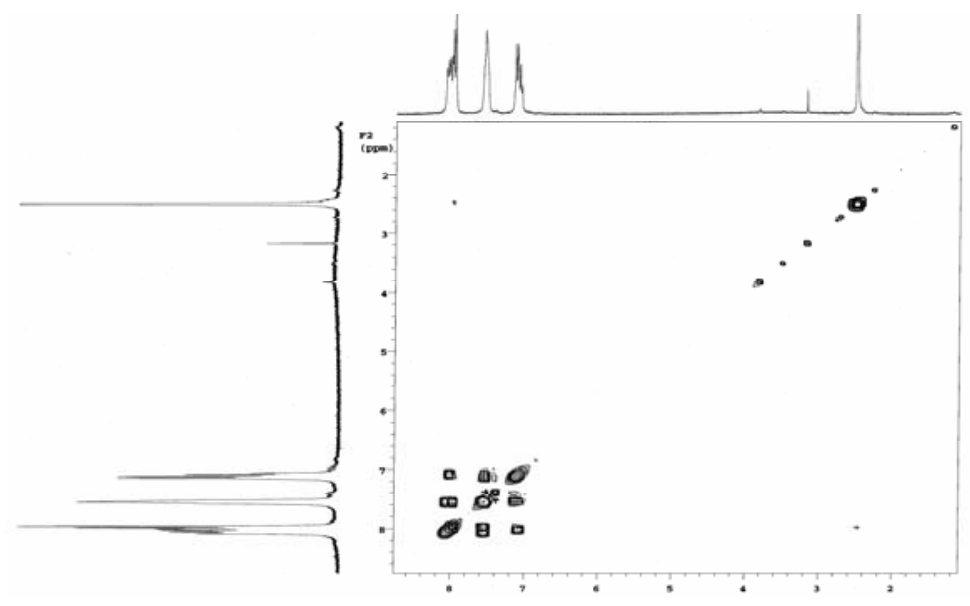

Figure 46: $\mathrm{H}-\mathrm{H}$ COSY spectrum ([D $\left.\left.\mathrm{D}_{6}\right] \mathrm{DMSO}, 500 \mathrm{MHz}\right)$ of phenylbenzoxazole-4carboxylic acid (63).

In the HMBC spectrum, the proton at $\delta 7.96$ showed a ${ }^{3} J$ correlation with the carbon at $\delta 163.4$, where another proton at $\delta 7.99$ showed correlation with the carbonyl at $\delta$ 165.5. In AntiBase, with the molecular formula and the substructures A and B along with ${ }^{13} \mathrm{C}$ NMR values, no respective compound was found, which indicated a new secondary metabolite. A search in the Chemical Abstract delivered, however, compound 63. ${ }^{80,81}$ The ${ }^{1} \mathrm{H}$ NMR, ${ }^{13} \mathrm{C}$ NMR and mass spectrometry as well as the colour of the compound were identical to 2-phenylbenzoxazole-4-carboxylic acid (63), which is now reported from a natural source for the first time. 


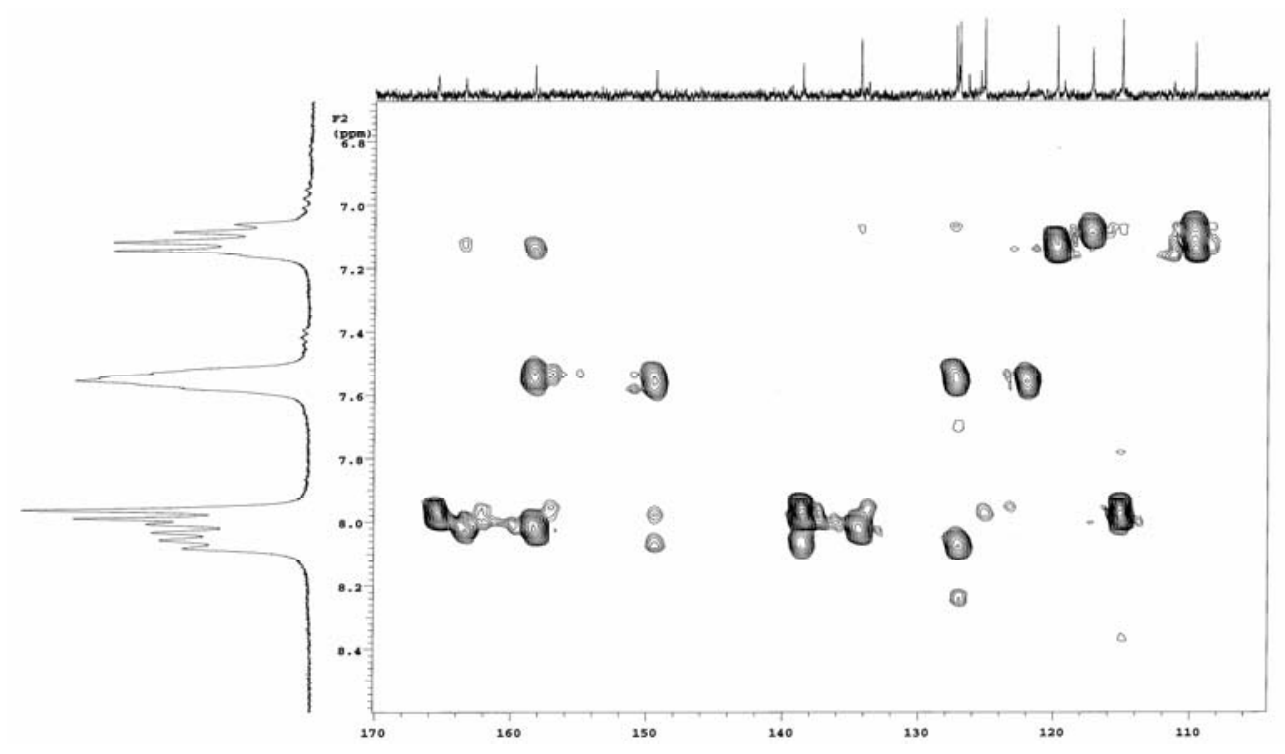

Figure 47: $\mathrm{HMBC}$ spectrum ([D 6 DMSO, $500 \mathrm{MHz})$ of 2-phenylbenzoxazole-4carboxylic acid (63).

Compound 63 showed activity as 5-HT inhibitor and exhibited central activity in treating chemotherapy-induced nausea and vomiting (CINV) as well as diarrhoea and irritable bowel syndrome (IBS-D).

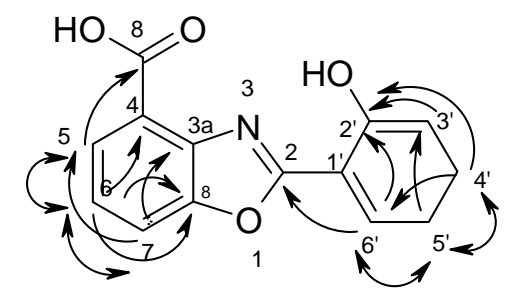

63

\subsubsection{2-(3-Chloro-2-hydroxy phenyl) benzoxazole-4-carbxylic acid}

2-(3-Chloro-2-hydroxyphenyl)-benzoxazole-4-carboxylic acid (64) is a derivative of $\mathbf{6 3}$ and was also isolated from the same fraction. It was obvious from ESI mass spectrometry that this compound contained chlorine. In ESIMS one signal was observed at $m / z 288[\mathrm{M}-\mathrm{H}]^{+}$, followed by another signal at $m / z, 290$ Dalton which was $1 / 3$ of the first signal at $m / z$ 288. The same pattern was also observed at $m / z, 244$ and $m / z 246$ Dalton. The signal at $m / z 244$ was the decarboxylation product of the parent signal at 288 Dalton $\left([\mathrm{M}-\mathrm{H}]^{+}\right)$. HRESIMS afforded the molecular formula $\mathrm{C}_{14} \mathrm{H}_{8} \mathrm{NO}_{4} \mathrm{Cl}$. Compound 64 showed 7 protons at $\delta 7-8$, while compound 63 showed only 6 protons in the same region. One of them was obviously replaced by a chlorine atom. 


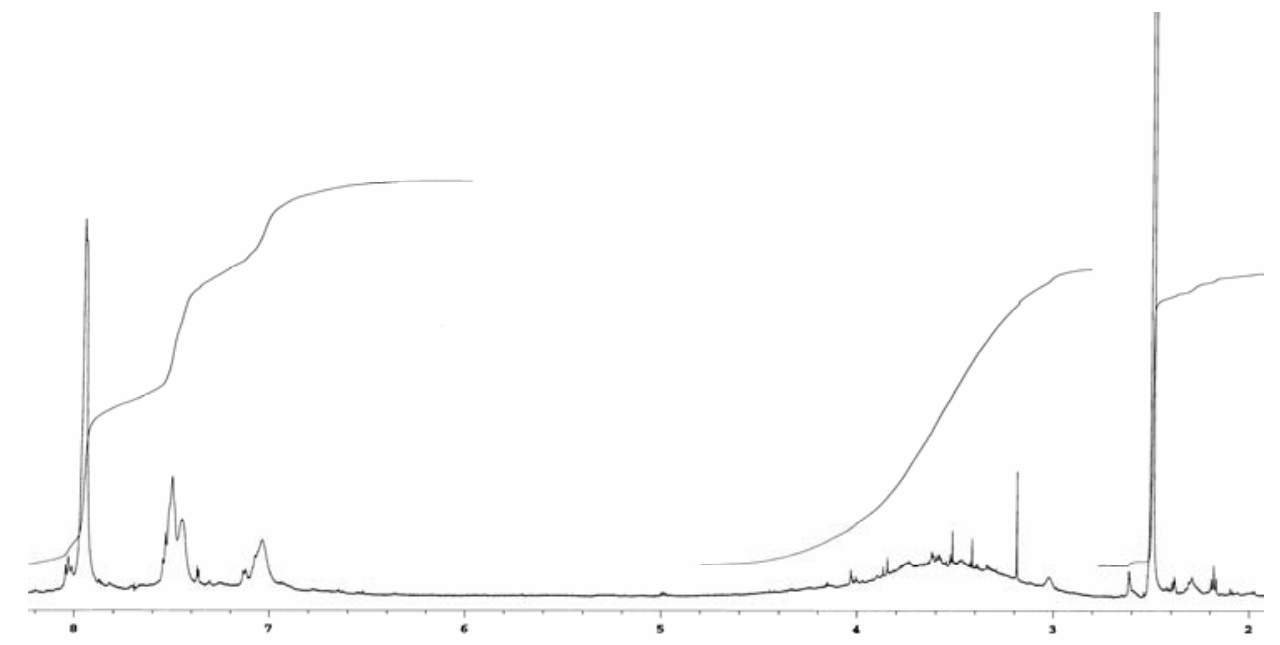

Figure 48: ${ }^{1} \mathrm{H}$ NMR spectrum $\left(\left[\mathrm{D}_{6}\right] \mathrm{DMSO}, 300 \mathrm{MHz}\right)$ of 2-(3-chloro-2-hydroxyphenyl)-benzoxazole-4-carboxylic acid (64).

In 63, there were two protons ( $3^{\prime}$ and $5^{\prime}$ ) at $\delta 7.10$, but in compound 64 there was only one proton at $\delta 7.10$. So, either the 3'- or 5'-position of this compound was occupied by chlorine. The H-H COSY spectrum showed correlations between the protons H-4', $5^{\prime}$ and 6'. Therefore, the chlorine was assigned to position 3 '. Based on mass, ${ }^{1} \mathrm{H}$ NMR and H-H COSY data, compound 64 was identified as 2-(3-chloro-2-hydroxy phenyl)benzoxazole-4-carboxylic acid.

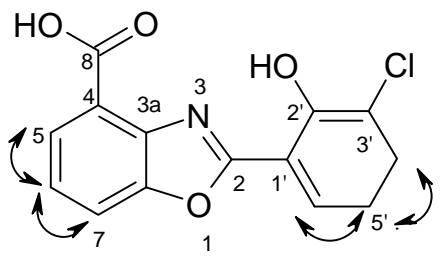

64

Synthesized UK-1 (67) and the analogue 68 showed cytotoxicity against human carcinoma cell lines. ${ }^{80}$ Synthetic 2-hydroxylpropylvalpromide (69) was useful for the treatment of neural psychotic, epilepsy, pain and migraine. ${ }^{82}$ Amides derived from ferulic acid showed stimulatory effects on insulin secretion. ${ }^{83}$ The synthetic azole $1 \mathrm{H}$ pyrazole-4-carboxamide (70) showed potential antifungal and herbicidal activity. ${ }^{84,85}$<smiles>COC(=O)c1cccc(NC(=O)c2cccc3oc(-c4cccc5[nH]c(-c6ccccc6OCc6ccccc6)nc45)nc23)c1N</smiles> 
<smiles>CCCC(CCC)C(=O)NCCC(C)O</smiles>

69<smiles>Cc1c(C(=O)N[C@@H](C)[C@](O)(CN2C=NCN2)c2ccc(F)cc2F)cnn1-c1cc(Cl)cc(Cl)c1</smiles>

70

\subsection{Terrestrial Actinomycete $\mathbf{N} \mathbf{4 3 5}$}

The goal in selecting this strain was to find active compounds against plant pathogenic fungi. N 435 showed moderate antifungal activity, and on TLC of the crude extract, several UV absorbing zones were visible; spraying with anisaldehyde/sulphuric acid showed a pink colour reaction. This behaviour, the UV absorbance together with the antifungal activity of the strain drew our attention for further investigation.

The strain was cultivated on a 20-liter scale shaker culture $(110 \mathrm{rpm})$ at $28{ }^{\circ} \mathrm{C}$ for 72 hours. After filtration using Celite the water phase was extracted with XAD-16 and the bio-mass with ethyl acetate. The brown extract was chromatographed using silica gel column chromatography and eluted with dichloromethane and methanol with a gradient of increasing polarity. As a result, seven fractions were obtained. Working up and purification of the fractions led to the isolation of many diketopiperazines along with a new secondary metabolite, ramthacin B (78). 


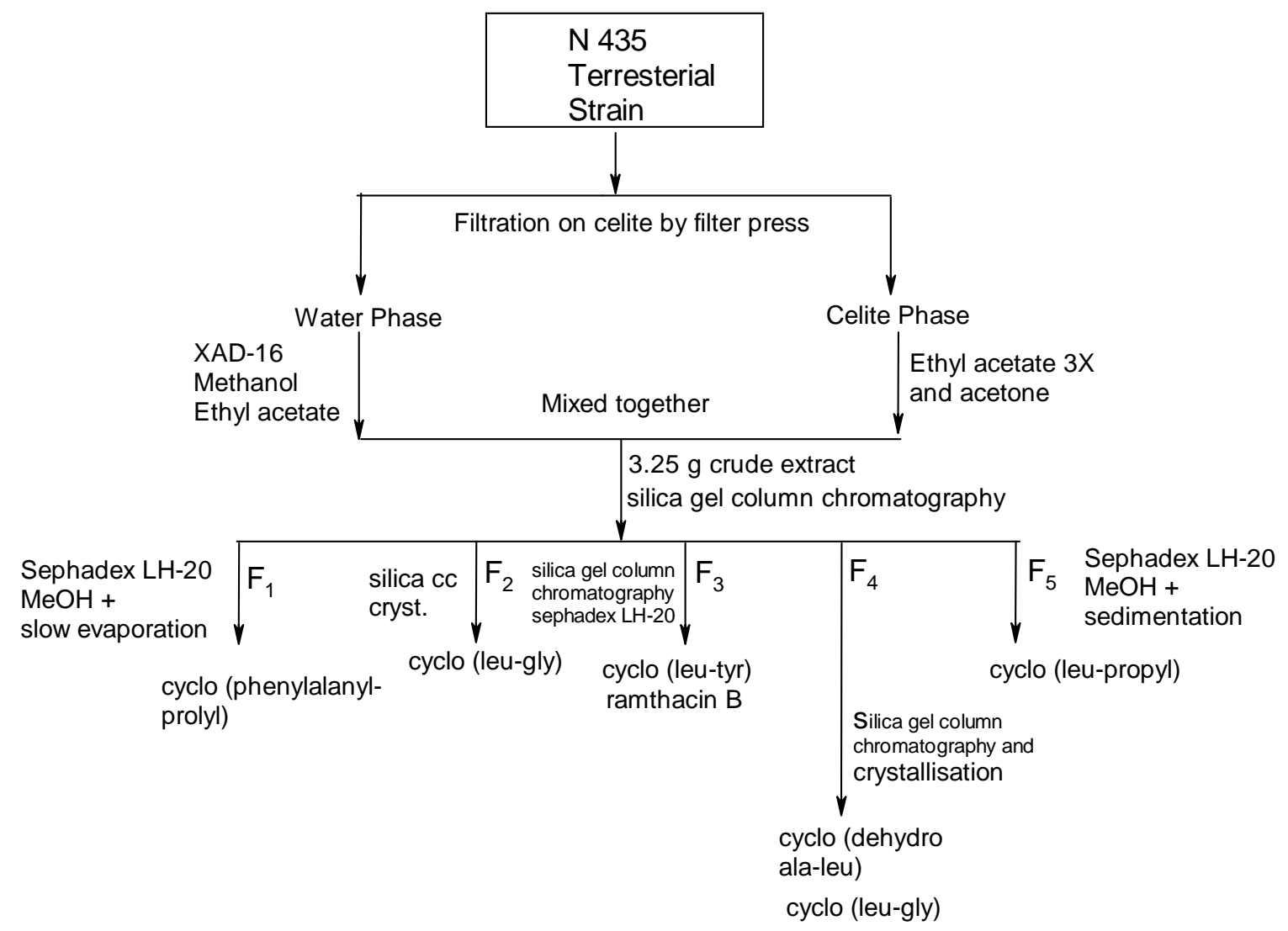

Figure 49: Work-up scheme for terrestrial Actinomycete N 435.

\subsubsection{Cyclo(dehydroalanyl-isoleucyl)}

Compound 71 was isolated from fraction 2 as a white colourless solid after fractionating by silica column chromatography followed by Sephadex LH-20 column chromatography. Compound $\mathbf{7 1}$ crystallized from dichloromethane/methanol as thin needles.<smiles>C=C1NC(=O)C(CC(C)C)NC1=O</smiles> 


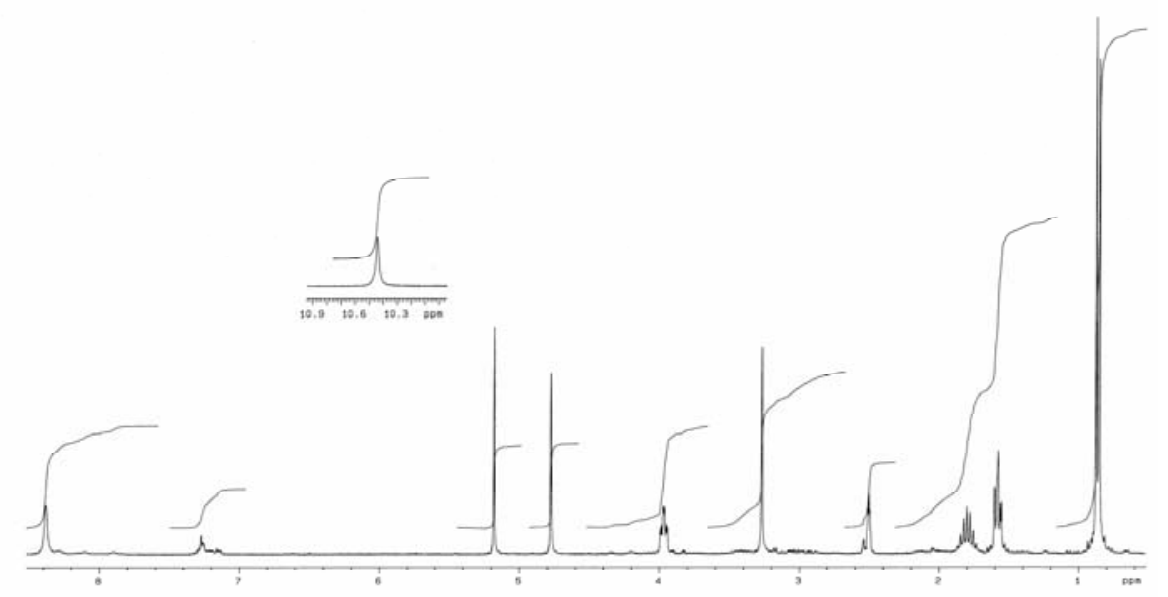

Figure 50: ${ }^{1} \mathrm{H}$ NMR spectrum ([D6]DMSO, $300 \mathrm{MHz}$ ) of cyclo(dehydroalanylisoleu-cyl) (71).

EI mass spectrum established the molecular weight as $m / z$ 170. The ${ }^{1} \mathrm{H}$ NMR spectrum showed two acidic proton signals at $\delta 10.43$ and 8.40 as broad singlets. There was an $s p^{2}$ methylene with two $1 \mathrm{H}$ singlets at $\delta 5.20$ and 4.78 , as well as an isobutyl group $[\delta 1.80(\mathrm{~m}, 1 \mathrm{H}, \mathrm{CH}), 1.77(\mathrm{dd}, 2 \mathrm{H})]$ and 2 methyl groups as a doublet at $\delta 0.85$. Another methine proton at $\delta 3.97$ was observed as a multiplet. Based on ${ }^{1} \mathrm{H}$ NMR spectrum mass, a search in AntiBase identified this compound as cyclo(dehydroalanyl-isoleucyl) (71); this was confirmed by comparison with an authentic spectrum.

\subsubsection{Cis-Cyclo(leucyl-tyrosyl)}

Compound 72 was isolated as colourless solid from fraction 3. Beside the $R_{f}$ value, it showed the same physical properties as compound 71. In the ${ }^{1} \mathrm{H}$ NMR spectrum there were two H/D exchangeable protons at $\delta 9.15$ and 8.00. A para-substituted benzene ring was observed at $\delta 6.91$ and 6.65, and two methine protons were visible at $\delta 4.06$ and 3.46 suggesting their attachment to hetero atoms. There was a methylene observed at $\delta 3.01$ and 2.73 near a chiral centre, an isopropyl group at $\delta 0.66$ (2 methyl) and 1.45 (methine multiplet) as well as another methylene at $\delta 0.80$ and 0.23 .<smiles>CC(C)CC1NC(=O)C(Cc2ccc(O)cc2)NC1=O</smiles> 


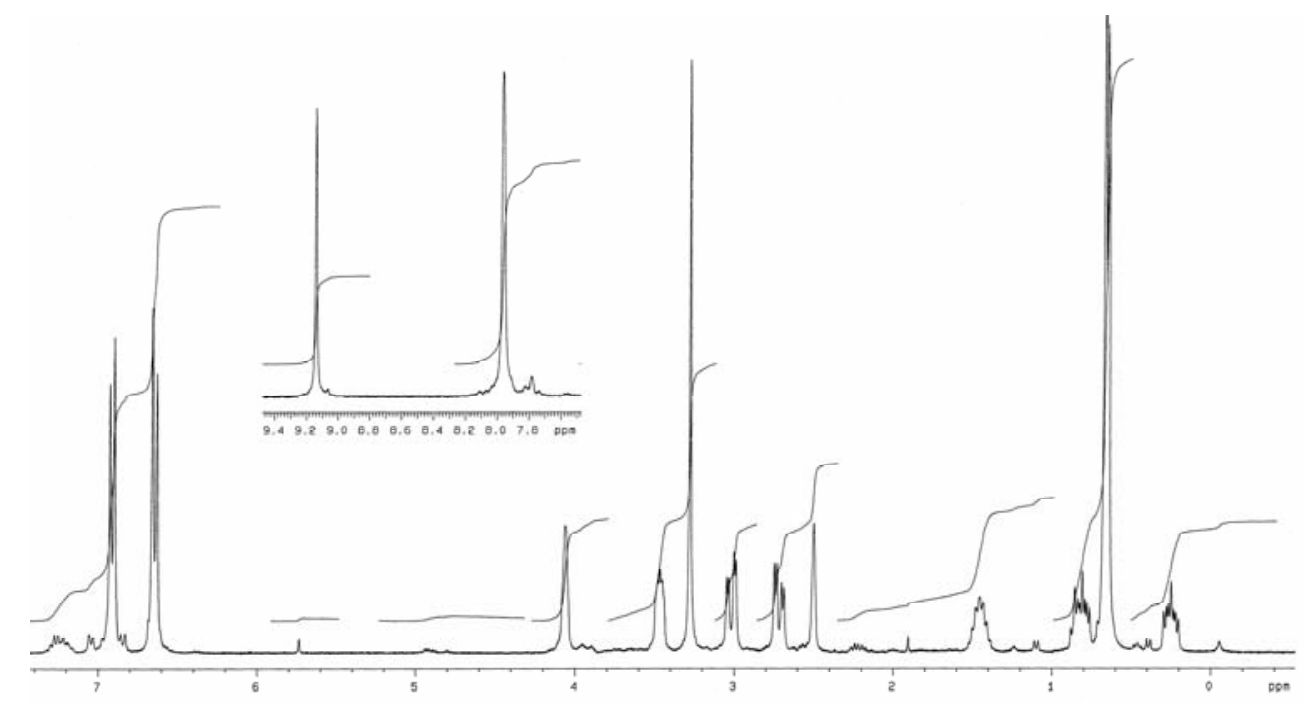

Figure 51: ${ }^{1} \mathrm{H}$ NMR spectrum ([D 6$]$ DMSO, $\left.300 \mathrm{MHz}\right)$ of cis-cyclo(leucyl-tyrosyl) (72).

HRESIMS established the mass as $m / z 275.1403$ ([M-H] $]^{-}$for $\mathrm{C}_{15} \mathrm{H}_{19} \mathrm{~N}_{2} \mathrm{O}_{3}$, indicating that there were seven double bond equivalents present.

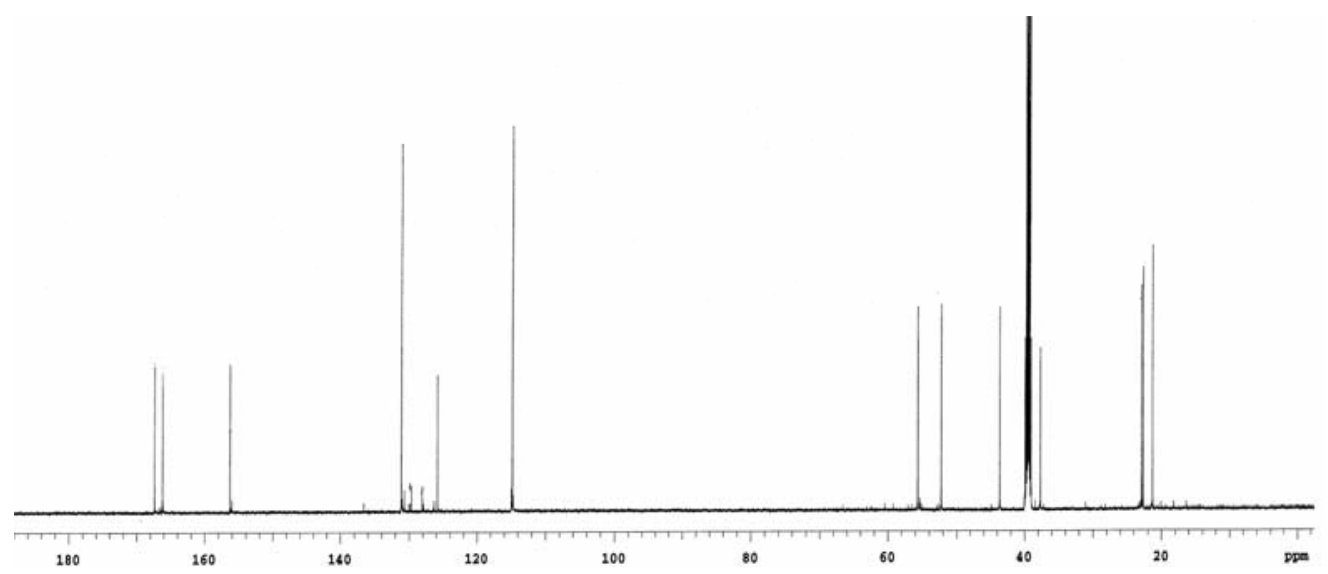

Figure 52: ${ }^{13} \mathrm{C}$ NMR spectrum ([D $]$ DMSO, $75 \mathrm{MHz}$ ) of cis-cyclo(leucyl-tyrosyl) (72).

In the ${ }^{13} \mathrm{C}$ NMR spectrum there were two carbonyl signals of an ester, acid or amide system at $\delta 167.4$ and 166.2. There was an oxygenated $s p^{2}$ carbon observed at $\delta 156.3$ as well as five carbon signals at $\delta 131.1(2 \mathrm{CH}), 125.9$ and $114.8(2 \mathrm{CH})$ for the rest of an aromatic system. Seven additional carbon signals were observed between $\delta$ 55.621.3. 


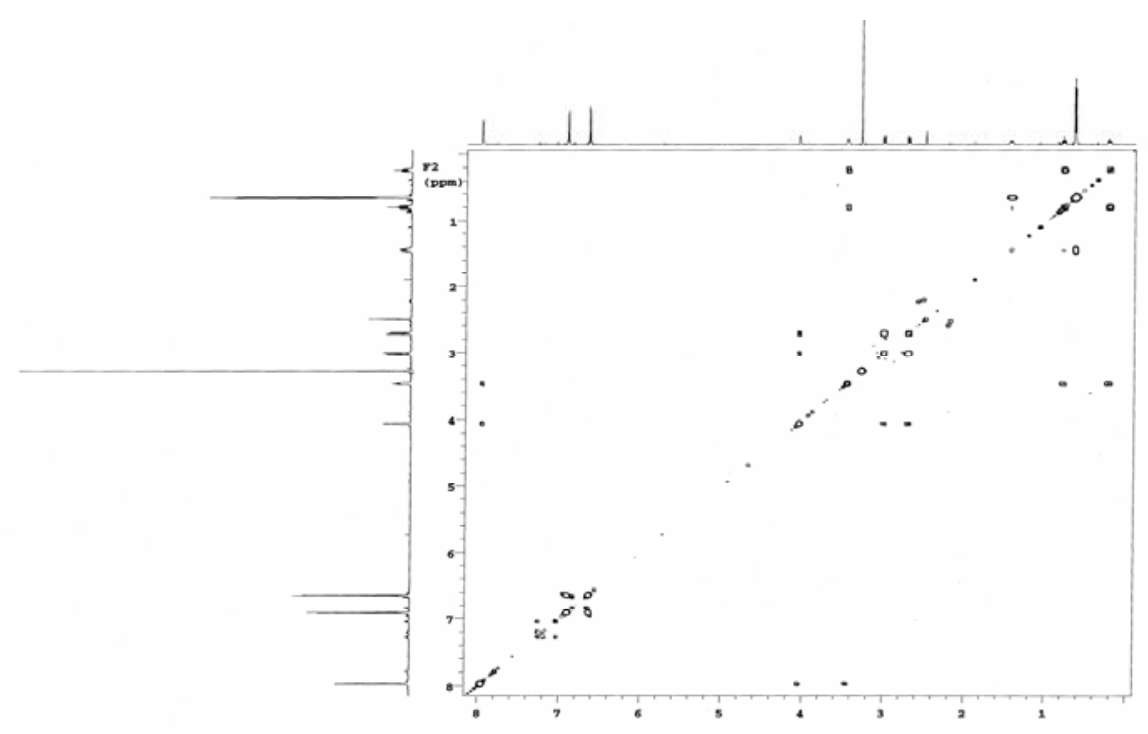

Figure 53: H-H COSY spectrum ([D $\left.\left.{ }_{6}\right] \mathrm{DMSO}, 300 \mathrm{MHz}\right)$ of cis-cyclo(leucyl-tyrosyl) (72).

From the H-H COSY, an isobutyl system was determined and the substructures A and $B$ resulted from the observed correlations.<smiles>CCCC</smiles>

A<smiles>CC(C)C(C)C(C)C</smiles>

B

Figure 54: Fragments (A and B) from the H-H COSY spectrum of cis-cyclo(leucyltyrosyl) (72).

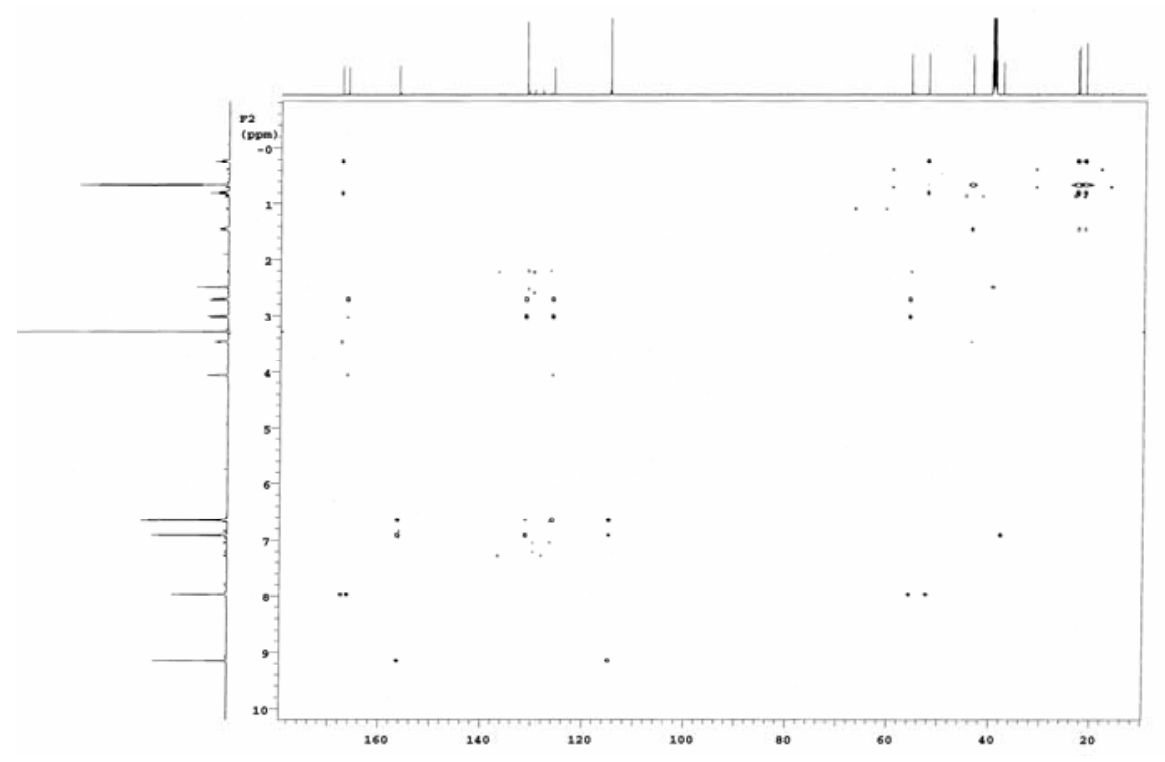

Figure 55: H-H COSY spectrum ([D $\left.\left.{ }_{6}\right] \mathrm{DMSO}, 300 \mathrm{MHz}\right)$ of cis-cyclo(leucyl-tyrosyl) (72). 
The methylene at $\delta 0.80$ and 0.23 showed correlation with the phenol moiety, which indicated that they were directly attached. This methylene also showed correlation with another methine. Additionally, the methine showed correlation with the carbonyl of an amide. The same type of correlation was observed for the isobutyl group. The EI mass spectrum showed the following fragmentation (Figure 56), which supported a further diketopiperazine. From the HMBC correlations and published data ${ }^{86}$ the structure was confirmed as cis-cyclo(leucyl-tyrosyl).

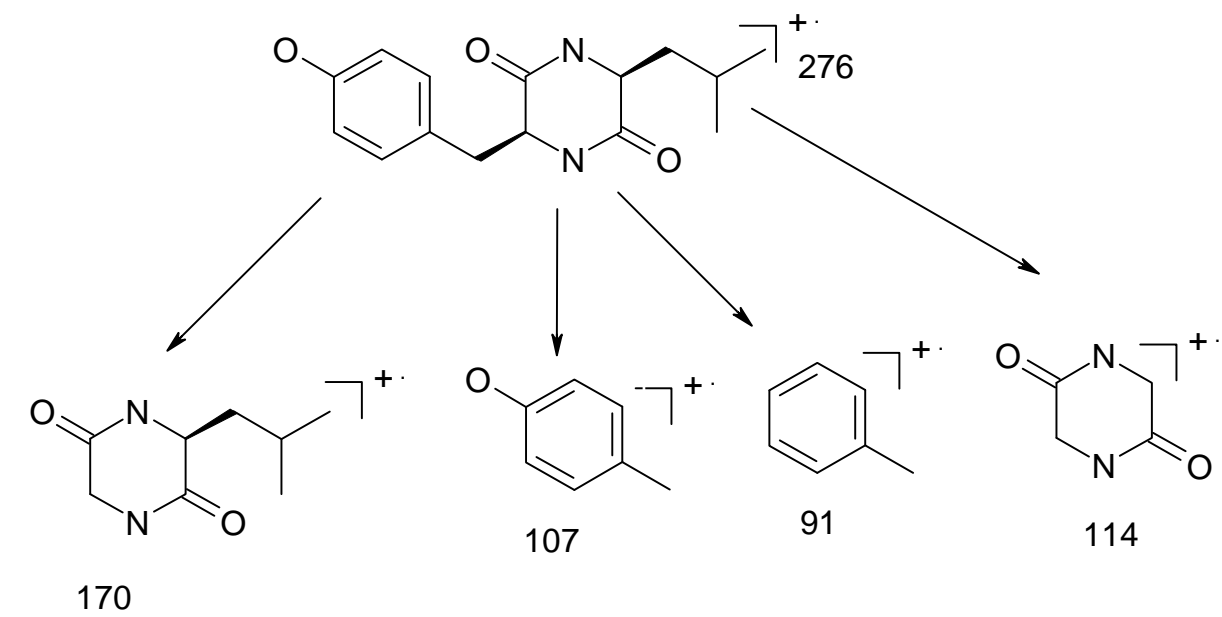

Figure 56: EI-MS fragmentation of cis-cyclo(leucyl-tyrosyl) (72).

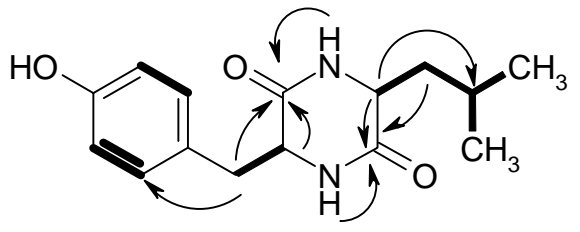

Figure 57: $\mathrm{HMBC}(\longrightarrow)$ and $\mathrm{H}-\mathrm{H}$ COSY $(-)$ correlations of cis-cyclo(leucyl-tyrosyl) (72).<smiles>O=C1N[C@@H](Cc2ccccc2)C(=O)N2CCC[C@H]12</smiles>

cyclo(phenylalanyl-prolyl) (73)<smiles>CC(C)/C=c1\[nH]c(=O)/c(=C/c2ccccc2)[nH]c1=O</smiles>

Albonoursin (74) 
<smiles>CC(C)CC1NC(=O)[C@H]2CCCN2C1=O</smiles>

trans-cyclo(leucyl-proplyl) (75)<smiles>CC(C)C1NC(=O)[C@H]2CCCN2C1=O</smiles>

trans-cyclo(glycyl-leucyl) (76)

Diketopiperazines are the smallest cyclic peptides known, commonly biosynthesised from amino acids by different organisms, including mammals, and are considered to be secondary functional metabolites or side products of terminal peptide cleavage. Cyclic dipeptides are extensively obtained by extraction from natural sources, but may be easily synthesized. ${ }^{87,88}$ They are generally biosynthesised from proteinogenic L- $\alpha$-amino acids having a cis-configuration and possess a wide spectrum of biological activities, which include: antitumor, antiviral, antifungal, antibacterial and antihyperglycaemic. In addition, they have been found to inhibit plasminogen activator inhibitor and alter cardiovascular and blood-clotting functions. Diketopiperazines are also described as signalling compounds however the exact role that they play in bacterial cell-to-cell communication has not been ascertained. In particular some diketopiperazines containing tyrosine have been found to bind $\mu$-opioid receptors ${ }^{89}$ while cyclo(leucyl-prolyl) inhibited the growth of Mycobacterium marinum, an experimental model for $M$. tuberculosis. ${ }^{90}$ It is quite unusual for such a variety of diketopiperazines to be isolated from one source.

\subsubsection{Ramthacin B}

Colourless oil with middle polarity was isolated from sub-fraction 3. It was UV absorbing at $254 \mathrm{~nm}$ and fluorescent at $366 \mathrm{~nm}$. After spraying the TLC plate with anisaldehyde/sulphuric acid, it gave a dark green colour. The molecular weight was established by EIMS to be $\mathrm{m} / \mathrm{z} 367$. In the aromatic region it showed three $1 \mathrm{H}$ signals, two of which were doublets of doublets at $\delta 7.79$ and $\delta 6.59$ indicating two orthocoupled protons; the third proton at $\delta 7.77$ showed a meta-coupling. In the aliphatic region three methyl groups were found as a singlet at $\delta 1.63$. The chemical shift for these three methyl groups indicated the attachment of $s p^{2}$ carbon atoms or influence inductive effect. At $\delta 4.91,4.60$ and 3.67, three methine protons were observed which were attached with hetero atoms. At $\delta 3.48$ and 3.11, one methylene was observed which was obviously near a chiral centre. The $1 \mathrm{H}$ NMR spectrum showed substructures A, B, C. With the help of ESIMS and 1H NMR spectrum the compound was searched in AntiBase resulted no hit. The substructures from the 1H NMR spectrum leaded to a high similarity to virantmycin (77). ${ }^{91} 1 \mathrm{H}$ NMR spectrum was found same 
with compound $\mathbf{7 8}$ which was recently isolated and the structure has already been established by 1D and 2D NMR (publication in process) from marine Streptomyces sp. B7801.92 The 1H NMR spectrum and mass were identical to that of the compound 78. Compound 78 has been named as ramthacin $\mathrm{B}$, which is a new secondary metabolite.<smiles>CC(C)(C)c1ccccc1</smiles>

A
$3 \times$<smiles>C</smiles>

B<smiles>CCOC</smiles>

$\mathrm{C}$

Figure 58: Substructures of compound $\mathbf{7 8}$ derived from ${ }^{1} \mathrm{H}$ NMR spectrum.

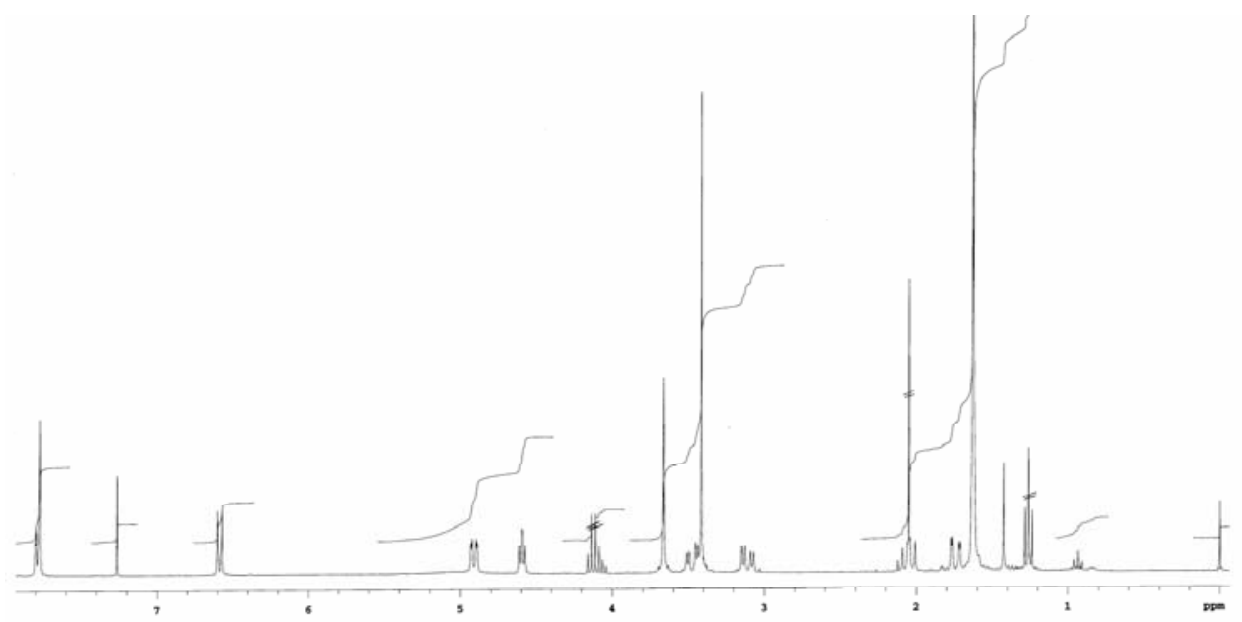

Figure 59: ${ }^{1} \mathrm{H}$ NMR spectrum $\left(\mathrm{CDCl}_{3}, 300 \mathrm{MHz}\right)$ of ramthacin $\mathrm{B}(\mathbf{7 8})$.<smiles>CO[C@]1(CCC(C)=C(C)C)Nc2ccc(C(=O)O)cc2CC1Cl</smiles> 


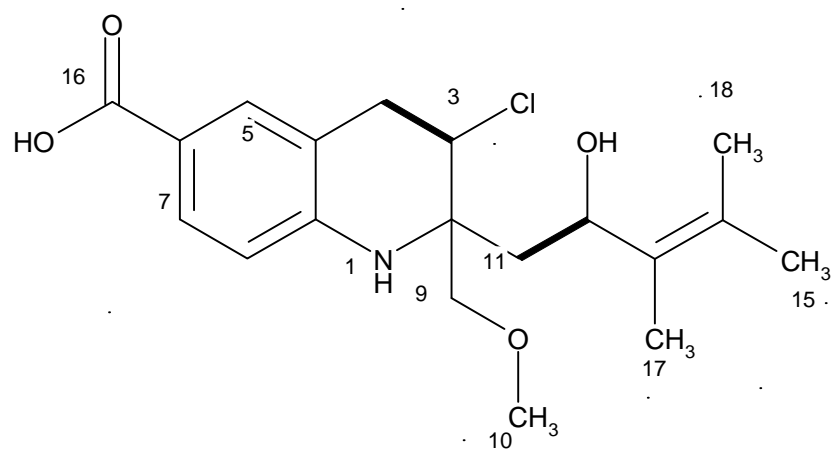

78

Virantmycin (77) was isolated from Streptomyces sp. by Japanese coworkers in 1980 which showed potential antiviral activity. ${ }^{93}$ Compound 78 was not stable enough to check antiviral activity. The benzastatin family and virantmycin (77) are a novel class of indoline and tetrahydroquinoline alkaloids isolated from Streptomyces nitrosporeous. ${ }^{94-96}$ Benzastatins show inhibitory activity against glutamate toxicity and lipid peroxidation in rat liver microsomes that can be used to prevent brain ischemia injury, and consists of indoline alkaloids such as benzastatin E (80), and tetrahydroquinoline alkaloids such as benzastatin $\mathrm{C}$ (79), which are structurally related to virantmycin. Virantmycin, a potent inhibitor towards RNA and DNA viruses, is a unique 2,2disubstituted tetrahydroquinoline alkaloid with contiguous quaternary and tertiary stereo centres. To date, several research groups have reported the total syntheses of virantmycin. ${ }^{97,98}$<smiles>CC[C@@]1(CCC(C)=C(C)C)Nc2ccc(C(N)=O)cc2C[C@H]1Cl</smiles>

79<smiles>CC[C@](O)(CCC(C)=C(C)C)[C@H]1Cc2cc(C(N)=O)ccc2N1</smiles>

80

\subsection{Streptomyces sp. $\mathbf{B 7 8 5 7}$}

The chemical investigation of the marine-derived Streptomyces sp. B 7857 delivered four new oxygenated $\alpha$-gorgonene sesquiterpenes named bacteriogorgonene $\mathrm{A}(\mathbf{8 1})$, (82), bacteriogorgonene B (83) and bacteriogorgonene C (84) and a few trivial compounds as well as ten known secondary metabolites, namely homononactic acid, 1acetyl- $\beta$-carbolin, polypropylenglycol, nonactic acid, uracil, thymidine, deoxy- 
uridine, and 2-hydroxy-1-(1H-indol-3-yl)-ethanone. The structures of the new constituents were elucidated by chemical and spectroscopic studies.

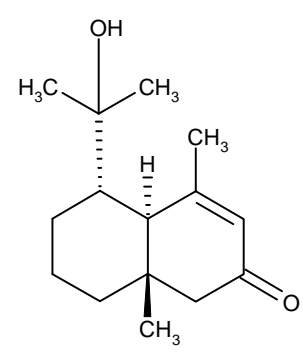

81

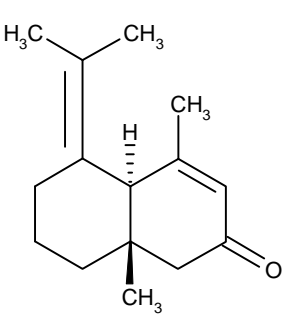

82

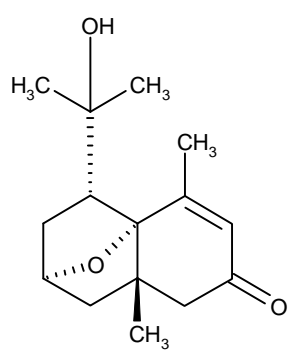

83

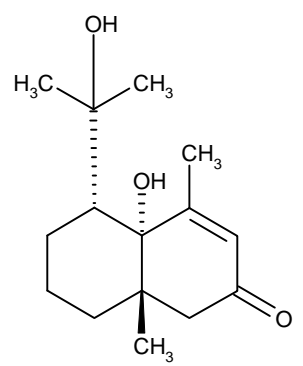

84

The strain Streptomyces sp. B 7857 was selected due to its moderate to high biological activity against Staphylococcus aureus, Streptomyces viridochromogenes (Tü 57), Chlorella vulgaris, and Artemia salina. It also showed several UV absorbing zones. Spraying with anisaldehyde/sulphuric acid on TLC showed a dark pink colour reaction, turned to green with time.

The strain was incubated using $\mathrm{M}_{2}$ medium on a $20 \mathrm{~L}$ scale. After 7 days, the wellgrown culture broth was mixed with Celite, and filtered under pressure. The filtrate was extracted with XAD-16 column and the resin was washed with demineralised water and eluted with methanol to get a dark brown extract.

On TLC the biomass and XAD extract did not show much difference and hence were combined. The combined crude extract was chromatographed on a silica gel column eluting with a dichloromethane/methanol gradient to give 4 fractions. Fraction 1 consisted mostly fats and lipids along with polypropylene glycol, whereas fraction 2 showed two main spots on TLC. Thus this fraction 2 was further purified by using Sephadex LH-20, silica column chromatography, PTLC and finally by RP-18 open column chromatography eluting with 50-60\% aqueous methanol to give compound 81. PTLC was most effective in separating the fractions as several compounds overlapped in column chromatography. After PTLC further purification was done by RP18 column chromatography. Using the same method, another two new terpenes, compounds 83 and 84 were isolated from the same fraction. Fraction 5 was further purified to give seven trivial compounds, namely 1 -acetyl- $\beta$-carbolin, 3-(2hydroxyethyl)indole, uracil, thymidine, deoxyuridine, and 2-hydroxy-1-(1H-indol-3yl)-ethanone. 


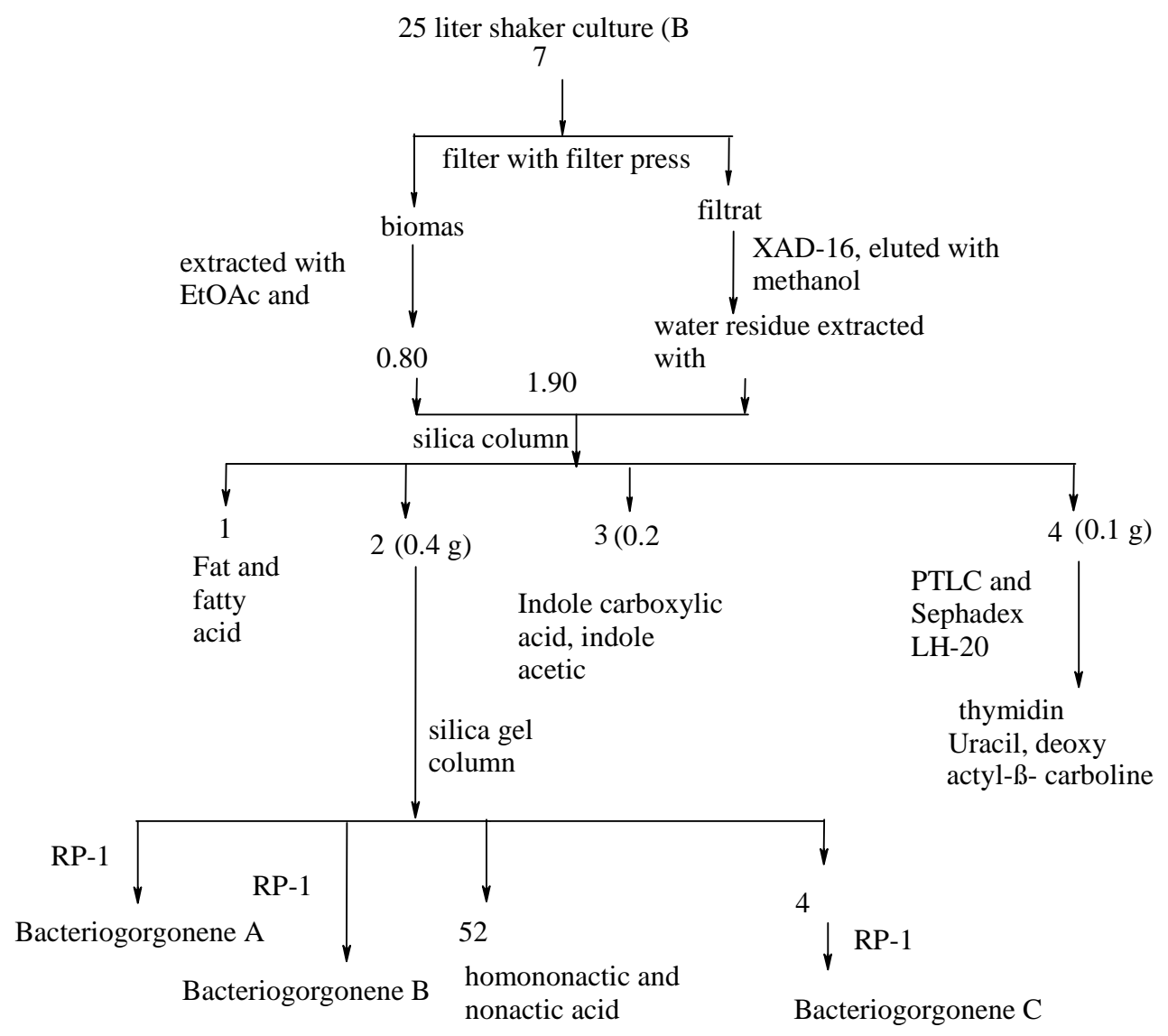

Figure 60: Working scheme of Streptomyces sp. B 7857.

\subsubsection{Bacteriogorgonene A}

Compound 81 was isolated as very unpolar colourless oil, which was difficult to separate from the fat fraction. PTLC was done by multiple developments, followed by RP-18 and Sephadex LH-20 column chromatography to isolate compound 81. In the ${ }^{1} \mathrm{H}$ NMR spectrum there were no protons observed in the aromatic region. Four methyl groups were visible between $\delta 1.00-2.00$. The methyl singlet at $\delta 0.94$ was easily determined as being attached to a quaternary carbon atom. Two further methyl groups appeared in the same place at $\delta 1.31$ as singlet, which indicated both the methyl groups were attached to a quaternary carbon. The remaining methyl at $\delta 1.95$ showed a very small coupling $(J=1.4 \mathrm{~Hz})$ indicating a ${ }^{4} J$ distance. The olefinic proton at $\delta 5.90$ also showed the ${ }^{4} J$ coupling with the methyl and was near an electronwithdrawing group. This proton was in the $\alpha$-position of a carbonyl group, indicating the $\beta$-position was substituted with the methyl group. From the spectra, the presence of at least 3 methylene groups was clear. Two of the methylene groups (at $\delta 2.25$ and 1.37) showed $A B X$ systems, indicating the presence of neighbouring chiral centres. 


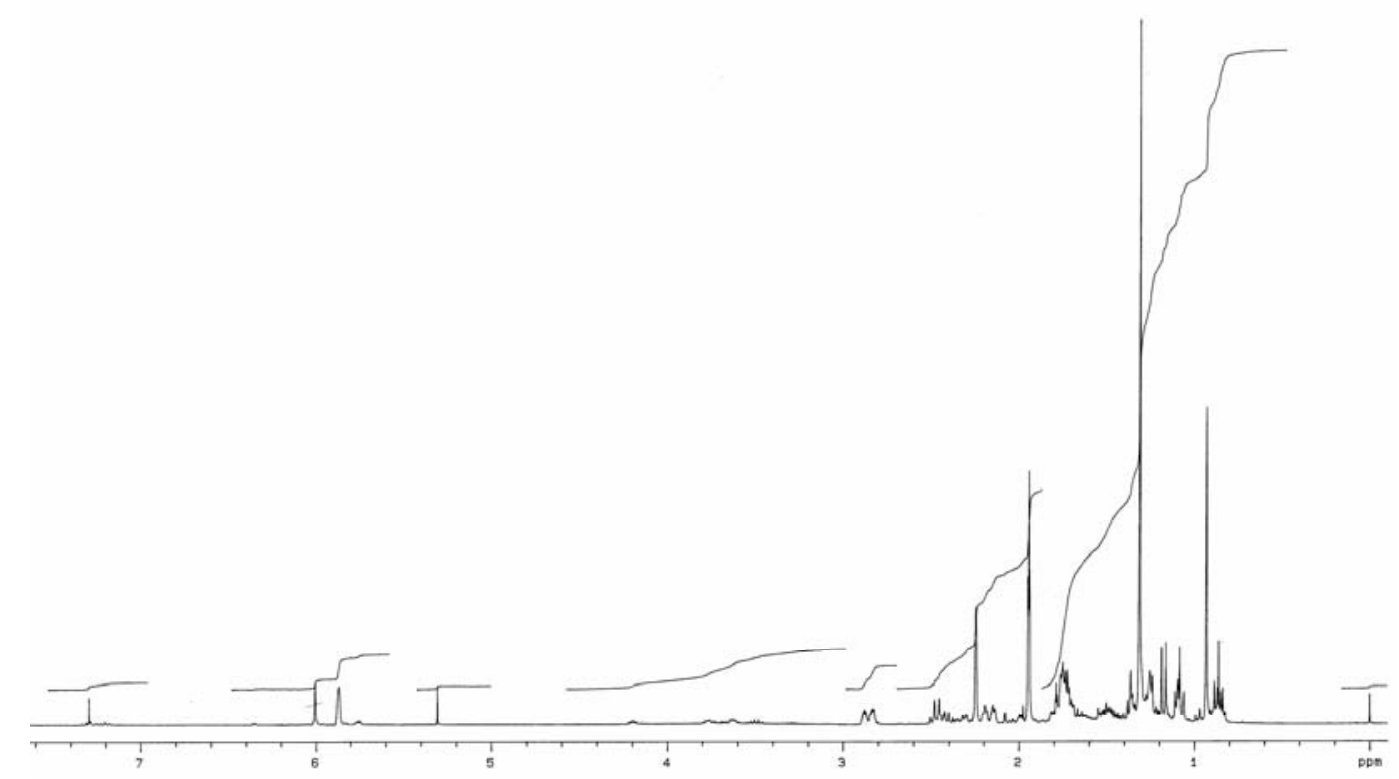

Figure 61: ${ }^{1} \mathrm{H}$ NMR spectrum $\left(300 \mathrm{MHz}, \mathrm{CDCl}_{3}\right)$ of bacteriogorgonene A (81).<smiles>CC(=O)C=C(C)C</smiles>

A<smiles>CC(C)(C)C</smiles>

B<smiles>CC(C)C</smiles>

C (4 times)

Figure 62: Substructures of bacteriogorgonene A (81).

The ${ }^{13} \mathrm{C}$ NMR spectrum revealed 15 carbon signals, among them 12 signals were observed in the $s p^{3}$ and 2 in the $s p^{2}$ region. One carbon signal at $\delta 200.1$ was the carbonyl of a ketone or aldehyde, while the carbon at $\delta 74.4$ was attached to an oxygen atom.

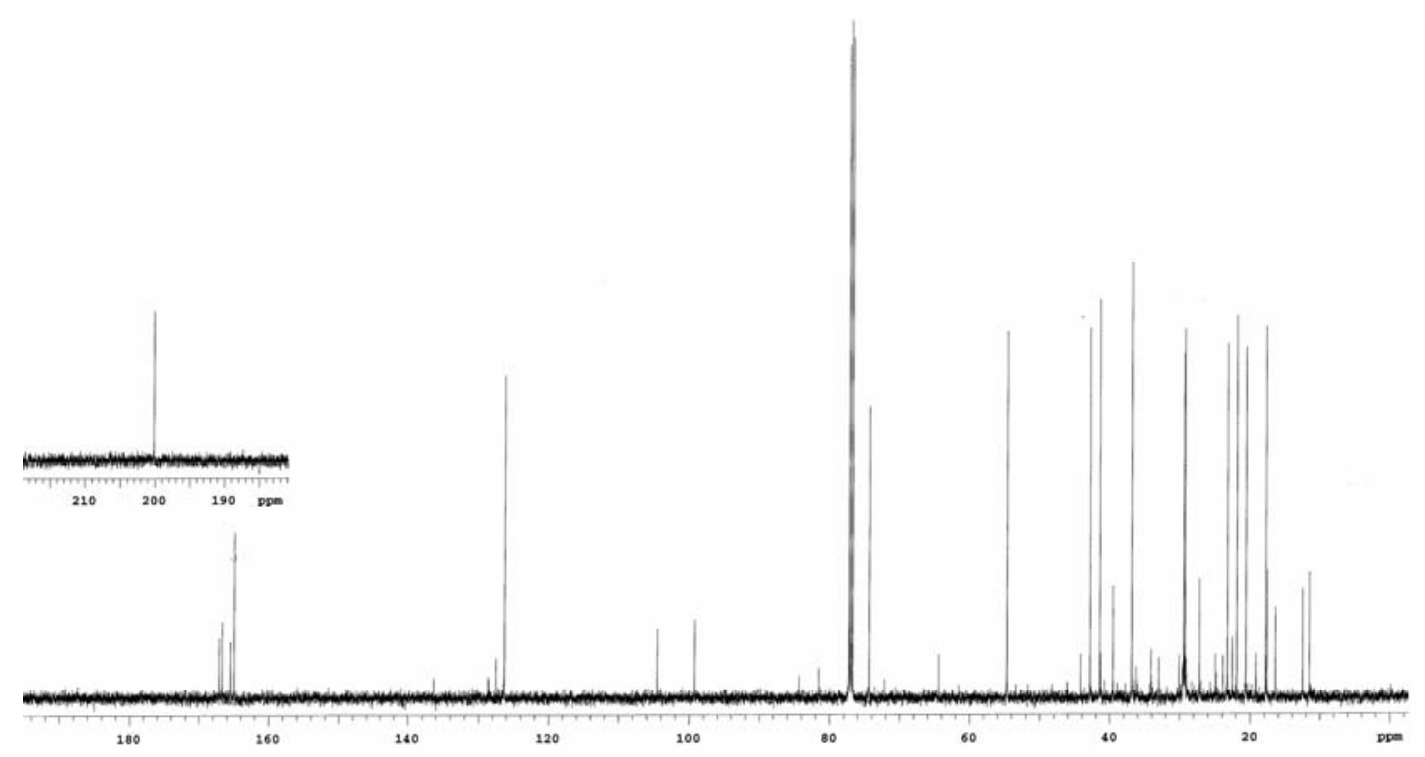

Figure 63: ${ }^{13} \mathrm{C}$ NMR spectrum $\left(\mathrm{CDCl}_{3}, 150 \mathrm{MHz}\right)$ of bacteriogorgonene $\mathrm{A}(\mathbf{8 1})$. 


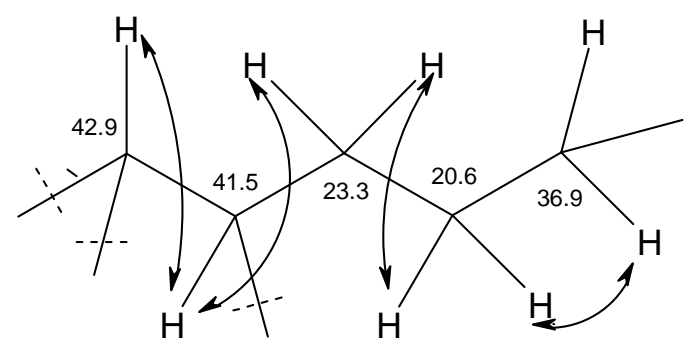

Figure 64: H-H COSY correlations of fragment D in bacteriogorgonene A (81).

In the HMBC spectrum, the proton at $\delta 5.90$ made a correlation with carbon signals at $\delta 41.5(\mathrm{CH})$ and $54.7\left(\mathrm{CH}_{2}\right)$. This proton also showed a ${ }^{2} J$ correlation with the carbonyl at $\delta 200.1$ which meant that this proton was located in $\alpha$-position to the carbonyl. The methyl group at $\delta 1.90$ showed a small correlation with the signal at $\delta$ 165.0 as well as correlations with $\delta 42.9(\mathrm{CH})$ and $\delta 126.3$. This indicated that the methyl at $\delta 1.90$ is attached to the carbon at $\delta 165.0$.

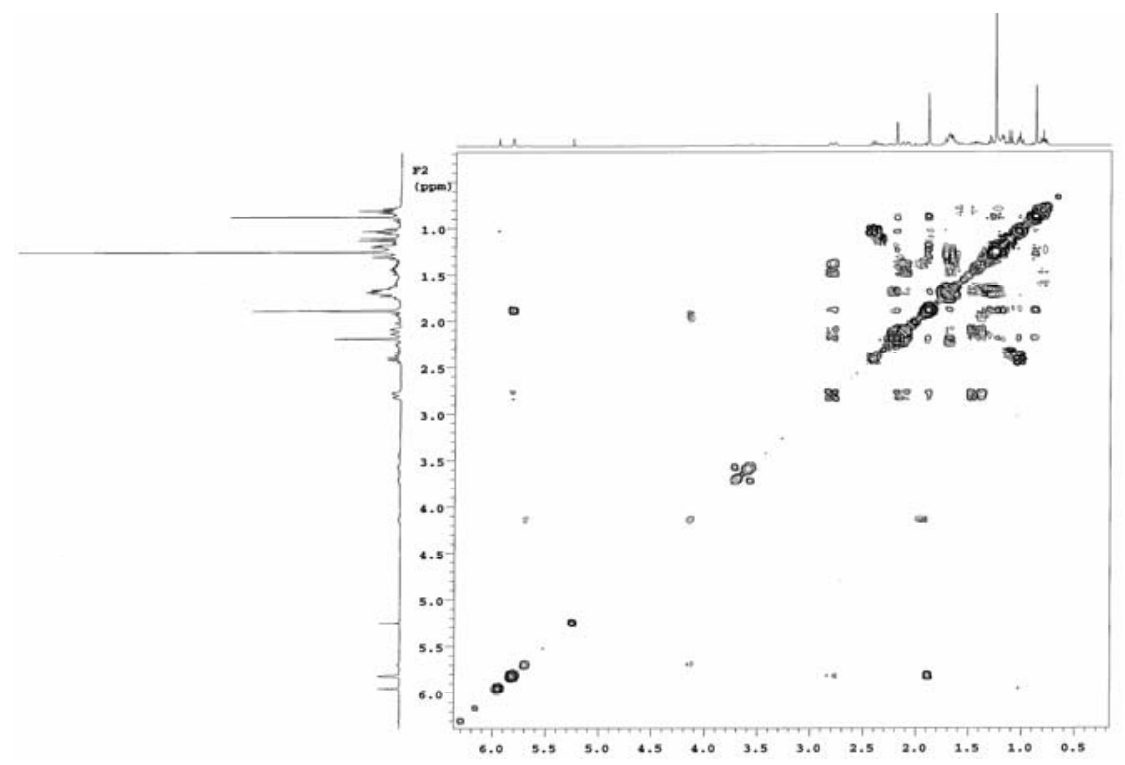

Figure 65: $\mathrm{H}-\mathrm{H}$ COSY spectrum $\left(\mathrm{CDCl}_{3}, 600 \mathrm{MHz}\right)$ of bacteriogorgonene $\mathrm{A}(\mathbf{8 1})$.

The methylene proton at $\delta 54.7$ showed many correlations to the carbon signals at $\delta$ 200.1, 126.3, 42.9, $36.9\left(\mathrm{C}_{\mathrm{q}}\right), 36.9\left(\mathrm{CH}_{2}\right)$ and $17.8\left(\mathrm{CH}_{3}\right)$. The methyl at $\delta 17.8$ made correlation with $\delta 36.9\left(\mathrm{C}_{\mathrm{q}}\right), \delta 36.9\left(\mathrm{CH}_{2}\right), 42.9$ and 54.7. Therefore, it was deduced that the methyl was directly attached to $36.9\left(\mathrm{C}_{\mathrm{q}}\right)$ as this was a singlet and $\delta 36.9$ $\left(\mathrm{CH}_{2}\right)$ and $42.9(\mathrm{CH})$ were adjacent to this quaternary carbon at $\delta 36.9$. So, a sixmembered ring is confirmed. The methyl at 21.9 also showed a correlation with $\delta 42.9$ $(\mathrm{CH})$. The two methyls at $\delta 1.25$ made correlation with each other as well as with the oxygenated quaternary carbon at $\delta 74.4$. 


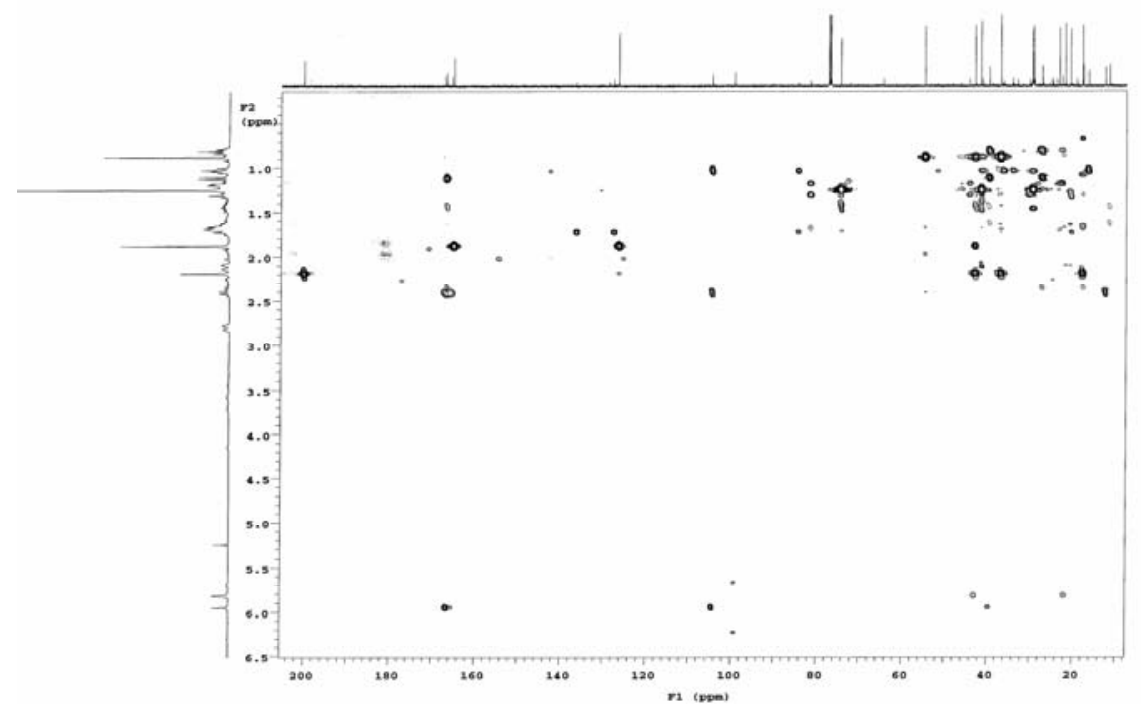

Figure 66: $\mathrm{HMBC}$ spectrum $\left(\mathrm{CDCl}_{3}, 600 \mathrm{MHz}\right)$ of bacteriogorgonene $\mathrm{A}(\mathbf{8 1})$.

The (+)-HRESIMS measurement delivered the formula $\mathrm{C}_{15} \mathrm{H}_{24} \mathrm{O}_{2}$, pointing to a sesquiterpene. By comparing the spectroscopic data with a similar structure (85), structure $\mathbf{8 1}$ was established. ${ }^{99-100}$

There were three stereo centres present at position $4 \mathrm{a}, 5$ and 8a. In the NOESY experiment of compound $\mathbf{8 1}$, the proton at position 4 a showed a correlation with the proton at position 5 , which confirmed that these two protons were on the same side. However, the methyl at position 4 a did not show any correlation with the methyl protons at position $8 \mathrm{a}$, which agrees better with the assumption that both were in a trans position than in a cis orientation.<smiles>C=C(C)[C@H]1CCC[C@@]2(C)CC(=O)C=C(C)[C@H]12</smiles> 


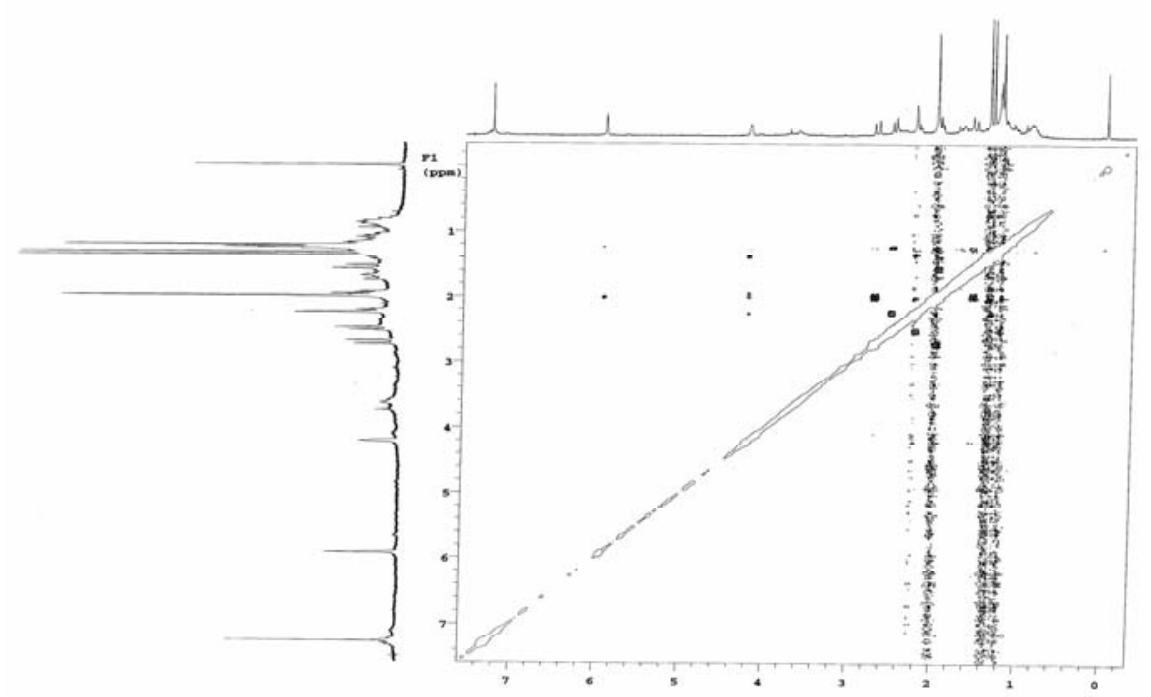

Figure 67: NOESY spectrum $\left(\mathrm{CDCl}_{3}, 300 \mathrm{MHz}\right)$ of bacteriogorgonene $\mathrm{A}(\mathbf{8 1})$.

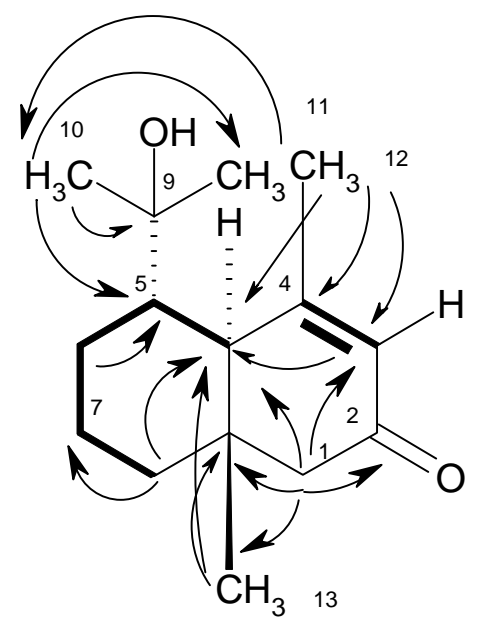

Figure 68: The H H COSY $(-)$ and $\mathrm{HMBC}(\rightarrow)$ correlations of bacteriogorgonene A $(\mathbf{8 1})$.

ESIMS showed two masses at $\mathrm{m} / \mathrm{z} 236$ and 218 Dalton, and HRMS established the molecular formula as $\mathrm{C}_{15} \mathrm{H}_{23} \mathrm{O}$ for compound 82, which was one molecule of water less than compound 81. In the ${ }^{1} \mathrm{H}$ NMR spectrum, one proton was observed at $\delta$ 5.90, together with another peak at $\delta 5.95$, where the integration was, however, not fitting; this suggested a mixture with another compound. This was established indeed by the ${ }^{13} \mathrm{C}$ NMR spectrum where two $s p^{2}$ carbon signals were detected at $\delta 99.1$ and 113.5 which were not part of compound 81. The second compound had obviously one double bond more than $\mathbf{8 1}$. Since only one hydroxyl group was present in compound $\mathbf{8 1}$ at position 9 , there were two possibilities to loose a water molecule. If the proton was taken from position 10 it would create a terminal alkene which would be less stable than that with the double bond in position 5 . Therefore, the most plausible structure for the compound with $m / z 218$ was 82 . 


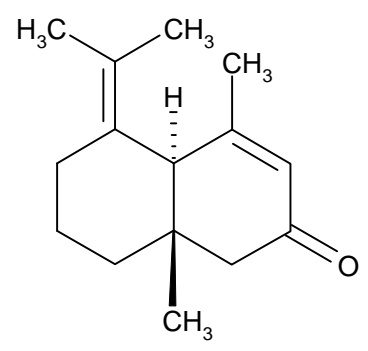

82

\subsubsection{Bacteriogorgonene $B$}

Another similar sesquiterpene was isolated from subfraction $2 b$, which was obviously closely related with the previous compounds. There was an additional oxygenated methine proton observed in the ${ }^{1} \mathrm{H}$ NMR spectrum of compound $\mathbf{8 3}$ at $\delta 4.21$, which was not visible in $\mathbf{8 1}$. In the ${ }^{13} \mathrm{C}$ NMR spectrum there were three quaternary $s p^{3}$ carbon signals appearing at $\delta 84.6,81.1$ and 67.7, whereas in compound 81, two carbon signals were observed in this region. This fact was also supported by the ${ }^{1} \mathrm{H}$ NMR spectrum, which showed an additional methine attached to oxygen. HRESIMS established the molecular formula as $\mathrm{C}_{15} \mathrm{H}_{22} \mathrm{O}_{3}$.

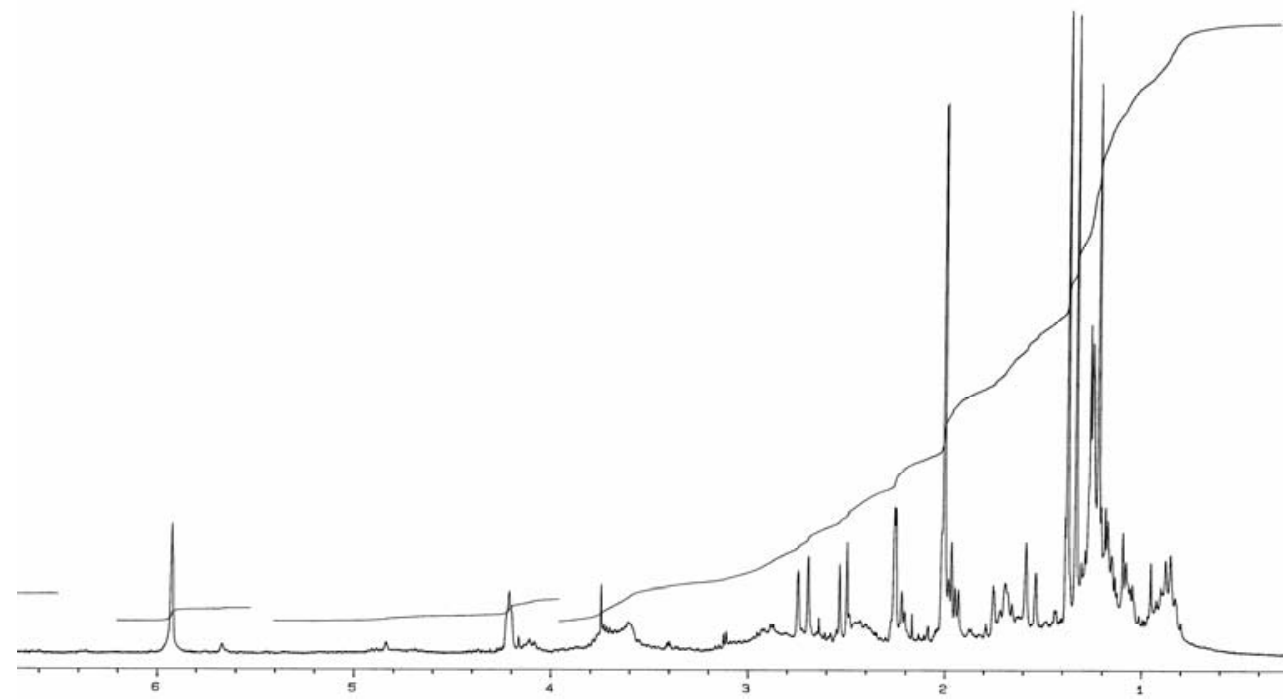

Figure 69: ${ }^{1} \mathrm{H}$ NMR spectrum $\left(\mathrm{CDCl}_{3}, 300 \mathrm{MHz}\right)$ of bacteriogorgonene $\mathrm{B}(\mathbf{8 3})$.

The methine carbon signal at $\delta 42.9$ in compound $\mathbf{8 1}$ was replaced by a quaternary carbon at 84.6. At $\delta 67.7$ there was another quaternary $s p^{3}$ carbon signal observed which could also be connected with oxygen. In compound 81 there were 4 double bonds equivalents whereas the molecular formula $\mathrm{C}_{15} \mathrm{H}_{22} \mathrm{O}_{3}$ of compound $\mathbf{8 3}$ showed 
five equivalents. Therefore, one oxygen atom was attached to both of the carbon atoms at $\delta 84.6$ and 67.7. One carbonyl at $\delta 199.3$ and an oxygenated methine at $\delta 81.1$ were also present in ${ }^{13} \mathrm{C}$ NMR spectrum of $\mathbf{8 3}$ which were also observed in the similar area in compound $\mathbf{8 1}$.

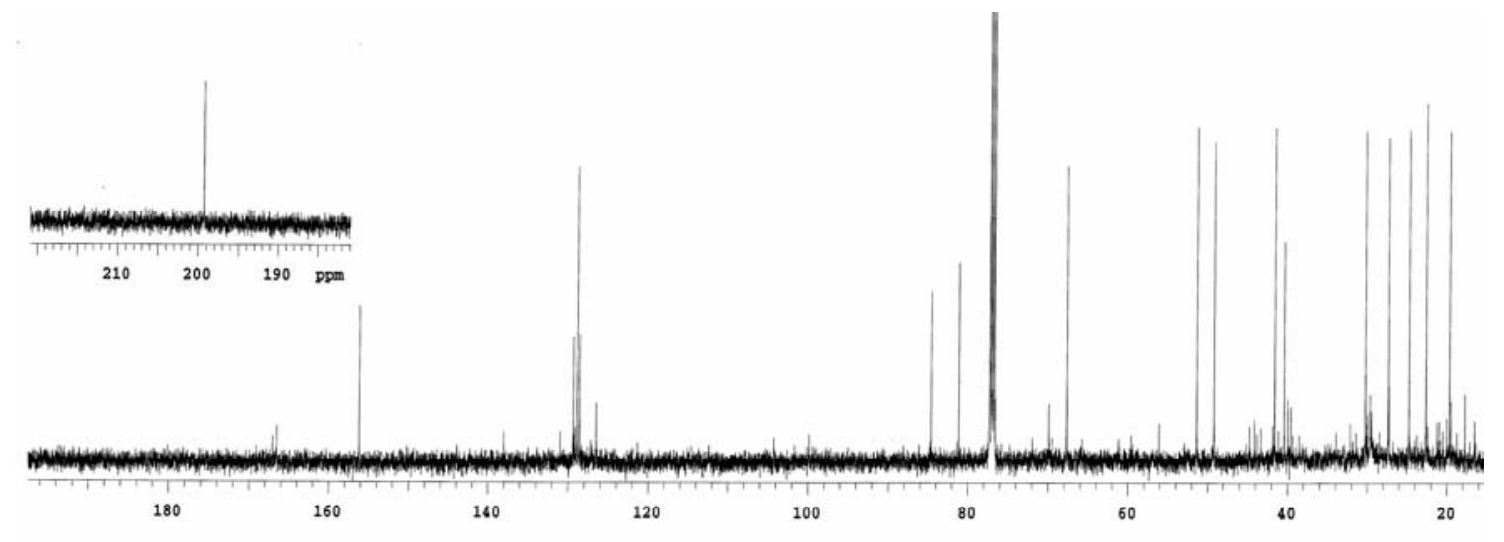

Figure 70: ${ }^{13} \mathrm{C} \mathrm{NMR}\left(\mathrm{CDCl}_{3}, 125 \mathrm{MHz}\right)$ spectrum of bacteriogorgonene $\mathrm{B}(\mathbf{8 3})$.

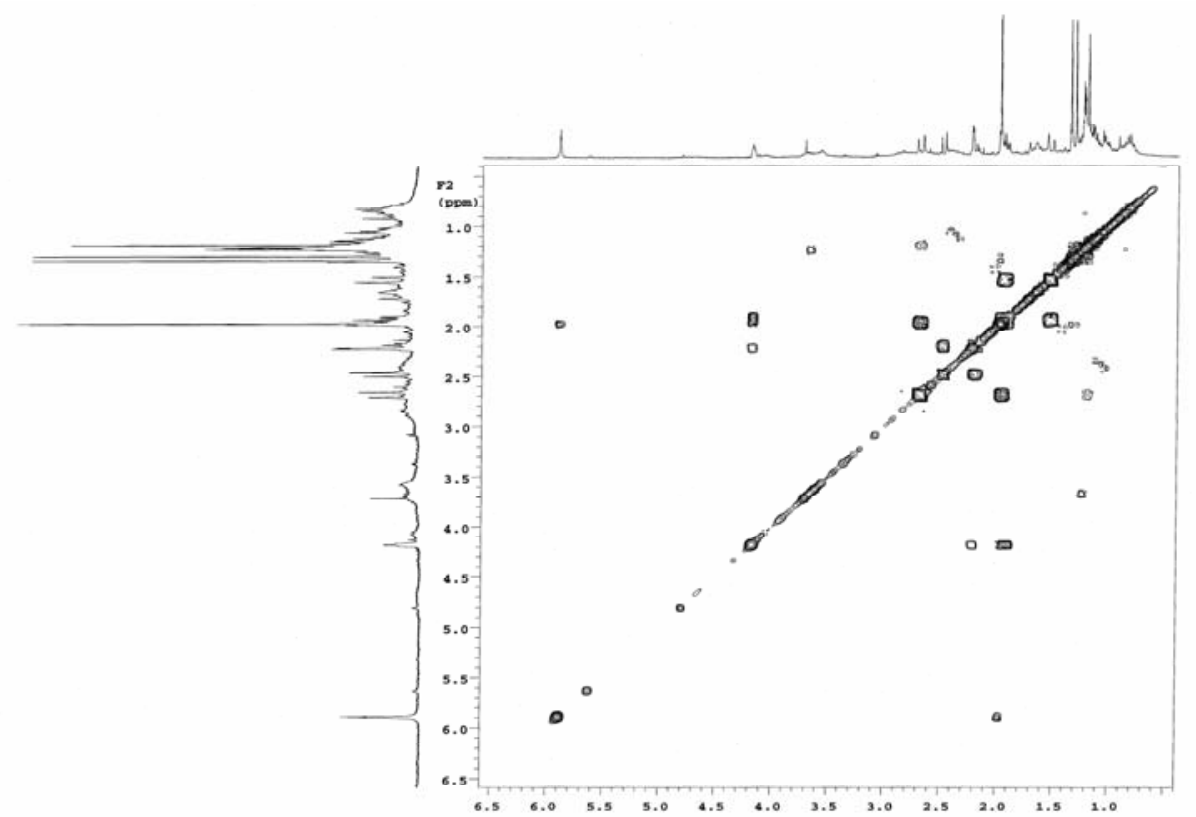

Figure 71: $\mathrm{H}-\mathrm{H}$ COSY spectrum $\left(\mathrm{CDCl}_{3}, 600 \mathrm{MHz}\right)$ of bacteriogorgonene $\mathrm{B}(\mathbf{8 3})$.

In the HMBC spectrum, the proton attached at carbon 6 showed a ${ }^{3} J$ coupling with the carbon at $\delta$ 84.6. This proton also showed a COSY correlation with the methylene attached with carbon 7 in compound $\mathbf{8 3}$, while the methylene at carbon 7 showed correlations with carbon $4 \mathrm{a}, 9$ and 11 . These correlations were similar to those observed in compound $\mathbf{8 1}$. 


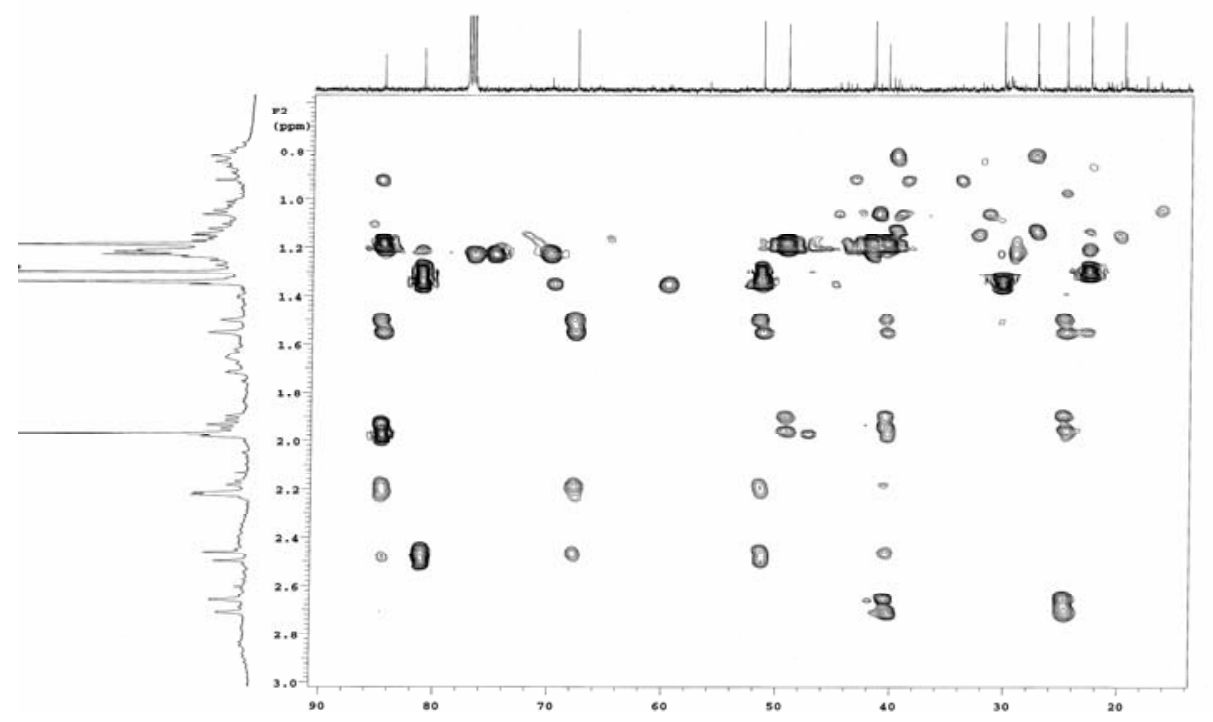

Figure 72: $\mathrm{HMBC}$ spectrum $\left(\mathrm{CDCl}_{3}, 600 \mathrm{MHz}\right)$ of bacteriogorgonene $\mathrm{B}(\mathbf{8 3})$.

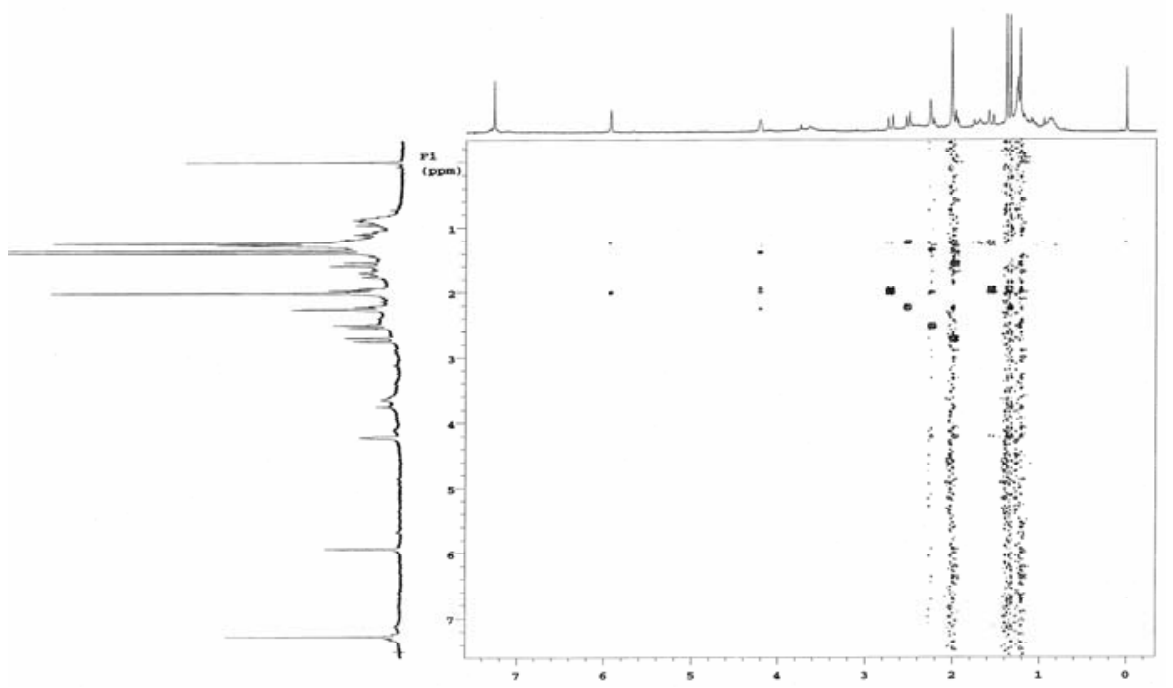

Figure 73: NOESY spectrum $\left(\mathrm{CDCl}_{3}, 300 \mathrm{MHz}\right)$ of bacteriogorgonene $\mathrm{B}(\mathbf{8 3})$.

With the help of 1D and 2D NMR as well as the correlation of $\mathbf{8 1}$, structure $\mathbf{8 3}$ was established. 


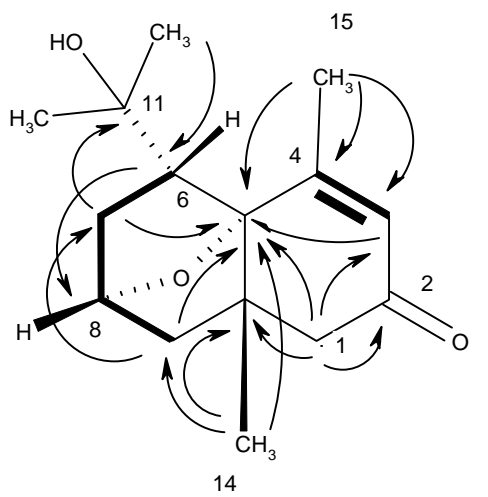

Figure 74: HMBC correlations of bacteriogorgonene B $(\mathbf{8 3})$.

\subsubsection{Bacteriogorgonene $\mathrm{C}$}

Compound 84 was isolated as a colourless oil and separated from the same fraction $2 \mathrm{D}$ as 83 . The mass was determined by CIMS as $m / z, 270\left[\mathrm{M}+\mathrm{NH}_{4}\right]^{+}$. The ${ }^{1} \mathrm{H}$ NMR spectrum had similarity to the other three sesquiterpenes, which already have been described. However, no oxymethine was observed in compound 84. HRESIMS delivered the molecular formula $\mathrm{C}_{15} \mathrm{H}_{24} \mathrm{O}_{3}$.

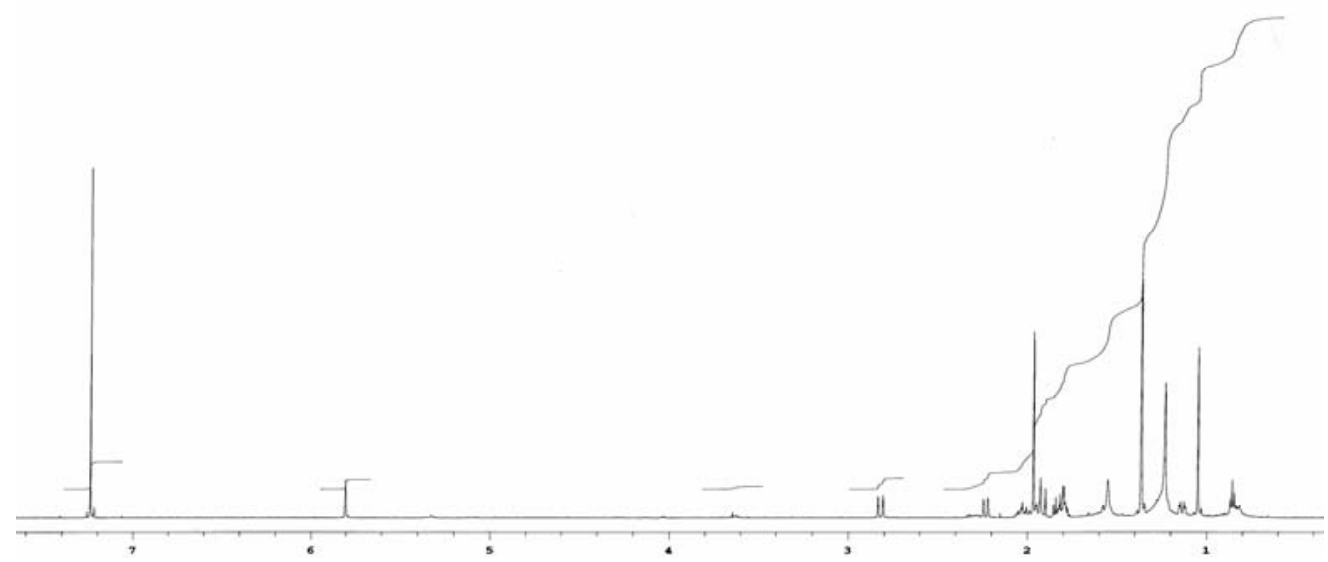

Figure 75: ${ }^{1} \mathrm{H}$ NMR spectrum $\left(\mathrm{CDCl}_{3}, 300 \mathrm{MHz}\right)$ of bacteriogorgonene $\mathrm{C}(\mathbf{8 4})$.

This compound presented one more oxygen atom than the compound 81. The ${ }^{1} \mathrm{H}$ NMR spectrum showed no proton signals in the region between $\delta 2.90-5.90$ as in compound 82. The ${ }^{13} \mathrm{C}$ NMR spectrum showed a carbon signal at $\delta 82.2$ along with the quaternary carbon at $\delta 74.4$ as in compound $\mathbf{8 1}$. 


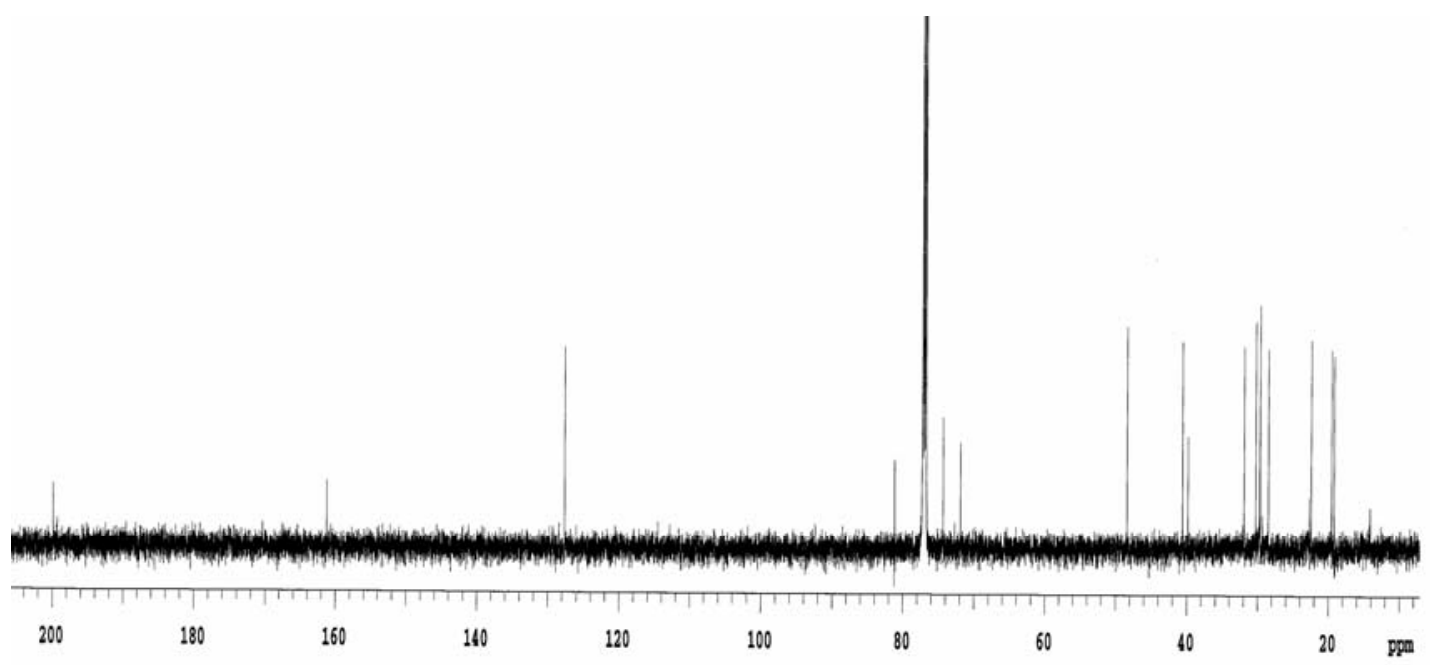

Figure 76: ${ }^{13} \mathrm{C}$ NMR spectrum $\left(\mathrm{CDCl}_{3}, 125 \mathrm{MHz}\right)$ of bacteriogorgonene $\mathrm{C}(\mathbf{8 4})$.

H-H COSY spectrum showed similar correlations as found in compound 81, but in compound $\mathbf{8 4}, \mathrm{C}-4 \mathrm{a}$ was a quaternary carbon, while it was a methine carbon in compound $\mathbf{8 1}$.

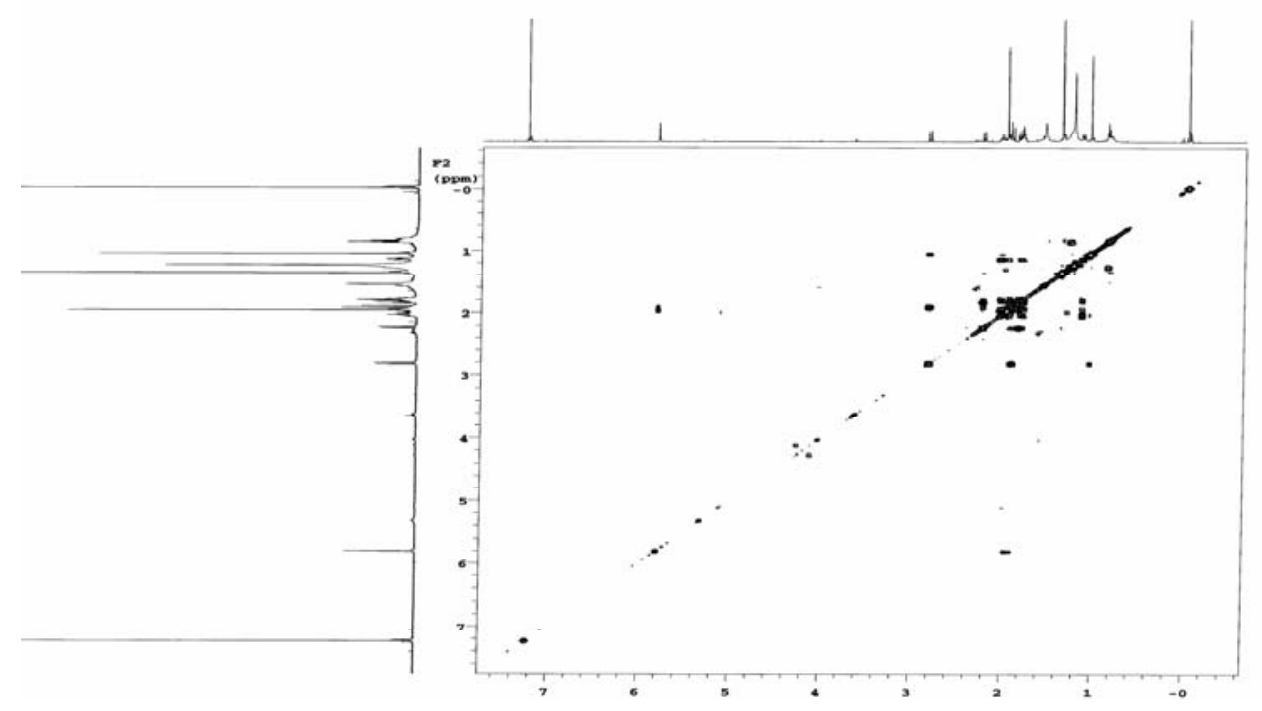

Figure 77: $\mathrm{H}-\mathrm{H}$ COSY spectrum $\left(\mathrm{CDCl}_{3}, 600 \mathrm{MHz}\right)$ of bacteriogorgonene $\mathrm{C}(\mathbf{8 4})$.

In the HMBC spectrum the methyl at $\delta 1.90$ showed a ${ }^{3} J$ coupling with the oxygenated quaternary carbon at $\delta$ 82.2. The other HMBC correlations were similar to compound 81. With the help of $1 \mathrm{D}$ and $2 \mathrm{D}$ spectra the structure 84 was elucidated. ${ }^{101-102}$ 


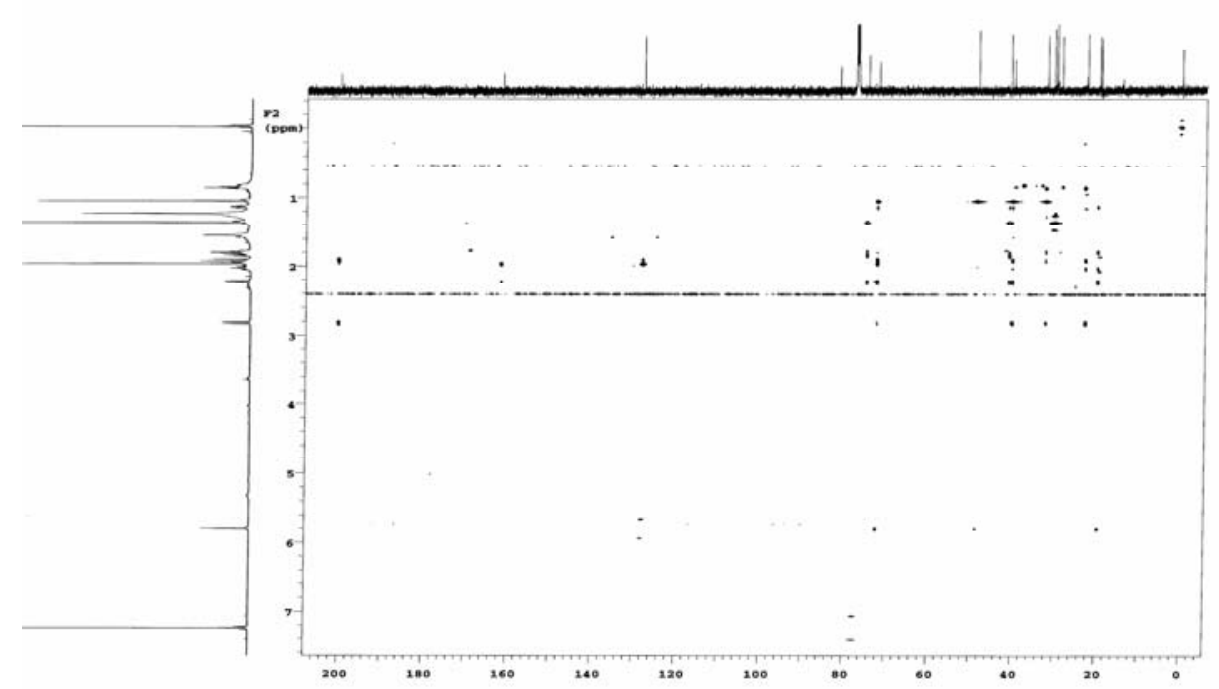

Figure 78: $\mathrm{HMBC}$ spectrum $\left(\mathrm{CDCl}_{3}, 600 \mathrm{MHz}\right)$ of bacteriogorgonene $\mathrm{C}(\mathbf{8 4})$.

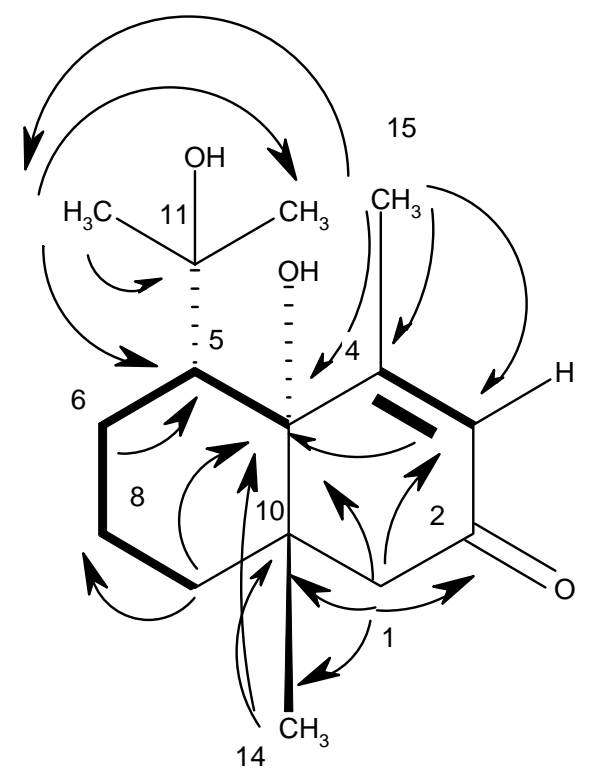

Figure 79: HMBC correlation of bacteriogorgonene C (84).

Gorgonene terpenes are a rare class of sesquiterpenes in comparison to other terpenes such as selinane, cadinane, calamane, eudesmane, amorphane etc. Gorgonene terpenes possess an isopropyl group in the unusual C-6 position and are apparently biosynthesised from two tail-to-tail fused isoprene units. The few known gorgonene terpenes were isolated from plant sources. This is the first report of bacteria producing gorgonene sesquiterpenes.

During the last two decades, sesquiterpenoids and their biological activities have been the focus of numerous phytochemical, pharmacological and synthetic studies. ${ }^{103-104}$ Because sesquiterpenes exhibit a wide range of biological activities, such as plant growth regulators, insect antifeedant, antifungal, anti-tumour and antibacterial activities, there has been much interest in relating structure and the relation between oxy- 
genation pattern and function ${ }^{105}$. Sesquiterpenes are common metabolites from plant or fungi, and only a few have been isolated from microorganism.

Fraction 3 and 4 constituted the major portion of this strain, which consisted of homononactic acid (86) and nonactic acid (87). Therefore, it could be concluded that the high biological activity of this strain was due to homononactic acid and its derivatives.

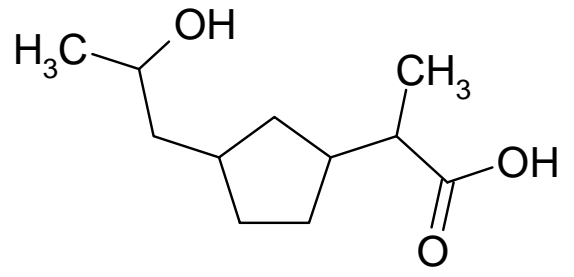

86

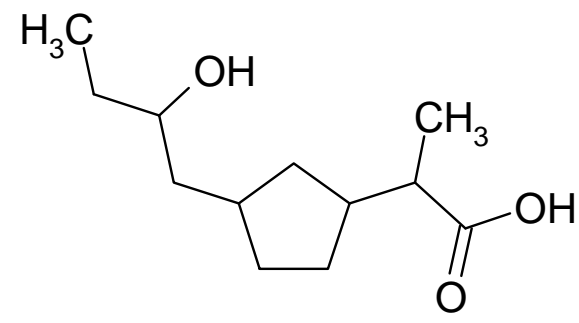

87

\subsection{Terrestrial Streptomycete GW5127}

This strain was cultivated on $\mathrm{M}_{2}$ medium using tap water. A 25 litre shaker culture of the terrestrial streptomycete strain GW5127 was to incubate a $28{ }^{\circ} \mathrm{C}$ using $\mathrm{M}_{2}$ medium. The fermentor broth was harvested after 8 days, mixed with Celite, and then filtered. The filtrate and mycelia were subjected to extraction separately using XAD-2 for the water phase, followed by elution with $\mathrm{MeOH} / \mathrm{H}_{2} \mathrm{O}$. The aqueous methanolic extract was concentrated and the water residue was again extracted with ethyl acetate. The mycelium was extracted with ethyl acetate (3 times). The extracts yielded three new metabolites, i.e. 9-ethyl-6,11-dihydroxy-4-methoxy-naphthacene-5,12-dione (88), 4-acetyl-1,3-dihydro-imidazo[4,5-b]pyridin-2-one (90), and $\mathrm{N}$-[2-(3-hydroxy-4methoxy-phenyl)-ethyl]-acetamide (93) along with three known compounds, 4-hydroxy-3-methoxy-tyrosol (94), $\beta$-rubromycin (95) and $\gamma$-rubromycin (96).

Silica gel column chromatography of the mycelial fraction followed by PTLC and size exclusion chromatography yielded $\beta$-rubromycin and $\gamma$-rubromycin as red solids. The extract from the water phase was separated into four fractions on silica gel. PTLC of fraction II followed by Sephadex LH-20 delivered compound $\mathbf{8 8}$ as a red solid. The middle polar fraction III (0.25 g) yielded on Sephadex LH-20 compound 90 and 3-hydroxy-4-methoxy- $\beta$-phenylethyl alcohol (94) as colourless solids. The polar fraction IV yielded on Sephadex LH-20 N-[2-(3-hydroxy-4-methoxy-phenyl)-ethyl]acetamide (93) as colourless oil. 


\subsubsection{3-Deoxy-1,3-dihydrodaunomycinone}

EIMS of compound $\mathbf{8 8}$ showed a molecular ion at $m / z$ 348, and HREIMS revealed the molecular formula as $\mathrm{C}_{21} \mathrm{H}_{16} \mathrm{O}_{5}$ corresponding to 14 double bond equivalents. The UV spectra $(\mathrm{MeOH})$ of $\mathbf{8 8}$ displayed four strong bands at $\lambda_{\max } 249,310,542$ and $577 \mathrm{~nm}$ in neutral solution. Under basic methanol conditions, the latter band showed a bathochromic shift to $\lambda_{\max } 621 \mathrm{~nm}$.

The ${ }^{1} \mathrm{H}$ NMR spectrum of $\mathbf{8 8}$ exhibited two singlets of chelated hydroxyl groups at $\delta$ 16.12 and 15.30. In the aromatic region, two spin systems were observed, the signals of a 1,2,3-trisubstituted benzene ring at $\delta 8.13,7.74$ and 7.29, and a second pattern of a 1,2,4-trisubstituted aromatic system at $\delta 8.39,7.63$, and 8.26. In the aliphatic region, the signals of a methoxy group $(\delta 4.08)$ and an ethyl group $(\delta 2.86, \mathrm{q} ; 1.38, \mathrm{t})$ were also detected.

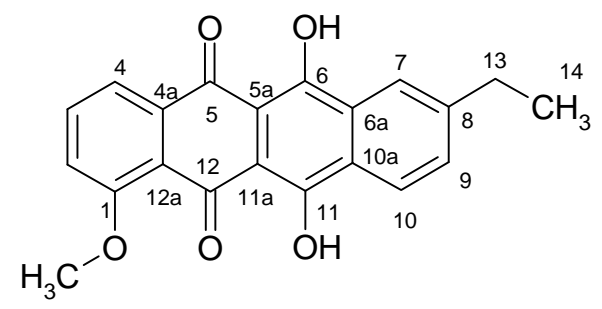

87
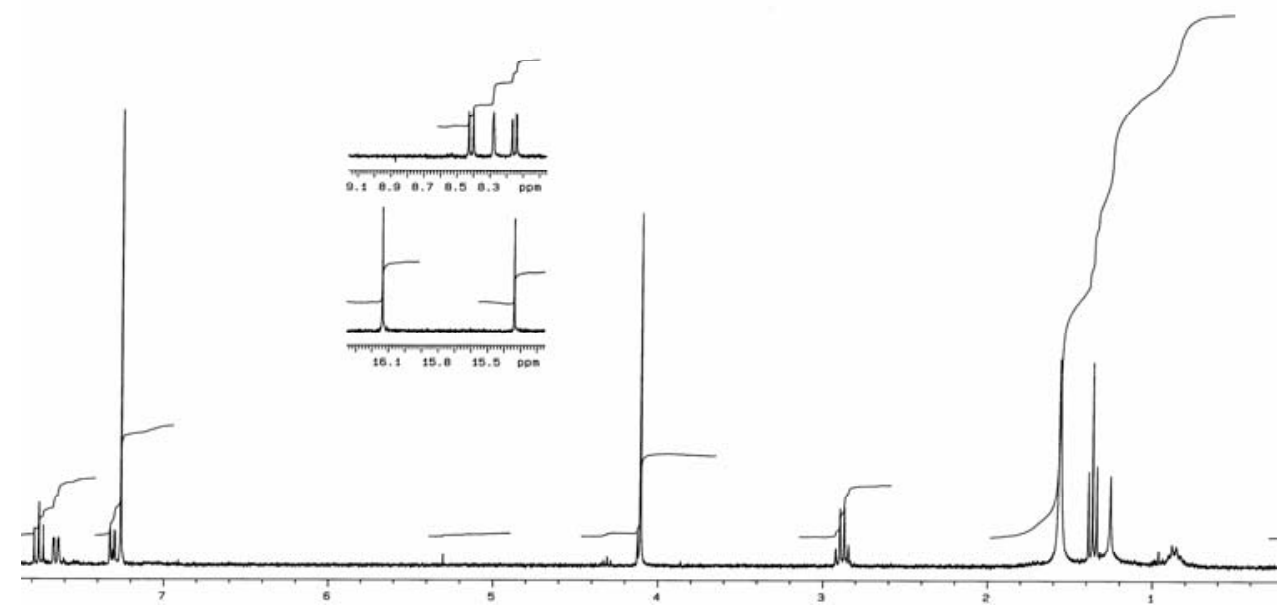

Figure 80: ${ }^{1} \mathrm{H} \mathrm{NMR}$ spectrum $\left(\mathrm{CDCl}_{3}, 300 \mathrm{MHz}\right)$ of 13-deoxy-1,3-dihydrodaunomycinone $(\mathbf{8 8})$.

The ${ }^{13} \mathrm{C} / \mathrm{APT}$ experiment afforded 21 carbon signals, which were classified into 12 quaternary carbons, 6 methines, 1 methylene and 2 methyl carbons. These data were comparable with those reported for a tetracycline, 1,3-dihydrodaunomycinone $(\mathbf{8 8})^{9-10}$ with only one exceptions. In the reference compound, C-13 contained a hydroxyl 
group, which was missing in compound $\mathbf{8 8}$ and therefore, the combination of C-13 and $\mathrm{C}-14$ composed an ethyl group.

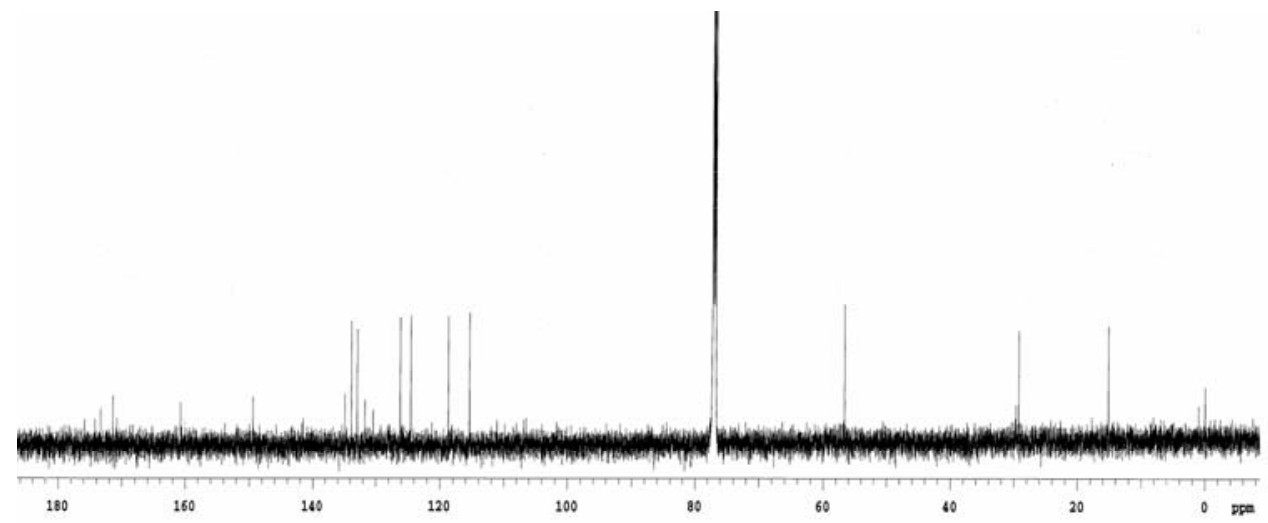

Figure 81: ${ }^{13} \mathrm{C}$ NMR spectrum $\left(\mathrm{CDCl}_{3}, 300 \mathrm{MHz}\right)$ of 13-deoxy-1,3-hydrodaunomycinone $(\mathbf{8 8})$.

The downfield shift of the carbon and HMBC couplings with aromatic carbon signals indicated the direct linkage to an aromatic system.

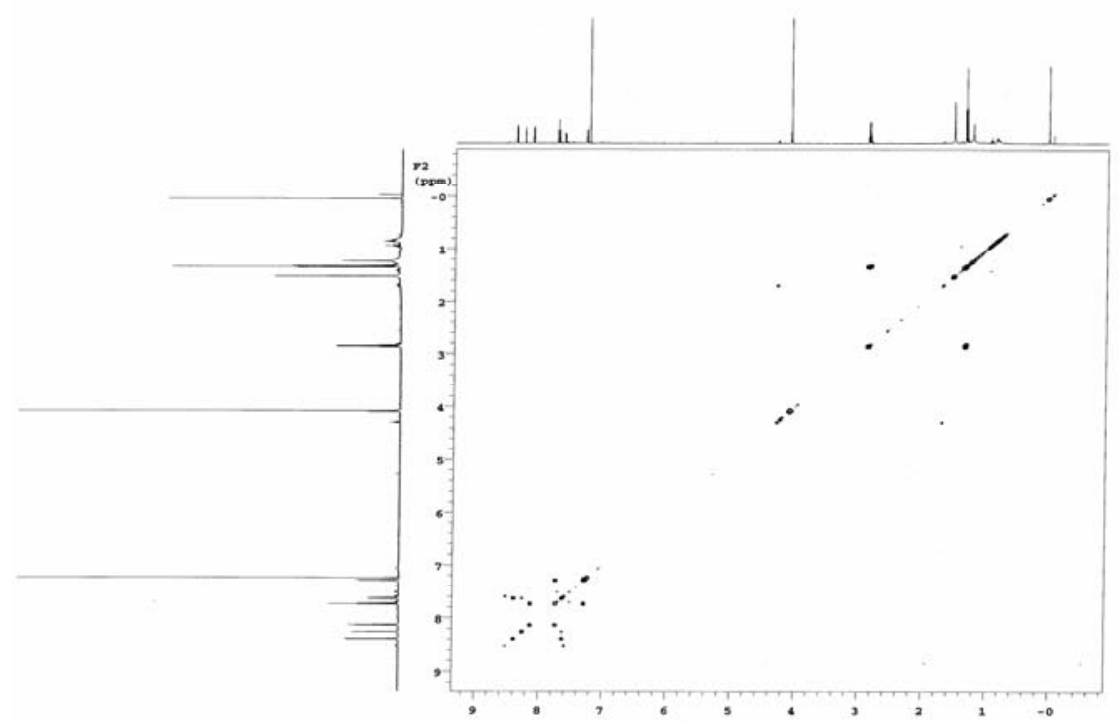

Figure 82: $\mathrm{H}-\mathrm{H}$ COSY spectrum $\left(\mathrm{CDCl}_{3}, 500 \mathrm{MHz}\right)$ of 13-deoxy-1,3-dihydrodaunomycinone (88).

These data and the ${ }^{13} \mathrm{C}$ NMR spectrum indicated clearly that $\mathbf{8 8}$ was a homologue of 6,11-dihydroxy-4-methoxy-9-methyl-naphthacene-5,12-dione (89), a quinone isolated recently from Streptomyces sp. GW10/1811. Structure $\mathbf{8 8}$ was confirmed by further HMBC data. A number of similar compounds have been reported from bacterial sources, and some of which are reported to show potent antitumor and antibiotic activities. Tetracenequinones can be formed as artefacts from corresponding anthracyclines, which are dehydrated easily during work-up, and so both types are often iso- 
lated together. It is worth to mention therefore, that neither feudomycines, nor feudomycinones or other anthracyclines were detectable here.<smiles>COc1cccc2c1C(=O)c1c(c(O)c3ccc(C)cc3c1O)C2=O</smiles>

89

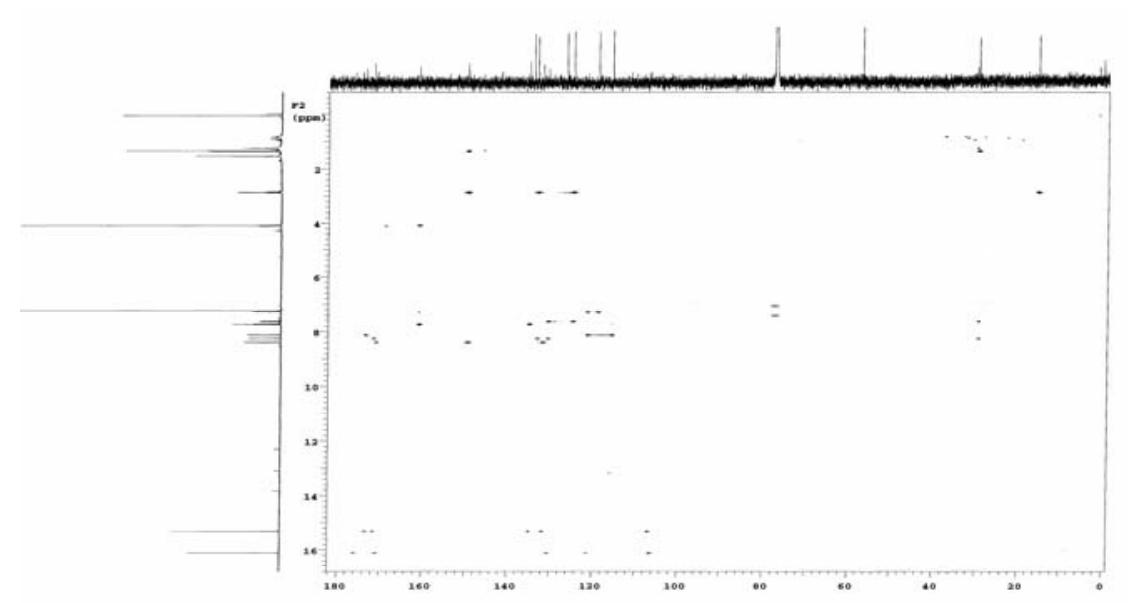

Figure 83: $\mathrm{HMBC}$ spectrum $\left(\mathrm{CDCl}_{3}, 500 \mathrm{MHz}\right)$ of 13-deoxy-1,3-dihydrodaunomycinone $(\mathbf{8 8})$

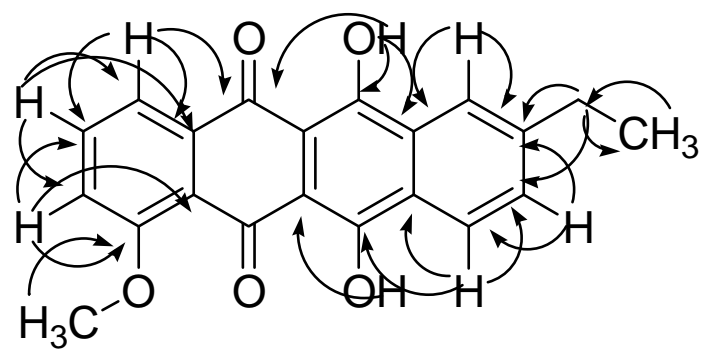

Figure 84: The HMBC correlations of 13-deoxy-1,3-dihydrodaunomycinone (88).

\subsubsection{4-Acetyl-1,3-dihydro-imidazo[4,5-b]pyridin-2-one}

The UV spectra of a second compound $\mathrm{C}_{8} \mathrm{H}_{7} \mathrm{~N}_{3} \mathrm{O}_{2}$ (HREIMS) showed two maxima at 262 and $316 \mathrm{~nm}$ of an aromatic system. The ${ }^{1} \mathrm{H}$ NMR spectrum displayed only three proton signals at $\delta 8.26(\mathrm{~d}, J=5.1 \mathrm{~Hz}, 1 \mathrm{H}), 7.23(\mathrm{~d}, J=5.1 \mathrm{~Hz}, 1 \mathrm{H})$ and $\delta 2.68(\mathrm{~s}$, $\mathrm{Me})$. 


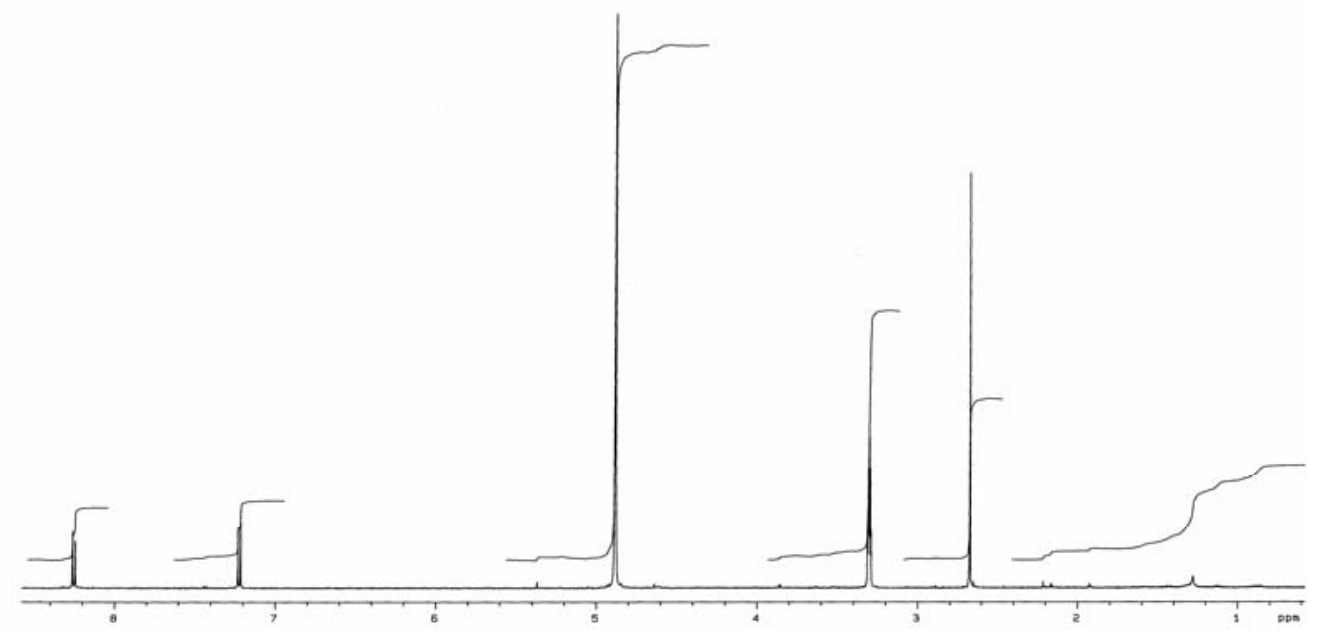

Figure 85: ${ }^{1} \mathrm{H}$ NMR $\left(\mathrm{CD}_{3} \mathrm{OD}, 300 \mathrm{MHz}\right)$ spectrum of 4-acetyl-1,3-dihydroimidazo[4,5-b]pyridin-2-one (90).

In the ${ }^{13} \mathrm{C}$ NMR spectrum, two aromatic methine signals were found at $\delta 142.7$ and 108.8; a methyl at $\delta 25.9$ and 5 quaternary carbon signals appeared at $\delta$ 201.4, 157.6, 139.6, 135.0, and 128.5.

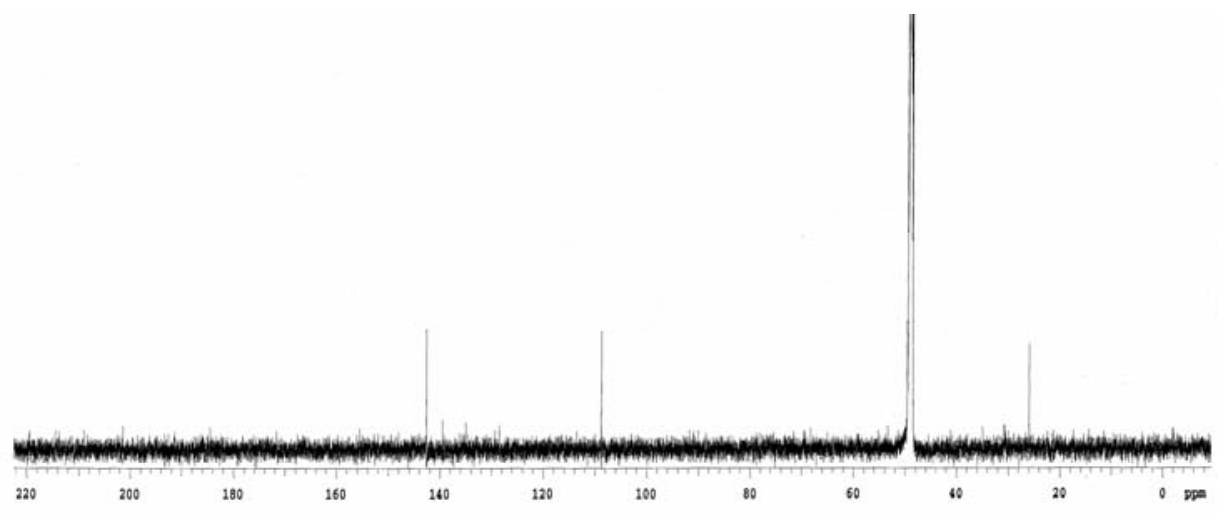

Figure 86: ${ }^{13} \mathrm{C}$ NMR $\left(\mathrm{CD}_{3} \mathrm{OD}, 125 \mathrm{MHz}\right)$ spectrum of 4-acetyl-1,3-dihydroimidazo[4,5-b]pyridin-2-one (90).

The presence of a benzene ring was ruled out on the basis of the low coupling constants $(5.1 \mathrm{~Hz})$ of two methine doublets; also because of the downfield shifts, a pyridine system was more plausible. An acetyl moiety was established on the basis of the methyl singlet in the ${ }^{1} \mathrm{H}$ NMR spectrum and the corresponding carbon signals at $\delta$ 25.9 as well as a carbonyl signal at $\delta 201.4$. The shift of the remaining carbon atom $(\delta$ 157.6) suggested a carbonyl group, which would form a 1,3-dihydro-imidazo[4,5c]pyridin-2-one (90) or a 1,3-dihydro-imidazo[4,5-b]pyridin-2-one (91) unit with the residual two $\mathrm{NH}$ fragments and the pyridine ring. 


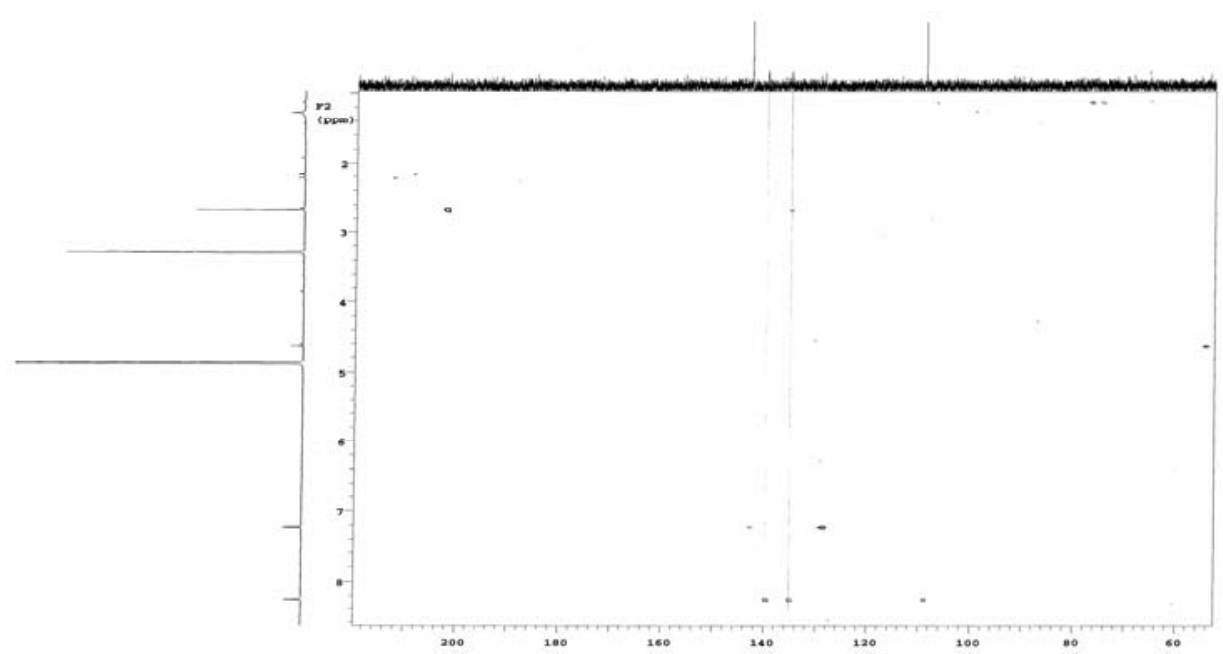

Figure 87: $\mathrm{HMBC}$ spectrum $\left(\mathrm{CD}_{3} \mathrm{OD}, 600 \mathrm{MHz}\right)$ of 4-acetyl-1,3-dihydroimidazo[4,5b]pyridin-2-one $(\mathbf{9 0})$.

Both alternatives explained the $\mathrm{CO}$ shift and also the additional $\mathrm{IR}_{\mathrm{CO}}$ signals at 1685 ( $\alpha, \beta$-unsaturated $\mathrm{CO}$ ) and $1619 \mathrm{~cm}^{-1}(\mathrm{NH}-\mathrm{CO}-\mathrm{NH})$. The data are comparable to the values of urea, which gives intense absorption bands at $v \sim 1680$ and $1620 \mathrm{~cm}^{-1}$.<smiles>CC(=O)c1nccc2[nH]c(=O)[nH]c12</smiles>

90<smiles>CC(=O)c1ccnc2[nH]c(=O)[nH]c12</smiles>

91

One of the two $o$-coupling protons was connected with a downfield-shifted carbon atom $(\delta 8.26, \mathrm{~d}, J=5.1 \mathrm{~Hz})$ and must therefore be placed in $\alpha$-position with respect to the pyridine nitrogen. This suggested that the acetyl group must be attached to C- 4 or to the other $\alpha$-position. The resulting isomers 90/91 were, however, indistinguishable on the basis of spectroscopic data, and only an indirect argument was favouring a 2acetyl-pyridine, that is the missing HMBC coupling between the aromatic protons and the acetyl-CO signal. The structure was confirmed on the basis of syntheses, which finally excluded structure $\mathbf{9 1}$. Compound $\mathbf{9 0}$ was later obtained also from the marine Streptomyces isolate B7967. ${ }^{106}$ 


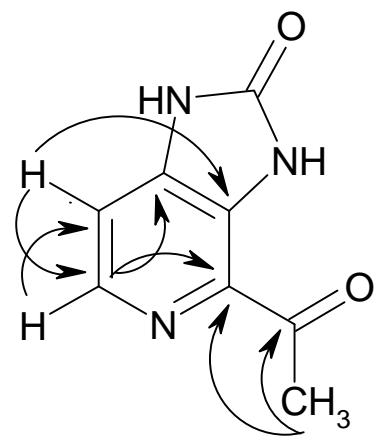

Figure 88: The HMBC correlations of 90.

A few simple dihydroimidazolones have been found linked to sugar moieties, e.g. in ureidobalhimycin, a glycopeptide antibiotic. Anellated imidazolones are rare in nature, and not more than $c a$. 40 derivatives have been reported so far, well-known purine derivatives like uric acid or guanin-8-ones being the predominant examples. The closest similarity with $\mathbf{9 0}$ is shown by the azobactin chromophor $\mathbf{9 2}$.<smiles>NC(=O)C1CCn2c(=O)[nH]c3cc4cc(O)c(O)cc4[n+]1c32</smiles>

92

Many imidazolones exhibit activity against gram-positive and gram-negative bacteria, are herbicidal or act as biotin antimetabolites and are antifungal agents. In our tests, 90 was inactive against microorganisms (Staphylococcus aureus, Escherichia coli, Bacillus subtilis, Streptomyces viridochromogenes, Candida albicans, Mucor miehei), and showed no cytotoxicity (brine shrimp test).

\subsubsection{N-[2-(3-Hydroxy-4-methoxy-phenyl)-ethyl]-acetamide}

The ${ }^{1} \mathrm{H}$ NMR data of compound 93 resembled those of $\mathrm{N}$-acetyl-tyramine and showed broad singlets of $\mathrm{D}_{2} \mathrm{O}$-exchangeable protons at $\delta 6.16(\mathrm{OH})$ and $5.75(\mathrm{NH})$, an acetyl group and an ethanediyl fragment. The signal pattern of a 1,2,4-trisubstituted benzene ring and an additional methoxy signal suggested 3-methoxy-N-acetyltyramine or the isomeric $N$-[2-(3-hydroxy-4-methoxy-phenyl)-ethyl]-acetamide (93), in accordance with the molecular formula by EIHRMS. 


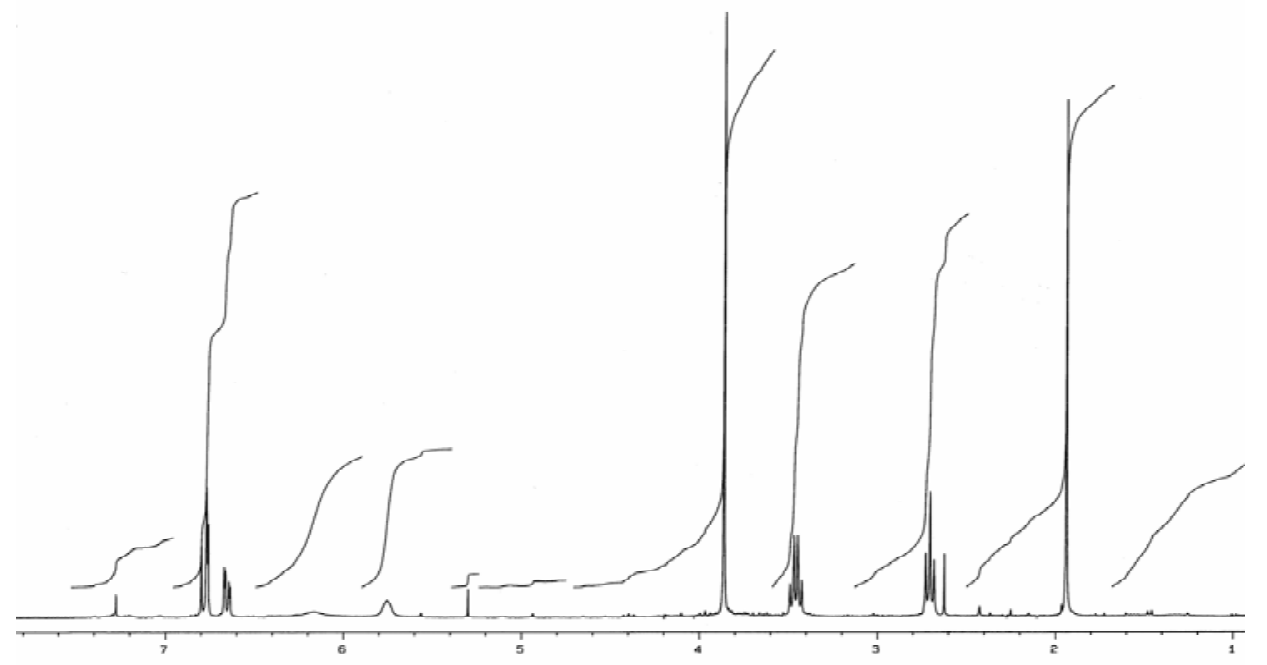

Figure 89: ${ }^{1} \mathrm{H}$ NMR $\left(\mathrm{CD}_{3} \mathrm{OD}, 300 \mathrm{MHz}\right)$ spectrum of $3 \mathrm{~N}$-[2-(3-hydroxy-4-methoxyphenyl)-ethyl]-acetamide (93).

The ${ }^{13}$ C/APT NMR spectrum of 93 afforded carbon signals which were identified as four quaternary carbons, three methines, 2 methylenes and two methyls.

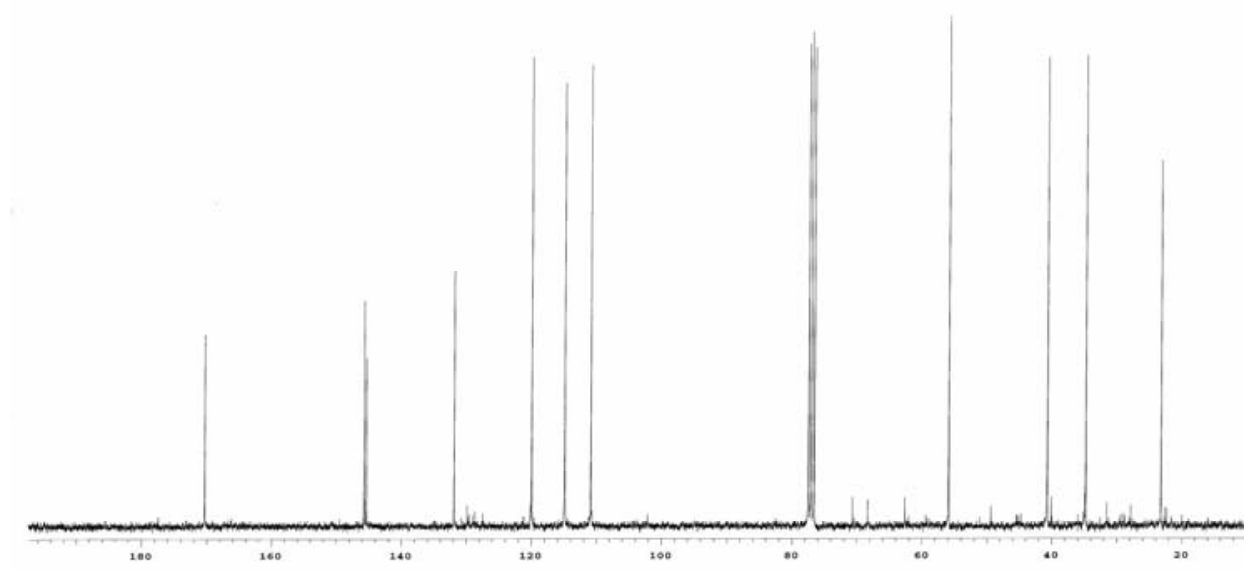

Figure 90: ${ }^{13} \mathrm{C}$ NMR $\left(\mathrm{CD}_{3} \mathrm{OD}, 125 \mathrm{MHz}\right)$ spectrum of $N$-[2-(3-hydroxy-4-methoxyphenyl)-ethyl]-acetamide (93).

Structure 93 was fully confirmed by the 2D NMR spectra. It is a synthetically known neuroprotectant and a metabolite of L-DOPA in rats, but this is the first report from a natural source. ${ }^{107}$<smiles>COc1cc(CCNC(C)=O)ccc1O</smiles> 


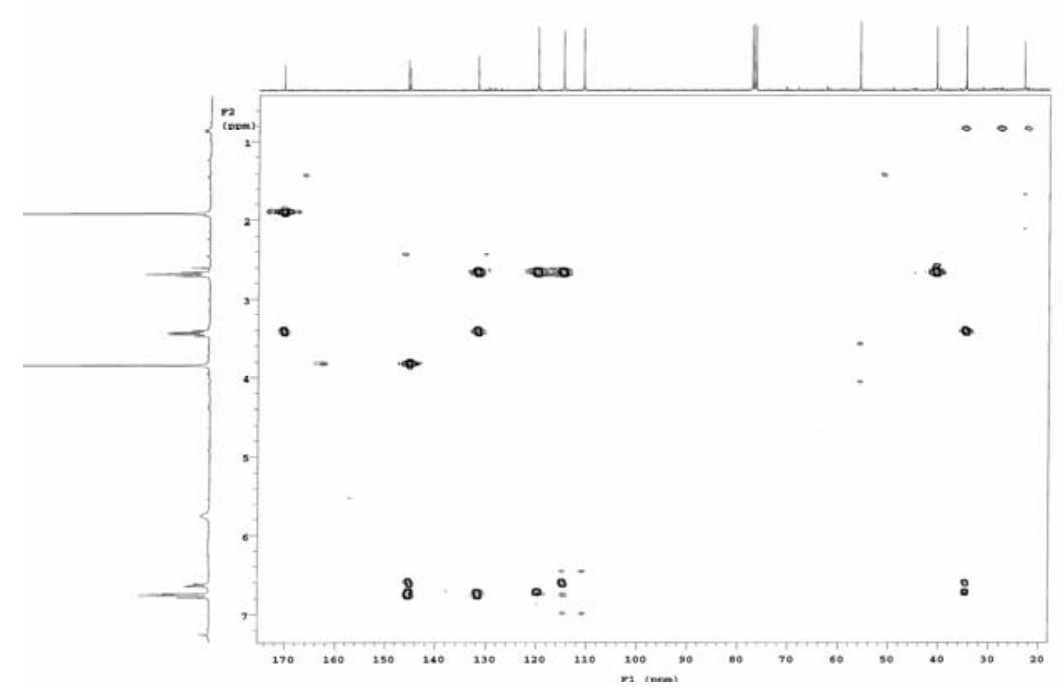

Figure 91: $\mathrm{HMBC}$ spectrum $\left(\mathrm{CD}_{3} \mathrm{OD}, 600 \mathrm{MHz}\right)$ of $\mathrm{N}$-[2-(3-hydroxy-4-methoxyphenyl)-ethyl]-acetamide (93).

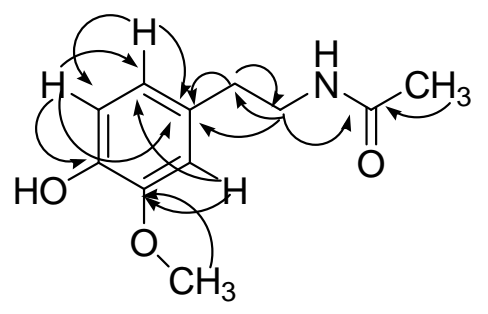

Figure 92: $\mathrm{HMBC}$ correlations observed for $N$-[2-(3-hydroxy-4-methoxy-phenyl)ethyl]-acetamide (93).

\subsubsection{4-(2-Hydroxyethyl)-2-methoxy-phenol}

The colourless UV active compound 94 showed a molecular weight of $\mathrm{m} / \mathrm{z} 168$ by EIMS. The ${ }^{1} \mathrm{H}$ NMR spectrum showed three protons attached to $s p^{2}$ carbon atoms at $\delta$ $6.80(2 \mathrm{H})$ and $6.72(1 \mathrm{H})$ of an aromatic ring. There was a singlet of methoxy protons at $\delta$ 3.90. Two methylene triplets appeared at $\delta 3.83$ and 2.75, and an acidic proton (H/D exchangeable) gave a broad singlet at $\delta$ 5.60. With the help of EIMS and ${ }^{1} \mathrm{H}$ NMR data, a search in AntiBase identified this compound as 4-(2-hydroxyethyl)-2methoxy-phenol (94). 


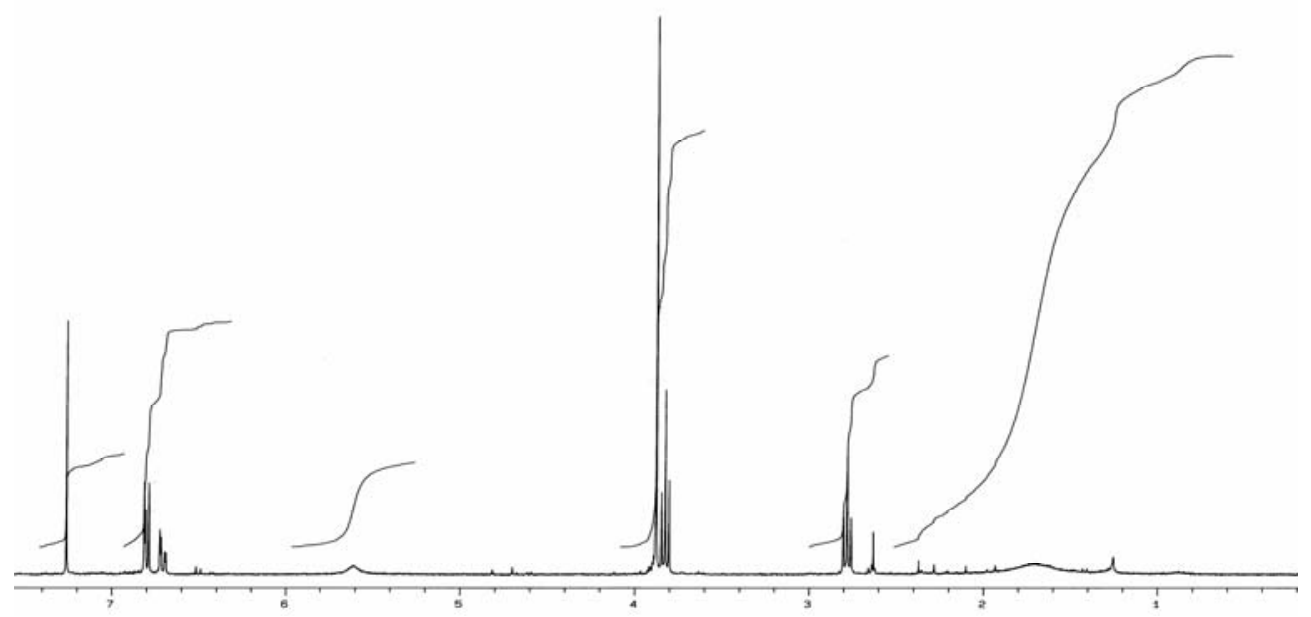

Figure 93: ${ }^{1} \mathrm{H} \mathrm{NMR}\left(\mathrm{CDCl}_{3}, 300 \mathrm{MHz}\right)$ spectrum of 4-(2-hydroxyethyl)-2-methoxyphenol (94).<smiles>COc1cc(CCO)ccc1O</smiles>

94

\subsection{5 $\beta$-Rubromycin}

TLC of the mycelium phase showed a major red compound, which did not change colour on spraying with anisaldehyde/sulphuric acid. On purification by PTLC followed by Sephadex LH-20, the metabolite was obtained as a red powder. The (+)-ESI mass spectrum indicated an $[\mathrm{M}+\mathrm{Na}]^{+}$ion at $m / z$ 559. In the ${ }^{1} \mathrm{H}$ NMR spectrum of this compound, two broad singlets of chelated hydroxyl groups were observed at $\delta 12.50$ and 10.66, three aromatic $1 \mathrm{H}$ singlets were found at $\delta 7.60,7.22$ and 7.04, three singlets in the range of $\delta 4.00-3.80$ were attributed to three methoxy groups, three methylene groups were seen in the aliphatic region. A search with this information in AntiBase led to the identification as $\beta$-rubromycin, which was confirmed by comparison with authentic spectra from our collection as well as published data from the literature. $^{108}$ 
<smiles>COC(=O)c1cc2cc3c(c(O)c2c(=O)o1)OC1(CC3)CC2=C(O1)C(=O)c1c(O)c(OC)cc(OC)c1C2=O</smiles>

95

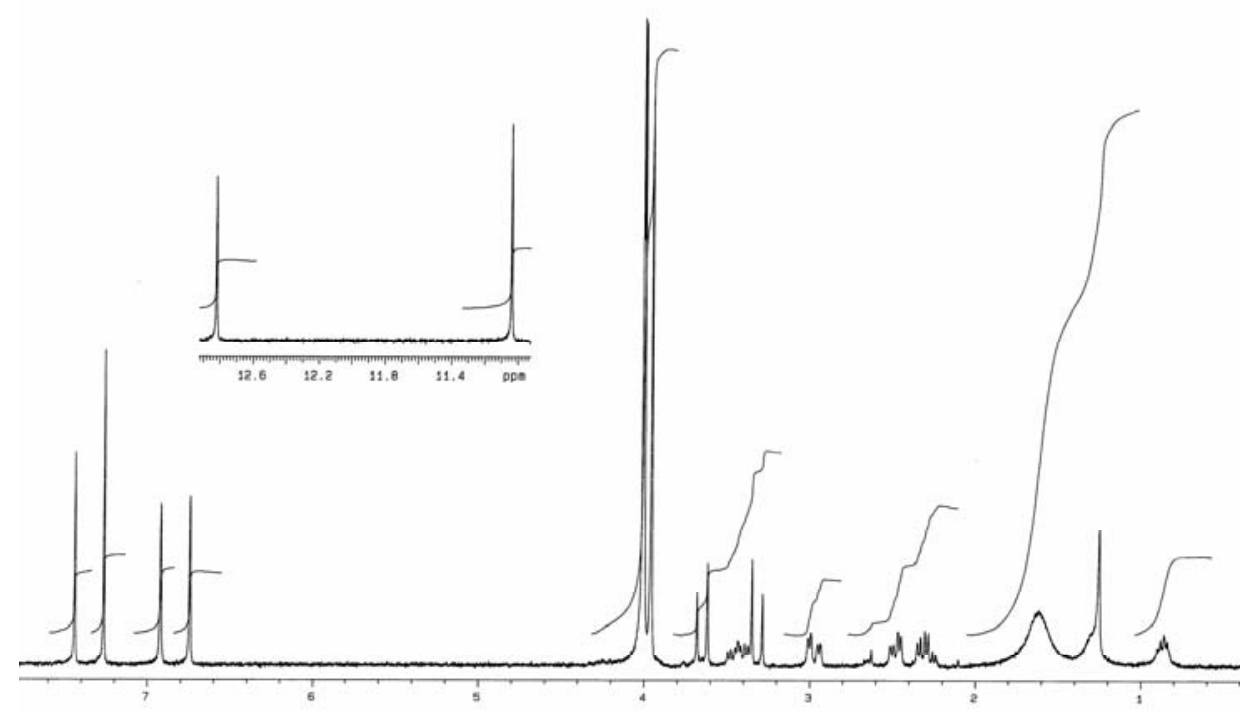

Figure 94: ${ }^{1} \mathrm{H} \mathrm{NMR}$ spectrum $\left(\mathrm{CDCl}_{3}, 300 \mathrm{MHz}\right)$ of $\beta$-rubromycin (95).

The red antibiotic $\beta$-rubromycin (95) was isolated as a mixture with $\gamma$-rubromycin by Brockmann et al. from Streptomyces collinus. Its structure was determined by degradation reactions, and the two carbonyl groups were initially placed in ortho position. Further structural and biosynthetic investigations led to the revision of its structure into 95. ${ }^{109}$

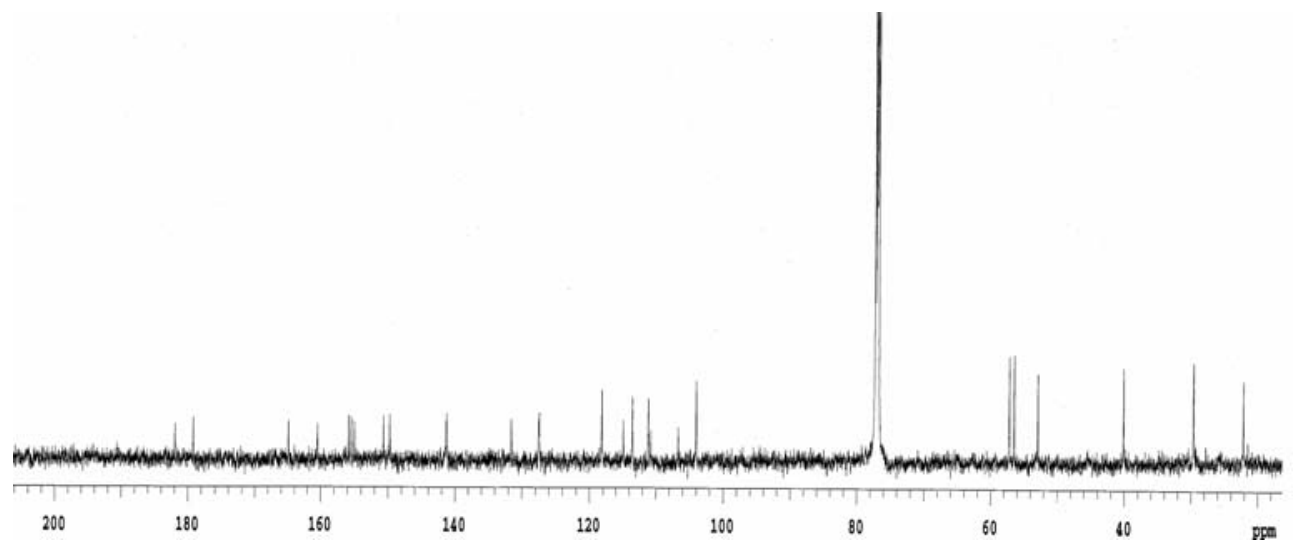

Figure 95: ${ }^{13} \mathrm{C}$ NMR spectrum $\left(\mathrm{CDCl}_{3}, 125 \mathrm{MHz}\right)$ of $\beta$-rubromycin (95) 


\subsection{6 $\gamma$-Rubromycin}

A second quinone 96 was also obtained as a red powder from the same mycelium phase, which exhibited similar characteristics as $\mathbf{9 5}$. The EI mass spectrum indicated a molecular weight of $m / z 522$ and the mass difference of $\Delta m=15$ indicated the loss of one methyl group. The ${ }^{1} \mathrm{H}$ NMR spectrum was also very similar to that of $\beta$ rubromycin, but in addition to the two chelated hydroxyl groups, a further one appeared at $\delta$ 13.14. In the aliphatic region the major difference was the presence of only two methoxy signals. The compound was identified as $\gamma$-rubromycin (96), which always occurs in mixture with $\beta$-rubromycin (95).

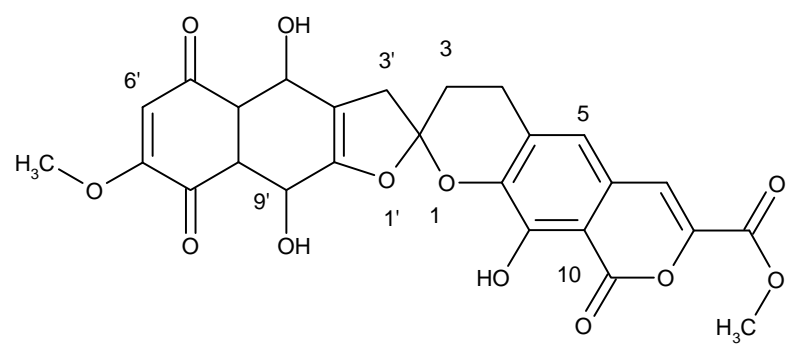

96

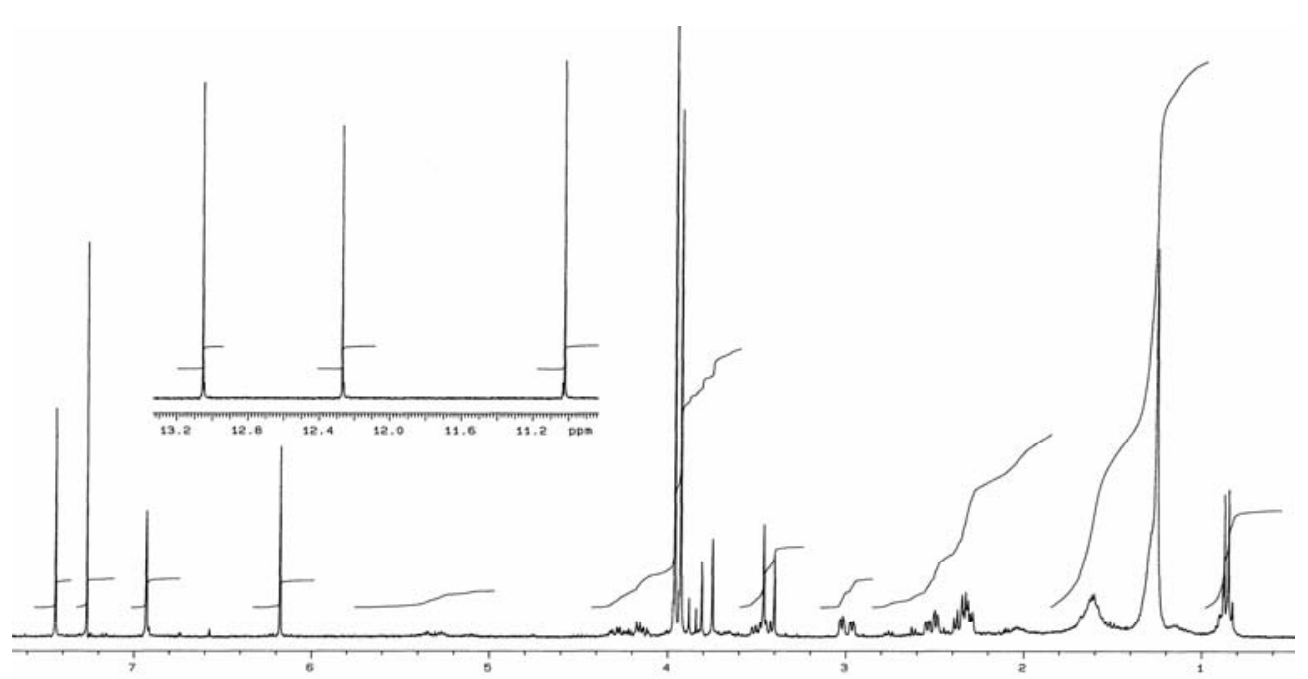

Figure 96: ${ }^{1} \mathrm{H} \mathrm{NMR}$ spectrum $\left(\mathrm{CDCl}_{3}, 300 \mathrm{MHz}\right)$ of $\gamma$-rubromycin (96).

$\beta$-Rubromycin (95) and $\gamma$-rubromycin (96) have been reported to show a marked growth inhibition of Bacillus subtilis, Staphylococcus aureus, Staphylococcus albus, Escherichia coli and to be active against other Gram positive germs. ${ }^{108}$ 


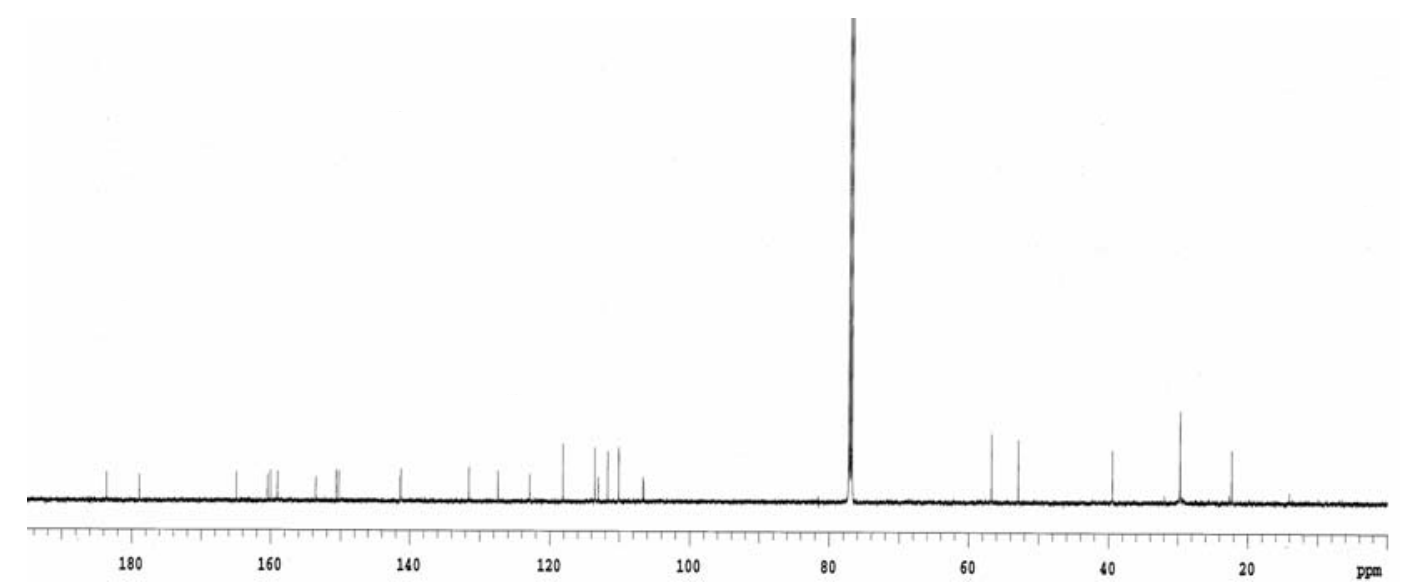

Figure 97: ${ }^{13} \mathrm{C}$ NMR spectrum $\left(\mathrm{CDCl}_{3}, 125 \mathrm{MHz}\right)$ of $\gamma$-rubromycin (96).

\subsection{Terrestrial Streptomyces sp. GW 18/1811}

The crude extract of the terrestrial Streptomyces sp. GW 18/1811 obtained from a $1 \mathrm{~L}$ culture exhibited biological activity against Streptomyces viridochromogenes (Tü 57), Escherichia coli, Bacillus subtilis, Staphylococcus aureus, Chlorella vulgaris, Chlorella sorokiniana, Candida albicans and Mucor miehei. TLC of the crude extract showed two relatively non-polar orange spots, however, on spraying with anisaldehyde/sulphuric acid, several colourless zones without UV absorption became visible as brown and violet spots. A 20 shaker culture in $\mathrm{M}_{2}$ medium became brown-yellow after four days of growth and delivered on work-up $1.8 \mathrm{~g}$ of a dark brown crude extract, which after defatting was chromatographed on silica gel and pre-separated into four fractions by TLC. 


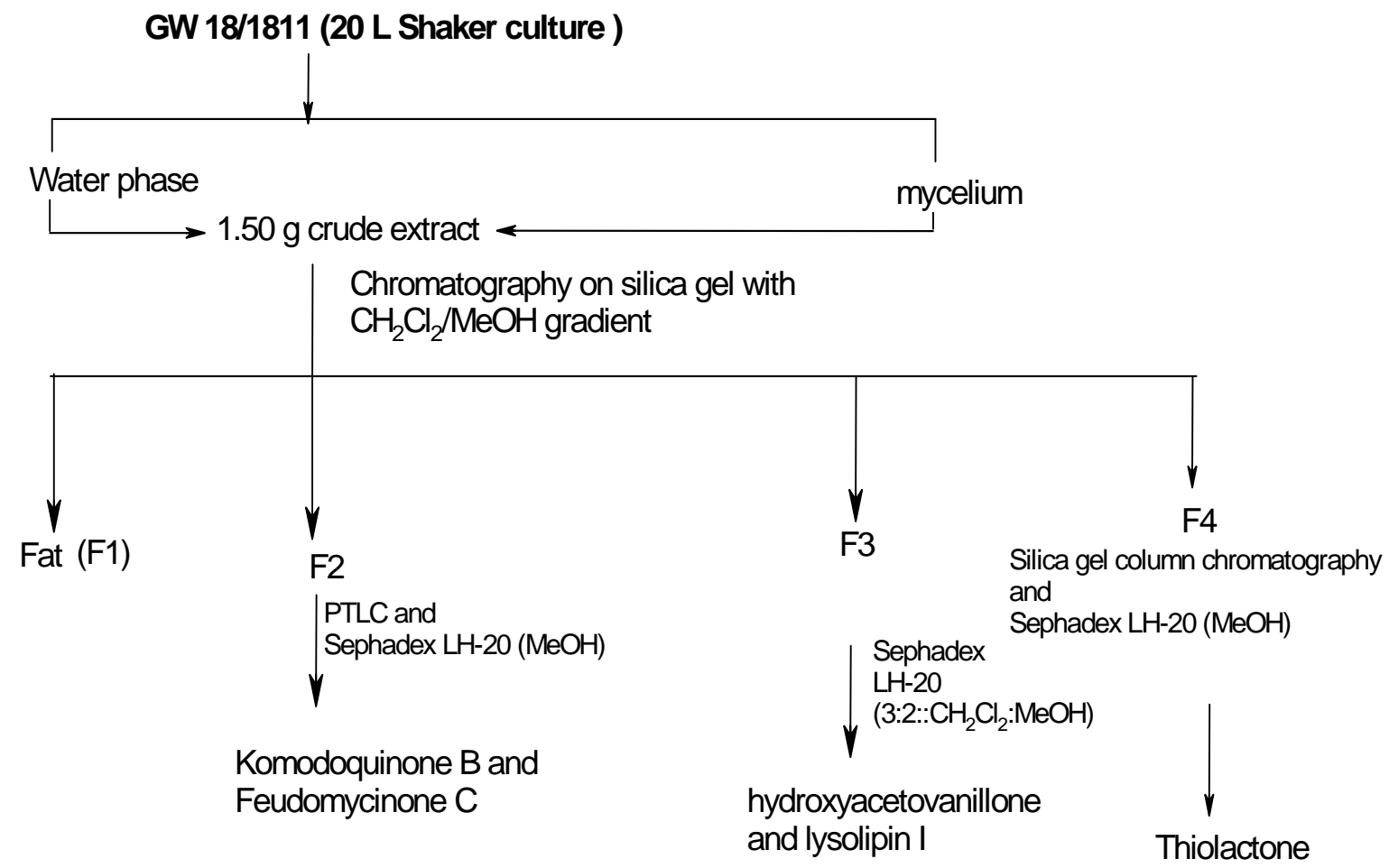

Figure 98 : Working scheme of terrestrial Streptomyces sp. GW 18/1811.

\subsubsection{Komodoquinone B}

Compound 97 was isolated as orange-red solid, which showed UV-absorbance at 254 $\mathrm{nm}$ and orange fluorescence at $366 \mathrm{~nm}$. Addition of sodium hydroxide on the TLC plate gave a colour change to violet pointing a peri-hydroxyquinone, while addition of anisaldehyde/sulphuric acid did not produce remarkable colour change. ESIMS showed a quas-molecular ion peak at $m / z 379[\mathrm{M}+\mathrm{Na}]^{+}$. The ${ }^{1} \mathrm{H}$ NMR spectrum indicated the presence of one methyl group at $\delta$ 1.36. There were two chelated H/D exchangeable protons at $\delta 13.40$ and 12.20 as broad singlets and two methylene groups near two chiral centres at $\delta 3.02,2.53,2.05$ and 1.84. Also one oxygenated methine at $\delta 4.94$ and a hydroxyl at $\delta 5.28$ were observed. The ${ }^{1} \mathrm{H}$ NMR spectrum led us to construct the partial structures assignable to the A-ring and D-ring parts of an anthracycline.<smiles>CC(C)(C)C(=O)c1c(O)cccc1C(C)(C)C</smiles><smiles>CC1(O)CC(O)C(C(C)(C)C)C(C)(C)C1</smiles>

Figure 99: Partial structures A and D of compound 97. 


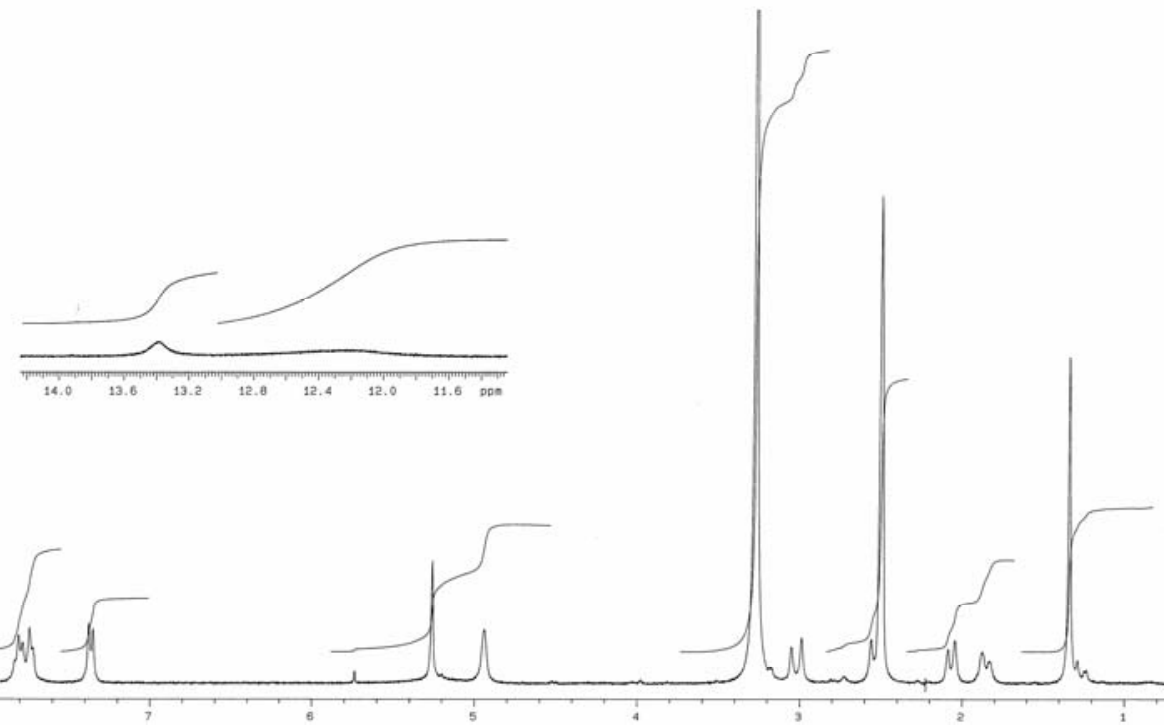

Figure 100: ${ }^{1} \mathrm{H}$ NMR spectrum $\left(\left[\mathrm{D}_{6}\right] \mathrm{DMSO}, 300 \mathrm{MHz}\right)$ of Komodoquinone B (97).

With the help of the mass and ${ }^{1} \mathrm{H}$ NMR data, a search in AntiBase led to komodoquinone B (97), which was confirmed with the data of the published literature. ${ }^{110} \mathrm{Ko}-$ modoquinone B (97) was isolated for the first time in 2003 as novel neuritogenic anthracycline from a marine Streptomyces sp. KS3. Komodoquinones belong to the rather rare class of 1,10-unsubstituted 9-methyl-anthracyclines. ${ }^{111}$<smiles></smiles>

97

\subsubsection{Feudomycinone $\mathrm{C}$}

Compound 98 was isolated as an orange solid, which showed UV absorbance at 254 $\mathrm{nm}$ and an orange fluorescence at $366 \mathrm{~nm}$. Addition of sodium hydroxide on the TLC plate changed the orange colour to violet pointing a peri-hydroxyquinone. Compound 98 was also isolated from the same fraction as Komodoquinone B (97) but showed a difference in the polarity on TLC. ESIMS showed an ion peak at $m / z 393[\mathrm{M}+\mathrm{Na}]^{+}$. The ${ }^{1} \mathrm{H}$ NMR spectra showed similarity with the ${ }^{1} \mathrm{H}$ NMR of komodoquinone B (97) except for the presence of one methoxy group at $\delta 4.11$ for compound $\mathbf{9 8}$. There were two chelated hydroxyl singlets at $\delta 14.10$ and 13.32 . ${ }^{1} \mathrm{H}$ NMR led to the partial structures assignable to the A-ring and D-ring parts of anthracycline. With the help of the mass and ${ }^{1} \mathrm{H}$ NMR data, a search in AntiBase led to feudomycinone $\mathrm{C}(\mathbf{9 8})$. The structure was confirmed by comparison of data with those in the literature. ${ }^{112}$ 


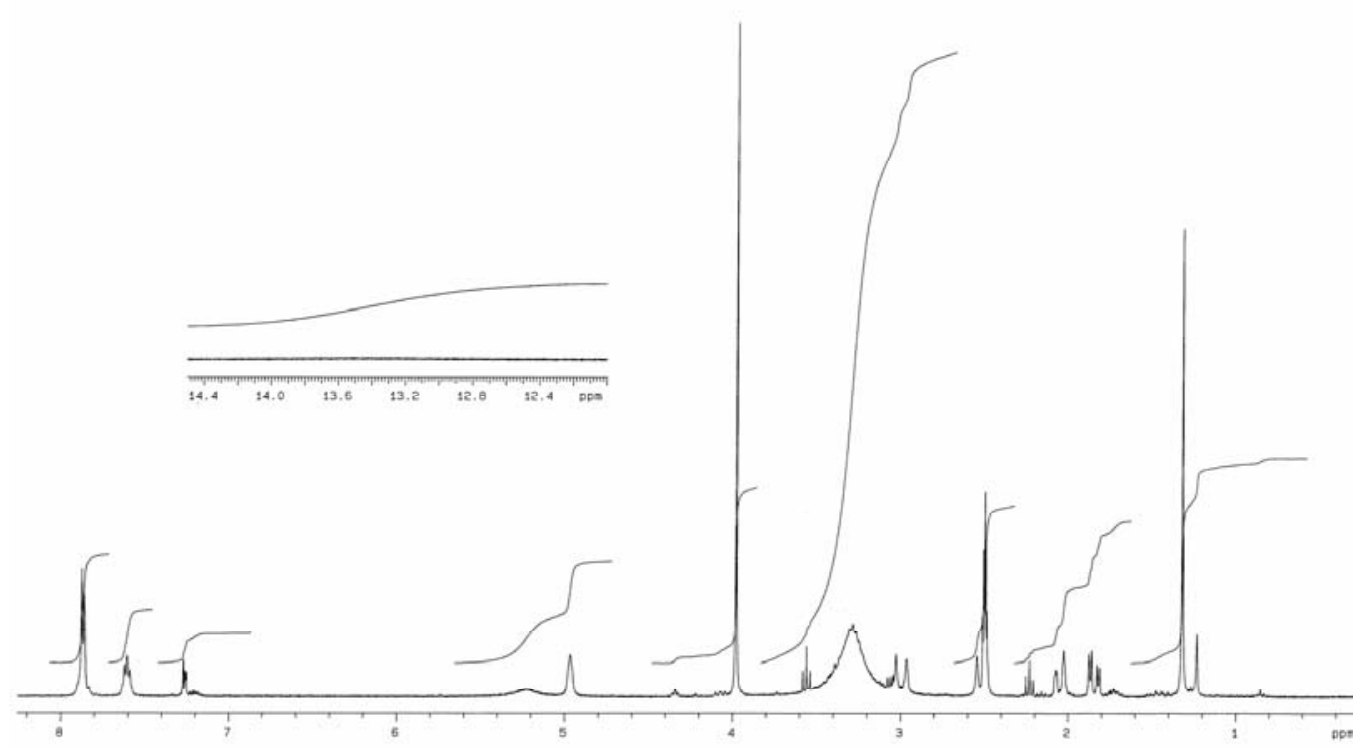

Figure 101: ${ }^{1} \mathrm{H}$ NMR spectrum $\left(\left[\mathrm{D}_{6}\right] \mathrm{DMSO}, 300 \mathrm{MHz}\right)$ of Feudomycinone $\mathrm{C}(\mathbf{9 8})$.<smiles>COc1cccc2c1C(=O)c1c(O)c3c(c(O)c1C2=O)C[C@@](C)(O)C[C@H]3O</smiles>

98

\subsection{3 $\alpha$-Hydroxyacetovanillone}

Compound 99 was isolated as colourless UV absorbing oil with an $R_{f}$ value of 0.43 $\left(\mathrm{CH}_{2} \mathrm{Cl}_{2} / 5 \% \mathrm{MeOH}\right)$. EIMS spectrum showed the mass as 182 . From the ${ }^{1} \mathrm{H}$ NMR spectrum, the presence of an $\mathrm{ABX}$ system $(\delta 7.52,7.43$, and 6.96) was observed. There was also one oxygenated methylene at $\delta 4.81$ and one methoxy group at $\delta 3.95$. 


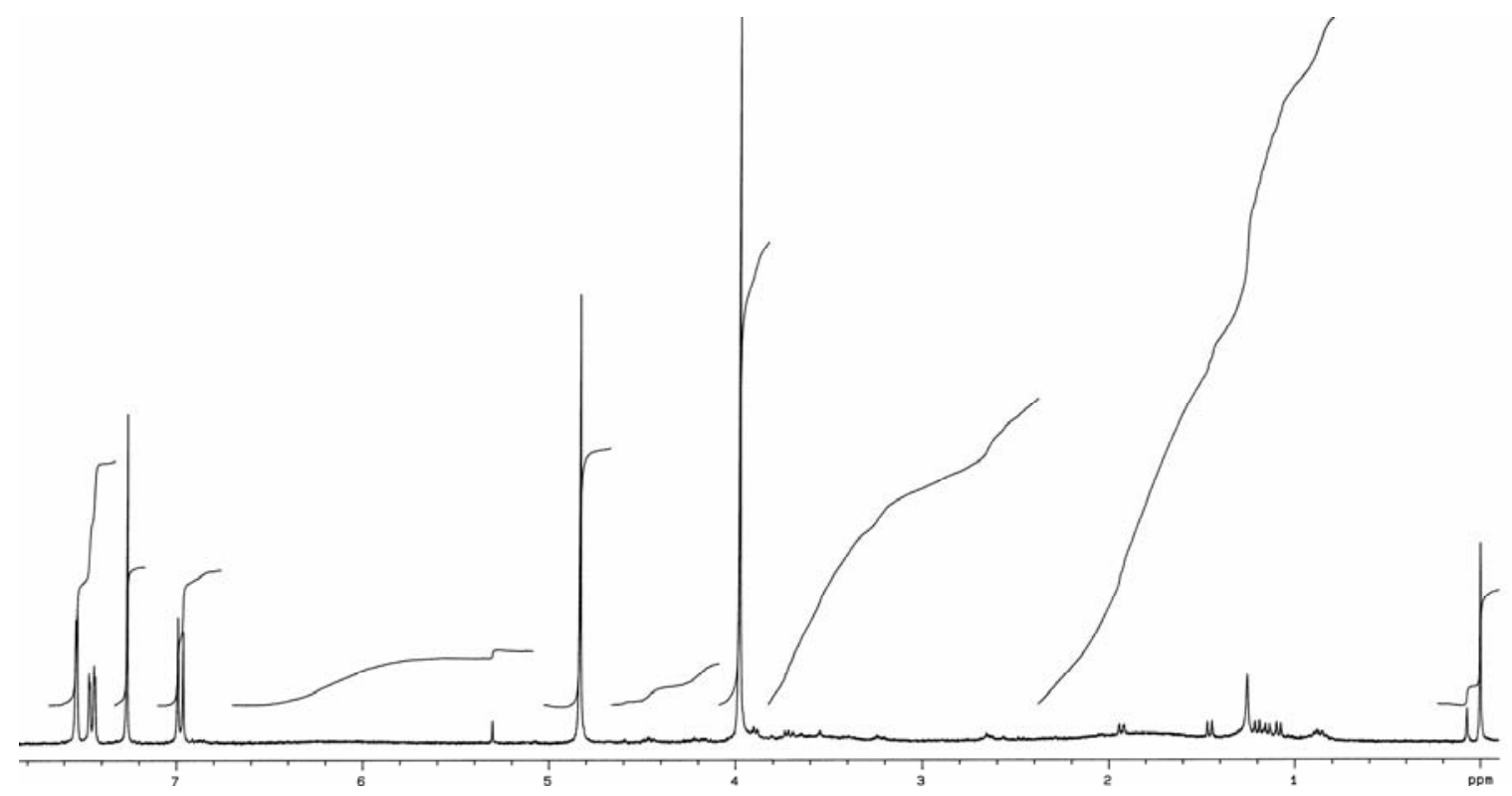

Figure 102: ${ }^{1} \mathrm{H} \mathrm{NMR}\left(\mathrm{CDCl}_{3}, 300 \mathrm{MHz}\right)$ of $\alpha$-hydroxyacetovanillone (99).

In the ${ }^{13} \mathrm{C}$ NMR spectrum, there were ten carbon signals visible, one carbonyl at $\delta$ 196.7, two oxygenated $s p^{2}$ carbons at $\delta 151.4$ and $\delta 146.9$ as well as further $4 s p^{2}$ carbons. Additionally, one methoxy group at $\delta 56.2$ and a methylene carbon at $\delta 64.9$ were present.

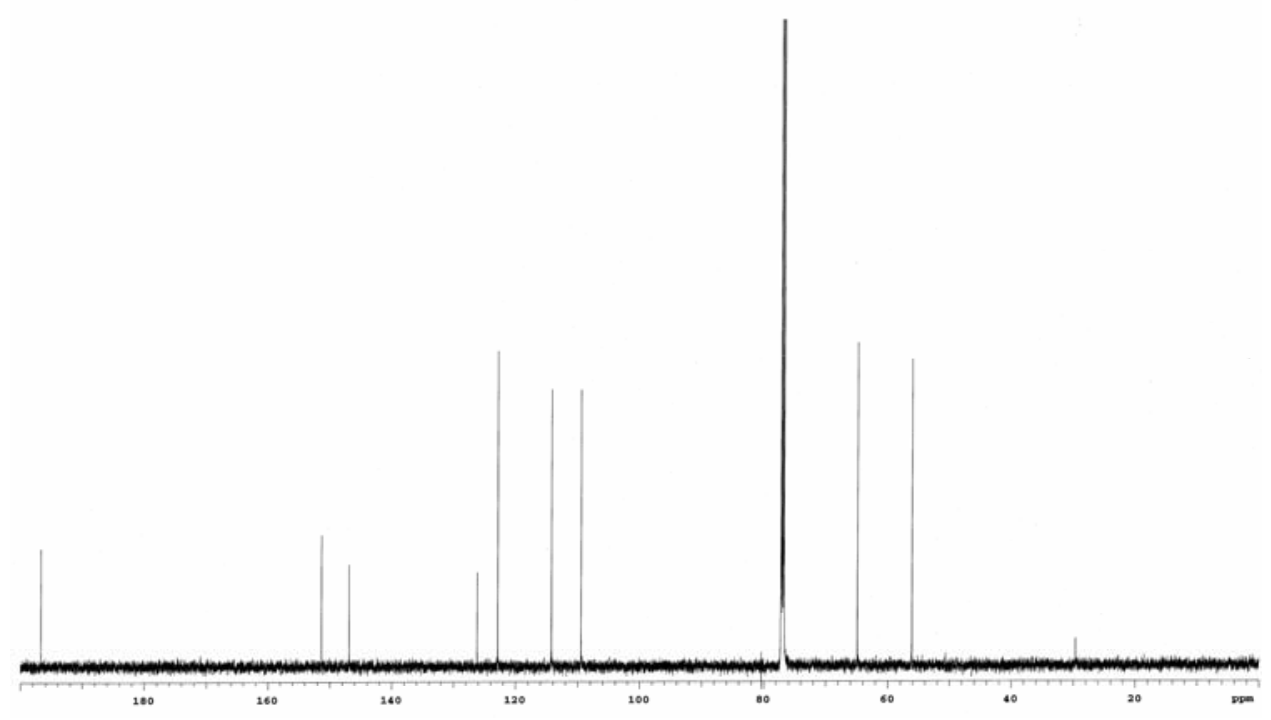

Figure 103: ${ }^{13} \mathrm{C} \mathrm{NMR}\left(\mathrm{CDCl}_{3}, 75 \mathrm{MHz}\right)$ of $\alpha$-hydroxyacetovanillone (99). 


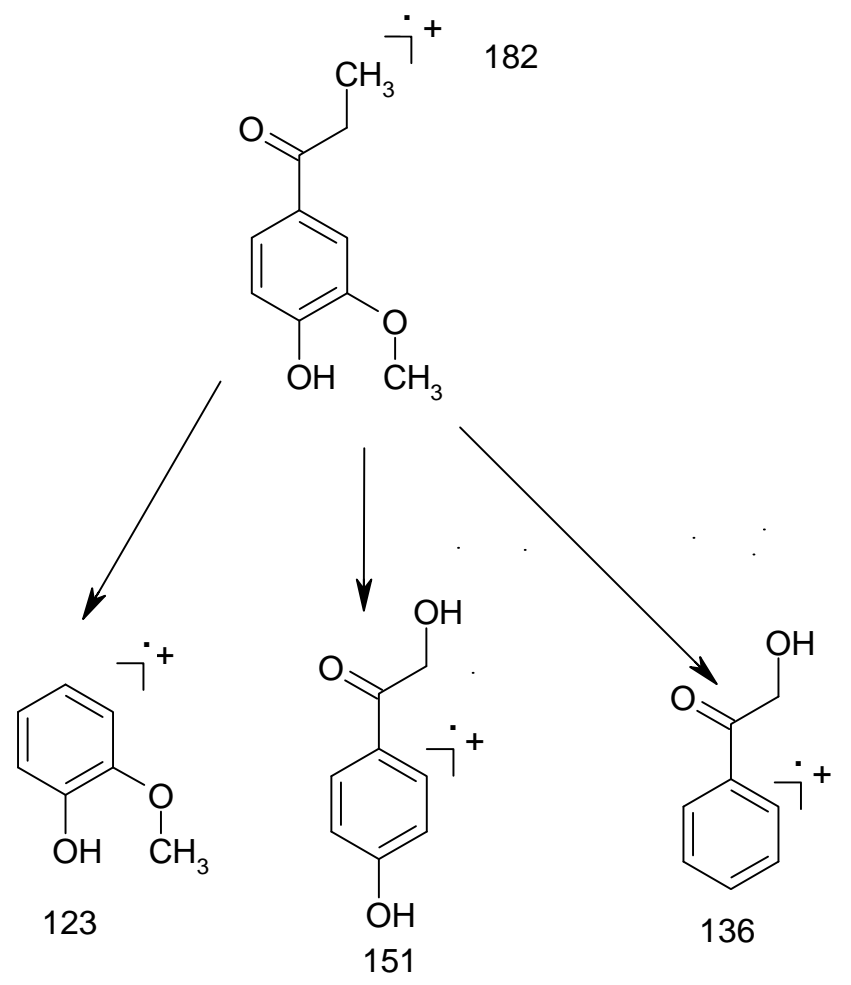

Figure 104: EIMS fragmentation pattern of $\alpha$-hydroxyacetovanillone (99).

The fragments from EIMS were helpful in determining the structure of compound 99. The molecular peak appeared at $m / z 182$ Dalton. At $m / z$ 151, the peak was [M $\left.\mathrm{OCH}_{3}\right]^{+}, m / z 136\left[\mathrm{M}-\mathrm{OH}-\mathrm{OCH}_{3}\right]^{+}, m / z, 123\left[\mathrm{M}-\mathrm{COCH}_{2} \mathrm{OH}\right]^{+}$(Figure 104). By using the NMR data and mass spectrum, the compound was searched in the Dictionary of Natural Products, which resulted in compound 99. The 1D and 2D correlations confirmed the structure as 3-methoxy-4-hydroxy-acetophenone ( $\alpha$-hydroxyacetovanillone, 99). Compound 99 was previously isolated from plants ${ }^{113}$ but there was no report from bacterial sources. 


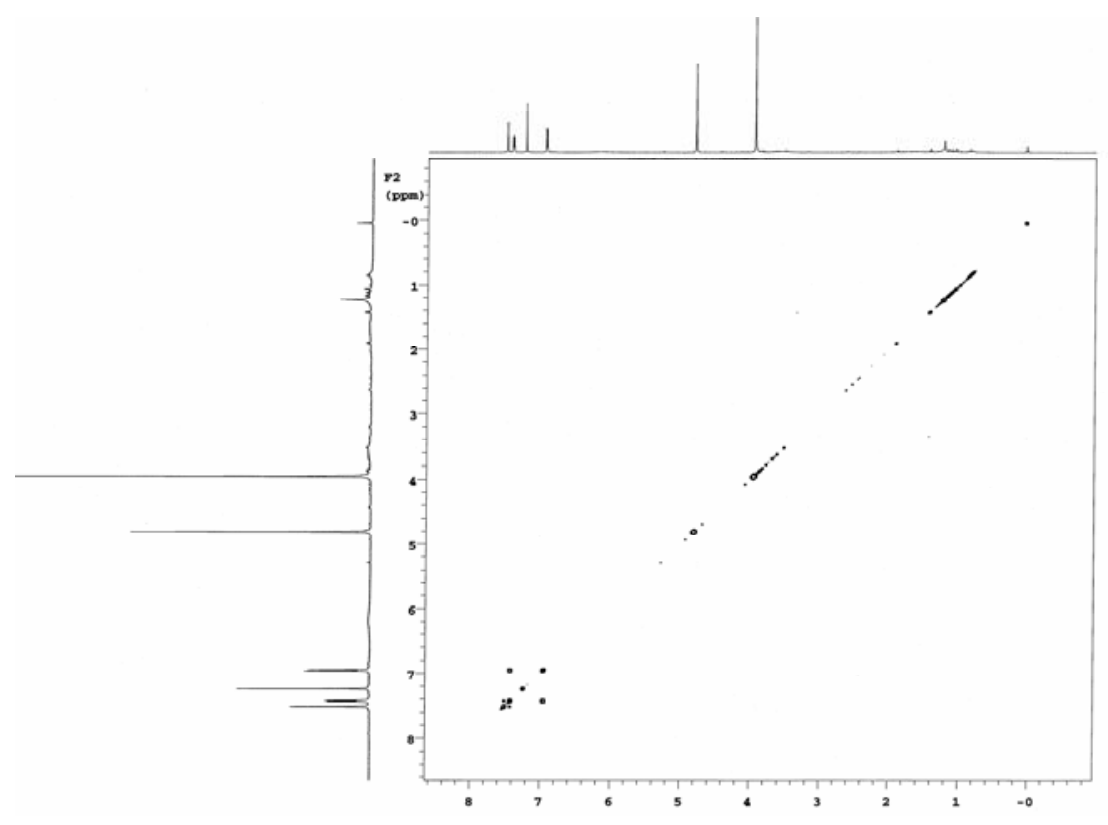

Figure 105: $\mathrm{H}-\mathrm{H}$ COSY spectrum $\left(\mathrm{CDCl}_{3}, 500 \mathrm{MHz}\right)$ of $\alpha$-hydroxyacetovanillone (99).<smiles>COc1cc(C(=O)CO)ccc1O</smiles>

99

$\alpha$-Hydroxyacetovanillone derivatives, e.g. $\operatorname{cis}-N$-p-coumaroyloctopamine (100), are known as wound inducible compounds, which activate virulence genes of Agrobacterium tumefaciens, especially in species within the Solanaceae ${ }^{114}$ family.<smiles>O=C(/C=C\c1ccc(O)cc1)NCC(O)c1ccc(O)cc1</smiles> 


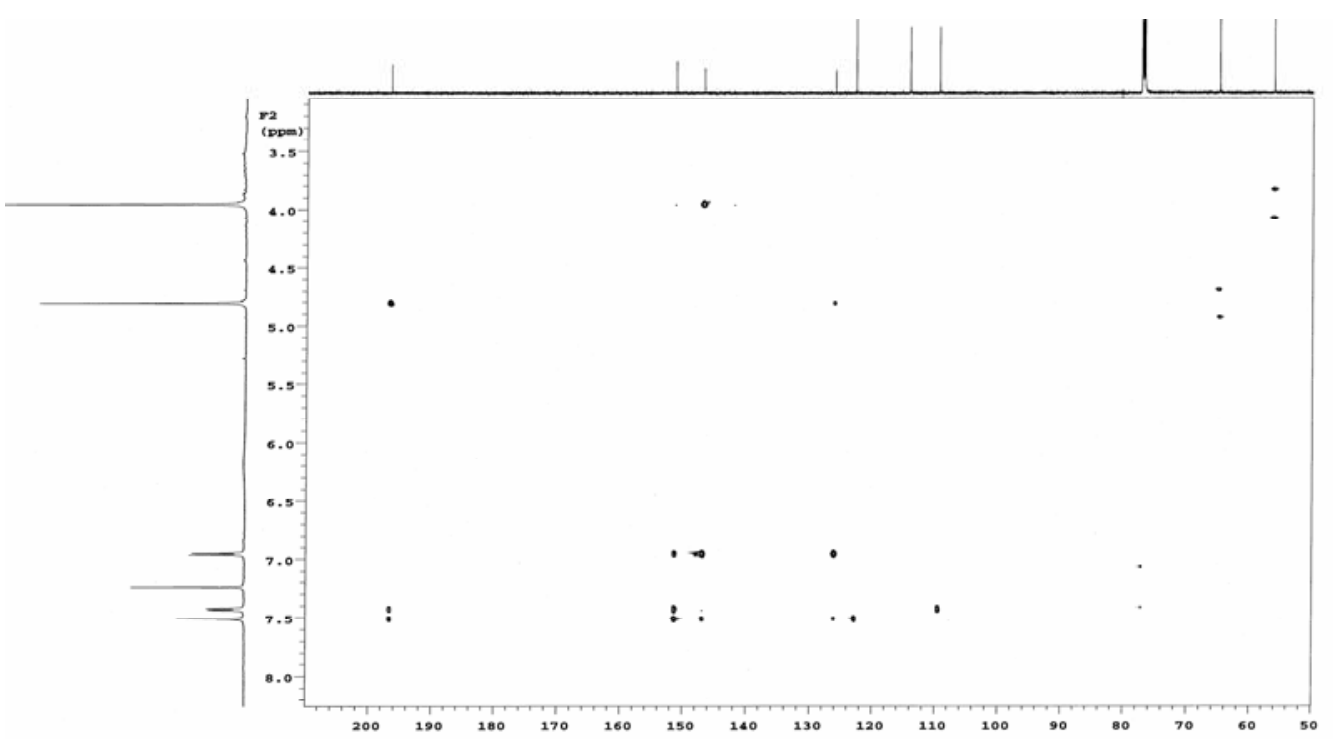

Figure 106: $\mathrm{HMBC}$ spectrum $\left(\mathrm{CDCl}_{3}, 500 \mathrm{MHz}\right)$ of $\alpha$-hydroxyacetovanillone (99).

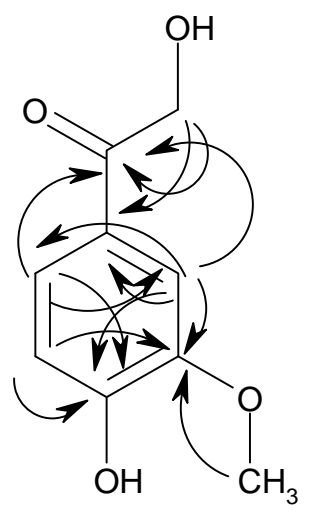

Figure 107: HMBC correlations of $\alpha$-hydroxyacetovanillone (99).

\subsubsection{N-Acetylhomocysteine thiolactone}

Compound 101 was isolated as non-UV absorbing colourless needles from fraction 4. ESIMS showed the mass as $m / z 159 .{ }^{1} \mathrm{H}$ NMR spectrum displayed one H/D exchangeable proton as a broad singlet at $\delta 6.10$. The methyl at $\delta 2.05$ was assumed to be a part of an acetyl group or to be attached to an $s p^{2}$ carbon atom or a nitrogen atom. The proton at $\delta 4.55$ indicated its attachment to a hetero atom. The pattern of the protons at $\delta$ 1.90 and 2.95 suggested a methylene group near a chiral centre. Another methylene group appeared at $\delta 3.30$ as a multiplet and was obviously not beside a chiral centre. 


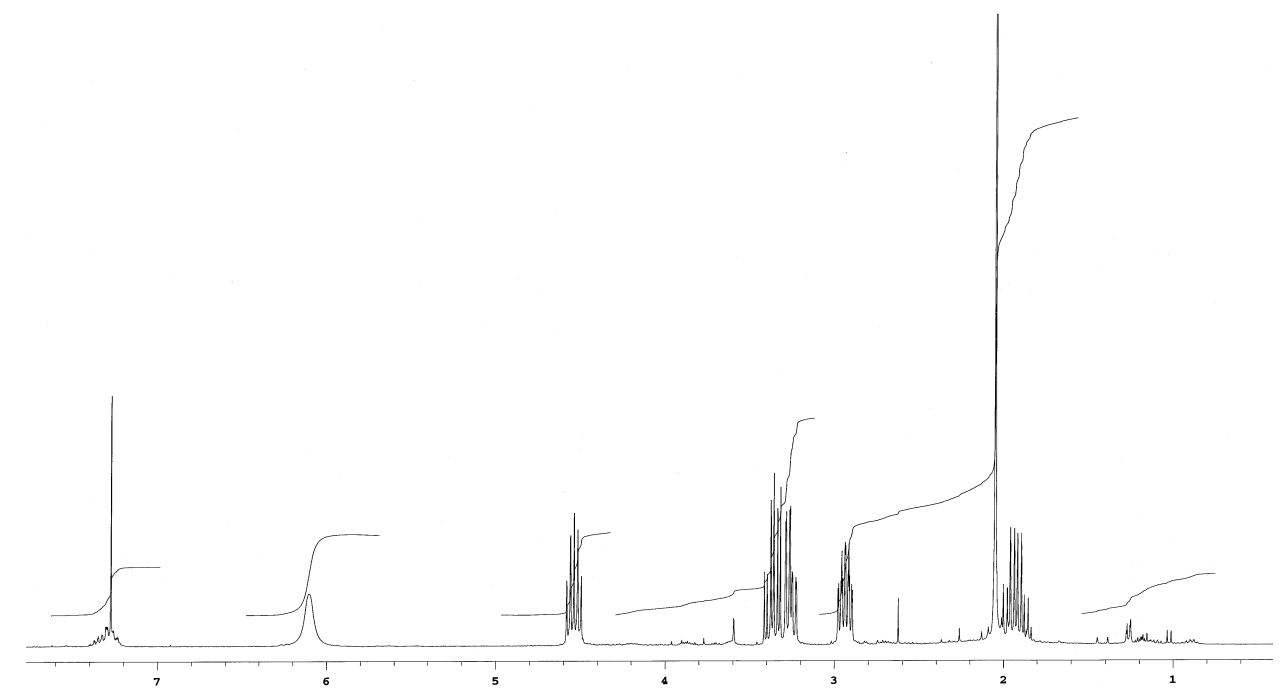

Figure 108: ${ }^{1} \mathrm{H}$ NMR $\left(\mathrm{CDCl}_{3}, 300 \mathrm{MHz}\right)$ of $\mathrm{N}$-acetylhomocysteine thiolactone (101).

The ${ }^{13} \mathrm{C}$ NMR spectrum showed only 6 carbon signals. There was a carbonyl signal of an aliphatic ketone observed at $\delta$ 205.8, a carbonyl signal of an ester, amide or acid at $\delta 170.8$ as well as a carbon at $\delta 59.4$, which was near a hetero atom, and three additional carbon signals at $\delta 31.7,27.5$ and 23.0.

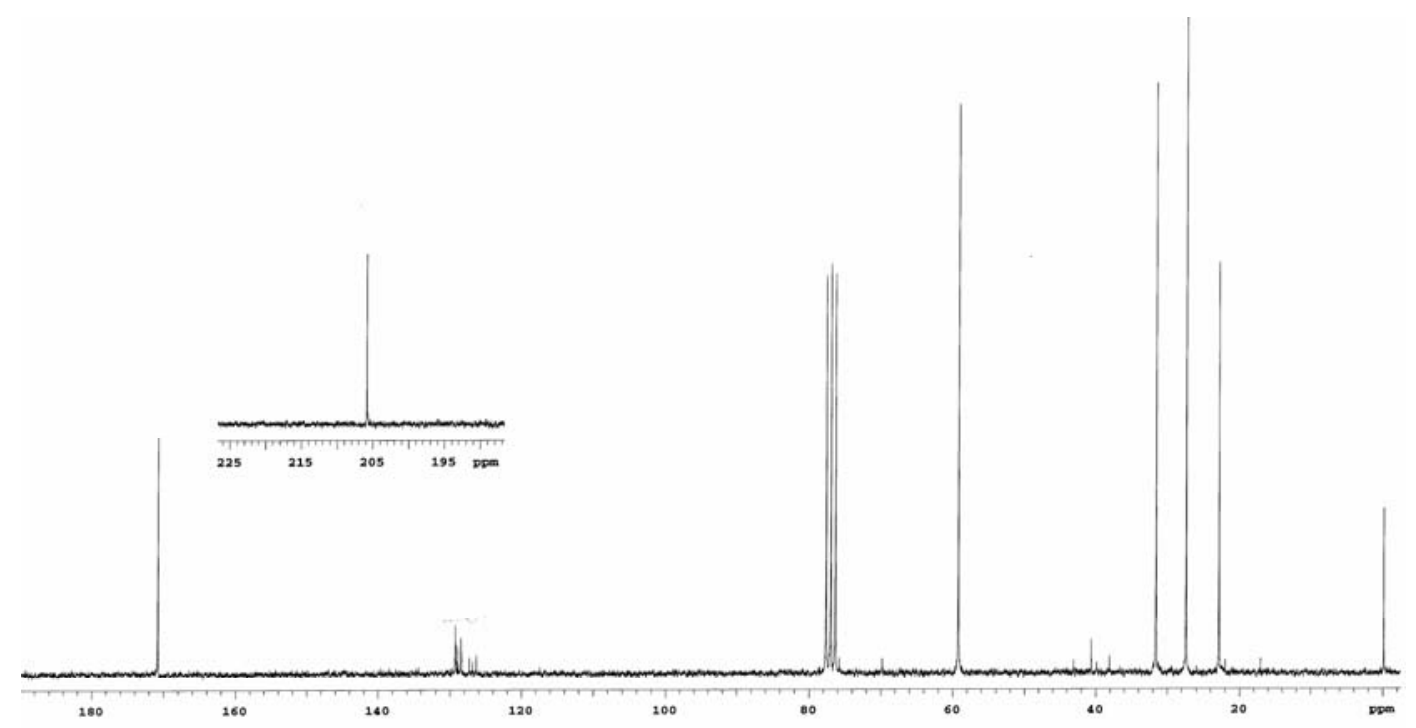

Figure 109: ${ }^{13} \mathrm{C} \mathrm{NMR}\left(\mathrm{CDCl}_{3}, 150 \mathrm{MHz}\right)$ of $\mathrm{N}$-acetylhomocysteine thiolactone (101).

Based on NMR and mass data, a search in AntiBase gave a thiolactone structure 101. ${ }^{115} \mathrm{~N}$-Acetylhomocysteine thiolactone (101) is commercially available and is included in some medical preparations ${ }^{116}$. It is also used as a reagent for insolubilizing antibodies. $^{117}$ 


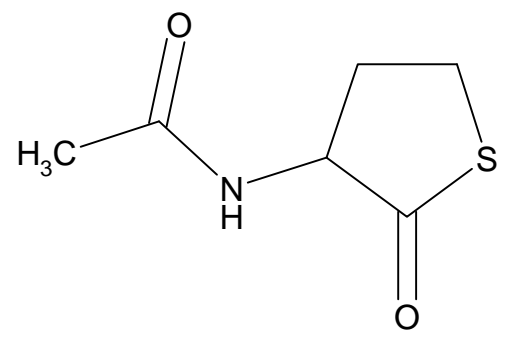

101

\subsubsection{Lysolipin I}

Compound 102 was isolated as UV absorbing bright yellow needles from the third fraction after silica column chromatography followed by Sephadex LH-20. (+)ESIMS showed the molecular peaks at $\mathrm{m} / z 597$ and 599 in the mass spectrum. The height of the signal at 599 Dalton was 33\% in comparison to 597 in the mass spectrum which was a clear indication that the compound contained chlorine. The ${ }^{1} \mathrm{H}$ NMR spectrum showed two chelated hydroxyl group at $\delta 13.13$ and 12.93 , as well as three aromatic protons at $\delta 7.94,7.37$ and 7.09. There were two protons observed at $\delta$ 5.63 and 5.40 and four methine protons at $\delta 5.03,5.02,4.70$ and 4.46, which were either attached with $s p^{2}$ carbon or with heteroatoms. Also three methyl signals were seen at $\delta 4.18,3.55$, and $3.37(6 \mathrm{H})$.

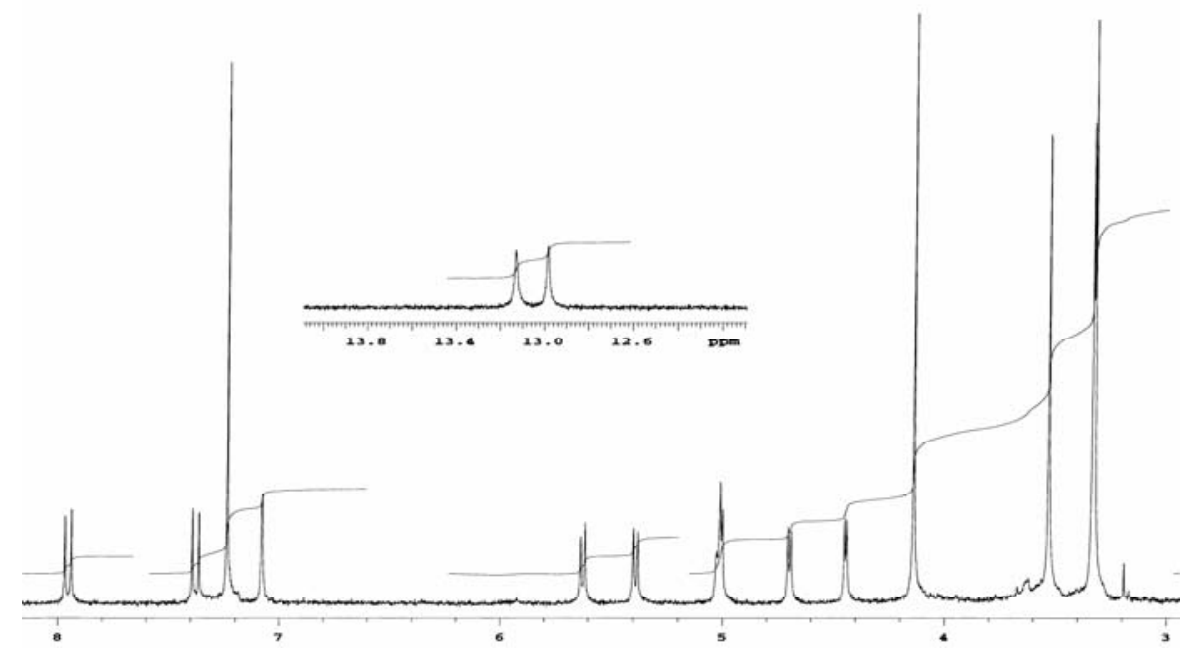

Figure 110: ${ }^{1} \mathrm{H} \mathrm{NMR}\left(\mathrm{CDCl}_{3}, 3 \mathrm{OO} \mathrm{MHz}\right)$ of lysolipin (102).

From ${ }^{13} \mathrm{C}$ NMR spectrum there were 29 carbons: two carbonyls at $\delta 181.6$ and 168.2, seven oxygenated quaternary $s p^{2}$ carbon signals were shown at $\delta 158.9,151.6,149.9$, $145.1,143.6,139.6$, and 139.0 and eleven more in the $s p^{2}$ region. From the data a search in AntiBase led to the identification as lysolipin I (102). The spectroscopic data were identical with the those previously published. ${ }^{118,119}$ 


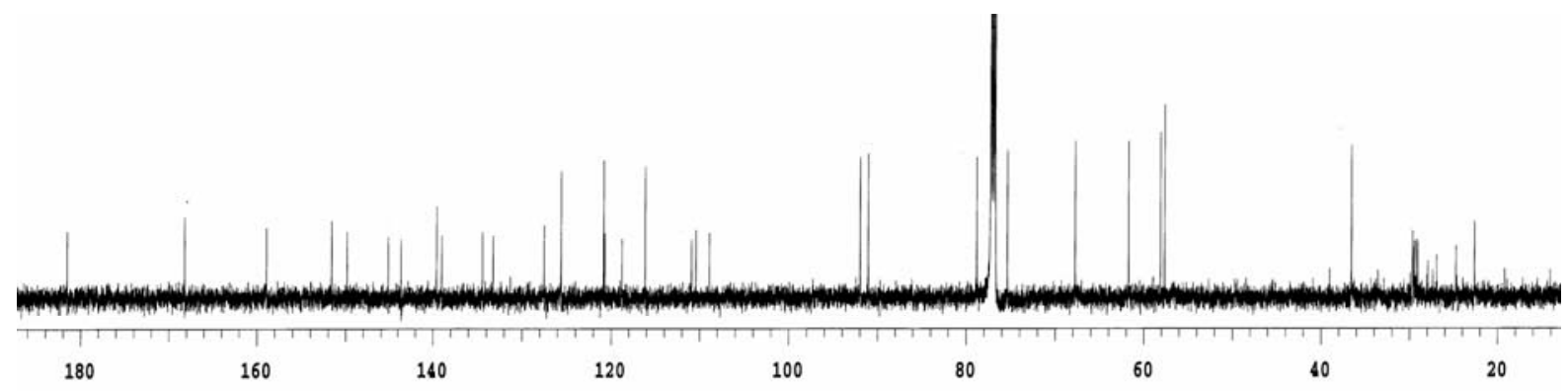

Figure 111: ${ }^{13} \mathrm{C} \mathrm{NMR}\left(\mathrm{CDCl}_{3}, 125 \mathrm{MHz}\right)$ of lysolipin (102).

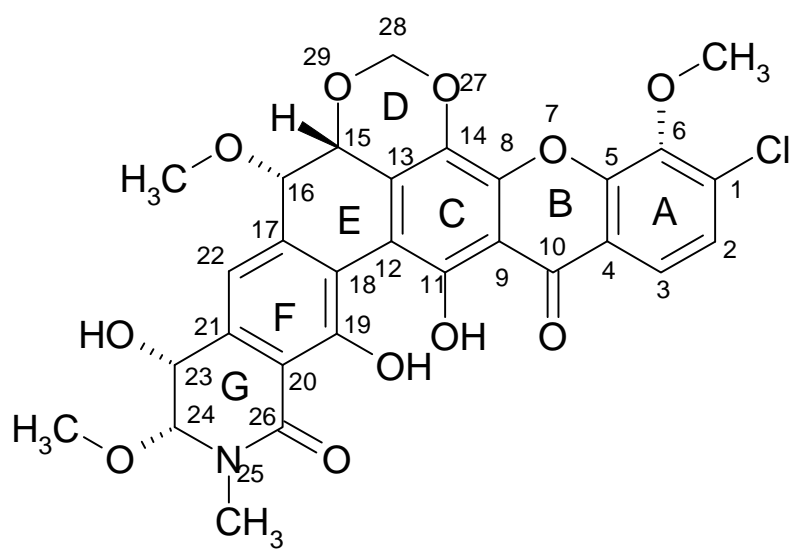




\section{$5 \quad$ Fungal metabolites}

\subsection{Bikaverin}

Compound 103 was isolated as a deep red solid from a crude sample sent by Prof. Dr. H. Anke, Institute for Biotechnology and Drug Research, Kaiserslautern. It was insoluble in methanol, chloroform, acetone, pyridine and even in DMSO. The ${ }^{1} \mathrm{H}$ NMR spectrum was measured in a mixture of chloroform and trifluoroacetic acid. The ${ }^{1} \mathrm{H}$ NMR spectrum showed three olefinic protons at $\delta 7.39,7.35$ and 6.80. There were two methoxy and one methyl group at $\delta 4.16$ and $\delta 2.97$, respectively.

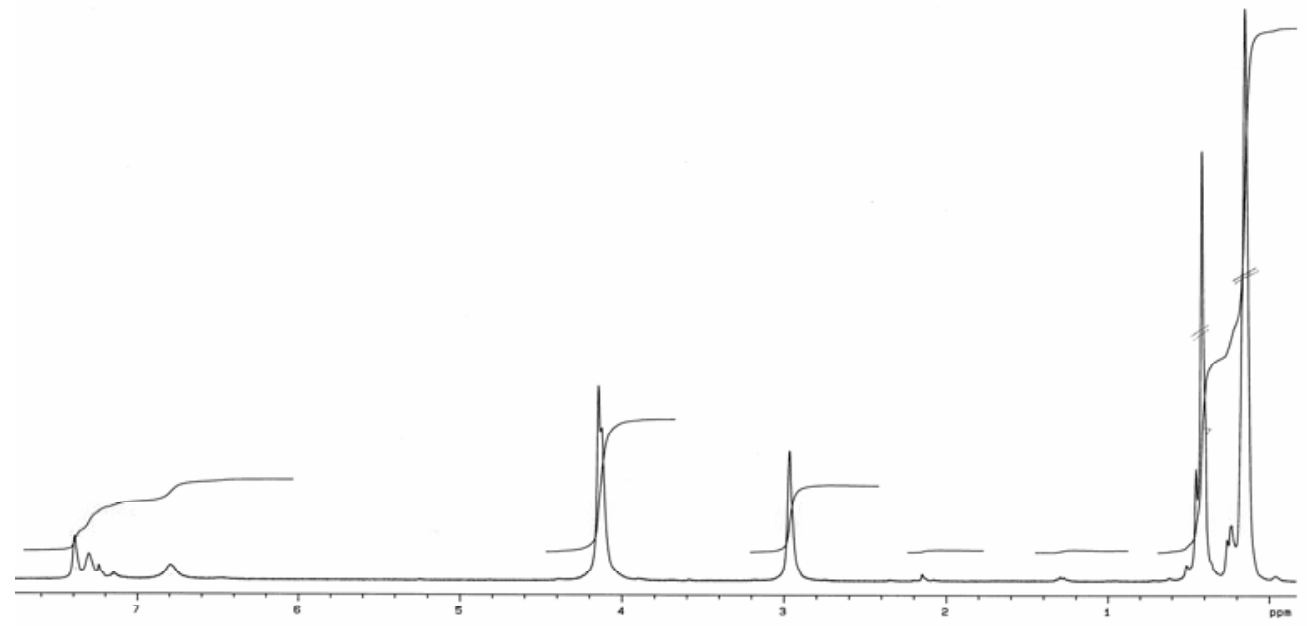

Figure 112: ${ }^{1} \mathrm{H}$ NMR spectrum $\left(300 \mathrm{MHz}, 50 \% \mathrm{CDCl}_{3}+50 \%\right.$ TFA) of bikaverin (103).

In the ${ }^{13} \mathrm{C}$ NMR spectrum there were 20 carbons visible. There was one methyl signal at $\delta$ 23.4, two methoxy at $\delta 57.5$ and 58.0, which were also obvious from the ${ }^{1} \mathrm{H}$ NMR spectrum. All other carbon signals were in the $s p^{2}$ region. There were probably 4 carbonyls at $\delta$ 179.5 178.2, 172.0 and 166.1. The ${ }^{1} \mathrm{H}$ NMR and ${ }^{13} \mathrm{C}$ NMR spectra and mass data showed identity with the literature values for bikaverin (103). 


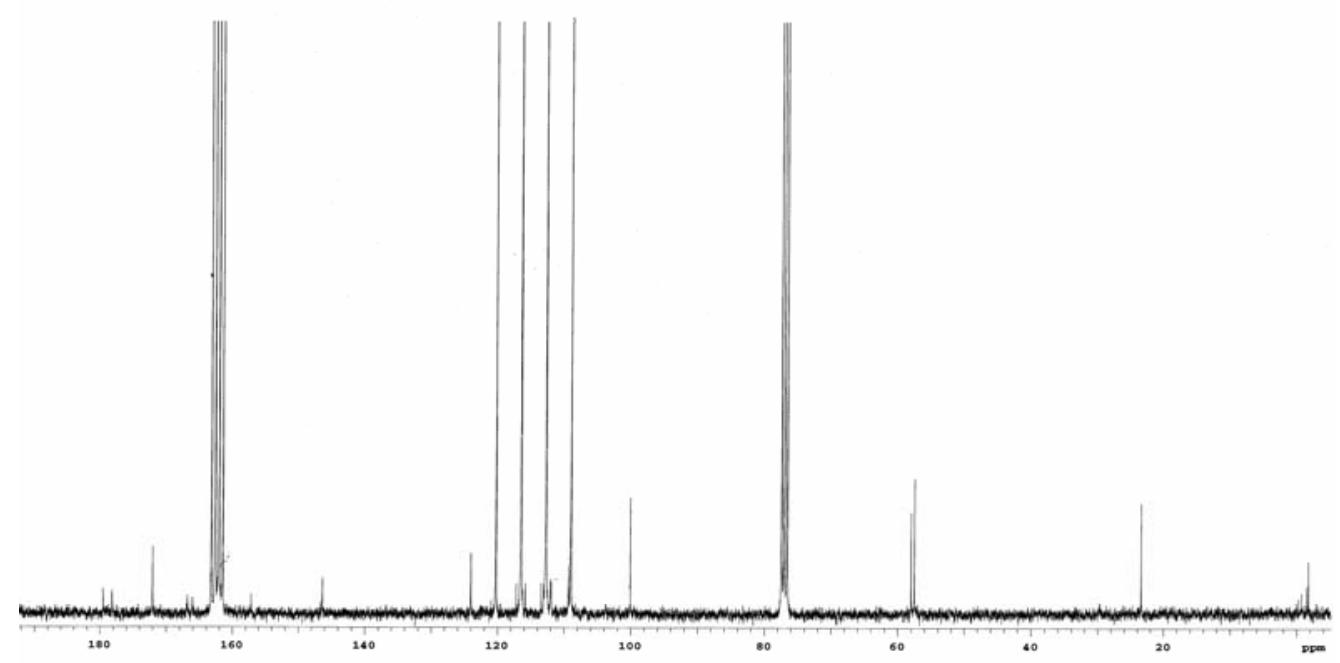

Figure 113: ${ }^{13} \mathrm{C}$ NMR spectrum $\left(75 \mathrm{MHz}, \mathrm{CDCl}_{3}+50 \%\right.$ TFA) of Bikaverin (103).<smiles>COC1=CC(=O)c2c(c(O)c3oc4cc(OC)cc(C)c4c(=O)c3c2O)C1=O</smiles>

103<smiles></smiles>

Figure 114: EIMS fragmentation of Bikaverin (103).

Bikaverin (103) was previously isolated from Fusarium oxysporum. ${ }^{120}$ Its benzoxanthone ring system is very rare among natural products. Due to its high bioactivity it has been synthesized and the way of biosynthesis has also been investigated by sev- 
eral research groups. ${ }^{121}$ McInnes A. G. et al. ${ }^{122}$ have found that bikaverin is an acetogenin and postulated a biosynthetic pathway.

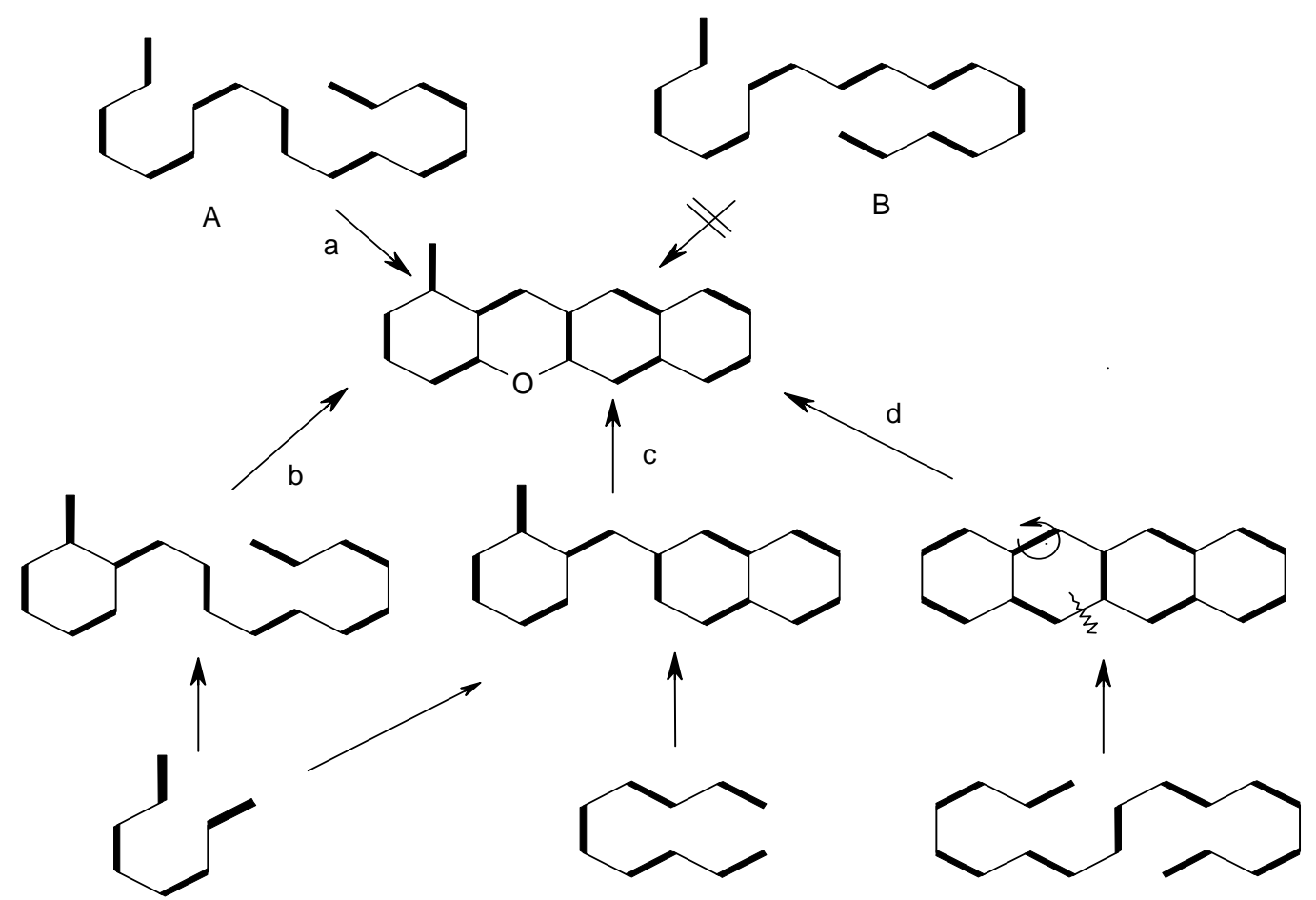

Figure 115: Suggested biosynthetic pathway of bikaverin (103) according to Mcinnes. ${ }^{122}$ Bold lines are indicating the acetate units. 


\section{Plant Metabolites from Tephrosia sp.}

Twenty five samples from the plant Tephrosia calophylla were obtained from one of our research collaborator, Professor Seru Ganapaty from the Pharmacognosy and Photochemistry Division, Andhra University, India. Twentyfive different compounds were confirmed in these samples; 21 of them were known and 4 were new compounds including the new benzil derivative calophione A (104).

Tephrosia is a tropical and subtropical genus of Leguminosae in the Fabaceae family, which forms perennial woody shrubs. Between 300 and 400 species are known, ${ }^{123}$ of which 35 occur in India, 30 are native to South America, 70 are found in South Africa and 50 in equatorial Africa. ${ }^{124}$ Tephrosia calophylla is a perennial undershrub found widely in Andhra Pradesh, south India. Many species in the Tephrosia genus are poisonous, particularly to fish, for their high concentration of rotenone. Tephrosia species have historically been used by many indigenous cultures as fish poisons. In the last century, several Tephrosia species have been studied in connection with the use of rotenone as an insecticide and pesticide. The plants are also used traditionally in folk medicine. According to Ayurveda, the plant is useful as an anthelmintic, antipyretic and as well as an alexiteric drug. It is also active against leprosy, ulcers, and used as alternative cures for diseases of the liver, spleen, heart and blood. According to the Unani system of medicine, the root is diuretic, allays thirst, enriches blood, cures diarrhoea, is useful in bronchitis, inflammations, boils and pimples. Leaves are tonic to intestines and a promising appetizer. The plants prevent the soil erosion and helps in fixing nitrogen. Their leaves are used as fodder and the seeds can be used as substitute for coffee.

\subsection{Spinoflavanone B}

Compound 103 was obtained as a yellow solid. The molecular weight was deduced from the (+)-ESI mass spectrum, which indicated a pseudomolecular peak at $\mathrm{m} / \mathrm{z} 393$ $[\mathrm{M}+\mathrm{H}]^{+}$; HRESIMS established the molecular formula $\mathrm{C}_{25} \mathrm{H}_{28} \mathrm{O}_{4}$. The ${ }^{1} \mathrm{H} \mathrm{NMR}$ spectrum showed the resonances typical of a saturated flavanone ring [7,8]. A set of peaks characteristic of two C-3-methylbut-2-enyl groups $[4,5]$ was also observed in its spectrum. The ${ }^{1} \mathrm{H}$ NMR spectrum showed two singlets, one at $\delta 12.35$ attributed to a chelated phenolic hydroxyl and another at $\delta 6.39$ for a free $\mathrm{OH}$ group. A broad singlet at $\delta 7.44$ integrating for five protons in the ${ }^{1} \mathrm{H}$ NMR spectrum was attributed to a monosubstituted benzene ring. 
<smiles>CC(C)=CCc1c(O)c(CC=C(C)C)c2c(c1O)C(=O)CC(c1ccccc1)O2</smiles>

104

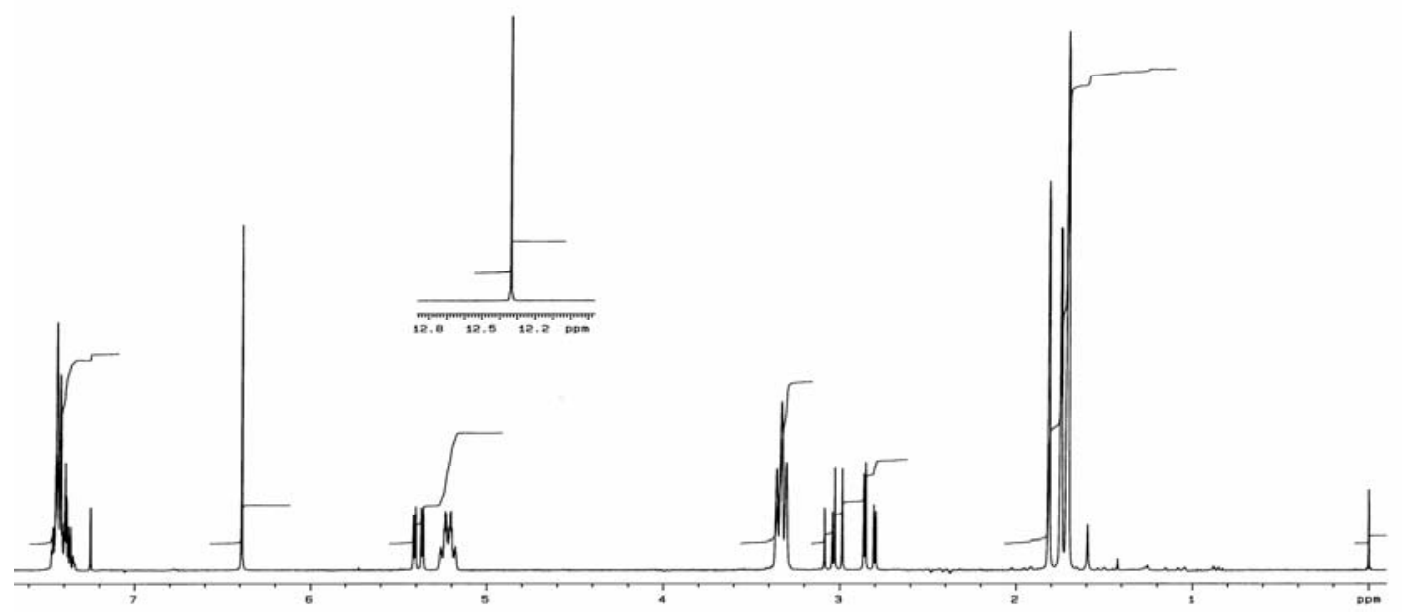

Figure 116: ${ }^{1} \mathrm{H} \mathrm{NMR}$ spectrum $\left(\mathrm{CDCl}_{3}, 300 \mathrm{MHz}\right)$ of spinoflavanone $\mathrm{B}(\mathbf{1 0 3})$.

In ${ }^{13} \mathrm{C}$ NMR spectrum, $17 s p^{2}$ carbon signals were observed; one of them at $\delta 196.2$ indicated the presence of a ketone or aldehyde and three appeared at $\delta 162.3,159.3$ and 157.6 indicating the attachment of oxygen. There was one methine carbon at $\delta$ 78.8 , which is also obviously attached with oxygen. Three methylene carbons were observed at $\delta 43.4\left(\mathrm{CH}_{2}-3\right), 21.9\left(\mathrm{CH}_{2}-1^{\prime \prime}, 1^{\prime \prime \prime}\right)$ as well as 4 methyl carbons.

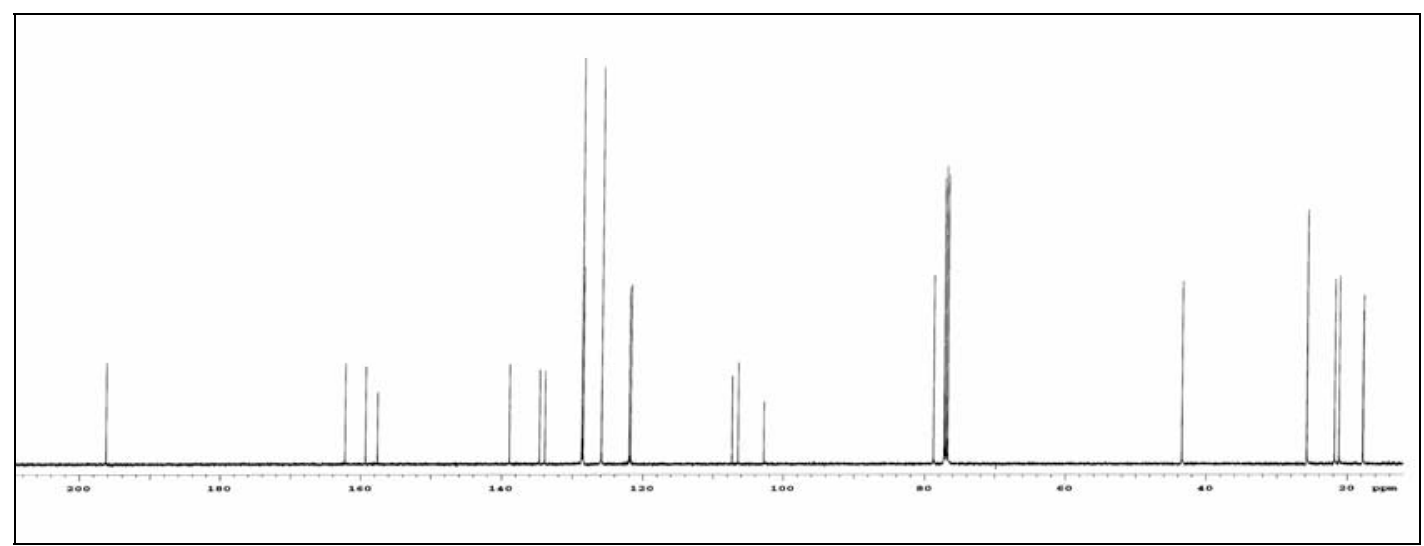

Figure 117: ${ }^{13} \mathrm{C}$ NMR spectrum $\left(\mathrm{CDCl}_{3}, 75 \mathrm{MHz}\right)$ of spinoflavanone $\mathrm{B}(\mathbf{1 0 3})$. 
In the H-H COSY spectrum, two isoprenyl units could be confirmed from the correlation along with other fragments (A-C).

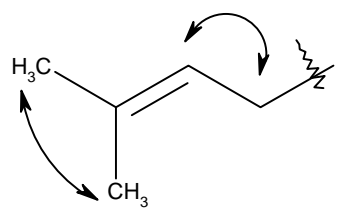

A

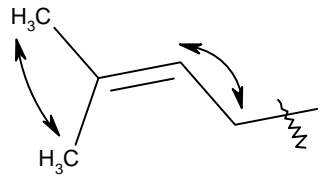

B<smiles>CC1(O)CCC(C(C)(C)C)C1</smiles>

C

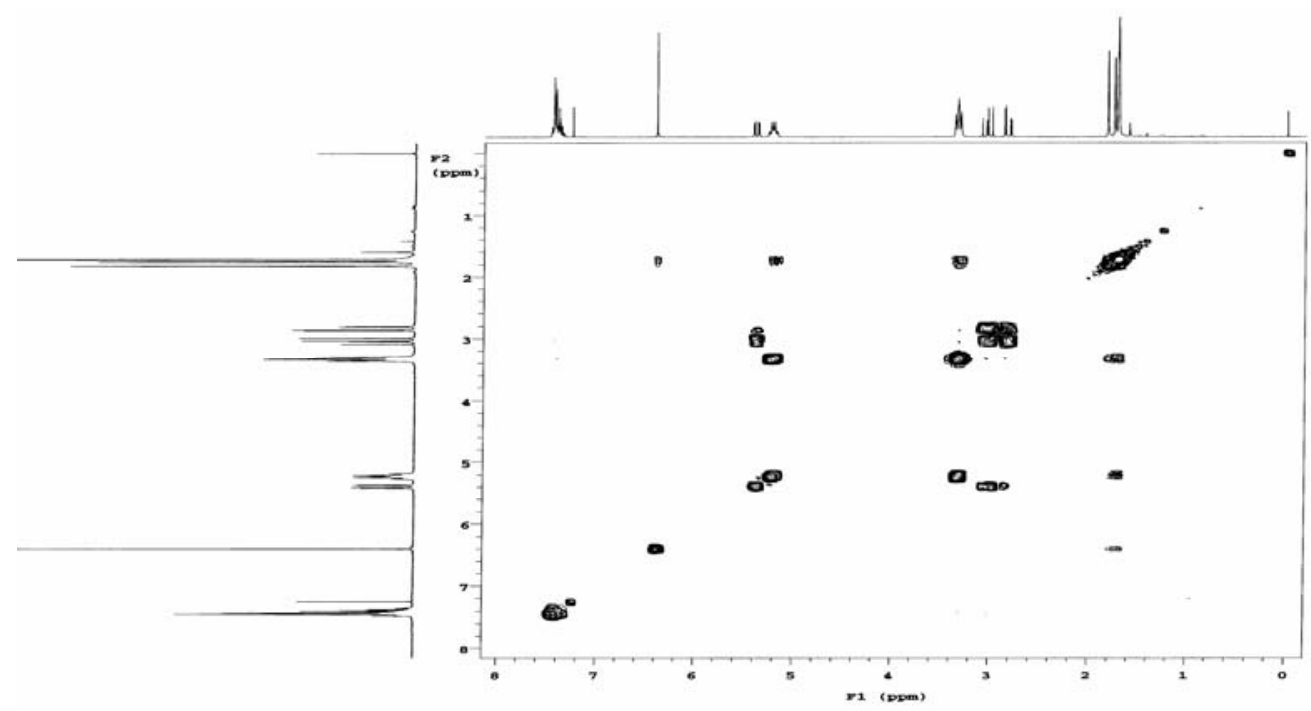

Figure 118: $\mathrm{H}-\mathrm{H}$ COSY spectrum $(300 \mathrm{MHz})$ of spinoflavanone $\mathrm{B}(\mathbf{1 0 3})$ in $\mathrm{CDCl}_{3}$.

From HMBC spectrum, the methylene protons at $\delta 43.4$ showed correlation with the only carbonyl group and with the phenyl group. The $\mathrm{OH}$ group $\delta 12.35$ was likely to be acid or chelated with the carbonyl group. All information derived from ${ }^{1} \mathrm{H}$ NMR, ${ }^{13} \mathrm{C}$ NMR, ESIMS and 2D spectroscopy supported the spinoflavanone B structure. The published spectroscopic and spectrometric data for spinoflavanone $\mathrm{B}^{125}$ were identical with that of the obtained structure $\mathbf{1 0 3}$. 


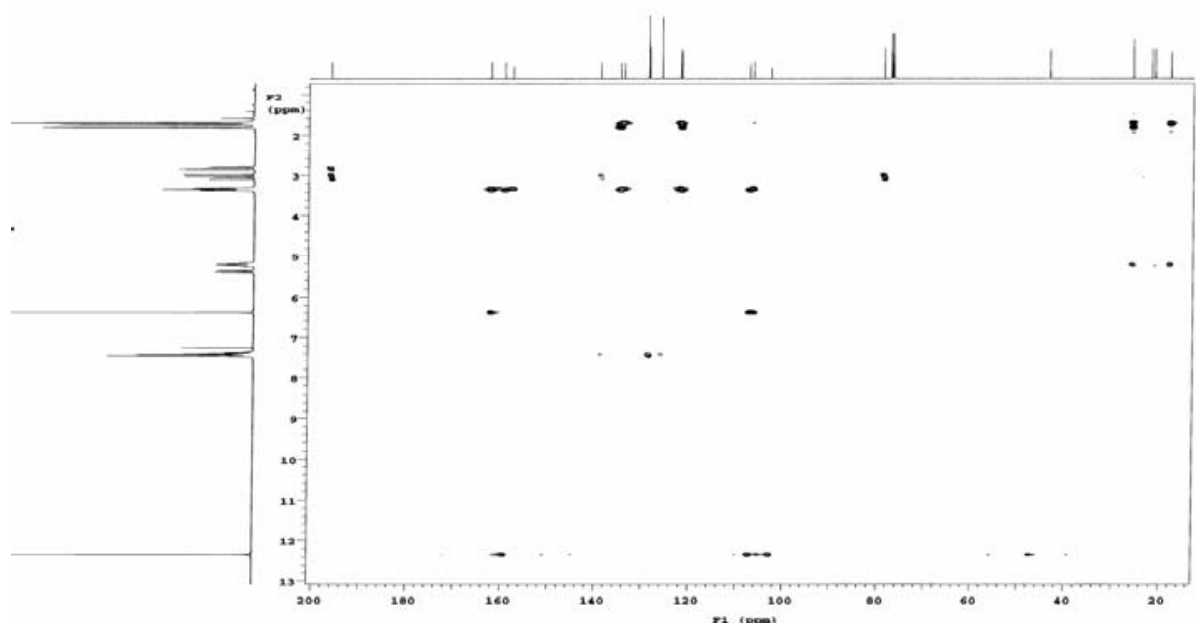

Figure 119: $\mathrm{HMBC}$ spectrum $(300 \mathrm{MHz})$ of spinoflavanone (103) in $\mathrm{CDCl}_{3}$.

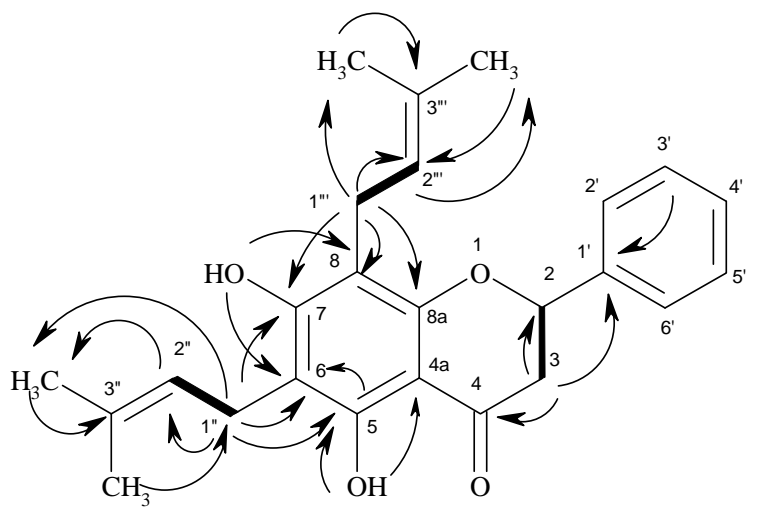

Figure 120: The HMBC $(\rightarrow)$ and $\mathrm{H}-\mathrm{H}$ COSY $(-)$ correlations of spinoflavone B (103).

\subsection{Caliphione A}

The molecular formula of compound $\mathbf{1 0 4}$ was obtained as white amorphous powder. HRESIMS $369.09699[\mathrm{M}+\mathrm{H}]^{+}$established the molecular formula as $\mathrm{C}_{20} \mathrm{H}_{17} \mathrm{O}_{7}$. The ${ }^{1} \mathrm{H}$ NMR spectrum displayed resonances for two hydrogen-bonded hydroxyls at $\delta_{\mathrm{H}}$ $12.24\left(\mathrm{OH}-6^{\prime}\right)$ and $\delta_{\mathrm{H}} 12.06(\mathrm{OH}-6 ")$, a singlet resonance for a methylenedioxy group at $\delta_{\mathrm{H}} 6.00\left(\mathrm{H}-2^{\prime}\right)$ and four well-separated $1 \mathrm{H}$ singlets for aromatic protons, $\delta_{\mathrm{H}} 7.23(\mathrm{H}-$ $4 "), \delta_{\mathrm{H}} 6.82\left(\mathrm{H}-4^{\prime}\right), \delta_{\mathrm{H}} 6.53\left(\mathrm{H}^{-} 7^{\prime}\right)$ and $\delta_{\mathrm{H}} 6.45$ (H- 7") clearly indicating that this compound consisted of two tetra-substituted rings. 


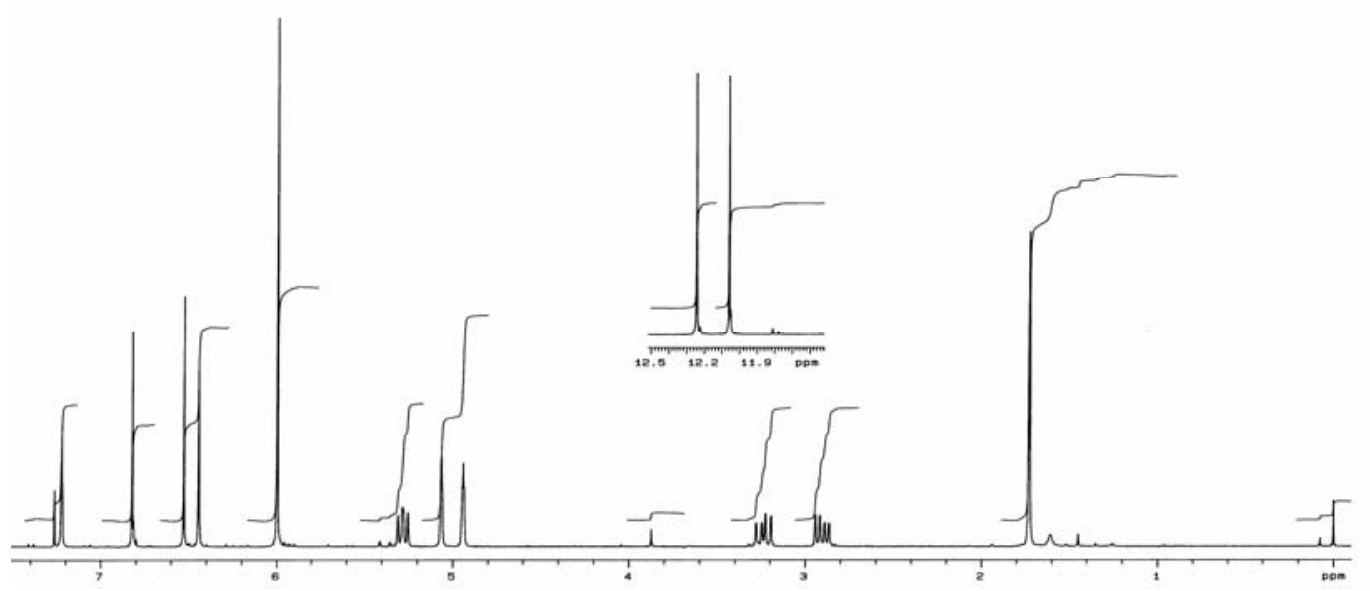

Figure 121: ${ }^{1} \mathrm{H}$ NMR spectrum $(300 \mathrm{MHz})$ of caliphione $\mathrm{A}(\mathbf{1 0 4})$ in $\mathrm{CDCl}_{3}$

In addition, the ${ }^{1} \mathrm{H}$ NMR spectrum exhibited an $\mathrm{ABX}$ system for a tri-substituted dihydrofuran ring comprising of an oxymethine at $\delta 5.30$ coupled to the gem-methylene protons at $\delta 3.30$ and signals for an exomethylene, $\delta 4.98$ and 5.10 as singlets and a methyl singlet at $\delta 1.72$ constituting the presence of an isopropenyl unit as part of the side chain.

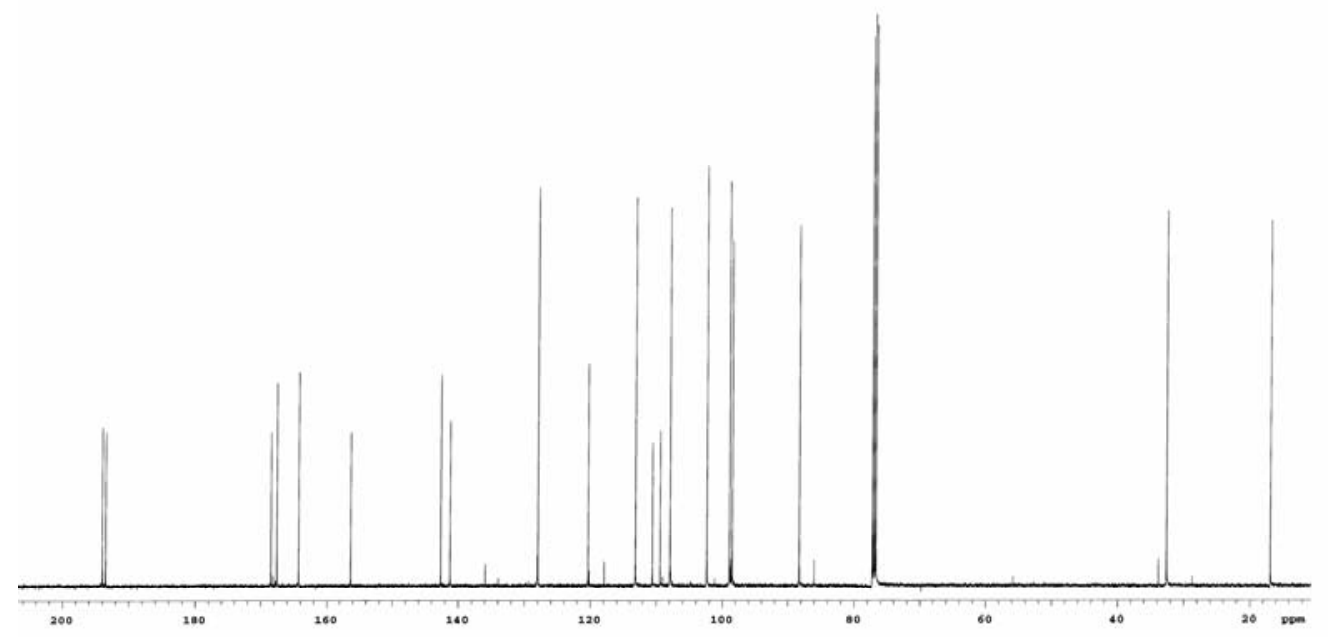

Figure 122: ${ }^{13} \mathrm{C}$ NMR spectrum $(125 \mathrm{MHz})$ of caliphione $\mathrm{A}(\mathbf{1 0 4})$ in $\mathrm{CDCl}_{3}$.

The ${ }^{13} \mathrm{C}$ NMR spectrum displayed resonances for two carbonyl signals at $\delta 194.0$ and 193.5 , together with signals corresponding to an oxymethine carbon $(\delta 88.3)$, an upfield methylene ( $\delta$ 32.6), eight quartenary carbons and a down-field $s p^{3}$ carbon at $\delta$ 102.4 , confirming to be associated with a benzil moiety, a benzofuran and a benzodioxy functionality.

From the H-H COSY spectrum the following substructure of the compound was elucidated. 

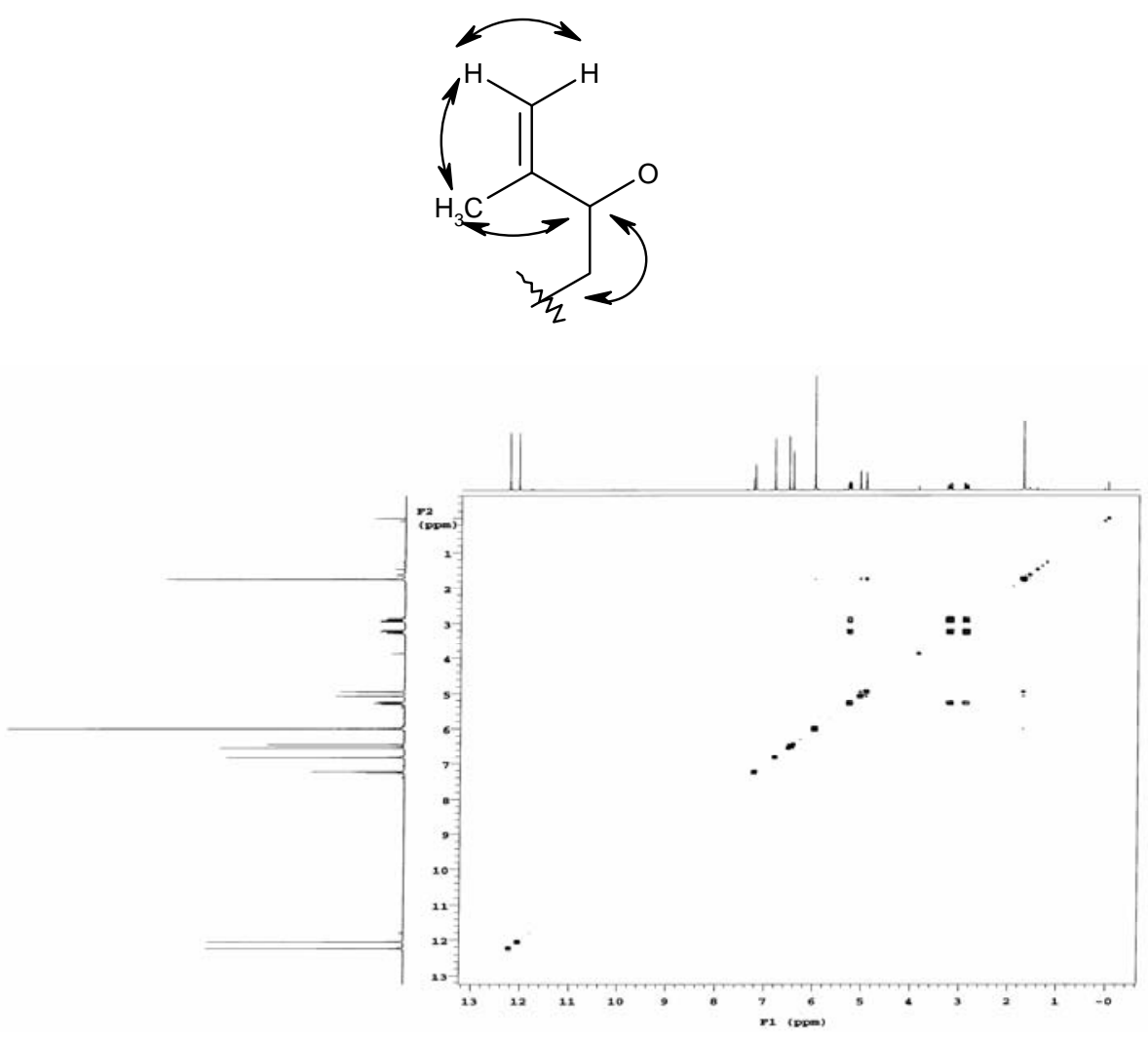

Figure 123: $\mathrm{H}-\mathrm{H}$ COSY spectrum $(600 \mathrm{MHz})$ of caliphione $\mathrm{A}(\mathbf{1 0 4})$ in $\mathrm{CDCl}_{3}$.

In HMBC spectrum the methylenedioxy protons showed correlation with the carbon C-3'a, 7a ( $\delta 141.2$ and 156.4). The protons H-4', 7' ( $\delta 6.82$ and $\delta 6.53)$ attached at C-4', $7^{\prime}(\delta 98.9$ and 108.0) showed correlation with both carbons at C-3'a, 7a $(\delta 141.2$ and $156.4)$, indicating that they are in the same aromatic ring. The proton at $\mathrm{H}-4$ ' $(\delta 6.82)$ also showed correlation with one oxygenated carbon at C-6' $\left(\delta\right.$ 167.6) and $\mathrm{C}_{\mathrm{q}}-5^{\prime}(\delta$ 109.4), while the proton H-7' ( $\delta 6.53$ ) showed correlation with one of the carbonyl at $\delta$ 194.0. From the value of the $\mathrm{OH}$ at $\delta 12.2$ it was clear that it was chelated.

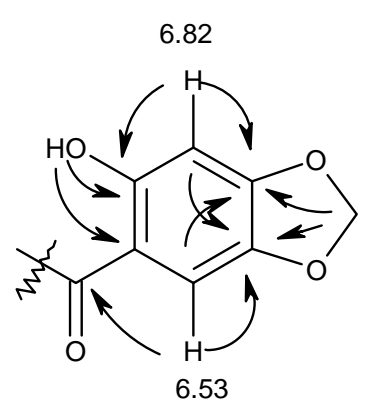

A

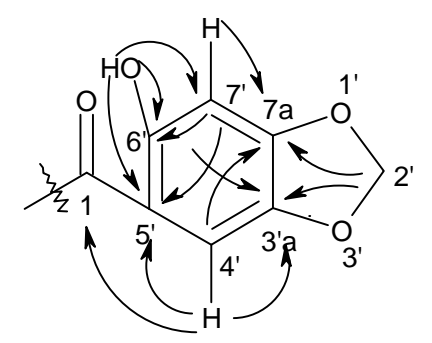

B

The HMBC spectrum showed another part (substructure C) with a similar pattern, which was connected with the substructures A and B already obtained from the $\mathrm{H}-\mathrm{H}$ COSY spectrum. 


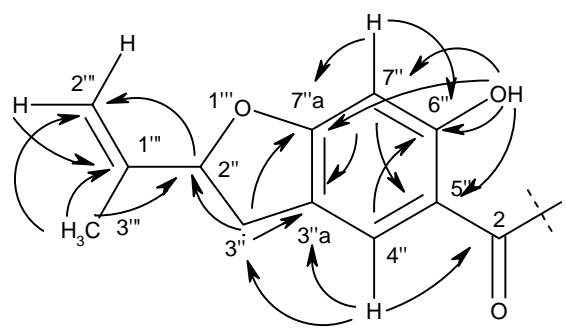

$\mathbf{C}$

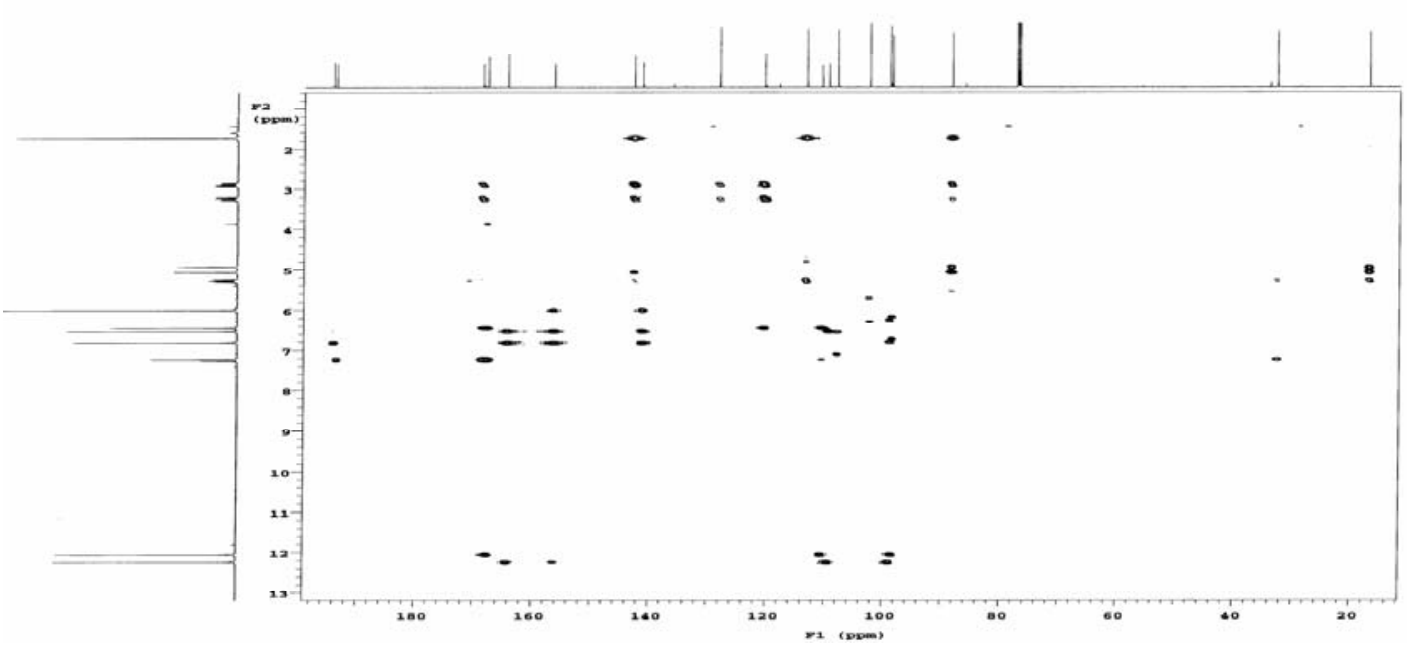

Figure 124: $\mathrm{HMBC}$ spectrum $(600 \mathrm{MHz})$ of caliphione $\mathrm{A}(\mathbf{1 0 4})$ in $\mathrm{CDCl}_{3}$.

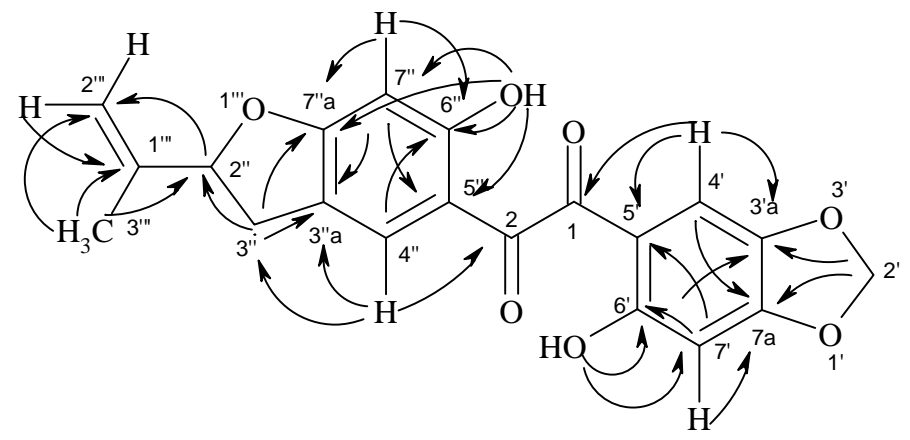

105<smiles>[R]Oc1cc2c(c(O)c1C(=O)Cc1ccc3c(c1)OCO3)CCC(C)(C)O2</smiles>

$\mathrm{R}=\mathrm{H}$ or $\mathrm{CH}_{3}$ 
From the 1D and 2D NMR data and comparison with those of published compound 106, ${ }^{126}$ structure 104 was confirmed.

\subsection{Tephcalostan}

Tephlocalostan (107) was obtained as colourless needles and showed a quasimolecular ion peak at $m / z 363.08641[\mathrm{M}+\mathrm{H}]^{+}$for $\mathrm{C}_{21} \mathrm{H}_{15} \mathrm{O}_{6}$. The ${ }^{1} \mathrm{H}$ NMR spectrum exhibited four aromatic singlet signals at $\delta 7.67,7.43,7.06$, and 6.87 , a methylenedioxy group at $\delta 6.05$ and an isopropenyl dihydrofuran moiety, visible from two diastereotropic proton signals at $\delta 3.45,3.13(A B X)$, an oxygenated methine proton signal at $\delta 5.33(A B X)$, two olefinic protons at $\delta 5.12\left(7^{\prime}-\mathrm{H}_{\mathrm{a}}\right), 4.94\left(7^{\prime}-\mathrm{H}_{\mathrm{b}}\right)$, and a methyl signal at $\delta 1.80\left(8^{\prime}-\mathrm{CH}_{3}\right)$.

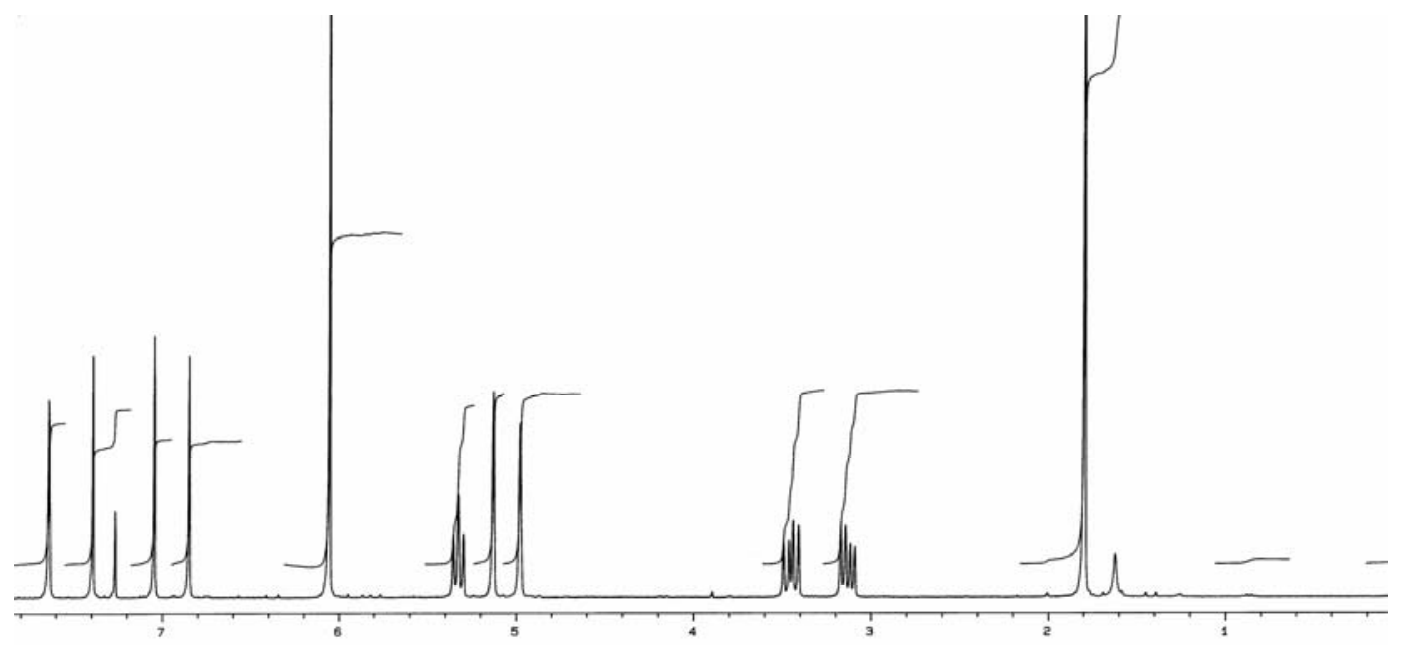

Figure 125: ${ }^{1} \mathrm{H}$ NMR spectrum $(300 \mathrm{MHz})$ of tephlocalostan (107) in $\mathrm{CDCl}_{3}$.

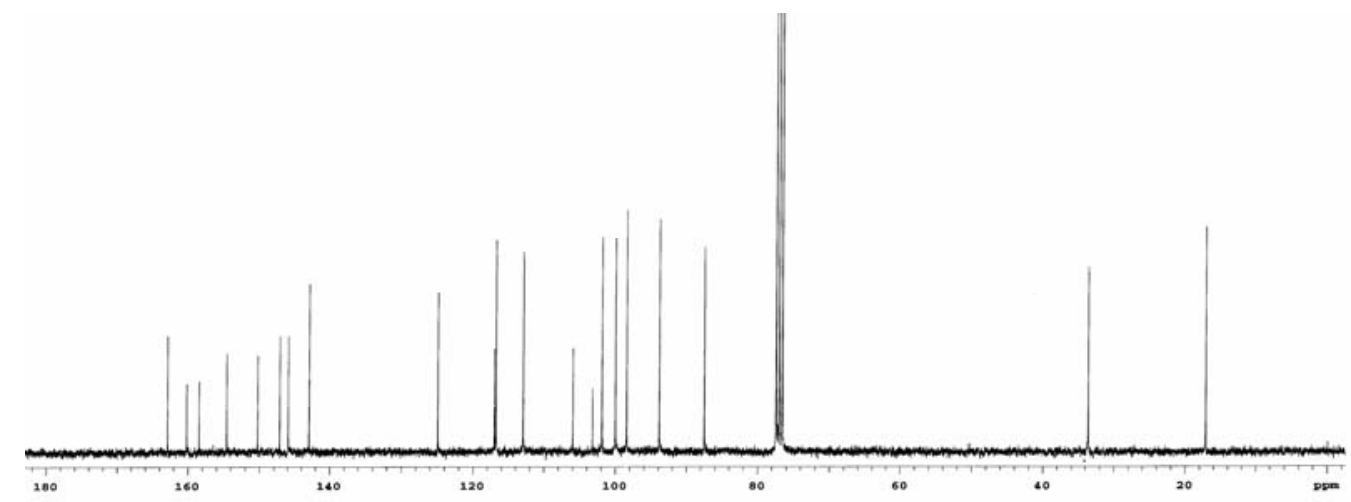

Figure 126: ${ }^{13} \mathrm{C}$ NMR spectrum $(75 \mathrm{MHz})$ of tephlocalostan (107) in $\mathrm{CDCl}_{3}$.

The ${ }^{13} \mathrm{C}$ NMR spectrum showed 21 carbon signals, of which 19 were placed in the $s p^{2}$ region. Since there was a methylenedioxy group $(\delta 6.05)$, this carbon signal was also 
observed in the $s p^{2}$ region. Based on the ${ }^{1} \mathrm{H}$ and ${ }^{13} \mathrm{C}$ NMR spectra and the substructures in addition to the molecular formula the compound was searched in Dictionary of Natural Products and Chemical Abstracts. The 1D and 2D NMR data was identical to the published values of tephlocalostan. ${ }^{127}$

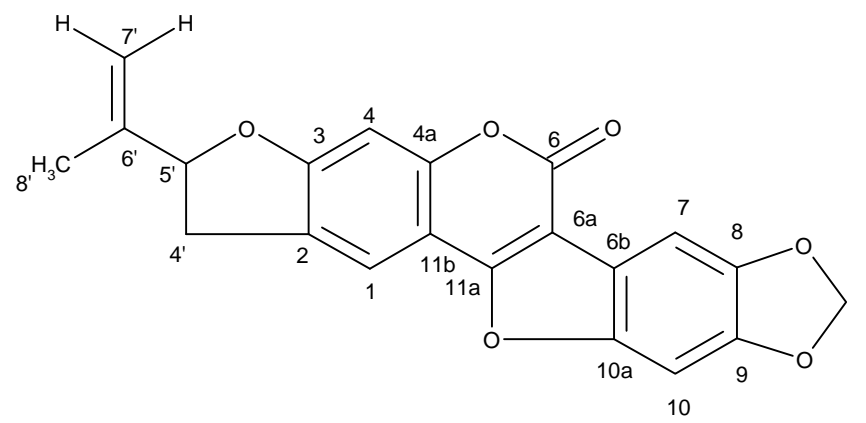

107

\subsection{Tephcalostan A}

A further new tephcalostan derivative $\mathbf{1 0 8}$ was obtained as colourless needles, which showed a $[\mathrm{M}+\mathrm{H}]^{+}$peak at $m / z 363.08641$ for $\mathrm{C}_{21} \mathrm{H}_{15} \mathrm{O}_{6}$. The ${ }^{1} \mathrm{H}$ NMR spectrum exhibited signals for four aromatic protons at $\delta 7.80,7.43,7.28$ and 6.96, a methylenedioxy group at $\delta 6.13$ and an allyl dihydrofuran moiety, differentiable from two diastereotopic proton signals $\delta 3.58,3.13(A B X)$, an oxygenated methane proton signal $\delta 5.43(\mathrm{AB} X)$ and two olefinic protons at $\delta 5.45\left(7^{\prime}-\mathrm{H}_{\mathrm{a}}\right)$ and $5.28\left(7^{\prime}-\mathrm{H}_{\mathrm{b}}\right)$.

The ${ }^{13} \mathrm{C}$ NMR spectrum showed 21 carbon signals, among which 19 were observed in the $s p^{2}$ region. As the methylenedioxy group $(\delta 6.05)$ is present, the respective carbon also appeared in the $s p^{2}$ region $(\delta 101.6)$.

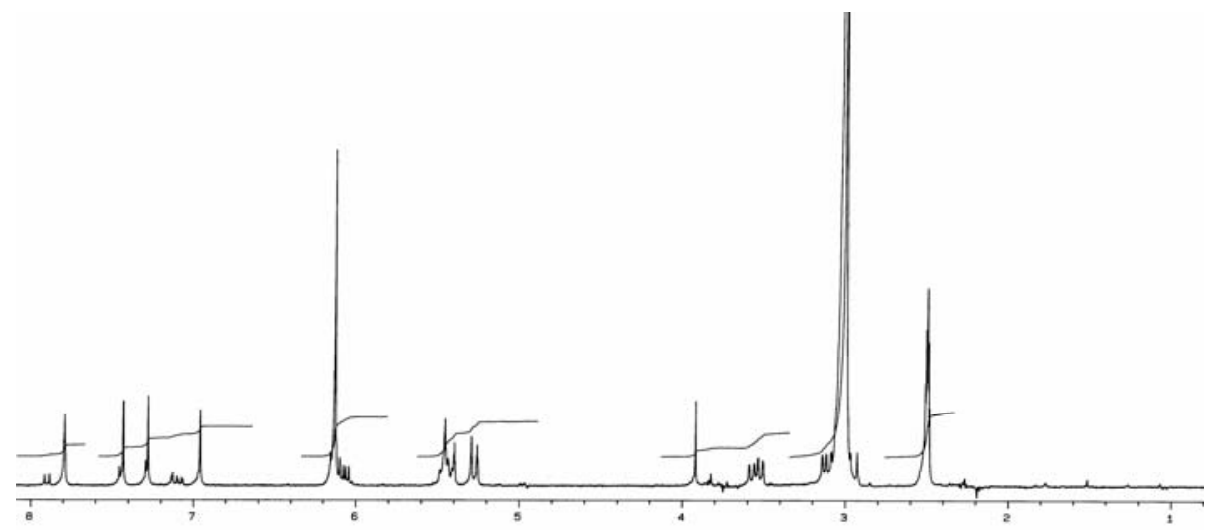

Figure 127: ${ }^{1} \mathrm{H}$ NMR spectrum $(300 \mathrm{MHz})$ of tephcalostan A (108) in $\left[\mathrm{D}_{6}\right] \mathrm{DMSO}$. 


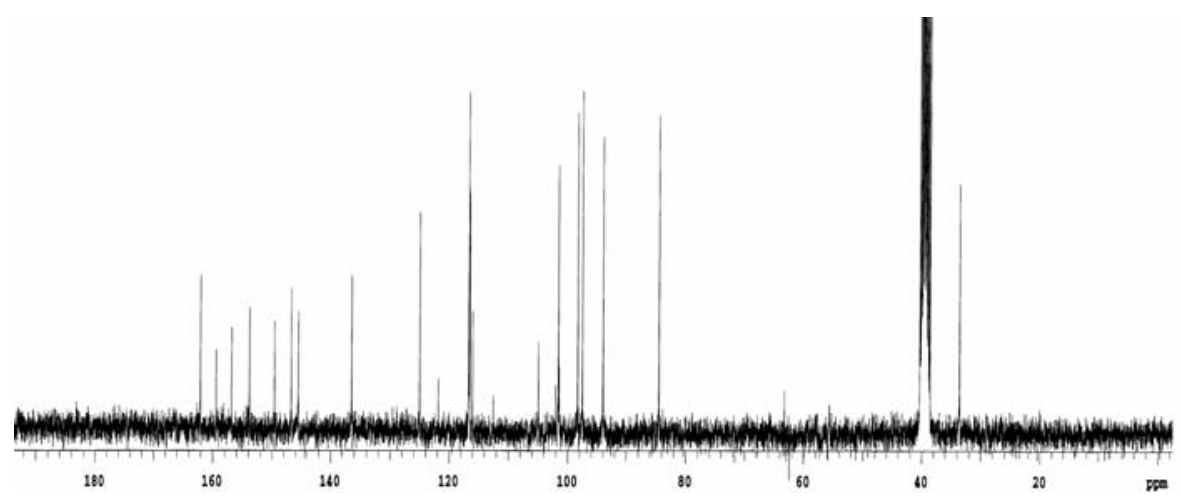

Figure 128: ${ }^{13} \mathrm{C}$ NMR spectrum $(125 \mathrm{MHz})$ of tephcalostan $\mathrm{A}(\mathbf{1 0 8})$ in $\left[\mathrm{D}_{6}\right] \mathrm{DMSO}$.

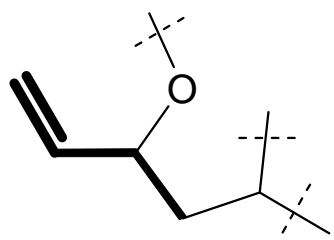

A

Figure 129: From the H-H COSY spectrum the fragment A was found.

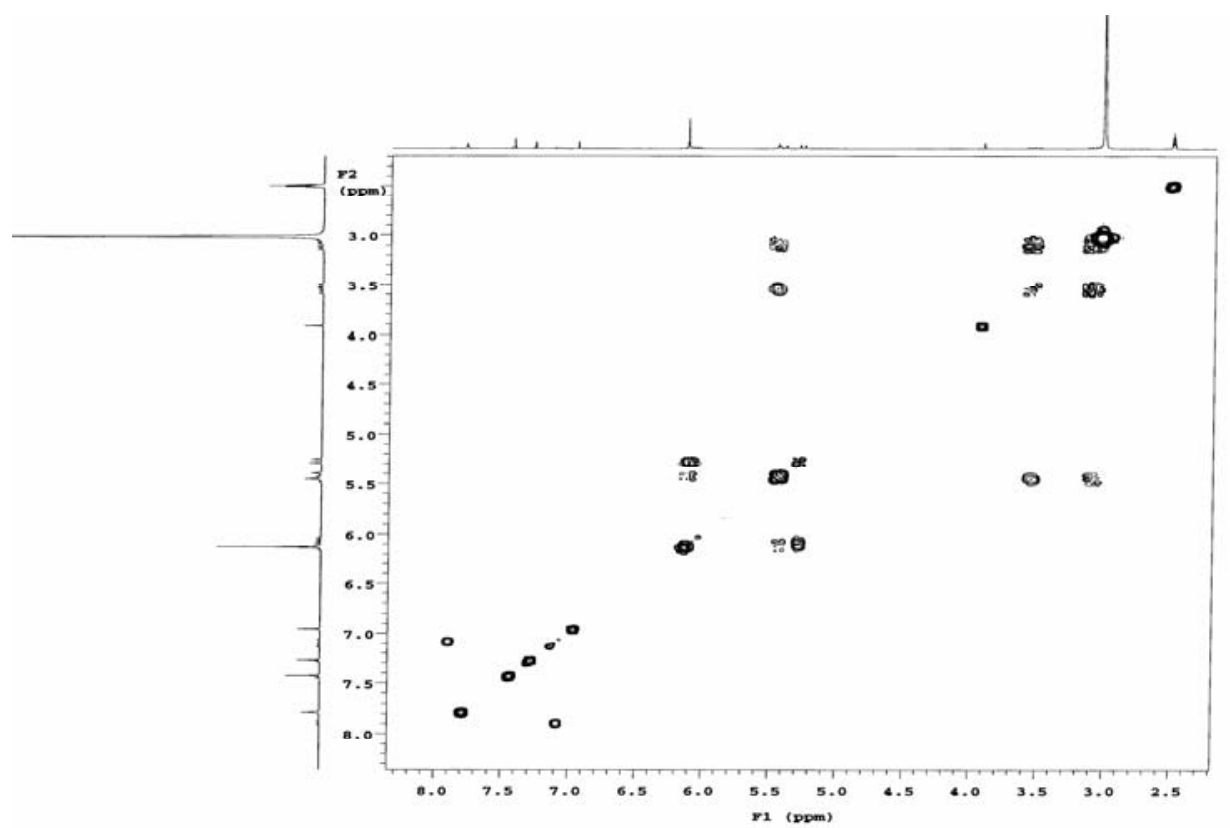

Figure 130: H-H COSY spectrum $(600 \mathrm{MHz})$ of tephcalostan A (108) in $\left[\mathrm{D}_{6}\right] \mathrm{DMSO}$.

Further confirmation was obtained from HMBC correlations between the exocyclic methylene protons at $\delta 5.28$ and 7'-H ( $\delta$ 5.45) and the olefinic carbon C-6' $(\delta$ 136.5) and ${ }^{2} J$ coupling between the oxymethine, H-5' $(\delta 5.43)$ of the dihydrofuran ring and the $s p^{2}$ carbon at $\delta 136.5$. 


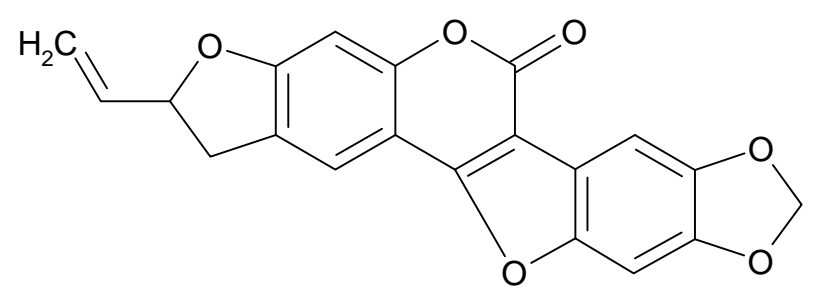

108

According to Chemical Abstracts this was a new compound. Tephlocastan A (108) was confirmed by comparing the $1 \mathrm{D}$ and $2 \mathrm{D}$ data with the related tephlocastan (109) from the same plant. ${ }^{127}$ NMR data are closely related to those of $\mathbf{1 0 9}$, which has one methyl group more in 6'-position. ${ }^{128}$<smiles></smiles>

109

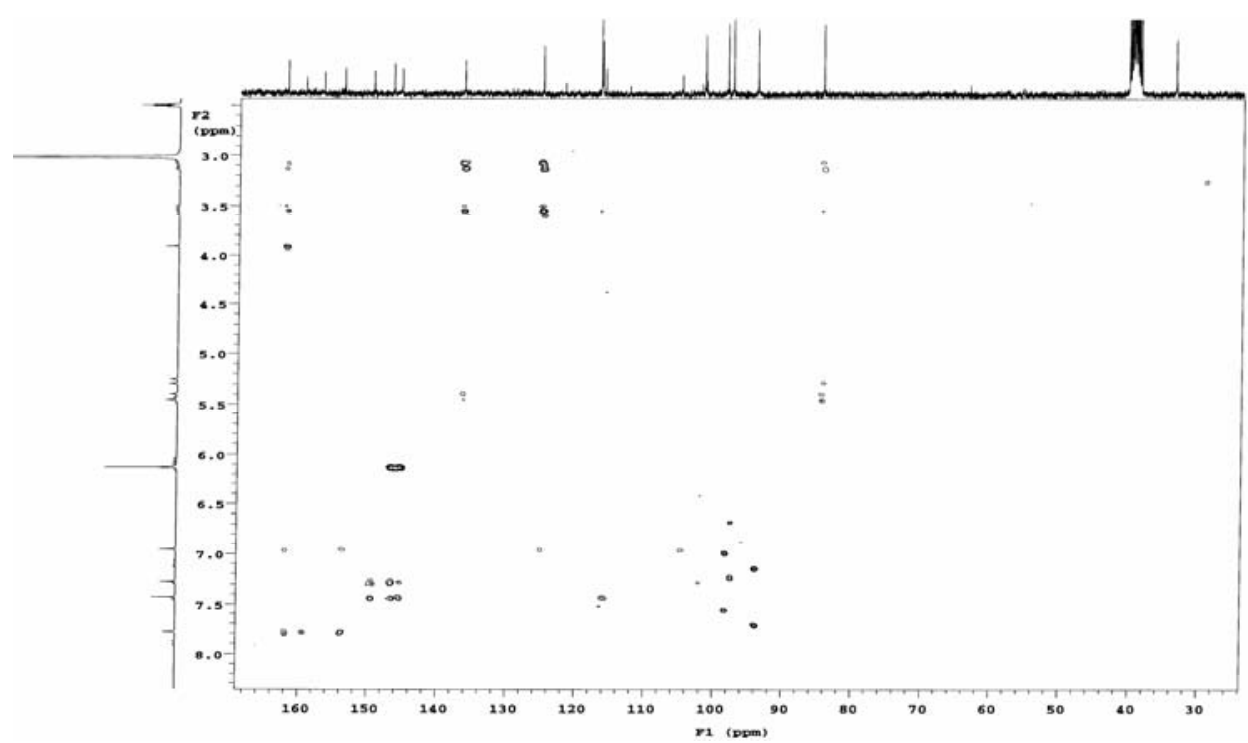

Figure 131: HMBC spectrum $(600 \mathrm{MHz})$ of tephcalostan A (108) in $\left[\mathrm{D}_{6}\right] \mathrm{DMSO}$.

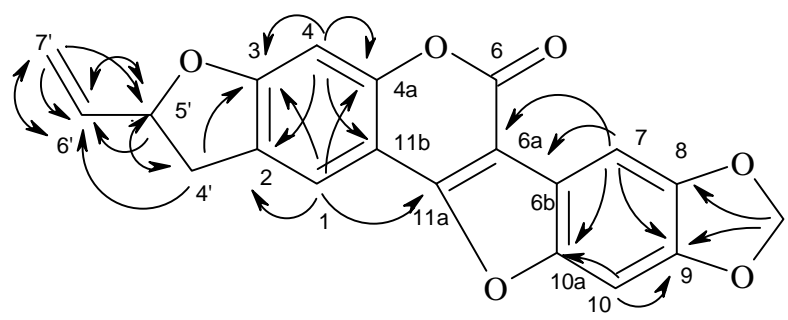




\subsection{Tephcalostan B and C}

Tephcalostan B (111) and C (112) were white solids with poor solubility in all common organic solvents including DMSO. However, at $100{ }^{\circ} \mathrm{C}$ the compound was dissolved in DMSO. From the ESIMS spectrum, two masses were found at $\mathrm{m} / z, 379$ ([M $\left.\mathrm{H}^{-}\right)$and $377\left(\left[\mathrm{M}-\mathrm{H}^{-}\right)\right.$. HRESIMS delivered the formulas $\mathrm{C}_{21} \mathrm{H}_{15} \mathrm{O}_{7}$ and $\mathrm{C}_{21} \mathrm{H}_{17} \mathrm{O}_{7}$. In the ${ }^{1} \mathrm{H}$ NMR spectrum from the integrations a mixture of two closely related compounds was assumed. From the mass difference and the ${ }^{1} \mathrm{H}$ NMR spectrum it was revealed that the two compounds differed only by one double bond.

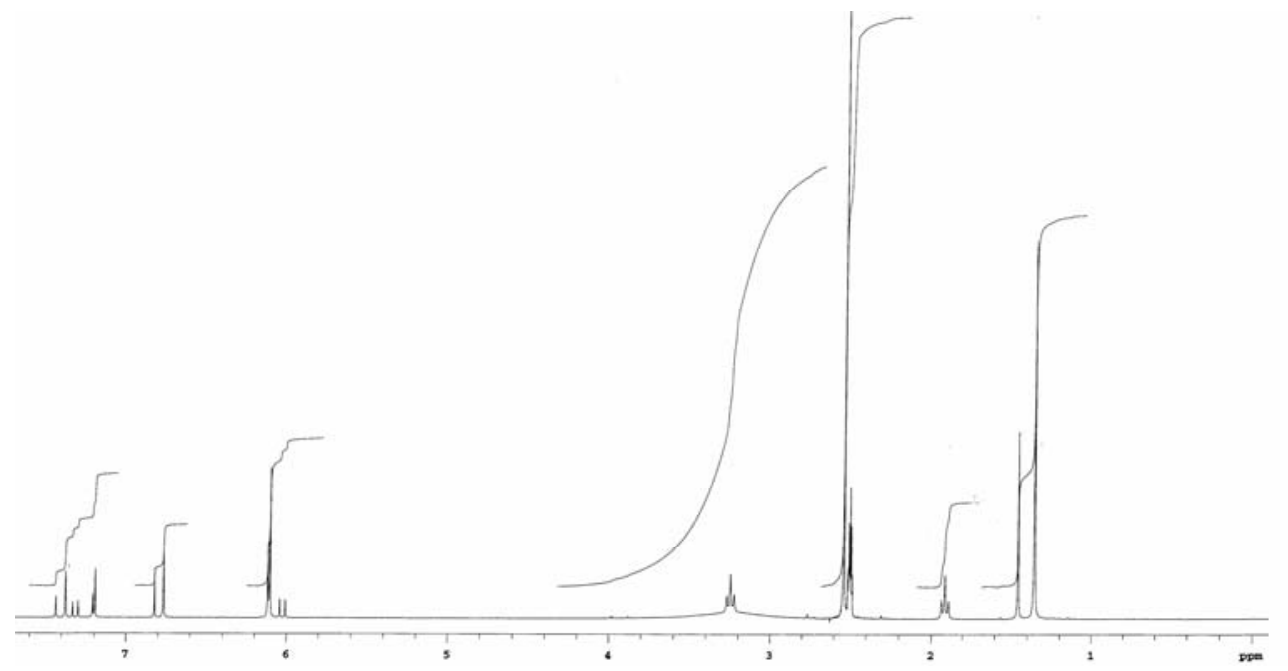

Figure 132: 1H NMR spectrum (125 MHz) of tephcalostan B (111) and C (112) in [D6]DMSO.

Also from the ${ }^{13} \mathrm{C}$ NMR spectrum the evidence of two different compounds was clear. Due to the poor solubility and the similarity in the mass we did not try to separate them but the two compounds could be easily distinguished from the spectra. A search using HRESIMS and ${ }^{1} \mathrm{H}$ NMR in the Dictionary of Natural Products and the Chemical Abstracts did not yield hits.

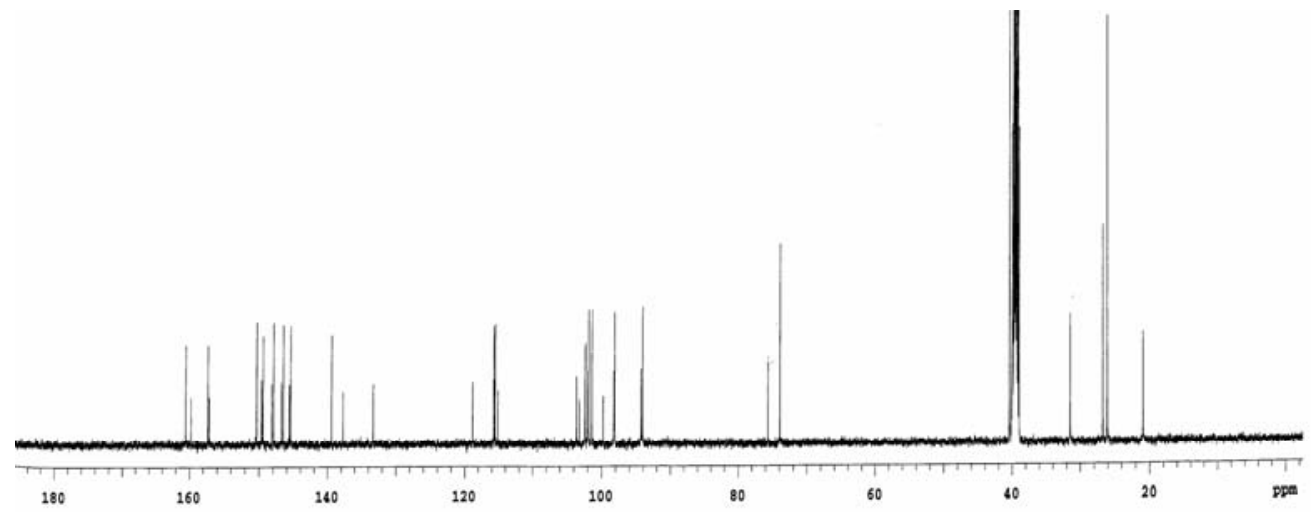

Figure 133: ${ }^{13} \mathrm{C}$ NMR spectrum $(300 \mathrm{MHz})$ of Tephcalostan $\mathrm{B}(\mathbf{1 1 1})$ and $\mathrm{C}(\mathbf{1 1 2})$ in $\left[\mathrm{D}_{6}\right] \mathrm{DMSO}$. 
In ${ }^{1} \mathrm{H}$ NMR and ${ }^{13} \mathrm{C}$ NMR spectrum the intensity of the signals were not proportional indicating a mixture of compounds. From the HSQC and H-H COSY it was clear that this a mixture of two closely related compounds.

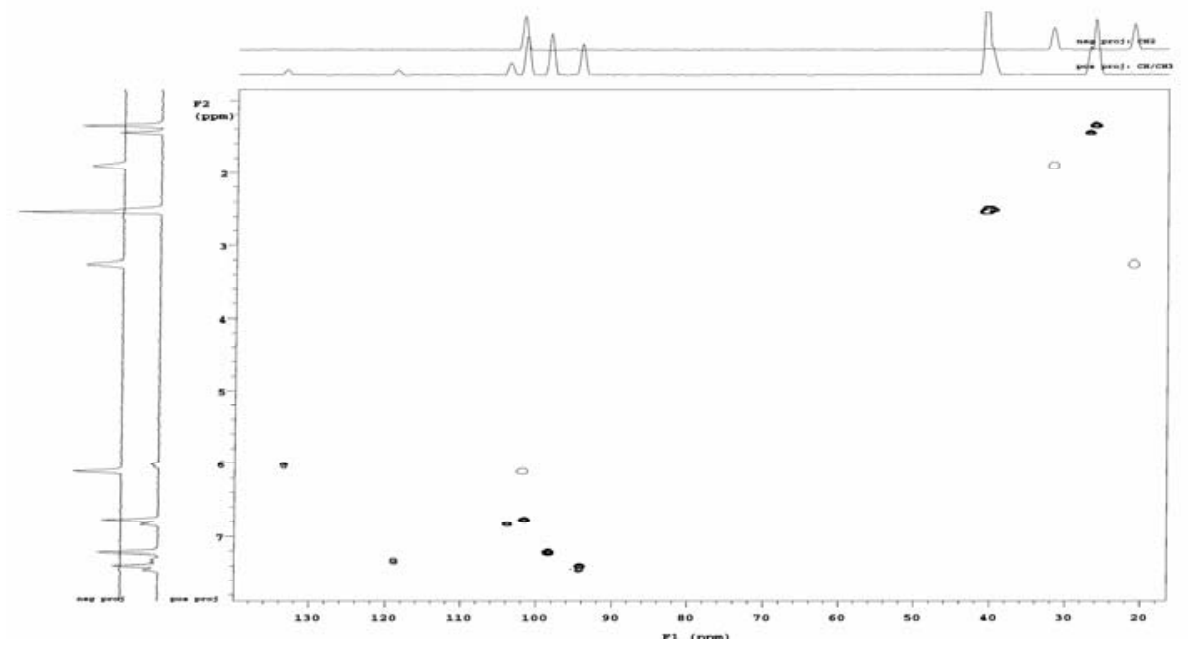

Figure 134:HSQC spectrum (600 MHz) of Tephcalostan B (111) and C (112) in $\left[\mathrm{D}_{6}\right] \mathrm{DMSO}$.

From the HMBC spectrum, a quaternary carbon at $\delta 157.2$ showed no correlation at all. The methylenedioxy at $\delta 6.10$ showed correlation with C-6 and C-7. Additionally, the protons at $\mathrm{H}-5$ and $\mathrm{H}-8$ showed correlations with $\mathrm{C}-6$ and $\mathrm{C}-7$. The proton at $\mathrm{H}-5$ showed two more correlations with $\mathrm{C}-3$ and C-4. The two methyl groups at $\delta 1.36$ showed correlation with carbon signal at C-2". The olefinic proton at H-5" also showed correlation with C-2". Considering all the HMBC correlations and with the help of COSY unit A was drawn. Unit B showed the similar correlations except the olefinic proton $\delta$ 6.02. This bond was saturated. Taking all the substructures, 1D and 2D NMR compounds 111 and 112 were drawn. They were further confirmed by comparison with the related isoglycyrol (110). ${ }^{128,129}$

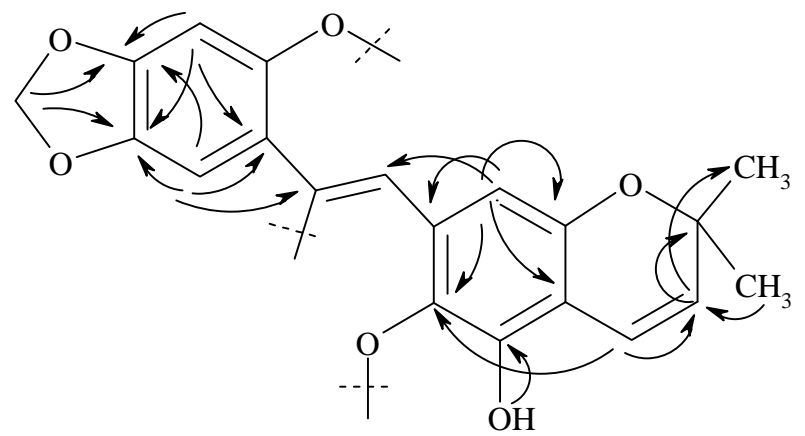

Figure 135: Fragment of tephcalostan B (Unit A). 


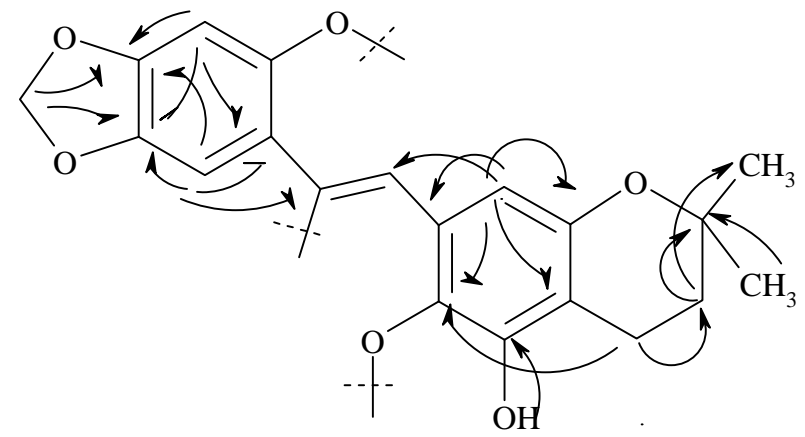

Figure 136: Fragment of tephcalostan C (Unit B).<smiles>COc1c2c(cc3c1C1Oc4cc(O)ccc4C1C(=O)O3)OC(C)(C)CC2</smiles>

110

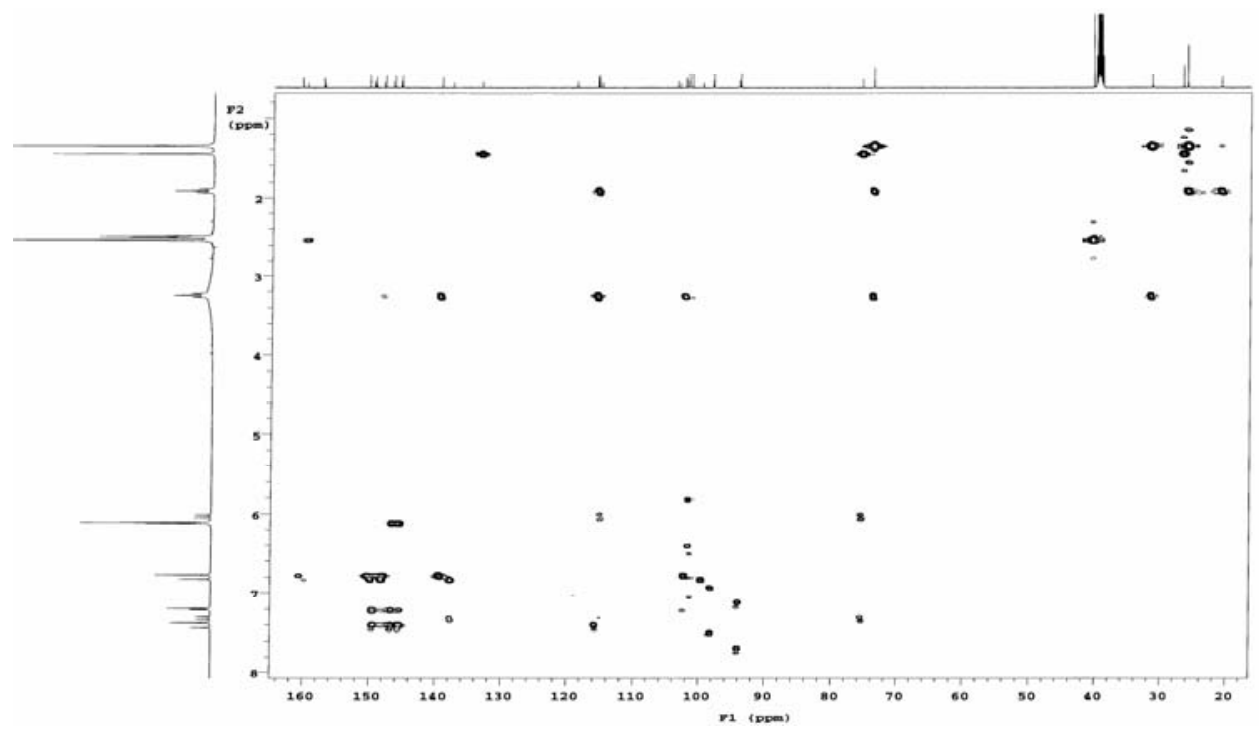

Figure 137: $\mathrm{HMBC}$ spectrum $(600 \mathrm{MHz})$ of tephcalostan $\mathrm{B}(\mathbf{1 1 1})$ and $\mathrm{C}(\mathbf{1 1 2})$ in $\left[\mathrm{D}_{6}\right] \mathrm{DMSO}$. 


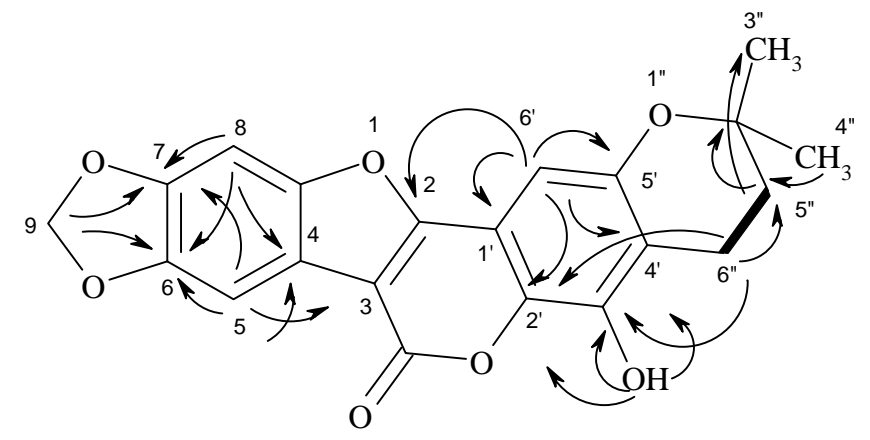

111

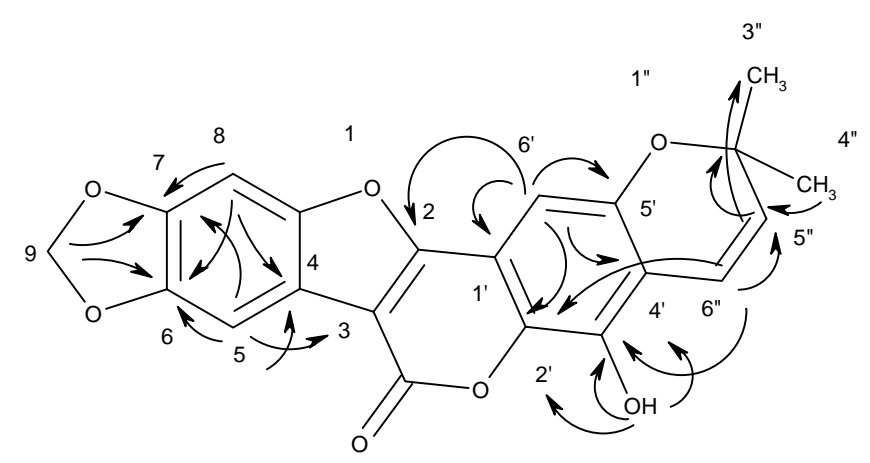

112

\subsection{Milletone}

Compound 113 was obtained as yellow needles, showed a $[\mathrm{M}+\mathrm{H}]^{+}$peak at $379.11766[\mathrm{M}+\mathrm{H}]^{+}$established the molecular formula $\mathrm{C}_{21} \mathrm{H}_{19} \mathrm{O}_{6}$ by HRESIMS. The ${ }^{1} \mathrm{H}$ NMR spectrum exhibited signals for six aromatic protons, while a methylenedioxy group was found at $\delta 5.85$. From the molecular formula there were 13 double bond equivalents calculated. The shift of two methine protons at $\delta 4.90$ and 3.79 indicated the attachment of oxygen. There were 2 methyl singlets and one methylene at $\delta 4.62$ and 4.17 also observed in ${ }^{1} \mathrm{H}$ NMR spectrum.

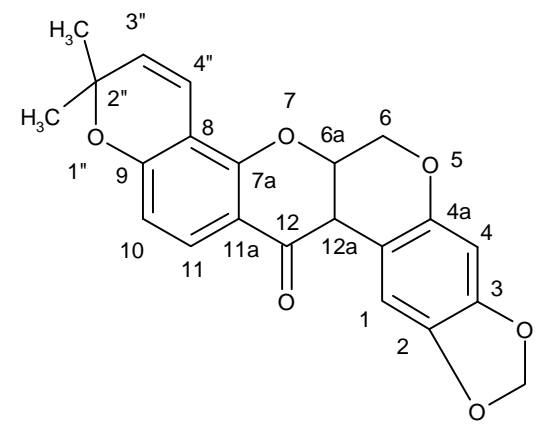




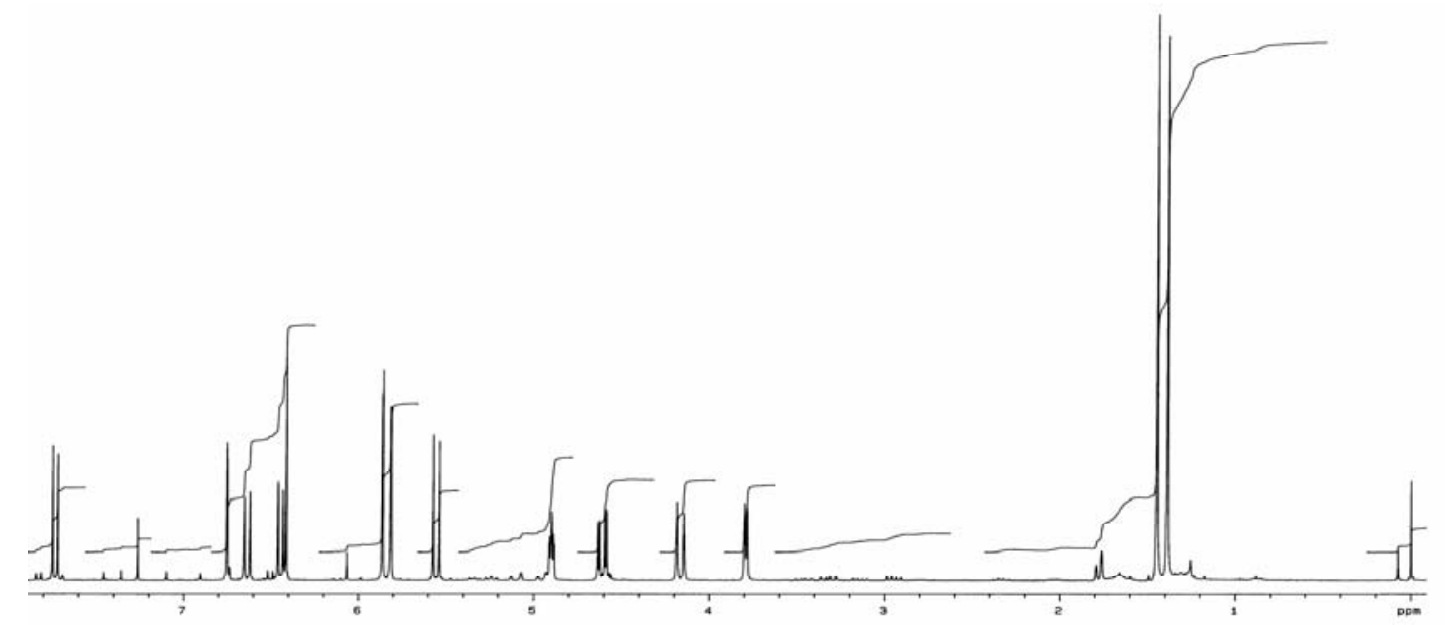

Figure 138: ${ }^{1} \mathrm{H}$ NMR spectrum $(300 \mathrm{MHz})$ of milletone (113) in $\mathrm{CDCl}_{3}$.

In the ${ }^{13} \mathrm{C}$ NMR spectrum there was a carbonyl at $\delta 189.1$, which is typical for flavones. There were five $s p^{2}$ carbons, which seemed to be attached to oxygen. There were also $3 s p^{3}$ carbon signals at $\delta 77.7\left(\mathrm{C}_{\mathrm{q}}\right), 72.1(\mathrm{CH})$, and $66.2\left(\mathrm{CH}_{2}\right)$.

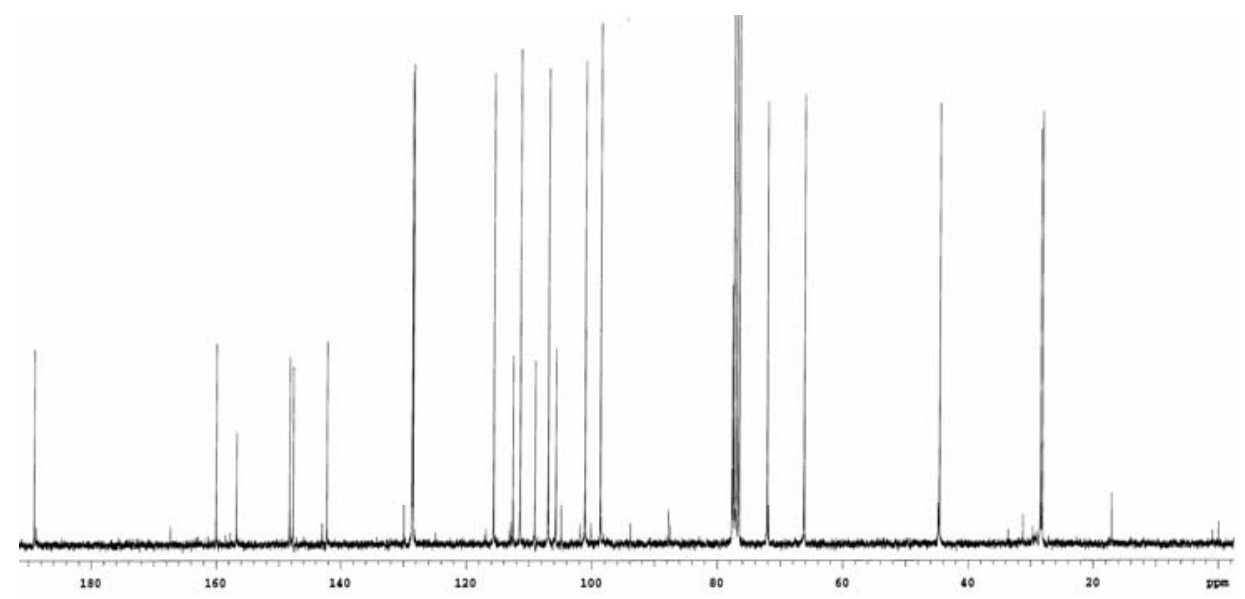

Figure 139: ${ }^{13} \mathrm{C}$ NMR spectrum $(125 \mathrm{MHz})$ of milletone (113) in $\mathrm{CDCl}_{3}$.

Five fragments (A-E) were derived from the H-H COSY spectrum. Substructures A and B were two pairs $o$-coupled olefinic protons, substructure C and D were also olefinic but without neighbouring proton.<smiles>CC=CC(C)(C)C</smiles>

A<smiles>CCC=CC(C)(C)C</smiles>

B<smiles>[Z]C=C(C)C(C)(C)C</smiles>

C<smiles>CC=C(CC)C(C)(C)C</smiles><smiles>[CH][C@H](C)[C@H](C)C(C)C</smiles>

D
$\mathbf{E}$ 


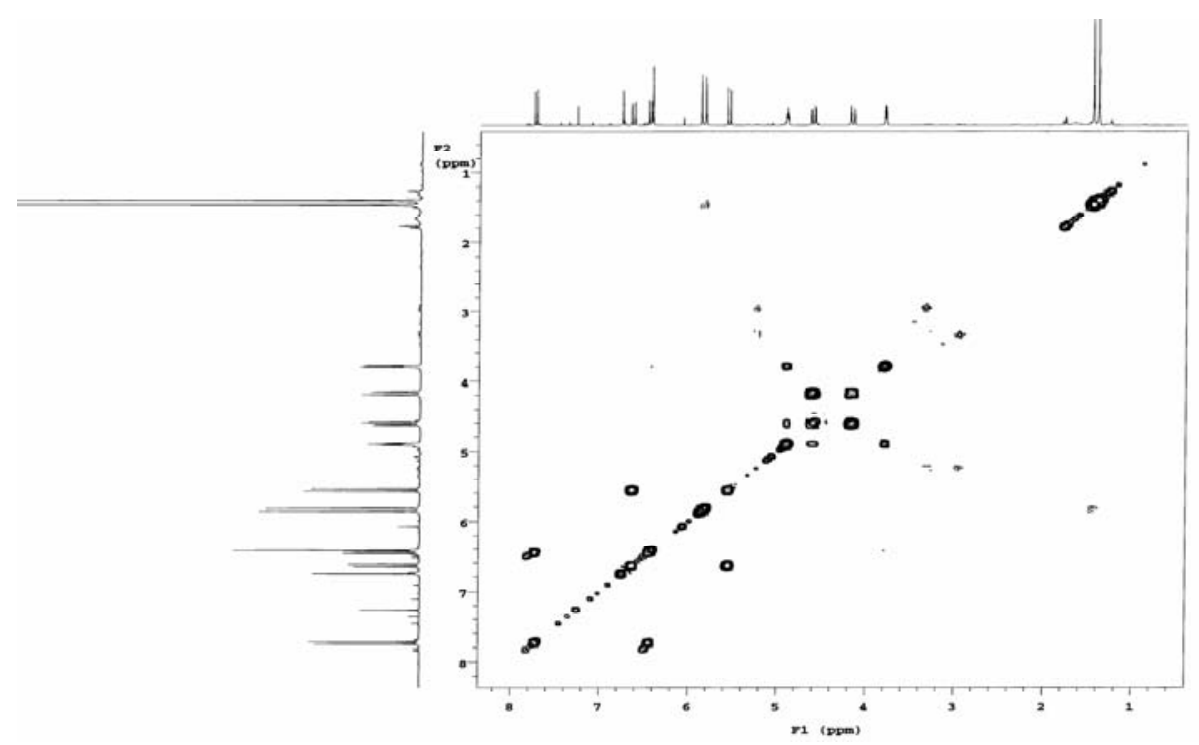

Figure 140: $\mathrm{H}-\mathrm{H}$ COSY spectrum $(600 \mathrm{MHz})$ of milletone (113) in $\mathrm{CDCl}_{3}$

In the HSQC spectrum a methylenedioxy group ( $\delta 5.85)$ was observed. However, it was displayed as a doublet in ${ }^{1} \mathrm{H}$ NMR spectrum, which was assumed to be due to 2 singlets of two different conformers in the ratio $1: 1$.

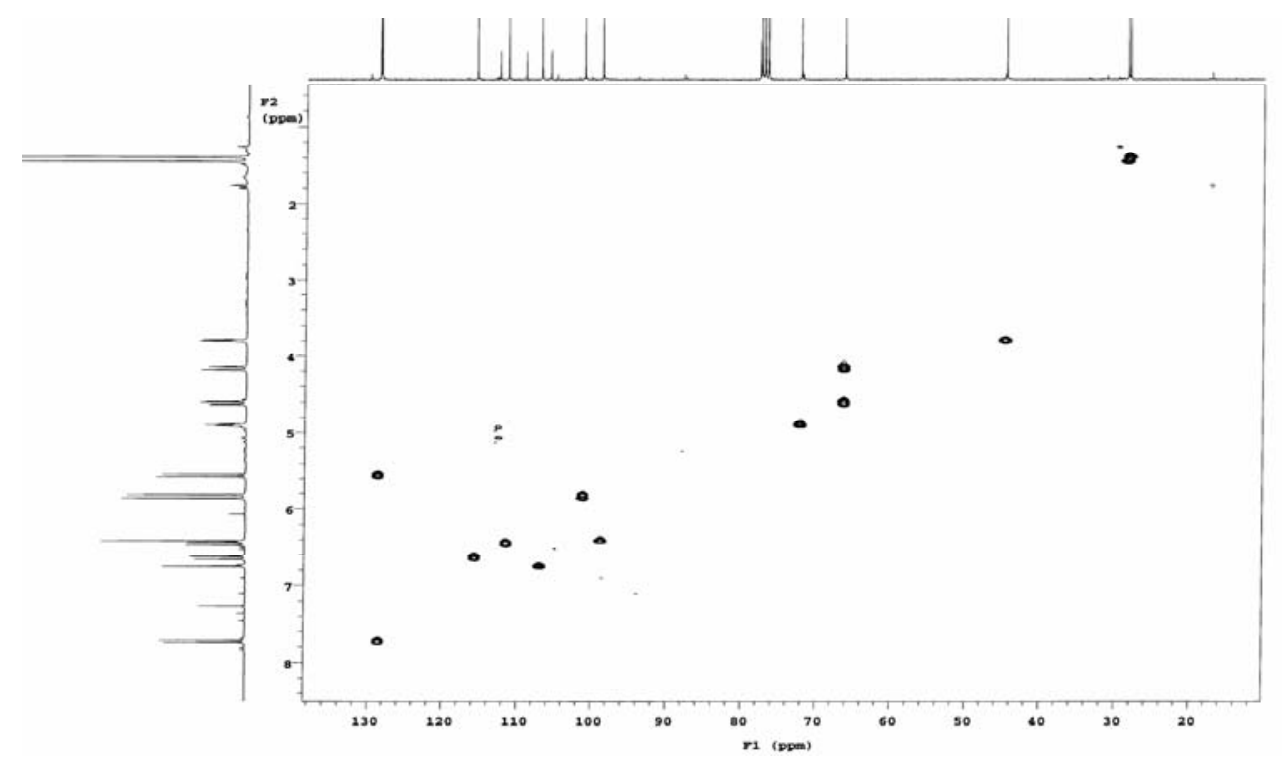

Figure 141: HSQC spectrum $(600 \mathrm{MHz})$ of milletone (113) in $\mathrm{CDCl}_{3}$.

In the HMBC spectrum the methylenedioxy group at $\delta 5.85$ showed correlations with signals at C-3 $(\delta$ 147.7) C-2 ( $\delta$ 142.2). Two aromatic protons at H-1 $(\delta 6.75)$ and H-4 $(\delta$ 6.41) also showed correlation with the same carbons indicating that they were in the same ring. As a result, the substructure A was derived from the HMBC spectrum. 


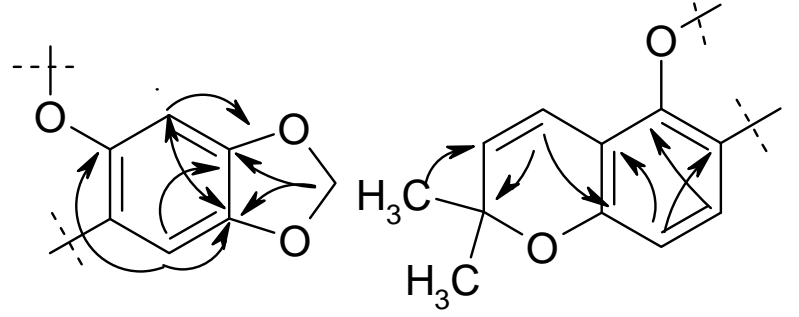

A

By using the HRMS, H-H COSY and HMBC spectra and the substructure A, the compound was searched in Dictionary of Natural Products and Chemical Abstract. The structure was confirmed as milletone after comparing with spectroscopic data published in the literature. ${ }^{130}$

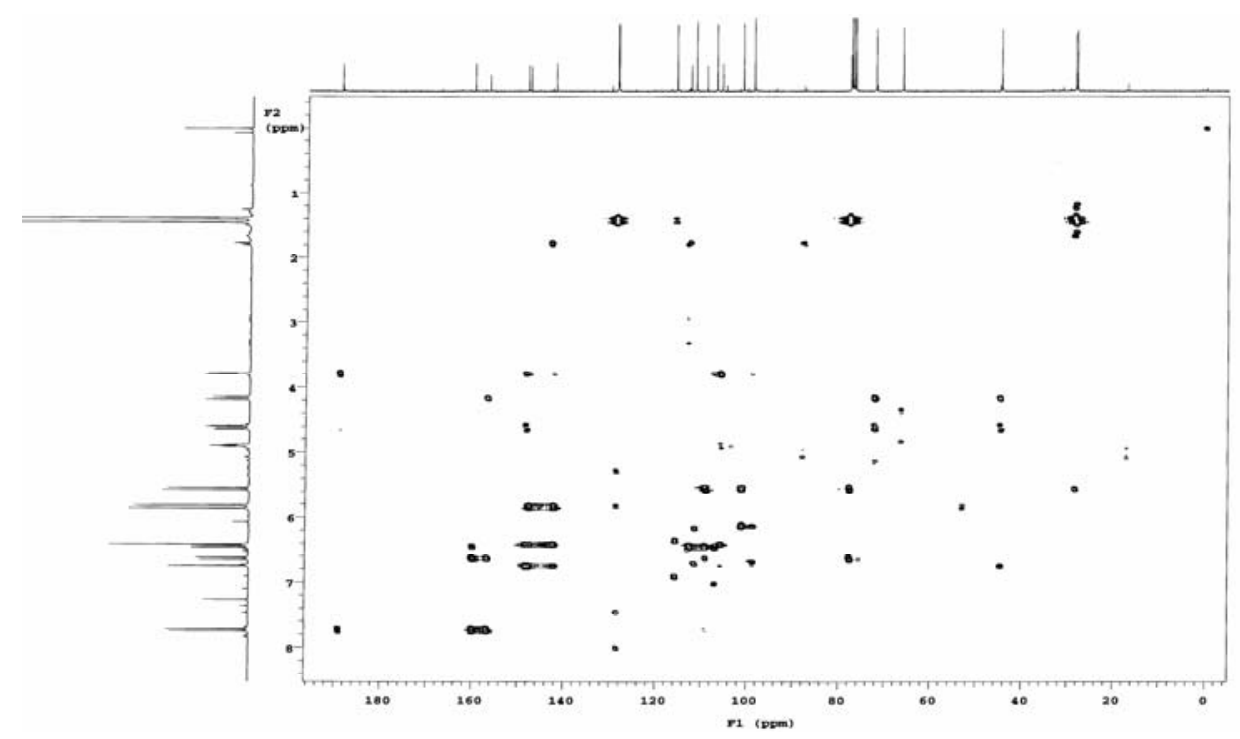

Figure 142: $\mathrm{HMBC}$ spectrum $(600 \mathrm{MHz})$ of milletone $(113)$ in $\mathrm{CDCl}_{3}$.

\subsection{Glabranine}

Glabranine (114) was isolated as a colourless solid. The ${ }^{1} \mathrm{H}$ NMR spectrum showed the resonances typical for the saturated ring of a flavanone. A set of peaks characteristic of a C-3-methylbut-2-enyl subunit was observed at $\delta 5.22(\mathrm{t}), \delta 3.32\left(\mathrm{~d}, \mathrm{CH}_{2}\right)$ and $\delta$ $\left.1.71\left(\mathrm{CH}_{3}\right)_{2}\right)$. The ${ }^{1} \mathrm{H}$ NMR spectrum showed two singlets, one at $\delta 12.00$ attributed to a chelated phenolic hydroxyl and another at $\delta 6.55$ attributed to a phenolic hydroxyl. A broad singlet at $\delta 7.45$ integrating for five protons was attributed to the unsubstituted B-ring. 


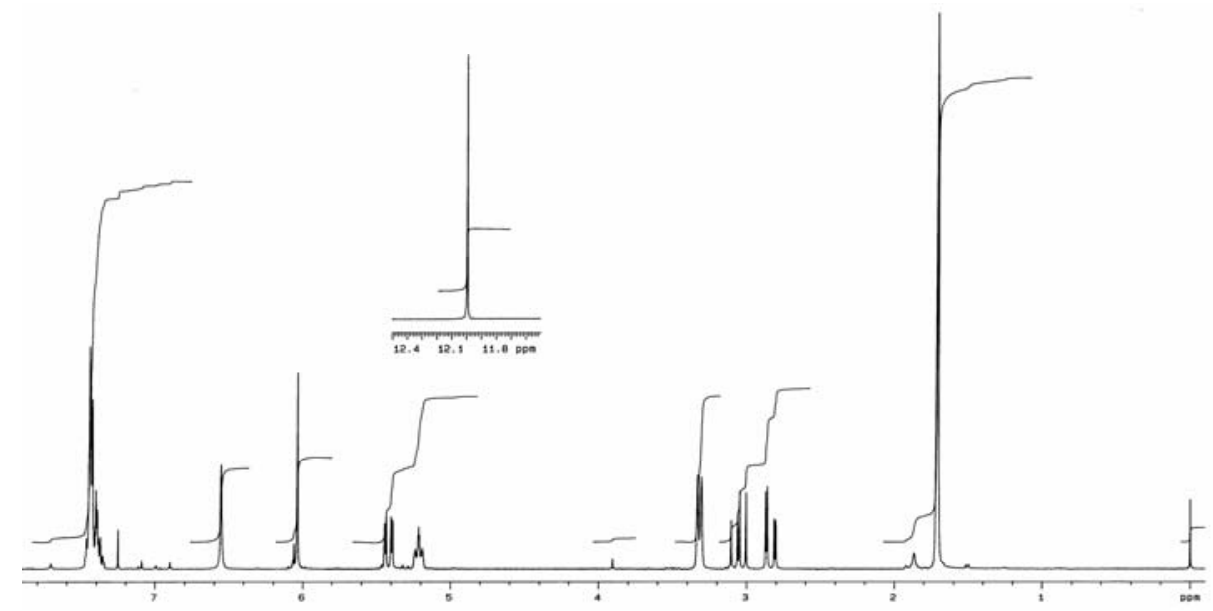

Figure 143: ${ }^{1} \mathrm{H} \mathrm{NMR}$ spectrum $\left(\mathrm{CDCl}_{3}, 300 \mathrm{MHz}\right)$ of glabranine (114).

In the ${ }^{13} \mathrm{C}$ NMR spectrum $17 s p^{2}$ carbons were observed, among which that at $\delta 196.3$ indicated the presence of a ketone or aldehyde and three at $\delta 163.8,162.2$, and 159.7 indicated the attachment of oxygen. There was also one methine carbon at $\delta 78.9$, which is also obviously attached to oxygen. Two methylene carbons were observed at $\delta 43.2$, and 21.8 (two) as well as 2 methyl carbons at $\delta 25.8$ and 17.8. The compound was identified as glabranine (114) by comparing the $1 \mathrm{D}$ and 2D NMR data with the literature. $^{131}$

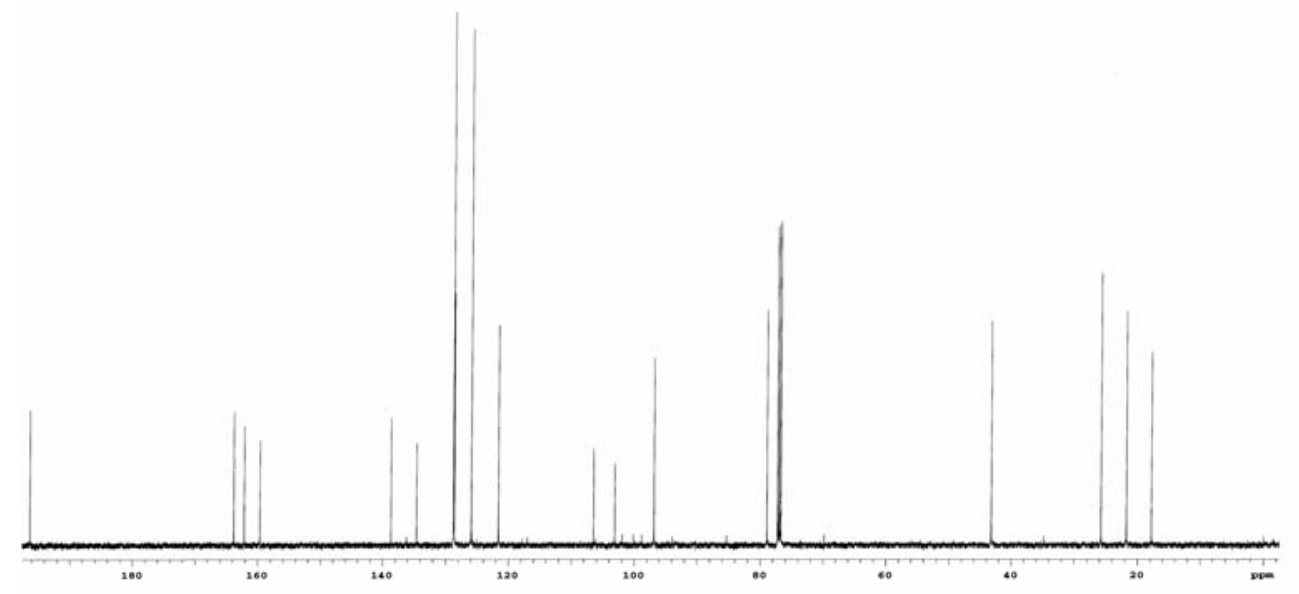

Figure 144: ${ }^{13} \mathrm{C}$ NMR spectrum $\left(\mathrm{CDCl}_{3}, 125 \mathrm{MHz}\right)$ of glabranine (114).<smiles>CC(C)=CCc1c(O)cc(O)c2c1OC(c1ccccc1)CC2=O</smiles> 


\subsection{Betulinic acid and Betulinic acid methyl ester}

The mass spectrum of compound 115 showed a molecular ion at $m / z 456$ by EIMS, which was in agreement with the molecular formula $\mathrm{C}_{30} \mathrm{H}_{48} \mathrm{O}_{3}$. Compound 116 was prepared by methylation of 115 with diazomethane. The ${ }^{1} \mathrm{H}$ and ${ }^{13} \mathrm{C}$ NMR data suggested that this compound could be a triterpene. This fact was confirmed by the similarity of the ${ }^{1} \mathrm{H}$ and ${ }^{13} \mathrm{C}$ NMR spectra of compound 115 with those of lupeol or betulinic acid (115). The ${ }^{1} \mathrm{H}$ NMR spectrum of compound 115 revealed the presence of six tertiary methyl groups at $\delta 0.65,0.77,0.98,0.87,0.93$ and an isopropenyl group at $\delta 1.65,4.70$ and 4.56 . The ${ }^{13} \mathrm{C}$ NMR spectrum showed the typical pattern ${ }^{132}(\delta$ $177.7,150.8,110.9$, and 77.3) of a 3-hydroxy lupeol or betulinic acid derivative. The other carbon signals were found in the $s p^{3}$ region. To determine the position of the carboxylic acid, the compound was methylated with diazomethane. In the proton spectrum, the expected methoxy group was visible, but did not show any NOESY correlations. The spectra were, however, identical with those of spectra and published values of authentic betulinic acid (115), so that also identity of their structures was assumed. ${ }^{133,134}$

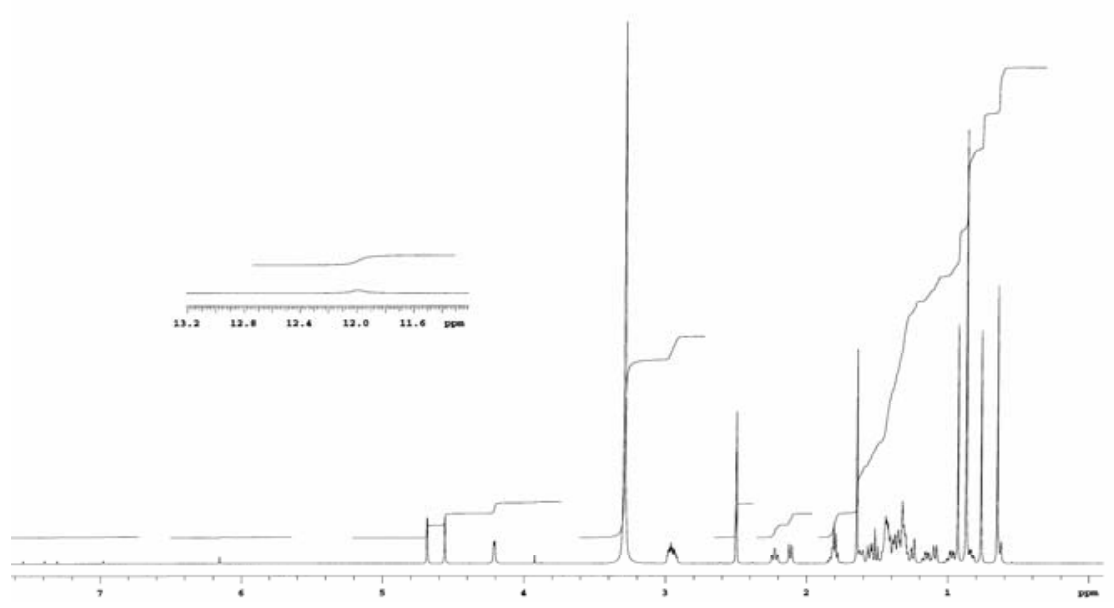

Figure 145: ${ }^{1} \mathrm{H}$ NMR $(300 \mathrm{MHz})$ spectrum of betulinic acid (115) in $\left[\mathrm{D}_{6}\right] \mathrm{DMSO}$. 


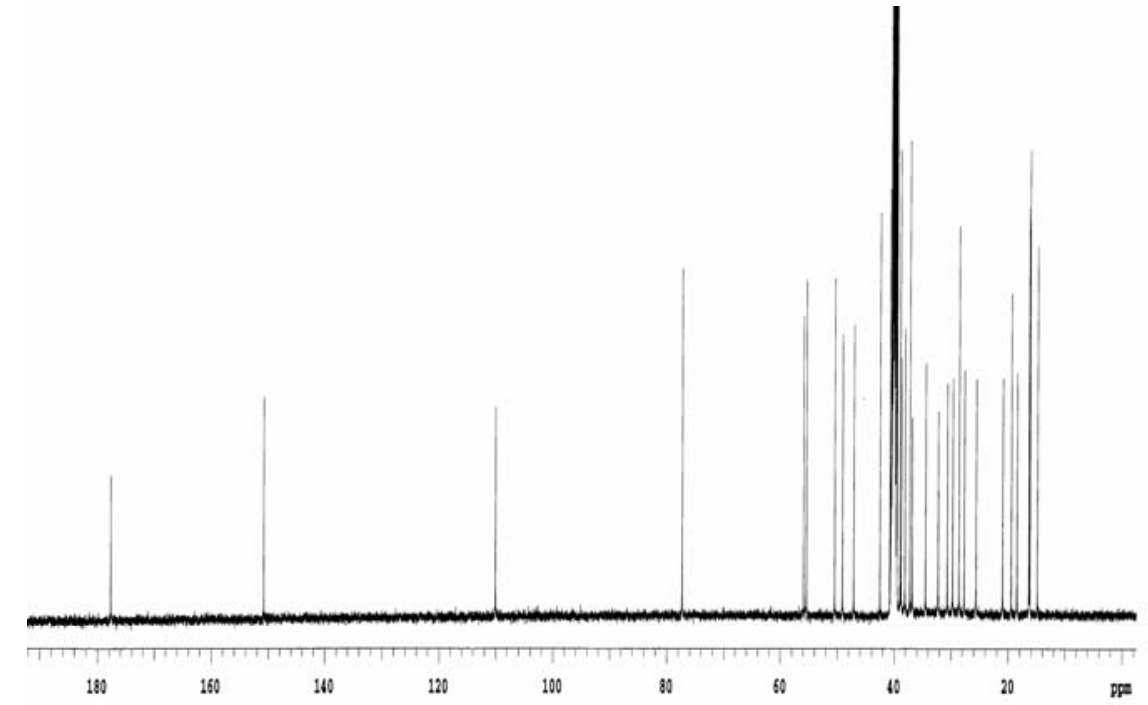

Figure 146: ${ }^{13} \mathrm{C}$ NMR (125 MHz) spectrum of betulinic acid (115) in $\left[\mathrm{D}_{6}\right] \mathrm{DMSO}$.<smiles>[3H]C(C(=C)C)C(C)(C)C</smiles>

Figure 147: The isoprenyl system was confirmed by from the H-H COSY spectrum.

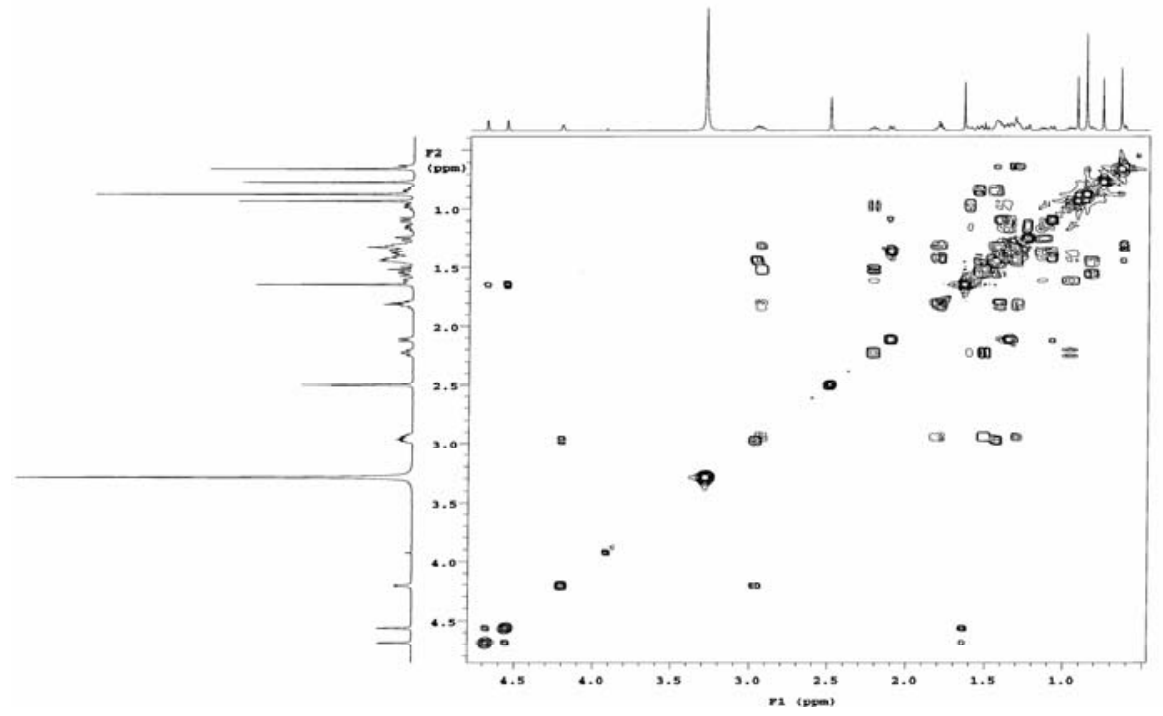

Figure 148: H-H COSY spectrum $(600 \mathrm{MHz})$ of betulinic acid (115) in [ $\left.\mathrm{D}_{6}\right] \mathrm{DMSO}$. 


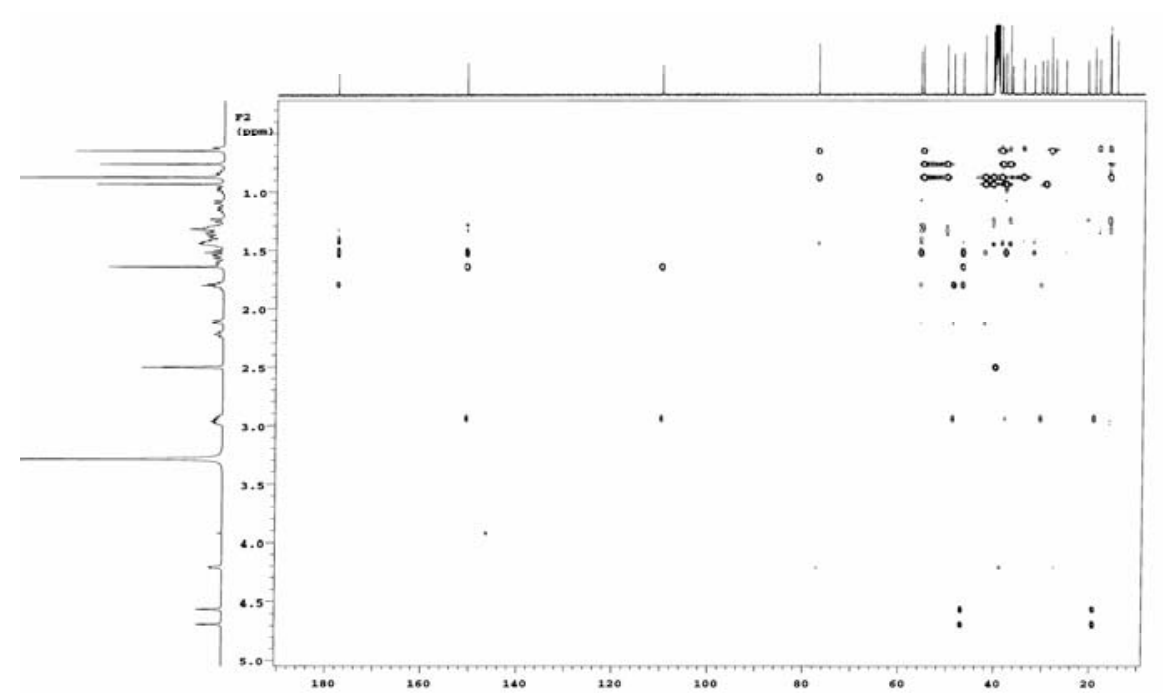

Figure 149: HMBC spectrum $(600 \mathrm{MHz})$ of betulinic acid (115) in [D 6 DMSO.

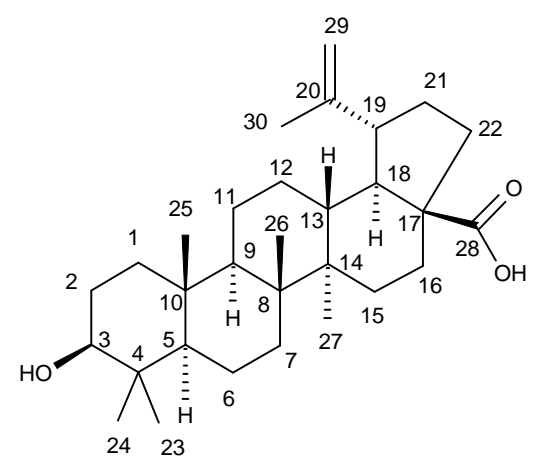

115

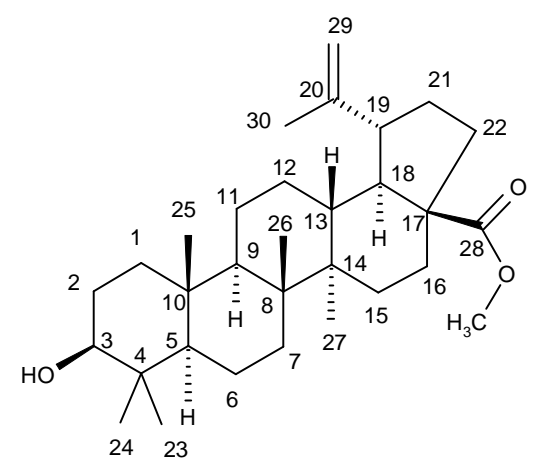

116

\subsection{Stigmasterol and $\gamma$-Sitosterol}

Stigmasterol (117) and $\gamma$-sitosterol (118) were isolated as a white crystalline mixture. From EIMS, the molecular weight was determined as $m / z 414$ and 412. It was thought that one of the compounds was present as impurity. In the ${ }^{1} \mathrm{H}$ NMR spectrum the multiplet at $\delta 3.50$ is typical for steroids with hydroxyl group at 3-position; at $\delta 5.00-5.40$ there was evidence of $3 s p^{2}$ protons. Using the mass and ${ }^{1} \mathrm{H}$ NMR data, 3 olefinic protons were observed. 


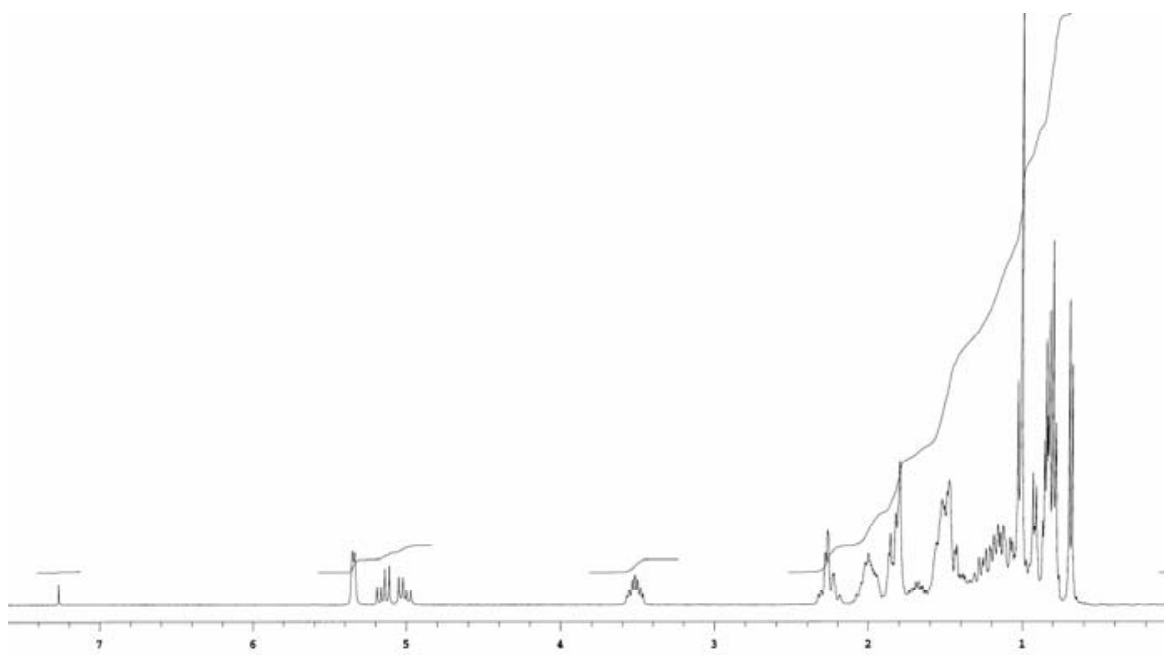

Figure 150: ${ }^{1} \mathrm{H}$ NMR $(300 \mathrm{MHz})$ spectrum of stigmasterol (117) and $\gamma$-sitosterol (118) in $\mathrm{CDCl}_{3}$.

In ${ }^{13} \mathrm{C}$ NMR spectrum, four carbon signals were appeared in the $s p^{2}$ region at $\delta 102$ 142, which indicated that there were at least two double bonds. There was the signal of an oxygenated methine carbon at $\delta$ 77.0. In $s p^{3}$ region, overlapping carbon signals appeared.

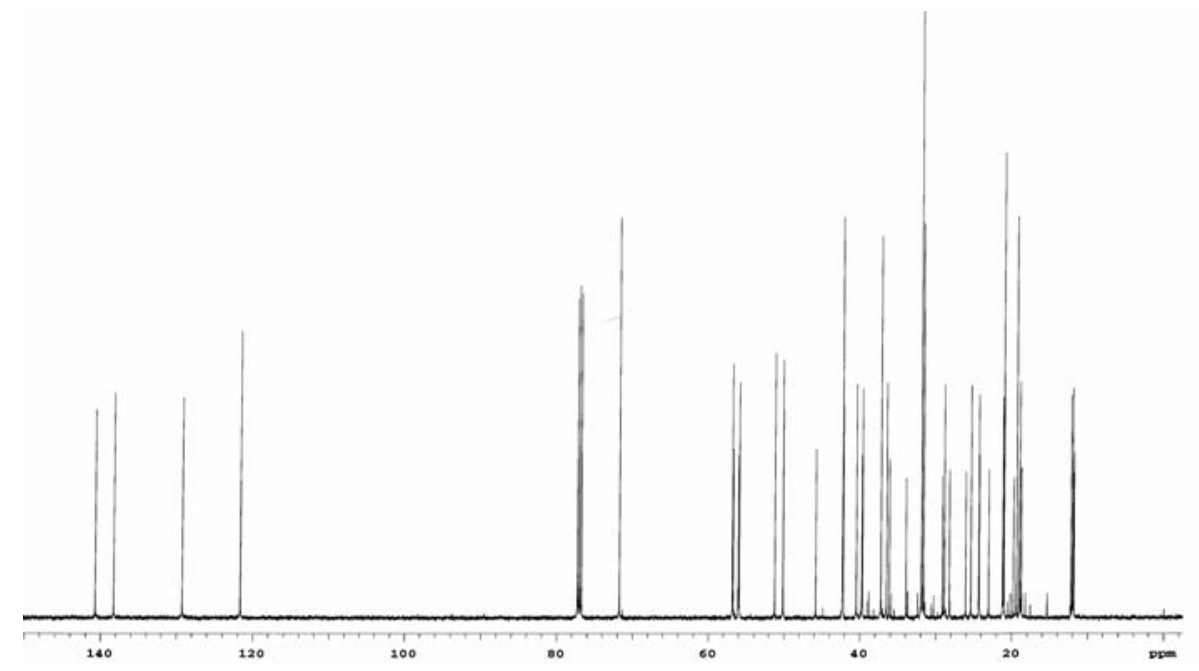

Figure 151: ${ }^{13} \mathrm{C}$ NMR (125 MHz) spectrum of stigmasterol (117) and $\gamma$-sitosterol (118) in $\mathrm{CDCl}_{3}$.

The NMR data were identical with those of a previously obtained stigmasterol (117) / $\gamma$-sitosterol (118) mixture. 
<smiles>CCC(/C=C/[C@H](C)C1CCC2[C@@H]3CC=C4C[C@@H](O)CC[C@]4(C)[C@H]3CC[C@]12C)C(C)C</smiles>

117

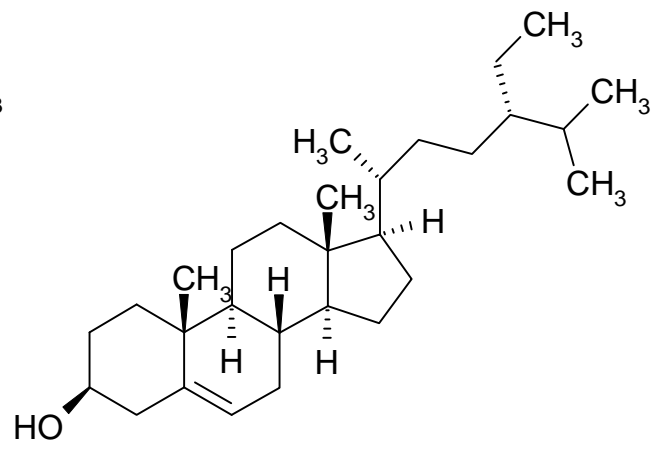

118

\subsection{2-Methoxymaackiain}

Compound 122 was isolated as a yellow powder. In the ${ }^{1} \mathrm{H}$ NMR spectrum showed signals at $\delta 3.45(\mathrm{~m})$ of one methane attached with a hetero atom and at $\delta 3.58(\mathrm{t}, J=$ $10.8 \mathrm{~Hz}), 4.20(\mathrm{dd}, J=5.4,4.9 \mathrm{~Hz}$ ) one methylene also attached with one hetero atom. At $\delta 5.45(\mathrm{~d}, J=6.9 \mathrm{~Hz})$, one proton was also observed which was either olefinic or attached to a hetero atom. The spectrum further showed the presence of a hydroxyl $(\delta$ 5.72), a methylenedioxy ( $\delta 5.91)$ and a methoxyl group $(\delta 3.91)$, and four aromatic $1 \mathrm{H}$ singlets $(\delta 6.43,6.53,6.71$ and 6.92).

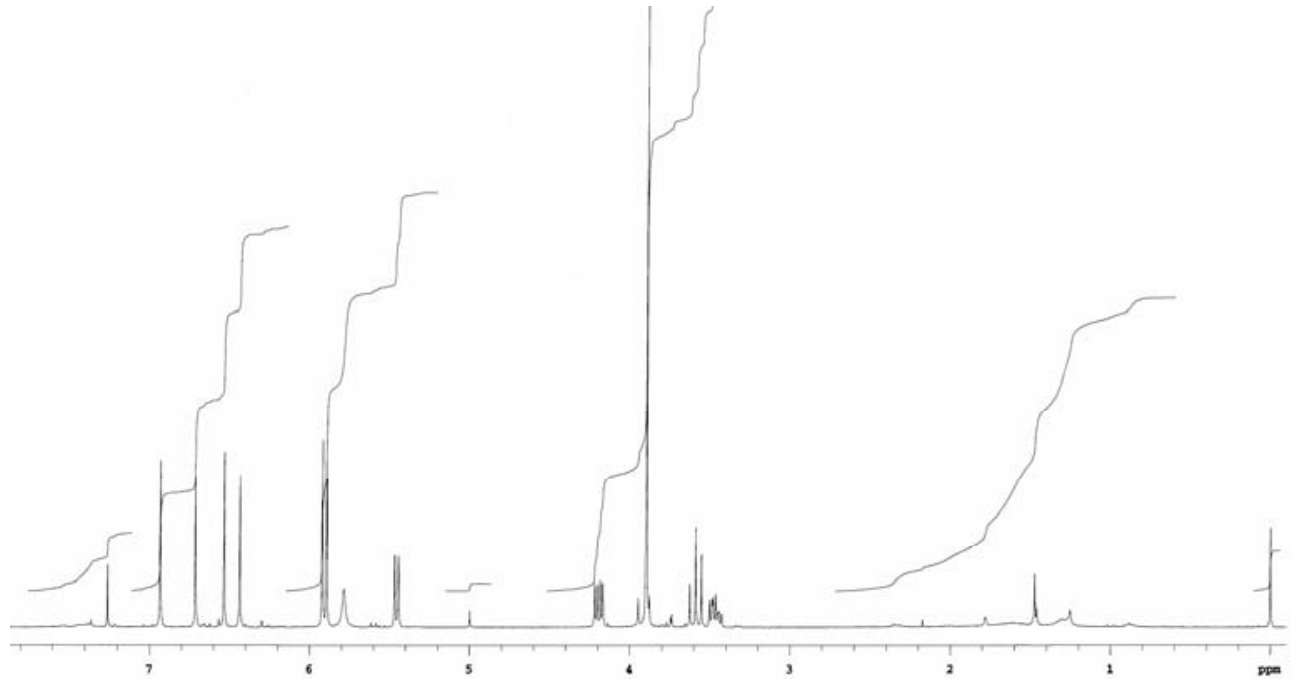

Figure 152: ${ }^{1} \mathrm{H}$ NMR (300 MHz) spectrum of 2-methoxymaackiain (122) in $\mathrm{CDCl}_{3}$.

In the ${ }^{13} \mathrm{C}$ NMR spectrum, there were 17 signals visible. Thirteen of them appeared in the $s p^{2}$ region, and six of them were observed between $\delta 140-155$ and could be connected with oxygen. 


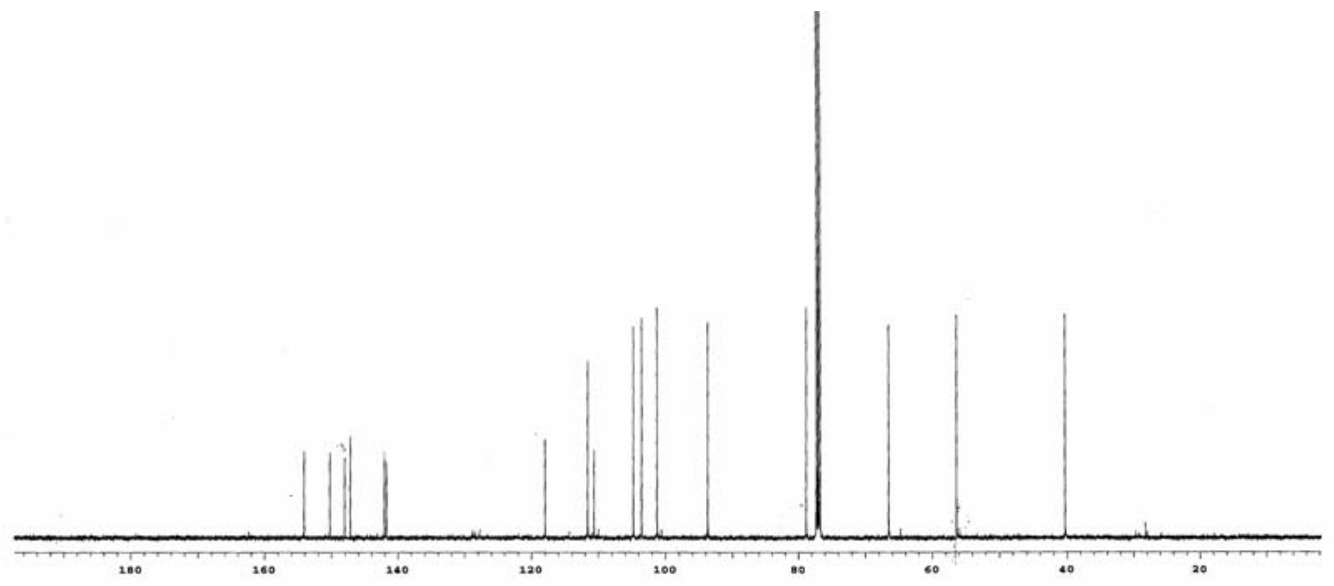

Figure 153: ${ }^{13} \mathrm{C}$ NMR (125 MHz) spectrum of 2-methoxymaackiain (122) in $\mathrm{CDCl}_{3}$.

One aromatic proton, a singlet at $\delta 6.92$, was assignable to $\mathrm{H}-1$ after comparison with the proton signals of maackiain (119), 2-hydroxypterocarpin (120) and 2methoxypterocarpin (121).<smiles>Oc1ccc2c(c1)OCC1c3cc4c(cc3OC21)OCO4</smiles><smiles>COc1cc2c(cc1O)C1Oc3cc4c(cc3C1CO2)OCO4</smiles>

119 120<smiles>COc1cc2c(cc1OC)C1Oc3cc4c(cc3C1CO2)OCO4</smiles>

121

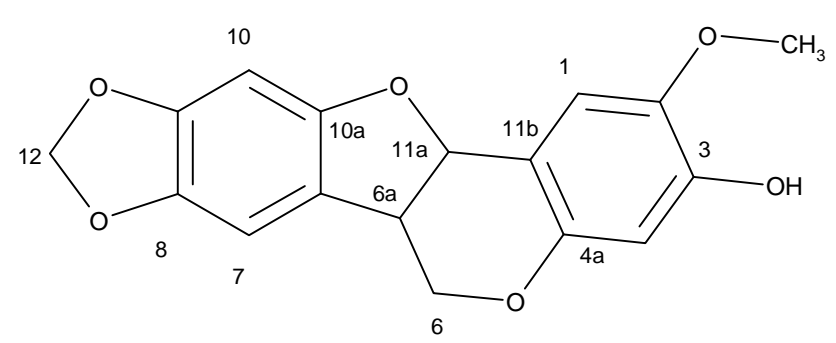




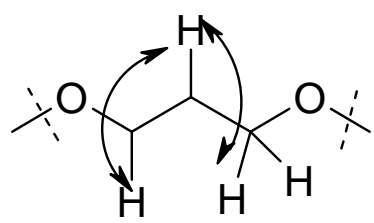

A

Figure 154: From the H-H COSY spectrum and based on ${ }^{13} \mathrm{C}$ NMR values substructure A was derived.

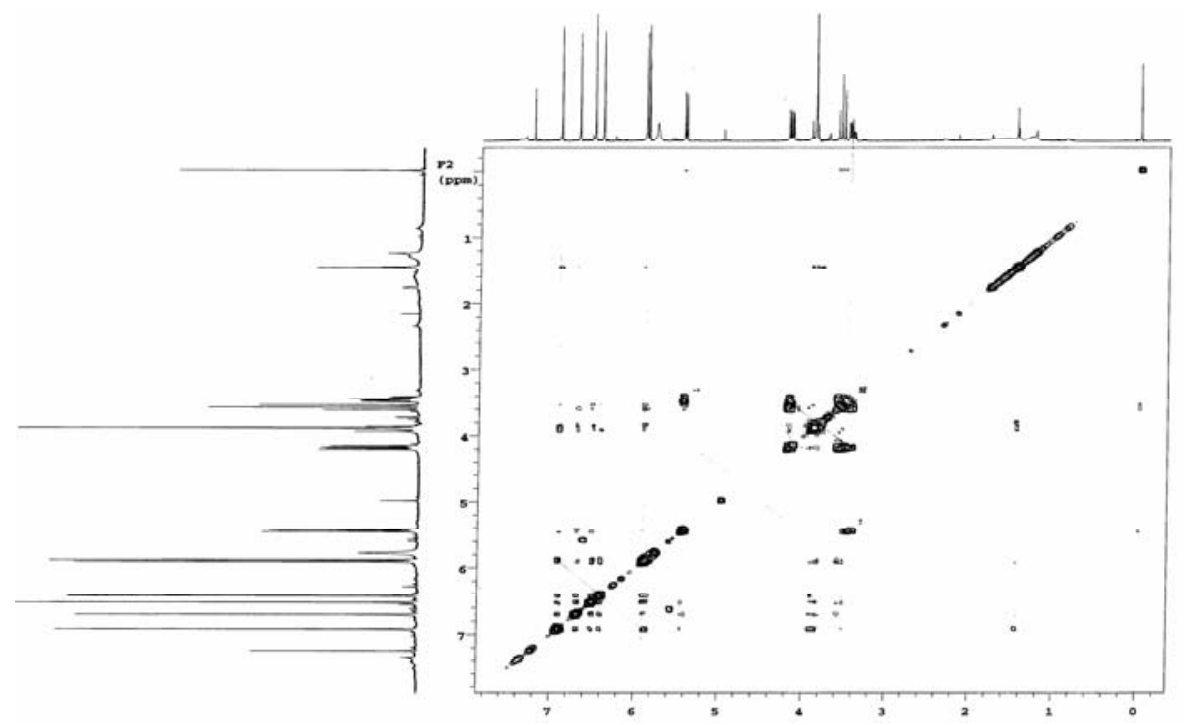

Figure 155: H-H COSY spectrum $(600 \mathrm{MHz})$ of 2-methoxymaackiain (122) in $\mathrm{CDCl}_{3}$.

The methoxy protons at $\mathrm{C}-2(\delta 3.90)$ showed only one correlation with the carbon signal at C-2 $(\delta$ 142.1). Therefore, the methoxy group was attached at position C-2. The methylenedioxy protons showed correlations with carbon signals at C-8 and C-) $(\delta 148.1,141.7)$.

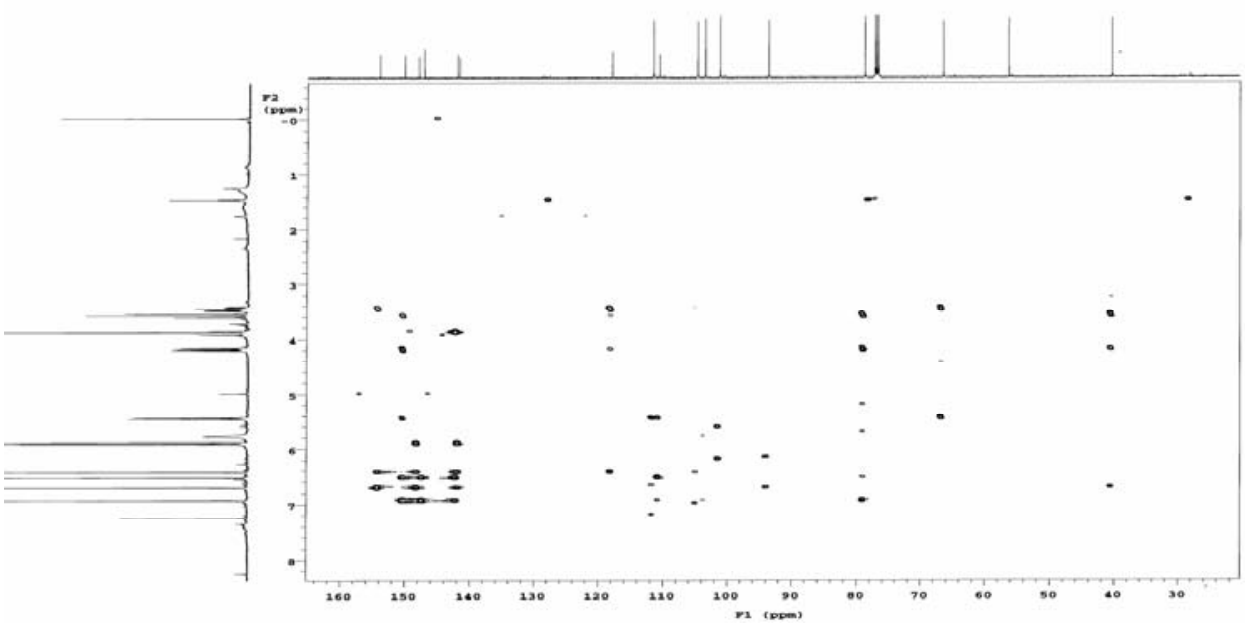

Figure 156: $\mathrm{HMBC}$ spectrum $(600 \mathrm{MHz})$ of 2-methoxymaackiain (122) in $\mathrm{CDCl}_{3}$. 
The methoxy group showed only one correlation with C-3. The hydroxyl group showed correlation with C-4. The value of carbon signal C-2 was $\delta 142.1$, which is also attached to oxygen as there was no other hetereo atom observed from HRESIMS. So, the hydroxyl group was attached with C-2. Using all the 1D and 2D NMR data, the compound was searched in the Dictionary of Natural Products and a pterocarpan, 2-methoxymaackiain was found identical. 2-methoxymaackiain was published ${ }^{135,136}$ in the year 1990 .

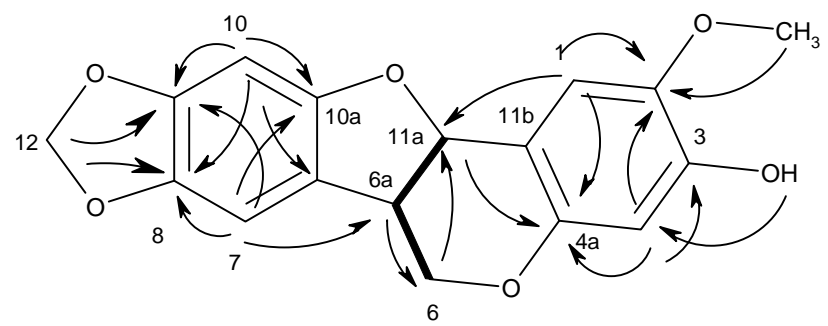

122

Figure 157: The H-H COSY $(-)$ and $\operatorname{HMBC}(\longrightarrow)$ correlations of 122.

\subsection{Tephrosol (2-methoxymedicagol)}

2-Methoxymedicagol (123) was isolated as amorphous powder, had NMR data similar to 2-methoxymaackiain. There were four aromatic protons at $\delta 7.41,7.23,7.16,6.89$ and a methylenedioxy group at $\delta 6.12$ as well as a methoxy signal at $\delta 3.90$. HRESIMS gave the formula $\mathrm{C}_{17} \mathrm{H}_{11} \mathrm{O}_{7}$ for $[\mathrm{M}+\mathrm{H}]^{+}$.

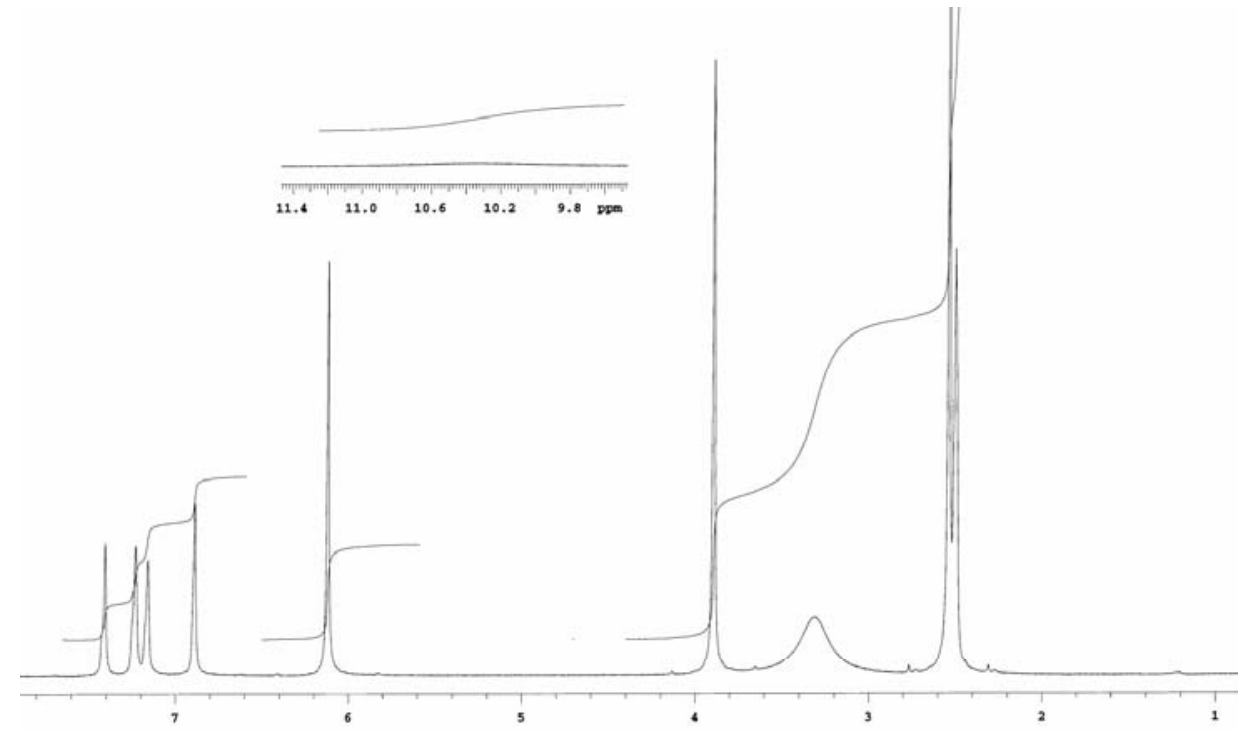

Figure 158: ${ }^{1} \mathrm{H}$ NMR (300 MHz) spectrum of 2-methoxymedicagol (123) in $\mathrm{CDCl}_{3}$.

${ }^{13} \mathrm{C}$ NMR spectrum $\left(\mathrm{CDCl}_{3}, 125 \mathrm{MHz}\right)$ showed 13 carbons signals. Among them, only one carbon signal was observed in the aliphatic region $(\delta 56.0)$ for a methoxy group 
and a methylenedioxy group at $\delta 102.0$. Four aromatic methine carbon signals were at $\delta 116.3,103.6,102.9$ and 102.4 visible. The remaining carbon signals were due to quaternary $s p^{2}$ carbons.

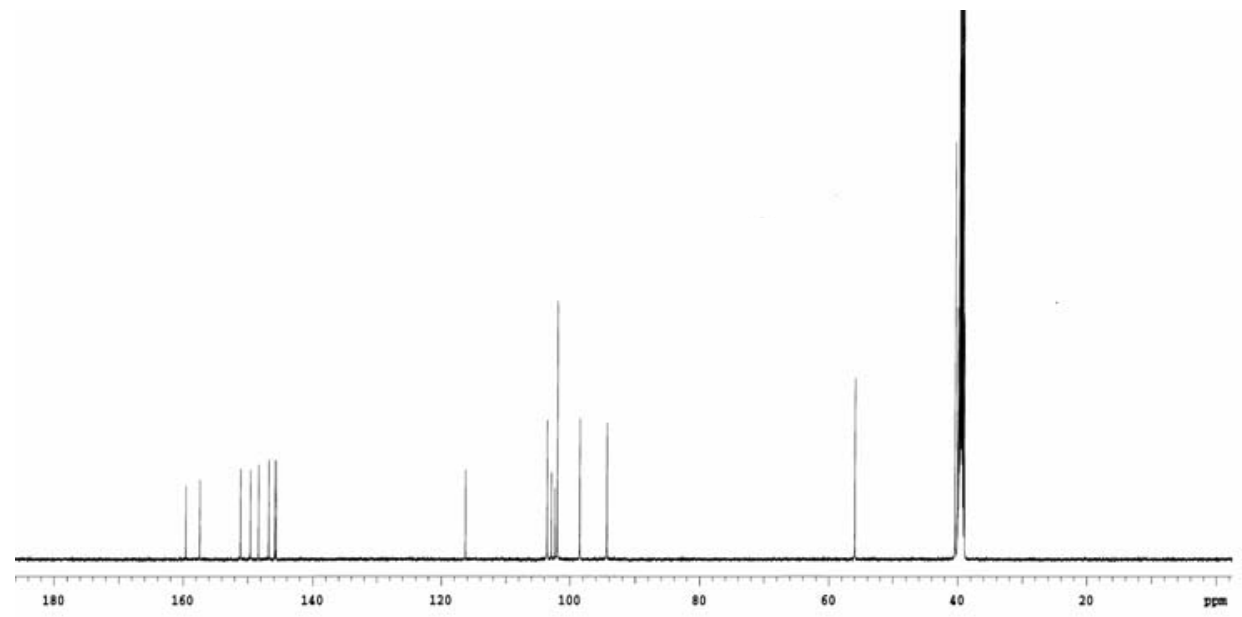

Figure 159: ${ }^{13} \mathrm{C}$ NMR (125 MHz) spectrum of 2-methoxymedicagol (123) in $\mathrm{CDCl}_{3}$.

In the HMBC spectrum, the methylenedioxy protons showed correlation with the carbon signal at C-8 and C-9. The only methoxy showed correlation with carbon signal C-4 and hydroxyl group showed correlation with carbon signal at C-4, which means the hydroxyl group is attached with carbon C-3 ( $\delta$ 151.1). Compound 123 showed similarity in the 2D correlations with already described 2-methoxymaackiain (122). But in compound $\mathbf{1 2 2}$ one methylene group is substituted by a carbonyl group to form a lactone. Compound 122 was confirmed as 2-methoxymedicagol (122). ${ }^{137,138}$

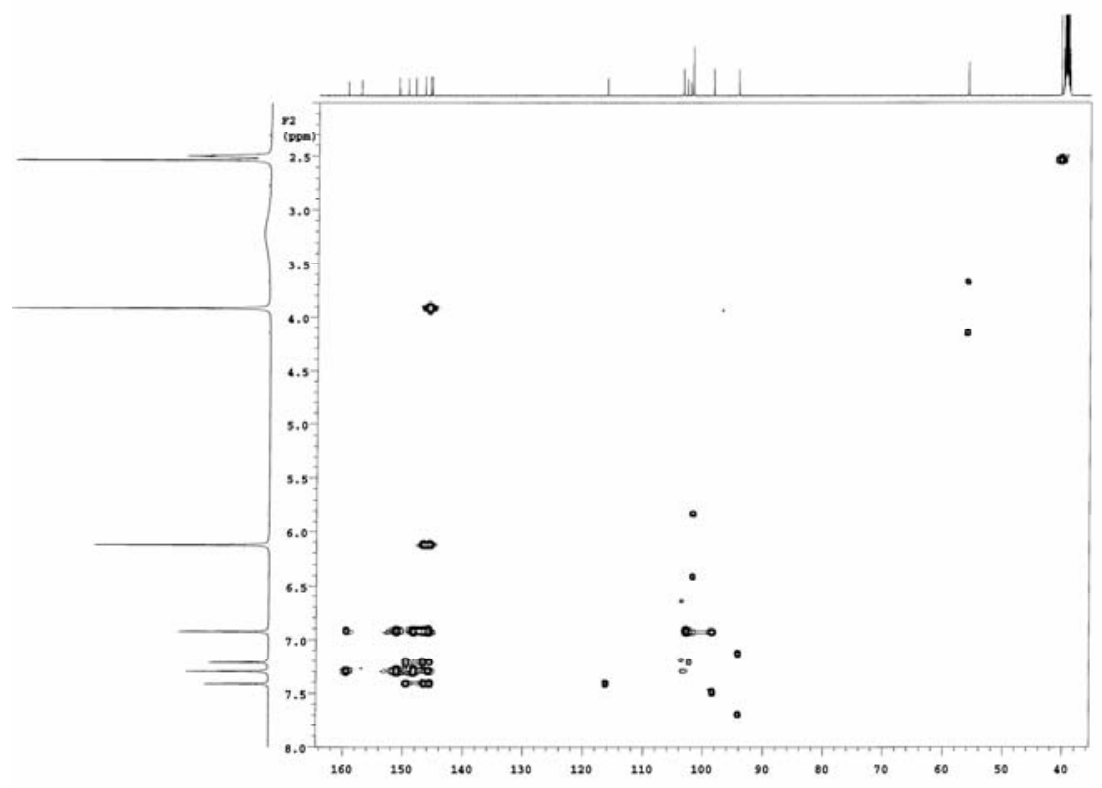

Figure 160: $\mathrm{HMBC}$ spectrum $(500 \mathrm{MHz})$ of 2-methoxymedicagol (123) in $\mathrm{CDCl}_{3}$. 


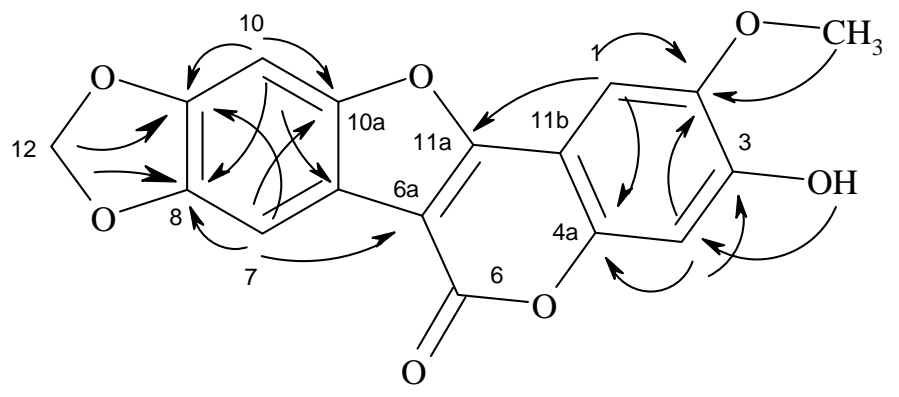

123

\subsection{Didehydrovillosin (mixture of two stereoisomers at C-6)}

Didehydrovillosin (124) was obtained as pale yellow pellets. (+)-ESIMS and HRMS resulted in $\mathrm{C}_{23} \mathrm{H}_{21} \mathrm{O}_{8}$ for $[\mathrm{M}+\mathrm{H}]^{+}$. The ${ }^{1} \mathrm{H}$ NMR spectrum exhibited signals due to a chelated hydroxyl group $(\delta 13.20)$, three aromatic protons at $\delta 8.33,6.75$, and 6.44 , an isopropenyldihydrofuran ring, two methoxy groups at $\delta 3.81$ and 3.78 and a hemiacetal proton at $\delta 6.20$. The ${ }^{1} \mathrm{H}$ NMR data of this compound were similar to villinol, a rotenoid, which exhibits a methoxy group at $\delta 3.65$, assigned to C-6. From the NMR data, it was clear that it was a mixture of two stereoisomers.<smiles>C=C(C)C1Cc2c(cc(O)c3c(=O)c4c(oc23)O[C@H](O)c2cc(OC)c(OC)cc2-4)O1</smiles> 


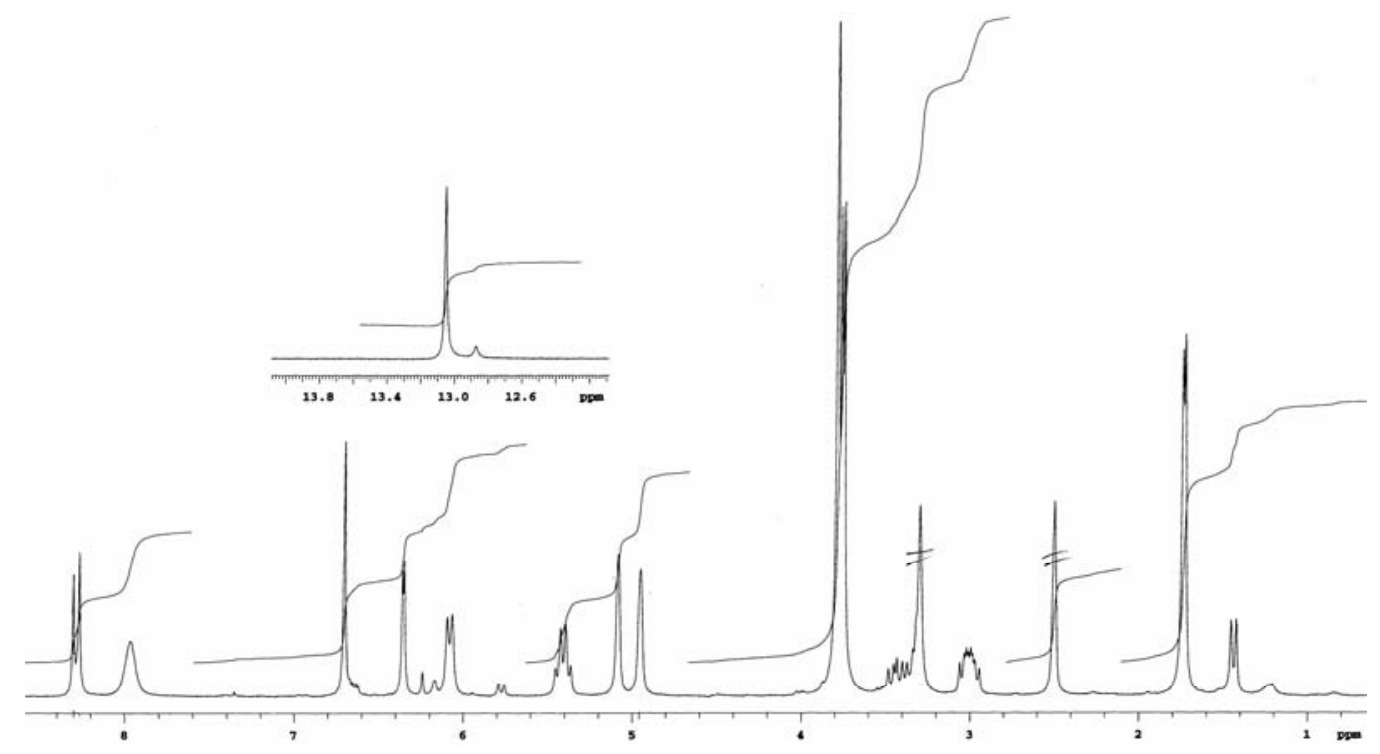

Figure 161: ${ }^{1} \mathrm{H}$ NMR $(300 \mathrm{MHz})$ spectrum of dehydrovillosin $(\mathbf{1 2 4})$ in $\left[\mathrm{D}_{6}\right] \mathrm{DMSO}$.

The ${ }^{13} \mathrm{C}$ NMR spectrum showed a signal at $\delta 88.5$, which could be attributed to a hemiacetal carbon (C-6). All carbon signals appeared as twins, due to the presence of two similar isomers. In the $s p^{3}$ region, there were two methoxy signals at $\delta 56.0$ and one methyl carbon signal at $\delta 56.0$, a methylene and a methyl signal. All remaining carbon signals were present in the $s p^{2}$ region.

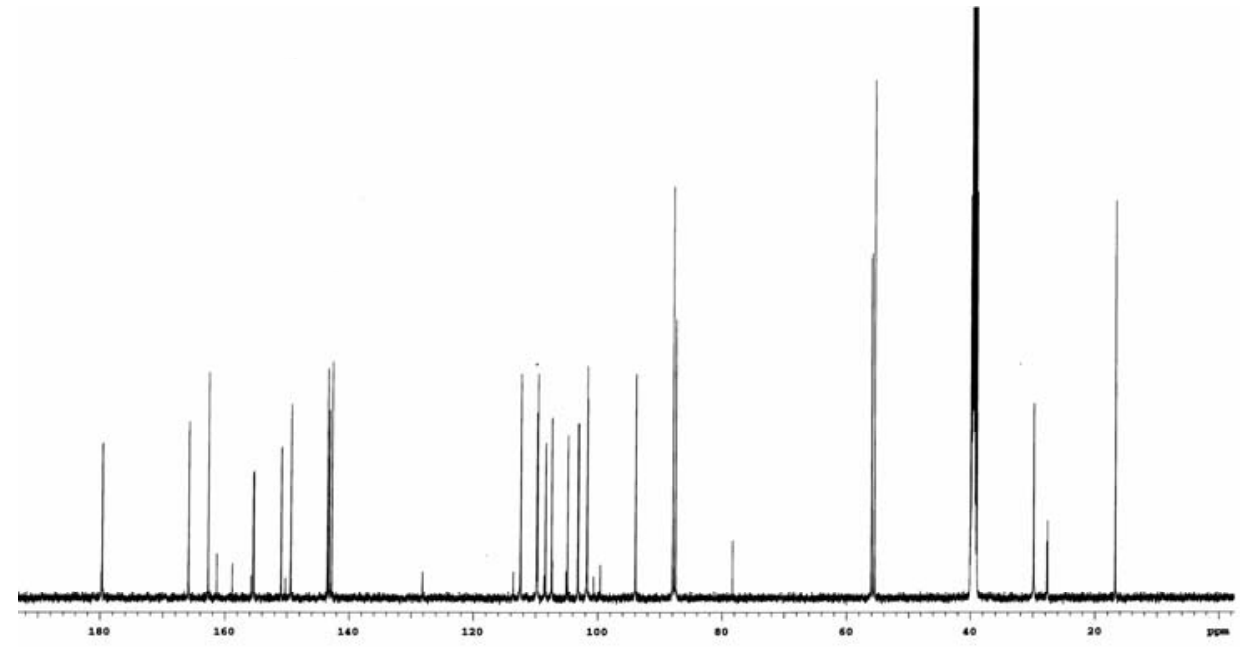

Figure 162: ${ }^{13} \mathrm{C}$ NMR (125 MHz) spectrum of dehydrovillosin (124) in [D $]$ DMSO.

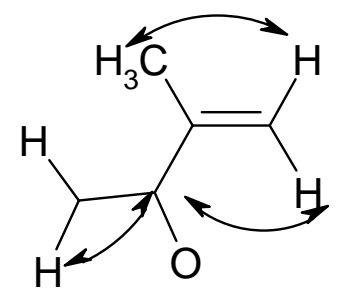

A

Figure 163: From H-H COSY spectrum substructure A was derived. 


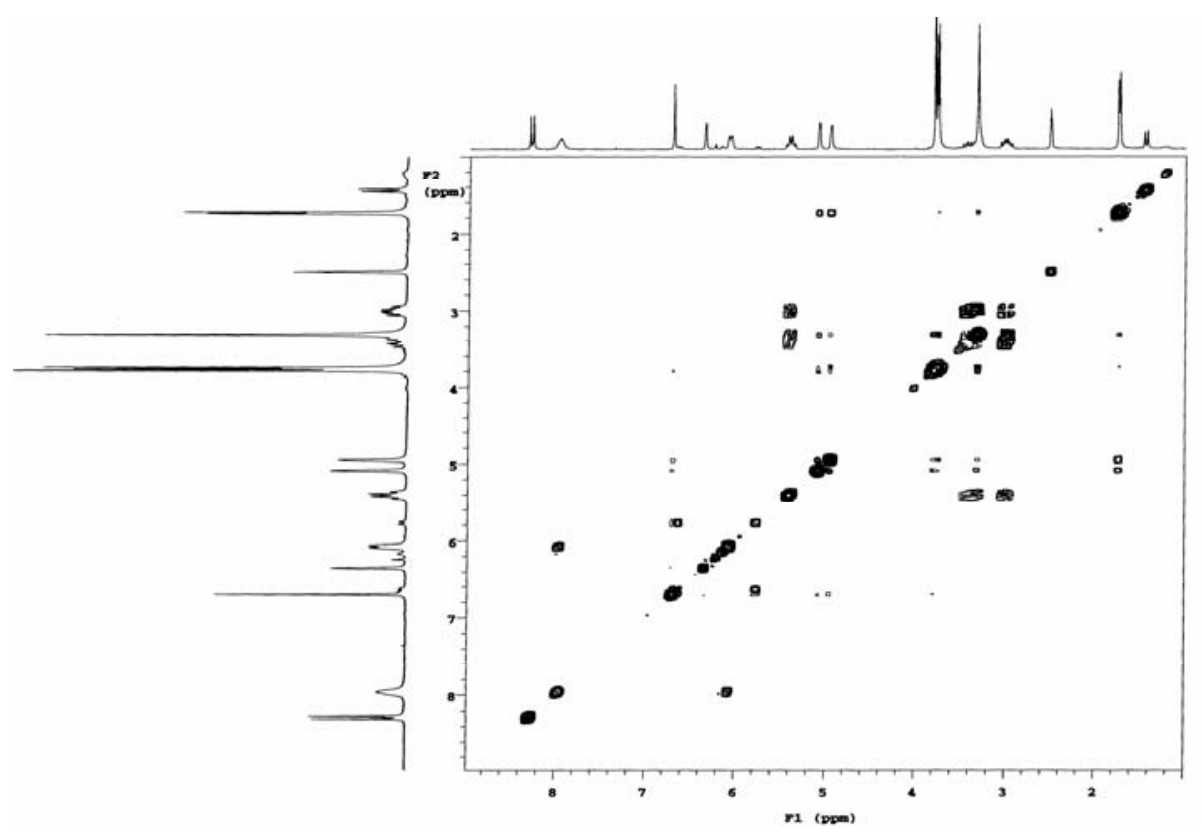

Figure 164: H-H COSY spectrum $(600 \mathrm{MHz})$ of dehydrovillosin (124) in [D6]DMSO.

The methoxy protons showed correlation with carbon signals C-2 and C-3. With the same carbon signals, the aromatic protons at H-1 and H-4 were correlating, but these two protons did not show COSY correlation. Therefore, they were in one benzene ring and the protons were in para-position as shown in substructure B. With the help of COSY and HMBC spectra, substructure $\mathrm{C}$ was developed.

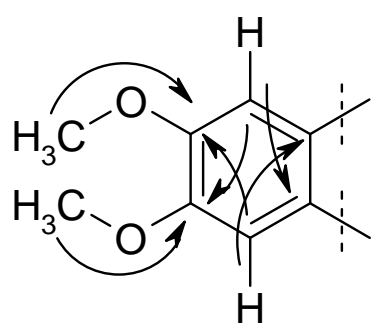

B

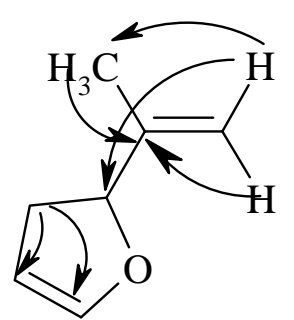

$\mathbf{C}$ 


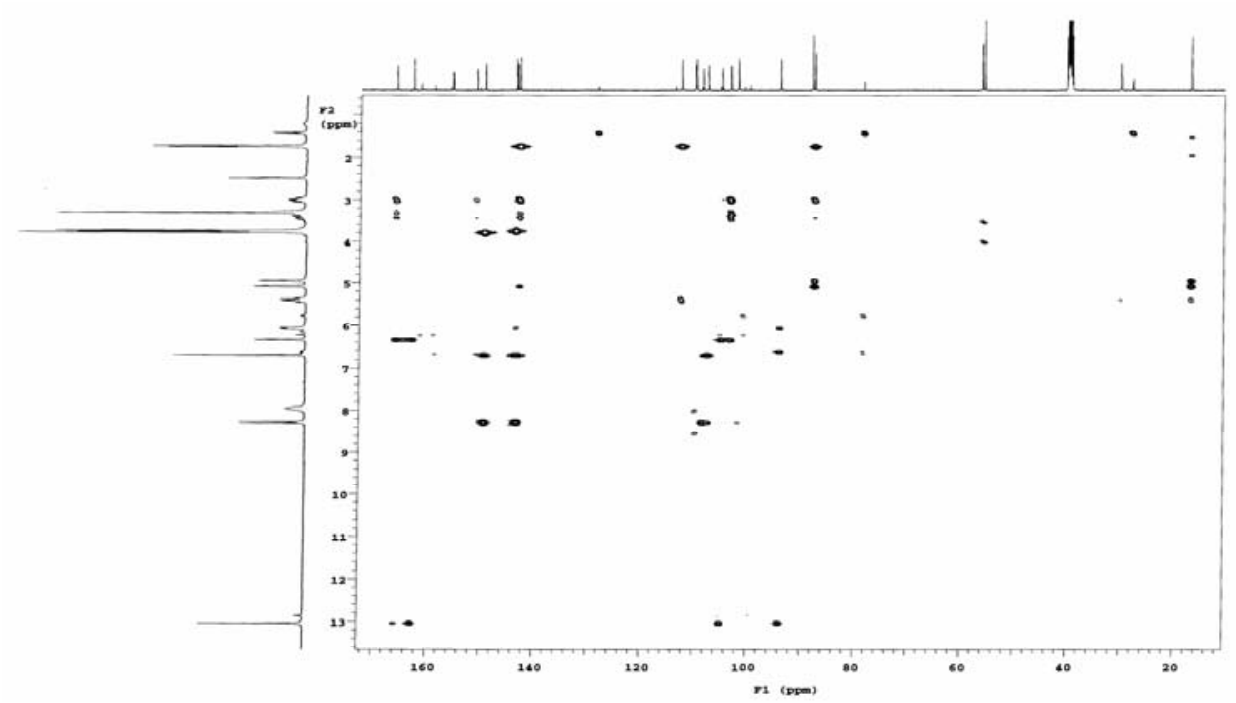

Figure 165: HMBC spectrum $(600 \mathrm{MHz})$ of dehydrovillosin (124) in [ $\left.\mathrm{D}_{6}\right] \mathrm{DMSO}$.

The hydroxyl at carbon 6 showed correlation with $4 \mathrm{a}$ and $12 \mathrm{~b}$. The proton at carbon 10 showed correlation with 8,9 and 11a. The hydroxyl signal showed correlation with 10, 11 and 11a. The 1D and 2D NMR data were compared with the published values for to confirm the structure as didehydrovillosin (124). ${ }^{139}$

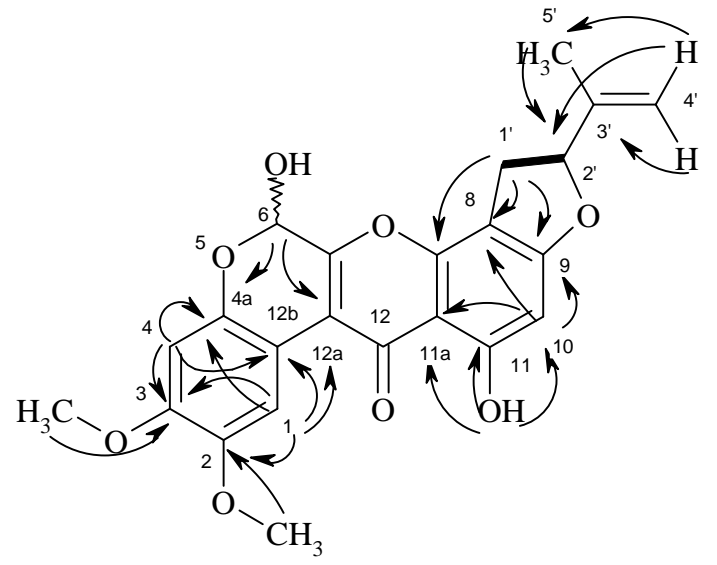

124

\subsection{Dehydrorotenone}

Compound 126 was only sufficiently soluble in pyridine and showed (+)-ESIMS ions at $\mathrm{m} / \mathrm{z} 807\left([2 \mathrm{M}+\mathrm{Na}]^{+}, 100\right)$ and $393\left([\mathrm{M}+\mathrm{H}]^{+}\right.$. The ${ }^{1} \mathrm{H}$ NMR spectrum of this compound showed similarity with the phenolic rotenoids. In the ${ }^{1} \mathrm{H}$ NMR spectrum there were four $s p^{2}$ signals at $\delta 9.32,8.74,7.41$ and 7.21. There was also an oxygenated isoprenyl system at $\delta 5.85,5.61$ and 5.38, an oxygenated methylene at $\delta 5.50$ (s, $\mathrm{CH}_{2}$ ) and two methoxy groups at $\delta 4.30$ and 4.17. There was another methylene at $\delta$ $3.83,3.56$ which was possibly near a chiral centre and a hetero atom. There was only one methyl at $\delta 1.87$. 


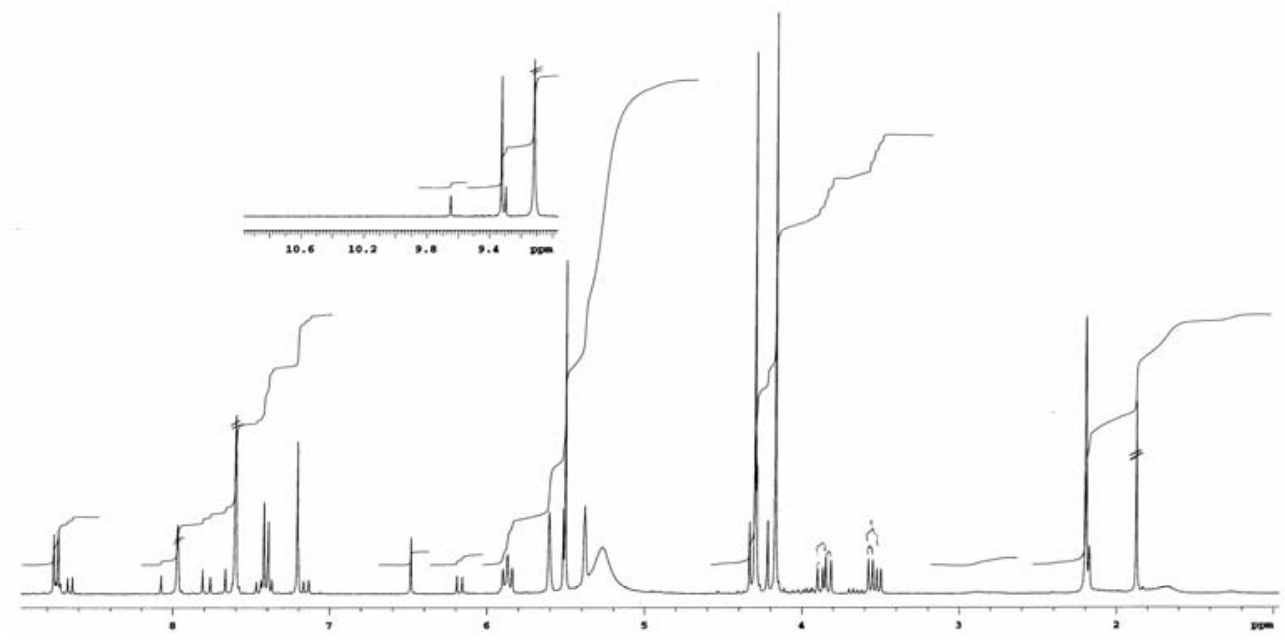

Figure 166: ${ }^{1} \mathrm{H}$ NMR spectrum $(300 \mathrm{MHz})$ spectrum of dehydrorotenone (126) in $\left[\mathrm{D}_{5}\right]$ pyridine.

In the ${ }^{13} \mathrm{C}$ NMR spectrum there was a carbonyl of an ester or acid at $\delta 174.1$, six oxygenated quaternary $s p^{2}$ carbons and $9 s p^{2}$ carbons attached with hydrogen were observed. Two methoxy signals were also present $\delta 56.7$ and 56.0.

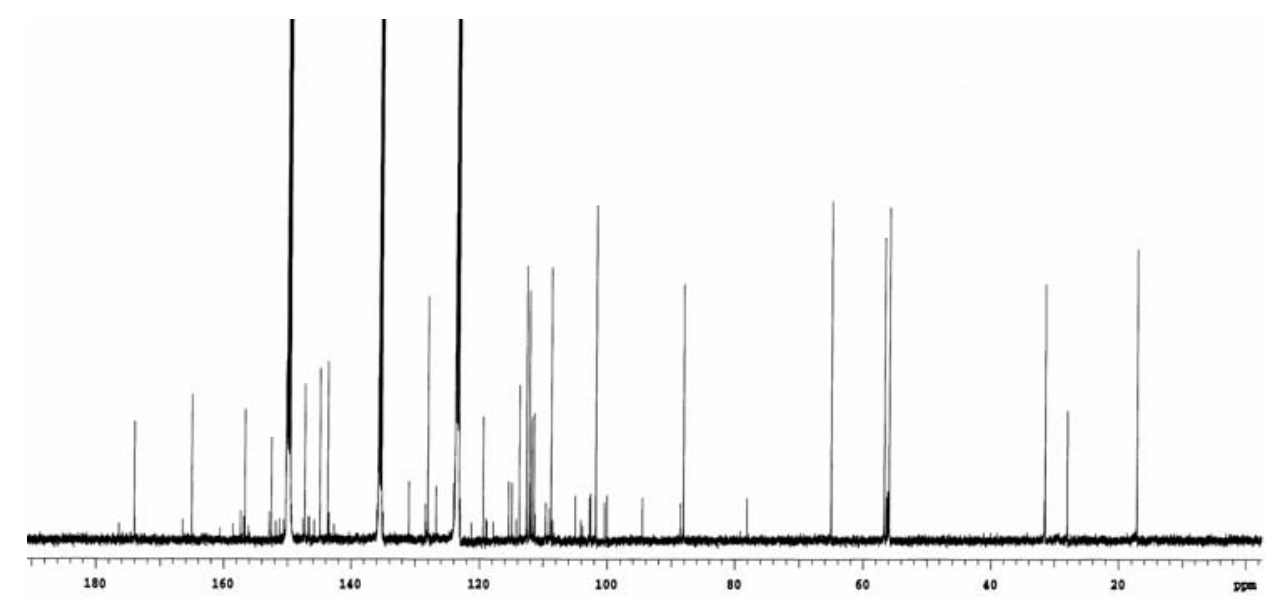

Figure 167: ${ }^{13} \mathrm{C}$ NMR $(125 \mathrm{MHz})$ spectrum of dehydrorotenone (126) in $\left[\mathrm{D}_{5}\right]$ pyridine From the H-H COSY spectrum, substructure A was derived.

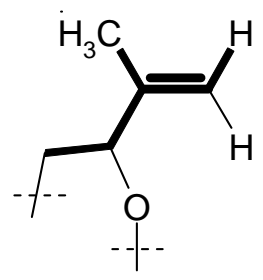

A 


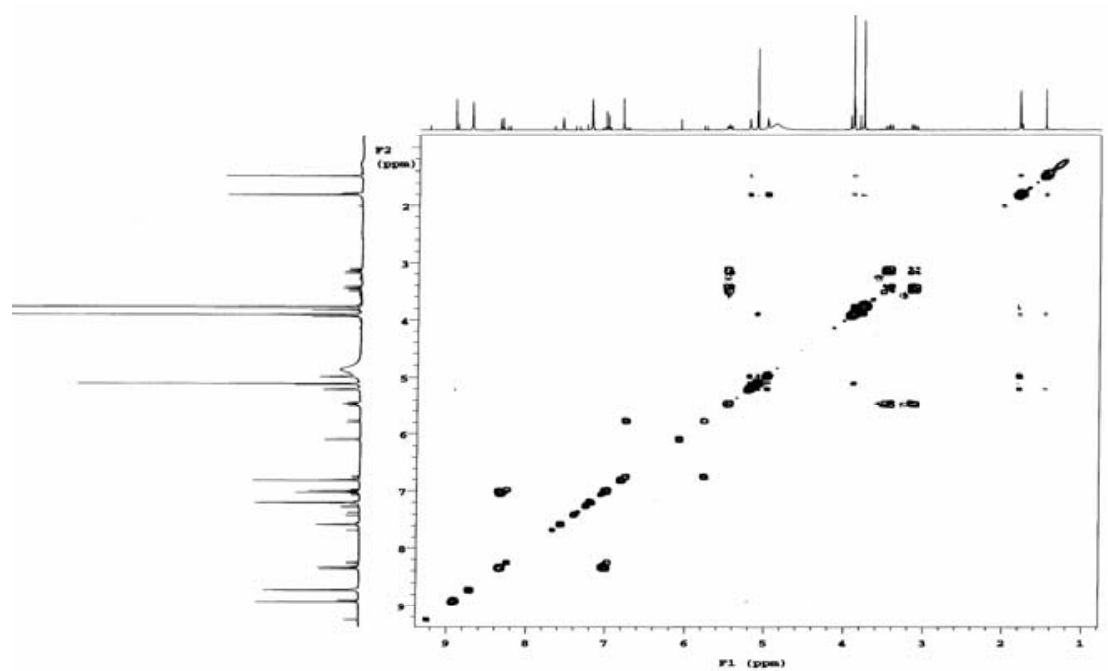

Figure 168: $\mathrm{H}-\mathrm{H}$ COSY spectrum $(600 \mathrm{MHz})$ of dehydrorotenone (126) in $\left[\mathrm{D}_{5}\right]$ pyridine.

Based on the molecular formula and the other spectroscopic data as well as the similarity with phenolic rotenoids, the compound was searched in the Dictionary of Natural Products and confirmed as dehydrorotenone (126). ${ }^{140,141}$

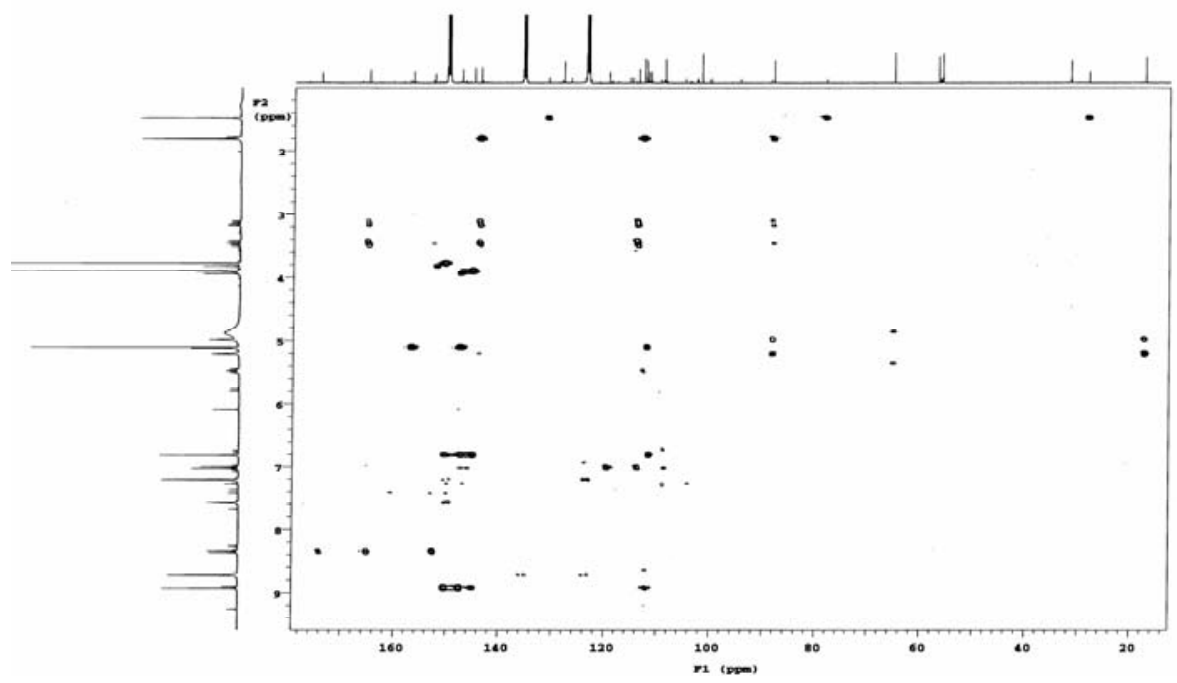

Figure 169: HMBC spectrum $(600 \mathrm{MHz})$ of dehydrorotenone (126) in $\left[\mathrm{D}_{5}\right]$ pyridine. 


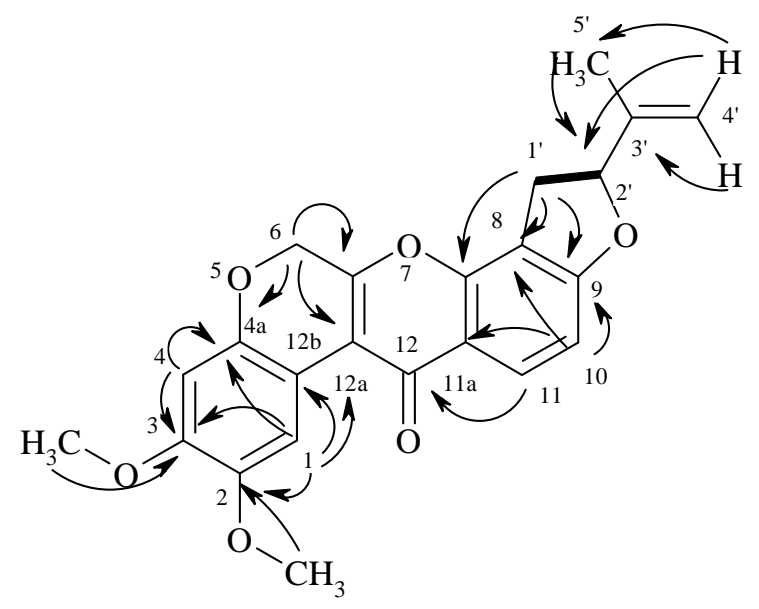

126

\subsection{Substituted Dichromen-7-one}

The rotenoid 127 was isolated as a yellow solid which showed $m / z 423\left([\mathrm{M}-\mathrm{H}]^{-}\right.$ (ESIMS) and HRESIMS $[\mathrm{M}+\mathrm{H}]^{+}$for $\mathrm{C}_{23} \mathrm{H}_{20} \mathrm{O}_{8}$. The ${ }^{1} \mathrm{H}$ NMR spectrum showed one chelated hydroxy group at $\delta 12.90$ (s); 7 further signals of protons which could be attached to $s p^{2}$ carbon atoms were present at $\delta 8.30(\mathrm{~s}), 8.00(\mathrm{~s}, \mathrm{OH}) 6.75,6.70,6.28$, $6.20,5.80$. There were two methoxy and two methyl groups observed.

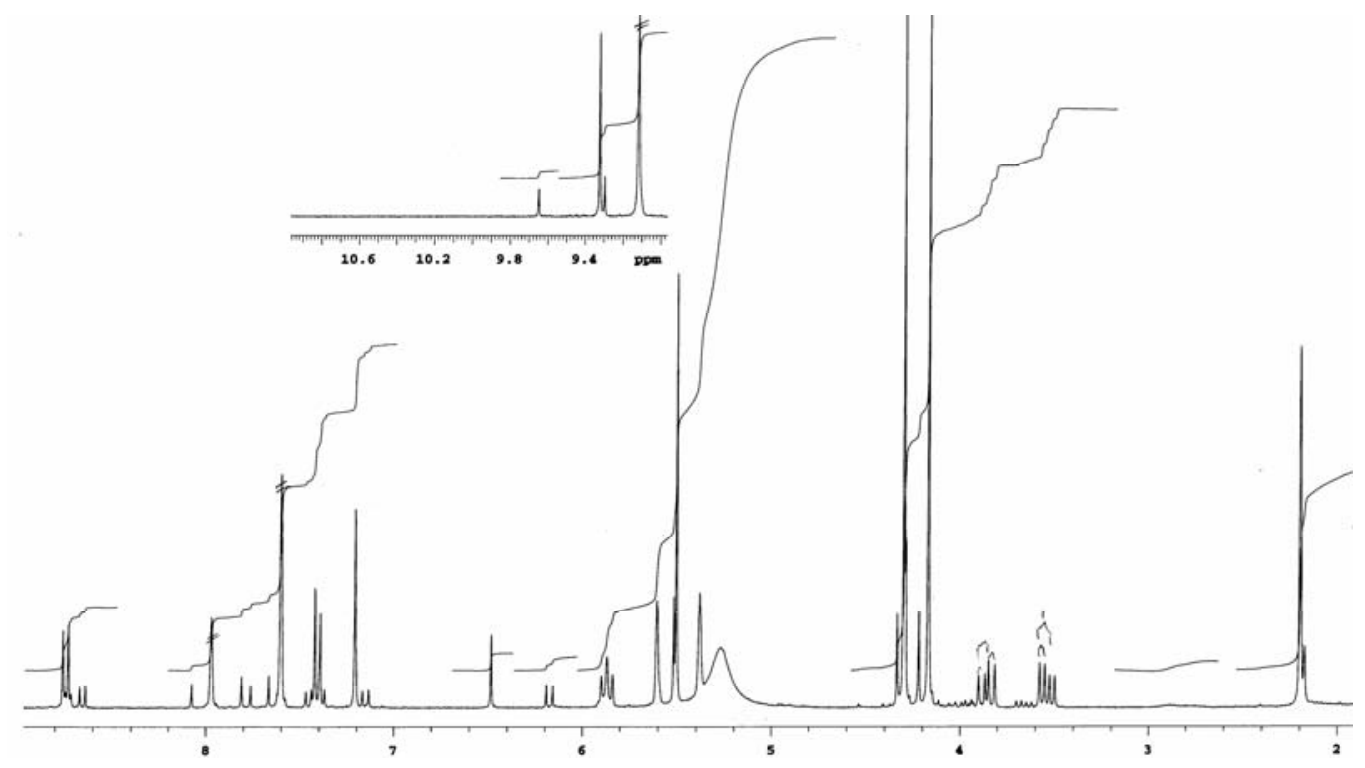

Figure 170: ${ }^{1} \mathrm{H}$ NMR (300 MHz) spectrum of 13-Hydroxy-9,10-dimethoxy-3,3dimethyl-3H,13H-pyrano[2,3-c;6,5-f']dichromen-7-one (127) in $\left[\mathrm{D}_{6}\right] \mathrm{DMSO}$.

The molecular formula $\mathrm{C}_{23} \mathrm{H}_{21} \mathrm{O}_{8}$ for rotenoid $\mathbf{1 2 7}$ provided fourteen double bond equivalents. 


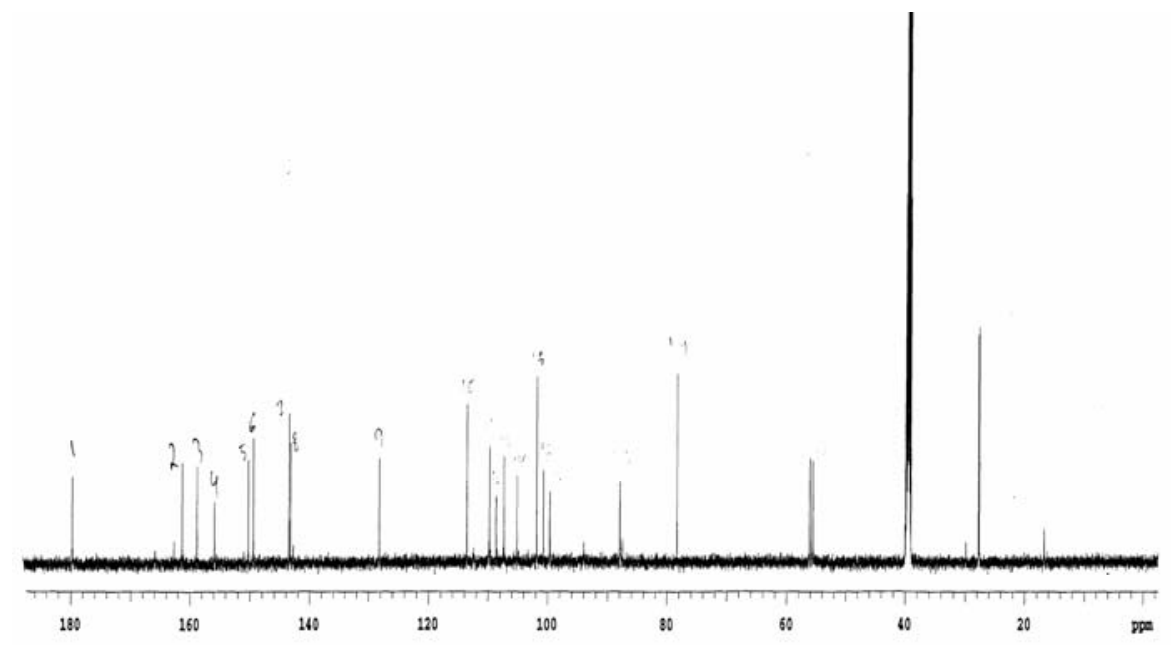

Figure 171: ${ }^{13} \mathrm{C}$ NMR (300 MHz) spectrum of 13-Hydroxy-9,10-dimethoxy-3,3dimethyl-3H,13H-pyrano[2,3-c;6,5-f']dichromen-7-one (127) in $\left[\mathrm{D}_{6}\right] \mathrm{DMSO}$.

From the H-H COSY spectrum there was a correlation between the proton at $\delta 6.20$ and the hydroxyl group at $\delta 8.00$. Another COSY correlation was also observed between the protons at $\delta 6.70$ and 5.80 .

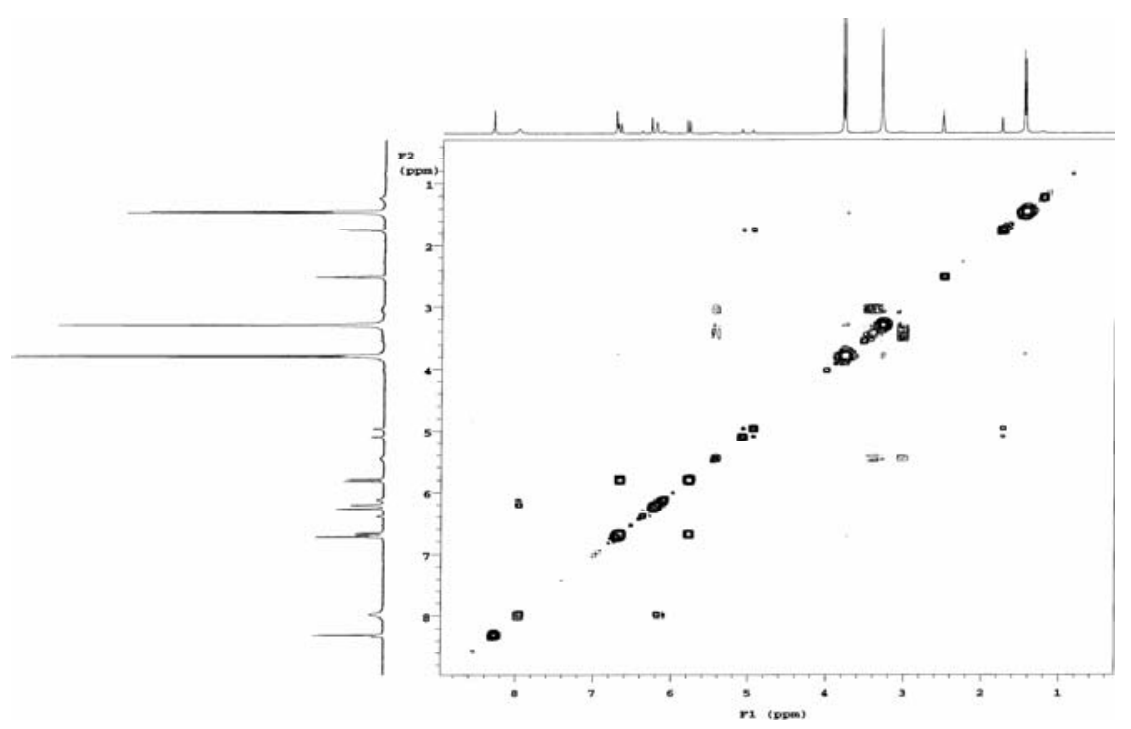

Figure 172:H-H COSY spectrum (600 MHz) of 13-Hydroxy-9,10-dimethoxy-3,3dimethyl-3H,13H-pyrano[2,3-c;6,5-f']dichromen-7-one (127) in [D 6 DMSO.

In the HMBC spectrum, correlations of $\mathrm{H}-1$ to $\mathrm{C}-12 \mathrm{a}, \mathrm{H}-6$ to $\mathrm{C}-4 \mathrm{a}$ and $\mathrm{C}-6 \mathrm{a}, \mathrm{OH}-11$ to C-11a, H-4' to C-2' and C-3', H-1' to C-8, 9 and 7a as well as $\mathrm{H}-10$ to C-11a and C8 revealed the presence of a rotenoid skeleton. Furthermore, two methoxy groups were found to be attached to C-2 and C-3, respectively, and the two methyl groups were attached to C-3'. Compound 127 was established by comparing the $1 \mathrm{D}$ and 2D 
NMR data with the published data. ${ }^{142-143}$ Rotenoids are known for their insecticidal and pesticidal activities. Their toxicity is caused by the inhibition of NADH oxidation in the respiratory chain. A known rotenoid derrisin was found highly active against Helicobacter pylori. ${ }^{144}$

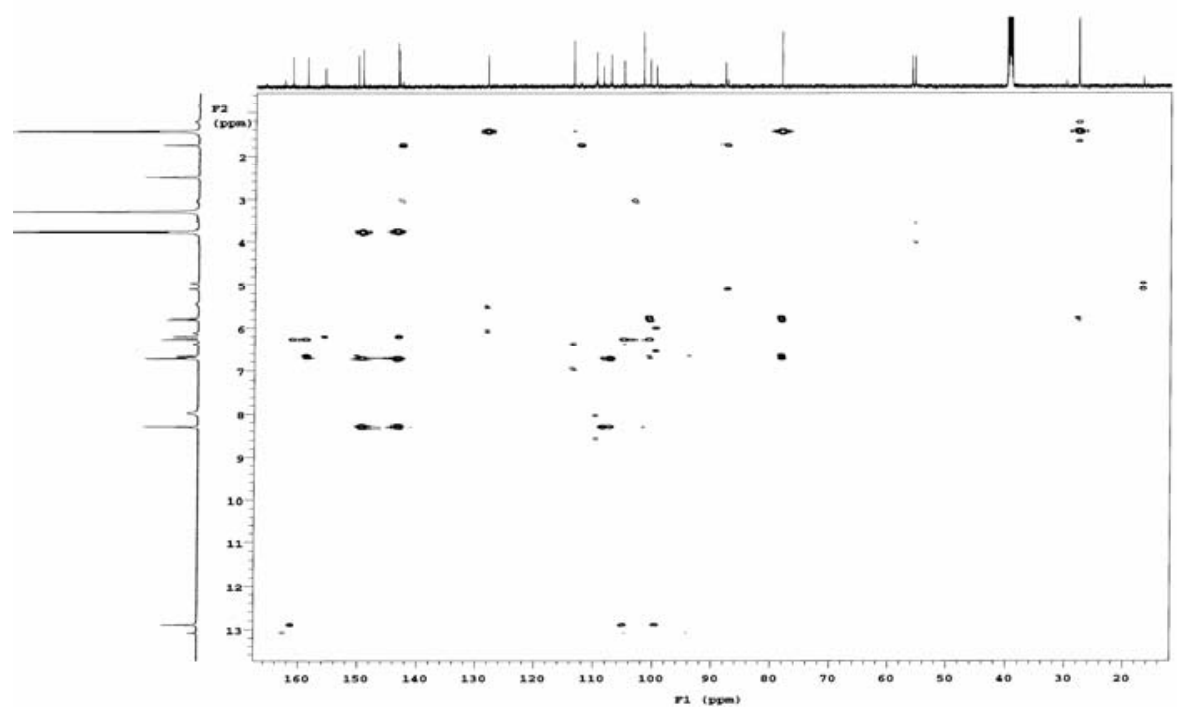

Figure 173: HMBC spectrum (600 MHz) of 13-Hydroxy-9,10-dimethoxy-3,3dimethyl-3H,13H-pyrano[2,3-c;6,5-f']dichromen-7-one (127) in $\left[\mathrm{D}_{6}\right] \mathrm{DMSO}$.

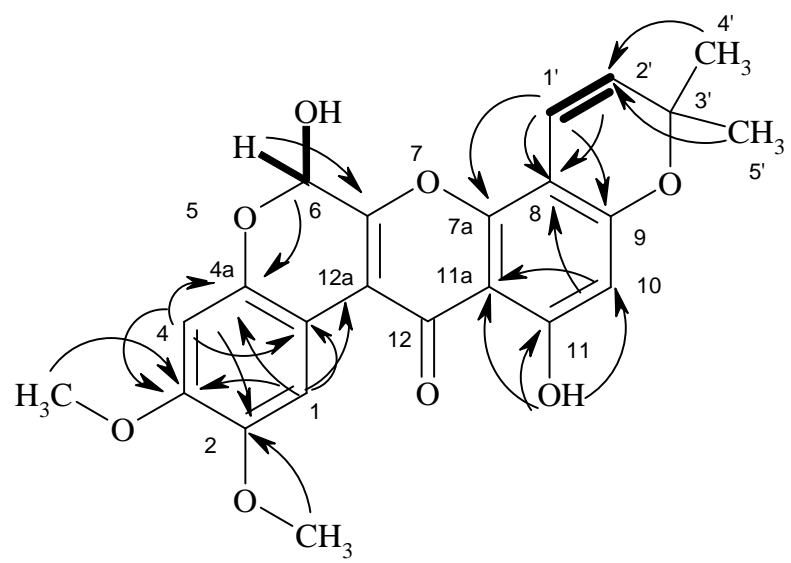

127

\subsection{Obovatin methyl ether}

The salient feature in the ${ }^{1} \mathrm{H}$ NMR spectrum of obovatin methyl ether (128) was the $A B X$ system, diagnostic for the $\mathrm{C}-2$ and $\mathrm{C}-3$ protons of a flavanone. The $\mathrm{C}-2$ proton $(\mathrm{X})$, appeared as a double doublet at $\delta 5.40(A B X)$, while the $\mathrm{AB}$ signal of the $\mathrm{C}-3$ protons appeared at $\delta 2.79(A B X)$. The aromatic region was defined by a sharp multiplet at $\delta 7.45(5 \mathrm{H})$ indicating the unsubstituted B-ring; a singlet at $\delta 6.10(1 \mathrm{H})$ could 
be assigned to either the C- 6 or the C- 8 proton. There was a methoxy signal at $\delta 3.89$ and two methyl singlets at $\delta 1.46$ and 1.44 , respectively.

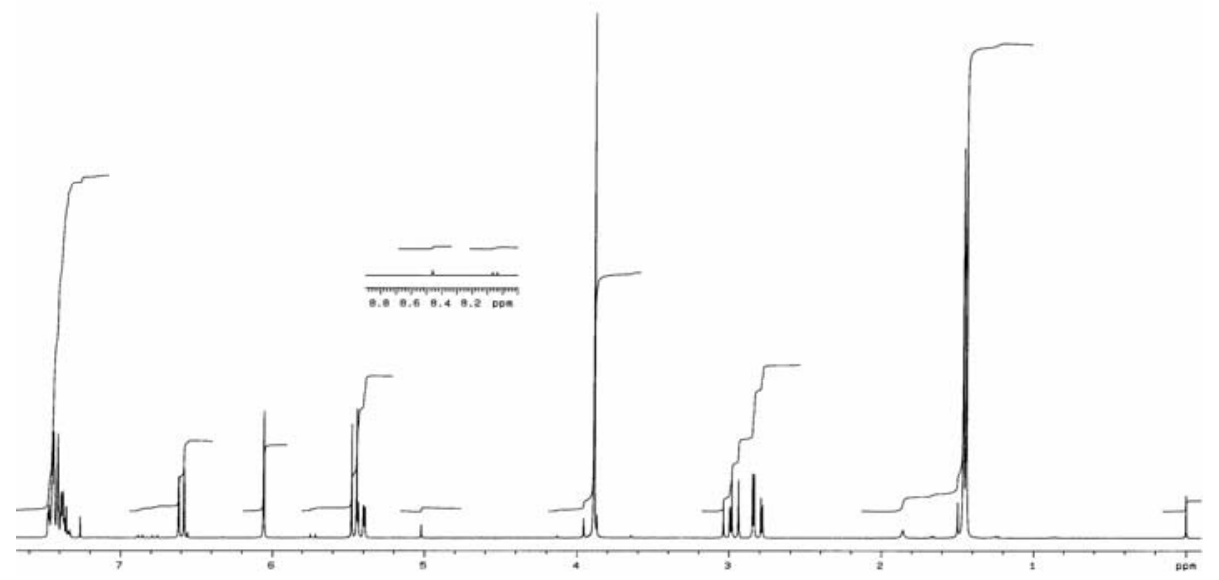

Figure 174: ${ }^{1} \mathrm{H}$ NMR $(300 \mathrm{MHz})$ spectrum of obovatin methyl ether (128) in $\mathrm{CDCl}_{3}$. The ${ }^{13} \mathrm{C}$ NMR spectrum showed 21 carbon signals. Among them 3 aliphatic carbons were observed at $\delta 45.6\left(\mathrm{CH}_{2}\right), 28.4\left(\mathrm{CH}_{3}\right)$ and $28.1\left(\mathrm{CH}_{3}\right)$; one methoxy carbon was at $\delta 56.1$ and two oxygenated carbon signals at $\delta 78.9,77.9$.

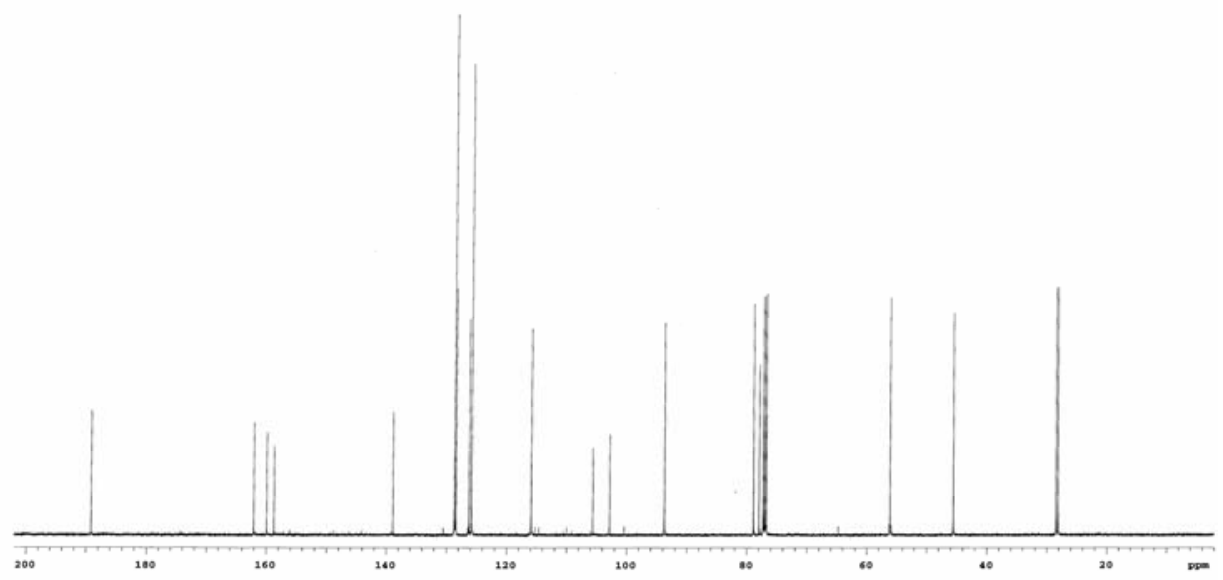

Figure 175: ${ }^{13} \mathrm{C}$ NMR $(125 \mathrm{MHz})$ spectrum of obovatin methyl ether (128) in $\mathrm{CDCl}_{3}$.

The H-H COSY spectrum confirmed the described $A B X$ system along with two orthocoupled protons, which were connected with $s p^{2}$ carbon atoms. These are shown as substructure A and B.
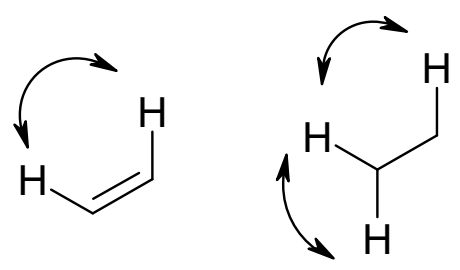

A 


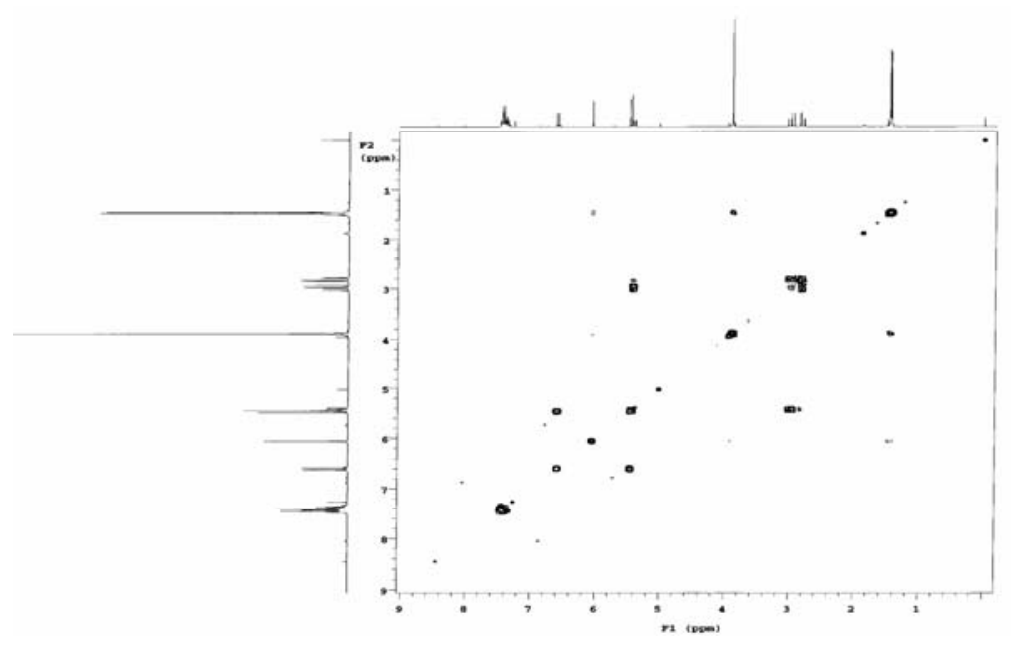

Figure 176: $\mathrm{H}-\mathrm{H}$ COSY spectrum $(600 \mathrm{MHz})$ of obovatin methyl ether (128) in $\mathrm{CDCl}_{3}$.

From the HMBC spectrum the proton at H-6 showed ${ }^{2} J$ correlation with the signal at C-5; with the same carbon, the methoxy protons at $\delta 3.89$ showed a correlation, and therefore this methoxy group was attached here. Two methyl groups, which appeared as a singlet at C-2", showed correlations with C-3" and C-7. The olefinic proton C-4" showed correlation with C-2", C-7, C-8 and C-8a. The methylene at H-3 showed correlation with carbon signal at $\mathrm{C}-1$ '.

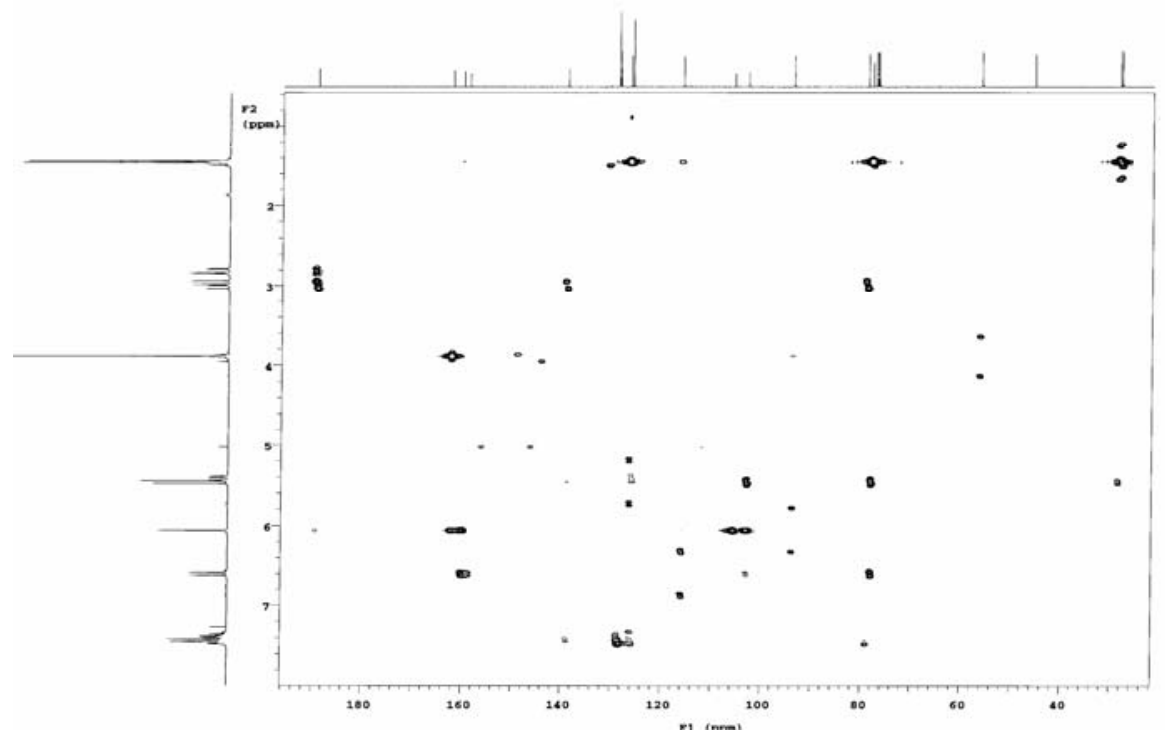

Figure 177: HMBC spectrum (600 MHz) of obovatin methyl ether (128) in $\mathrm{CDCl}_{3}$.

By using the 1D and 2D NMR data, the compound was searched in the Dictionary of Natural Products. Obovatin methyl ether (128) was found identical with respect to 1D and 2D NMR data. ${ }^{145,146}$ 


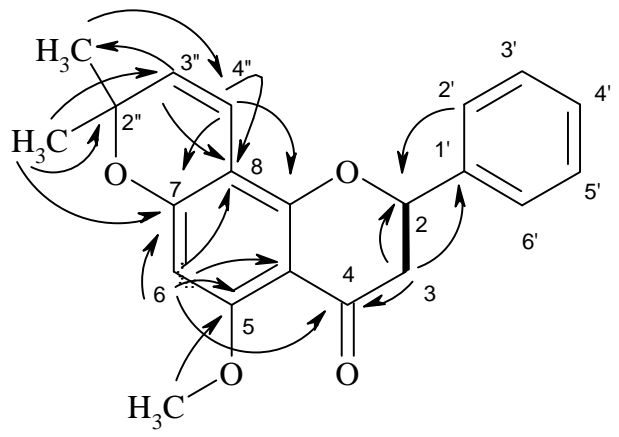

128

\subsection{Obovatin}

Obovatin (129) showed similarity in 1D and 2D NMR with that of obovatin methyl ether (128), which was previously described and elucidated. In obovatin, the 5- $\mathrm{OCH}_{3}$ group is replaced by an $\mathrm{OH}$ group. All the other correlations were the same.

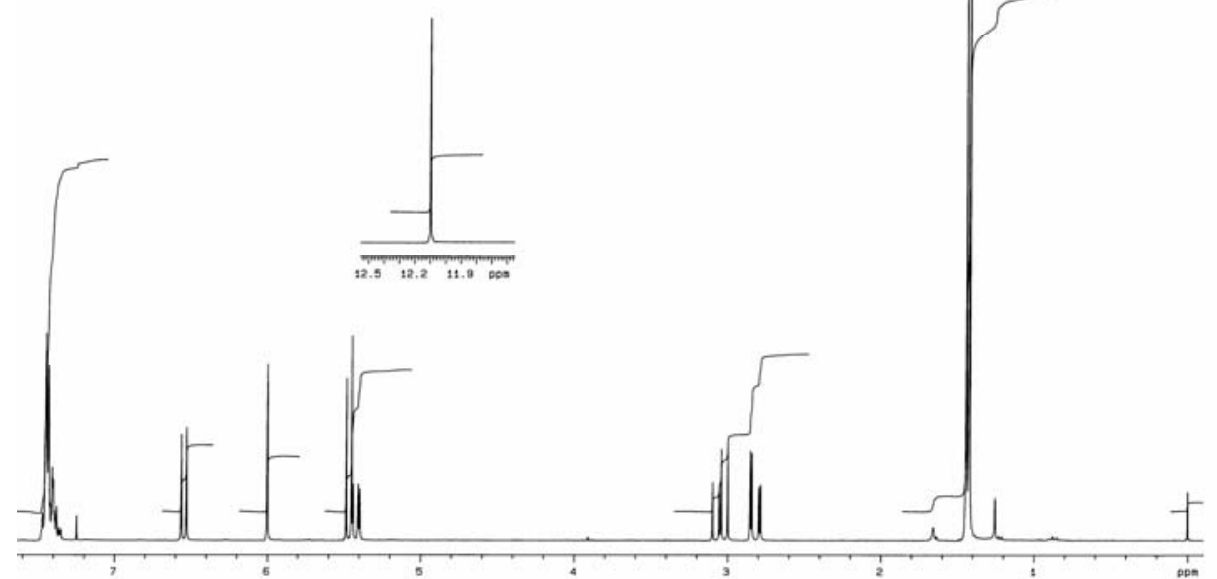

Figure 178: ${ }^{1} \mathrm{H}$ NMR (300 MHz) spectrum of obovatin (129) in $\mathrm{CDCl}_{3}$.

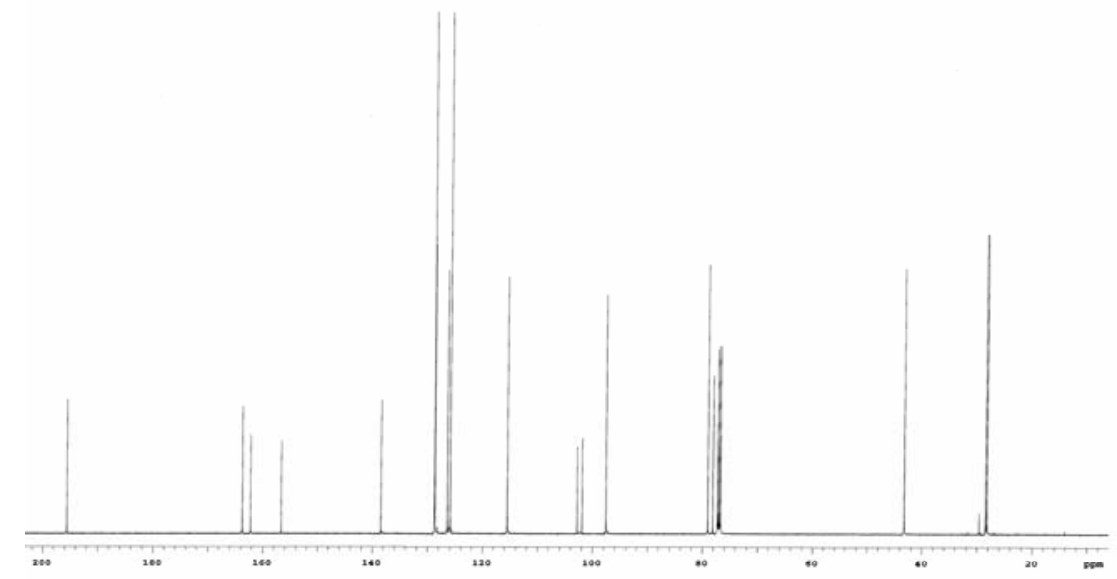

Figure 179: ${ }^{13} \mathrm{C}$ NMR $(125 \mathrm{MHz})$ spectrum of obovatin (129) in $\mathrm{CDCl}_{3}$. 


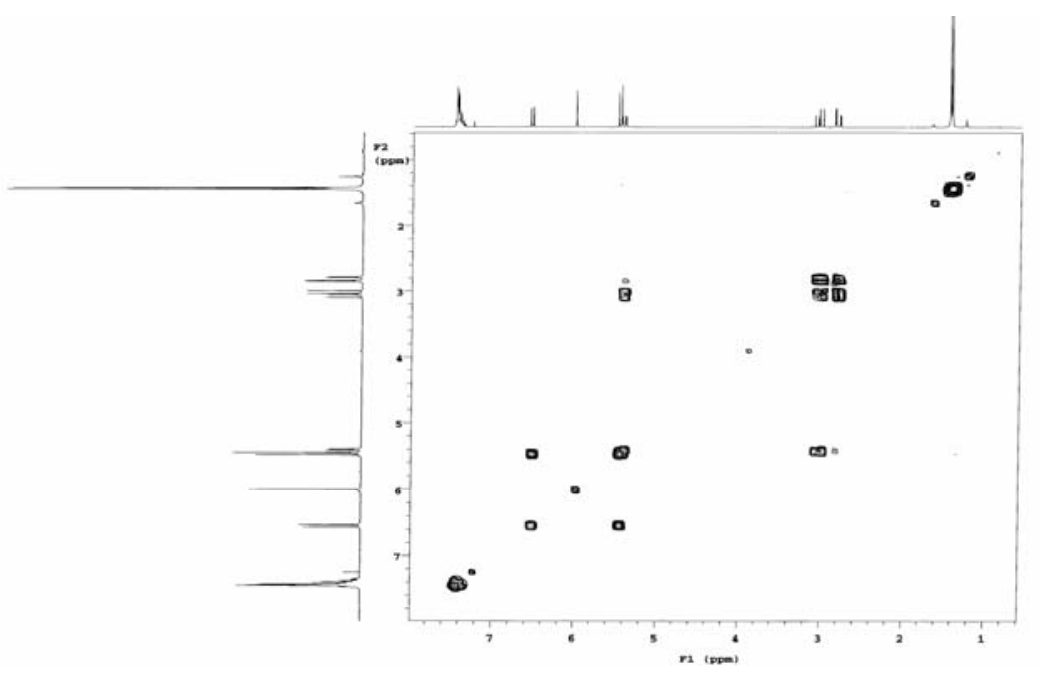

Figure 180: $\mathrm{H}-\mathrm{H}$ COSY spectrum $(600 \mathrm{MHz})$ of obovatin (129) in $\mathrm{CDCl}_{3}$.

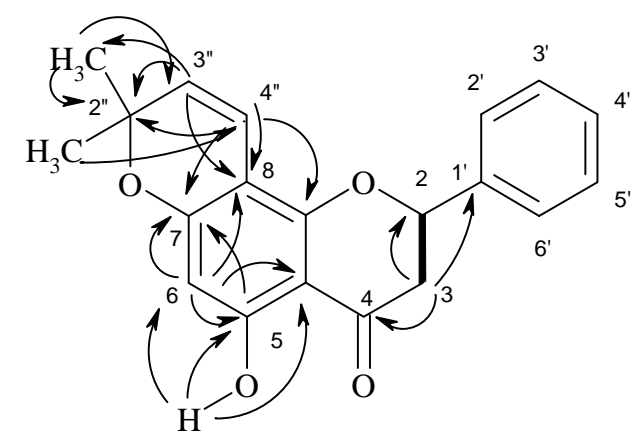

129

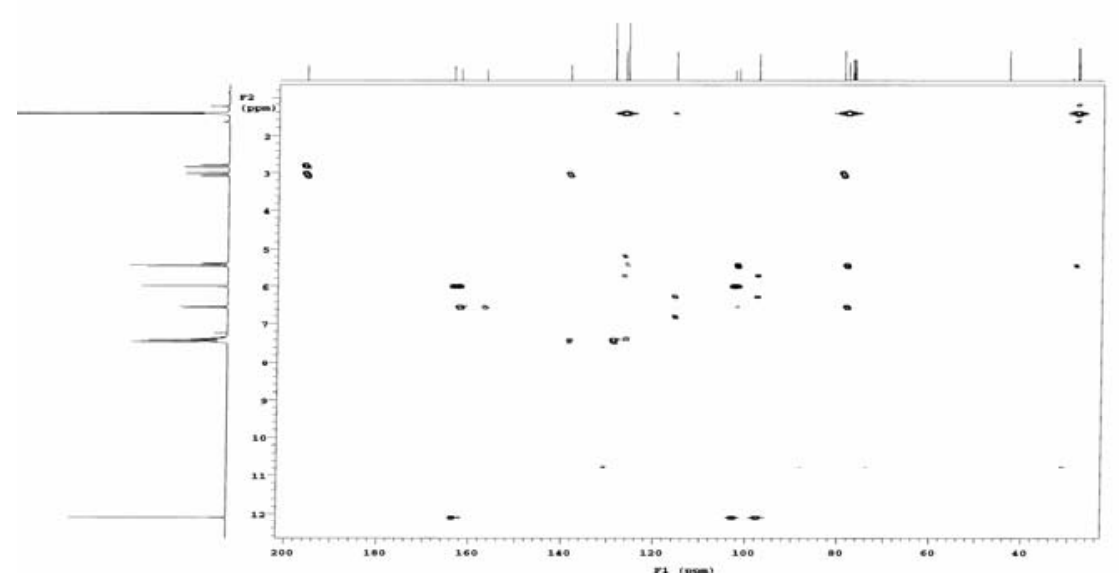

Figure 181: $\mathrm{HMBC}$ spectrum $(600 \mathrm{MHz})$ of obovatin (129) in $\mathrm{CDCl}_{3}$.

\subsection{7-Methylglabranin}

The molecular formula of 7-methylglabranin (130) was established as $\mathrm{C}_{21} \mathrm{H}_{22} \mathrm{O}_{4}$ by HRESIMS. The ${ }^{1} \mathrm{H}$ NMR spectrum showed the presence of one methoxy and one chelated hydroxyl group by singlets at $\delta 3.84(3 \mathrm{H})$ and $\delta 12.14$ respectively and also the presence of a dimethylallyl side chain by a triplet at $5.15(1 \mathrm{H})$, a doublet at $\delta 3.22$ and 
a sharp singlet at $\delta 1.62(6 \mathrm{H})$. The aromatic region was dominated by a multiplet at $\delta$ $7.44(5 \mathrm{H})$ indicating the unsubstituted B-ring and a singlet at $\delta 6.09(1 \mathrm{H})$ which could be assigned to either the C- 6 or the C- 8 proton. The salient feature of the high resolution ${ }^{1} \mathrm{H}$ NMR spectrum was the ABX system, diagnostic for the $\mathrm{C}-2$ and $\mathrm{C}-3$ protons of a flavanone. The C-2 proton (X partial signal) appeared at $\delta 5.41$, while the C-3 protons $(\mathrm{AB})$ appeared at $\delta 3.05$ and $\delta 2.85\left(\mathrm{ABX}, 2 \mathrm{H}, J_{\mathrm{AB}}=17.1, J_{\mathrm{AX}}=12.6, J_{\mathrm{BX}}=\right.$ $3.2 \mathrm{~Hz}, 3-\mathrm{CH}_{2}$ ). The value of $J=12.6 \mathrm{~Hz}$ ) for the coupling constant $J_{\mathrm{AX}}$ was indicative of an axial coupling. Therefore, the C-2 hydrogen was axial and ring B was equatorial. The position of the hydroxyl group followed from the low-field phenolic proton signal in the ${ }^{1} \mathrm{H}$ NMR spectrum.

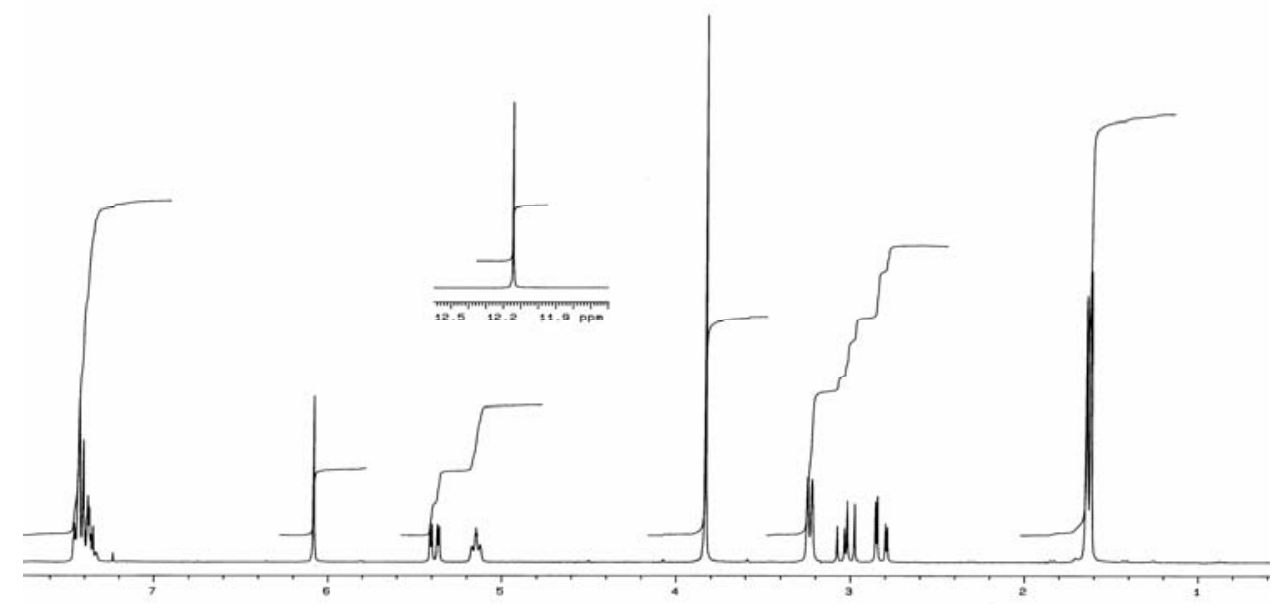

Figure 182: ${ }^{1} \mathrm{H}$ NMR $(300 \mathrm{MHz})$ spectrum of 7-methylglabranin (130) in $\mathrm{CDCl}_{3}$.

In the ${ }^{13} \mathrm{C}$ NMR spectrum a ketone signal was present at $\delta$ 196.2. The monosubstituted phenyl ring appeared at C-2', C-6' ( $\delta$ 128.6), C-3', C-5' $(\delta$ 125.9) and C-4' $(\delta$ 128.4). There was one methoxy signal at $\delta 55.8$.

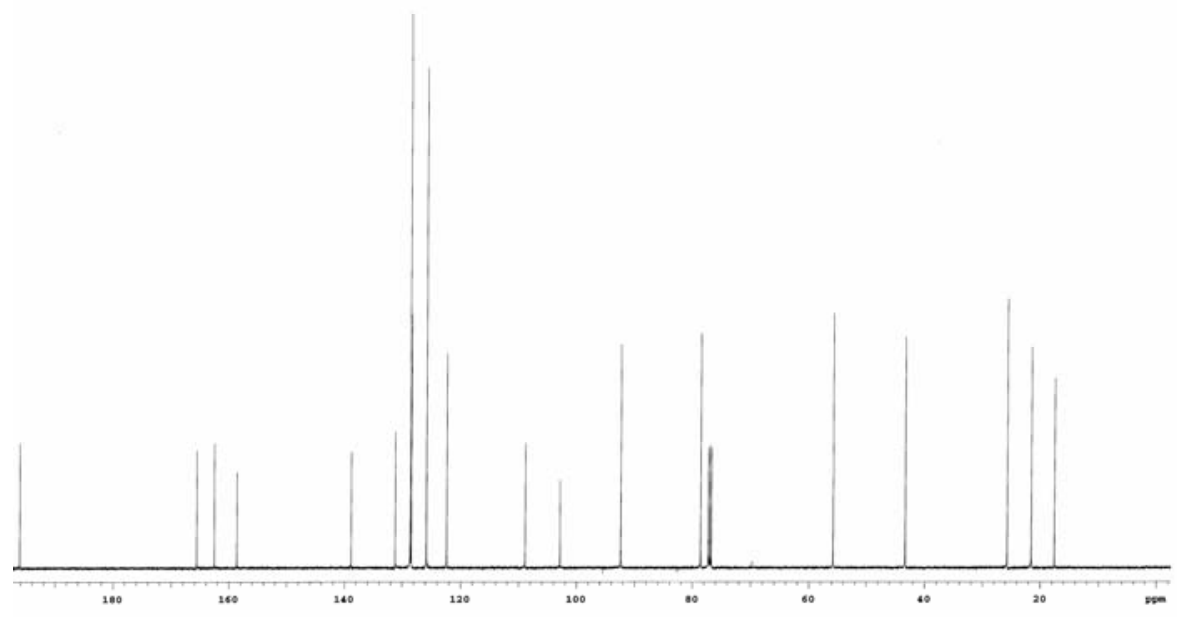

Figure 183: ${ }^{1} \mathrm{H}$ NMR (125 MHz) spectrum of 7-methylglabranin (130) in $\mathrm{CDCl}_{3}$. 
The ${ }^{1} \mathrm{H}$ NMR and ${ }^{13} \mathrm{C}$ NMR values were identical with the published ${ }^{147}$ values of 7 methylglabranin (130), which was also isolated from Tephrosia sp.<smiles>COc1cc(O)c2c(c1CC=C(C)C)OC(c1ccccc1)CC2=O</smiles>

130

\subsection{Candidone}

Candidone (131) was obtained as a white solid. The molecular formula was established as $\mathrm{C}_{22} \mathrm{H}_{24} \mathrm{O}_{4}$ by HRESIMS. The ${ }^{1} \mathrm{H}$ NMR spectrum showed the presence of two methoxy groups by singlets at $\delta 3.92$ and 3.87, respectively and also the presence of a dimethylallyl side chain was indicated by a triplet at $\delta 5.15(\mathrm{lH})$, a doublet at $\delta 3.22$ and two singlets at $\delta 1.67$ and 1.62 . The aromatic region was defined by a multiplet at $\delta 7.41(5 \mathrm{H})$ indicating the unsubstituted B-ring, and a singlet at $\delta 6.13(1 \mathrm{H})$, which could be assigned to either the C-6 or the C-8 proton. The salient feature of the ${ }^{1} \mathrm{H}$ NMR spectrum was the $A B X$ system, diagnostic for the C-2 and C-3 protons of a flavanone. The $\mathrm{C}-2$ proton, the $\mathrm{X}$ part, appeared as a double doublet at $\delta 5.40$, while the C-3 protons $(\mathrm{AB})$ appeared at $\delta 2.98,2.85\left(A B X, J_{\mathrm{AB}}=17.1, J_{\mathrm{AX}}=12.8, J_{\mathrm{BX}}=3.2\right.$ $\mathrm{Hz}, \mathrm{CH}_{2}$ ). The ${ }^{1} \mathrm{H}$ NMR spectrum showed similarity to $\mathbf{1 3 0}$, which was previously described.

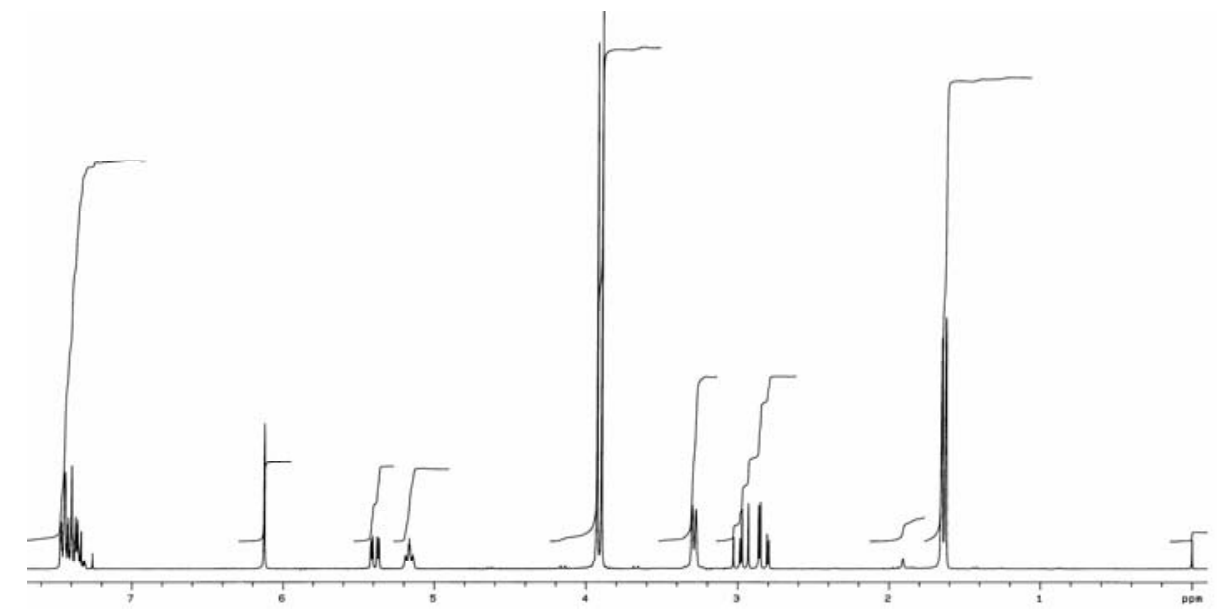

Figure 184: ${ }^{1} \mathrm{H}$ NMR spectrum $(300 \mathrm{MHz})$ of candidone (131) in $\mathrm{CDCl}_{3}$.

The ${ }^{13} \mathrm{C}$ NMR spectrum $\left(\mathrm{CDCl}_{3}, 125 \mathrm{MHz}\right)$ showed similarity with the previously described 7-methylglabranin (130). In compound 131 there were, however, two methoxy 
units instead of one methoxy unit as in 7-methylglabranin (130). The ${ }^{1} \mathrm{H}$ NMR and ${ }^{13} \mathrm{C}$ NMR spectra showed values identical with the published data of candidone (131) which was also isolated from Tephrosia sp. ${ }^{148,149}$

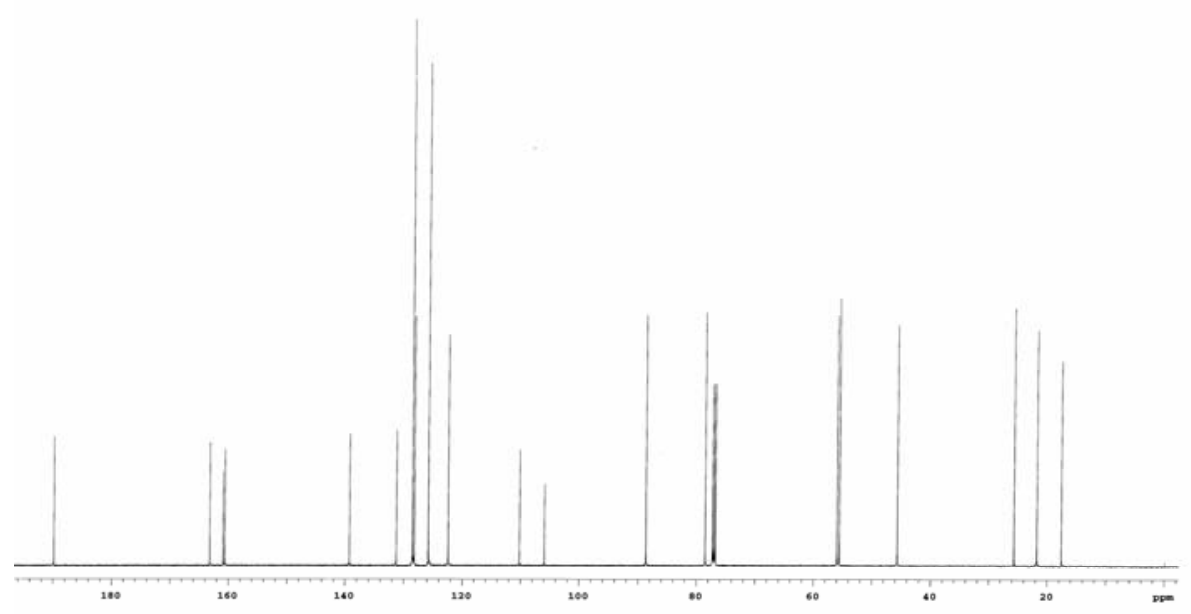

Figure 185: ${ }^{1} \mathrm{H}$ NMR spectrum $(300 \mathrm{MHz})$ of candidone (131) in $\mathrm{CDCl}_{3}$.

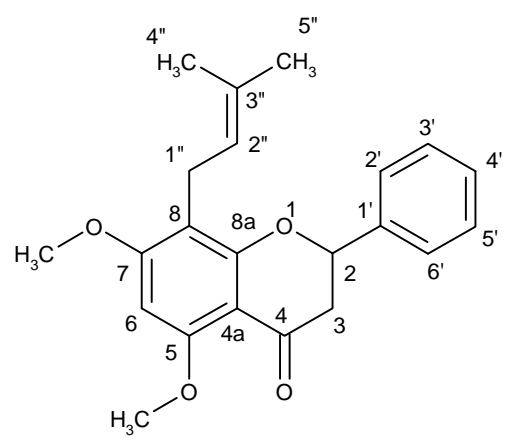

131

\subsection{Ovalichalcone}

Ovalichalcone (132) showed a molecular formula of $\mathrm{C}_{21} \mathrm{H}_{20} \mathrm{O}_{5}$ from HRESIMS. In the ${ }^{1} \mathrm{H}$ NMR spectrum there were two chelated hydroxy groups at $\delta 15.47$ and 13.66 visible; additionally, six protons attached to $s p^{2}$ carbon atoms along with one methoxy protons and two methyl groups were observed in the ${ }^{1} \mathrm{H}$ NMR spectrum. 


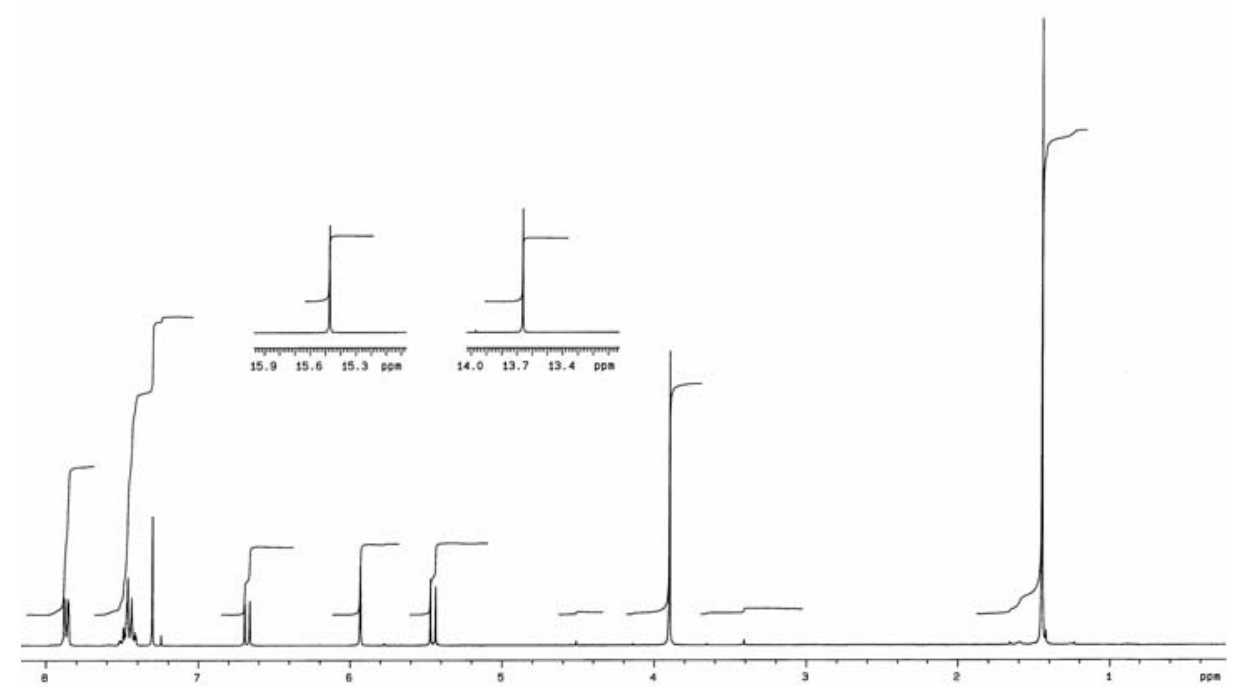

Figure 186: ${ }^{1} \mathrm{H}$ NMR $(300 \mathrm{MHz})$ spectrum of ovalichalcone (132) in $\mathrm{CDCl}_{3}$.

In the ${ }^{13} \mathrm{C}$ NMR spectrum there was many more carbon signals found than expected from the formula. The sample seemed very pure from ${ }^{1} \mathrm{H}$ NMR spectrum but ${ }^{13} \mathrm{C}$ NMR spectrum gave a different picture. After searching in Dictionary of Natural Products with the help of the ${ }^{1} \mathrm{H}$ NMR spectrum and the molecular formula, ovalichalcone (132) was found. Structure 132 was confirmed by comparing the data with published compounds. ${ }^{150}$

During ${ }^{1} \mathrm{H}$ NMR spectrum measurement, a portion of the enol was obviously converted into the keto form which was visible in the ${ }^{13} \mathrm{C}$ NMR spectrum (3 CO groups), but not in the ${ }^{1} \mathrm{H}$ spectrum. The spectral data are similar to the published data, where also this keto-enol tautomerism is mentioned.

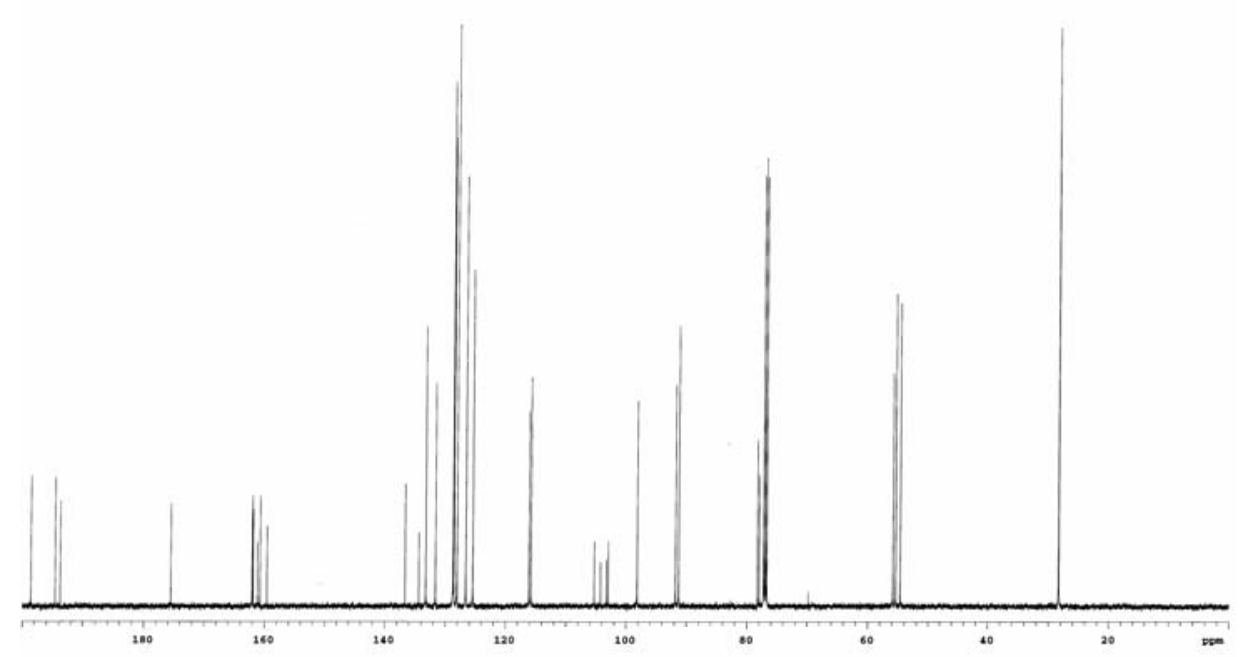

Figure 187: ${ }^{13} \mathrm{C}$ NMR spectrum $(300 \mathrm{MHz})$ spectrum of ovalichalcone (132) and its diketo tautomer $(\mathbf{1 3 3})$ in $\mathrm{CDCl}_{3}$. 
<smiles>COc1cc2c(c(O)c1C(=O)/C=C(\O)c1ccccc1)C=CC(C)(C)O2</smiles>

132<smiles>COc1cc2c(c(O)c1C(=O)CC(=O)c1ccccc1)C=CC(C)(C)O2</smiles>

133

\subsection{Praecansone B}

Compound 134 was another chalcone derivative which showed the molecular formula $\mathrm{C}_{22} \mathrm{H}_{22} \mathrm{O}_{4}$. There were 12 double bond equivalents calculated from the molecular formula. From the coupling constant of an olefinic double bond at $\delta 7.40$ and $7.00(\mathrm{~d}, J=$ $16.1 \mathrm{~Hz}$ ) the existence of an $\alpha, \beta$-unsaturated carbonyl system was clear; additionally, a mono substituted benzene ring was found. At $\delta 6.26$ there was a singlet as well as further proton signals observed at H-6 $(\delta 6.54)$ and $\mathrm{H}-3$ "-H $(\delta 5.54)$, two methoxy signals at $\delta 3.74,3.73$ and two methyls as a singlet $\delta 1.46$.

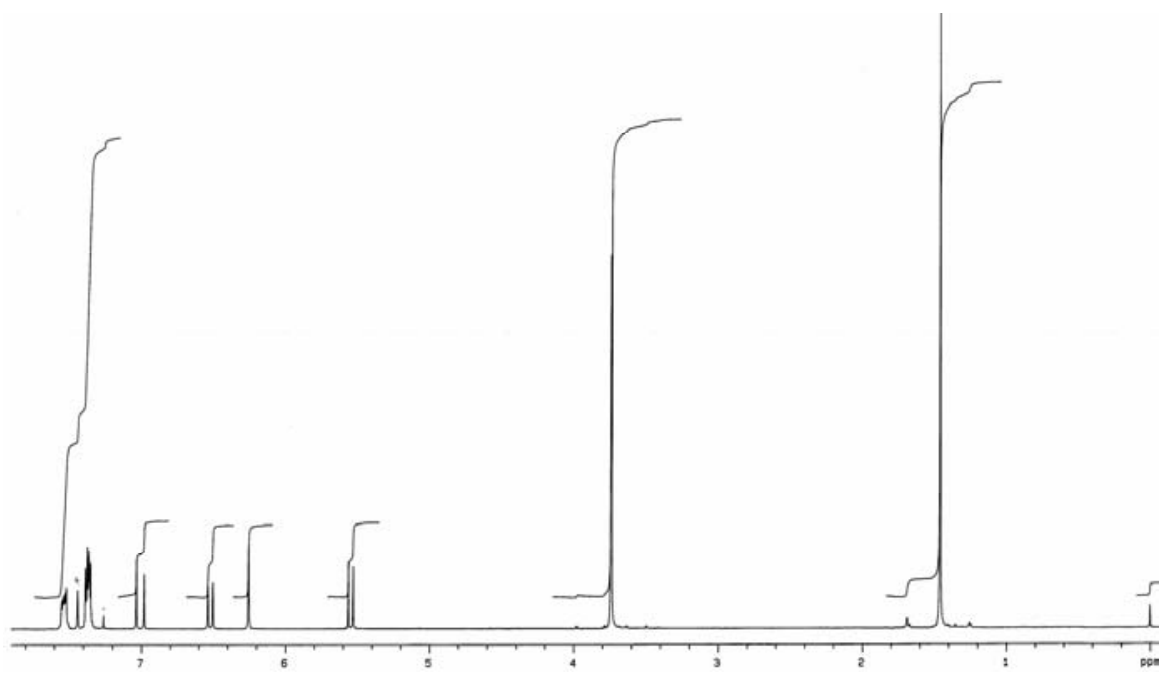

Figure 188: ${ }^{1} \mathrm{H}$ NMR $(300 \mathrm{MHz})$ spectrum of praecansone $\mathrm{B}(\mathbf{1 3 4})$ in $\mathrm{CDCl}_{3}$.

In the ${ }^{13} \mathrm{C}$ NMR spectrum there was a carbonyl of ketone or aldehyde at $\delta$ 194.1. There were six $s p^{2}$ quaternary carbon signals and ten methine carbons observed. There was an oxygenated methine at $\delta 76.8$, two methoxy groups at $\delta 63.3$ and 55.8 as well as two methyls at $\delta$ 27.9. 


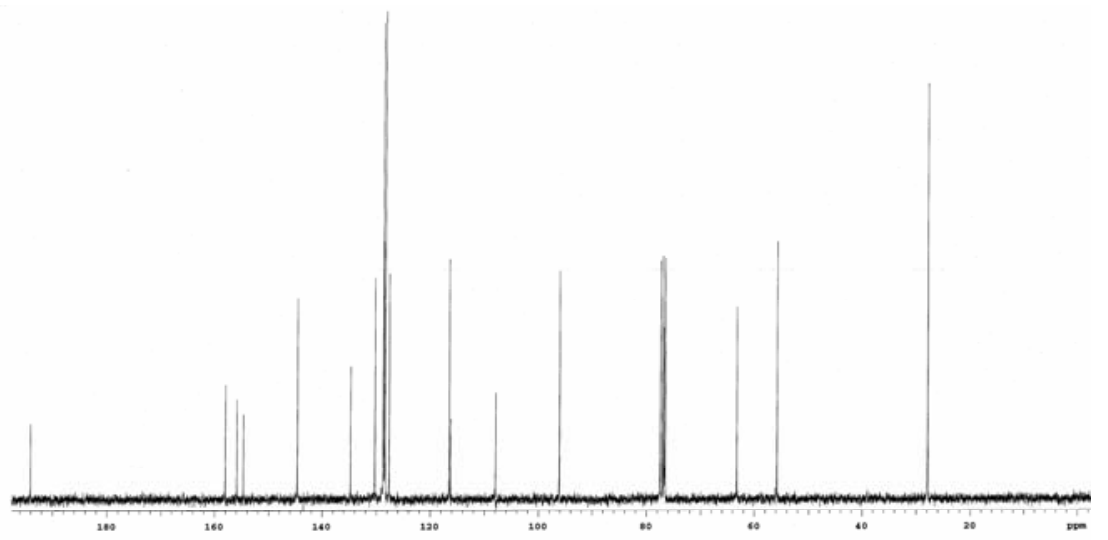

Figure 189: ${ }^{13} \mathrm{C}$ NMR (125 MHz) spectrum of praecansone $\mathrm{B}(\mathbf{1 3 4})$ in $\mathrm{CDCl}_{3}$.

From the H-H COSY spectrum substructures A-C were confirmed.

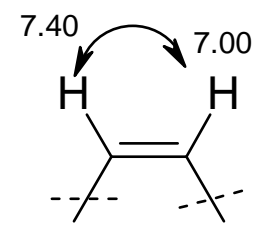

A

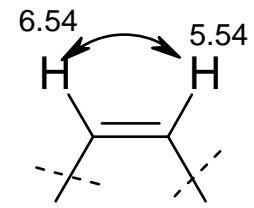

B<smiles>Ic1ccccc1</smiles>

$\mathbf{C}$

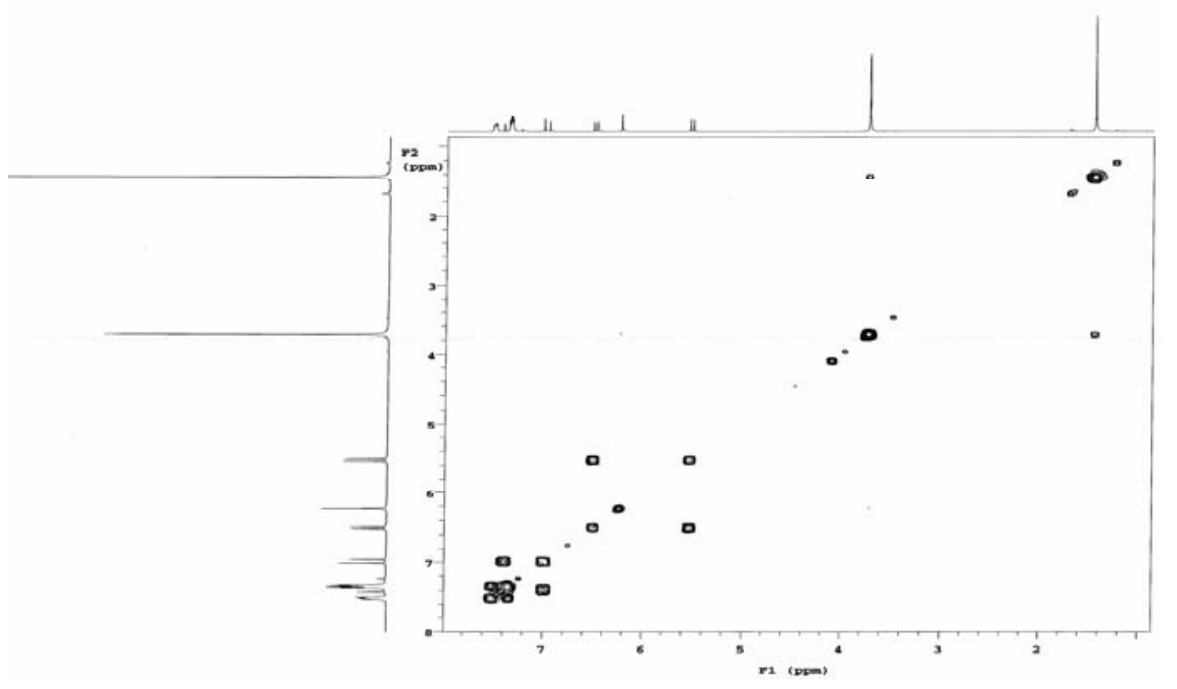

Figure 190: $\mathrm{H}-\mathrm{H}$ COSY spectrum $(600 \mathrm{MHz})$ of praecansone $\mathrm{B}(\mathbf{1 3 4})$ in $\mathrm{CDCl}_{3}$.

In the HMBC spectrum, the proton at H-6 $(\delta$ 6.26) showed correlation with two oxygenated $s p^{2}$ carbons at C-5, $7(\delta 158.1,156.0)$ as well as two more quaternary $s p^{2}$ carbons at $\delta 116.2,107.9$. Two methyl groups at $\delta 1.46$ showed correlation with an oxygenated methine at $\delta$ 76.8. The proton $\mathrm{H}-4 "$ at $\delta 6.54$ showed correlation with $\mathrm{C}-7,8$ and 9. The olefinic protons $\mathrm{H}-1$ and $\mathrm{H}-2$ at $\delta 7.40$ and 7.00 respectively showed correlation with $\mathrm{C}_{\mathrm{q}}-1^{\prime}\left(\delta\right.$ 134.8) which is a $s p^{2}$ carbon from the monosubstituted phenyl group. These two protons also showed correlations with carbonyl at $\delta 194.1$. By using the HMBC correlation drawn above as well as HRESIMS the compound was searched 
in the Dictionary of Natural Products. The structure of compound 134 was confirmed by comparing with the published data. ${ }^{151}$

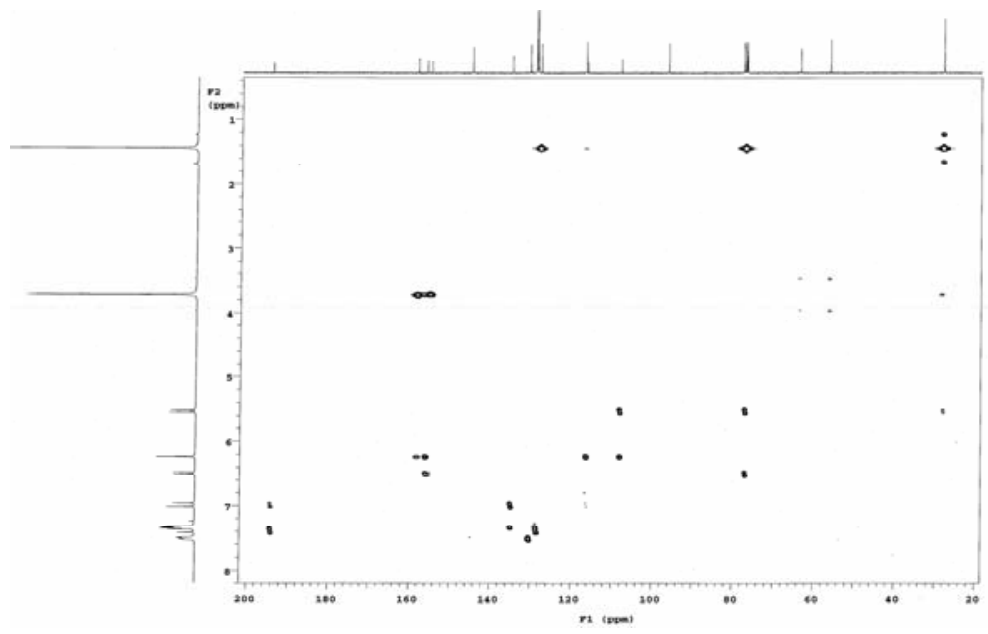

Figure 191: $\mathrm{HMBC}$ spectrum $(600 \mathrm{MHz})$ of praecansone $\mathrm{B}(134)$ in $\mathrm{CDCl}_{3}$.

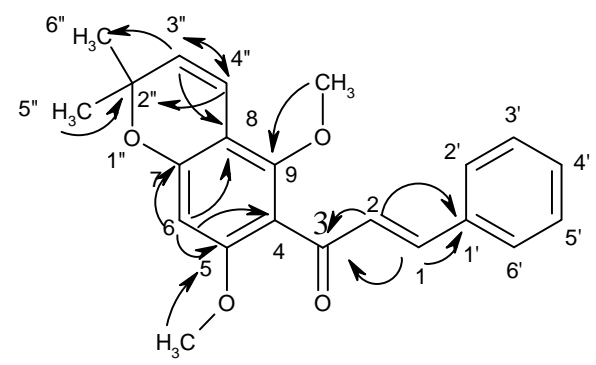

134

Praecansone B (134) was cytotoxic and showed activity against protozoa, e.g. Leishmania parasites and against leukaemia in cell cultures. ${ }^{152}$ 


\section{Summary}

An increasing number of infectious diseases is still not curable as many organisms are showing antibiotic resistance which urges the development of new medicaments to combat them. Natural products are playing an important role for this purpose. Even though the research on secondary bioactive metabolites is comparatively old, there are still unexplored areas. Especially in marine natural products research, microorganisms are considered to be a productive and successful focus. For about two decades, microbial marine natural products have been the subject of chemical and pharmacological interest and have established themselves as a group of biomedical importance. To date, more than 2700 microbial compounds have been reported from marine sources (AntiBase).

In the research work, six terrestrial Streptomyces sp. and three marine-derived bacteria strains were selected and based on their chemical and biological screening, fermented under standard conditions. One fungal strain and extracts from a plant, a tropical Tephrosia sp. were additionally analysed.

After a pre-screening to select strains with interesting properties, the bacteria were cultured in the usual way and extracted with solvents or adsorber resins. A sequence of chromatographic steps delivered the individual compounds, which underwent a dereplication step to sort out known metabolites. The structures of new compounds were elucidated by mass data and 1D and 2D NMR spectra.

\subsection{Terrestrial Streptomyces sp. ADM 14}

The terrestrial Streptomyces sp. ADM 14 was found to inhibit the growth of Staphylococcus aureus, Bacillus subtilis, Escherichia coli, Streptomyces viridochromogenes, and the fungus Candida albicans in the agar diffusion test. This strain produced many trivial compounds, among them uridine, 5'-methyl-thioadenosine, adenine, uracil, indole-3-acetic acid, indole-3-carboxylic acid, tryptophol, tyrosol, 1-hydroxy-4methoxy-naphthalene-2-caboxylic acid, phenyl acetic acid, and anthranilic acid. 5'Methyl-thioadenosine has anticancer activity, 1-hydroxy-4-methoxy-naphthalene-2caboxylic acid (43) is a herbicidal compound firstly isolated from Streptosporangium cinnabarinum, but is also known from synthesis. ${ }^{54}$ 5,7,9,11-Tetrahydroxy-4,6,8,10tetramethyl-tridec-2-enoic acid (45) was the only new metabolite of this streptomycete. Compound $\mathbf{4 5}$ is a part of macrolides like erythromycin and may be a biosynthetic intermediate of the macrolides. A relevant compound named 10-deoxymethynolide (46) showed significant antibiotic activity. ${ }^{57}$ 
<smiles>COc1cc(C(=O)O)c(O)c2ccccc12</smiles>

43<smiles>CCC(OC(=O)C(C)C(O)C(C)CC(C)C(O)C=CC(C)C(C)C)C(C)C</smiles>

46<smiles>CCC(O)C(C)C(O)C(C)C(O)C(C)C(O)C(C)/C=C/C(=O)O</smiles>

45

\subsection{Marine Streptomycete B7880}

The marine-derived streptomycete isolate B7880 is a producer of $\zeta$-pyrromycinone (47) cinerubin B A (49), cinerubin (51), and cinerubin X (52). Cinerubins are known antibiotics with a common galirubinone (47) aglycone, but differ in the sugar residues.<smiles>CC[C@]1(O)CCc2c1cc1c(c2O)C(=O)c2c(O)ccc(O)c2C1=O</smiles>

47

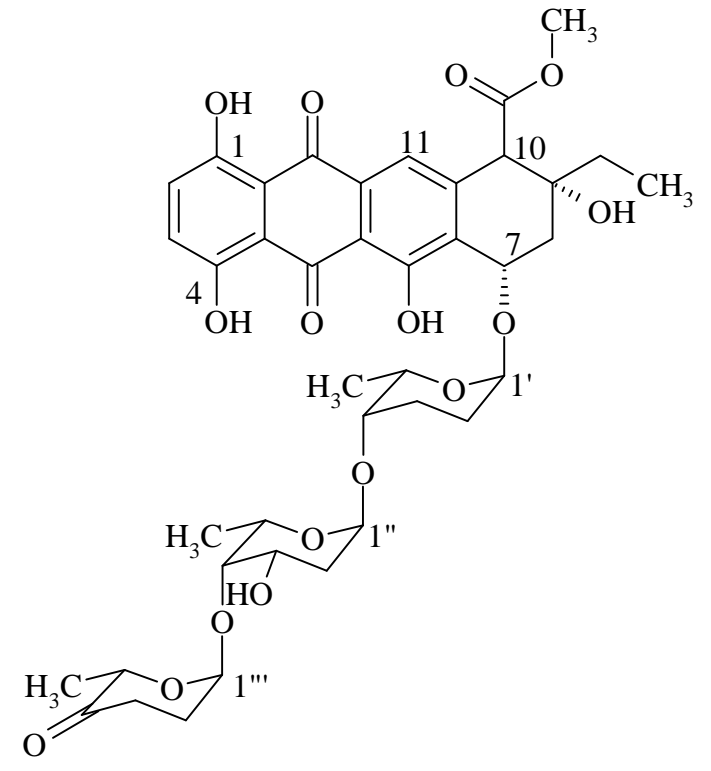

52 


\subsection{Terrestrial Streptomyces sp. ACT 7619}

The terrestrial Streptomyces sp. isolate ACT 7619 was found to be highly active against bacteria and fungi. This strain produced mainly homononactic acid and its derivatives like dinanctin. A known butenoloide, 4,10-dihydroxy-10-methyl-dodec-2-en1,4-olide, (56) was also isolated. The activity of this strain was due to the presence of dinactin (53).

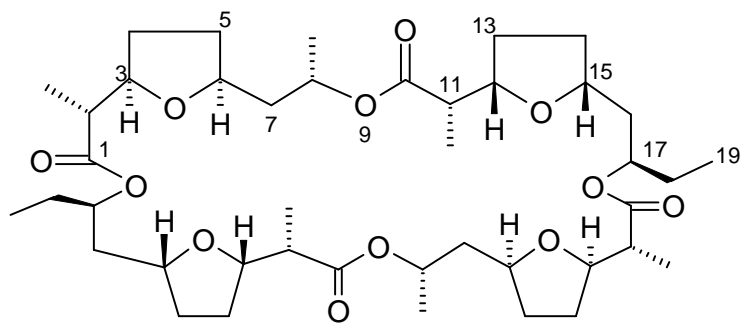

53

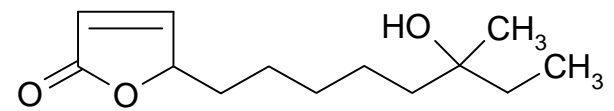

56

\subsection{Terrestrial Streptomycete GW6311}

The extract from the terrestrial streptomycete GW6311 exhibited two low-polar redorange bands, which turned to blue/violet by treatment with sodium hydroxide, pointing to peri-hydroxyquinones. Further purification provided two bioactive quinones, namely $\beta$-indomycinone (57) and saptomycin A (58).<smiles>C/C=C/CC(C)(O)c1cc(=O)c2c(C)cc3c(c2o1)C(=O)c1cccc(O)c1C3=O</smiles>

57<smiles>C/C=C/C(O)C(C)c1cc(=O)c2c(C)cc3c(c2o1)C(=O)c1c(O)cccc1C3=O</smiles>

58

\subsection{Terrestrial Streptomyces sp. GT 2005/079}

The crude extract of the terrestrial Streptomyces sp. GT 2005/079 exhibited in the biological screening pronounced antibacterial activity against Staphylococcus aureus, Bacillus subtilis, and Escherichia coli. Chromatography delivered six new compounds: Three of them were straight-chain amides (59-62), one was a homononactic acid amide and two new natural oxazole antibiotics $(63,64)$ were isolated. The straight-chain amides were moderately active against Mucor miehei. Compounds $\mathbf{6 3}$ 
and 64 were active against Streptomyces viridochromogenes (Tü 57), Staphylococcus aureus and Escherichia coli.<smiles>CC(=O)NCCCCCCC(C)O</smiles>

59<smiles>CC(=O)NCCCCCC(C)(C)O</smiles>

61<smiles>O=C(O)c1cccc2oc(-c3ccccc3O)nc12</smiles>

63<smiles>CC(=O)NCCCCCCC(C)O</smiles>

60<smiles>CCC(O)CC1CCC(C(C)C(N)=O)O1</smiles>

62<smiles>O=C(O)c1cccc2oc(-c3cccc(Cl)c3O)nc12</smiles>

64

Fairfax et al. published the use of benzoxazolecarboxamides, their preparation and pharmaceutical compositions for treating chemotherapy-induced nausea and vomiting (CINV) and Irritable Bowel Syndrome (IBS-D). ${ }^{153}$ Most of them are synthetic, but a few are known also from natural sources. Taniguchi et al. isolated the bis(benzoxazole) UK-1 (135) from the mycelial cake of an actinomycete strain with potential activity against B16, HeLa and P338 cells. ${ }^{154}$ UK-1 analogues show catalytic inhibition of human topoisomerase II. ${ }^{155}$<smiles>Cc1c(-c2nc3c(C(=O)O)cccc3o2)ccc2oc(-c3ccccc3O)nc12</smiles>

135

Homononactic acid amide is a close derivative of homononactic acid which is known for its antifungal and pesticidal activity ${ }^{156}$ and is a building block for other anticancer compounds. ${ }^{157}$ Yoshihito Tanouchi et al. showed the immunosuppressive effects of oligonactins (tetranactin, trinactin and dinactin) on experimental autoimmune uveoretinitis in rats. ${ }^{158}$ Shelby $\mathrm{P}$ Umland et al. showed the effects of cyclosporin A and dinactin on T-cell proliferation, interleukin-5 production, and murine pulmonary inflammation. ${ }^{159}$ 


\subsection{Terrestrial Actinomyces sp. $\mathbf{N} 435$}

The terrestrial Actinomyces sp. N 435 was investigated with the goal to find active compounds against plant-phathogenic fungi, as the crude extracts showed moderate antifungal activity. Working up and purification of the fractions led to the isolation of diketopiperazines along with a new secondary metabolite, ramthacin B (78), which is structurally closely related to the antiviral natural compound virantmycin (77). Ramthacin B (78) was not so stable. After ${ }^{1} \mathrm{H}$ NMR and MS spectrum, this compound was degraded in the NMR tube. So, the bioactivity could not be measured.<smiles>CO[C@]1(CCC(C)=C(C)C)Nc2ccc(C(=O)O)cc2CC1Cl</smiles>

77<smiles>COCC1(C[C@H](O)C(C)=C(C)C)Nc2ccc(C(=O)O)cc2C[C@H]1Cl</smiles>

78

\subsection{Marine-derived Streptomyces sp. B 7857}

The chemical investigation of the marine-derived Streptomyces sp. B 7857 delivered four new oxygenated $\alpha$-gorgonene sesquiterpenes derivatives named bacteriogorgonene (A-D) (81-84) as well as ten known secondary metabolites, namely homononactic acid, 1-acetyl- $\beta$-carbolin, 3-(2-hydroxyethyl)indole, polypropylenglycole, nonactic acid, uracil, thymidine, deoxyuridine, and 2-hydroxy-1-(indol-3-yl)ethanone.

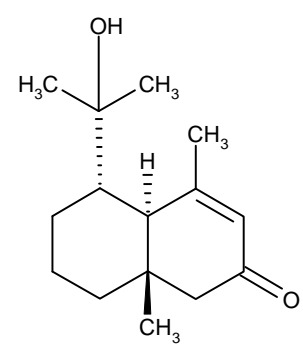

81

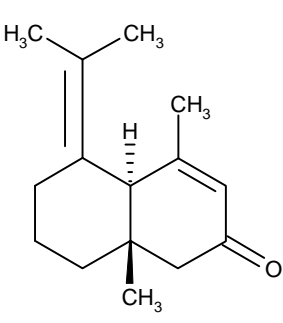

82

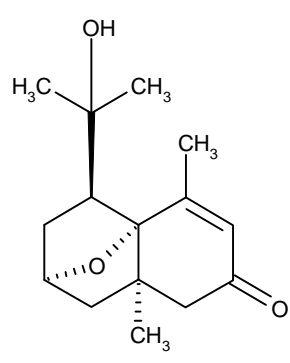

83<smiles>CC1=CC(=O)C[C@]2(C)CCCC(C(C)(C)O)C12O</smiles>

84

Gorgonene terpenes are a rare class of sesquiterpenes, which possess an isopropyl group in the unusual C-6 position, an indication that they apparently contain two tailto-tail fused isoprene units. This is the first report that bacteria contain oxygenated gorgonene sequiterpenes. Until recently; only 50 sesquiterpenoids had been isolated from micro organisms. 


\subsection{Terrestrial streptomycete GW5127}

The terrestrial streptomycete GW5127 yielded three new metabolites, i.e. 9-ethyl6,11-dihydroxy-4-methoxy-naphthacene-5,12-dione (88), 4-acetyl-1,3-dihydroimidazo[4,5-b]pyridin-2-one (90), 5 and N-[2-(3-hydroxy-4-methoxy-phenyl)-ethyl]acetamide (93), along with three known compounds, 4-hydroxy-3-methoxy-tyrosol, $\beta$ rubromycin (95) and $\gamma$-rubromycin (96).<smiles>CCc1ccc2c(O)c3c(c(O)c2c1)C(=O)c1cccc(OC)c1C3=O</smiles>

88<smiles>CC(=O)c1nccc2[nH]c(=O)[nH]c12</smiles>

90<smiles>COc1cc(CCNC(C)=O)ccc1O</smiles>

93<smiles>COC(=O)c1cc2cc3c(c(O)c2c(=O)o1)OC1(CC3)CC2=C(O1)C(=O)c1c(O)c(OC)cc(OC)c1C2=O</smiles>

95<smiles>COC(=O)c1cc2cc3c(c(O)c2c(=O)o1)OC1(CC3)CC2=C(O1)C(O)C1C(=O)C(OC)=CC(=O)C1C2O</smiles>

96

\subsection{Terrestrial Streptomyces sp. GW 18/1811}

The terrestrial Streptomyces sp. GW 18/1811 exhibited good biological activity against bacteria, fungi and algae. This strain produced komodoquinone B (97), feudomycinone C (98), a new bacterial metabolite $\alpha$-hydroxyacetovanillone (99), $N$ acetylhomocysteine thiolactone (99) and a chlorinated known antibiotic lysolipin I (102). 
<smiles>COc1cccc2c1C(=O)c1c(O)c3c(c(O)c1C2=O)C[C@@](C)(O)C[C@@H]3O</smiles>

97<smiles>C[C@]1(O)Cc2c(O)c3c(c(O)c2[C@@H](O)C1)C(=O)c1cccc(O)c1C3=O</smiles>

98<smiles>COc1cc(C(=O)CO)ccc1O</smiles>

99<smiles>CC(=O)NC1CCSC1=O</smiles>

101

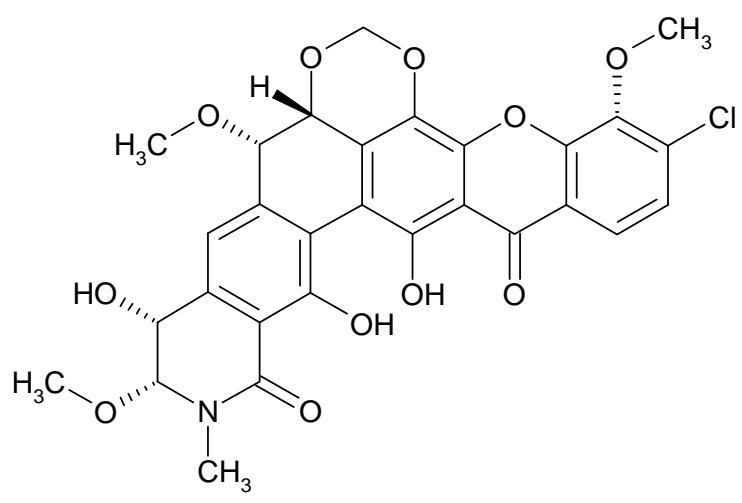

102

The antitumor-active komodoquinone B showed reduced side effects of neuropathy and they are useful for controlling multiple drug resistant tumors. ${ }^{160}$ Lysolipin I is active against gram-pos. and gram-neg. bacteria. ${ }^{118}$

\subsection{Fungal metabolite}

The fungal antibiotic bikaverin (103) was received from Prof. Dr. H. Anke, Institute for Biotechnology and Drug Research, Kaiserslautern, Germany.<smiles>COC1=CC(=O)c2c(c(O)c3oc4cc(OC)cc(C)c4c(=O)c3c2O)C1=O</smiles>

103

Bikaverin inhibited the incorporation of purine bases into nucleic acids and acid soluble nucleotides in tumour cells and inhibits ATP formation at the level of the oxidative phosphorylation. ${ }^{161}$ Bikaverin showed inhibition of mitochondrial functions also an efficient hemolytic agent. ${ }^{162}$ 


\subsection{Plant metabolites from Tephrosia calophylla}

Twentyeight samples from the plant source (Tephrosia calophylla) were obtained from one of our research collaborator, Professor Seru Ganapaty from Andhra University, India. Twentyfive different compounds were confirmed in those samples. A novel benzil derivative calophione A (104) together with three new coumestan derivatives, tephcalostan A $(\mathbf{1 0 8}), C$ and D $(\mathbf{1 1 1}, \mathbf{1 1 2})$ were isolated from the roots of Tephrosia calophylla. The All compounds were evaluated for their cytotoxic activity against RAW and HT-29 cancer cell lines and antiprotozoal activity against various parasitic protozoa. Calophione A exhibited significant cytotoxicity with $\mathrm{IC}_{50}$ values of 5.00 (RAW) and $2.90 \mu \mathrm{M}(\mathrm{HT}-29)$ respectively.<smiles>C=C(C)C1Cc2cc(C(=O)C(=O)c3cc4c(cc3O)OCO4)c(O)cc2O1</smiles>

104<smiles></smiles>

111<smiles></smiles>

108<smiles></smiles>

112

In this thesis 9 terrestrial and marine-derived streptomycetes, one plant and one fungal extracts were studied. From the terrestrial and marine strains, 47 compounds were isolated, from which 14 compounds can be considered as new secondary metabolites. From the plant Tephrosia sp., 25 compounds were isolated, from which 4 compounds can be considered as new secondary metabolites. The altogether 73 metabolites contained different groups such as peptides, quinones, macrolides, and indoles.

Table 3: Total number of isolated compounds from bacteria in this thesis.

\begin{tabular}{llll}
\hline Source & $\begin{array}{l}\text { No of Strains } \\
\text { or plant } \boldsymbol{s p} \text {. }\end{array}$ & No of compounds & $\begin{array}{l}\text { No of new } \\
\text { compounds }\end{array}$ \\
\hline $\begin{array}{l}\text { Marine and terrestrial } \\
\text { streptomycetes }\end{array}$ & 10 & 47 & 14 \\
$\begin{array}{l}\text { Fungal metabolites } \\
\text { Plant metabolites }\end{array}$ & 1 & 1 & - \\
\hline
\end{tabular}




\section{Experimental Part}

\subsection{General}

IR spectra: Perkin-Elmer 1600 Series FT-IR; Perkin-Elmer 297 Infrared Spectrophotometer; Beckman DU-640; Shimadzu FT-IR; (KBr tablet and film). - UV/VIS spectra: Perkin-Elmer Lambda 15 UV/VIS spectrometer. - Optical rotations: Polarimeter (Perkin-Elmer, model 241). - ${ }^{1} \mathrm{H}$ NMR spectra: Varian Unity 300 (300.542 MHz), Bruker AMX 300 (300.542 MHz), Varian Inova 500 (499.8 MHz). Coupling constants $(J)$ in Hz. Abbreviations: $\mathrm{s}=$ singlet, $\mathrm{d}=$ doublet, $\mathrm{dd}=$ doublet doublet, $\mathrm{t}=$ triplet, $\mathrm{q}=$ quartet, quint $=$ quintet, $\mathrm{m}=$ multiplet, $\mathrm{br}=$ broad. $-{ }^{13} \mathrm{C}$ NMR spectra: Varian Unity 300 (75.5 MHz), Varian Inova 500 (125.7 MHz). Chemical shifts were measured relatively to tetramethylsilane as internal standard. Abbreviations: APT (Attached Proton Test): $\mathrm{CH} / \mathrm{CH}_{3}$ up and $\mathrm{C}_{\mathrm{q}} / \mathrm{CH}_{2}$ down. - 2D NMR spectra: $\mathrm{H}, \mathrm{H}$ COSY spectra $\left({ }^{1} \mathrm{H},{ }^{1} \mathrm{H}\right.$-Correlated Spectroscopy), HMBC spectra (Heteronuclear Multiple Bond Connectivity), HMQC spectra (Heteronuclear Multiple Quantum Coherence) and NOESY spectra (Nuclear Overhauser Effect Spectroscopy). - Mass spectra: EIMS at $70 \mathrm{eV}$ with Varian MAT 731, Varian 311A, AMD-402, high resolution with perflurokerosene as standard. ESIMS with Quattro Triple Quadruple mass spectrometer Finigan MAT-Incos 50, ESIMS LCQ (Finnigan).

\subsection{Materials}

Thin layer chromatography (TLC): DC-Folien Polygram SIL G/UV 254 (MachereyNagel \& Co.). - Glass plates for chemical screening: Merck silica gel 60 F254, $(10 \times$ $20 \mathrm{~cm}$ ). - Preparative thin layer chromatography (PTLC): $55 \mathrm{~g}$ silica gel P/UV 254 (Macherey-Nagel \& Co.) is added to $120 \mathrm{ml}$ of demineralised water with continuous stirring for 15 minutes. $60 \mathrm{ml}$ of the homogenous suspension is poured on a horizontal held $(20 \times 20 \mathrm{~cm})$ glass plate and the unfilled spaces are covered by distributing the suspension. The plates are air dried for 24 hours and activated by heating for 3 hours at $130{ }^{\circ} \mathrm{C}$. - Column chromatography (CC): MN silica gel 60: 0.05-0.2 mm, 70-270 mesh (Macherey-Nagel \& Co). Sephadex LH-20 (Pharmacia) was used for size exclusion chromatography.

\subsection{Spray reagents}

Anisaldehyde/sulphuric acid: $1 \mathrm{ml}$ anisaldehyde was added to $100 \mathrm{ml}$ of a stock solution containing $85 \mathrm{ml}$ methanol, $14 \mathrm{ml}$ acetic acid and $1 \mathrm{ml}$ sulphuric acid. - Ehrlich's 
reagent: $1 \mathrm{~g}$ 4-dimethylaminobenzaldehyde was dissolved in a mixture of $25 \mathrm{ml}$ hydrochloric acid (37\%) and $75 \mathrm{ml}$ methanol; it gives a red to violet colouration with indoles, a yellow colour with anthranilic acid derivatives and a grey colour reaction with some N-heterocycles. - Ninhydrin: 0.3 g ninhydrin (2,2-dihydroxyindan-1,3dione) was dissolved in $95 \mathrm{ml}$ iso-propanol. The mixture was added to $5 \mathrm{ml}$ collidin (2,4,6-trimethylpyridin) and $5 \mathrm{ml}$ acetic acid (96\%). This reagent gives a blue to a violet colouration with amino acids, peptides and polypeptides with free amino groups.

Chlorine/o-dianisidin reaction: The reagent was prepared from $100 \mathrm{ml} o$-dianisidin $(0.032 \%)$ in $1 \mathrm{~N}$ acetic acid, $1.5 \mathrm{~g} \mathrm{Na}_{2} \mathrm{WO}_{4} \cdot 2 \mathrm{H}_{2} \mathrm{O}$ in $10 \mathrm{ml}$ water, $115 \mathrm{ml}$ acetone and $450 \mathrm{mg}$ KI. The moistened TLC plate was kept ca. $30 \mathrm{~min}$ in a chlorine atmosphere (from $0.5 \mathrm{~g} \mathrm{KClO}_{3}+2 \mathrm{ml}$ conc. $\mathrm{HCl}$ ) and then dried for ca. $1 \mathrm{~h}$, till the excess of chlorine was evaporated and then dipped into the reagent. The reagent is specific for peptides as universal spraying reagent.

$\mathrm{NaOH}$ or $\mathrm{KOH}: 2 \mathrm{~N} \mathrm{NaOH}$ or $\mathrm{KOH}$ solutions are used to identify perihydroxyquinones by deepening of the colour from orange to violet or blue.

\subsection{Microbiological materials}

Storage of strains: Deep-freeze storage in a Dewar vessel, 1'Air liquid type BT 37 A. - Capillaries for deep-freeze storage: diameter $1.75 \mathrm{~mm}$, length $80 \mathrm{~mm}$, Hirschmann Laborgeräte Eberstadt. - Soil for soil culture: Luvos Heilerde LU-VOS JUST GmbH \& Co. Friedrichshof (from the health shop). - Ultraturrax: Janke \& Munkel KG. Shaker : Infors AG (CH 4103 Einbach) type ITE. - Laboratory shaker: IKA-shaker type S50 (max. $6000 \mathrm{Upm}$ ). - Autoclave: Albert Dargatz Autoclave volume 119 1, working temperature $121{ }^{\circ} \mathrm{C}$, working pressure $1.2 \mathrm{~kg} / \mathrm{cm}^{2}$. - Antibiotic assay discs: 9 mm diameter, Schleicher \& Schüll No. 321 261. - Culture media: glucose, bacto peptone, bacto agar, dextrose, soybean flour, mannitol, yeast extract and malt extract were purchased from Merck, Darmstadt. - Petri-dishes: $94 \mathrm{~mm}$ diameter, $16 \mathrm{~mm}$ height, Fa. Greiner Labortechnik, Nürtingen. - Celite: Celite France S. A., RueilMalmaison Cedex. - Sterile filters: Midisart 2000, $0.2 \mu \mathrm{m}$, PTFE-Filter, Sartorius, Göttingen. - Laminar-Flow-Box: Kojar KR-125, Reinraumtechnik GmbH, Rielasingen-Worblingen 1. - Brine shrimp eggs (Artemia salina): SERA Artemia Salinenkrebseier, SERA Heinsberg. - Salinenkrebsfutter: microcell DOHSE Aquaristik KG Bonn (brine shrimp eggs and food can be obtained from aquaristic shops). 


\subsection{Recipes}

All culture media were autoclaved at 1.2 bar and $120{ }^{\circ} \mathrm{C}$. Sterilisation time for $1 \mathrm{~L}$ shaker culture: $33 \mathrm{~min}$.

Artificial sea water

Iron(III) citrate

$\mathrm{NaCl}$

$\mathrm{MgCl}_{2} \cdot 6 \mathrm{H}_{2} \mathrm{O}$

$\mathrm{Na}_{2} \mathrm{SO}_{4}$

$\mathrm{CaCl}_{2}$

$\mathrm{Na}_{2} \mathrm{HPO}_{4}$

$\mathrm{SiO}_{2}$

Trace element stock soln.

Stock soln.

tap water

Trace element stock solution

$\begin{array}{ll}\mathrm{H}_{3} \mathrm{BO}_{3} & 0.611 \mathrm{~g} \\ \mathrm{MnCl}_{2} & 0.389 \mathrm{~g} \\ \mathrm{CuSO}_{4} & 0.056 \mathrm{~g} \\ \mathrm{ZnSO}_{4} \cdot 7 \mathrm{H}_{2} \mathrm{O} & 0.056 \mathrm{~g} \\ \mathrm{Al}_{2}\left(\mathrm{SO}_{4}\right)_{3} \cdot 18 \mathrm{H}_{2} \mathrm{O} & 0.056 \mathrm{~g} \\ \mathrm{NiSO}_{4} \cdot 6 \mathrm{H}_{2} \mathrm{O} & 0.056 \mathrm{~g} \\ \mathrm{CO}_{\left(\mathrm{NO}_{3}\right)_{3} \cdot 6 \mathrm{H}_{2} \mathrm{O}} & 0.056 \mathrm{~g} \\ \mathrm{TiO}_{2} & 0.056 \mathrm{~g} \\ \left(\mathrm{NH}_{4}\right)_{6} \mathrm{Mo}_{7} \mathrm{O}_{24} \cdot 4 \mathrm{H}_{2} \mathrm{O} & 0.056 \mathrm{~g} \\ \mathrm{LiCl}^{\mathrm{SnCl}} & 0.028 \mathrm{~g} \\ \mathrm{KI} & 0.028 \mathrm{~g}\end{array}$

$2 \mathrm{~g}$ (powder)

$389 \mathrm{~g}$

$176 \mathrm{~g}$

$68.8 \mathrm{~g}$

$36.0 \mathrm{~g}$

$0.16 \mathrm{~g}$

$0.30 \mathrm{~g}$

$20 \mathrm{~mL}$

$200 \mathrm{~mL}$

add $20 \mathrm{~L}$ 
tap water

Stock solution

$\mathrm{KCl}$

$\mathrm{NaHCO}_{3}$

$\mathrm{KBr}$

$\mathrm{SrCl}_{2} \cdot 6 \mathrm{H}_{2} \mathrm{O}$

$\mathrm{H}_{3} \mathrm{BO}_{3}$

$\mathrm{NaF}$

$\mathrm{NH}_{4} \mathrm{NO}_{3}$

tap water ad $1 \mathrm{~L}$

$110 \mathrm{~g}$

$32 \mathrm{~g}$

$16 \mathrm{~g}$

$6.8 \mathrm{~g}$ (dissolved separately)

$4.4 \mathrm{~g}$

$0.48 \mathrm{~g}$

$0.32 \mathrm{~g}$

ad $2 \mathrm{~L}$

\subsubsection{Nutrients}

$\mathrm{M}_{2}$ medium (without sea water)

$\begin{array}{ll}\text { Malt extract } & 10 \mathrm{~g} \\ \text { Glucose } & 4 \mathrm{~g} \\ \text { Yeast extract } & 4 \mathrm{~g} \\ \text { Tap water } & \operatorname{ad} 1 \mathrm{~L}\end{array}$

The $\mathrm{pH}$ was adjusted to 7.8 using $2 \mathrm{~N} \mathrm{NaOH}$. Solid medium was prepared by adding $18 \mathrm{~g}$ of agar

$\mathrm{M}_{2}^{+}$medium $\left(\mathrm{M}_{2}\right.$ medium with sea water $)$

Malt extract

Glucose

Yeast extract

Artificial seawater

Tap water
$10 \mathrm{~g}$

$4 \mathrm{~g}$

$4 \mathrm{~g}$

$500 \mathrm{~mL}$

$500 \mathrm{~mL}$

The $\mathrm{pH}$ was adjusted to 7.8 using $2 \mathrm{~N} \mathrm{NaOH}$. Solid medium was prepared by adding $18 \mathrm{~g}$ of agar. 
Luria-Bertani-Medium (LB)

$\begin{array}{ll}\text { Trypton } & 10 \mathrm{~g} \\ \text { Yeast extract } & 5 \mathrm{~g} \\ \mathrm{NaCl} & 10 \mathrm{~g}\end{array}$

Tap water $\quad 1000 \mathrm{~mL}$

The $\mathrm{pH}$ was adjusted to 7.8 using $2 \mathrm{~N} \mathrm{NaOH}$. Solid medium was prepared by adding $18 \mathrm{~g}$ of agar.

Soja-Mannitol Medium

Soybean meal (defatted) $\quad 20 \mathrm{~g}$

D (-)-Mannitol $20 \mathrm{~g}$

Tap water $\quad 1000 \mathrm{ml}$

The $\mathrm{pH}$ was adjusted to 7.8 using $2 \mathrm{~N} \mathrm{NaOH}$. Solid medium was prepared by adding $18 \mathrm{~g}$ of agar.

M Test Agar (for test organisms Escherichia coli, Bacillus subtilis (ATCC 6051), Staphylococcus aureus, Mucor miehei (Tü 284):

$\begin{array}{ll}\text { Malt extract } & 10 \mathrm{~g} \\ \text { Yeast extract } & 4 \mathrm{~g} \\ \text { Glucose } & 4 \mathrm{~g} \\ \text { Bacto agar } & 20 \mathrm{~g} \\ \text { Demineralised water } & 1000 \mathrm{~mL}\end{array}$

The $\mathrm{pH}$ was adjusted to 7.8 using $2 \mathrm{~N} \mathrm{NaOH}$.

Sabouraud-Agar

(for test organism Candida albicans)

Glucose $40 \mathrm{~g}$

Bacto peptone $10 \mathrm{~g}$

Bacto agar $20 \mathrm{~g}$ 
Demineralised water

$1000 \mathrm{~mL}$

The $\mathrm{pH}$ was adjusted to 7.8 using $2 \mathrm{~N} \mathrm{NaOH}$.

Nutritional solution A

Soybean meal (defatted) $\quad 30 \mathrm{~g}$

Glycerol $30 \mathrm{~g}$

$\mathrm{CaCO}_{3} \quad 2 \mathrm{~g}$

Artificial sea water $\quad 750 \mathrm{ml}$

Demineralised water $\quad 250 \mathrm{ml}$

Nutritional solution B

Starch $10 \mathrm{~g}$

NZ-Amine $5 \mathrm{~g}$

Soybean meal $\quad 2 \mathrm{~g}$

Yeast extract $5 \mathrm{~g}$

$\mathrm{KNO}_{3} \quad 3 \mathrm{~g}$

Algal extract $\quad 2.5 \mathrm{ml}$

Artificial sea water $\quad 750 \mathrm{ml}$

Demineralised water $\quad 250 \mathrm{ml}$

Stock solutions and media for cultivation of algae

Fe-EDTA

$0.7 \mathrm{~g}$ of $\mathrm{FeSO}_{4} \cdot 7 \mathrm{H}_{2} \mathrm{O}$ and $0.93 \mathrm{~g}$ EDTA (Titriplex III) are dissolved in $80 \mathrm{ml}$ of demineralised water at $60{ }^{\circ} \mathrm{C}$ and then diluted to $100 \mathrm{ml}$.

Trace element Solution II:

Solution A:

$\mathrm{MnSO}_{4} \cdot \mathrm{H}_{2} \mathrm{O} \quad 16.9 \mathrm{mg}$

$\mathrm{Na}_{2} \mathrm{MoO}_{4} \cdot 2 \mathrm{H}_{2} \mathrm{O} \quad 13.0 \mathrm{mg}$ 
$\mathrm{Co}\left(\mathrm{NO}_{3}\right)_{2} \cdot 6 \mathrm{H}_{2} \mathrm{O} \quad 10.0 \mathrm{mg}$

Salts are dissolved in $10 \mathrm{ml}$ of demineralised water.

Solution B:
$\mathrm{CuSO}_{4} \cdot 5 \mathrm{H}_{2} \mathrm{O}$
$5.0 \mathrm{mg}$
$\mathrm{H}_{3} \mathrm{BO}_{3}$
$10.0 \mathrm{mg}$
$\mathrm{ZnSO}_{4} \cdot 7 \mathrm{H}_{2} \mathrm{O}$
$10.0 \mathrm{mg}$

Salts are dissolved each in $10 \mathrm{ml}$ of demineralised water. Solutions A is added to B and diluted to $100 \mathrm{ml}$ with demineralised water.

Bold's Basal medium (BBM): (for algae Chlorella vulgaris, Chlorella sorokiniana and Scenedesmus subspicatus.

$\begin{array}{ll}\mathrm{NaNO}_{3} & 0.250 \mathrm{~g} \\ \mathrm{KH}_{2} \mathrm{PO}_{4} & 0.175 \mathrm{~g} \\ \mathrm{~K}_{2} \mathrm{HPO}_{4} & 0.075 \mathrm{~g} \\ \mathrm{MgSO}_{4} \cdot 7 \mathrm{H}_{2} \mathrm{O} & 0.075 \mathrm{~g} \\ \mathrm{NaCl} & 0.025 \mathrm{~g} \\ \mathrm{CaCl} \cdot 2 \mathrm{H}_{2} \mathrm{O} & 0.025 \mathrm{~g} \\ \mathrm{Fe}_{-} \mathrm{EDTA} & 1.0 \mathrm{ml} \\ \text { Trace element solution II } & 0.1 \mathrm{ml}\end{array}$

Salts are dissolved in $10 \mathrm{ml}$ of demineralised water and added to Fe-EDTA and trace element solution II. The mixture made to one litre with demineralised water. Solid medium was prepared by adding $18 \mathrm{~g}$ of agar. 


\subsection{Microbiological and analytical methods}

\subsubsection{Storage of Strains}

All bacteria strains were stored in liquid nitrogen for long time. The strains were used to inoculate agar plates with the suitable media at room temperature.

\subsubsection{Pre-Screening}

The microbial isolates (obtained from culture collections) were cultured in a $1 \mathrm{~L}$ scale in $1 \mathrm{~L}-E r l e n m e y e r$ flasks each containing $200 \sim 250 \mathrm{ml}$ of $\mathrm{M}_{2}$ or (for marine strains) $\mathrm{M}_{2}{ }^{+}$medium. The flasks were shaken for 3-5 days at $28{ }^{\circ} \mathrm{C}$, after which the entire fermentation broth was freeze-dried and the residue extracted with ethyl acetate. The extracts were evaporated to dryness and used for the antimicrobial tests in a concentration of $50 \mu \mathrm{g} / \mathrm{ml}$.

\subsubsection{Biological screening}

The crude extract was dissolved in $\mathrm{CHCl}_{3} / 10 \% \mathrm{MeOH}$ (concentration $50 \mu \mathrm{g} / \mathrm{mL}$ ), in which the paper disks were dipped, dried under sterile conditions (flow box) and put on an agar plates inoculated with Bacillus subtilis (ATCC6051), Staphylococcus aureus, Streptomyces viridochromogenes (Tü 57), Escherichia coli, Chlorella vulgaris, Chlorella sorokiniana, Scenedesmus subspicatus, Candida albicans and Mucor miehei (Tü 284).

The plates were incubated at $37^{\circ} \mathrm{C}$ for bacteria ( 12 hours), $27{ }^{\circ} \mathrm{C}$ for fungi ( 24 hours), and $24-26{ }^{\circ} \mathrm{C}$ under day-light for micro-algae (96 hours). The diameter of the inhibition zones was measured by ruler.

\subsubsection{Chemical and pharmacological screening}

Samples of the extracts were separated on silica gel glass plates $(10 \times 20 \mathrm{~cm})$ with two solvent systems $\mathrm{CHCl}_{3} / 5 \% \mathrm{MeOH}$ and $\mathrm{CHCl}_{3} / 10 \% \mathrm{MeOH}$. After drying, the plates were photographed under UV light at $254 \mathrm{~nm}$ and marked at $366 \mathrm{~nm}$, and subsequently stained by anisaldehyde and Ehrlich's reagent. Finally, the plates were scanned for documentation. For the pharmacological investigations, approximately 25 mg of the crude extract was sent to industrial partners. 


\subsubsection{Brine shrimp microwell cytotoxicity assay}

To a $500 \mathrm{ml}$ separating funnel, filled with $400 \mathrm{ml}$ of artificial seawater, $1 \mathrm{~g}$ of dried eggs of Artemia salina and $1 \mathrm{~g}$ food were added. The suspension was aerated by bubbling air into the funnel and kept for 24 to 48 hours at room temperature. After aeration had been removed, the suspension was kept for $1 \mathrm{~h}$ undisturbed, whereby the remaining unhatched eggs dropped. In order to get a higher density of larvae, one side of the separating funnel was covered with aluminium foil and the other illuminated with a lamp, whereby the phototropic larvae were gathering at the illuminated side and could be collected by pipette. 30 to 40 shrimp larvae were transferred to a deepwell microtiter plate (wells diameter $1.8 \mathrm{~cm}$, depth $2 \mathrm{~cm}$ ) filled with $0.2 \mathrm{ml}$ of salt water and the dead larvae counted (number $\mathrm{N}$ ). A solution of $20 \mu \mathrm{g}$ of the crude extract in 5 to $10 \mu \mathrm{l}$ DMSO was added and the plate kept at r.t. in the dark. After $24 \mathrm{~h}$, the dead larvae were counted in each well under the microscope (number A). The still living larvae were killed by addition of ca. $0.5 \mathrm{ml}$ methanol so that subsequently the total number of the animals could be determined (number $\mathrm{G}$ ). The mortality rate $\mathrm{M}$ was calculated in \%. Each test row was accompanied by a blind sample with pure DMSO (number B) and a control sample with $1 \mu \mathrm{g} /$ test actinomycin D. The mortality rate $\mathrm{M}$ was calculated using the following formula:

$M=\left[\frac{(A-B-N)}{(G-N)}\right] \cdot 100$

with
$\mathrm{M}=\quad$ percent of the dead larvae after $24 \mathrm{~h}$.
$\mathrm{A}=\quad$ number of the dead larvae after $24 \mathrm{~h}$.
$\mathrm{B}=\quad$ average number of the dead larvae in the blind samples after $24 \mathrm{~h}$
$\mathrm{N}=\quad$ number of the dead larvae before starting of the test.
$\mathrm{G}=\quad$ total number of brine shrimps

\subsubsection{Primary screening}

Antibiotic screening (disk diffusion test): The test is performed using paper discs with a diameter of $8 \mathrm{~mm}$ under standardized conditions. If the inhibition zone is ranging from 11 to $20 \mathrm{~mm}$, the compound is considered to be weakly active (+), from 21 to 30 
$\mathrm{mm}$ designated as active (++) and over $30 \mathrm{~mm}$ is highly active (+++). - Chemical screening: evaluation of the separated bands by the number, intensity and colour reactions with different staining reagents on TLC. - Toxicity test: By counting survivors after $24 \mathrm{hrs}$, the mortality of the extracts was calculated (see above). The extracts, fractions or isolated compounds were considered inactive when the mortality rate was lower than $10 \%$ (-), from 10 to $59 \%$ as weakly active (+), from 60 to $95 \%$ as active $(++)$ and over $95 \%$ as strongly active $(+++)$.

\subsection{Terrestrial Streptomyces sp. ADM 14}

\subsubsection{Primary Screening}

The semiquantitative results of antibacterial, antifungal and phytotoxic tests are listed in the Table 4. The TLC of the crude extract exhibited a light yellow spot and three colourless UV inactive bands, which became first brown and later turned to violet with anisaldehyde/sulphuric acid.

Table 4: Biological activity of the crude extract of the strain ADM 14.

\begin{tabular}{ll}
\hline Test organisms & Inhibition zone $(\varnothing \mathbf{m m})$ \\
\hline Bacillus subtilis & 17 \\
Staphylococcus aureus & 25 \\
Streptomyces viridochromogenes (Tü 57) & 15 \\
Escherichia coli & 13 \\
Candida albicans & 10 \\
Scenedesmus subspicatus & 22 \\
\hline
\end{tabular}

\subsubsection{Cultivation, work-up and isolation}

For the cultivation, well grown agar plates of the strain ADM 14 were used to inoculate 20 litres of $\mathrm{M}_{2}$ medium, which was cultivated as shaker culture for 5 days at 28 ${ }^{\circ} \mathrm{C}$. The obtained broth was filtered over Celite, the water phase was extracted with XAD-16, and the resin eluted with methanol; the biomass was extracted with ethyl acetate. The combined extracts were evaporated to dryness under vacuum to afford a brown crude extract $(2.1 \mathrm{~g})$. The extract was defatted with cyclohexane and the methanolic part was pre-separated by column chromatography into five fractions. Further purification on Sephadex LH-20 followed by PTLC and silica gel column chromatography afforded 14 compounds: 1-hydroxy-4-methoxy-naphthalene-2-caboxylic 
acid (43, $2.5 \mathrm{mg}), 5$ '-methyl-thioadenosine (44, $75 \mathrm{mg})$, and 5,7,9,11-tetrahydroxy4,6,8,10-tetramethyl-tridec-2-enoic acid $(\mathbf{4 5}, 6.7 \mathrm{mg})$.

1-Hydroxy-4-methoxynaphthoic acid (43): light grey coloured solid, $R_{f}=0.66$ $\left(\mathrm{CH}_{2} \mathrm{Cl}_{2} / 5 \% \mathrm{MeOH}\right)$, blue UV fluorescent. - ${ }^{1} \mathrm{H} \mathrm{NMR}\left(\mathrm{CD}_{3} \mathrm{OD}, 300 \mathrm{MHz}\right) \delta 8.27$ $\left(\mathrm{dd}, 1 \mathrm{H},{ }^{3} \mathrm{~J}=8.0,{ }^{4} \mathrm{~J}=1.4 \mathrm{~Hz}, 8-\mathrm{H}\right), 8.12\left(\mathrm{dd}, 1 \mathrm{H},{ }^{3} \mathrm{~J}=8.0,{ }^{4} \mathrm{~J}=1.4 \mathrm{~Hz}, 8-\mathrm{H}\right), 7.50$ (m, 2H, 7, 8-H), 7.29 (s, 1H, 3-H), 3.96 (s, 3H, 4- $\left.\mathrm{OCH}_{3}\right)$; - (-)-ESIMS m/z (217 [M-H]', 100), (217 [2M-2H+ Na]',55).

5'-Methyl-thioadenosine (44): Colourless solid, $R_{f}=0.41\left(\mathrm{CHCl}_{3} / 10 \% \mathrm{MeOH}\right), \mathrm{UV}$ absorbing, dark grey with anisaldehyde/sulphuric acid. - ${ }^{1} \mathrm{H}$ NMR ([D 6 DMSO, 300 MHz) $\delta 8.38(\mathrm{~s}, 1 \mathrm{H}, 2-\mathrm{H}), 8.17(\mathrm{~s}, 1 \mathrm{H}, 6-\mathrm{H}), 7.23$ ( $b r \mathrm{~s}, \mathrm{H} / \mathrm{D}$ exchangeable, $2 \mathrm{H}, \mathrm{NH}_{2}$ ), $5.92\left(\mathrm{~d},{ }^{3} J=6 \mathrm{~Hz}, 1 \mathrm{H}, 11^{\prime}-\mathrm{H}\right), 5.82-5.55(\mathrm{br} \mathrm{s}, \mathrm{H} / \mathrm{D}$ exchangeable, $2 \mathrm{H}, \mathrm{OH}), 4.73\left(\mathrm{t},{ }^{3} J\right.$ $\left.=6.3 \mathrm{~Hz}, 1 \mathrm{H}, 2^{\prime}-\mathrm{H}\right), 4.16\left(\mathrm{~m}, 1 \mathrm{H}, 3^{\prime}-\mathrm{H}\right), 4.03\left(\mathrm{ABX}, 1 \mathrm{H}, 4^{\prime}-\mathrm{H}\right), 2.90\left(\mathrm{ABX}, 1 \mathrm{H}, 5^{\prime}-\right.$ Ha), 2.75 (ABX, 1H, 5'-Hb), 2.07 (s, 3H, 5'-SCH$)_{3}$. - (+)-ESIMS m/z 617 ([2M $\left.+\mathrm{Na}]^{+}, 100\right), 320\left([\mathrm{M}+\mathrm{Na}]^{+}, 93\right), 298\left([\mathrm{M}+\mathrm{H}]^{+}, 10\right) ;-(-)-E S I M S ~ m / z 593\left([2 \mathrm{M}-\mathrm{H}]^{-}\right.$, 100), 296 ([M-H] $\left.]^{-}, 10\right)$; - EIMS (70 eV) m/z 297 ([M] $\left.]^{+}, 12\right), 250$ (6), 194 (28), 166 (8), 164 (100), 138 (8), 136 (100), 61 (54).

5,7,9,11-Tetrahydroxy-4,6,8,10-tetramethyl-tridec-2-enoic acid (45): Grey solid, $R_{f}=0.38\left(\mathrm{CHCl}_{3} / 7 \% \mathrm{MeOH}\right), \mathrm{UV}$ absorbing, violet with anisaldehyde/sulphuric acid.

$-{ }^{1} \mathrm{H}$ NMR $\left(\mathrm{CD}_{3} \mathrm{OD}, 300 \mathrm{MHz}\right) \delta 6.32\left(\mathrm{dd}, 1 \mathrm{H},{ }^{3} \mathrm{~J}=16.0,9.5 \mathrm{~Hz}, 3-\mathrm{H}\right), 5.86(\mathrm{~d}, 1 \mathrm{H}$, $\left.{ }^{3} J=15.7 \mathrm{~Hz}, 2-\mathrm{H}\right), 3.41-3.74(\mathrm{~m}, 4 \mathrm{H}, 5,7,9,11-\mathrm{H}), 2.43(\mathrm{~m}, 1 \mathrm{H}, 4-\mathrm{H}), 1.86(\mathrm{~m}, 1 \mathrm{H}, 8-$ $\mathrm{H}), 1.84(\mathrm{~m}, 1 \mathrm{H}, 6-\mathrm{H}), 1.77(\mathrm{~m}, 1 \mathrm{H}, 10-\mathrm{H}), 1.58\left(\mathrm{~m}, 2 \mathrm{H}, 12-\mathrm{CH}_{2 \mathrm{a}}\right), 1.32(\mathrm{~m}, 2 \mathrm{H}, 12-$ $\left.\mathrm{CH}_{2 \mathrm{~b}}\right), 0.98\left(\mathrm{t}, 3 \mathrm{H},{ }^{3} \mathrm{~J}=7.0 \mathrm{~Hz}, 12-\mathrm{CH}_{3}\right), 0.98\left(\mathrm{t}, 3 \mathrm{H},{ }^{3} \mathrm{~J}=7.0 \mathrm{~Hz}, 4-\mathrm{CH}_{3}\right), 0.92(\mathrm{~d}$, $\left.3 \mathrm{H},{ }^{3} \mathrm{~J}=6.9 \mathrm{~Hz}, 8-\mathrm{CH}_{3}\right), 0.85\left(\mathrm{~d}, 3 \mathrm{H},{ }^{3} \mathrm{~J}=7.0 \mathrm{~Hz}, 6-\mathrm{CH}_{3}\right), 0.78\left(\mathrm{~d}, 3 \mathrm{H},{ }^{3} \mathrm{~J}=6.9 \mathrm{~Hz}\right.$, 10- $\left.\mathrm{CH}_{3}\right) .-{ }^{13} \mathrm{C}$ NMR $\left(\mathrm{CD}_{3} \mathrm{OD}, 125 \mathrm{MHz}\right) \delta 171.7$ (1-CO), 150.4 (2-CH), 126.4 (3$\mathrm{CH}), 80.3$ (7-CH), 79.2 (9-CH), $76.4(5-\mathrm{CH}), 74.6$ (11-CH), 42.6 (4-CH), 41.7 (10$\mathrm{CH}), 37.9(8-\mathrm{CH}), 36.8(6-\mathrm{CH}), 26.2\left(12-\mathrm{CH}_{2}\right), 16.9\left(4-\mathrm{CH}_{3}\right), 11.8\left(8-\mathrm{CH}_{3}\right), 10.5$ (6$\left.\mathrm{CH}_{3}\right), 9.7\left(10-\mathrm{CH}_{3}\right), 5.9\left(12-\mathrm{CH}_{3}\right) .-(+)$-ESIMS $m / z .333\left([\mathrm{M}+\mathrm{H}]^{+}\right) ;-(+)$-HRESIMS $\mathrm{m} / \mathrm{z} 333.20610[\mathrm{M}+\mathrm{H}]^{+}$, (calcd for $\left.\mathrm{C}_{17} \mathrm{H}_{33} \mathrm{O}_{6}, 333.20603\right)$.

\subsubsection{Synthesis of 1-hydroxy-4-methoxynaphthoic acid (43)}

1-Hydroxy-4-methoxynaphthol (42) (8.7 g, $0.05 \mathrm{M})$ and freshly heated $\mathrm{K}_{2} \mathrm{CO}_{3}(17.2$ $\mathrm{g}, 0.125 \mathrm{M}$, preheated on a Bunsen burner to about $500{ }^{\circ} \mathrm{C}$ for 45 minutes) were mixed homogeneously. It was then taken in a $250 \mathrm{ml}$ autoclave and dry ice $(\sim 50 \mathrm{~g})$ was added. The reaction mixture was heated in the sealed autoclave to $150{ }^{\circ} \mathrm{C}$ for 6 hours under the resulting pressure of 70-80 bar. After cooling to r.t., the product was mixed with $50 \mathrm{ml}$ water and air was passed through the solution for 15 minutes, to 
oxidize unreacted naphthol. The deep blue precipitation formed was separated by filtration and discarded. The filtrate was acidified with $6 \mathrm{~N} \mathrm{HCl}$, whereby a light blue precipitate was formed. The solid was separated again by filtration and the filtrate was discarded. The product ( $1.4 \mathrm{~g}$ ) was purified by crystallization from methanol/dichloromethane. The NMR data were identical with those of the natural product (see theoretical part).

\subsection{Marine Streptomyces sp. B 7880}

\subsubsection{Primary screening}

The ethyl acetate extract of the streptomycete isolate B8969 showed the presence of several pink to red coloured, UV absorbing zones. The strain exhibited high biological activity against Mucor miehei (Tü284), Candida albicans, and the alga Chlorella sorokiniana.

Table 5: Biological activity of the crude extract of the strain B 7880

\begin{tabular}{ll}
\hline Test organisms & Inhibition zone $(\varnothing \mathbf{~ m m})$ \\
\hline Bacillus subtilis & 0 \\
Staphylococcus aureus & 17 \\
Streptomyces viridochromogenes (Tü 57) & 11 \\
Escherichia coli & 0 \\
Candida albicans & 12 \\
Mucor miehei & 14 \\
Chlorella vulgaris & 13 \\
Chlorella sorokiniana & 17 \\
Scenedesmus subspicatus & 0 \\
\hline
\end{tabular}

\subsubsection{Isolation and cultivation}

The strain B7880 was cultivated on a 20-litre scale for 7 days at $28{ }^{\circ} \mathrm{C}$ as shaker culture $(110 \mathrm{rpm})$. After filtration, the dark red broth was filtered to separate the water phase and mycelium. The water phase was extracted by XAD-16 and the mycelium was extracted with ethyl acetate. The extracts from both phases $(2.58 \mathrm{~g})$ were combined, as TLC did not show much difference. For isolation and purification of the metabolites, the resulting product was chromatographed on silica gel column using a chloroform-methanol gradient. After fractionation of the crude extract through silica gel column chromatography, fraction 2 was further purified by PTLC and Sephadex 
LH-20 to isolate $\zeta$-pyrromycinone $(47,1.6 \mathrm{mg})$ and cinerubin $\mathrm{B}(\mathbf{4 9}, 1.8 \mathrm{mg})$ as low polar, orange fluorescent $(366 \mathrm{~nm})$, which turned to violet by treating with sodium hydroxide. Following the same method cinerubin X (51, $2.1 \mathrm{mg})$ and cinerubin A (52, $1.1 \mathrm{mg}$ ) were separated from fraction 4.

S-Pyrromycinone (47): Low polar orange-red solid, $R_{f}=0.57\left(\mathrm{CHCl}_{3} / 5 \% \mathrm{MeOH}\right)$, violet with $\mathrm{NaOH} .-{ }^{1} \mathrm{H} \mathrm{NMR}\left(\mathrm{CDCl}_{3}, 300 \mathrm{MHz}\right) \delta 13.02(\mathrm{~s}, 1 \mathrm{H}, \mathrm{OH}), 12.61(\mathrm{~s}, 1 \mathrm{H}$, $\mathrm{OH}), 12.28$ (s, 1H, OH), 7.69 (s, 1H, 11-H), 7.29, 7.25 (AB, $\left.{ }^{3} J=9.4 \mathrm{~Hz}, 2 \mathrm{H}, 2,3-\mathrm{H}\right)$, $3.96(\mathrm{~s}, 1 \mathrm{H}, 10-\mathrm{H}), 3.74\left(\mathrm{~s}, 3 \mathrm{H}, 15-\mathrm{OCH}_{3}\right), 3.07,2.86\left(\mathrm{ABX}, J_{\mathrm{AB}}=14.1, J_{\mathrm{AX}}=2.2\right.$ $\left.\mathrm{Hz}, J_{\mathrm{AX}}=2.4 \mathrm{~Hz}, 2 \mathrm{H}, 7_{\mathrm{a}}, 7_{\mathrm{b}}-\mathrm{H}\right), 2.33\left(\mathrm{~m}, 1 \mathrm{H}, 8-\mathrm{H}_{\mathrm{A}}\right), 1.95\left(\mathrm{~m}, 1 \mathrm{H}, 8-\mathrm{H}_{\mathrm{B}}\right), 1.72,1.62$ $\left(\mathrm{m}, 2 \mathrm{H}, 12-\mathrm{CH}_{2}\right), 1.09\left(\mathrm{t},{ }^{3} \mathrm{~J}=7.6 \mathrm{~Hz}, 3 \mathrm{H}, 13-\mathrm{CH}_{3}\right) ;-(+)$-ESIMS $m / z 845([2 \mathrm{M}+$ $\left.\mathrm{Na}]^{-}, 100\right),-(-)-E S I M S ~ m / z 411\left([\mathrm{M}-\mathrm{H}]^{-}, 100\right)$.

Cinerubin B (49): Middle polar red amorphous solid, $R_{f}=0.60\left(\mathrm{CHCl}_{3} / 10 \% \mathrm{MeOH}\right)$, orange UV fluorescent, turned to blue-violet with $2 \mathrm{~N} \mathrm{NaOH} .-{ }^{1} \mathrm{H} \mathrm{NMR}\left(\mathrm{CDCl}_{3}, 300\right.$ $\mathrm{MHz}) \delta 12.97(b r \mathrm{~s}, 1 \mathrm{H}, \mathrm{OH}), 12.80(b r \mathrm{~s}, 1 \mathrm{H}, \mathrm{OH}), 12.21(\mathrm{~s}, \mathrm{br}, 1 \mathrm{H}, \mathrm{OH}), 7.73(\mathrm{~s}$, $1 \mathrm{H}, 11-\mathrm{H}), 7.32,7.28\left(\mathrm{AB},{ }^{3} J=9.4 \mathrm{~Hz}, 2 \mathrm{H}, 2,3-\mathrm{H}\right), 5.51\left(\mathrm{~d},{ }^{3} J=2.4 \mathrm{~Hz}, 1^{\prime}-\mathrm{H}\right), 5.28$ $\left(\mathrm{d},{ }^{3} J=2.3 \mathrm{~Hz}, 1 \mathrm{H}, 7-\mathrm{H}\right), 5.20$ (d, $\left.{ }^{3} J=2.4 \mathrm{~Hz}, 1 \mathrm{H}, 1 "-\mathrm{H}\right), 5.17\left(\mathrm{~d},{ }^{3} J=2.1 \mathrm{~Hz}, 1{ }^{\prime \prime}-\mathrm{H}\right)$, 4.81 (q, $\left.{ }^{3} J=6.4 \mathrm{~Hz}, 1 \mathrm{H}, 5^{\prime}-\mathrm{H}\right), 4.68$ (q, $\left.{ }^{3} J=5.2 \mathrm{~Hz}, 1 \mathrm{H}, 5^{\prime}-\mathrm{H}\right), 4.38$ (m, 3H, 3',4",5"'H), 4.13 (s, 1H, 10-H), 4.04 (m, 2H, 5",2"'-H), 3.83 (s, 1H, 4'-H), 3.72 (s, 15- $\mathrm{OCH}_{3}$ ), $2.60\left(\mathrm{~d},{ }^{3} \mathrm{~J}=3.4 \mathrm{~Hz}, 2 \mathrm{H}, 3^{\prime}{ }^{\prime}-\mathrm{CH}_{2}\right), 2.48\left(\mathrm{~m}, 2 \mathrm{H}, 8-\mathrm{H}_{\mathrm{eq}}, 2^{\prime}-\mathrm{H}_{\mathrm{eq}}\right), 2.38-2.17$ [m, 7H, 8-H, $\left.\mathrm{NMe}_{2}\right), 1.95\left(\mathrm{~m}, 3 \mathrm{H}, 2^{\prime}-\mathrm{CH}_{2}, 2 '-\mathrm{H}\right), 1.75\left(\mathrm{~m}, 1 \mathrm{H}, 13-\mathrm{H}_{\mathrm{A}}\right), 1.53\left(\mathrm{~m}, 1 \mathrm{H}, 13-\mathrm{H}_{\mathrm{B}}\right), 1.37$ (d, $\left.{ }^{3} J=7.2,3 \mathrm{H}, 6{ }^{6} '-\mathrm{CH}_{3}\right), 1.30\left(\mathrm{~d},{ }^{3} J=7.2 \mathrm{~Hz}, 3 \mathrm{H}, 6^{\prime}-\mathrm{CH}_{3}\right), 1.23\left(\mathrm{~d},{ }^{3} J=7.3 \mathrm{~Hz}, 3 \mathrm{H}\right.$, 6"--H$), 1.09\left(\mathrm{t},{ }^{3} J=7.4 \mathrm{~Hz}, 3 \mathrm{H}, 14-\mathrm{CH}_{3}\right) .-(+)$-ESIMS $m / z 826\left([\mathrm{M}+\mathrm{H}]^{+}, 100\right)$; - (-)-ESIMS $m / z 1671\left([2 \mathrm{M}+\mathrm{Na}-2 \mathrm{H}]^{-}, 100\right), 824\left([\mathrm{M}-\mathrm{H}]^{-}, 75\right), 411$ (90).

Cinerubin A (51): Red amorphous, middle polar substance, $R_{f}=0.54\left(\mathrm{CHCl}_{3} / 10 \%\right.$ $\mathrm{MeOH})$, UV orange fluorescence, turned to blue-violet with $2 \mathrm{~N} \mathrm{NaOH}-{ }^{1} \mathrm{H} \mathrm{NMR}$ $\left(\mathrm{CDCl}_{3}, 300 \mathrm{MHz}\right) \delta 12.92(\mathrm{~s}, 1 \mathrm{H}, 1-\mathrm{OH}), 12.76(\mathrm{~s}, 1 \mathrm{H}, 4-\mathrm{OH}), 12.17$ (s, 1H, 6-OH), 7.69 (s, 1H,11-H), 7.29 (d, $\left.{ }^{3} J=9.4 \mathrm{~Hz}, 1 \mathrm{H}, 3-\mathrm{H}\right), 7.27$ (d, $\left.{ }^{3} J=9.4 \mathrm{~Hz}, 1 \mathrm{H}, 2-\mathrm{H}\right), 5.56$ (br s, 1H, 1'-H), $5.30\left(b r \mathrm{~d},{ }^{4} J=4.2 \mathrm{~Hz}, 1 \mathrm{H}, 7-\mathrm{H}\right), 5.12\left(\mathrm{t},{ }^{3} J=6.4 \mathrm{~Hz}, 1{ }^{\prime \prime}-\mathrm{H}\right), 5.08$ (d, $\left.{ }^{4} J=3.5 \mathrm{~Hz}, 1^{\prime \prime}-\mathrm{H}\right), 4.62$ (q, $\left.{ }^{3} J=6.4 \mathrm{~Hz}, 5^{\prime \prime}-\mathrm{H}\right), 4.54$ (q, 1H, $J=6.2 \mathrm{~Hz}, 5$ '-H), 4.19 (q, $J=6.8 \mathrm{~Hz}, 5 "-\mathrm{H}), 4.16(\mathrm{~m}, 1 \mathrm{H}, 3 "-\mathrm{H}), 4.11$ (s, 1H, 10-H), 3.78 (m, 1H, 3'-H), 3.76 (s,

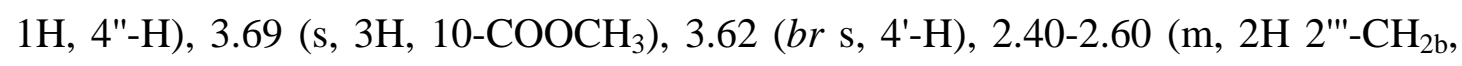
3'"-H), 2.40 (d, 1H, $J=15.0 \mathrm{~Hz}, 8-\mathrm{CH}_{2 \mathrm{a}}$ ), 2.35 (dd, 1H, $J=15.0,4.2 \mathrm{~Hz}, 8-\mathrm{CH}_{2 \mathrm{~b}}$ ), 2.20 [s, 6H, N $\left.\left(\mathrm{CH}_{3}\right)_{2}\right], 2,16\left(\mathrm{~m}, 2^{2 '-}-\mathrm{CH}_{2 \mathrm{a}}\right), 2.15\left(\mathrm{~m}, 1 \mathrm{H}, 2^{2}-\mathrm{CH}_{2 \mathrm{~b}}\right), 2.10\left(\mathrm{~m}, 1 \mathrm{H}, 2\right.$ "- $\left.\mathrm{CH}_{2 \mathrm{a}}\right)$, $1.95\left(\mathrm{~m}, 1 \mathrm{H}, 2^{\prime}-\mathrm{CH}_{2 \mathrm{~b}}\right), 1.81\left(\mathrm{~m}, 1 \mathrm{H}, 2^{\prime}-\mathrm{CH}_{2 \mathrm{a}}\right), 1.75\left(\mathrm{~m}, 1 \mathrm{H}, 13-\mathrm{CH}_{2 \mathrm{~b}}\right), 1.51(\mathrm{~m}, 1 \mathrm{H}, 13-$ $\mathrm{CH}_{2 \mathrm{a}}$ ), 1.38 (d, $\left.J=6.7 \mathrm{~Hz}, 6^{\prime \prime}-\mathrm{H}\right), 1.34$ (d, $J=6.4 \mathrm{~Hz}, 6$ '-H), 1.22 (d, $\left.J=6.4 \mathrm{~Hz}, 6 "-\mathrm{H}\right)$, $1.15\left(\mathrm{t}, J=7.3 \mathrm{~Hz}, 14-\mathrm{CH}_{3}\right) .-(+)$-ESIMS $m / z, 828[\mathrm{M}+\mathrm{H}]^{+} ;-(-)$-ESIMS $m / z, 826$ $[\mathrm{M}-\mathrm{H}]^{-}$. 
Cinerubin X (52): Red amorphous, middle polar substance $R_{f}=0.48\left(\mathrm{CHCl}_{3} / 10 \%\right.$ $\mathrm{MeOH}$ ), an orange UV fluorescence, turned to blue-violet with $2 \mathrm{~N} \mathrm{NaOH} .-{ }^{1} \mathrm{H}$ NMR $\left(\mathrm{CDCl}_{3}, 300 \mathrm{MHz}\right) \delta 12.92(\mathrm{~s}, 1 \mathrm{H}, 1-\mathrm{OH}), 12.76(\mathrm{~s}, 1 \mathrm{H}, 4-\mathrm{OH}), 12.24(\mathrm{~s}, 1 \mathrm{H}, 6-\mathrm{OH})$, 7.72 (s, 1H,11-H), 7.29 (d, $J=9.4 \mathrm{~Hz}, 1 \mathrm{H}, 9.4,3), 7.27$ (d, $J=9.4 \mathrm{~Hz}, 1 \mathrm{H}, 2-\mathrm{H}), 5.56$ (br s, 1H, 1'-H), $5.30(b r \mathrm{~d}, J=4.2 \mathrm{~Hz}, 1 \mathrm{H}, 7-\mathrm{H}), 5.12$ (t, $\left.J=6.4 \mathrm{~Hz}, 1{ }^{\prime \prime}-\mathrm{H}\right), 5.08$ (d, $J$ $\left.=3.5 \mathrm{~Hz}, 1^{\prime \prime}-\mathrm{H}\right), 4.62$ (q, $\left.J=6.4 \mathrm{~Hz}, 5^{\prime \prime}-\mathrm{H}\right), 4.54$ (q, 1H, $\left.J=6.2 \mathrm{~Hz}, 5 '-\mathrm{H}\right), 4.19$ (q, $J=$ $6.8 \mathrm{~Hz}, 5 "-\mathrm{H}), 4.16$ (m, 1H, 3"-H), 4.11 (s, 1H, 10-H), 3.78 (m, 1H, 3'-H), 3.76 (s, 1H,

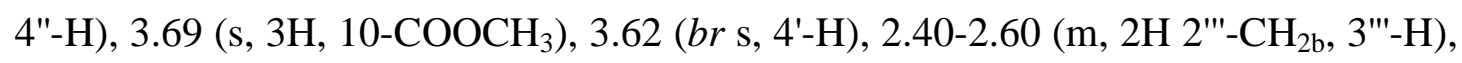
2.40 (d, 1H, $J=15.0 \mathrm{~Hz}, 8-\mathrm{CH}_{2 \mathrm{a}}$ ), 2.35 (dd, 1H, $J=15.0,4.2 \mathrm{~Hz}, 8-\mathrm{CH}_{2 \mathrm{~b}}$ ), 2, 16 (m, 2"'$\mathrm{CH}_{2 \mathrm{a}}$ ), 2.15 (m, 1H, 2"-CH $\left.\mathrm{CH}_{2 \mathrm{~b}}\right), 2.10\left(\mathrm{~m}, 1 \mathrm{H}, 2\right.$ "- $\left.\mathrm{CH}_{2 \mathrm{a}}\right), 1.95\left(\mathrm{~m}, 1 \mathrm{H}, 2^{\prime}-\mathrm{CH}_{2 \mathrm{~b}}\right), 1.81$ (m, $\left.1 \mathrm{H}, 2^{\prime}-\mathrm{CH}_{2 \mathrm{a}}\right), 1.75\left(\mathrm{~m}, 1 \mathrm{H}, 13-\mathrm{CH}_{2 \mathrm{~b}}\right), 1.51\left(\mathrm{~m}, 1 \mathrm{H}, 13-\mathrm{CH}_{2 \mathrm{a}}\right), 1.38\left(\mathrm{~d}, J=6.7 \mathrm{~Hz}, 6{ }^{\prime \prime}-\right.$ H), $1.34\left(\mathrm{~d}, J=6.4 \mathrm{~Hz}, 6{ }^{\prime}-\mathrm{H}\right), 1.22(\mathrm{~d}, J=6.4 \mathrm{~Hz}, 6 "-\mathrm{H}), 1.15\left(\mathrm{t}, J=7.3 \mathrm{~Hz}, 14-\mathrm{CH}_{3}\right)$. - (+)-ESIMS m/z $807\left([\mathrm{M}+\mathrm{Na}]^{+}, 100\right), 1591\left([2 \mathrm{M}+\mathrm{Na}]^{+}, 70\right) ;-(-)-E S I M S ~ m / z, 783$ ([M - H] $\left.]^{-}, 100\right)$.

\subsection{Terrestrial Streptomyces sp. ACT 7619}

\subsubsection{Primary Screening}

The ethyl acetate extract of the terrestrial Streptomyces sp. isolate ACT 7619 showed high activity against bacteria and fungi. Some colourless weakly UV absorbing band gave violet colouration with anisaldehyde/sulphuric acid.

Table 6: Biological activity of the crude extract of the strain Act 7619

\begin{tabular}{ll}
\hline Test organisms & Inhibition zone $(\varnothing \mathbf{m m})$ \\
\hline Bacillus subtilis & 11 \\
Staphylococcus aureus & 12 \\
Streptomyces viridochromogenes (Tü 57) & 0 \\
Escherichia coli & 11 \\
Candida albicans & 11 \\
Mucor miehei & 11 \\
\hline
\end{tabular}

\subsubsection{Fermentation and isolation}

The $25 \mathrm{~L}$ fermentation of the strain ACT 7619 was performed as a shaker culture using $\mathrm{M}_{2}$ medium during five days at $28^{\circ} \mathrm{C}$ on a linear shaker. The brown mycelium of the growth culture was separated by filtration with the aid of Celite into mycelia cake and filtrate. Both were extracted with ethyl acetate and combined after the analysis of 
the TLC to give a dark oily crude extract $(3.23 \mathrm{~g})$. The crude extract was subjected to silica column chromatography with a dichloromethane-methanol gradient to separate four fractions. Fraction 2 was further purified by Sephadex LH-20 and silica gel column chromatography to find bonactin $(\mathbf{5 4}, 3.0 \mathrm{mg})$. Fraction 3 was further purified in the same way as $\mathbf{5 4}$ to obtain nonactic acid $(\mathbf{8 6}, 36 \mathrm{mg})$, homononactic acid $(\mathbf{8 7}, 120$ $\mathrm{mg}$ ), and dinactin $(\mathbf{5 3}, 15 \mathrm{mg})$. After passing fraction 4 through Sephadex LH-20, 4,10-dihydroxy-10-methyl-11-oxo-dodec-2-en-1,4-olid (56, $1.0 \mathrm{mg})$ was obtained.

Dinactin (53): Colourless oil, $R_{f}=0.27\left(\mathrm{CHCl}_{3} / 10 \% \mathrm{MeOH}\right)$, violet band on spraying with anisaldehyde/sulphuric acid. $-{ }^{1} \mathrm{H} \mathrm{NMR}\left(\mathrm{CDCl}_{3}, 300 \mathrm{MHz}\right) \delta 4.93(\mathrm{~m}, 2 \mathrm{H}$, 17-H, 8-H), 4.02 ( br q, 2H, 3-H, 12-H), 3.87 (m, 2H, 6-H, 15-H), 2.52 (m, 2H, 2-H, 11-H), $2.01\left(\mathrm{~m}, 2 \mathrm{H}, 5-\mathrm{CH}_{2}, 14-\mathrm{CH}_{2}\right), 1.92\left(\mathrm{~m}, 2 \mathrm{H}, 4-\mathrm{H}_{2}, 13-\mathrm{CH}_{2}\right), 1.67$ (m, 2H, 7$\mathrm{CH}_{2}$ ), 1.56 (m, 2H, 4- $\left.\mathrm{CH}_{2}, 13-\mathrm{CH}_{2}\right), 1.56$ (m, 2H, 18- $\left.\mathrm{CH}_{2}\right), 1.49$ (m, 2H, 5-H2, 14$\left.\mathrm{CH}_{2}\right), 1.23\left(\mathrm{~d},{ }^{3} \mathrm{~J}=6.2 \mathrm{~Hz}, 3 \mathrm{H}, 8-\mathrm{CH}_{3}\right), 1.10\left(\mathrm{~d},{ }^{3} \mathrm{~J}=7.0 \mathrm{~Hz}, 3 \mathrm{H}, 11-\mathrm{CH}_{3}\right), 1.08\left(\mathrm{~d},{ }^{3} J\right.$ $\left.=7.0 \mathrm{~Hz}, 3 \mathrm{H}, 2-\mathrm{CH}_{3}\right), 0.87\left(\mathrm{t},{ }^{3} \mathrm{~J}=7.4 \mathrm{~Hz}, 3 \mathrm{H}, 19-\mathrm{CH}_{3}\right) ;-(+)-\mathrm{ESIMS} \mathrm{m} / \mathrm{z} 787([\mathrm{M}+$ $\left.\mathrm{Na}]^{+}, 100\right)$.

Bonactin (54): Colourless oil, $R_{f}=0.2\left(\mathrm{CH}_{2} \mathrm{Cl}_{2} / 5 \% \mathrm{MeOH}\right)$, turns first brown and then violet with anisaldehyde/sulphuric acid. $-{ }^{1} \mathrm{H} \mathrm{NMR}\left(\mathrm{CDCl}_{3}, 300 \mathrm{MHz}\right) \delta 6.10(\mathrm{br}$ s, 2H, $2 \mathrm{OH}), 5.02(\mathrm{~m}, 1 \mathrm{H}, 8-\mathrm{H}), 4.18\left(\mathrm{~m}, 1 \mathrm{H}, 6{ }^{\prime}-\mathrm{H}\right), 4.00\left(\mathrm{~m}, 3 \mathrm{H}, 3-\mathrm{H}, 3{ }^{\prime}-\mathrm{H}, 6-\mathrm{H}\right)$, $3.78\left(\mathrm{~m}, 1 \mathrm{H}, 8^{\prime}-\mathrm{H}\right), 2.50\left(\mathrm{dq},{ }^{3} J=8.3,7.0 \mathrm{~Hz}, 2 \mathrm{H}, 2-\mathrm{H}, 2^{\prime}-\mathrm{H}\right), 2.05-1.90(\mathrm{~m}, 4 \mathrm{H})$, 1.40-1.80 (m, 10H), $1.22\left(\mathrm{~d},{ }^{3} J=6.3 \mathrm{~Hz}, 3 \mathrm{H}, 9-\mathrm{CH}_{3}\right), 1.10\left(\mathrm{~d},{ }^{3} J=7.0 \mathrm{~Hz}, 3 \mathrm{H}, 2^{2}-\right.$ $\left.\mathrm{CH}_{3}\right), 1.15\left(\mathrm{~d},{ }^{3} J=7.0 \mathrm{~Hz}, 3 \mathrm{H}, 2-\mathrm{CH}_{3}\right), 0.93\left(\mathrm{t},{ }^{3} J=7.4 \mathrm{~Hz}, 3 \mathrm{H}, 10-\mathrm{CH}_{3}\right) .-(+)-$ ESIMS $m / z 423\left([\mathrm{M}+\mathrm{Na}]^{+}, 48\right) ;-(-)-\operatorname{ESIMS~} m / z 399\left([\mathrm{M}-\mathrm{H}]^{-}, 45\right)$.

4,10-Dihydroxy-10-methyl-11-oxo-dodec-2-en-1,4-olide (56): Colourless solid, $R_{f}=$ $0.33\left(\mathrm{CH}_{2} \mathrm{Cl}_{2} / 5 \% \mathrm{MeOH}\right)$, blue colouration with anisaldehyde/sulphuric acid. $-{ }^{1} \mathrm{H}$ $\operatorname{NMR}\left(\mathrm{CDCl}_{3}, 300 \mathrm{MHz}\right) \delta 7.43\left(\mathrm{dd},{ }^{3} J=5.6 \mathrm{~Hz},{ }^{4} J=1.5 \mathrm{~Hz}, 1 \mathrm{H}, 3-\mathrm{H}\right), 6.11\left(\mathrm{dd},{ }^{3} J=\right.$ $\left.5.6 \mathrm{~Hz},{ }^{4} \mathrm{~J}=1.9 \mathrm{~Hz}, 1 \mathrm{H}, 2-\mathrm{H}\right), 5.03(\mathrm{~m}, 1 \mathrm{H}, 4-\mathrm{H}), 3.84$ (s, 1H, 10-OH), 2.20 (s, 3H, $\left.12-\mathrm{CH}_{3}\right), 1.78-1.30\left(\mathrm{~m}, 10 \mathrm{H}, 5,6,7,8,9-\mathrm{CH}_{2}\right), 1.36\left(\mathrm{~s}, 3 \mathrm{H}, 10-\mathrm{CH}_{3}\right)$.

\subsection{Terrestrial Streptomyces sp. GW 6311}

\subsubsection{Primary Screening}

The pale yellow crude extract exhibited a moderate activity only against micro algae. TLC using $\mathrm{CH}_{2} \mathrm{Cl}_{2} / 5 \% \mathrm{MeOH}$ and $\mathrm{CH}_{2} \mathrm{Cl}_{2} / 10 \% \mathrm{MeOH}$ indicated the presence of yellow bands, which changed to red with diluted sodium hydroxide indicating peri- 
hydroxy-quinones. Further purification of the crude extract provided $\beta$-indomycinone (57) and saptomycinone A (58).

Table 7: Biological activity of the crude extract of the strain GW 6311.

\begin{tabular}{ll}
\hline Test organisms & Inhibition zone $(\varnothing \mathbf{~ m m})$ \\
\hline Bacillus subtilis & 0 \\
Staphylococcus aureus & 0 \\
Streptomyces viridochromogenes (Tü 57) & 0 \\
Escherichia coli & 0 \\
Candida albicans & 0 \\
Mucor miehei & 0 \\
Chlorella vulgaris & 15 \\
Chlorella sorokiniana & 15 \\
Scenedesmus subspicatus & 16 \\
\hline
\end{tabular}

\subsubsection{Cultivation and isolation}

The fermentation was performed on a 201 scale at $28{ }^{\circ} \mathrm{C}$ for 5 days using $\mathrm{M}_{2}$ medium as linear shaker culture. In each 11 Erlenmeyer flask was inoculated with $1 \mathrm{~cm}^{2}$ pieces from a well grown agar plate. After 5 days the culture was pink-yellow and was separated by filtration with the aid of Celite into mycelial cake and filtrate. Both were extracted with ethyl acetate and combined after the analysis by TLC to give $1.57 \mathrm{~g}$ crude oily extract. The crude extract was subjected to silica gel column chromatography using dichloromethane/methanol gradient to find 2 fractions. Fraction 2 was further separated by Sephadex LH-20 and PTLC into a mixture of $\beta$-indomycinone (57) and saptomycin A $(\mathbf{5 8}, 3.2 \mathrm{mg})$.

$\boldsymbol{\beta}$-Indomycinone (57): Yellowish-orange amorphous powder, $R_{f}=0.78\left(\mathrm{CHCl}_{3} / 5 \%\right.$ $\mathrm{MeOH}$ ), orange UV fluorescent, colour change to red with $\mathrm{NaOH}$, brown with sulphuric. $-{ }^{1} \mathrm{H} \mathrm{NMR}\left(\mathrm{CDCl}_{3}, 300 \mathrm{MHz}\right) \delta 12.85$ (br s, 1H, OH), 8.08 (s, 1H, 6-H), 7.83 $\left(\mathrm{dd},{ }^{3} J=7.6,1.3 \mathrm{~Hz}, 1 \mathrm{H}, 8-\mathrm{H}\right), 7.68\left(\mathrm{t},{ }^{3} J=8.0 \mathrm{~Hz}, 1 \mathrm{H}, 9-\mathrm{H}\right), 7.38\left(\mathrm{dd},{ }^{3} J=8.4,1.3\right.$ Hz, 10-H), 6.56 (s, 1H, 3-H), 5.74 (m, 1H, 18-H), 5.39 (m, 1H, 17-H), 3.02 (s, 3H, 13$\left.\mathrm{CH}_{3}\right), 2.91\left(\mathrm{dd},{ }^{3} J=14.4,{ }^{3} J=8.5 \mathrm{~Hz}, 1 \mathrm{H}, 16-\mathrm{H}_{\mathrm{A}}\right), 2.78\left(\mathrm{dd},{ }^{3} J=14.4,{ }^{3} J=7.2\right.$ $\left.\mathrm{Hz}, 1 \mathrm{H}, 16-\mathrm{H}_{\mathrm{B}}\right), 1.68$ (s, 3H, 15- $\left.\mathrm{CH}_{3}\right), 1.64\left(\mathrm{~d},{ }^{3} \mathrm{~J}=6.8 \mathrm{~Hz}, 19-\mathrm{CH}_{3}\right) .-(+)-E S I M S ~ m / z$ $405\left([\mathrm{M}+\mathrm{H}]^{+}, 8\right), 405\left([2 \mathrm{M}+\mathrm{Na}]^{+}, 100\right) ;-(-)-E S I M S ~ m / z, 403\left([\mathrm{M}-\mathrm{H}]^{-}, 100\right)$.

Saptomycin A (58): Yellow amorphous powder, $R_{f}=0.70\left(\mathrm{CHCl}_{3} / 5 \% \mathrm{MeOH}\right)$, orange UV fluorescence on TLC, red by treatment with $\mathrm{NaOH}$, brown by sulphuric acid. $-{ }^{1} \mathrm{H} \mathrm{NMR}\left(\mathrm{CDCl}_{3}, 300 \mathrm{MHz}\right) \delta 12.64$ (br s, $\left.1 \mathrm{H}, \mathrm{OH}\right), 8.09$ (s, 1H, 6-H), 7.83 $\left(\mathrm{dd},{ }^{3} J=7.6 \mathrm{~Hz}, 1.3 \mathrm{~Hz}, 1 \mathrm{H}, 8-\mathrm{H}\right), 7.69\left(\mathrm{t},{ }^{3} J=8.0 \mathrm{~Hz}, 1 \mathrm{H}, 9-\mathrm{H}\right), 7.38\left(\mathrm{dd},{ }^{3} J=8.4\right.$, 
$1.3 \mathrm{~Hz}, 10-\mathrm{H}), 6.28(\mathrm{~s}, 1 \mathrm{H}, 3-\mathrm{H}), 5.65(\mathrm{~m}, 1 \mathrm{H}, 18-\mathrm{H}), 5.53\left(\mathrm{td},{ }^{3} \mathrm{~J}=10.5,{ }^{3} \mathrm{~J}=6.8 \mathrm{~Hz}\right.$, $1 \mathrm{H}, 17-\mathrm{H}), 5.01\left(\mathrm{dd},{ }^{3} \mathrm{~J}=8.5,{ }^{3} \mathrm{~J}=3.8 \mathrm{~Hz}, 1 \mathrm{H}, 16-\mathrm{H}\right), 3.02$ (s, 3H, 13- $\left.\mathrm{CH}_{3}\right), 2.99(\mathrm{dq}$, $\left.{ }^{3} J=3.8,{ }^{3} J=7.5 \mathrm{~Hz}, 1 \mathrm{H}, 14-\mathrm{H}\right), 1.71\left(\mathrm{~d},{ }^{3} J=7.0 \mathrm{~Hz}, 3 \mathrm{H}, 19-\mathrm{CH}_{3}\right), 1.45\left(\mathrm{~d},{ }^{3} J=7.0\right.$, $\left.\mathrm{Hz}, 3 \mathrm{H}, 15-\mathrm{CH}_{3}\right) .-(+)-\mathrm{ESIMS} m / z 405\left([\mathrm{M}+\mathrm{H}]^{+}, 8\right), 427\left([2 \mathrm{M}+\mathrm{Na}]^{+}, 100\right) ;-(-)-$ ESIMS $m / z, 403$ ([M - H] $\left.]^{-}, 100\right)$.

\subsection{Streptomyces sp. GT 2005/079}

\subsubsection{Primary screening}

The crude extract of the terrestrial Streptomyces sp. GT 2005/079 showed antibacterial activity against Staphylococcus aureus, Bacillus subtilis and Escherichia coli. The TLC screening revealed several non-polar non-UV absorbing compounds, which changed to green with anisaldehyde/sulphuric acid.

Table 8: Biological activity of the crude extract of Streptomyces sp. 2005/079.

\begin{tabular}{ll}
\hline Test organisms & Inhibition zone $(\varnothing \mathbf{~ m m})$ \\
\hline Bacillus subtilis & 0 \\
Staphylococcus aureus & 11 \\
Streptomyces viridochromogenes (Tü 57) & 17 \\
Escherichia coli & 0 \\
Candida albicans & 0 \\
Mucor miehei & 14 \\
Chlorella vulgaris & 0 \\
Chlorella sorokiniana & 0 \\
Scenedesmus subspicatus & 0 \\
Artemia salina & $100 \%$ \\
\hline
\end{tabular}

\subsubsection{Cultivation and isolation}

20.0 L of $\mathrm{M}_{2}$ medium was prepared and transferred to $1.0 \mathrm{~L}$ Erlenmeyer flasks (250 $\mathrm{ml}$ each) and inoculated by the pre-cultured terrestrial streptomycete GT 2005/079. The flasks were then incubated on a linear shaker $(110 \mathrm{rpm})$ at $28^{\circ} \mathrm{C}$ for 8 days until the broth attained dark brown colour. The culture broth was mixed with Celite and filtered off under pressure. The filtrate was extracted with XAD-16 column and the resin eluted with methanol to get dark brown extract. The biomass was extracted with ethyl acetate (3 times). Both extracts were combined due to their identical chromatograms to give an oily crude extract (4.25 g). Chromatography on a silica gel (col- 
umn, $\mathrm{CH}_{2} \mathrm{Cl}_{2} / 0-50 \% \mathrm{MeOH}$ gradient) gave four main fractions. Fraction 1 consisted mostly of fats and lipids, whereas fraction 2 showed three non UV absorbing spots which showed green colour with anisaldehyde/sulphuric acid on TLC. Fraction 2 was further purified on silica gel column chromatography, Sephadex LH-20 and followed by RP-8 open column eluting with 50-60\% aqueous methanol to get 59 (15 mg) which gave bright green colour with anisaldehyde-sulphuric acid. Fraction 3 was separated on silica gel and Sephadex LH-20 into 3 subfractions. The subtractions afforded on an RP-8 column eluting with 40\% aqueous methanol 60 (6 mg) and 61 (16 $\mathrm{mg})$. Fraction 3 from the main column was subjected to a RP-phenyl column $(12 \times 1$ $\mathrm{cm}$ ) and eluted with $30 \%$ aqueous methanol to get three sub-fractions. Further purification with methanol on Sephadex LH-20 yielded 62 (1.2 mg). Fraction 4 contained a highly fluorescent polar compound. It was separated on Sephadex LH-20 into two sub-fractions. $\mathrm{F}_{4 \mathrm{a}}$ and $\mathrm{F}_{4 \mathrm{~b}}$ were sparingly soluble in dichloromethane or methanol, and even in a mixture of dichloromethane and methanol. The fractions were washed several times with a mixture of dichloromethane and methanol to obtain 63 (14 mg) and 64 (3 mg).

$N$-(7-Hydroxy-6-methyl-octyl)-acetamide (59): Colourless oil, $R_{f}=0.65\left(\mathrm{CHCl}_{3} / 5\right.$ $\% \mathrm{MeOH})$, green colouration with anisaldehyde/sulphuric acid. $-{ }^{1} \mathrm{H} \mathrm{NMR}\left(\mathrm{CDCl}_{3}\right.$, $300 \mathrm{MHz}) \delta 5.63$ (br s, 1H, 1-NH), 3.66 (quint, $1 \mathrm{H}, J=6.7 \mathrm{~Hz}, 7-\mathrm{CH}), 3.22$ (q, 2H, $\left.{ }^{3} J=6.2 \mathrm{~Hz}, 1-\mathrm{CH}_{2}\right), 1.97\left(\mathrm{~s}, 3 \mathrm{H}, \mathrm{COCH}_{3}\right), 1.25-1.38\left(\mathrm{~m}, 8 \mathrm{H}, 2,3,4,5-\mathrm{CH}_{2}\right), 1.18(\mathrm{~s}$, 6H, 6- $\left.\left(\mathrm{CH}_{3}\right)_{2}\right) .-{ }^{13} \mathrm{C} \mathrm{NMR}\left(\mathrm{CDCl}_{3}, 125 \mathrm{MHz}\right) \delta 170.1(\mathrm{CO}-1), 71.6\left(\mathrm{C}_{\mathrm{q}}-6\right), 39.9$ $\left(\mathrm{CH}_{2}-5\right), 39.6\left(\mathrm{CH}_{2}-1\right), 32.3\left(\mathrm{CH}_{2}-4\right), 29.5\left(\mathrm{CH}_{2}-3\right), 27.1\left(\mathrm{CH}_{3}-6\right), 26.7\left(\mathrm{CH}_{2}-2\right), 23.3$ $\left(\mathrm{CH}_{2}-5\right), 19.4\left(-\mathrm{COCH}_{3}\right), 14.5\left(7-\mathrm{CH}_{3}\right) .-(+)-\mathrm{ESIMS} \mathrm{m} / \mathrm{z} 202[\mathrm{M}+\mathrm{H}]^{+}, 224[\mathrm{M}+$ $\mathrm{Na}]^{+}, 425[2 \mathrm{M}+\mathrm{Na}]^{+}$; - (+)-ESI-HRMS m/z 224.16218 $[\mathrm{M}+\mathrm{Na}]^{+}$(calcd for $\mathrm{C}_{11} \mathrm{H}_{23} \mathrm{NO}_{2} \mathrm{Na}, 224.16210$ ).

$N$-(7-Hydroxy-octyl)-acetamide (60): Colourless oil, $R_{f}=0.60\left(\mathrm{CHCl}_{3} / 5 \% \mathrm{MeOH}\right)$, green colouration with anisaldehyde/sulphuric acid. - ${ }^{1} \mathrm{H} \mathrm{NMR}\left(\mathrm{CDCl}_{3}, 300 \mathrm{MHz}\right) \delta$ $5.49(b r \mathrm{~s}, 1 \mathrm{H}, 1-\mathrm{NH}), 3.66(\mathrm{q}, 1 \mathrm{H}, J=6.2 \mathrm{~Hz}, 7-\mathrm{CH}), 3.06\left(\mathrm{q}, 2 \mathrm{H},{ }^{3} J=6.2 \mathrm{~Hz}, 1-\right.$ $\left.\mathrm{CH}_{2}\right), 1.96\left(\mathrm{~s}, 3 \mathrm{H}, \mathrm{COCH}_{3}\right), 1.25-1.38\left(\mathrm{~m}, 10 \mathrm{H}, 2,3,4,5,6-\mathrm{CH}_{2}\right), 1.13\left(\mathrm{~d}, 3 \mathrm{H},{ }^{3} \mathrm{~J}=6.38\right.$ $\left.\mathrm{Hz}, \mathrm{COCH}_{3}\right), 0.86\left(\mathrm{t}, 3 \mathrm{H},{ }^{3} \mathrm{~J}=6.74 \mathrm{~Hz}, 7-\mathrm{CH}_{3}\right) .-{ }^{13} \mathrm{C} \mathrm{NMR}\left(\mathrm{CDCl}_{3}, 125 \mathrm{MHz}\right) \delta$ $170.1(\mathrm{CO}), 68.2(7-\mathrm{CH}), 41.0\left(6-\mathrm{CH}_{2}\right), 39.6\left(1-\mathrm{CH}_{2}\right), 29.2\left(3-\mathrm{CH}_{2}\right), 27.2\left(2-\mathrm{CH}_{2}\right)$, $23.7\left(5-\mathrm{CH}_{2}\right), 23.4\left(-\mathrm{COCH}_{3}\right), 16.6\left(8-\mathrm{CH}_{3}\right) .-(+)-\mathrm{ESIMS} m / z 201[\mathrm{M}+\mathrm{Na}]^{+}$; - (+)HRESIMS $m / z 210.14653[\mathrm{M}+\mathrm{Na}]^{+}$, (calcd for $\mathrm{C}_{10} \mathrm{H}_{21} \mathrm{NO}_{2} \mathrm{Na}, 210.14645$ ).

$N$-(6-Hydroxy-6-methyl-heptyl)-acetamide (61): Colourless oil, $R_{f}=0.55\left(\mathrm{CHCl}_{3} / 5\right.$ $\% \mathrm{MeOH})$, green colouration with anisaldehyde/sulphuric acid. - ${ }^{1} \mathrm{H} \mathrm{NMR}\left(\mathrm{CDCl}_{3}\right.$, $300 \mathrm{MHz}) \delta 6.66(b r \mathrm{~s}, 1 \mathrm{H}, 1-\mathrm{NH}), 5.33(\mathrm{~s}, 1 \mathrm{H}, 6-\mathrm{OH}), 3.20\left(\mathrm{q},{ }^{3} J=6.2 \mathrm{~Hz}, 2 \mathrm{H}, 1-\right.$ $\left.\mathrm{CH}_{2}\right), 1.97$ (s, 3H, $\left.\mathrm{COCH}_{3}\right), 1.25-1.38\left(\mathrm{~m}, 8 \mathrm{H}, 2,3,4,5-\mathrm{CH}_{2}\right), 1.18$ [s, 6H, 6- $\left.\left(\mathrm{CH}_{3}\right)_{2}\right]$. - 
${ }^{13} \mathrm{C} \mathrm{NMR}\left(\mathrm{CDCl}_{3}, 125 \mathrm{MHz}\right) \delta 170.4(\mathrm{CO}), 70.5\left(\mathrm{C}_{\mathrm{q}}-6\right), 43.4\left(\mathrm{CH}_{2}-5\right), 39.3\left(\mathrm{CH}_{2}-1\right)$, $29.2\left(\mathrm{CH}_{2}-3\right), 28.9\left(\left(\mathrm{CH}_{3}\right)_{2}-6\right), 27.2\left(\mathrm{CH}_{2}-2\right), 23.7\left(\mathrm{CH}_{2}-5\right), 22.9\left(\mathrm{COCH}_{3}\right)$. - (+)ESIMS m/z $210\left([\mathrm{M}+\mathrm{Na}]^{+}, 100\right) ;-(+)-E S I-H R M S ~ m / z ~ 210.14653[\mathrm{M}+\mathrm{Na}]^{+}$(calcd 210. 14645 for $\mathrm{C}_{10} \mathrm{H}_{21} \mathrm{NO}_{2} \mathrm{Na}$ ).

Homononactic acid amide (62): Colourless oil, $R_{f}=0.47\left(\mathrm{CHCl}_{3} / 5 \% \mathrm{MeOH}\right)$, violet colouration with anisaldehyde/sulphuric acid $-{ }^{1} \mathrm{H}$ NMR $\left(\mathrm{CDCl}_{3}, 300 \mathrm{MHz}\right) \delta 6.27(b r$ $\mathrm{s}, 1 \mathrm{H}, \mathrm{NH} / \mathrm{OH}), 5.50(b r \mathrm{~s}, 1 \mathrm{H}, \mathrm{NH} / \mathrm{OH}), 4.18(\mathrm{~m}, 1 \mathrm{H}, 5-\mathrm{H}), 3.90(\mathrm{q}, J=7.7 \mathrm{~Hz}, 2-\mathrm{H})$, $3.80(\mathrm{~m}, 1 \mathrm{H}, 9-\mathrm{H}), 2.71$ ( $b r \mathrm{~s}, 1 \mathrm{H}, \mathrm{NH} / \mathrm{OH}), 2.36$ (quint, $\left.{ }^{3} J=7.2 \mathrm{~Hz}, 9-\mathrm{H}\right), 2.00$ (m, $2 \mathrm{H}, 3,4-\mathrm{H}), 1.71\left(\mathrm{~m}, 2 \mathrm{H}, 8-\mathrm{H}_{2}\right), 1.62(\mathrm{~m}, 2 \mathrm{H}, 3,4-\mathrm{H}), 1.50(\mathrm{~m}, 2 \mathrm{H}, 10-\mathrm{H} 2), 1.17$ (d, $\left.3 \mathrm{H},{ }^{3} J=7.0 \mathrm{~Hz}, 6-\mathrm{CH}_{3}\right), 0.94\left(\mathrm{t}, 3 \mathrm{H},{ }^{3} \mathrm{~J}=7.4 \mathrm{~Hz}, 10-\mathrm{CH}_{3}\right) \cdot-{ }^{13} \mathrm{C} \mathrm{NMR}_{\left(\mathrm{CDCl}_{3}, 125\right.}$ MHz) $\delta 176.9$ (CO-7), 81.1 (CH-2), 77.0 (CH-5), 70.6 (CH-9), 46.0 (CH-6), 41.1 $\left(\mathrm{CH}_{2}-8\right), 30.6\left(\mathrm{CH}_{2}-4\right), 30.2\left(\mathrm{CH}_{2}-10\right), 29.6\left(\mathrm{CH}_{2}-3\right), 14.2(6-\mathrm{CH} 3), 14.2\left(10-\mathrm{CH}_{3}\right)$. $(+)$-ESIMS $m / z 216\left([\mathrm{M}+\mathrm{H}]^{+}, 25\right), 238\left([\mathrm{M}+\mathrm{Na}]^{+}, 75\right) .-(+)-H R E S I M S ~ m / z$ $216.15946[\mathrm{M}+\mathrm{H}]^{+}$(calcd for $\mathrm{C}_{11} \mathrm{H}_{22} \mathrm{NO}_{3}, 216.15942$ ).

2-(2'-Hydroxy-phenyl)-benzoxazole-4-carboxylic acid (63) Pale yellow solid, $R_{f}=$ $0.34\left(\mathrm{CHCl}_{3} / 15 \% \mathrm{MeOH}\right)$, green fluorescent. - ${ }^{1} \mathrm{H} \mathrm{NMR}\left(\mathrm{CDCl}_{3}, 300 \mathrm{MHz}\right) \delta 12.03$ (br s, 1H, 8-OH), 7.96-8.08 (m, 3H, 5,7,6'-H), 7.55 (br s, 2H, 4',6-H), 7.06-7.15 (m, $\left.2 \mathrm{H}, 33^{\prime}, 5^{\prime}-\mathrm{H}\right) .-{ }^{13} \mathrm{C}$ NMR $\left(\mathrm{CDCl}_{3}, 125 \mathrm{MHz}\right) \delta 165.5(\mathrm{CO}-8), 163.4\left(2-\mathrm{C}_{\mathrm{q}}\right), 158.3\left(2^{\prime}-\right.$ $\left.\mathrm{C}_{\mathrm{q}}\right), 149.4\left(7 \mathrm{a}-\mathrm{C}_{\mathrm{q}}\right), 138.6\left(3 \mathrm{a}-\mathrm{C}_{\mathrm{q}}\right), 134.3(4-\mathrm{CH}), 127.3(6-\mathrm{CH}), 127.1(5-\mathrm{CH}), 125.2$ (6-CH), $122.4\left(4-\mathrm{C}_{\mathrm{q}}\right), 119.8\left(5^{\prime}-\mathrm{CH}\right), 117.2\left(3^{\prime}-\mathrm{CH}\right), 115.0(7-\mathrm{CH}), 109.7\left(1-\mathrm{C}_{\mathrm{q}}\right) .-$ $(+)-E S I M S ~ m / z 278\left([\mathrm{M}+\mathrm{Na}]^{+}, 58\right) .-(+)-H R E S I M S m / z 278.04238\left([\mathrm{M}+\mathrm{Na}]^{+}\right)$ (calcd for $\mathrm{C}_{14} \mathrm{H}_{9} \mathrm{NO}_{4} \mathrm{Na}, 278.04239$ ).

2-(2'-Hydroxy-4'-chloro-phenyl)benzoxazole-4-carboxylic acid (64) Pale yellow solid, green fluorescent. $R_{f}=0.30\left(\mathrm{CHCl}_{3} / 15 \% \mathrm{MeOH}\right) .-{ }^{1} \mathrm{H} \mathrm{NMR}\left(\mathrm{CDCl}_{3}, 300\right.$ MHz) $\delta 12.03$ ( $b r \mathrm{~s}, 1 \mathrm{H}, 8-\mathrm{OH}), 7.96-8.08$ (m, 3H, 5,7,6'-H), 7.55 (br s, 2H, 4',6-H),

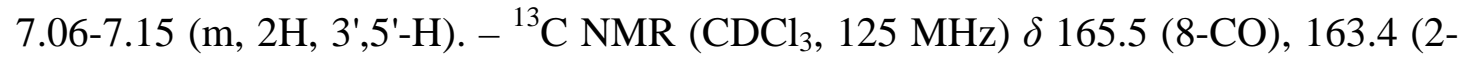
$\left.\mathrm{C}_{\mathrm{q}}\right), 158.3\left(2^{\prime}-\mathrm{C}_{\mathrm{q}}\right), 149.4\left(7 \mathrm{a}-\mathrm{C}_{\mathrm{q}}\right), 138.6\left(3 \mathrm{a}-\mathrm{C}_{\mathrm{q}}\right), 134.3\left(4^{\prime}-\mathrm{CH}\right), 127.3\left(6^{\prime}-\mathrm{CH}\right), 127.1$ (5-CH), 125.2 (6-CH), $122.4\left(4-\mathrm{C}_{\mathrm{q}}\right), 119.8\left(5^{\prime}-\mathrm{CH}\right), 117.2\left(3^{\prime}-\mathrm{CH}\right), 115.0(7-\mathrm{CH})$, $109.7\left(1^{\prime}-\mathrm{C}_{\mathrm{q}}\right)-(-)-$ ESIMS $\mathrm{m} / \mathrm{z} 288\left([\mathrm{M}-\mathrm{H}]^{-}, 100\right), 290\left([\mathrm{M}-\mathrm{H}]^{-}, 30\right) .-(-)-$ HRESIMS $m / z 288.00691$ ([M - H] $]^{-}$) (calcd for $\mathrm{C}_{14} \mathrm{H}_{8} \mathrm{NO}_{4} \mathrm{Cl}, 288.00636$ ). 


\subsection{Terrestrial Actinomycete N 435}

\subsubsection{Primary Screening}

TLC of the crude extract of the terrestrial actinomycete N 435 showed several UV absorbing zones. Anisaldehyde/sulphuric acid on TLC showed a pink colour reaction.

Table 9: Biological activity of the crude extract of the strain N 435.

\begin{tabular}{ll}
\hline Test organisms & Inhibition zone $(\varnothing \mathbf{~ m m})$ \\
\hline Bacillus subtilis & 0 \\
Staphylococcus aureus & 0 \\
Streptomyces viridochromogenes (Tü 57) & 0 \\
Escherichia coli & 0 \\
Candida albicans & 0 \\
Mucor miehei & 0 \\
Chlorella vulgaris & 20 \\
Chlorella sorokiniana & 20 \\
Scenedesmus subspicatus & 0 \\
\hline
\end{tabular}

\subsubsection{Fermentation and Isolation}

The strain was cultivated on a 20-liter scale shaker culture $(110 \mathrm{rpm})$ at $28{ }^{\circ} \mathrm{C}$ for 72 hours. After filtration using Celite, the water phase was extracted with XAD-16 and the bio-mass with ethyl acetate. Both extracts were combined according to the TLC analysis, to deliver an oily brown crude extract (3.25 g). The extract was chromatographed using silica gel column chromatography and eluted with dichloromethane and methanol with a gradient of increasing polarity. As a result, seven fractions were obtained. Working up and purification of the fractions led to the isolation of diketopiperazines $(\mathbf{7 1}, 6.5 \mathrm{mg} ; \mathbf{7 2}, 12 \mathrm{mg})$ along with ramthacin $\mathrm{B}(\mathbf{7 8}, 1.50 \mathrm{mg})$.

Cyclo(dehydroalanyl-isoleucyl) (71): Colourless solid, $R_{f}=0.53\left(\mathrm{CHCl}_{3} / 5 \%\right.$ $\mathrm{MeOH})$, with anisaldehyde/sulphuric acid orange. $-{ }^{1} \mathrm{H}$ NMR ([D $]$ DMSO, $\left.300 \mathrm{MHz}\right)$ $\delta 10.43(\mathrm{~s}, 1 \mathrm{H}, \mathrm{NH}), 8.40(\mathrm{~s}, 1 \mathrm{H}, \mathrm{NH}), 5.20\left(\mathrm{~s}, 1 \mathrm{H}, 3-\mathrm{H}_{2 \mathrm{a}}\right), 4.78\left(\mathrm{~s}, 1 \mathrm{H}, 3-\mathrm{H}_{2 \mathrm{~b}}\right), 3.97$ (m, 1H, 6-H), $1.80(\mathrm{~m}, 1 \mathrm{H}, 8-\mathrm{H}), 1.77\left(\mathrm{dd}, 2 \mathrm{H}, J=8.4,3.3 \mathrm{~Hz}, 7-\mathrm{H}_{2}\right) 0.85$ [d, 6H, $J=$ $\left.10.3 \mathrm{~Hz}, 9,10-\left(\mathrm{CH}_{3}\right)_{2}\right]$. - EIMS (70 eV) m/z $170\left([\mathrm{M}]^{+}, 100\right), 114$ (M-isobutyl, 25).

cis-Cyclo(leucyl-tyrosyl) (72): Colourless solid, $R_{f}=0.60\left(\mathrm{CHCl}_{3} / 10 \% \mathrm{MeOH}\right)$, with anisaldehyde/sulphuric acid orange. $-{ }^{1} \mathrm{H}$ NMR ([D $]$ DMSO, $\left.300 \mathrm{MHz}\right) \delta 9.14(\mathrm{~s}, 1 \mathrm{H}$, $\mathrm{NH}), 7.97$ (s, 1H, NH), 6.91 (d, 2H, J=8.5 Hz, 3', 5'-H), 6.65 (d, 2H, $J=8.5 \mathrm{~Hz}, 3$ ', 5'-H), 4.06 (quint, $J=4.0 \mathrm{~Hz}, 3-\mathrm{H}), 3.03\left(\mathrm{dd}, 1 \mathrm{H}, J=13.7,4.0 \mathrm{~Hz}, 7-\mathrm{CH}_{2 \mathrm{a}}\right.$ ), 2.71 (dd, 
$\left.1 \mathrm{H}, J=13.7,4.0 \mathrm{~Hz}, 7-\mathrm{CH}_{2 \mathrm{~b}}\right), 1.45\left(\mathrm{~m}, 1 \mathrm{H}, 11-\mathrm{H}_{2 \mathrm{a}}\right), 0.66(\mathrm{dd}, 6 \mathrm{H}, J=4.9,1.7 \mathrm{~Hz}$, 9,10- $\left.\mathrm{CH}_{3}\right), 0.23\left(\mathrm{~m}, 1 \mathrm{H}, 11-\mathrm{H}_{2 \mathrm{a}}\right) .-{ }^{13} \mathrm{C} \mathrm{NMR}\left(\left[\mathrm{D}_{6}\right] \mathrm{DMSO}, 300 \mathrm{MHz}\right) \delta 167.4\left(\mathrm{C}_{\mathrm{q}}, 2-\right.$ $\mathrm{CO}), 166.2\left(\mathrm{C}_{\mathrm{q}}, 5-\mathrm{CO}\right), 156.3\left(\mathrm{C}_{\mathrm{q}}, 4^{\prime}-\mathrm{OH}\right), 131.1\left(2 \mathrm{CH}, 3^{\prime}, 55^{\prime}-\mathrm{H}\right), 125.9\left(\mathrm{C}_{\mathrm{q}}-1^{\prime}\right) 114.8$ (2CH, 2',6'-H), $55.6(\mathrm{CH}, 6-\mathrm{H}), 52.3(\mathrm{CH}, 3-\mathrm{H}), 43.6\left(\mathrm{CH}_{2}, 11-\mathrm{CH}_{2}\right), 37.7\left(\mathrm{CH}_{2}, 7-\mathrm{H} 2\right)$, $22.9(\mathrm{CH}, 8-\mathrm{H}), 22.7\left(\mathrm{CH}_{3}, 9-\mathrm{H}_{3}\right), 21.3\left(\mathrm{CH}_{3}, 10-\mathrm{H}_{3}\right)$. - EIMS $m / z 276\left([\mathrm{M}]^{+}, 15\right), 170$ (100), 114 (36), 107 (98) 44 (52). - (-)-ESIMS m/z 275 ([M - H]', 47), 551 ([2 M - H] , 100); - (-)-HRESIMS 275.13974 (calcd for $\mathrm{C}_{15} \mathrm{H}_{20} \mathrm{~N}_{2} \mathrm{O}_{3}, 275.13956$ ).

Ramthacin B (78): UV absorbing, colourless oil, $R_{f}=0.73$ (cyclohexane/60\% ethylacetate), dark green colouration with anisaldehyde/sulphuric acid. - ${ }^{1} \mathrm{H}$ NMR $\left(\mathrm{CDCl}_{3}, 300 \mathrm{MHz}\right) \delta 7.79\left(\mathrm{dd},{ }^{3} J=9.1,{ }^{4} \mathrm{~J}=1.8 \mathrm{~Hz}, 1 \mathrm{H}, 7-\mathrm{H}\right), 7.77\left(\mathrm{~d},{ }^{4} J=1.8 \mathrm{~Hz}\right.$, $1 \mathrm{H}, 5-\mathrm{H}), 6.59\left(\mathrm{~d},{ }^{3} J=9.1 \mathrm{~Hz}, 1 \mathrm{H}, 8-\mathrm{H}\right), 4.91\left(\mathrm{dd},{ }^{3} J=10.0,{ }^{3} J=2.4 \mathrm{~Hz} 1 \mathrm{H}, 12-\mathrm{H}\right)$, $4.60\left(\mathrm{t},{ }^{3} J=11.1 \mathrm{~Hz}, 1 \mathrm{H}, 3-\mathrm{H}\right), 3.67(\mathrm{~s}, 2 \mathrm{H}, 17-\mathrm{H}), 3.48\left(\mathrm{dd},{ }^{3} J=17.5,{ }^{2} J=4.9 \mathrm{~Hz}\right.$, $\left.1 \mathrm{H}, 4-\mathrm{H}_{\mathrm{A}}\right), 3.42\left(\mathrm{~s}, 1 \mathrm{H}, 3-\mathrm{H} 17-\mathrm{OCH}_{3}\right), 3.11\left(\mathrm{dd},{ }^{3} J=17.3,{ }^{2} J=6.2 \mathrm{~Hz}, 1 \mathrm{H}, 4-\mathrm{H}_{\mathrm{B}}\right)$, $2.05\left(\mathrm{~m}, 1 \mathrm{H}, 11-\mathrm{H}_{\mathrm{A}}\right), 1.74\left(\mathrm{dd},{ }^{3} J=14.4,{ }^{2} J=2.4 \mathrm{~Hz}, 1 \mathrm{H}, 11-\mathrm{H}_{\mathrm{B}}\right), 1.63\left(\mathrm{~s}, 9 \mathrm{H}, 3 \mathrm{CH}_{3}\right.$, 15- $\left.\mathrm{CH}_{3}, 18-\mathrm{CH}_{3}, 19-\mathrm{CH}_{3}\right)$. - EIMS m/z 367 ([M] $\left.{ }^{+}, 10\right), 322$ (12), 304 (40), 258 (100), 224 (68), 99 (34). - (+)-ESIMS $m / z 801\left([2 \mathrm{M}-2 \mathrm{H}+3 \mathrm{Na}]^{+}, 50\right), 412[\mathrm{M}-\mathrm{H}+2$ $\left.\mathrm{Na}]^{+}, 100\right) .-(-)$-ESIMS $m / z 755$ ([2 M - $\left.\left.2 \mathrm{H}+\mathrm{Na}\right]^{-}, 100\right), 733$ ([2 M - H] $\left.]^{-}, 70\right), 366$ ([M - H] $]^{-}, 10$ ), - HREIMS $m / z$ (calcd for $\mathrm{C}_{19} \mathrm{H}_{26} \mathrm{NO}_{4} \mathrm{Cl}, 367.15500$ ).

\subsection{Marine Streptomyces sp. B 7857}

\subsubsection{Primary screening}

Streptomyces sp. B 7857 showed moderate biological activity (Table 9). It also showed several UV fluorescent zones on TLC. Some non-UV absorbing spots showed green colour with spraying with anisaldehyde/sulphuric acid. The crude extract showed $34 \%$ activity against Artemia salina.

Table 10: Biological activity of the crude extract of the strain B7857.

\begin{tabular}{ll}
\hline Test organisms & Inhibition zone $(\varnothing \mathbf{~ m m})$ \\
\hline Bacillus subtilis & 0 \\
Staphylococcus aureus & 17 \\
Streptomyces viridochromogenes (Tü 57) & 14 \\
Escherichia coli & 0 \\
Candida albicans & 0 \\
Mucor miehei & 0 \\
Chlorella vulgaris & 11
\end{tabular}


Chlorella sorokiniana $\quad 11$

Scenedesmus subspicatus

\subsubsection{Fermentation and Isolation}

The terrestrial Streptomyces sp. B 7857 was cultivated using $\mathrm{M}_{2}$ medium in Erlenmeyer flasks (250 ml each) at a 201 scale on a linear shaker $(110 \mathrm{rpm})$ at $28{ }^{\circ} \mathrm{C}$ for 7 days. The culture broth was mixed with Celite, and filtered off under pressure. The filtrate was extracted with XAD-16 column and the resin was eluted with methanol to get a dark brown extract. The biomass was extracted with ethyl acetate (3 times). From the TLC the biomass and XAD extract did not show much difference and were mixed together. The total crude extract $(2.57 \mathrm{~g})$ was chromatographed on a silica gel column eluting with a $\mathrm{CH}_{2} \mathrm{Cl}_{2} / \mathrm{MeOH}$ gradient to get four fractions. Fraction 1 was further purified by using Sephadex LH-20, silica column chromatography, PTLC and finally by an RP-8 gravity column eluting with 50-60 \% aqueous methanol to get compound 81 (15.8 $\mathrm{mg}$ ) and $\mathbf{8 3}(9 \mathrm{mg})$, which gave first bright pink colour and turned to green with anisaldehyde/sulphuric acid. In the same way compound $84(1.1 \mathrm{mg})$ was isolated from fraction 3. Fraction 3 and 4 contained the major components of this strain, which were homononactic acid $(\mathbf{8 7}, 175 \mathrm{mg})$ and nonactic acid $(\mathbf{8 6}, 23 \mathrm{mg})$.

Bacteriogorgonene A (81): Colourless oil, $R_{f}=0.40\left(\mathrm{CHCl}_{3} / 5 \% \mathrm{MeOH}\right) .-{ }^{1} \mathrm{H} \mathrm{NMR}$ $\left(\mathrm{CDCl}_{3}, 300 \mathrm{MHz}\right) \delta 5.86(\mathrm{~s}, 1 \mathrm{H}, \mathrm{NH}), 2.25\left(\mathrm{~s}, 2 \mathrm{H}, 1-\mathrm{CH}_{2}\right), 2.25\left(\mathrm{t},{ }^{4} J=1.1 \mathrm{~Hz}, 1 \mathrm{H}\right.$, 3-H), 2.84, 2.89 (m, 1H, 5-H), $1.37(\mathrm{~m}, 1 \mathrm{H}, 6-\mathrm{H}), 2.14,2.21\left(\mathrm{~m}, 2 \mathrm{H}, 7-\mathrm{CH}_{2}\right), 1.37$ (m, $\left.4 \mathrm{H}, 8,9-\left(\mathrm{CH}_{2}\right)_{2}\right), 1.31(\mathrm{~s}, 6 \mathrm{H}, 12,13-\mathrm{Me}) 0.94\left(\mathrm{~s}, 3 \mathrm{H}, 14-\mathrm{CH}_{3}\right), 1.95\left(\mathrm{t},{ }^{4} \mathrm{~J}=1.4,15-\right.$ $\left.\mathrm{CH}_{3}\right) .-{ }^{13} \mathrm{C}$ NMR $\left(\mathrm{CDCl}_{3}, 150 \mathrm{MHz}\right) \delta 200.1\left(2-\mathrm{C}_{\mathrm{q}}\right), 165.0\left(4-\mathrm{C}_{\mathrm{q}}\right), 126.3(3-\mathrm{CH})$, $74.4\left(11-\mathrm{C}_{\mathrm{q}}\right), 54.7\left(1-\mathrm{CH}_{2}\right), 42.9(5-\mathrm{CH}), 41.5(6-\mathrm{CH}), 36.9\left(9-\mathrm{CH}_{2}\right), 36.9\left(10-\mathrm{C}_{\mathrm{q}}\right)$, $29.3\left(12,13-\left(\mathrm{CH}_{3}\right)_{2}\right), 23.3\left(7-\mathrm{CH}_{2}\right), 21.9\left(15-\mathrm{CH}_{3}\right), 20.6\left(8-\mathrm{CH}_{2}\right), 17.8\left(14-\mathrm{CH}_{3}\right)$; (+)-ESIMS $m / z, 237[\mathrm{M}+\mathrm{H}]^{+} .-(+)$- HRESIMS $m / z, 237.18510$ (calcd. for $\mathrm{C}_{15} \mathrm{H}_{25} \mathrm{O}_{2}$, 237.18510).

82- (+)-ESIMS m/z, $219[\mathrm{M}+\mathrm{H}]^{+}-(+)-$HRESIMS m/z 219.17447 (219.17444 calcd for $\mathrm{C}_{15} \mathrm{H}_{23} \mathrm{O}$ ).

Bacteriogorgonene B (83): Colourless oil, $R_{f}=0.50\left(\mathrm{CHCl}_{3} / 5 \% \mathrm{MeOH}\right) .-{ }^{1} \mathrm{H} \mathrm{NMR}$ $\left(\mathrm{CDCl}_{3}, 300 \mathrm{MHz}\right) \delta 5.93(\mathrm{t}, J=1.1 \mathrm{~Hz}, 1 \mathrm{H}, 3-\mathrm{H}), 4.21(b r \mathrm{~s}, 1 \mathrm{H}, 8-\mathrm{H}), 2.72(\mathrm{~d}, 1 \mathrm{H}, J$ $\left.=15.6 \mathrm{~Hz}, 1-\mathrm{CH}_{2 \mathrm{~b}}\right), 2.52\left(\mathrm{t},{ }^{3} J=11.3 \mathrm{~Hz}, 1 \mathrm{H}, 6-\mathrm{H}\right), 2.21\left(\mathrm{~d}, J=2.2 \mathrm{~Hz}, 1 \mathrm{H}, 1-\mathrm{CH}_{2 \mathrm{a}}\right)$, $\left.2.21\left(\mathrm{~m}, 1 \mathrm{H}, 7-\mathrm{CH}_{2 \mathrm{~b}}\right), 1 \mathrm{H}, 1-\mathrm{CH}_{2 \mathrm{~b}}\right), 2.00\left(\mathrm{~s}, 3 \mathrm{H}, 15-\mathrm{CH}_{3}\right), 1.97\left(\mathrm{~m}, 2 \mathrm{H}, 8-\mathrm{CH}_{2}\right), 1.96$ $\left(\mathrm{m}, 1 \mathrm{H}, 7-\mathrm{CH}_{2 \mathrm{a}}\right), 1.56\left(\mathrm{dd}, J=15.0,2.5 \mathrm{~Hz}, 2 \mathrm{H}, 9-\mathrm{CH}_{2}\right), 1.38\left(\mathrm{~s}, 3 \mathrm{H}, 13-\mathrm{CH}_{3}\right), 1.34$

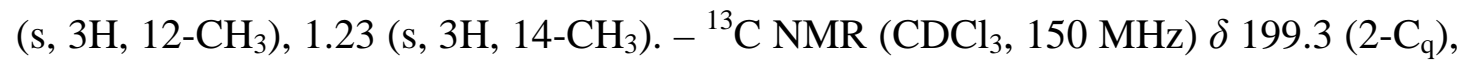
$156.2\left(4-\mathrm{C}_{\mathrm{q}}\right), 128.9(3-\mathrm{CH}), 84.6\left(5-\mathrm{C}_{\mathrm{q}}\right), 81.1\left(11-\mathrm{C}_{\mathrm{q}}\right), 67.7\left(8-\mathrm{CH}_{2}\right), 51.4(6-\mathrm{CH})$, 
$49.2\left(1-\mathrm{CH}_{2}\right), 41.7\left(9-\mathrm{CH}_{2}\right), 40.4\left(10-\mathrm{C}_{\mathrm{q}}\right), 27.4\left(7-\mathrm{CH}_{2}\right), 24.8\left(14-\mathrm{CH}_{3}\right), 22.6$ (12$\left.\mathrm{CH}_{3}\right), 20.3\left(13-\mathrm{CH}_{3}\right), 19.7$ (15-CH). - CIMS $\left(\mathrm{NH}_{3}\right) \mathrm{m} / z .518\left(\left[2 \mathrm{M}+\mathrm{NH}_{4}\right]^{+}, 24\right), 285$ $\left(\left[\mathrm{M}+\mathrm{NH}_{3}+\mathrm{NH}_{4}\right]^{+}, 24\right), 268\left(\left[\mathrm{M}+\mathrm{NH}_{4}\right]^{+}, 100\right) ;-(+)-H R E S I M S m / z 273.14640$ (calcd for $\mathrm{C}_{15} \mathrm{H}_{22} \mathrm{O}_{3} \mathrm{Na}, 273.14642$ ).

Bacteriogorgonene C (84): Colourless oil, $R_{f}=0.35\left(\mathrm{CHCl}_{3} / 5 \% \mathrm{MeOH}\right) .-{ }^{1} \mathrm{H} \mathrm{NMR}$ $\left(\mathrm{CDCl}_{3}, 300 \mathrm{MHz}\right) \delta 5.80(\mathrm{t}, J=1.1 \mathrm{~Hz}, 1 \mathrm{H}, 3-\mathrm{H}), 2.82\left(1 \mathrm{H}, 1-\mathrm{CH}_{2 \mathrm{~b}}\right), 1.90(1 \mathrm{H}, 1-$ $\left.\mathrm{CH}_{2 \mathrm{a}}\right), 2.25\left(1 \mathrm{H}, 7-\mathrm{CH}_{2 \mathrm{~b}}\right), 2.05\left(1 \mathrm{H}, 9-\mathrm{CH}_{2 \mathrm{~b}}\right), 1.95\left(1 \mathrm{H}, 8-\mathrm{CH}_{2 \mathrm{~b}}\right), 1.90(\mathrm{~s}, 3 \mathrm{H}, 14-$ $\left.\mathrm{CH}_{3}\right), 1.85\left(1 \mathrm{H}, 7-\mathrm{CH}_{2 \mathrm{a}}\right), 1.80\left(2 \mathrm{H}, 6-\mathrm{CH}_{2}\right), 1.80\left(1 \mathrm{H}, 8-\mathrm{CH}_{2 \mathrm{a}}\right), 1.40(\mathrm{~s}, 6 \mathrm{H}, 12,13-$ $\left.\left(\mathrm{CH}_{3}\right)_{2}\right), 1.15\left(1 \mathrm{H}, 9-\mathrm{CH}_{2 \mathrm{a}}\right), 1.02\left(\mathrm{~s}, 3 \mathrm{H}, 15-\mathrm{CH}_{3}\right) .-{ }^{13} \mathrm{C} \mathrm{NMR}\left(\mathrm{CDCl}_{3}, 150 \mathrm{MHz}\right) \delta$ $199.9\left(2-\mathrm{C}_{\mathrm{q}}\right), 161.2\left(4-\mathrm{C}_{\mathrm{q}}\right), 127.6(3-\mathrm{CH}), 74.4\left(11-\mathrm{C}_{\mathrm{q}}\right), 71.9(5-\mathrm{CH}), 48.4\left(1-\mathrm{CH}_{2}\right)$, $40.6(6-\mathrm{CH}), 39.8,\left(10-\mathrm{C}_{\mathrm{q}}\right), 31.9\left(9-\mathrm{CH}_{2}\right), 28.5\left(7-\mathrm{CH}_{2}\right), 28.5\left(13-\mathrm{CH}_{3}\right), 28.3(12-$ $\left.\mathrm{CH}_{3}\right), 22.4\left(15-\mathrm{CH}_{3}\right), 19.6\left(8-\mathrm{CH}_{2}\right), 19.2\left(14-\mathrm{CH}_{3}\right) .-$ DCIMS $m / z 270\left(\left[2 \mathrm{M}+\mathrm{NH}_{4}\right]^{+}\right.$, 42); - (+)-HRESIMS m/z 275.16176 (calcd for $\mathrm{C}_{15} \mathrm{H}_{24} \mathrm{O}_{3} \mathrm{Na}, 275.16177$ ).

\subsection{Terrestrial Streptomyces sp. GW 5127}

\subsubsection{Primary screening}

The crude extract of terrestrial Streptomyces sp. GW 5127 was found to inhibit the growth of Gram-positive and Gram-negative bacteria, fungi and algae. TLC of the crude extract exhibited several red spots, which turned to violet with anisaldehyde/sulphuric acid and some colourless UV inactive bands, which became first brown and later turned to violet with anisaldehyde/sulphuric acid.

Table 11: Biological activity of the crude extract of the strain GW 5127.

\begin{tabular}{ll}
\hline Test organisms & Inhibition zone $(\varnothing \mathbf{m m})$ \\
\hline Bacillus subtilis & 11 \\
Staphylococcus aureus & 11 \\
Streptomyces viridochromogenes (Tü 57) & 13 \\
Escherichia coli & 11 \\
Candida albicans & 10.5 \\
Mucor miehei & 12 \\
\hline
\end{tabular}

\subsubsection{Fermentation and isolation}

A sub-culture of the terrestrial strain Streptomycetes GW5127 was used to incubate a 251 shaker culture using $\mathrm{M}_{2}$ medium at $28^{\circ} \mathrm{C}$ in a linear shaker culture. The culture 
broth was harvested after 8 days, mixed with Celite, and filtered off. The filtrate and mycelia were separately extracted using XAD-16 for the water phase, followed by elution with $\mathrm{MeOH}-\mathrm{H}_{2} \mathrm{O}$. The methanolic extract was evaporated and the water residue was again extracted with ethyl acetate. The biomass was extracted with ethyl acetate (3 times).

Silica gel column chromatography of the mycelial fraction $(0.91 \mathrm{~g})$ followed by PTLC and size exclusion chromatography yielded $\beta$-rubromycin $(95,25 \mathrm{mg}$ ) and $\gamma$ rubromycin $(\mathbf{9 6}, 20 \mathrm{mg})$ as red solids. The extract from the water phase $(1.84 \mathrm{~g})$ was separated into four fractions on silica gel (column, $\mathrm{CH}_{2} \mathrm{Cl}_{2}-\mathrm{MeOH}$ gradient). PTLC of fraction $2(0.31 \mathrm{~g})$ followed by Sephadex $\mathrm{LH}-20\left(\mathrm{CH}_{2} \mathrm{Cl}_{2}-\mathrm{MeOH}\right.$ 6:4) gave $2 \mathrm{mg} \mathbf{8 8}$ as a red solid. The middle polar fraction $3(0.25 \mathrm{~g})$ yielded on Sephadex LH-20 $\left(\mathrm{CH}_{2} \mathrm{Cl}_{2}-\mathrm{MeOH}\right.$ 6:4 and $\left.\mathrm{MeOH}\right) \quad 90(1.5 \mathrm{mg})$ and 3-hydroxy-4-methoxy- $\beta$ phenylethyl alcohol $(\mathbf{9 4}, 7.2 \mathrm{mg})$ as colourless solids. The polar fraction $4(0.21 \mathrm{~g})$ yielded on Sephadex LH-20 (MeOH) $N$-[2-(3-hydroxy-4-methoxy-phenyl)-ethyl]acetamide $(\mathbf{9 3}, 3.7 \mathrm{mg})$ as colourless oil.

8-Ethyl-6,11-diydroxy-1-methoxy-naphthacene-5,12-dione (88): Red amorphous solid, $R_{f}=0.50\left(\mathrm{CH}_{2} \mathrm{Cl}_{2}\right)$, pink UV fluorescent ; UV (MeOH): $\lambda_{\max } 310$ (4.07), $510 \mathrm{sh}$ (3.38), 542 (3.58), 577 (3.53); $\lambda_{\max }(\mathrm{MeOH} / \mathrm{HCl}) \mathrm{nm}(\log \varepsilon): 310$ (4.07), 510 (3.43), 542 (3.64), 578 (3.57); $\lambda_{\max }(\mathrm{MeOH} / \mathrm{NaOH}) \mathrm{nm}(\log \varepsilon): 313$ (4.03), 621 (3.52). ; IR $(\mathrm{KBr}): v_{\max } 3440,2924,1635,1557,1419,1384,1271,1042 \mathrm{~cm}^{-1} .-{ }^{1} \mathrm{H}$ NMR $\left(\mathrm{CDCl}_{3}, 600 \mathrm{MHz}\right) \delta 16.10(1 \mathrm{H}, \mathrm{s}, 6-\mathrm{OH}), 15.31(1 \mathrm{H}, \mathrm{s}, 11-\mathrm{OH}), 8.39(1 \mathrm{H}, \mathrm{d}, J=8.2$ $\mathrm{Hz}, 7-\mathrm{H}), 8.26(1 \mathrm{H}, \mathrm{d}, J=1.8 \mathrm{~Hz}, 10-\mathrm{H}), 8.13(\mathrm{dd}, J=7.8,1.0 \mathrm{~Hz}, 1-\mathrm{H}), 7.74(1 \mathrm{H}, \mathrm{t}, J$ $=8.1 \mathrm{~Hz}, 2-\mathrm{H}), 7.63(1 \mathrm{H}, \mathrm{dd}, J=8.2,1.8 \mathrm{~Hz}, 8-\mathrm{H}), 7.29(1 \mathrm{H}, \mathrm{d}, J=8.1 \mathrm{~Hz}, 3-\mathrm{H}), 4.08$ $\left(3 \mathrm{H}, \mathrm{s}, 4-\mathrm{OCH}_{3}\right), 2.86(2 \mathrm{H}, \mathrm{q}, J=7.7 \mathrm{~Hz}, 13-\mathrm{H}), 1.34(3 \mathrm{H}, \mathrm{t}, J=7.7 \mathrm{~Hz}, 14-\mathrm{H}) .-{ }^{13} \mathrm{C}$ $\mathrm{NMR}\left(\mathrm{CDCl}_{3}, 150 \mathrm{MHz}\right) \delta 176.0$ (5-CO), 174.0 (12-CO), $171.4\left(11-\mathrm{C}_{\mathrm{q}}\right), 170.5$ (6$\left.\mathrm{C}_{\mathrm{q}}\right), 160.0\left(4-\mathrm{C}_{\mathrm{q}}\right), 149.4\left(9-\mathrm{C}_{\mathrm{q}}\right), 134.9\left(12 \mathrm{a}-\mathrm{C}_{\mathrm{q}}\right), 133.9(2-\mathrm{CH}), 133.0(8-\mathrm{CH}), 131.5$ $\left(10 \mathrm{a}-\mathrm{C}_{\mathrm{q}}\right), 130.5\left(6 \mathrm{a}-\mathrm{C}_{\mathrm{q}}\right), 126.2(7-\mathrm{CH}), 124.5(10-\mathrm{CH}), 121.1\left(4 \mathrm{a}-\mathrm{C}_{\mathrm{q}}\right), 118.7(1-\mathrm{CH})$, $115.4(3-\mathrm{CH}), 108.2\left(11 \mathrm{a}-\mathrm{C}_{\mathrm{q}}\right), 107.1\left(5 \mathrm{a}-\mathrm{C}_{\mathrm{q}}\right), 56.6\left(4-\mathrm{OCH}_{3}\right), 29.3\left(13-\mathrm{CH}_{2}\right), 15.2(14-$ $\left.\mathrm{CH}_{3}\right) .-(+)$-ESI MS: $m / z(\%) 349\left([\mathrm{M}+\mathrm{H}]^{+}, 35\right), 719\left([2 \mathrm{M}+\mathrm{Na}]^{+}, 100\right) .-\mathrm{EI} \mathrm{MS}$ $(70 \mathrm{eV}) \mathrm{m} / \mathrm{z} 348\left([\mathrm{M}]^{+}, 100\right), 330\left(\left[\mathrm{M}-\mathrm{H}_{2} \mathrm{O}\right]^{+}, 65\right), 302\left(\left[\mathrm{M}-\left(\mathrm{CO}+\mathrm{H}_{2} \mathrm{O}\right)\right]^{+}, 12\right), 262$ (6), 205 (4), 189 (5), 165 (5); - HREIMS m/z [M] ${ }^{+}$(calcd for $\mathrm{C}_{21} \mathrm{H}_{16} \mathrm{O}_{5}, 348.09977$; found: 348.0998).

4-Acetyl-1,3-dihydro-imidazo[4,5-b]pyridin-2-one (90): White amorphous powder, $R_{f}=0.66\left(\mathrm{CH}_{2} \mathrm{Cl}_{2}-10 \% \mathrm{MeOH}\right): \mathrm{UV} \lambda_{\max }(\mathrm{MeOH}) \mathrm{nm}(\log \varepsilon): 264$ (3.86), 349 (3.32), 379 (3.15); $\lambda_{\max }(\mathrm{MeOH} / \mathrm{HCl}) \mathrm{nm}(\log \varepsilon): 265$ (4.05), 327 (3.56), 373 (2.97); $\lambda_{\max }(\mathrm{MeOH} / \mathrm{NaOH}) \mathrm{nm}(\log \varepsilon): 272$ (3.73), 341 (3.38), 369 (3.33), 379 (3.36). - IR $\nu_{\max }(\mathrm{KBr}): 3437(\mathrm{NH}), 2967(\mathrm{CH}), 1685(\mathrm{C}=\mathrm{O}), 1619,1460,1383,1268,1160,638$ 
$\mathrm{cm}^{-}{ }^{1} .-{ }^{1} \mathrm{H}$ NMR $\left(\mathrm{CD}_{3} \mathrm{OD}, 300 \mathrm{MHz}\right) \delta 8.26(1 \mathrm{H}, \mathrm{d}, J=5.1 \mathrm{~Hz}, 6-\mathrm{H}), 7.23(1 \mathrm{H}, \mathrm{d}, J=$ $5.1 \mathrm{~Hz}, 7-\mathrm{H}), 2.68(3 \mathrm{H}, \mathrm{s}, 9-\mathrm{H}) .-{ }^{13} \mathrm{C} \mathrm{NMR}\left(\mathrm{CD}_{3} \mathrm{OD}, 125 \mathrm{MHz}\right) \delta 201.4(8-\mathrm{CO})$, $157.6(2-\mathrm{CO}), 142.7(6-\mathrm{CH}), 139.6\left(7 \mathrm{a}-\mathrm{C}_{\mathrm{q}}\right), 135.0\left(4-\mathrm{C}_{\mathrm{q}}\right), 128.5\left(3 \mathrm{a}-\mathrm{C}_{\mathrm{q}}\right), 108.8(7-$ $\mathrm{CH}), 25.9\left(9-\mathrm{CH}_{3}\right)$. -EIMS: $m / z(\%)=177\left([\mathrm{M}]^{++}, 78\right), 149$ (38), 135 (83), 107 (40), 43 (100). - HREIMS $m / z, 177.0534$ (calcd for $\mathrm{C}_{8} \mathrm{H}_{7} \mathrm{~N}_{3} \mathrm{O}_{2}, 177.0538$ ).

$N$-[2-(3-Hydroxy-4-methoxy-phenyl)-ethyl]-acetamide (93): Colourless oil, $R_{\mathrm{f}}=$ $0.30\left(\mathrm{CH}_{2} \mathrm{Cl}_{2}-5 \% \mathrm{MeOH}\right)$. - IR (KBr): $v_{\max } 3441(\mathrm{OH}), 2932,1651$ (amide), 1513, 1440, 1383, 1276, 1132, $1027 \mathrm{~cm}^{-1}$. UV $\lambda_{\max }(\mathrm{MeOH}) \mathrm{nm}(\log \varepsilon): 325$ (3.71); $\lambda_{\max }(\mathrm{MeOH} / \mathrm{HCl}) \mathrm{nm}(\log \varepsilon): 325$ (3.73); $\lambda_{\max }(\mathrm{MeOH} / \mathrm{NaOH}) \mathrm{nm}(\log \varepsilon): 334$ (3.72). $-{ }^{1} \mathrm{H}$ NMR $\left(\mathrm{CDCl}_{3}, 300 \mathrm{MHz}\right) \delta 6.78\left(1 \mathrm{H}, \mathrm{d},{ }^{3} J=8.1 \mathrm{~Hz}, 5-\mathrm{H}\right), 6.77(1 \mathrm{H}, \mathrm{d}, J=2.1$ $\mathrm{Hz}, 2-\mathrm{H}), 6.65(1 \mathrm{H}, \mathrm{dd}, J=8.1,2.1 \mathrm{~Hz}, 6-\mathrm{H}), 6.16(b r \mathrm{~s}, 1 \mathrm{H}, 3-\mathrm{OH}), 5.75(b r \mathrm{~s}, 1 \mathrm{H}$, 3'-NH), $3.84\left(3 \mathrm{H}, \mathrm{s}, 4-\mathrm{OCH}_{3}\right), 3.44\left(2 \mathrm{H}, \mathrm{q}, J=6.8 \mathrm{~Hz}, 2^{\prime}-\mathrm{H}\right), 2.68(2 \mathrm{H}, \mathrm{t}, J=6.8 \mathrm{~Hz}$, $\left.1^{\prime}-\mathrm{H}\right), 1.92\left(3 \mathrm{H}, \mathrm{s}, 5^{\prime}-\mathrm{H}_{3}\right) .-{ }^{13} \mathrm{C} / \mathrm{APT} \mathrm{NMR}\left(\mathrm{CDCl}_{3}, 150 \mathrm{MHz}\right) \delta 170.4\left(4^{\prime}-\mathrm{C}_{\mathrm{q}}\right), 145.7$ $\left(3-\mathrm{C}_{\mathrm{q}}\right), 145.4\left(4-\mathrm{C}_{\mathrm{q}}\right), 131.9\left(1-\mathrm{C}_{\mathrm{q}}\right), 120.0(6-\mathrm{CH}), 114.9(2-\mathrm{CH}), 110.9(5-\mathrm{CH}), 55.9$ $\left(4-\mathrm{OCH}_{3}\right), 40.7\left(1^{\prime}-\mathrm{CH}_{2}\right), 34.8\left(2^{\prime}-\mathrm{CH}_{2}\right), 23.2\left(5^{\prime}-\mathrm{CH}_{3}\right)$. EIMS $(70 \mathrm{eV}) \mathrm{m} / z 209[\mathrm{M}]^{+}$ (18), 150 (100), 137 (39), 135 (20), - HREIMS $\mathrm{m} / \mathrm{z}[\mathrm{M}]^{+}$(calcd for $\mathrm{C}_{11} \mathrm{H}_{15} \mathrm{NO}_{3}$, 209.1052; found: 209.1052).

4-(2-Hydroxy ethyl)-2-methoxy-phenol (94): colourless oil, $R_{f}=0.430\left(\mathrm{CH}_{2} \mathrm{Cl}_{2}\right.$ $10 \% \mathrm{MeOH})-{ }^{1} \mathrm{H}$ NMR $\left(\mathrm{CD}_{3} \mathrm{OD}, 300 \mathrm{MHz}\right): \delta 6.82(\mathrm{dd}, J=8.1,1.8 \mathrm{~Hz}, 1 \mathrm{H}, 5-\mathrm{H})$, $6.81(\mathrm{~d}, J=1.8 \mathrm{~Hz}, 1 \mathrm{H}, 2-\mathrm{H}), 6.73(\mathrm{dd}, J=8.1,1.9 \mathrm{~Hz}, 1 \mathrm{H}, 6-\mathrm{H}), 3.90$ (s, 3H, 3OCH3), 3.86 (t, $\left.J=7.4 \mathrm{~Hz}, 2 \mathrm{H}, 8-\mathrm{CH}_{2}\right), 2.78\left(\mathrm{t}, J=7.4 \mathrm{~Hz}, 2 \mathrm{H}, 7-\mathrm{CH}_{2}\right)$.

$\boldsymbol{\beta}$-Rubromycin (95): Red powder, $R_{f}=0.60\left(\mathrm{CH}_{2} \mathrm{Cl}_{2} / 10 \% \mathrm{MeOH}\right) . \quad-{ }^{1} \mathrm{H} \mathrm{NMR}$ $\left(\mathrm{CDCl}_{3}, 300.0 \mathrm{MHz}\right) \delta 12.50($ br s, 1H, OH), 10.64 (br s, 1H, OH), 7.61 (s, 1H, 6-H), $7.22(\mathrm{~s}, 1 \mathrm{H}, 5-\mathrm{H}), 7.04(\mathrm{~s}, 1 \mathrm{H}, 6 \mathrm{\prime}-\mathrm{H}), 3.98\left(\mathrm{~s}, 3 \mathrm{H}, \mathrm{OCH}_{3}\right), 3.90\left(\mathrm{~s}, 3 \mathrm{H}, \mathrm{OCH}_{3}\right), 3.86(\mathrm{~s}$, $\left.3 \mathrm{H}, \mathrm{OCH}_{3}\right), 3.40,3.38\left(\mathrm{AB},{ }^{3} \mathrm{~J}=18.0 \mathrm{~Hz}, 1 \mathrm{H}, 3^{\prime}-\mathrm{H}_{\mathrm{a}, \mathrm{b}}\right), 3.20-2.98\left(\mathrm{~m}, 2 \mathrm{H}, 4-\mathrm{H}_{2}\right), 2.55$, $2.30\left(2 \mathrm{~m}, 2 \mathrm{H}, 3-\mathrm{H}_{2}\right) .-{ }^{13} \mathrm{C}$ NMR (125 MHz, $\left.\mathrm{CDCl}_{3}\right) \delta 181.1\left(9^{\prime}-\mathrm{C}_{\mathrm{q}}\right), 177.9\left(4^{\prime}-\mathrm{C}_{\mathrm{q}}\right)$, $163.4\left(9-\mathrm{C}_{\mathrm{q}}\right), 159.7\left(7-\mathrm{C}_{\mathrm{q}}\right), 155.3\left(9^{\prime} \mathrm{a}-\mathrm{C}_{\mathrm{q}}\right), 154.7\left(5^{\prime}-\mathrm{CH}\right), 153.9\left(7^{\prime}-\mathrm{C}_{\mathrm{q}}\right), 148.8\left(10-\mathrm{C}_{\mathrm{q}}\right)$, $148.4\left(8^{\prime}-\mathrm{C}_{\mathrm{q}}\right), 140.4\left(10 \mathrm{a}-\mathrm{C}_{\mathrm{q}}\right), 140.3\left(7-\mathrm{C}_{\mathrm{q}}\right), 131.5\left(4 \mathrm{a}-\mathrm{C}_{\mathrm{q}}\right), 127.2\left(3^{\prime} \mathrm{a}-\mathrm{C}_{\mathrm{q}}\right), 127.0(5 \mathrm{a}-$ $\left.\mathrm{C}_{\mathrm{q}}\right), 118.6(5-\mathrm{CH}), 114.1\left(8^{\prime} \mathrm{a}-\mathrm{C}_{\mathrm{q}}\right), 113.2(6-\mathrm{CH}), 111.2\left(2-\mathrm{C}_{\mathrm{q}}\right), 109.5\left(6{ }^{\prime}-\mathrm{C}_{\mathrm{q}}\right), 106.2$ $\left(9 \mathrm{a}-\mathrm{C}_{\mathrm{q}}\right), 105.2\left(6^{\prime}-\mathrm{C}_{\mathrm{q}}\right), 56.7\left(7^{\prime}-\mathrm{OCH}_{3}\right), 56.3\left(5^{\prime}-\mathrm{OCH}_{3}\right), 52.4\left(7-\mathrm{CO}_{2} \mathrm{CH}_{3}\right), 38.7\left(3^{\prime}-\right.$ $\left.\mathrm{CH}_{2}\right), 28.2\left(3-\mathrm{CH}_{2}\right), 21.4\left(4-\mathrm{CH}_{2}\right) .-(+)-E S I M S ~ m / z 1632\left([3 \mathrm{M}+\mathrm{Na}]^{+}, 68\right), 1095$ $\left([2 \mathrm{M}+\mathrm{Na}]^{+}, 100\right), 559\left([\mathrm{M}+\mathrm{Na}]^{+}, 10\right) ;-(-)-E S I M S ~ m / z, 535\left([\mathrm{M}-\mathrm{H}]^{-}\right)$.

$\gamma$-Rubromycin (96): Red powder, $R_{f}=0.63\left(\mathrm{CH}_{2} \mathrm{Cl}_{2} / 10 \% \mathrm{MeOH}\right) . \quad-{ }^{1} \mathrm{H} \mathrm{NMR}$ $\left(\mathrm{CDCl}_{3}, 300.0 \mathrm{MHz}\right) \delta 13.07(\mathrm{~s}, 1 \mathrm{H}, \mathrm{OH}), 12.28(\mathrm{~s}, 1 \mathrm{H}, \mathrm{OH}), 11.05(\mathrm{~s}, 1 \mathrm{H}, \mathrm{OH}), 7.63$ (s, 1H, 6-H), 7.22 (s, 1H, 5-H), 6.37 (s, 1H, 6'-H), 3.85 (s, 6H, 7'-OCH, $\left.7-\mathrm{CO}_{2} \mathrm{CH}_{3}\right)$, 3.60-3.00 (m, 4H, $\left.\mathrm{CH}_{2}\right), 2.40-2.20\left(\mathrm{~m}, 2 \mathrm{H}, \mathrm{CH}_{2}\right) .-{ }^{13} \mathrm{C} \mathrm{NMR}\left(125 \mathrm{MHz}, \mathrm{CDCl}_{3}\right) \delta$ 
22.3 (C-4), 29.7 (C-3), $39.5\left(\mathrm{C}-3\right.$ '), 52.9 (7- $\left.\mathrm{CO}_{2} \mathrm{CH}_{3}\right), 56.7$ (7'- $\left.\mathrm{OCH}_{3}\right), 106.5(\mathrm{C}-4$ 'a), 106.6 (C-9a), 110.1 (C-6'), 111.7 (C-2), 113.0 (C-8'a), 113.5 (C-6), 118.2 (C-5), 122.9 (C- 3'a), 127.4 (C 5a), 131.5 (C-4a), 141.2 (C-7), 141.3 (C- 10a), 150.1 (C-9'), 150.6 (C-10), 153.5 (C-9'a), 158.9 (C- 4'), 160.0 (C-7'), 160.5 (7- $\left.\mathrm{CO}_{2} \mathrm{CH}_{3}\right), 164.9$ (C-9), $179.0\left(\mathrm{C}-8^{\prime}\right), 183.7\left(\mathrm{C}-5^{\prime}\right) .-(+)$-ESIMS $m / z 1589\left([3 \mathrm{M}+\mathrm{Na}]^{+}, 100\right), 523\left([\mathrm{M}+\mathrm{H}]^{+}\right.$, 2).

\subsection{Terrestrial Streptomyces sp. GW 18/1811}

\subsubsection{Primary screening}

The crude extract of the terrestrial Streptomyces sp. GW 18/1811 obtained from a $1 \mathrm{~L}$ culture exhibited biological activity against Streptomyces viridochromogenes (Tü 57), Escherichia coli, Bacillus subtilis, Staphylococcus aureus, Chlorella vulgaris, Chlorella sorokiniana, Candida albicans, and Mucor miehei.

Table 12: Biological activity of the crude extract of the strain GW 18/1811.

\begin{tabular}{ll}
\hline Test organisms & Inhibition zone $(\varnothing \mathbf{m m})$ \\
\hline Bacillus subtilis & 14 \\
Staphylococcus aureus & 11 \\
Streptomyces viridochromogenes (Tü 57) & 11 \\
Chlorella vulgaris & 28 \\
Chlorella sorokiniana & 13 \\
Scenedesmus subspicatus & 10 \\
\hline
\end{tabular}

\subsubsection{Cultivation and isolation}

With a well grown agar culture of GW 18/1811, 100 of 1 L Erlenmeyer flasks each containing $250 \mathrm{ml}$ of $\mathrm{M}_{2}$ medium were inoculated and incubated for 6 days at $28{ }^{\circ} \mathrm{C}$ on a linear shaker (110 rpm). The culture broth was mixed with Celite, and filtered off. The filtrate and mycelia were separately extracted using XAD-16 for the water phase, followed by elution with $\mathrm{MeOH}-\mathrm{H}_{2} \mathrm{O}$. The methanolic extract was evaporated and the water residue was again extracted with ethyl acetate. The biomass was extracted with ethyl acetate (3 times). Evaporation of the organic solvent gave $1.5 \mathrm{~g}$ of a brown oily extract. The crude extract was subjected to silica column chromatography using $\mathrm{CH}_{2} \mathrm{Cl}_{2} / \mathrm{MeOH}$ gradient to separate four fractions. Fraction 1 contained fat and fatty acids. After purifying fraction 2 through Sephadex LH-20 and PTLC, feudomycinone $(\mathbf{9 8}, 3.5 \mathrm{mg})$ and komodoquinone B $(\mathbf{9 7}, 7 \mathrm{mg})$ were isolated. Separation of 
fraction 3 on Sephadex LH-20 provided $\alpha$-hydroxyacetovanillone (99, $3.0 \mathrm{mg})$ and lysolipin I (102, $2.5 \mathrm{mg})$. PTLC and Sephadex LH-20 of fraction 5 gave $\mathrm{N}$ acetylhomocysteine thiolactone $(\mathbf{1 0 1}, 30 \mathrm{mg})$.

Komodoquinone B (97): Red solid, UV absorbing (254 nm) and orange fluorescent $(366 \mathrm{~nm}), R_{f}=0.42\left(\mathrm{CH}_{2} \mathrm{Cl}_{2} / 5 \% \mathrm{MeOH}\right) .-{ }^{1} \mathrm{H}-\mathrm{NMR}\left(\left[\mathrm{D}_{6}\right] \mathrm{DMSO}, 300 \mathrm{MHz}\right) \delta 13.40$ (br s, 1H, 11-OH), $12.20(b r \mathrm{~s}, 1 \mathrm{H}, 4-\mathrm{OH}), 7.81\left(\mathrm{dd},{ }^{3} J=7.7 \mathrm{~Hz}, 7.5 \mathrm{~Hz}, 1 \mathrm{H}, 2-\mathrm{H}\right)$, $7.75\left(\mathrm{dd},{ }^{3} J=7.5 \mathrm{~Hz},{ }^{4} J=1.5 \mathrm{~Hz}, 1 \mathrm{H}, 1-\mathrm{H}\right), 7.37\left(\mathrm{dd},{ }^{3} J=7.7 \mathrm{~Hz},{ }^{4} J=1.5 \mathrm{~Hz}, 1 \mathrm{H}, 3-\right.$ $\mathrm{H}), 5.28(\mathrm{~s}, 1 \mathrm{H}, \mathrm{OH}), 4.94(\mathrm{br} \mathrm{s}, 1 \mathrm{H}, 7-\mathrm{H}), 3.02\left(\mathrm{~d},{ }^{2} \mathrm{~J}=18.5 \mathrm{~Hz}, 1 \mathrm{H}, 10-\mathrm{H}_{\mathrm{A}}\right), 2.53(\mathrm{~d}$, $\left.{ }^{2} J=18.5 \mathrm{~Hz}, 1 \mathrm{H}, 10-\mathrm{H}_{\mathrm{B}}\right), 2.05\left(\mathrm{dd},{ }^{2} J=13.7 \mathrm{~Hz}, 1 \mathrm{H}, 8-\mathrm{H}_{\mathrm{A}}\right), 1.84\left(\mathrm{dd},{ }^{2} J=13.7 \mathrm{~Hz}\right.$, $\left.1 \mathrm{H}, 8 \mathrm{H}_{\mathrm{B}}\right), 1.36(\mathrm{~s}, 3 \mathrm{H}, 13-\mathrm{H}) .-(+)-\mathrm{ESIMS} m / z 1091[3 \mathrm{M}+\mathrm{Na}]^{+}(45), 735([2 \mathrm{M}+$ $\left.\mathrm{Na}]^{+}, 100\right), 379\left([\mathrm{M}+\mathrm{Na}]^{+}, 7\right) \cdot-(-)-E S I M S m / z 733\left([2 \mathrm{M}+\mathrm{Na}-2 \mathrm{H}]^{+}, 100\right), 355$ ([M - H] $]^{-},(52)$.

Feudomycinone C (98): Red solid, $R_{f}=0.48\left(\mathrm{CH}_{2} \mathrm{Cl}_{2} / 5 \% \mathrm{MeOH}\right), \mathrm{UV}$ absorbing $(254 \mathrm{~nm})$ and orange fluorescent $(366 \mathrm{~nm}) .-{ }^{1} \mathrm{H} \mathrm{NMR}\left(\mathrm{CDCl}_{3}, 300 \mathrm{MHz}\right) \delta 14.10(\mathrm{br}$ s, $1 \mathrm{H}, 11-\mathrm{OH}), 13.32(\mathrm{~s}, 1 \mathrm{H}, 6-\mathrm{OH}), 8.05\left(\mathrm{dd},{ }^{3} \mathrm{~J}=7.7 \mathrm{~Hz}, 1 \mathrm{H}, 1-\mathrm{H}\right), 7.78\left(\mathrm{t},{ }^{3} J=7.7\right.$ $\mathrm{Hz}, 1 \mathrm{H}, 2-\mathrm{H}), 7.40\left(\mathrm{~d},{ }^{3} \mathrm{~J}=7.7 \mathrm{~Hz}, 1 \mathrm{H}, 3-\mathrm{H}\right), 5.28(\mathrm{~s}, 1 \mathrm{H}, \mathrm{OH}), 4.30\left(\mathrm{t}, 1 \mathrm{H},{ }^{3} J=7.7\right.$ $\mathrm{Hz}, 7-\mathrm{CH}), 4.07$ (s, 3H, 4-OCH 3$), 3.02$ (d, $\left.{ }^{2} J=18.5 \mathrm{~Hz}, 1 \mathrm{H}, 10-\mathrm{HA}\right), 2.53$ (d, ${ }^{2} J=$ $\left.18.5 \mathrm{~Hz}, 1 \mathrm{H}, 10-\mathrm{H}_{\mathrm{B}}\right), 2.05\left(\mathrm{dd},{ }^{2} J=13.7 \mathrm{~Hz}, 1 \mathrm{H}, 8-\mathrm{H}_{\mathrm{A}}\right), 1.84\left(\mathrm{dd},{ }^{2} J=13.7 \mathrm{~Hz}, 1 \mathrm{H}, 8-\right.$ $\left.\mathrm{H}_{\mathrm{B}}\right), 1.36$ (s, 3H, 13-H). - (+)-ESIMS m/z 763 ([2 M + Na $\left.]^{+}, 100\right), 393\left([\mathrm{M}+\mathrm{Na}]^{+}\right.$, 40); - (-)-ESIMS $m / z, 761$ ([2 M + Na - 2 H $\left.]^{+}, 30\right), 369$ ([M - H] $\left.]^{-}, 100\right)$.

$\boldsymbol{\alpha}$-Hydroxyacetovanillone (99): Colourless oil, $\boldsymbol{R}_{f}=0.43\left(\mathrm{CH}_{2} \mathrm{Cl}_{2} / 5 \% \mathrm{MeOH}\right), \mathrm{UV}$ absorbing at $254 \mathrm{~nm} .-{ }^{1} \mathrm{H}$ NMR $\left(\mathrm{CDCl}_{3}, 300 \mathrm{MHz}\right): \delta 7.52(\mathrm{~d}, 1 \mathrm{H}, J=1.8 \mathrm{~Hz}, 2-\mathrm{H})$, $7.43(\mathrm{dd}, J=8.3,1.8 \mathrm{~Hz}, 1 \mathrm{H}, 6-\mathrm{H}), 6.96(\mathrm{~d}, J=8.3 \mathrm{~Hz}, 1 \mathrm{H}, 5-\mathrm{H}), 4.81(\mathrm{~s}, 2 \mathrm{H}$, $\left.\mathrm{COCH}_{2} \mathrm{OH}\right), 3.95\left(\mathrm{~s}, 3 \mathrm{H}, 3-\mathrm{OCH}_{3}\right) .-{ }^{13} \mathrm{C} \mathrm{NMR}\left(\mathrm{CDCl}_{3}, 150 \mathrm{MHz}\right) \delta 196.7$ (1'-CO), $151.4\left(4-\mathrm{C}_{\mathrm{q}}\right), 146.9$ (3-Cq), $126.2\left(1-\mathrm{C}_{\mathrm{q}}\right), 122.9(6-\mathrm{CH}), 114.3(5-\mathrm{CH}), 109.5(2-\mathrm{CH})$, $64.9\left(2^{\prime}-\mathrm{CH}_{2}\right), 56.2\left(3-\mathrm{OCH}_{3}\right)$. - EIMS (70 eV) m/z 182 (18), 151 (100), 136 (8), 123 (20).

$N$-Acetylhomocysteine thiolactone (101): White solid, $R_{f}=0.57 \quad\left(\mathrm{CH}_{2} \mathrm{Cl}_{2} /\right.$ $7 \% \mathrm{MeOH}){ }^{1} \mathrm{H} \mathrm{NMR}\left(\mathrm{CDCl}_{3}, 300 \mathrm{MHz}\right) \delta 6.10($ br s, $1 \mathrm{H}, \mathrm{NH}), 4.55(\mathrm{~m}, 1 \mathrm{H}, 3-\mathrm{H})$, 3.20-3.40 (m, 2H, 5- $\left.\mathrm{CH}_{2}\right), 2.95\left(\mathrm{~m}, 1 \mathrm{H}, 4-\mathrm{CH}_{2 \mathrm{a}}\right), 2.05\left(\mathrm{~s}, 3 \mathrm{H}, \mathrm{COCH}_{3}\right)$ and $1.90(\mathrm{~m}$, $\left.1 \mathrm{H}, 4-\mathrm{CH}_{2 \mathrm{~b}}\right) \cdot-{ }^{13} \mathrm{C} \mathrm{NMR}\left(\mathrm{CDCl}_{3}, 150 \mathrm{MHz}\right) \delta 205.8$ (CO-2), 170.8 (1-CO), $\delta 59.4$ $(\mathrm{CH}-3), 31.7\left(4-\mathrm{CH}_{2}\right), 27.5\left(5-\mathrm{CH}_{2}\right), 23.0\left(1-\mathrm{COCH}_{3}\right) .-(+)-$ ESIMS m/z $341([2 \mathrm{M}+$ $\left.\mathrm{Na}]^{+}, 100\right), 182\left([\mathrm{M}+\mathrm{Na}]^{+}, 68\right)$.

Lysolipin I (102): Yellow solid, UV absorbing, $R_{f}=0.65\left(\mathrm{CH}_{2} \mathrm{Cl}_{2} / 10 \% \mathrm{MeOH}\right)$, on TLC pink with $\mathrm{NaOH}-{ }^{1} \mathrm{H} \mathrm{NMR}\left(\mathrm{CDCl}_{3}, 300 \mathrm{MHz}\right) \delta 13.13(\mathrm{~s}, 1 \mathrm{H}, 11-\mathrm{OH}), 12.93$ (s, 1H, 19-OH), 7.94 (d, $J=8.0 \mathrm{~Hz}, 1 \mathrm{H}, 3-\mathrm{CH}), 7.37$ (d, $J=8.0 \mathrm{~Hz}, 1 \mathrm{H}, 2-\mathrm{CH}), 7.09$ 
(s, 1H, 22-CH), $5.63\left(\mathrm{~d}, J=5.4 \mathrm{~Hz}, 1 \mathrm{H}, 28-\mathrm{CH}_{2 \mathrm{a}}\right.$ ), $5.40\left(\mathrm{~d}, J=5.4 \mathrm{~Hz}, 1 \mathrm{H}, 28-\mathrm{CH}_{2 \mathrm{~b}}\right.$ ), $5.03(\mathrm{~m}, 1 \mathrm{H}, 23-\mathrm{CH}), 5.02(\mathrm{~d}, J=3.0 \mathrm{~Hz}, 1 \mathrm{H}, 16-\mathrm{CH}), 4.70(\mathrm{~d}, J=4.1 \mathrm{~Hz}, 1 \mathrm{H}, 24-$ $\mathrm{CH}), 4.46(\mathrm{~d}, J=3.0 \mathrm{~Hz}, 1 \mathrm{H}, 15-\mathrm{CH}), 4.18\left(\mathrm{~s}, 3 \mathrm{H}, 6-\mathrm{OCH}_{3}\right), 3.55$ (s, 3H, 24- $\left.\mathrm{OCH}_{3}\right)$, $3.37\left(\mathrm{~s}, 6 \mathrm{H}, 16-\mathrm{OCH}_{3}, \mathrm{NCH}_{3}\right) .-{ }^{13} \mathrm{C} \mathrm{NMR}\left(\mathrm{CDCl}_{3}, 150 \mathrm{MHz}\right) \delta 181.6(10-\mathrm{CO}), 168.2$ (26-CO), $158.9\left(6-\mathrm{C}_{\mathrm{q}}\right), 151.6\left(14-\mathrm{C}_{\mathrm{q}}\right), 149.9\left(8-\mathrm{C}_{\mathrm{q}}\right), 145.1\left(11-\mathrm{C}_{\mathrm{q}}\right), 143.6\left(5-\mathrm{C}_{\mathrm{q}}\right), 139.6$ $\left(19-\mathrm{C}_{\mathrm{q}}\right), 139.0\left(1-\mathrm{C}_{\mathrm{q}}\right), 134.4\left(20-\mathrm{C}_{\mathrm{q}}\right), 133.3\left(9-\mathrm{C}_{\mathrm{q}}\right), 127.5\left(4-\mathrm{C}_{\mathrm{q}}\right), 125.6(2-\mathrm{CH}), 120.8$ $(3-\mathrm{CH}), 120.7\left(21-\mathrm{C}_{\mathrm{q}}\right), 118.8\left(17-\mathrm{C}_{\mathrm{q}}\right), 116.2(22-\mathrm{CH} 2), 110.9\left(13-\mathrm{C}_{\mathrm{q}}\right) 110.4\left(18-\mathrm{C}_{\mathrm{q}}\right)$, $108.9\left(12-\mathrm{C}_{\mathrm{q}}\right), 92.0\left(28-\mathrm{CH}_{2}\right), 91.1(24-\mathrm{CH}), 78.8(15-\mathrm{CH}), 75.4(16-\mathrm{CH}), 67.8$ (23$\mathrm{CH}), 61.7\left(6-\mathrm{OCH}_{3}\right), 58.1\left(24-\mathrm{OCH}_{3}\right), 57.7\left(23-\mathrm{OCH}_{3}\right), 36.6\left(\mathrm{~N}^{-} \mathrm{CH}_{3}\right) .-(+)-\mathrm{ESIMS}$ $m / z 620[\mathrm{M}+\mathrm{Na}]^{+}, 1217[2 \mathrm{M}+\mathrm{Na}]^{+},-(-)-E S I M S ~ m / z, 596[\mathrm{M}-\mathrm{H}]^{-}$.

\subsection{Fungal metabolite bikaverin}

Bikaverin (103). Prof. H. Anke had sent a dark red sparingly soluble material for further identification. The sample (75 mg) was suspended in $\mathrm{CHCl}_{3}$ and methanol and centrifuged (2000 rpm, $10 \mathrm{~min}$ ) to separate easily soluble impurities. - ${ }^{1} \mathrm{H}$ NMR (300 $\left.\mathrm{MHz},\left[\mathrm{CHCl}_{3}+\mathrm{TFA}\right]\right) \delta 7.39(b r \mathrm{~s}, 1 \mathrm{H}, 4-\mathrm{H}), 7.35(b r \mathrm{~s}, 1 \mathrm{H}, 2-\mathrm{H}), 6.80(b r \mathrm{~s}, 1 \mathrm{H}, 9-$ $\mathrm{H}), 4.16\left(b r \mathrm{~s}, 6 \mathrm{H}, 8-\left(\mathrm{OCH}_{3}\right)_{2}\right), 2.97\left(b r \mathrm{~s}, 3 \mathrm{H}, 1-\mathrm{CH}_{3}\right) .-{ }^{13} \mathrm{C} \mathrm{NMR}(75 \mathrm{MHz}$, $\left.\mathrm{CHCl}_{3}+\mathrm{TFA}\right) \delta 179.5$ (12-CO), $178.2\left(11-\mathrm{C}_{\mathrm{q}}\right), 172.0\left(3-\mathrm{C}_{\mathrm{q}}\right), 166.8\left(6-\mathrm{C}_{\mathrm{q}}\right), 166.1$ (7$\left.\mathrm{C}_{\mathrm{q}}\right), 163.4\left(8-\mathrm{C}_{\mathrm{q}}\right), 162.4\left(4 \mathrm{a}-\mathrm{C}_{\mathrm{q}}\right), 157.1\left(5 \mathrm{a}-\mathrm{C}_{\mathrm{q}}\right), 146.3\left(1-\mathrm{C}_{\mathrm{q}}\right), 124.0(2-\mathrm{CH}), 113.4$ $\left(6 \mathrm{a}-\mathrm{C}_{\mathrm{q}}\right), 112.4\left(11 \mathrm{a}-\mathrm{C}_{\mathrm{q}}\right), 112.1\left(12 \mathrm{a}-\mathrm{C}_{\mathrm{q}}\right), 109.3(9-\mathrm{CH}), 103.7\left(10 \mathrm{a}-\mathrm{C}_{\mathrm{q}}\right), 100.1(4-\mathrm{CH})$, $58.0\left(3-\mathrm{OCH}_{3}\right), 57.5\left(8-\mathrm{OCH}_{3}\right), 23.4\left(1-\mathrm{CH}_{3}\right)$. - EIMS (70 eV) $m / z .382\left([\mathrm{M}]^{+}, 100\right)$, 368 (56), 352 (84), $324(36)$.

\subsection{Plant metabolites}

The root parts of Tephrosia calophylla were collected from Khailasa hills, Visakhapatnam, India, during February 2006. Air-dried and finely powdered roots (1.5 kg) of T. calophylla were macerated at room temperature for $72 \mathrm{~h}$ with chloroform $(3 \times 4.5$ L). The crude extracts were subjected to column chromatography on silica gel.

Spinoflavanone B (103): Yellow solid. - ${ }^{1} \mathrm{H}$ NMR $\left(\mathrm{CDCl}_{3}, 300 \mathrm{MHz}\right) \delta 12.35(\mathrm{~s}, 1 \mathrm{H}$, 5-OH), 7.44 (m, 5H, phe), 6.39 (s, 1H, 7-OH), 5.39 (ABX, 1H, 2-H), 5.22, 5.20 (2 t, $J$ $=7.2 \mathrm{~Hz}$, each 1H, 2", 2', '-H), 3.35 (t, $J=7 \mathrm{~Hz}, 4 \mathrm{H}, 1$ ', 1', $\left.{ }^{-} \mathrm{CH}_{2}\right), 3.05,2.85(A B \mathrm{X}$, $\left.J_{\mathrm{AB}}=16.0, J_{\mathrm{AX}}=12.7, J_{\mathrm{BX}}=3.5 \mathrm{~Hz}, 2 \mathrm{H}, 3-\mathrm{CH}_{2}\right), 1.82,1.75(2 \mathrm{~s}$, each $3 \mathrm{H}, 2 \mathrm{Me})$, $1.70(\mathrm{~s}, 6 \mathrm{H}, 2 \mathrm{Me})-{ }^{13} \mathrm{C} \mathrm{NMR}\left(\mathrm{CDCl}_{3}, 125 \mathrm{MHz}\right) \delta 196.2(4-\mathrm{CO}), 162.3\left(5-\mathrm{C}_{\mathrm{q}}\right)$, $159.3\left(7-\mathrm{C}_{\mathrm{q}}\right), 157.6\left(8 \mathrm{a}-\mathrm{C}_{\mathrm{q}}\right), 138.9\left(1^{\prime}-\mathrm{C}_{\mathrm{q}}\right), 134.7\left(3^{\prime \prime}-\mathrm{C}_{\mathrm{q}}\right), 133.9\left(3^{\prime \prime}-\mathrm{C}_{\mathrm{q}}\right), 128.7\left(2^{\prime}, 6^{\prime}-\right.$ $\mathrm{CH}), 128.5$ (4'-CH), 125.9 (3',5'-CH), 122.0/121.7 (2",2"'-CH), 107.3 (6-CH), 106.5 (8-CH), $102.8\left(4 \mathrm{a}-\mathrm{C}_{\mathrm{q}}\right), 78.8(2-\mathrm{CH}), 43.4\left(3-\mathrm{CH}_{2}\right), 25.82 / 25.80\left(3 "-\mathrm{CH}_{3}, 2 \mathrm{Me}\right), 21.9$ 


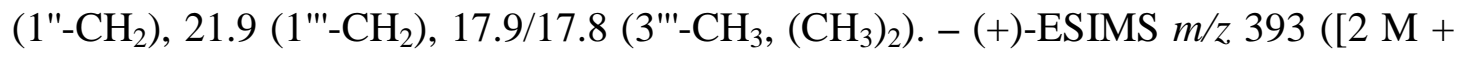
$\mathrm{H}]^{+}, 78$ ). - (+)-HRESIMS $m / z$ 393.20610 [M + H $]^{+}$, (calcd for $\mathrm{C}_{25} \mathrm{H}_{29} \mathrm{O}_{4}, 393.20603$ ).

Calophione A (104): White amorphous powder. - ${ }^{\mathbf{1}} \mathbf{H} \mathbf{~ N M R}\left(\mathrm{CDCl}_{3}, 300 \mathrm{MHz}\right) \delta$ 12.24 (s, 1H, 6'-OH), 12.06 (s,1H, 6"-OH), 7.23 (br s, 1H, 4"-H), 6.82 (s, 1H, 4'-H), 6.53 (s, 1H, 7'-H), 6.45 (s, 1H, 7"-H), 6.00 (s, 2H, 2'-CH $), 5.30$ (ABX, 1H, 2"-H), $5.10\left(\right.$ br s, $1 \mathrm{H}, 2$ ''- $\left.\mathrm{H}_{\mathrm{a}}\right), 5.10\left(\mathrm{sbr}, 1 \mathrm{H}, 2^{\prime \prime}-\mathrm{H}_{\mathrm{b}}\right), 3.30,2.90\left(A B \mathrm{X}, J_{\mathrm{AB}}=15.6, J_{A X}=10.6\right.$, $\left.J_{B X}=8.7 \mathrm{~Hz} ; 2 \mathrm{H}, 3 "-\mathrm{CH}_{2}\right), 1.72(\mathrm{~s}, 3 \mathrm{H}, \mathrm{Me}) .-{ }^{13} \mathbf{C ~ N M R}\left(\mathrm{CDCl}_{3}, 125 \mathrm{MHz}\right) \delta 194.0$ (1-CO), 193.5 (2-CO), $168.5\left(6 "-C_{q}\right), 167.6\left(6^{\prime}-C_{q}\right), 164.3\left(7 " a-C_{q}\right), 156.4\left(7^{\prime} a-C_{q}\right)$, $142.7\left(1{ }^{\prime \prime}-\mathrm{C}_{\mathrm{q}}\right), 141.2\left(3^{\prime} \mathrm{a}-\mathrm{C}_{\mathrm{q}}\right), 128.0(4 "-\mathrm{CH}), 120.3(3$ "a-C $), 113.2\left(2^{\prime \prime \prime}-\mathrm{CH}_{2}\right), 110.6$ $\left(5^{\prime \prime}-\mathrm{C}_{\mathrm{q}}\right), 109.4\left(5^{\prime}-\mathrm{C}_{\mathrm{q}}\right), 108.0$ (4'-CH), $102.4\left(2^{\prime}-\mathrm{CH}_{2}\right), 98.9$ (7'-CH), 98.5 (7"-CH), 88.3 (2"-CH), $32.6\left(3^{\prime \prime-} \mathrm{CH}_{2}\right), 16.9\left(3^{\prime \prime}-\mathrm{CH}_{3}\right) .-(+)$-ESIMS $\mathrm{m} / \mathrm{z} 391\left([\mathrm{M}+\mathrm{Na}]^{+}\right)$; - (+)HRESIMS $m / z 369.09699[\mathrm{M}+\mathrm{H}]^{+}$, (calcd for $\left.\mathrm{C}_{20} \mathrm{H}_{17} \mathrm{O}_{7}, 369.09688\right)$.

Tephcalostan (107): Colourless needles. $-{ }^{1} \mathrm{H} \mathrm{NMR}\left(\mathrm{CDCl}_{3}, 300 \mathrm{MHz}\right) \delta 7.67(\mathrm{~s}, 1 \mathrm{H}$, 1-H), 7.43 (s, 1H, 7-H), 7.06 (s, 1H, 10-H), 6.87 (s, 1H, 4-H), 6.05 (s, 2H, O-CH2-O), $5.33\left(\mathrm{ABX}, 1 \mathrm{H}, J=8.6 \mathrm{~Hz}, 1 \mathrm{H}, 5\right.$ '-H), 5.12 ( $\left.b r \mathrm{~s}, 1 \mathrm{H}, 7^{\prime}-\mathrm{Ha}\right), 4.94$ ( $b r \mathrm{~s}, 1 \mathrm{H}, 7$ ' $^{-\mathrm{Hb}}$ ), 3.45, $3.13\left(A B X, J_{A B}=16.2, J_{A X}=9.3, J_{B X}=7.8 \mathrm{~Hz}, 2 \mathrm{H}, 4^{\prime}-\mathrm{H}_{2}\right), 1.80$ (s, 3H, 8'-CH 3 ). $-{ }^{13} \mathrm{C} \mathrm{NMR}\left(\mathrm{CHCl}_{3}, 125 \mathrm{MHz}\right) \delta 162.9\left(3-\mathrm{C}_{\mathrm{q}}\right), 160.2\left(11 \mathrm{a}-\mathrm{C}_{\mathrm{q}}\right), 158.5$ (6-CO), 154.6 $\left(4 \mathrm{a}-\mathrm{C}_{\mathrm{q}}\right) 150.3\left(10 \mathrm{a}-\mathrm{C}_{\mathrm{q}}\right), 147.2\left(9-\mathrm{C}_{\mathrm{q}}\right), 146.0\left(8-\mathrm{C}_{\mathrm{q}}\right), 142.9(6-\mathrm{CH}), 124.9(2-\mathrm{CH})$, $117.0\left(6 \mathrm{~b}-\mathrm{C}_{\mathrm{q}}\right), 116.8(8-\mathrm{CH}), 112.9\left(7-\mathrm{CH}_{2}\right), 106.0\left(11 \mathrm{~b}-\mathrm{C}_{\mathrm{q}}\right), 103.2\left(6 \mathrm{a}-\mathrm{C}_{\mathrm{q}}\right), 101.9(\mathrm{O}-$ $\left.\mathrm{CH}_{2}-\mathrm{O}\right), 100.0$ (7- $\left.\mathrm{C}_{\mathrm{q}}\right), 98.5$ (4-CH), 93.9 (10-CH), 87.5 (5'-CH), $33.6\left(4^{\prime}-\mathrm{C}_{\mathrm{q}}\right), 17.1$ (8'$\left.\mathrm{CH}_{3}\right) .-(+)$-ESIMS $m / z 747\left([2 \mathrm{M}+\mathrm{Na}]^{+}\right) ;-(+)-H R E S I M S ~ m / z 363.08641[\mathrm{M}+\mathrm{H}]^{+}$, (calcd 363.08632 for $\mathrm{C}_{21} \mathrm{H}_{15} \mathrm{O}_{6}$ ).

Tephcalostan A (108): Colourless powder. $-{ }^{1} \mathrm{H}$ NMR ([D $]$ DMSO, $300 \mathrm{MHz}, 100$ $\left.{ }^{\circ} \mathrm{C}\right) \delta 7.80(\mathrm{~s}, 1 \mathrm{H}, 1-\mathrm{H}), 7.43(\mathrm{~s}, 1 \mathrm{H}, 10-\mathrm{H}), 7.28(\mathrm{~s}, 1 \mathrm{H}, 7-\mathrm{H}), 6.96(\mathrm{~s}, 1 \mathrm{H}, 4-\mathrm{H}), 6.13(\mathrm{~s}$, $\left.2 \mathrm{H},-\mathrm{O}-\mathrm{CH}_{2}-\mathrm{O}-\right), 6.08\left(\mathrm{~m}, 1 \mathrm{H}, 6{ }^{\prime}-\mathrm{H}\right), 5.45\left(\mathrm{~m}, 1 \mathrm{H}, 7^{\prime}-\mathrm{CH}_{2 \mathrm{a}}\right), 5.43(\mathrm{~m}, 1 \mathrm{H}, 5 '-\mathrm{H}), 5.28$ $\left(\mathrm{m}, 1 \mathrm{H}, 7^{\prime}-\mathrm{CH}_{2 \mathrm{~b}}\right), 3.58,3.13\left(A B X, J_{A B}=15.1, J_{A X}=9.2, J_{B X}=7.3 \mathrm{~Hz}, 2 \mathrm{H}, 4^{\prime}-\mathrm{H}_{2}\right)$. ${ }^{13} \mathrm{C}$ NMR ([D 6 DMSO, $\left.125 \mathrm{MHz}, 100{ }^{\circ} \mathrm{C}\right) \delta 162.1\left(3-\mathrm{C}_{\mathrm{q}}\right), 159.4\left(11 \mathrm{a}-\mathrm{C}_{\mathrm{q}}\right), 156.8(6-$ $\mathrm{CO}), 153.8\left(4 \mathrm{a}-\mathrm{C}_{\mathrm{q}}\right) 149.6\left(10 \mathrm{a}-\mathrm{C}_{\mathrm{q}}\right), 146.7\left(9-\mathrm{C}_{\mathrm{q}}\right), 145.5\left(8-\mathrm{C}_{\mathrm{q}}\right), 136.5(6-\mathrm{CH}), 125.0$ $(2-\mathrm{CH}), 116.7(1-\mathrm{CH}), 116.5\left(7^{\prime}-\mathrm{CH}_{2}\right), 116.0\left(6 \mathrm{~b}-\mathrm{C}_{\mathrm{q}}\right), 105.0\left(6 \mathrm{a}-\mathrm{C}_{\mathrm{q}}\right), 101.6\left(\mathrm{O}-\mathrm{CH}_{2}-\right.$ O), $101.5\left(11 \mathrm{~b}-\mathrm{C}_{\mathrm{q}}\right), 98.3(10-\mathrm{CH}), 97.6(4-\mathrm{CH}), 94.0$ (7-CH), 84.5 (5'-CH), 33.6 (4'$\left.\mathrm{C}_{\mathrm{q}}\right) .-(+)$-ESIMS $m / z 719\left([2 \mathrm{M}+\mathrm{Na}]^{+}\right) .-(+)-H R E S I M S ~ m / z, 349.07073[\mathrm{M}+\mathrm{H}]^{+}$, (calcd for $\mathrm{C}_{20} \mathrm{H}_{13} \mathrm{O}_{6}, 349.07067$ ).

Tephcalostan B (111): Colourless solid. - ${ }^{1} \mathrm{H}$ NMR (DMSO, $\left.300 \mathrm{MHz}\right) \delta 7.37$ (s, $1 \mathrm{H}, 5-\mathrm{H}), 7.20\left(\mathrm{~s}, 1 \mathrm{H}, 66^{\prime}-\mathrm{H}\right), 6.77(\mathrm{~s}, 1 \mathrm{H}, 8-\mathrm{H}), 6.10\left(\mathrm{~s}, 2 \mathrm{H}, 9-\mathrm{CH}_{2}\right), 3.25(\mathrm{t}, J=6.8$ $\left.\mathrm{Hz}, 2 \mathrm{H}, 6 "-\mathrm{CH}_{2}\right), 1.90$ (t, $\left.J=6.8 \mathrm{~Hz}, 2 \mathrm{H}, 5 "-\mathrm{CH}_{2}\right), 1.36\left(\mathrm{~s}, 6 \mathrm{H}, 2 "-\mathrm{CH}_{3}\right) .-{ }^{13} \mathrm{C} \mathrm{NMR}$ $\left(\mathrm{CDCl}_{3}, 125 \mathrm{MHz}\right) \delta 160.6\left(2-\mathrm{C}_{\mathrm{q}}\right), 157.3(3 \mathrm{a}-\mathrm{CO}), 150.4\left(5^{\prime}-\mathrm{C}_{\mathrm{q}}\right), 149.4\left(8 \mathrm{a}-\mathrm{C}_{\mathrm{q}}\right) 148.0$ 
$\left(3^{\prime}-\mathrm{C}_{\mathrm{q}}\right), 146.5\left(7-\mathrm{C}_{\mathrm{q}}\right), 145.5\left(6-\mathrm{C}_{\mathrm{q}}\right), 139.4\left(2^{\prime}-\mathrm{C}_{\mathrm{q}}\right), 115.7\left(4^{\prime}-\mathrm{C}_{\mathrm{q}}\right), 115.6\left(4-\mathrm{C}_{\mathrm{q}}\right), 102.4$ $\left(1^{\prime}-\mathrm{C}_{\mathrm{q}}\right), 102.3\left(3-\mathrm{C}_{\mathrm{q}}\right), 101.9\left(9-\mathrm{CH}_{2}\right), 101.4(6 '-\mathrm{CH}), 98.2(8-\mathrm{CH}), 94.1(5-\mathrm{CH}), 73.9$

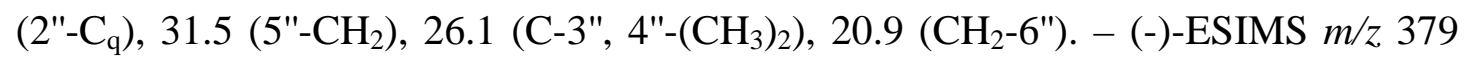
$\left([\mathrm{M}-\mathrm{H}]^{-}, 70\right)$; - (+)-HRESIMS $m / z 381.0967080[\mathrm{M}+\mathrm{H}]^{+}$, (calcd for $\mathrm{C}_{21} \mathrm{H}_{17} \mathrm{O}_{7}$, 381.09688).

Tephcalostan C (112): Colourless solid. - ${ }^{1} \mathrm{H}$ NMR ([D $]$ DMSO, $\left.300 \mathrm{MHz}\right) \delta 7.44$ (s, 1H, 5-H), 7.32 (d, $J=10.1 \mathrm{~Hz}, 1 \mathrm{H}, 6 "-\mathrm{H}), 7.21$ (s, 1H, 6'-H), 6.82 (s, 1H, 8-H), $6.11\left(\mathrm{~s}, 2 \mathrm{H}, 9-\mathrm{CH}_{2}\right), 6.02(\mathrm{~d}, J=10.1 \mathrm{~Hz}, 1 \mathrm{H}, 5 "-\mathrm{H}), 1.46\left(\mathrm{~s}, 6 \mathrm{H}, 2\right.$ "- $\left.\mathrm{CH}_{3}\right) .-{ }^{13} \mathrm{C} \mathrm{NMR}$ $\left(\mathrm{CDCl}_{3}, 125 \mathrm{MHz}\right) \delta 159.8\left(4-\mathrm{C}_{\mathrm{q}}\right), 157.2(3 \mathrm{a}-\mathrm{CO}), 149.7\left(5^{\prime}-\mathrm{C}_{\mathrm{q}}\right), 149.6\left(8 \mathrm{a}-\mathrm{C}_{\mathrm{q}}\right) 148.2$ $\left(3^{\prime}-\mathrm{C}_{\mathrm{q}}\right), 146.8\left(7-\mathrm{C}_{\mathrm{q}}\right), 145.7\left(6-\mathrm{C}_{\mathrm{q}}\right), 137.7\left(2^{\prime}-\mathrm{C}_{\mathrm{q}}\right), 133.3$ (5"-CH), $118.9(6 "-\mathrm{CH}), 115.7$ $\left(1^{\prime} \mathrm{C}_{\mathrm{q}}\right), 103.6\left(3-\mathrm{C}_{\mathrm{q}}\right), 103.2\left(3 \mathrm{a}-\mathrm{C}_{\mathrm{q}}\right), 101.9\left(9-\mathrm{CH}_{2}\right), 98.8(6 '-\mathrm{C}), 98.3(8-\mathrm{CH}), 94.3$ (5$\mathrm{CH}), 94.3\left(4^{\prime}-\mathrm{C}_{\mathrm{q}}\right), 75.7\left(2^{\prime \prime}-\mathrm{C}_{\mathrm{q}}\right), 26.8$ ( 3",4"--CH $)$. - (-)-ESIMS m/z 377 ([M - H]', 70); - (+)-HRESIMS m/z $379.08113[\mathrm{M}+\mathrm{H}]^{+}$, (calcd 379.08177 for $\left.\mathrm{C}_{21} \mathrm{H}_{15} \mathrm{O}_{7}\right)$.

Milletone (113): Yellow needles. $-{ }^{1} \mathrm{H}$ NMR $\left(\mathrm{CDCl}_{3}, 300 \mathrm{MHz}\right) \delta 7.74(\mathrm{~d}, J=8.7$ $\mathrm{Hz}, 1 \mathrm{H}, 11-\mathrm{H}), 6.75$ (s, 1H, 1-H), 6.64 (d, $J=10.1 \mathrm{~Hz}, 1 \mathrm{H}, 4 "-\mathrm{H}), 6.45$ (d, $J=8.7 \mathrm{~Hz}$, $1 \mathrm{H}, 10-\mathrm{H}), 6.41$ (s, 1H, 4-H), 5.85 ("d", $\left.J=14.4 \mathrm{~Hz}, 2 \mathrm{H}, \mathrm{OCH}_{2} \mathrm{O}\right), 5.56(\mathrm{~d}, J=10.1$ $\mathrm{Hz}, 1 \mathrm{H}, 3 "-\mathrm{H}), 4.90(\mathrm{~m}, 1 \mathrm{H}, 6 \mathrm{a}-\mathrm{H}), 4.62$ (dd, $\left.J=12.1, J=3.2 \mathrm{~Hz}, 1 \mathrm{H}, 6-\mathrm{CH}_{2 \mathrm{a}}\right), 4.17$ (dd, $\left.J=12.1, J=1.4 \mathrm{~Hz}, 1 \mathrm{H}, 6-\mathrm{CH}_{2 \mathrm{~b}}\right), 3.79$ (d, $\left.1 \mathrm{H}, J=4.1 \mathrm{~Hz}, 12 \mathrm{a}-\mathrm{H}\right), 1.45,1.39$ (2s, each 3H, 2 2"- $\left.\mathrm{CH}_{3}\right) .-{ }^{13} \mathrm{C} \mathrm{NMR}\left(\mathrm{CDCl}_{3}, 125 \mathrm{MHz}\right) \delta 189.1$ (12-CO), 160.0 (9$\mathrm{C}_{\mathrm{q}}$ ), $156.8\left(7 \mathrm{a}-\mathrm{C}_{\mathrm{q}}\right), 148.3\left(4 \mathrm{a}-\mathrm{C}_{\mathrm{q}}\right), 147.7(3-\mathrm{CH}), 142.2\left(2-\mathrm{C}_{\mathrm{q}}\right), 128.7$ (11-CH), 128.5 (3"-CH), 115.7 (4"-CH), 112.6 (11a-CH), $111.4(10-\mathrm{CH}), 109.1$ (8-CH), 107.0 (1$\mathrm{CH}), 105.7\left(12 \mathrm{~b}-\mathrm{C}_{\mathrm{q}}\right), 101.1\left(\mathrm{O}-\mathrm{CH}_{2}-\mathrm{O}\right), 98.7(4-\mathrm{CH}), 77.7\left(2^{\prime \prime}-\mathrm{C}_{\mathrm{q}}\right), 72.1\left(6 \mathrm{a}-\mathrm{C}_{\mathrm{q}}\right), 66.2$ $\left(6-\mathrm{C}_{\mathrm{q}}\right), 44.6(12 \mathrm{a}-\mathrm{C}), 28.4\left(2^{\prime \prime}-\mathrm{CH}_{3}\right), 28.1\left(2 "-\mathrm{CH}_{3}\right) .-(+)$-ESIMS m/z $377\left([\mathrm{M}-\mathrm{H}]^{+}\right)$. - (+)-HRESIMS $m / z 379.11766[\mathrm{M}+\mathrm{H}]^{+}$, (calcd for $\left.\mathrm{C}_{21} \mathrm{H}_{19} \mathrm{O}_{6}, 379.07067\right)$.

Glabranine (114): Colourless solid. - ${ }^{1} \mathrm{H} \mathrm{NMR}\left(\mathrm{CDCl}_{3}, 300 \mathrm{MHz}\right) \delta 12.00(\mathrm{~s}, 1 \mathrm{H}, 5-$ $\mathrm{OH}), 7.45$ (m, 5H, Phe), $6.55(\mathrm{~s}, 1 \mathrm{H}, 7-\mathrm{OH}), 6.04(\mathrm{~s}, 1 \mathrm{H}, 6-\mathrm{H}), 5.43(\mathrm{ABX}, 1 \mathrm{H}, 2-\mathrm{H})$, $5.22(\mathrm{t}, J=7.3 \mathrm{~Hz}, 1 \mathrm{H}, 2 "-\mathrm{H}), 3.32\left(\mathrm{~d}, J=7.3,2 \mathrm{H}, 1 "-\mathrm{CH}_{2}\right), 3.08,2.85\left(A B X, J_{\mathrm{AB}}=\right.$ 17.1, $\left.J_{\mathrm{AX}}=12.8, J_{\mathrm{BX}}=3.2 \mathrm{~Hz}, 3-\mathrm{CH}_{2}\right), 1.71\left(\mathrm{~s}, 6 \mathrm{H}, 4 ", 5 "-\mathrm{CH}_{3}\right) .-{ }^{13} \mathrm{C} \mathrm{NMR}\left(\mathrm{CDCl}_{3}\right.$, $125 \mathrm{MHz}) \delta 196.3(3-\mathrm{CO}), 163.8\left(7-\mathrm{C}_{\mathrm{q}}\right), 162.2\left(8 \mathrm{a}-\mathrm{C}_{\mathrm{q}}\right), 159.7\left(5-\mathrm{C}_{\mathrm{q}}\right), 138.7\left(1^{\prime}-\mathrm{CH}\right)$, 134.6 (4'-C $\left.\mathrm{C}_{\mathrm{q}}\right), 128.8$ (2',6'-CH), 128.6 (4'-CH), 125.9 (3',5'-CH), 121.6 (2"-CH), 106.5 (8-CH), $103.1\left(4 \mathrm{a}-\mathrm{C}_{\mathrm{q}}\right), 96.9$ (6-CH), 78.9 (2-C), $43.2\left(1 "-\mathrm{CH}_{2}\right), 25.8\left(4 "-\mathrm{CH}_{3}{ }^{*}\right), 21.8$ $\left(\mathrm{CH}_{2}-3\right), 17.8\left(5^{\prime \prime}-\mathrm{CH}_{3}\right) ;{ }^{*}$ assignment may be exchanged. - (+)-ESIMS $m / z, 325$ ([2 $\left.\mathrm{M}+\mathrm{H}]^{+}\right) .-(+)$-HRESIMS $m / z, 325.14350[\mathrm{M}+\mathrm{H}]^{+}$, (calcd for $\mathrm{C}_{20} \mathrm{H}_{21} \mathrm{O}_{4}, 325.14351$ ).

Betulinic acid (115): White needles. - ${ }^{1} \mathrm{H}$ NMR ([D $]$ DMSO, $\left.300 \mathrm{MHz}\right) \delta 12.0$ (bs, 1H, 28-OH), 4.70 (s, 1H, 30-Ha), 4.56 (s, 1H, 30-Hb), 4.20 (d, J = 4.9 Hz, 3-OH), 2.97 (m, 1H, 3-H), 2.22 (dt, $J=14.1,3.7 \mathrm{~Hz}, 1 \mathrm{H}, 19-\mathrm{H}), 1.80$ (m, 2H, 16-H), 1.65 (s, $\left.3 \mathrm{H}, 30-\mathrm{CH}_{3}\right), 0.65-1.85\left(\mathrm{~m}, 1,2,6,7,11,12,15,21,22-\mathrm{CH}_{2}, 5-\mathrm{H}\right), 0.93$ (s, 3H, 23- 
$\left.\mathrm{CH}_{3}\right), 0.87$ (s, 6H, 26, 27- $\left.\left(\mathrm{CH}_{3}\right)_{2}\right), 0.77$ (s, 3H, $\left.-\mathrm{CH}_{3}\right), 0.65$ (s, 3H, 23- $\left.\mathrm{CH}_{3}\right) .-{ }^{13} \mathrm{C}$ NMR ([D 6 DMSO, $125 \mathrm{MHz}) \delta 177.7,150.8,110.9,77.3,56.0,55.4,50.5,49.1,47.1$, 42.5, 40.8, 39.0, 38.8, 38.2, 37.3, 36.9, 34.5, 32.3, 30.7, 29.7, 28.6, 27.7, 25.6, 21.0, 19.5, 18.5, 16.5, 16.29, 16.27, 14.9.-EIMS m/z 456 ([M] $\left.]^{+}, 98\right), 438$ (24), 248 (75), 220 (28), 207 (65), 189 (100), 175 (23), 147 (20), 135 (32), 119 (26), 95 (32), 81 (28), 69 (24) - (-)-ESIMS m/z $911\left([2 \mathrm{M}-\mathrm{H}]^{-}\right)$; - (+)-HRESIMS 457.36757 (calcd for $\left.\mathrm{C}_{30} \mathrm{H}_{48} \mathrm{O}_{3}, 457.36761\right)$.

Betulinic acid methyl ester (116): White solid. - ${ }^{1} \mathrm{H} \mathrm{NMR}\left(\mathrm{CDCl}_{3}, 300 \mathrm{MHz}\right) \delta 4.78$ (bs, 1H, 30-Ha), 4.60 (bs, 1H, 30-Hb), 3.86 (s, 3H, 28-O CH $), 3.20$ (m, 1H, 3-H), 3.00 (m, 1H, 19-H), 2.20 (m, 2H, 16-H), 1.70 (s, 3H, 30- $\left.\mathrm{CH}_{3}\right), 0.65-1.85$ (m, $9 \mathrm{CH}_{2}$,1,2,6,7, 11, 12, 15, 21, 22 and $1 \mathrm{CH}, 5-\mathrm{H}), 0.97$ (s, 6H, 26, 27- $\left.\left(\mathrm{CH}_{3}\right)_{2}\right), 0.93(\mathrm{~s}, 3 \mathrm{H}$, $\left.23-\mathrm{CH}_{3}\right), 0.82\left(\mathrm{~s}, 3 \mathrm{H},-\mathrm{CH}_{3}\right), 0.73\left(\mathrm{~s}, 3 \mathrm{H}, 23-\mathrm{CH}_{3}\right)$.

Stigmasterol (117): White crystalline solid.- ${ }^{1} \mathrm{H} \mathrm{NMR}\left(\mathrm{CDCl}_{3}, 300 \mathrm{MHz}\right) \delta 5.35(\mathrm{br}$ $\mathrm{d}, J=5.6 \mathrm{~Hz}, 1 \mathrm{H}, 6-\mathrm{H}), 3.53(1 \mathrm{H}, \mathrm{m}, 3-\mathrm{H}), 1.01$ (s, 3H, 19-H3), 0.93 (d, $J=6.4 \mathrm{~Hz}$, $\left.3 \mathrm{H}, 21-\mathrm{H}_{3}\right), 0.85\left(\mathrm{~d}, J=6.0 \mathrm{~Hz}, 3 \mathrm{H}, 29-\mathrm{H}_{3}\right), 0.83$ (d, $\left.J=7.6 \mathrm{~Hz}, 3 \mathrm{H}, 27-\mathrm{H}_{3}\right), 0.81(\mathrm{~d}, J$ $\left.=6.8 \mathrm{~Hz}, 3 \mathrm{H}, 26-\mathrm{H}_{3}\right), 0.68\left(\mathrm{~s}, 3 \mathrm{H}, 18-\mathrm{H}_{3}\right) .-{ }^{13} \mathrm{CNMR}\left(\mathrm{CDCl}_{3}, 125 \mathrm{MHz}\right) \delta 140.7(5-$ C), 121.7 (6-C), 71.7 (3-C), 56.8 (14-C), 55.9 (17-C), 50.1 (9-C), 45.8 (24-C), 42.30 (13-C), 42.26 (4-C), 39.8 (12-C), 37.3 (1-C), 36.5 (10-C), 36.3 (20-C), 33.9 (22-C), 31.90 (8-C), 31.91 (7-C), 31.7 (2-C), 28.9 (25-C), 28.2 (16-C), 26.4 (23-C), 24.3 (15C), 21.1 (11-C), 19.6 (26-C), 19.4 (19-C), 19.0 (27-C), 18.8 (21-C), 12.2 (29-C), 11.9 (18-C). - EIMS (70 eV) m/z $414\left([\mathrm{M}]^{+}, 100\right), 396$ (32), 255 (55). - HREIMS 414.3862 (calcd for $\mathrm{C}_{29} \mathrm{H}_{50} \mathrm{O}, 414.3857$ ).

2-Methoxymaackiain (122): Yellow powder. - ${ }^{1} \mathrm{H} \mathrm{NMR}\left(\mathrm{CDCl}_{3}, 300 \mathrm{MHz}\right) \delta 6.94$ (s,1H,1-H), $6.72(\mathrm{~s}, 1 \mathrm{H}, 7-\mathrm{H}), 6.54(\mathrm{~s}, 1 \mathrm{H}, 10-\mathrm{H}), 6.44(\mathrm{~s}, 1 \mathrm{H}, 4-\mathrm{H}), 5.91\left(\mathrm{~d}, \mathrm{CH}_{2}, 2 \mathrm{H}\right.$, $\left.12-\mathrm{CH}_{2}\right), 5.79$ (s, $\left.1 \mathrm{OH}, 3-\mathrm{OH}\right), 5.45(\mathrm{~d}, J=6.9 \mathrm{~Hz}, 11 \mathrm{a}-\mathrm{CH}), 4.20$ (dd, $J=5.4,4.9$ $\mathrm{Hz}, 1 \mathrm{H}, 6-\mathrm{CH}_{2 \mathrm{a}}$ ), 3.90 (s, 3H, 2- $\left.\mathrm{OCH}_{3}\right), 3.58\left(\mathrm{t}, J=10.8 \mathrm{~Hz}, 1 \mathrm{H}, 6-\mathrm{CH}_{2 \mathrm{~b}}\right), 3.45$ (m, $1 \mathrm{H}, 6 \mathrm{a}-\mathrm{CH}) .-{ }^{13} \mathrm{C} \mathrm{NMR}\left(\mathrm{CDCl}_{3}, 125 \mathrm{MHz}\right) \delta 154.1(10 \mathrm{a}-\mathrm{CO}), 150.2\left(4 \mathrm{a}-\mathrm{C}_{\mathrm{q}}\right), 148.1$ $\left(9-\mathrm{C}_{\mathrm{q}}\right), 147.2\left(3-\mathrm{C}_{\mathrm{q}}\right) 142.1\left(2-\mathrm{C}_{\mathrm{q}}\right), 141.7\left(8-\mathrm{C}_{\mathrm{q}}\right), 118.0\left(6 \mathrm{~b}-\mathrm{C}_{\mathrm{q}}\right), 111.6(1-\mathrm{CH}), 110.6$ $\left(11 \mathrm{~b}-\mathrm{C}_{\mathrm{q}}\right), 104.8$ (7-CH), $103.5(4-\mathrm{CH}), 101.3\left(12-\mathrm{CH}_{2}\right), 93.7(10-\mathrm{CH}), 78.8(11 \mathrm{a}-\mathrm{CH})$, $66.5\left(6-\mathrm{CH}_{2}\right), 56.4\left(2-\mathrm{OCH}_{3}\right), 40.3(6 \mathrm{a}-\mathrm{CH})$.

Tephrosol (2-methoxymedicagol) (123): Amorphous powder $-{ }^{1} \mathrm{H}$ NMR ([D $\left.\mathrm{D}_{5}\right]$ pyridine, $\left.300 \mathrm{MHz}\right) \delta 7.41(\mathrm{~s}, 1 \mathrm{H}, 10-\mathrm{H}), 7.23(\mathrm{~s}, 1 \mathrm{H}, 1-\mathrm{H}), 7.16(\mathrm{~s}, 1 \mathrm{H}, 7-\mathrm{H})$, 6.89 (s, $1 \mathrm{H}, 4-\mathrm{H}), 6.12\left(\mathrm{~s}, 2 \mathrm{H}, 12-\mathrm{CH}_{2}\right), 3.90\left(\mathrm{~s}, \mathrm{C} 2-\mathrm{OCH}_{3}\right) .-{ }^{13} \mathrm{C} \mathrm{NMR}_{\left(\mathrm{CDCl}_{3}, 125\right.}$ $\mathrm{MHz}) \delta 159.6\left(11 \mathrm{a}-\mathrm{C}_{\mathrm{q}}\right), 157.4(6-\mathrm{CO}), 151.1\left(3-\mathrm{C}_{\mathrm{q}}\right), 149.6\left(10 \mathrm{a}-\mathrm{C}_{\mathrm{q}}\right) 148.4(4 \mathrm{a}-\mathrm{C})$, $146.8\left(9-\mathrm{C}_{\mathrm{q}}\right), 145.9\left(8-\mathrm{C}_{\mathrm{q}}\right), 145.7$ (2-C), $116.3(6 \mathrm{~b}-\mathrm{CH}), 103.6(4-\mathrm{CH}), 102.9$ (11b$\left.\mathrm{C}_{\mathrm{q}}\right), 102.4(1-\mathrm{CH}), 102.0\left(12-\mathrm{CH}_{2}\right), 102.0\left(6 \mathrm{a}-\mathrm{C}_{\mathrm{q}}\right), 98.5(7-\mathrm{CH}), 94.4(10-\mathrm{CH}), 56.0$ 
(2-OCH 3 ). - (-)-ESIMS m/z 325 ([M -H], 100$)$; - (+)-HRESIMS m/z 327.049844 [M $+\mathrm{H}]^{+}$, (calcd for $\left.\mathrm{C}_{17} \mathrm{H}_{11} \mathrm{O}_{7}, 327.04994\right)$.

Didehydrovillosin (124): Yellow pellets. - ${ }^{1} \mathrm{H}$ NMR ([D $]$ DMSO, $\left.300 \mathrm{MHz}\right) \delta 13.05$ $(\mathrm{s}, 1 \mathrm{H}, 11-\mathrm{OH}), 8.30 / 8.27,(\mathrm{~s}, 1 \mathrm{H}, 1-\mathrm{H}), 7.97(\mathrm{~s}, 1 \mathrm{H}, 6-\mathrm{OH}), 6.70(\mathrm{~s}, 1 \mathrm{H}, 4-\mathrm{H})$, $6.36 / 6.35(\mathrm{~s}, 1 \mathrm{H}, 10-\mathrm{H}), 6.10(\mathrm{~d}, J=8.7 \mathrm{~Hz}, 1 \mathrm{H}, 6-\mathrm{H}$; instead of a double by coupling with $\mathrm{OH}$, the signal could also be explained by two singlets of the individual isomers.), $5.42 / 5.40$ (q, $J=8.5 \mathrm{~Hz}, 2^{\prime}-\mathrm{H}$; quartet due to overlapping of two triplets from the two isomers), $5.10\left(\mathrm{~s}, \mathrm{CH}, 4-\mathrm{CH}_{2 \mathrm{a}}\right), 4.98\left(\mathrm{~s}, \mathrm{CH}, 4-\mathrm{CH}_{2 \mathrm{~b}}\right), 3.79\left(\mathrm{~s}, 3 \mathrm{H}, 3-\mathrm{OCH}_{3}\right)$, $3.77 / 3.75$ (s, 3H, 2- $\left.-\mathrm{OCH}_{3}\right), 3.40\left(\mathrm{~m}, \mathrm{CH}, 1^{\prime}-\mathrm{CH}_{2 \mathrm{a}}\right), 3.02\left(\mathrm{~m}, \mathrm{CH}, 1^{\prime}-\mathrm{CH}_{2 \mathrm{~b}}\right), 1.74$ (d, $J$ $\left.=1.7 \mathrm{~Hz}, 3 \mathrm{H}, 5{ }^{\prime}-\mathrm{CH}_{3}\right) .-{ }^{13} \mathrm{C} \mathrm{NMR}\left(\mathrm{CDCl}_{3}, 125 \mathrm{MHz}\right) \delta 179.72 / 179.65$ (12-C), 165.93/165.89 (9-C), 162.77/162.75 (11-C $\left.\mathrm{q}_{\mathrm{q}}\right), 155.5 / 155.4$ (6a-C), 151.0/150.9 (7a-C $\mathrm{C}_{\mathrm{q}}$ ), $149.43 / 149.37\left(3-\mathrm{C}_{\mathrm{q}}\right), 143.53 / 143.50\left(2-\mathrm{C}_{\mathrm{q}}\right), 143.3 / 143.2\left(4 \mathrm{a}-\mathrm{C}_{\mathrm{q}}\right), 142.84 / 142.81(\mathrm{CH}-$ 3'), 112.47/112.45 (CH-4'), 109.9/109.7 (CH-10), 108.5/108.4 (1- $\left.\mathrm{C}_{\mathrm{q}}\right), 107.48 / 107.45$ $\left(12 \mathrm{~b}-\mathrm{C}_{\mathrm{q}}\right), \quad 104.90 / 104.87 \quad(12 \mathrm{a}-\mathrm{C}), \quad 103.3 / 103.2 \quad\left(11 \mathrm{a}-\mathrm{C}_{\mathrm{q}}\right), \quad 101.81 / 101.75 \quad(4-\mathrm{C})$, 93.99/93.97 (10-CH), 88.0 (6-CH), 87.56/87.54 (2'-CH), 56.07/56.00 (3- $\left.\mathrm{OCH}_{3}\right), 55.6$ $\left(2-\mathrm{OCH}_{3}\right), 29.91 / 29.87\left(1^{\prime}-\mathrm{CH}_{2}\right), 16.78 / 16.73\left(5^{\prime}-\mathrm{CH}_{3}\right)$. - (-)-ESIMS m/z $423\left([\mathrm{M}-\mathrm{H}]^{-}\right.$ , 80); - (+)-HRESIMS $m / z$ 425.12307 [M + H] $]^{+}$, (calcd for $\left.\mathrm{C}_{23} \mathrm{H}_{21} \mathrm{O}_{8}, 425.12310\right)$.

Dehydrorotenone (126): Pale yellow solid. - ${ }^{1} \mathrm{H}$ NMR ([D $]$ pyridine, $\left.300 \mathrm{MHz}\right) \delta$ $9.32(\mathrm{~s}, 1 \mathrm{H}, 1-\mathrm{H}), 8.74(\mathrm{~d}, J=8.6 \mathrm{~Hz}, 1 \mathrm{H}, 11-\mathrm{H}), 7.41(\mathrm{~d}, J=8.6 \mathrm{~Hz}, 1 \mathrm{H}, 10-\mathrm{H}), 7.21$ $(\mathrm{s}, 1 \mathrm{H}, 4-\mathrm{H}), 5.85\left(\mathrm{ABX}, 1 \mathrm{H}, 2^{\prime}-\mathrm{H}\right), 5.61\left(\mathrm{~s}, 1 \mathrm{H}, 4\right.$ '-H), $5.50\left(\mathrm{~s}, 6-\mathrm{CH}_{2}\right), 5.38(\mathrm{~s}, 1 \mathrm{H}, 4$ '$\mathrm{H}), 4.30\left(\mathrm{~s}, 3 \mathrm{H}, 3-\mathrm{OCH}_{3}\right), 4.17\left(\mathrm{~s}, 3 \mathrm{H}, 2-\mathrm{OCH}_{3}\right), 3.83\left(A B \mathrm{X}, J_{A B}=15.8, J_{A X}=9.9 \mathrm{~Hz}\right.$, $\left.1 \mathrm{H}, 1^{\prime}-\mathrm{CH}_{2 \mathrm{a}}\right), 3.56\left(1 \mathrm{H}, J_{A B}=15.8, J_{B X}=7.9 \mathrm{~Hz}, 1 \mathrm{H}, 1^{\prime}-\mathrm{CH}_{2 \mathrm{~b}}\right), 1.87\left(\mathrm{~s}, 3 \mathrm{H}, 5^{\prime}-\mathrm{CH}_{3}\right)$. ${ }^{13} \mathrm{C}$ NMR ([D $]$ pyridine, $\left.125 \mathrm{MHz}\right) \delta 174.1(12-\mathrm{CO}), 165.1\left(9-\mathrm{C}_{\mathrm{q}}\right), 156.7\left(6 \mathrm{a}-\mathrm{C}_{\mathrm{q}}\right)$, $152.6(7 \mathrm{a}-\mathrm{C}), 150.2\left(3-\mathrm{C}_{\mathrm{q}}\right), 147.4\left(4 \mathrm{a}-\mathrm{C}_{\mathrm{q}}\right), 145.9\left(2-\mathrm{C}_{\mathrm{q}}\right), 143.7\left(3^{\prime}-\mathrm{C}_{\mathrm{q}}\right), 128.1(11-\mathrm{CH})$, $119.5\left(11 \mathrm{a}-\mathrm{C}_{\mathrm{q}}\right), 113.8(8-\mathrm{CH}), 112.7\left(4^{\prime}-\mathrm{CH}_{2}\right), 112.2(1-\mathrm{CH}), 111.8\left(12 \mathrm{~b}-\mathrm{C}_{\mathrm{q}}\right), 111.5$ (12a-CH), $108.8(10-\mathrm{CH}), 101.8(4-\mathrm{CH}), 88.2(2 '-\mathrm{CH}), 65.1\left(6-\mathrm{CH}_{2}\right), 56.7\left(3-\mathrm{OCH}_{3}\right)$, $56.0\left(2-\mathrm{OCH}_{3}\right), 31.5\left(1^{\prime}-\mathrm{CH}_{2}\right), 17.2\left(5^{\prime}-\mathrm{CH}_{3}\right) .-(+)$-ESIMS $\mathrm{m} / \mathrm{z} 807\left([2 \mathrm{M}+\mathrm{Na}]^{+}\right.$, 100), $393\left([\mathrm{M}+\mathrm{H}]^{+}, 48\right)$

\section{3-Hydroxy-9,10-dimethoxy-3,3-dimethyl-3H,13H-pyrano[2,3-c;6,5-f']dichro-}

men-7-one (127): - ${ }^{1} \mathrm{H}$ NMR ([D 6 DMSO, $\left.300 \mathrm{MHz}\right) \delta 12.90$ (s, 1H, 11-OH), 8.30 (s, $1 \mathrm{H}, 1-\mathrm{H}), 8.00$ (s, 1H, 6-OH), $6.75(\mathrm{~s}, 1 \mathrm{H}, 4-\mathrm{H}), 6.70$ (d, J = 7.8 Hz, 1H, 1'-H), 6.28 (s, 1H, 10-H), 6.20 (s, 1H, 6-H), 5.80 (d, J=7.8 Hz, 1H, 2'-H), 3.80 (s, 3H, 2-OCH ${ }_{3}$ ), $3.77\left(\mathrm{~s}, 3 \mathrm{H}, 3-\mathrm{OCH}_{3}\right), 1.45\left(\mathrm{~s}, 3 \mathrm{H}, 4^{\prime}-\mathrm{CH}_{3}\right), 1.43\left(\mathrm{~s}, 3 \mathrm{H}, 5^{\prime}-\mathrm{CH}_{3}\right) .-{ }^{13} \mathrm{C} \mathrm{NMR}\left(\mathrm{CDCl}_{3}\right.$, $125 \mathrm{MHz}) \delta 179.9(12-\mathrm{CO}), 161.4\left(11-\mathrm{C}_{\mathrm{q}}\right), 158.9\left(9-\mathrm{C}_{\mathrm{q}}\right), 155.9\left(6 \mathrm{a}-\mathrm{C}_{\mathrm{q}}\right) 150.3\left(7 \mathrm{a}-\mathrm{C}_{\mathrm{q}}\right)$, $149.5\left(3-\mathrm{C}_{\mathrm{q}}\right), 143.6\left(2-\mathrm{C}_{\mathrm{q}}\right), 143.3\left(4 \mathrm{a}-\mathrm{C}_{\mathrm{q}}\right), 128.2\left(2^{\prime}-\mathrm{CH}\right), 113.6\left(1^{\prime}-\mathrm{CH}\right), 109.8(1-$ $\mathrm{CH}), 108.7\left(12 \mathrm{a}-\mathrm{C}_{\mathrm{q}}\right), 107.4(12 \mathrm{~b}-\mathrm{C}), 105.2\left(11 \mathrm{a}-\mathrm{C}_{\mathrm{q}}\right), 101.9(4-\mathrm{CH}), 110.7\left(8-\mathrm{C}_{\mathrm{q}}\right), 99.7$ $(10-\mathrm{CH}), 88.0(6-\mathrm{CH}), 78.3\left(3 '-\mathrm{C}_{\mathrm{q}}\right), 56.1\left(3-\mathrm{OCH}_{3}\right), 55.6\left(2-\mathrm{OCH}_{3}\right), 27.8\left(4-\mathrm{CH}_{3}\right)$, 
27.6 (5'-CH 3 ). - (-)-ESIMS m/z 423 ([M - H] ' , 100); - (+)-HRESIMS m/z 425.12307 $[\mathrm{M}+\mathrm{H}]^{+}$, (calcd for $\left.\mathrm{C}_{23} \mathrm{H}_{21} \mathrm{O}_{8}, 425.12310\right)$.

Obovatin methyl ether (128): White soild. - ${ }^{1} \mathrm{H} \mathrm{NMR}\left(\mathrm{CDCl}_{3}, 300 \mathrm{MHz}\right) \delta 7.45(\mathrm{~m}$, $5 \mathrm{H}, \mathrm{Phe}), 6.60$ (d, $J=9.6 \mathrm{~Hz}, 1 \mathrm{H}, 4$ "-H), 6.10 (s, 1H, 6-H), 5.46 (d, $J=10.0 \mathrm{~Hz}, 1 \mathrm{H}$, 3"-H), $5.40(\mathrm{ABX}, 1 \mathrm{H}, 2-\mathrm{H}), 3.89\left(\mathrm{~s}, 3 \mathrm{H}, 5-\mathrm{OCH}_{3}\right), 2.99\left(A B X, J_{A B}=16.5, J_{A X}=12.9\right.$ $\mathrm{Hz}, 1 \mathrm{H}, 3-\mathrm{H}_{2 \mathrm{a}}$ ), $2.79\left(A B X, J_{A B}=16.5, J_{B X}=3.2 \mathrm{~Hz}, 1 \mathrm{H}, 3-\mathrm{H}_{2 \mathrm{~b}}\right), 1.46\left(\mathrm{~s}, 3 \mathrm{H}, 2 "-\mathrm{CH}_{3}\right)$, $1.44\left(\mathrm{~s}, 3 \mathrm{H}, 2 "-\mathrm{CH}_{3}\right) .-{ }^{13} \mathrm{C} \mathrm{NMR}\left(\mathrm{CDCl}_{3}, 125 \mathrm{MHz}\right) \delta 189.1$ (4-CO), $162.1\left(5-\mathrm{C}_{\mathrm{q}}\right)$, $159.9\left(7-\mathrm{C}_{\mathrm{q}}\right), 158.7\left(8 \mathrm{a}-\mathrm{C}_{\mathrm{q}}\right), 138.9$ (1'-CH), 128.6 (3',5'-CH), $128.4\left(4^{\prime}-\mathrm{CH}\right), 126.2$ (4"$\mathrm{CH}), 125.9$ (2',6'-CH), 115.9 (3"-CH), $105.6\left(4 \mathrm{a}-\mathrm{C}_{\mathrm{q}}\right), 102.8\left(8-\mathrm{C}_{\mathrm{q}}\right), 93.7(6-\mathrm{CH}), 78.9$ (2-CH), $77.9\left(2 "-\mathrm{C}_{\mathrm{q}}\right), 56.1\left(5-\mathrm{OCH}_{3}\right), 45.6\left(\mathrm{CH}_{2}-3\right), 28.4\left(2 "-\mathrm{CH}_{3}\right), 28.1\left(2 "-\mathrm{CH}_{3}\right)-$ (+)-ESIMS $m / z 695\left([2 \mathrm{M}+\mathrm{Na}]^{+}, 100\right), 337\left([\mathrm{M}+\mathrm{H}]^{+}, 15\right) .-(+)-H R E S I M S ~ m / z$ $337.14357[\mathrm{M}+\mathrm{H}]^{+}$, (calcd for $\mathrm{C}_{21} \mathrm{H}_{21} \mathrm{O}_{4}, 337.14344$ ).

Obovatin (129): White soild. $-{ }^{1} \mathrm{H} \mathrm{NMR}\left(\mathrm{CDCl}_{3}, 300 \mathrm{MHz}\right) \delta 12.10(\mathrm{~s}, \mathrm{OH}, 5-\mathrm{OH})$, 7.45 (m, 5H, Phe), 6.55 (d, $J=10.1 \mathrm{~Hz}, 1 \mathrm{H}, 4 "-\mathrm{H}), 6.00$ (s, 1H, 6-H), 5.45 (d, $J=10.1$ $\mathrm{Hz}, 1 \mathrm{H}, 3 "-\mathrm{H}), 5.40(\mathrm{dd}, J=9.8,3.1 \mathrm{~Hz}, 1 \mathrm{H}, 2-\mathrm{H}), 3.05\left(\mathrm{ABX}, J_{\mathrm{AB}}=17.1 J_{\mathrm{AX}}=12.9\right.$ $\mathrm{Hz}, 1 \mathrm{H}, 3-\mathrm{H}_{2 \mathrm{a}}$ ), $2.82\left(\mathrm{ABX}, J_{\mathrm{AB}}=17.1, J_{\mathrm{BX}}=3.2 \mathrm{~Hz}, 1 \mathrm{H}, 3-\mathrm{H}_{2 \mathrm{~b}}\right), 1.44$ (s, 3H, 2"-CH $\mathrm{CH}_{3}$, $1.42\left(\mathrm{~s}, 3 \mathrm{H}, 2 "-\mathrm{CH}_{3}\right) .-{ }^{13} \mathrm{C} \mathrm{NMR}\left(\mathrm{CDCl}_{3}, 125 \mathrm{MHz}\right) \delta 195.6$ (4-CO), $163.7\left(5-\mathrm{C}_{\mathrm{q}}\right)$, $162.2\left(7-\mathrm{C}_{\mathrm{q}}\right), 156.7\left(8 \mathrm{a}-\mathrm{C}_{\mathrm{q}}\right), 138.5$ (1'-CH), 128.8 (3',5'-CH), 128.7 (4'-CH), 126.4 (4"$\mathrm{CH}), 125.9$ (2',6'-CH), 115.5 (3"-CH), $102.8\left(8-\mathrm{C}_{\mathrm{q}}\right), 101.9\left(4 \mathrm{a}-\mathrm{C}_{\mathrm{q}}\right), 97.6(6-\mathrm{CH}), 79.0$ (2-CH), $78.1\left(2 "-\mathrm{C}_{\mathrm{q}}\right), 43.3\left(3-\mathrm{CH}_{2}\right), 28.5\left(2 "-\mathrm{CH}_{3}\right), 28.2\left(2 "-\mathrm{CH}_{3}\right)-(-)-\mathrm{ESIMS} \mathrm{m/z}$ $665\left([2 \mathrm{M}-2 \mathrm{H}+\mathrm{Na}]^{+}, 100\right), 321\left([\mathrm{M}-\mathrm{H}]^{+}, 50\right) .-(+)-H R E S I M S ~ m / z ~ 323.12797[\mathrm{M}+$ $\mathrm{H}]^{+}$, (calcd for $\mathrm{C}_{20} \mathrm{H}_{19} \mathrm{O}_{4}, 323.12833$ ).

7-Methylglabranin (130): White solid. - ${ }^{1} \mathrm{H} \mathrm{NMR}\left(\mathrm{CDCl}_{3}, 300 \mathrm{MHz}\right) \delta 12.14(\mathrm{~s}, 1 \mathrm{H}$, 5-OH), 7.44 (m, 5H, Phe), 6.09 (s, 1H, 6-H), $5.41(\mathrm{ABX}, 1 \mathrm{H}, 2-\mathrm{H}), 5.16(\mathrm{t}, 1 \mathrm{H}, J=$ $\left.7.2 \mathrm{~Hz}, 2^{\prime \prime-H}\right), 3.84$ (s, 3H, 7-OMe), 3.23 (d, $\left.J=7.2 \mathrm{~Hz}, 1 \mathrm{H}, 1 "-\mathrm{H}\right), 3.05,2.85$ (ABX, $\left.2 \mathrm{H}, J_{\mathrm{AB}}=17.1, J_{\mathrm{AX}}=12.6, J_{\mathrm{BX}}=3.2 \mathrm{~Hz}, 3-\mathrm{CH}_{2}\right), 1.64,1.62(2 \mathrm{~s}$, each $3 \mathrm{H}, 4 ", 5 "-$ $\left.\mathrm{CH}_{3}\right) .-{ }^{13} \mathrm{C} \mathrm{NMR}\left(\mathrm{CDCl}_{3}, 125 \mathrm{MHz}\right) \delta 196.2(4-\mathrm{CO}), 165.6\left(7-\mathrm{C}_{\mathrm{q}}\right), 162.6\left(8 \mathrm{a}-\mathrm{C}_{\mathrm{q}}\right)$, $158.7\left(5-\mathrm{C}_{\mathrm{q}}\right), 138.9\left(3^{\prime \prime}-\mathrm{C}_{\mathrm{q}}\right), 131.2\left(1^{\prime}-\mathrm{C}_{\mathrm{q}}\right), 128.6\left(2^{\prime}, 6^{\prime}-\mathrm{CH}\right), 128.4\left(4^{\prime}-\mathrm{CH}\right), 125.9$ (3',5'-CH), 122.4 (2'-CH), $108.9\left(8-\mathrm{C}_{\mathrm{q}}\right), 102.9\left(8 \mathrm{a}-\mathrm{C}_{\mathrm{q}}\right), 92.4(6-\mathrm{CH}), 78.6(2-\mathrm{C}), 55.8$ (7-OMe), $43.3\left(1 "-\mathrm{CH}_{2}\right), 25.7$ (4"-- $\left.\mathrm{CH}_{3}\right), 21.6\left(3-\mathrm{CH}_{2}\right), 17.6\left(5 "-\mathrm{CH}_{3}\right) .-(+)-\mathrm{ESIMS}$ $\mathrm{m} / \mathrm{z} 339\left([\mathrm{M}+\mathrm{H}]^{+}\right) .-(+)$-HRESIMS $\mathrm{m} / z 339.15913[\mathrm{M}+\mathrm{H}]^{+}$, (calcd for $\mathrm{C}_{21} \mathrm{H}_{23} \mathrm{O}_{4}$, 339.15991).

Candidone (131): White solid. - ${ }^{1} \mathrm{H}$ NMR $\left(\mathrm{CDCl}_{3}, 300 \mathrm{MHz}\right) \delta 7.41(\mathrm{~m}, 5 \mathrm{H}$, Phe), 6.13 (s, 1H, 6-H), 5.40 (ABX, 1H, 2-H), 5.18 (t, 1H, J = $7.3 \mathrm{~Hz}, 2$ "-H), 3.92 (s, 3H, 7$\mathrm{OMe}), 3.87$ (s, 3H, 5-OMe), $3.32\left(\mathrm{~d}, 2 \mathrm{H}, J=7.3 \mathrm{~Hz}, 1 "-\mathrm{CH}_{2}\right), 2.98,2.85\left(A B \mathrm{X}, J_{\mathrm{AB}}=\right.$ $\left.17.1, J_{\mathrm{AX}}=12.8, J_{\mathrm{BX}}=3.2 \mathrm{~Hz}, 3-\mathrm{CH}_{2}\right), 1.67,1.62\left(2 \mathrm{~s}, 6 \mathrm{H}, 4 ", 5 "-\mathrm{CH}_{3}\right) .-{ }^{13} \mathrm{C} \mathrm{NMR}$ 
$\left(\mathrm{CDCl}_{3}, 125 \mathrm{MHz}\right) \delta 189.8$ (4-CO), $163.2\left(8 \mathrm{a}-\mathrm{C}_{\mathrm{q}}\right), 160.9\left(7-\mathrm{C}_{\mathrm{q}}\right), 160.6\left(5-\mathrm{C}_{\mathrm{q}}\right), 139.2$ (3"-CH), $131.2\left(1^{\prime}-\mathrm{C}_{\mathrm{q}}\right), 128.5$ (2',6'-CH), 128.2 (4'-CH), 125.8 (3',5'-CH), 122.4 (2"$\mathrm{CH}-2), 110.2\left(8-\mathrm{C}_{\mathrm{q}}\right), 105.9$ (4a-CH), 88.6 (6-CH), 78.5 (2-CH), 56.0 (7-OMe), 55.6 (5-OMe), $45.6\left(1 "-\mathrm{CH}_{2}\right), 25.7\left(4{ }^{\prime *}-\mathrm{CH}_{3}\right), 21.8\left(3-\mathrm{CH}_{2}\right), 17.6\left(5^{\prime \prime}{ }^{*}-\mathrm{CH}_{3}\right)$; * assignment may be exchanged. - (+)-ESIMS $m / z 727\left([2 \mathrm{M}+\mathrm{H}]^{+}\right) .-(+)$-HRESIMS $\mathrm{m} / \mathrm{z}$ $353.17485[\mathrm{M}+\mathrm{H}]^{+}$, (calcd for $\left.\mathrm{C}_{22} \mathrm{H}_{25} \mathrm{O}_{4}, 353.17474\right)$.

Ovalichalcone ( $\beta$-hydroxychalcone) and its diketo tautomer (132): Yellow solid. ${ }^{1} \mathrm{H} \mathrm{NMR}\left(\mathrm{CHCl}_{3}, 300 \mathrm{MHz}\right) \delta 15.47(\mathrm{~s}, 1 \mathrm{H}, \beta-\mathrm{OH}), 13.66\left(\mathrm{~s}, 1 \mathrm{H}, 6{ }^{\prime}-\mathrm{OH}\right), 7.85(\mathrm{dd}, J=$ 7.8, $1.4 \mathrm{~Hz} ; 2 \mathrm{H}, 2,6-\mathrm{H}), 7.45(\mathrm{~m}, 3 \mathrm{H}, 3,4,5-\mathrm{H}), 7.31(\mathrm{~s}, 1 \mathrm{H}, \alpha-\mathrm{H}), 6.68(\mathrm{~d}, J=10 \mathrm{~Hz}$, 1H, 3"-H), 5.93 (s, 1H, 3'-H), 5.45 (d, J = $10 \mathrm{~Hz}, 1 \mathrm{H}, 4$ "-H), 3.90 (s, 3H, 2'-OMe), $1.45\left(\mathrm{~s}, 6 \mathrm{H}, 2^{2}-\left(\mathrm{CH}_{3}\right)_{2}\right)$. - (+)-ESIMS $m / z 727\left([2 \mathrm{M}+\mathrm{Na}]^{+}, 47\right) ;-(+)-\operatorname{HRESIMS~} m / z$ $353.13846[\mathrm{M}+\mathrm{H}]^{+}$, (calcd for $\left.\mathrm{C}_{21} \mathrm{H}_{21} \mathrm{O}_{5}, 363.13834\right)$.

Praecansone B (134): Pale yellow solid. - ${ }^{1} \mathrm{H} \mathrm{NMR}\left(\mathrm{CDCl}_{3}, 300 \mathrm{MHz}\right) \delta 7.52(\mathrm{~m}$, 2H, 2',6'-H), 7.40 (d, 1H, $J=16.1,1-\mathrm{H}), 7.38$ (m, 3H, 3', 4',5'-H), 7.00 (d, 1H, $J=$ 16.1, 2-H), 6.54 (d, 1H, J = 9.2, 4"-H), 6.26 (s, 1H, 6-H), 5.54 (d, 1H, J = 9.2 Hz, 3"$\mathrm{H}), 3.74,3.73$ (2 s, 6H, 5,9-OMe), 1.46 (s, 6H, 5",6"-- $\left.\mathrm{CH}_{3}\right) .-{ }^{13} \mathrm{C} \mathrm{NMR}\left(\mathrm{CDCl}_{3}, 125\right.$ $\mathrm{MHz}) \delta 194.1$ (3-CO), $158.1\left(5-\mathrm{C}_{\mathrm{q}}\right), 156.0\left(7-\mathrm{C}_{\mathrm{q}}\right), 154.7\left(9-\mathrm{C}_{\mathrm{q}}\right), 144.7(1-\mathrm{CH}), 134.8$ $\left(1^{\prime}-\mathrm{C}_{\mathrm{q}}\right), 130.3\left(4^{\prime}-\mathrm{CH}\right), 128.8\left(2^{\prime}, 6^{\prime}-\mathrm{CH}\right), 128.7$ (2-CH), $128.4\left(3^{\prime}, 5^{\prime}-\mathrm{CH}\right), 127.7$ (3"$\mathrm{CH}), 116.5$ (4"-CH), $116.2\left(4-\mathrm{C}_{\mathrm{q}}\right), 107.9\left(8-\mathrm{C}_{\mathrm{q}}\right), 96.1(6-\mathrm{CH}), 76.8$ (2"-C), 63.3 (9$\left.\mathrm{OCH}_{3}\right), 55.8\left(5-\mathrm{OCH}_{3}\right), 27.9(5 ", 6 "-\mathrm{CH}) .-(+)$-ESIMS $\mathrm{m} / z .723\left([2 \mathrm{M}+\mathrm{Na}]^{+}\right) ;-(+)-$ HRESIMS $m / z 351.15919[\mathrm{M}+\mathrm{H}]^{+}$, (calcd for $\left.\mathrm{C}_{22} \mathrm{H}_{23} \mathrm{O}_{4}, 351.15910\right)$. 
9 References

(1) Saleem, M.; Ali M. S.; Hussain S.; Jabbar A.; Ashraf M.; Lee Y. S. Nat. Prod. Rep. 2007, 24, 1142-1152.

(2) Faulkner, D. J. Nat. Prod. Rep. 2002, 19, 1.

(3) Carvalho, L. R. D.; Fujii, M. T.; Roque N. F.; Lago, J. H. G Phytochemistry 2006, 67, 1331.

(4) Andersen R. J.; Williams D. E.; "Chemistry in the Marine Environment," ed. by Hester R. E.; Harrison R. M.; The Royal Society of Chemistry, Cambridge, 2000, 55-79.

(5) Kelecom, A. An. Acad. Bras. Cienc. 2002, 74, 151.

(6) Strobel, G.; Daisy, B.; Castillo U.; Harper, J. J. Nat. Prod. 2004, 67, $257-$ 268 ,

(7) Newman D. J.; Cragg, G.M. Curr. Med. Chem. 2004, 11, 1689.

(8) Rogers, B. L. Curr. Opin. Drug Discovery Dev. 2004, 7, 211-222.

(9) Larsen, T. O.; Lange, L.; Schnorr, K.; Stender, S.; J. C. Frisvad Tetrehedron Lett. 2007, 48, 1261-1264.

(10) Araki, Y.; Konoike T. J. Org. Chem. 1997, 62, 5299 -5309.

(11) Endo A. J Lipid Res. 1992, 33, 1569-1582.

(12) Shu, Y.-Z. J. Nat. Prod. 1998, 61, 1053-1071.

(13) Lee, D. H.; Kim, S. W.; Bae, K. S. ; Hong, J. S.; Suh, C.; Kang, Y. K.; Lee, J. S. Clinical Cancer Research 2007, 13, 6182-6186.

(14) Butler M. S. Nat. Prod. Rep. 2005, 22,162-195.

(15) Bechthold, A.; Domann, S.; Faust, B.; Hoffmeister, D.; Stockert, S.; Trefzer, A.; Weitnauer G.; Westrich, L. Chemotherapie J. 1999, 4, 130-135.

(16) Oshtari, T.; Shibasaki, M.; Yoshzawa, T.; Tomita, M.; Takao K. I.; Kobayashi, S. Tetrahedron 1997, 53, 10993-11006.

(17) Fujii, A. J. Antibiot. 1973, 26, 396; 1978, 31, 801-804.

(18) Martin, S. F.; Hida, T.; Kym, P. R.; Loft M.; Holgson, A. J. Am. Chem. Soc. 1997, 119, 3193-3194.

(19) Katzung, B. G. Pharmacology, 9e Section VIII. Chemotherapeutic Drugs Chapter 44. 2007.

(20) Ajito, K.; Kurihara, K.; Hara, O.; Okonogi, T.; Kikuchi, N.; Araake, M.; Suzuki, H.; Omoto S.; Inouye, S. J. Antibiot, 1997, 50, 150-61. 
(21) Malthum, K.; Coque, T. M.; Singh K. V.; Murray, B. E. Antimicrob. Agents Chemother. 1999, 43, 930-936; (b) L. M. Ednie, M. R. Jacobs, P. C. Appelbaum, Antimicrob. Agents Chemother 1997, 41, 2019-2022.

(22) Harris C. R.; Thorarensen, A. Curr. Med. Chem., 2004, 11, 2213-2243.

(23) Grond, S.; Langer, H.; Henne, H.; Sattler, I.; Thiericke, R.; Grabley, S.; Zähner H.; Zeeck A. Eur. J. Org. Chem., 2000, 39, 929 -937.

(24) Biabani, M. A.; Laatsch, H: J. Prakt. Chemie, 1998, 340, 589-607.

(25) Yoshikawa, K.; Takadera, T.; Adachi, K., Nishijima, M; Sano, H. J. Antibiot. 1997, 50, 949-953.

(26) Maskey, R. P.; Sevvana, M.; Usón, I.; Helmke E.; Laatsch, H. Angew. Chemie, 2004, 43 1281-3.

(27) Jaruchoktaweechai, C.; Suwanborirux, K.; Tanasupawatt, S.; Kittakoop, P.; Menasveta, P. J. Nat. Prod., 2000, 63, 984 -986.

(28) Burkholder, P. R. M.; Leitz, F. H.; Appl. Microbiol. 1966, 14, 649.

(29) Wang, J.; Soisson, S. M.; Young, K.; Shoop, W.; Kodali, S.; Galgoci, A.; Painter, R.; Parthasarathy, G.; Tang, Y. S.; Cummings, R.; Ha, S.; Dorso, K.; Motyl, M.; Jayasuriya, H.; Ondeyka, J.; Herath, K.; Zhang, C.; Hernandez, L.; Allocco, J.; Basilio, Á.; Tormo, J. R.; Genilloud, O.; Vicente, F.; Pelaez, F.; Colwell, L.; Lee, S. H.; Michael, B.; Felcetto, T.; Gill, C.; Silver, L. L.; Hermes, J. D.; Bartizal, K.; Barrett, J.; Schmatz, D.; Becker, J. W.; Cully, D.; Singh S. B. Nature 2006, 441, 358-361.

(30) Abdelfattah M. S. New Secondary Metabolites from Bacteria: Seitomycin with high Anti-Helicobacter pylori Activity, Exfoliazone B, new Steffimycinones, Espicufolin B, Flavomarine A and B, and BS-46 with a Novel Carbon Skeleton, PhD Thesis, Universität Göttingen 2005.

(31) Fostel J. M.; Lartey, P. A. Drug Discovery Today, 2000, 5, 25-32.

(32) Kidwai, M.; Venkataramanan, R.; Rastogi S.; Sapra, P. Curr. Med. Chem. Anti-infect. Agents, 2003, 2, 27-71.

(33) Girmenia C.; Martino, P. Curr. Opin. Oncol., 2003, 15, 283-288.

(34) Wiederhold N. P.; Lewis, R. E. Expert Opin. Invest. Drugs, 2003, 12, 13131333.

(35) Denning, D. W. Lancet, 2003, 362, 1142-1151.

(36) Kartsonis, N. A.; Nielsen J.; Douglas, C. M. Drug Resist. Update, 2003, 6, 197-218.

(37) Jarvis, B.; Friggitt D. P.; Scott, L. J. Drugs, 2004, 64, 969-982. 
(38) Chan, P. F.; Holmes, D. J.; Payne, D. J. Drug Discovery Today, 2004, 1, 519527.

(39) Bogdanovich, T.; Ednie, L. M.; Shapiro, S.; Appelbaum P. C. Antimicrob Agents Chemother. 2005, 49, 4210-4219.

(40) Fu, Y.; Hsieh, T. C.; Guo, J.; Lee M. Y.; Darzynkiewicz, Z.; Wu, J. M. Biochem. Biophys Res Commun. 2004, 322, 263-70.

(41) Tweit, R. C.; Pandey, R. C.; Rinehart Jr. K. L. J. Antibiot. 1982, 35, $997-$ 1012.

(42) Clercq, E. D. Mini Rev. Med. Chem., 2002, 2, 163-175.

(43) Xu, Z.; Buckheit R. W.; Hollingshead, M. G.; Flavin, M. T. Antimicrob Agents Chemother. 1999, 39, 315.

(44) Flavin, M.T.; Rizzio, Z. D.; Khilevich, A.; Kucherenko, A.; Sheinkman, A. K.; Vilaychank, V.; Lin, L.; Wei, M. J. Med. Chem. 1996, 39, 1303-13

(45) Xu, Z. Q.; Norris, K. N.; Weinberg, D. S.; Kardatzke, J.; Wertz, P.; Frank, P.; Flavin, M. T. J. Chromatography, 2000, 742, 267-175.

(46) Rinehart K. L., Kobayashi J, Harbour G. C., Hughes R. G Jr., Mizsak S. A., Schill T. A. J. Am. Chem. Soc. 1984, 106, 1524-6.

(47) Vanmiddlesworth. F.; Cannell, R. J. P. Methods in Biotechnology: Natural products isolation, eds. R. J. P. Cannell, Humana Press, Totowa, New Jersey, 1998.

(48) Bukingham J.; Thompson, S. Dictionary of natural products and other information sources for natural products scientists, Royal Society of Chemistry, London, 1997, 53-67.

(49) Laatsch, H. AntiBase, A Data Base for Rapid Structural Determination of Microbial Natural Products, 2008 and annual updates, Wiley VCH, Weinheim,Germany.

(50) Dictionary of Natural Products on CD-ROM, Chapman \& Hall, Chemical Database, 2008.

(51) Bringmann, G.; Lang, G. Sponges (porifers), Eds. Mueller, E. G. Werner, Springer-Verlag, Berlin, 2003, 89-116.

(52) Bringmann, G.; Messer, K.; Saeb, W.;. Peters E. M.; Peters, K. Phytochemistry 2001, 56, 387-391.

(53) Oka, I.; Frauendorf, F.; Laatsch, H. poster, 37. Diskussionstagung der Deutschen Gesellschaft für Massenspektrometrie, Leipzig 2004.

(54) Pfefferle C., Breinholt J., Gurtler H. and Fiedler H. P. J. Antibiot. 1997, 50, 1067-68. 
(55) Chu, T. M.; Mallette, M. F.; Mumma, R. O. Biochemistry 1968, 7, 1399-406.

(56) Pilli, R. A.; Andrade, C. K. Z.d.; Souto, C. R. O.; Meijere, A. d. J. Org. Chem. 1998, 63, 7811 -7819.

(57) Omura, S.; Takeshima, H.; Nakagawa, A.; Miyazawa, J., J. Antibiot. 1976, $29,316$.

(58) Brockmann, H., Jr.; Legrand, M. Tetrahedron 1963, 19, 395-400.

(59) Richle, W.; Winkler, E. K.; Hawley, D. M.; Dobler, M.; Keller-Schierlein, W. Helvetica Chimica Acta 1972, 55, 467-80.

(60) Geckeler, K.; Mutter, M. Zeitschrift fuer Naturforschung 1979, 34, 1024-5.

(61) Nakagawa, M.; Furihata, K.; Furihata, K.; Adachi, K.; Seto, H.; Otake, N. J. Antibiot. 1986, 39, 1178-9.

(62) Haneda M.; Nawata Y.; Hayashi T.; Ando K,; J. Antibiot. 1974, 7, 555-557.

(63) Stanley N. Graven, Lardy, H. A.; Rutter. A. Biochemistry 1966, 5, 1735 1742.

(64) Schumacher, R. W.; Talmage, S. C.; Miller, S. A.; Sarris, K. E.; Davidson, B. S.; Goldberg, A. J. Nat. Prod. 2003, 66, 1291-1293.

(65) Kim, W. H.; Jung, J. H.; Lee T. S.; Sang M. L.; Lee, E. Org. Lett. 2005, 6, 1085-1087.

(66) Kim, W. H.; Jung, J. H.; Lee. E.; J. Org. Chem. 2005, 70, 8190-8192.

(67) Mukku, V. J. R. V.; Speitling, M.; Laatsch, H.; Helmke, E. J. Nat. Prod. 2000, 63, 1570-1572.

(68) Cho, K.W.; Lee, H. S.; Rho, J. R.; Kim, T. S. Mo, S. J. J. Nat. Prod. 2001, 64, 664-667.

(69) Nadig, H.; Sequin, U. Helv. Chim. Acta 1987, 70, 1217-1228.

(70) Schumacher, R. W.; Davidson, B. S.; Montenegro D. A.; Bernan, V. S. J. Nat. Prod. 1995, 58, 613-617.

(71) Abe, N.; Enoki, N.; Nakakita, Y.; Uchida, H.; Nakamura T.; Munekata, M. J. Antibiot. 1993, 46, 1536-1549.

(72) Naoki, A.; Yasukazu, N.; Takehiko, N.; Nobuyasu, E.; Hideki, U.; Masanobu, M. J. Antibiot. 1993, 46, 1530-5.

(73) Ziegler, T. Science of Synthesis 2005, 21, 43-75.

(74) Masato, I.; Sakaguchi, A.; Kobayashi, C.; Ikariya, T. J. Am. Chem. Soc. 2007, 129, 290-229.

(75) Gelain, A.; Bettinelli, I.; Barlocco, D.; Kwon, B.; Jeong, T.; Cho, K. H.; Toma, L. J. Med. Chem. 2005, 48, 7708-7713. 
(76) Fernandez, S.; Menendez, E.; Gotor, V. Synthesis 1991, 9, 713-716

(77) Matsumoto, K.; Hashimoto, S.; Otami, S. Angew. Chem. 1986, 98, 569.

(78) Ikeda, Y.; H. Naganawa, Kondo, S.; Takeuchi, T. J. Antibiot. 1992, 45.

(79) Tang, Y.; Sattler, I.; Thiericke R.; Grabley, S. J. Antibiot. 2000, 53, 934-943.

(80) Huang, S.T.; I-Jen, H.; Chen, C. Bioorg. Med. Chem. 2006, 14, 6106-6119.

(81) Wang, B. B.; Maghami, N.; Goodlin, L.; Vanessa; Smith, P. J. Bioorg. Med. Chem. Lett. 2004, 14, 3221-3226.

(82) Bialer, M.; Yagen, B.; Papo, N. Israeli 2002, 10 pp. CODEN: ISXXAQ IL 130666 A 20020912.

(83) Fusaka, T.; Yamato, S.; Kajiwara, T.; Kamiyama, H.; Itoh, S.; Tanaka, Y. J Pesticide Sci. 2004, 29, 339-347.

(84) Nomura, E.; Kashiwada, A.; Hosoda, A.; Nakamura, K.; Morishita, H.; Tsuno, T.; Taniguchi H. Biorg. Med. Chem. 2003, 17, 3807-3813.

(85) Bartroli, J.; Turmo, E.; Alguero, M.; Boncompte, E.; Vericat, M. L.; Conte, L.; Ramis, J.; Merlos, M.; Garcia-Rafanell, J.; Forn, J. J. Med. Chem. 1998, $41,1855-1868$.

(86) Huang, H.; She, Z.; Lin, Y.; Vrijmoed, L. L. P.; Lin W. J. Nat. Prod. 2007, 70,1696-1699

(87) Folkes, A.; Roe, M. B.; Sohal, S.; Golec, J.; Faint, R.; Brooks, T.; Charlton, P. Bioorg. Med. Chem. Lett. 2001, 11, 2589.

(88) Wang, S.; Golec, J.; Miller, W.; Milutinovic, S.; Folkes, A.; Williams, S.; Brooks, T.; Hardman, K.; Charlton, P.; Wren, S.; Spencer, J. Bioorg. Med. Chem. Lett. 2002, 12, 2367.

(89) Martins, M.B.; Carvalho, I. Tetrahedron, 2007, 63, 9923-9932.

(90) Kilian, G.; Jamie, H.; Brauns, S. C. A.; Dyason, K.; Milne, P. J. Pharmazie 2005, 60, 305.

(91) Pearce C.M.; Sanders K.M. J. Chem. Soc. 1990, 409-412

(92) Al-Refai, M., New and Bioactive Secondary Metabolites from Marine and Terrestrial Bacteria: Ramthacin A, B, C, and Polyene Macrolides from Genetically Modified Bacteria. PhD Thesis, Universität Göttingen 2009.

(93) Omura, S.; Nakagawa, A.; Hashimoto, H.; Oiwa, R.; Iwai, Y.; Hirano, A.; Shibukawa, N.; Kojima, Y. J Antibiot. 1980, 33, 1395-1396.

(94) Kim, W. G.; Kim, J. P.; Kim, C. J.; Lee, K. H.; Yoo, I. D. J. Antibiot. 1996, 49, 20. 
(95) Kim, W. G.; Kim, J. P.; Koshino, H.; Shin-Ya, K.; Seto, H.; Yoo, I. D. Tetrahedron 1997, 53, 4309.

(96) Nakagawa, A.; Iwai, Y.; Hashimoto, H.; Miyazaki, N.; Oiwa, R.; Takahashi, Y.; Hirano, A.; Shibukawa, N.; Kojima, Y.; mura, S. J. Antiobiot. 1981, 34, 1408

(97) Mura, S.; Nakagawa, A. Tetrahedron Lett. 1981, 22, 2199.

(98) Hill, M. L.; Raphael, R. A. Tetrahedron 1990, 46, 4587.

(99) Todorova M. N. and Tsankova E. T., Phytochemistry 1999, 52, 1515.

(100) Wu S. J.; Fotso S.; Fuchao L.; Qin S.; Laatsch H. J. Nat. Prod. 2007, 70, 304-306

(101) Zhao Y.; Yue J., He Y.; Lin Z.; Sun H. Phytochemistry 1997, 44, 459.

(102) Babidge P. J.; Westropp, M; Ralph, A. Aust. J. Chem. 1984, 37, 629-33

(103) Gonzales A.G.; Barrera J. B.; Yanes A. C.; Diaz J.G. ; Perez E. M. R. Phytochemistry 1989, 28, 2520-2522

(104) Munoz-Martinez F.; Mendoza, C. R.; Bazzocchi, I. L.; Castanys S.; Jimenez I. A.; Gamarro F. J Med. Chem. 2005, 48, 4266-4275.

(105) Wu, Q.; Shi, Y.; Jia Z.; Nat. Prod. Rep. 2006, 23, 699-734.

(106) Rahman, H;, Shaaban, M.; Shaaban, K. A.; Saleem, M.; Helmke, E.; GrünWollny, I.; Laatsch, H. Nat. Prod. Comm. 2008 submitted.

(107) Rosecke, J; König, W.A. Flavour Fragrance J. 2000, 15, 315-319.

(108) Brockmann, H.; Lenk, W.; Schwantje, G.; Zeeck, A. Chem. Ber. 1969, 102,126-151.

(109) Puder, C.; Loya, S.; Hizi, A.; Zeeck, A.Eur. J. Org. Chem. 2000, 729-735.

(110) Itoh, T.; Kinoshita, M.; Aoki, S.; Kobayashi, M. J. Nat. Prod. 2003, 13731377.

(111) Laatsch, H.; Fotso, S. Naturally Occurring Anthracyclines, Topics Curr. Chem. 2008, 282 (Ed. K. Krohn), Springer, Berlin, Heidelberg, pp. 3-74.

(112) Oki, T.; Matsuzawa, Y.; Kiyoshima, K.; Yoshimoto, A.; Naganawa, H.; Takeuchi, T.; Umezawa, H. J. Antibiot. 1981, 34, 783-790.

(113) Mühlenbeck, U.; Kortenbusch, A.; Barz, W.; Phytochemistry 1996, 42, 1573 1579 .

(114) Chabot, S.; Bel-Rhlid, R.; Chenevert R.; Piche, Y. New Phytologist, 1992, 122, 461-467.

(115) Jisba, J. V.; Sedmera P.; Vanek, Z. O.; Drautz, H.; Zähner, H. J. Antibiot. 1985, 1, 111-113. 
(116) Meyer, F.; Grasedyck, K.; Peters K.; Lindner J. Arzeneimittelforsch. 1980, $30,635-40$.

(117) Chidlow, J. W.; Stephen J.; Smith, H. Nature 1968, 218, 773 - 774.

(118) Drautz, H.; Keller-Schierlein W.; Zahner, H. Arch. Microbiol. 1975, 106, 175.

(119) Dobler M.; Schierlein W. K. Helv. Chim. 1977, 60, 178-185.

(120) Kjaer, D.; Kjaer, A.; Pederson, C.; Bu'Lock J. D.; Smith, J. R. J. Chem. Soc. 1971, 2788-2791.

(121) Balan, J.; Fuska, J.; Kuhr, I.; Kuhrova, V. Folia Microbiol. 1970, 15, 479484.

(122) McInnes, A. G.; Walter, J. A.; Smith, D. G. J. Antbiot. 1976, 10, 1050-1057.

(123) Willis, J.C., 1973. “The Dictionary of Flowering Plants and Ferns”. (revised by Shaw, H.K.A), $8^{\text {th }}$ edition, Cambridge University Press, Cambridge, pp 1135 .

(124) Allen, O.N., Allen, E.K., 1981. The Leguminoseae. The University of Wisconsin Press, Wisconsin, pp. 645-649.

(125) Rao, E. V.; Prasad Y. R. Phytochemistry 1993, 32, 83-185.

(126) Lyer P. R; Iyer C. S. R. J. Nat. Prod. 1989, 52, 711-15.

(127) Pennaka, H.K.; Mopuru, V. B. R.; Duvvuru, G.; Madugala M. M.; Cristelle, C.; Bernard B. Chem. Pharm. Bull. 2003, 51, 194-196.

(128) Narkhede, D.D.; Iyer, P.R.; Rukmaniiyer, C.S. J. Nat. Prod.1989, 52, 502505.

(129) Jian, H.; Chen, L.; Heber, D.; Wenyuan, S.; LQing; Y. J. Nat. Prod. 2006, 69, 121-124.

(130) Yenesew, A.; Midiwo, O.J.; Waterman, P.G. Phytochemistry 1998, 47, 295300.

(131) Labbiento, L .; Menichini, F.; Monache, F. D. Phytochemistry 1986, 25, 1505-1506.

(132) Kikuchi, H.; Tensho, A.; Shimizu, I.; Shokawa, H.; Kuno, A.; Yamada, S.; Fuji wara, T.; Tomita Ken-chi. Chem. Lett. 1983, 603-606.

(133) Lopes, D.; Villela, C. T.; Kaplan M. A. C.; Carauta, J. P.P. Phytochemistry 1993, 34, 279-280

(134) Misra, T. N.; Singh, R. S.; Upadhyay, J. Phytochemistry 1983, 22, $2557-$ 2558. 
(135) Mizuno, M.; Tanaka, T.; Kohichi, T.; Nobuyasu, M.; Munekazu, I.; Chamlong, P. Phytochemistry 1990, 29, 2663-5.

(136) Patricia, M.; Lourenc, A. Phytochemistry 1998, 48, 359-362.

(137) M. Mizuo; T. Toshiyuki, T. Kohichi, M. Nobuyasu, I. Munekazu, P. Chamlong; Phytochemistry 1990, 29, 2663-5.

(138) Pulla, R. P.; Srimannarayana, G. Phytochemistry 1980, 19, 1272-3.

(139) Prashant, A.; Krupadanam, G. L. David; Phytochemistry 1993, 32, 484-6.

(140) Krupadanam, G. L D.; Sarma, P. N.; Srimannarayana, G.; Subba Roa, N. V. Ind. J. Chem. 1978, 16, 770-2.

(141) Krupadanam, G. L D.; Sarma, P. N.; Srimannarayana, G.; Subba Roa, N. V. Tetrahedron Lett. 1977, 24, 2125-8.

(142) Yun-Lian, K. L.; Yueh-Hsiung Heterocycles, 1995, 41, 1959-65.

(143) Petkov, E ; Uzunov P.; Kostova, I.; Somleva, T.; Ognyanov, I. Planta Medica 1983, 47, 237-9.

(144) Takashima, J.; Chiba, N.; Yoneda, K.; Ohsaki, A. J. Nat. Prod. 2002, 65, 611-613.

(145) Waterman, P. G.; Mahmoud E. N. Phytochemistry 1985, 24, 571-574.

(146) Manchanda, V. P.; Batta, A. K., Khanna, P. L., Khanna, R. N. Current Science 1976, 45, 322-4.

(147) Dagne, E.; Mammo, W.; Sterner, O. Phytochemistry 1992, 31, 3662-3663.

(148) Indrani, J.; Ghanim, A.; Khan, H. A. Phytochemistry 1980, 19, 1267-1268.

(149) Roy, M.; Mitra, S. R.; Bhattacharyya, A.; Adityachaudhury, N. Phytochemistry 1986, 25, 961-2.

(150) Gupta, R. K.; Krishnamurti, M. Phytochemistry 1977, 16, 293.

(151) Subrahmanyam, K.; Madhusudhana, J.; Jagannadha R. K. V. Ind. J. Chem. 1977, 15, 12-15.

(152) Borges-Argaez, R.; Balnbury, L.; Flowers, A.; Gimenez-Turba, A.; Ruiz, G.; Waterman, P. G.; Pena-Rodriguez, L. M. Phytomedicine 2007, 14, 530-533.

(153) Fairfax, D. J.; Yang, Z. U.S. Pat. Appl. Publ. 2006, US 2006183769 A1 20060817.

(154) Ueki, M.; Ueno, K.; Miyadoh, S.; Abe, K.; Shibata, K.; Taniguchi M.; Oi S. J. Antibiot. 1993, 46, 1089.

(155) Wang J. C. J. Mol. Biol. 1971, 55, 523-533.

(156) Jizba’ J.; Přikrylová, V.; Ujhelyiová, L.; Varkonda, S. Folia Microbiologica 1994, 39, 119-28. 
(157) Briggs, R. W; Hinton, J.F. J Magnetic Resonance 1979, 33, 363-77.

(158) Tanouchi, Y.; Shichi, H. Japanese J. Ophthalmology 1987, 31, 218-29.

(159) Umland, S. P.; Shah, H.; Jakway, J. P.; Shortall, J.; Razac, S.; Garlisi, C. G.; Falcone, A.; Kung, T. T.; Stelts, D.; Hegde, V.; Patel, M.; Billah, M. M.; Egan, R. W. Am. J. Resp. Cell Mol. Biol. 1999, 20, 481-492.

(160) Kobayashi, S.; Kamoshita, K. Jpn. Kokai Tokkyo Koho 2005, 15. JP $2003-$ 319126.

(161) Frank, H. J.; Battell, M. L.; Zombor, G.; Fuska, J.; Nemec, P. Biochem. Pharmacol. 1977, 26, 1973-1977.

(162) Kovac, L.; Bohmerova, E.; Fuska, J. J. Antibiot. 1978, 31, 616-20. 


\section{Acknowledgements}

I owe a huge debt of gratitude to my PhD supervisor, Professor Dr. Hartmut Laatsch who supported me in every aspect throughout the whole $\mathrm{PhD}$ period. He offered encouragement, sound advice, excellent teaching and a lot of good ideas which made the chemistry and my research interesting to me.

I am grateful to Professor Dr. A. Zeeck for accepting to read this work and for being my co-referent.

I am indebted to all my lab colleagues for providing a stimulating and fun environment in which to learn and grow. In particular, I would like to thank Dr. Petrea Facey, Mahmoud Al-Refai a nd Hnin Yu Win for their invaluable support. I would like to thank Mrs. F. Lissy, Mrs. Petra Lappe and Mr. Andreas Kohl for the technical support and administrative help.

I am grateful to Dr. F. Frauendorf and Mrs. G. Udavarnoki for the mass spectrometry and Dipl. Chem. R. Machinek, Mr, M. Weitemeyer, Mrs. U. Leonhardt, Mrs. C. Zolke, Mrs. C. Silbert in the NMR department for their indispensable contribution to this research.

I wish to thank my entire family whose love and support still sustain me today. A heartfelt acknowledgement goes to my wife Dr. Kulsum Ara. Lastly and most importantly, I wish to thank my parents Mr. Md. Fazlur Rahman and Mrs. Fazilatunnessa. They raised me, supported me, taught me and loved me. To them I dedicated this thesis. 


\section{Curriculum Vitae}

I was born in $2^{\text {nd }}$ December, 1974 in Munshipara, Satkhira, Bangladesh as the second son of my parents Mr. Md. Fazlur Rahman and Mrs. Fazilatunessa. From 1980 to 1984 I attended the Sultanpur primary school, Satkhira. From 1985 to 1989, I attended the Satkhira Government High School where I completed my secondary school certificate. From 1989 to 1991, I was in Satkhira Government College where I completed my higher secondary certificate. From 1993 to 1997 I completed my BSc (Honours) in Applied Chemistry and Chemical Technology from University of Dhaka, Bangladesh. In 1999 I received my MSc (research) from the same department as my BSc. The thesis title of my MSc was "Preparation of solar selective surface". From 1999 to 2003, I worked as an environmental analytical chemist in International Centre for Diarrhoeal Disease Research, Bangladesh (ICDDR,B). From April 2003 to October 2008, I completed my MSc equivalence in the Faculty of Chemistry, University of Goettingen, Germany. From $1^{\text {st }}$ October to $31^{\text {st }}$ October 2007, I was in the National Centre for Biotechnology, Madrid, Spain for a research project with DAAD scholarship. In $7^{\text {th }}$ December, 2007 I married the gynaecologist Dr. Kulsum Ara. From 2004 to October 2008, I worked as a PhD student in the Institute of Organic and Biomolecular Chemisty, University of Goettingen, Germany under the direct supervision of Professor Dr. Hartmut Laatsch. 